Universidad deValladolid

PROGRAMA DE DOCTORADO EN INGENIERÍA

TERMODINÁMICA DE FLUIDOS

TESIS DOCTORAL:

\title{
CARACTERIZACIÓN TERMODINÁMICA DE MEZCLAS LÍQUIDO + GAS PARA EL DESARROLLO DE TECNOLOGÍAS DE CAPTURA DE $\mathrm{CO}_{2}$
}

Presentada por Jorge Rubio Gallego para optar al grado de Doctor/a por la Universidad de Valladolid

Dirigida por:

Dr. José Juan Segovia Puras

Dr. Alejandro Moreau Ortega

Valladolid, Octubre 2019 



\section{RESUMEN}

La combustión de recursos fósiles es cada vez mayor, como consecuencia la concentración de dióxido de carbono en la atmosfera va aumentando, recientemente se han sobrepasado las $400 \mathrm{ppm}$. Esta tendencia es la causa principal de un proceso continuo de cambio climático, que ha provocado un aumento de la temperatura media de la superficie del planeta de aproximadamente $1^{\circ} \mathrm{C}$ desde la época preindustrial. La comunidad científica ha propuesto no superar $\operatorname{los} 2^{\circ} \mathrm{C}$ de incremento medio en la temperatura global.

En la presente investigación nos centramos en los procesos de post-combustión, para los cuales existen diferentes métodos para la separación del $\mathrm{CO}_{2}$ en una corriente de gas. Hoy en día, una de las tecnologías más aplicadas, es la captura del $\mathrm{CO}_{2}$ mediante la utilización de técnicas de separación físicas o químicas. La tecnología de captura más desarrollada es mediante la absorción química con aminas. La principal reacción en la absorción con aminas depende del tipo de amina. Cuando son aminas primarias o secundarias se produce la formación de un carbamato.

Un mayor conocimiento de las propiedades termofísicas que intervienen en los procesos de captura de $\mathrm{CO}_{2}$ resulta necesario. Por ello, es importante conocer los parámetros de operación para determinar la viabilidad técnica y económica de los procesos de captura, como por ejemplo: elección de una amina adecuada, la mezcla óptima de esta y sus propiedades como la densidad, necesaria para el cálculo de la solubilidad.

En este trabajo se presentan datos de propiedades termodinámicas tanto de disoluciones de aminas utilizadas en procesos de captura de $\mathrm{CO}_{2}$, como de mezclas de hidrocarburo + $\mathrm{CO}_{2}$. Además se aportan otros datos de interés como la solubilidad de $\mathrm{CO}_{2}$ en disoluciones acuosas de aminas como son: la Monoetanolamina (MEA), Dimetiletanolamina (DMAE) y la Trietanolamina (TEA). Además, se aportan datos de equilibrios liquido-vapor de compuestos involucrados en la manufactura del Nylon.

Las medidas de equilibrios se llevaron a cabo utilizando una celda de equilibrio isoterma desarrollada en el laboratorio TERMOCAL de la Universidad de Valladolid, basado en el diseño de Gibbs y Van Ness. Los datos se obtuvieron dentro del intervalo de temperaturas entre $313.15 \mathrm{~K}$ y $393.15 \mathrm{~K}$. Los valores de equilibrios líquido-vapor fueron ajustados a la ecuación de Margules y a diferentes modelos (Wilson, NRTL y UNIQUAC).

Las medidas de densidad se han realizado mediante un densímetro de tubo vibrante Anton Paar DMA HPM en un rango de 0.54 a $0.90 \mathrm{~g} \cdot \mathrm{cm}^{-3}$ para mezclas $\mathrm{CO}_{2}+$ hidrocarburo y de 1.02 a $1.16 \mathrm{~g} \cdot \mathrm{cm}^{-3}$ para disoluciones acuosas de aminas $+\mathrm{CO}_{2}$. La resolución del equipo es de $1 \cdot 10^{-5} \mathrm{~g} \cdot \mathrm{cm}^{-3}$, con una incertidumbre expandida de $7 \cdot 10^{-4} \mathrm{~g} \cdot \mathrm{cm}^{-3}$ en el intervalo de temperaturas entre $273.15 \mathrm{~K}$ y $373.15 \mathrm{~K}$ y en el rango de presiones de 0.1 hasta $100 \mathrm{MPa}$.

Las medidas de viscosidad de las mezclas de hidrocarburo $+\mathrm{CO}_{2}$ se efectuaron mediante un viscosímetro de hilo vibrante en un rango de viscosidades de 0.10 a $0.53 \mathrm{mPa} \cdot \mathrm{s}$. La incertidumbre expandida fue de $0.0032 \mathrm{mPa} \cdot \mathrm{s}$ en el intervalo de temperaturas entre $293.15 \mathrm{~K}$ y $353.15 \mathrm{~K}$ y en el rango de presiones de 0.1 hasta $100 \mathrm{MPa}$.

En el caso de la densidad y viscosidad, los diferentes sistemas se han correlacionado con la ecuación de Tammann-Tait y VFT que permiten representar el comportamiento de dichas 
propiedades en función de la temperatura y la presión. Los datos de solubilidad de las disoluciones acuosas de aminas $+\mathrm{CO}_{2}$ se ajustaron a una ecuación de $\mathrm{Xu}$ y Rochelle, que representa la presión parcial del $\mathrm{CO}_{2}$ en función de la temperatura y la composición del $\mathrm{CO}_{2}$. 


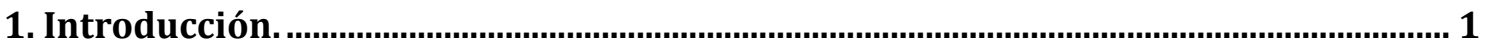

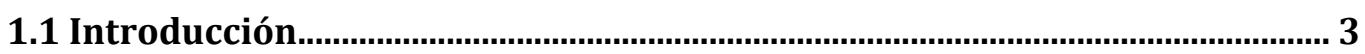

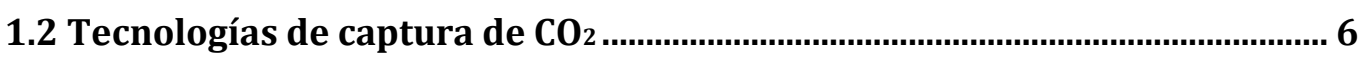

1.3 Tecnologías de post-combustión, absorción de $\mathrm{CO}_{2}$ con aminas...................10

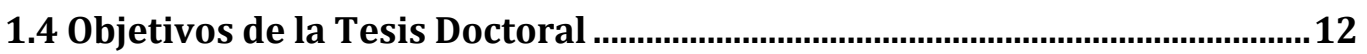

1.5 Estructura de la Tesis Doctoral ...................................................................... 14

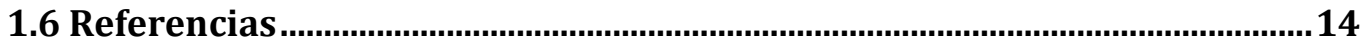

2. Determinación experimental del equilibrio líquido-vapor a diferentes

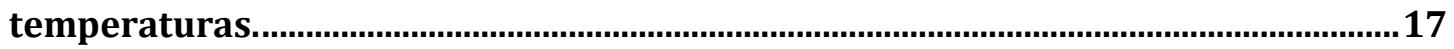

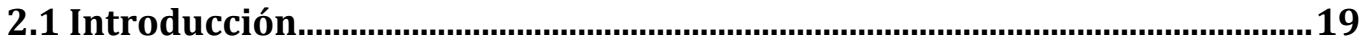

2.2 Descripción del método de medida utilizado para la determinación del

equilibrio líquido-vapor ....................................................................................... 19

2.3 Desgasificación de los compuestos puros .......................................................26

2.4 Reducción termodinámica de los datos de equilibrio líquido-vapor..........27

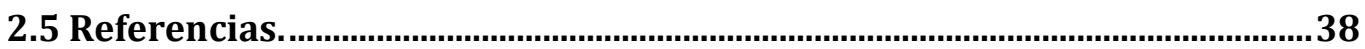

3. Resultados experimentales del equilibrio líquido-vapor isotermo...........................41

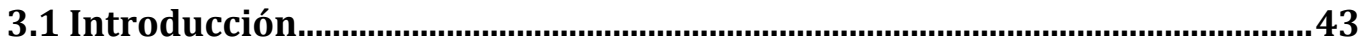

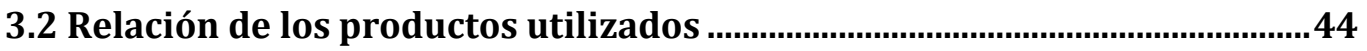


3.3 Presentación de los resultados experimentales del equilibrio líquido-

vapor

3.4 Discusión de los resultados

3.5 Referencias

4. Determinación de medidas de las densidades y solubilidades de $\mathrm{CO}_{2}$ en soluciones acuosas de aminas 79

4.1 Medidas de Solubilidad. Introducción .81

4.2 Descripción del método de medida utilizado para la determinación de la solubilidad del $\mathrm{CO}_{2}$ en mezclas acuosas de amina. .81

4.3 Procedimiento experimental para la medida de la solubilidad .82

4.4 Cálculo de la concentración en la mezcla. 84

4.5 Medidas de densidad. Introducción. 85

4.6 Principio de funcionamiento del densímetro de tubo vibrante. .86

4.7 Descripción del método de medida utilizado para la determinación de la

densidad.

4.8 Procedimiento experimental para medida de la densidad .90

4.9 Calibración del densímetro .93

4.10 Incertidumbre en la medida. .85

4.11 Validación del equipo. .96

4.12 Referencias .96

5. Resultado de medidas experimentales de las densidades y solubilidades de $\mathrm{CO}_{2}$ en soluciones acuosas de aminas. 99 
5.2 Ajuste de los datos de densidad (ecuación de Tammann-Tait modificada)..

5.3 Ajuste de los datos de solubilidad.

5.4 Relación de los productos utilizados.

5.5 Presentación de los resultados experimentales de las medidas de

densidad 105

5.6 Discusión de resultados de la densidad experimental. 129

5.7 Presentación de los resultados experimentales de las medidas de solubilidad 130

5.8 Discusión de resultados de la solubilidad experimental 138

5.9 Referencias 139

6. Determinación de medidas de densidades y viscosidades en mezclas de $\mathrm{CO}_{2}+$

hidrocarburo.

6.1 Introducción 143

6.2 Técnicas experimentales para la medida de la viscosidad 144

6.3 Principio de funcionamiento del Viscosímetro de Hilo Vibrante 145

6.4 Ecuaciones de trabajo 146

6.5 Descripción del equipo 148

6.6 Procedimiento de medida de la viscosidad de mezclas complejas 154

6.7 Descripción del método de medida utilizado para la determinación de la densidad de mezclas $\mathrm{CO}_{2}+$ hidrocarburo 161 
7. Resultados experimentales de las medidas de densidades y viscosidades en

mezclas de $\mathrm{CO}_{2}+$ hidrocarburo.

7.1 Introducción.

7.2 Ajuste de los datos de densidad (ecuación de Tammann-Tait

modificada).

7.3 Ajuste de los datos de viscosidad (modelo VFT) 168

7.4 Relación de los productos utilizados 169

7.5 Presentación de los resultados experimentales de las medidas de densidad 169

7.6 Discusión de resultados de la densidad experimental. 185

7.7 Presentación de los resultados experimentales de las medidas de

viscosidad. 185

7.8 Discusión de resultados de la viscosidad experimental 194

7.9 Referencias 195

8. Conclusiones. 


\section{Notación}

A Parámetro ajustable de la ecuación de Margules. Constante de calibración del densímetro. Parámetro ajustable, ecuación Tammann-Tait. Parámetro ajustable, ecuación Xu y Rochelle. Parámetro ajustable, modelo VFT.

AAD\% Desviación media obtenida en porcentaje.

a

Energía de interacción entre pares de moléculas. Parámetro de ajuste.

B

Segundo coeficiente del virial. Constante de calibración del densímetro. Parámetro ajustable, ecuación Tammann-Tait. Parámetro ajustable, modelo VFT.

$\mathrm{b}$

Parámetro de ajuste.

C

Parámetro ajustable, ecuación Tammann-Tait. Parámetro ajustable, modelo VFT.

c

Parámetro de ajuste.

$\mathrm{D}$

Índice de fragilidad (o parámetro de Angell).

d

Parámetro de ajuste.

E

Parámetro ajustable, modelo VFT.

$\mathrm{e}$

Parámetro de ajuste.

F

Parámetro ajustable, modelo VFT.

$\mathrm{F}_{0}$

Amplitud de la fuerza impulsora.

$\mathrm{f}$

Fugacidad. Parámetro de ajuste. Frecuencia del movimiento del hilo.

$f_{b}$

Ancho de banda de la señal.

$\mathrm{f}_{0}$

Frecuencia de resonancia en el vacío.

G

Energía de Gibbs molar.

g

Función adimensional de la energía de Gibbs molar de exceso, GE/RT. Energía de interacción entre pares de moléculas, modelo NRTL.

H Entalpía molar.

i Componente i puro. Parte imaginaria de la ecuación.

j Componente j puro.

k Módulo de elasticidad. Coeficiente de amortiguación.

L $\quad$ Longitud. Longitud del hilo. 
Número de parámetros de la ecuación de ajuste. Masa.

$\dot{\mathrm{m}}$

$\mathrm{m}_{0}$

MD\%

$\mathrm{N}$

$\mathrm{n}$

$\dot{\mathrm{n}}$

$\mathrm{P}_{\mathrm{C}}$

PI

$\mathrm{PM}$

$\Delta \mathrm{P}$

$\mathrm{p}$

Q

q

$\mathrm{R}$

r

SP

$\mathrm{T}$

$\mathrm{T}_{\mathrm{C}}$

$\mathrm{T}_{0}$

$\mathrm{t}$

$\mathrm{u}$

$\mathrm{w}$

$\% \mathrm{w}$
Flujo másico.

Masa del tubo vibrante en vacío.

Máxima desviación en porcentaje.

Número de componentes. Número de datos experimentales.

Número de moles. Número de puntos experimentales.

Flujo molar.

Presión crítica.

Medidor-indicador de presión.

Peso molecular.

Diferencial de presión.

Presión.

Parámetro del grupo funcional, modelo UNIQUAC.

Parámetro del modelo UNIQUAC, área de la superficie molecular.

Constante universal de los gases. Parámetro del grupo funcional, modelo UNIQUAC. Radio de una sección circular. Resistencia.

Parámetro del modelo UNIQUAC, volumen molecular de van der Waals.

Punto de consigna.

Temperatura absoluta.

Temperatura crítica.

Temperatura de Vogel.

Tiempo.

Energía de interacción entre pares de moléculas, modelo UNIQUAC. Incertidumbre de la variable observada.

Volumen. Volumen molar. Volumen de la celda. Tensión.

Número de grupos, modelo UNIQUAC. Volumen molar.

Volumen molar a presión atmosférica.

Factor acéntrico.

Tanto por ciento en peso. 
Fracción molar de la fase líquida. Fracción molar local, modelo NRTL. Fracción molar. Desplazamiento.

Fracción molar de la fase vapor.

$\mathrm{Z}$

Número de coordinación. Factor de compresibilidad.

\section{Letras griegas}

$\alpha$

Parámetro de no distribución al azar, modelo NRTL. Índice de carga en moles de $\mathrm{CO}_{2} /$ mol de amina.

$\beta \quad$ Masa adicional del fluido.

$\beta^{\prime} \quad$ Amortiguación debido a la viscosidad del fluido.

$\gamma \quad$ Coeficiente de actividad.

$\Delta_{0} \quad$ Amortiguación natural del hilo en el vacío.

$\varepsilon \quad$ Valor de tolerancia. Coeficiente de expansión de la celda de medida.

$\zeta \quad$ Fracción local en Volumen. Modelo de Wilson.

$\eta \quad$ Parámetro de la ecuación de Margules. Viscosidad dinámica.

$\vartheta \quad$ Fracción superficial modelo UNIQUAC.

$\Lambda \quad$ Parámetro ajustable del modelo de Wilson. Amplitud.

$\lambda \quad$ Parámetro de la ecuación de Margules. Parámetro en la ecuación de Wilson.

$\mu \quad$ Potencial químico.

$\rho \quad$ Densidad.

$\rho_{\mathrm{s}} \quad$ Densidad del hilo.

$\rho_{\mathrm{w}} \quad$ Densidad del agua.

$\sigma$

Desviación estandar.

$\tau \quad$ Parámetro del modelo NRTL. Período de vibración.

v Viscosidad Cinemática

$\Phi \quad$ Coeficiente de fugacidad.

$\varphi$

Fracción volumétrica modelo UNIQUAC. Desfase.

$\Omega \quad$ Relación de la viscosidad en función de la frecuencia.

$\omega_{0} \quad$ Frecuencia natural del sistema. 


\section{Subíndices}

$\mathrm{AC}$

Corriente alterna.

amina aq. Amina acuosa.

añad Cantidad añadida.

cal Valor calculado.

evac Cantidad evacuada.

exp Valor experimental.

fase vapor Propiedad de la fase vapor.

i Componente i-ésimo en una mezcla.

j Componente j-ésimo en una mezcla.

k Componente k-ésimo en una mezcla.

L Fase líquida.

l Componente l-ésimo en una mezcla.

lit Datos obtenidos de la bibliografía.

m Propiedad del campo magnético.

med Valor medio.

mezcla Propiedad de la mezcla.

r Resonancia.

ref Valor de la propiedad de referencia.

total líquido Cantidad total de la fase líquida.

v, vacío Valor en el vacío.

w Valor para el agua. Propiedad del hilo.

\% $\quad$ Tanto por ciento en peso de la propiedad medida.

x Fracción molar de la propiedad medida.

$\alpha \quad$ Índice de carga en moles de $\mathrm{CO}_{2} / \mathrm{mol}$ de amina. 


\section{Superíndices}

C Parte combinatorial.

E Propiedad de exceso.

i Exponente i-ésimo de la ecuación.

l Fase líquida.

R Propiedad residual.

sat Propiedad de saturación.

total Valor total.

V, v Fase vapor.

\section{Abreviaturas}

AC Corriente alterna.

amina aq Amina acuosa.

BIMP Oficina Internacional de Pesas y Medidas.

BioEcoUva Instituto Universitario de Investigación en Bioeconomía.

CCS Carbon Capture and Storage (captura y almacenamiento de carbono).

DMAE Metildietanolamina.

EOR Enhanced Oil Recovery

exp Experimental.

FEM Fuerza electromotriz.

F. $0 . \quad$ Función objetivo.

FR Modo de oscilación forzada.

FSE Fondo Social Europeo.

GC Cromatografía de gases.

GUM Guía para la Expresión de la Incertidumbre de Medida.

GWP Global Warming Potencial.

HFCs Hidrofluorocarburos.

MEA Monoetanolamina. 
min Mínimo.

NETs Tecnologías de emisión negativa.

NRTL Non-Randon-Two-Liquid.

rms Desviación cuadrática media.

TEA Trietanolamina.

TR Modulo de oscilación transitorio.

UNIFAC UNIQUAC Functional-group Activity Coefficients.

UNIQUAC UNIversal QUAsi-Chemical.

VFT Vogel-Fulcher-Tammann. 
Capítulo 1

INTRODUCCIÓN 



\subsection{Introducción.}

Los gases que más contribuyen al efecto invernadero son, en orden decreciente de contribución: dióxido de carbono, metano, óxido de nitrógeno y HFCs (hidrofluorocarburos).

Los productos químicos que absorben radiación a la misma longitud de onda que el agua pueden ser gases de efecto invernadero muy potentes, pero su efecto sería pequeño porque el agua ya está presente en la atmosfera. Sin embargo, el $\mathrm{CO}_{2}$ y el metano absorben radiación a longitudes de onda diferentes al agua. Estos gases aumentan la cantidad de radiación absorbida por la atmosfera, y por lo tanto incrementan la capacidad de la atmosfera de producir un efecto invernadero [1].

El potencial de calentamiento global (por sus siglas en inglés GWP) se define como el calor absorbido por una sustancia determinada en relación con el gas de referencia, $\mathrm{CO}_{2}$ [2]. El GWP se calcula en un tiempo determinado, normalmente 20, 100 ó 500 años. El GWP del $\mathrm{CO}_{2}$ es igual a 1. En la tabla 1.1 se presentan los valores del GWP y la vida útil de diferentes gases que contribuyen al efecto invernadero, tomando el R-134a como un ejemplo de HFCs.

Otro factor importante es el tiempo de permanencia de los gases en la atmosfera. Un producto químico gaseoso puede tener un efecto invernadero muy fuerte, pero si el tiempo de permanencia de este compuesto es pequeño, su efecto será mucho menor en comparación con otros productos químicos que persisten durante años [1].

Tabla 1.1. Valores del GWP relativos al $\mathrm{CO}_{2}$ de los principales gases que contribuyen al efecto invernadero y su vida en la atmósfera [2].

\begin{tabular}{|c|c|c|c|}
\hline Gas & GWP $_{\mathbf{2 0}}$ & GWP $_{\mathbf{1 0 0}}$ & Vida útil (años) \\
\hline Dióxido de carbono & 1 & 1 & $100-1.000$ \\
\hline Metano & 84 & 28 & 12.4 \\
\hline $\mathrm{N}_{2} \mathrm{O}$ & 264 & 265 & 121.0 \\
\hline $\mathrm{R}-134 \mathrm{a}$ & 3710 & 1300 & 13.4 \\
\hline
\end{tabular}

Como consecuencia de la combustión de recursos fósiles, la concentración de dióxido de carbono en la atmósfera ha estado aumentando y, recientemente se ha sobrepasado los $400 \mathrm{ppm}$. Esta tendencia al alza es la principal causa de un proceso continuo de cambio climático que, hasta la fecha, ha provocado un aumento de la temperatura media de la superficie del planeta de aproximadamente $1{ }^{\circ} \mathrm{C}$ desde la época preindustrial [3]. El objetivo del Acuerdo de París es "mantener el aumento de la temperatura media mundial muy por debajo de los $2{ }^{\circ} \mathrm{C}$ sobre los niveles preindustriales y procurar limitar el aumento de la temperatura a $1.5^{\circ} \mathrm{C} "$ [4]. 


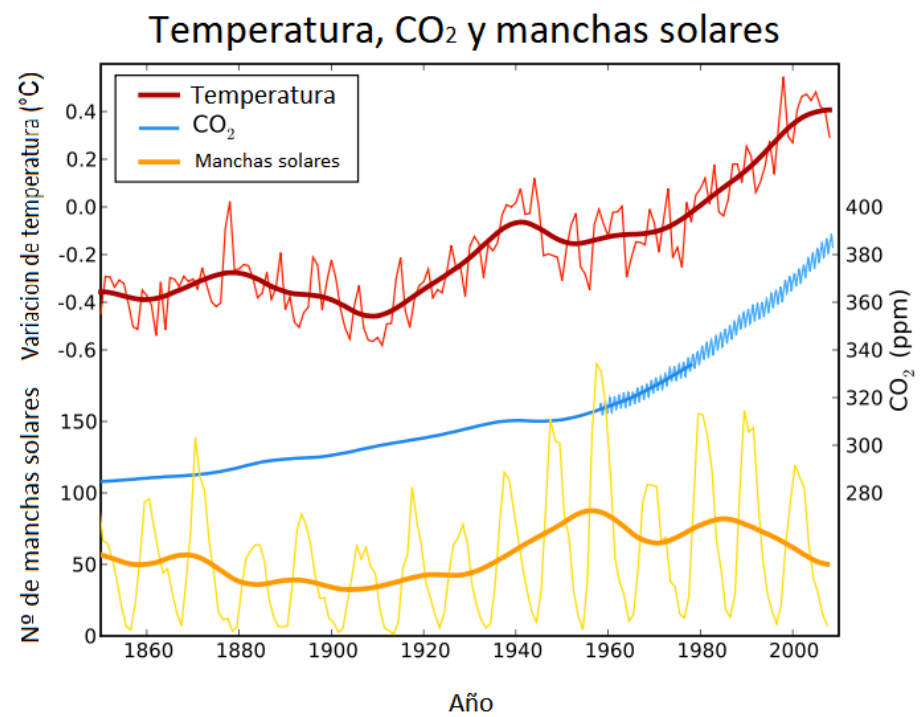

Figura 1.1. Crecimiento de la temperatura y concentración del $\mathrm{CO}_{2}$ con el tiempo [5].

Las concentraciones de dióxido de carbono $\left(\mathrm{CO}_{2}\right)$, metano $\left(\mathrm{CH}_{4}\right)$ y óxido nitroso $\left(\mathrm{N}_{2} \mathrm{O}\right)$ en la atmósfera han aumentado en un 40\% (391 ppm), 150\% (1.803 ppb) y 20\% (324 ppb) respectivamente, desde la era preindustrial al año 2011. Todos estos compuestos se encuentran en su mayor concentración desde hace 800.000 años. Durante el período comprendido entre 1880 y 2012, la temperatura media de la superficie terrestre y oceánica aumentó en $0.85{ }^{\circ} \mathrm{C}[2]$.

A nivel de porcentaje, un $80 \%$ del volumen de los gases de efecto invernadero corresponde al $\mathrm{CO}_{2}$. La evolución global empieza a mostrar una desaceleración en la concentración de estos gases, mayormente debido a la reducción de las emisiones. Como ejemplo, la Unión Europea ha conseguido reducir en un 18\% sus emisiones desde 1995. Por países, los mayores contaminantes son China, EE.UU. y la India, pero si se atiende al tamaño de la población los países más perjudicados son Catar, Trinidad y Tobago y Kuwait. En la Figura 1.2. se puede ver el volumen y la evolución de las emisiones de $\mathrm{CO}_{2}$ de los principales países y regiones contaminantes.

\section{Emisiones de $\mathrm{CO} 2$}

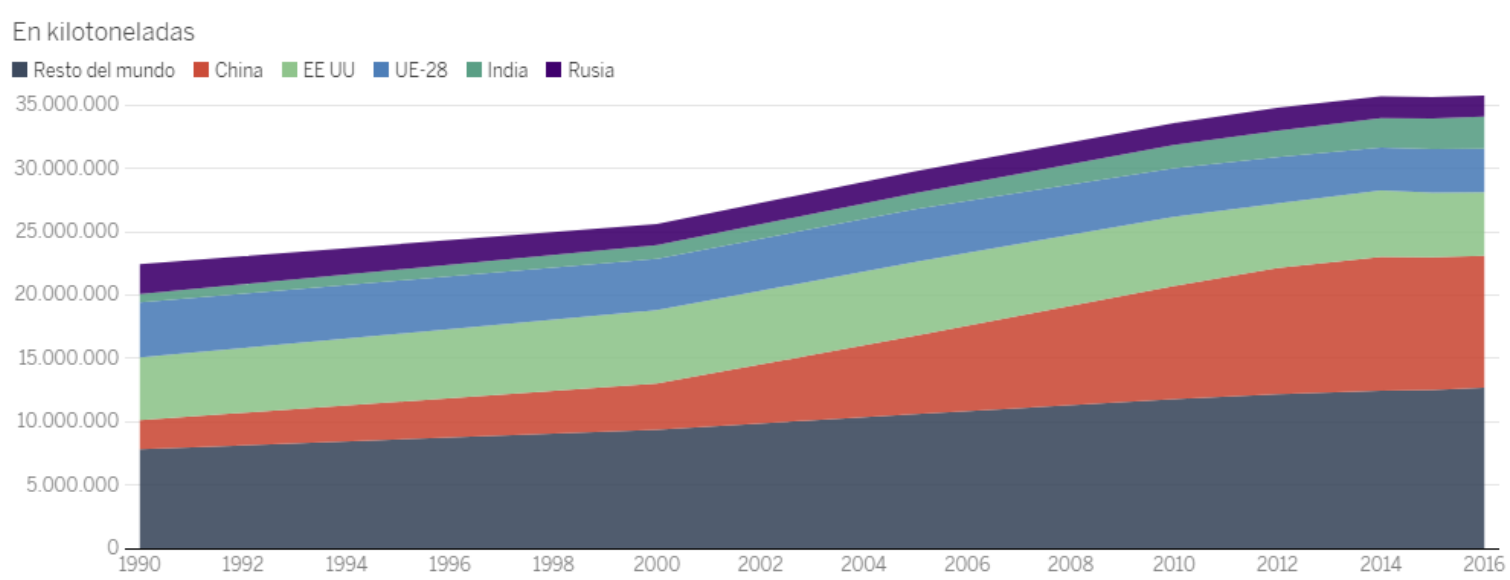

Figura 1.2. Volumen y la evolución de las emisiones del $\mathrm{CO}_{2}$ por países [6]. 
Con el objetivo de sustituir el carbón como principal fuente de energía, se instalarán millones de aerogeneradores y cientos de centrales nucleares. Añadiendo a esto el auge de la energía solar, hará posible que las fuentes no contaminantes sean prioritarias al carbón como fuente de energía en estos comienzos del siglo XXI. En el año 2020, las plantas y los suelos serán capaces de almacenar más de 17.000 millones de toneladas adicionales de dióxido de carbono al año, compensando de esta manera el 50\% de las emisiones mundiales de $\mathrm{CO}_{2}[7]$.

Sin embargo, estas medidas no serán suficientes. Los gobiernos de muchos países se verán obligados por diferentes convenios a emitir un volumen negativo de $\mathrm{CO}_{2}$, absorbiendo esencialmente los gases de efecto invernadero generados $\mathrm{y}$, además, aumentando enormemente el uso de la bioenergía. Se estima que las concentraciones de $\mathrm{CO}_{2}$ alcanzará su punto máximo en el año 2060, muy por encima del objetivo de 450 ppm [7].

Por el acuerdo de París, se ha propuesto no aumentar más de $2{ }^{\circ} \mathrm{C}$ la temperatura global. Sin embargo, algunos investigadores han cuestionado la viabilidad del uso de la bioenergía a gran escala con captura y almacenamiento de carbono (CCS) para evitar este aumento de temperatura, debido a que muchos modelos utilizados dependen de proporcionar emisiones negativas sustanciales de una forma relativamente barata. Todo ello ha generado controversia en la comunidad científica [7].

El Premio Nobel de Economía, William Nordhaus de la Universidad de Yale, sostiene que los impuestos al carbono serían la mejor manera de abordar los problemas creados por las emisiones de gases de efecto invernadero. El impuesto al carbono es un gravamen sobre la combustión de recursos fósiles como el carbón, el petróleo y el gas natural, que producen dióxido de carbono. El impuesto tendría por objeto hacer que los usuarios de esos combustibles paguen por el daño ambiental que causan. El objetivo final del impuesto sería eliminar los combustibles fósiles del mercado, e incentivar el uso de fuentes de energía que tengan poco o ningún efecto en el clima [8].

Una alternativa a la tecnología de secuestro de $\mathrm{CO}_{2}$ es ver el $\mathrm{CO}_{2}$ como un recurso y no como un desecho. El Comité para el Desarrollo de un Programa de Investigación para la Utilización de las Corrientes de Residuos de Carbono Gaseoso examinó los obstáculos a la comercialización de tecnologías de utilización de los gases de efecto invernadero. Estas tecnologías se encuentran en su mayor parte en vías de desarrollo, pero si tienen éxito, podrían mitigar la emisión de gases de efecto invernadero funcionando a bajo costo $o$ incluso proporcionar un valor añadido.

Ya existen tecnologías comerciales que operan a una escala relativamente pequeña, que utilizan o podrían utilizar $\mathrm{CO}_{2}$ como materia prima, como es el caso de la conversión química o biológica (a través de la fotosíntesis) en productos químicos o combustibles. La investigación fundamental y el desarrollo de nuevos procesos podrían aumentar las posibilidades de utilización del $\mathrm{CO}_{2}$ a escalas del orden de una gigatonelada por año. Sin embargo, la utilización del $\mathrm{CO}_{2}$ en esas cantidades masivas requeriría no sólo avances fundamentales de desarrollo de procesos, sino también la creación de sistemas que permitan la purificación y el transporte [9].

La mayoría de los esfuerzos para mitigar el cambio climático tienen por objeto reducir la velocidad a la que se emite $\mathrm{CO}_{2}$, desde los yacimientos de combustibles fósiles hasta que 
llegan a la atmósfera. Estas tecnologías incluyen las fuentes de energía renovables, el aumento de la eficiencia energética y la captura y almacenamiento de $\mathrm{CO}_{2}$ procedente de las emisiones de las plantas energéticas convencionales, es decir, que usan combustibles fósiles. Las tecnologías de emisión negativa (por sus siglas en inglés NETs), se centran en tecnologías que eliminan el $\mathrm{CO}_{2}$ de la atmósfera y lo devuelven a los reservorios geológicos $\mathrm{y}$ a los ecosistemas terrestres.

El Comité para el Desarrollo de una Agenda de Investigación para la Eliminación y Secuestro Seguro de Dióxido de Carbono fue creado para recomendar un plan detallado a seguir en la investigación y desarrollo para las tecnologías NETs. Estas recomendaciones incluyen: (1) Utilización de procesos biológicos para el aumento de las reservas de carbono en el suelo, bosques (biomasa vegetal) y grandes acumulaciones de agua como océanos, ríos y lagos; (2) producir energía a partir de la biomasa, al mismo tiempo que se capturan y almacenan las emisiones de $\mathrm{CO}_{2}$ resultantes de producir esa energía; (3) utilizar procesos químicos para capturar el $\mathrm{CO}_{2}$ directamente del aire y luego almacenarlo en reservorios geológicos; y (4) mejorar los procesos geológicos que capturan el $\mathrm{CO}_{2}$ de la atmósfera y lo almacenan permanentemente en las formaciones rocosas. Estas tecnologías se encuentran en etapas muy diferentes de desarrollo, algunas están prácticamente listas para su despliegue a gran escala, mientras que otras requieren todavía una investigación científica básica [10].

\subsection{Tecnologías de captura de $\mathrm{CO}_{2}$.}

La captura de carbono es la separación del $\mathrm{CO}_{2}$ de otros componentes gaseosos mediante técnicas físicas o químicas. La capacidad de almacenamiento, el coste de la energía y otros costes asociados imposibilitan el transporte y almacenamiento de gases con bajas concentraciones de $\mathrm{CO}_{2}$. Por lo tanto, es necesaria una tecnología de captura que sea capaz de producir una corriente gaseosa casi pura de $\mathrm{CO}_{2}$.

La captura se realiza normalmente en los principales puntos de emisión del $\mathrm{CO}_{2} \mathrm{Como}$, por ejemplo, las centrales termoeléctricas, donde sería más rentable y tendría el mayor impacto ambiental. Capturar $\mathrm{CO}_{2}$ proveniente de fuentes pequeñas y móviles como el producido por transporte, las residencias y los comercios es difícil y costoso comparado con fuentes grandes y localizadas. Las estrategias para la reducción de estas fuentes de $\mathrm{CO}_{2}$ son: el uso de hidrógeno, la electricidad producida en grandes centrales basadas en combustibles fósiles con captura de $\mathrm{CO}_{2}$, o mediante el uso de fuentes de energía renovables.

La captura desde el aire es posible, pero su aplicación es limitada debido a la baja presión parcial del $\mathrm{CO}_{2}$. Uno de los métodos más eficientes para capturar el $\mathrm{CO}_{2}$ del aire es cultivar biomasa y utilizarlo en plantas industriales con captura de carbono, logrando emisiones de carbono negativas.

Se pueden aplicar varias tecnologías para la captura de $\mathrm{CO}_{2}$ en función del proceso de producción. Las principales tecnologías para la captura de $\mathrm{CO}_{2}$ generado a partir de un combustible fósil (carbón, gas natural o petróleo), biomasa o una combinación de estos combustibles, son tres: 
$>$ Sistemas de post-combustión.

$>$ Sistemas de pre-combustión.

$>$ Sistemas de oxicombustión.

En la Figura 1.3 se muestra un diagrama con las principales tecnologías de captura de $\mathrm{CO}_{2}$.

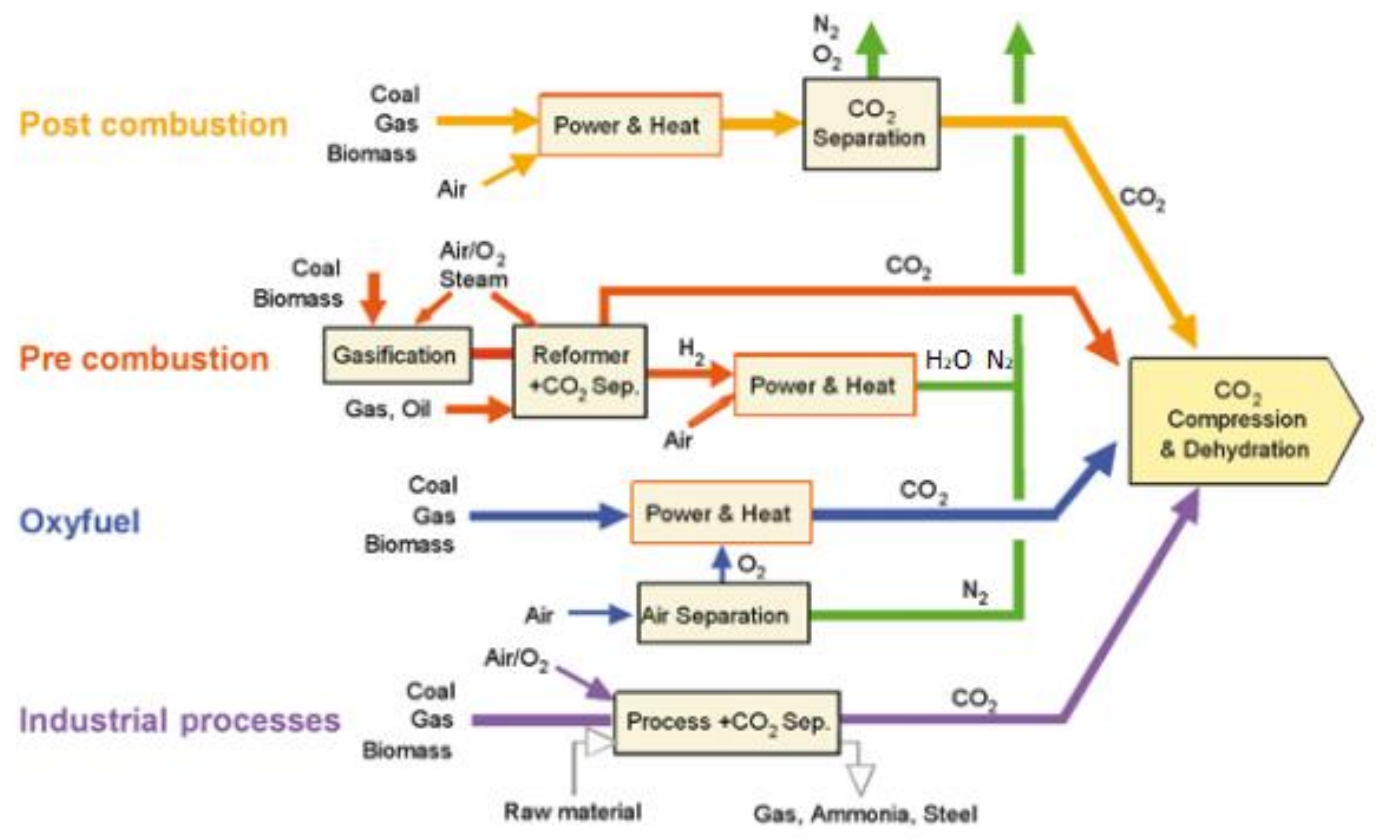

Figura 1.3. Visión general de los procesos y sistemas de captura de $\mathrm{CO}_{2}$ [11].

Sistemas de post-combustión. La captura de postcombustión es una tecnología de "etapa final", que consiste en separar el $\mathrm{CO}_{2}$ de un gas de combustión compuesto principalmente de nitrógeno, agua, $\mathrm{CO}_{2}$ y otras impurezas. La ventaja inherente es que es potencialmente adecuada para adaptarse al proceso sin afectar drásticamente a las operaciones del mismo [12]. Estos sistemas normalmente utilizan un solvente líquido para capturar la fracción de $\mathrm{CO}_{2}$ (típicamente 3-15\% en volumen) presente en el gas de combustión [11]. La separación se realiza mediante el empleo de diferentes métodos: absorción física o química, adsorción con membranas, separación criogénica, etc. Los sistemas de captura posterior a la combustión más desarrollados utilizan un disolvente orgánico (aminas), que se rocían en contracorriente a los humos de combustión. Después, es necesaria una etapa de regeneración del disolvente la cual requiere mucha energía (Figura 1.4). 


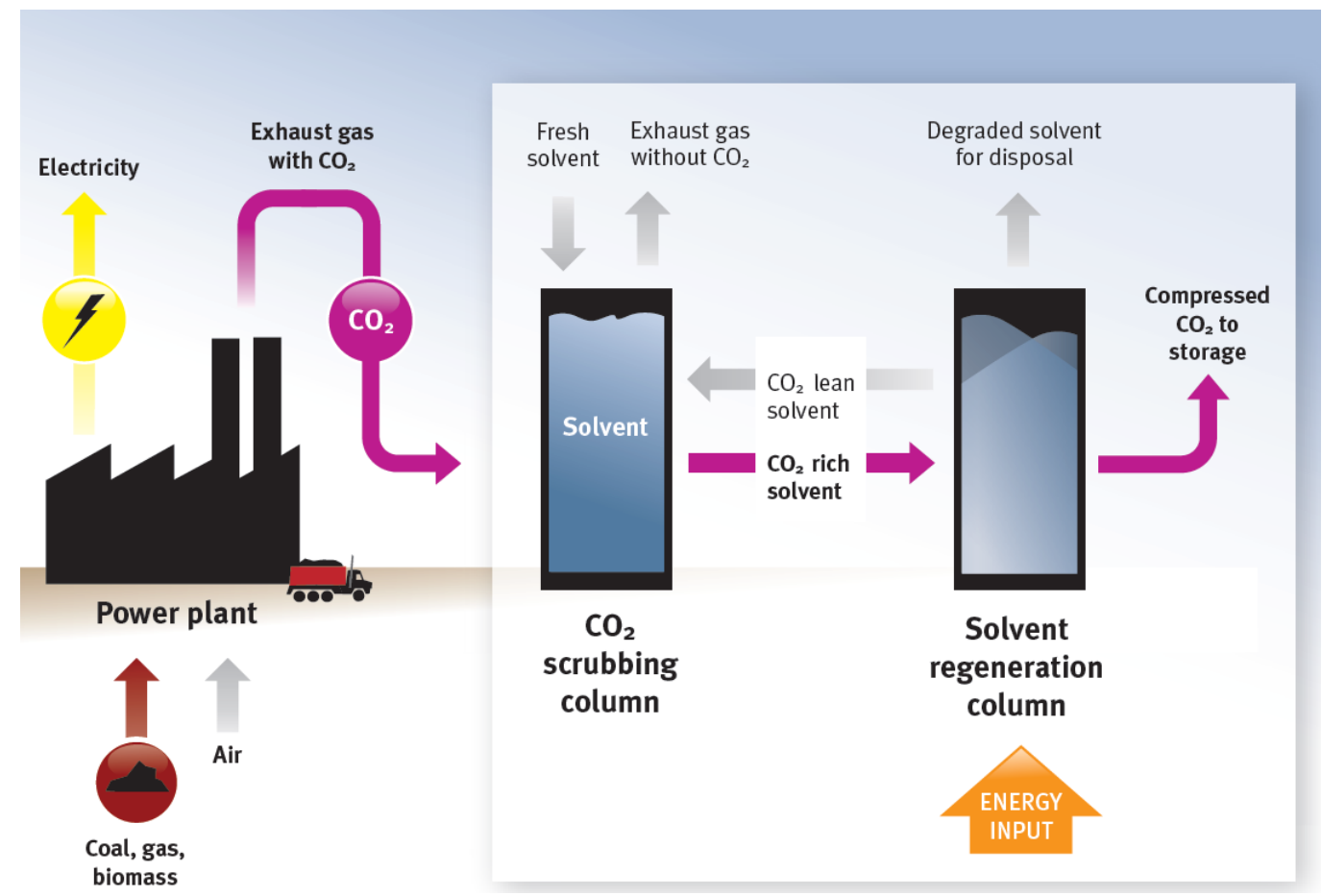

Figura 1.4. Proceso de captura de post-combustión con disolventes a base de aminas. El ejemplo más común es el proceso de absorción de monoetanolamina (MEA) acuosa al 30\% en peso [12].

Sistemas de pre-combustión. La captura previa a la combustión implica un proceso llamado gasificación, por el cual el combustible reacciona con déficit de oxígeno estequiométrico, produciendo una mezcla conocida como gas de síntesis o "syngas", formado principalmente por monóxido de carbono, metano, hidrógeno y $\mathrm{CO}_{2}$. A continuación, una serie de reacciones convierten estos gases en una mezcla de $\mathrm{CO}_{2} \mathrm{e}$ hidrógeno. Después de separarse de $\mathrm{CO}_{2}$, el gas rico en $\mathrm{H}_{2}$ puede ser utilizado en una turbina de gas o una pila de combustible (Figura 1.5).

$$
\begin{gathered}
\mathrm{CH}_{4}+\mathrm{H}_{2} \mathrm{O} \leftrightarrow \mathrm{CO}+3 \mathrm{H}_{2} \\
\mathrm{C}_{\mathrm{x}} \mathrm{H}_{\mathrm{y}}+\frac{\mathrm{x}}{2} \mathrm{O}_{2} \leftrightarrow \mathrm{xCO}+\left(\frac{\mathrm{y}}{2}\right) \mathrm{H}_{2} \\
\mathrm{CO}+\mathrm{H}_{2} \mathrm{O} \leftrightarrow \mathrm{CO}_{2}+\mathrm{H}_{2}
\end{gathered}
$$

La flexibilidad operativa está limitada por el coste del gasificador, lo que significa que para ser rentable tiene que funcionar a pleno rendimiento, produciendo constantemente gas de síntesis. Las condiciones para la captura de $\mathrm{CO}_{2}$ en este sistema son muy diferentes comparadas con la captura post-combustión debido a que el gas está a una presión elevada (2-7 $\mathrm{MPa}$ ) y la concentración de $\mathrm{CO}_{2}$ es mayor (15$60 \%$ en volumen). Esta presión parcial más alta de $\mathrm{CO}_{2}$ permite el uso de disolventes físicos (Selexol, Rectisol, etc.) en lugar de disolventes químicos para separar los dos gases [12]. 


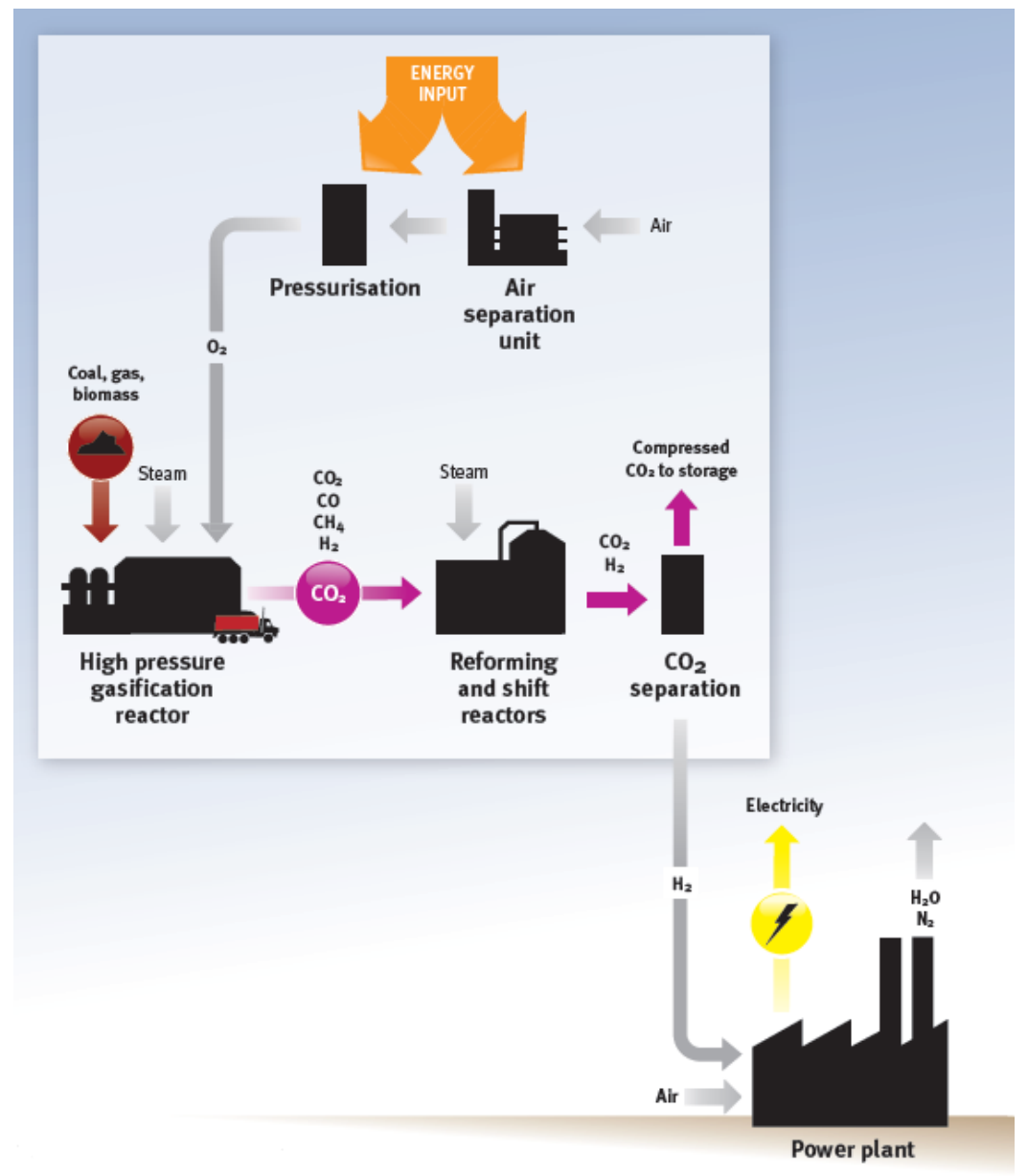

Figura 1.5. Proceso de captura de pre-combustión [12].

Sistemas de oxicombustión. La oxicombustión consiste en quemar combustible en una mezcla de $\mathrm{CO}_{2}$ reciclado y $\mathrm{O}_{2}$ puro en lugar de aire, resultando un gas de combustión que se compone principalmente de $\mathrm{CO}_{2}$ y vapor de agua, el cual es fácilmente separado mediante condensación [12]. Esto da como resultado un gas de combustión con altas concentraciones de $\mathrm{CO}_{2}$ (más del $80 \%$ en volumen). Una corriente de $\mathrm{CO}_{2}$ de alta pureza, permite la compresión inmediata para su almacenamiento (Figura 1.6).

La mayor desventaja de la oxicombustión es el costo de separar el oxígeno del aire, pero esto podría ser compensado realizando la combustión a alta presión, reduciendo los costos de la compresión subsiguiente. 


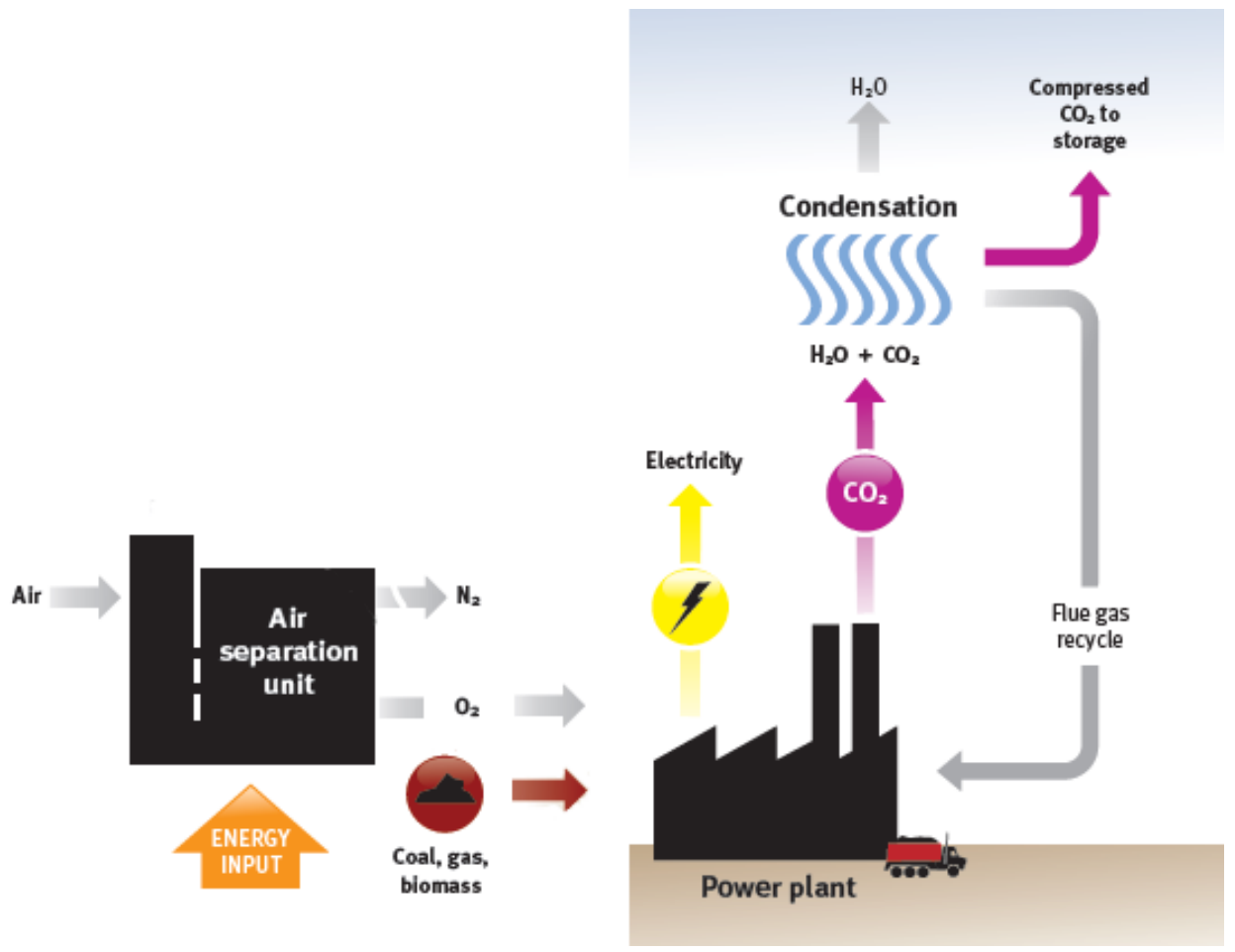

Figura 1.6. Proceso de captura de oxicombustión [12].

\subsection{Tecnologías de post-combustión, absorción de $\mathrm{CO}_{2}$ con aminas.}

En la presente investigación nos centraremos en los procesos de post-combustión, para los cuales existen diferentes métodos para la separación del $\mathrm{CO}_{2}$ de una corriente de gas, siendo los más comunes la absorción en líquidos (absorción química o física), la adsorción en un sólido, uso de membranas, la destilación criogénica o el bucle del calcio. Una de las tecnologías más aplicadas hoy en día para la captura de $\mathrm{CO}_{2}$ en el proceso de postcombustión es mediante la absorción química, siendo la absorción con aminas una de las tecnologías de captura más desarrolladas.

La tecnología se basa en la reversibilidad de las reacciones de neutralización ácido-base, de los solventes acuosos alcalinos con un gas ácido, como el $\mathrm{CO}_{2}$. Este proceso fue desarrollado para depurar gas natural y utilizarlo posteriormente en la extracción mejorada de petróleo (EOR, en sus iniciales en inglés Enhanced Oil Recovery). También ha sido utilizado en reacciones de carbonatación de agua salobre, en la producción de urea y hielo seco, o en la industria alimenticia [13].

La principal reacción en la absorción con aminas depende del tipo de amina a reaccionar, en el caso de soluciones acuosas de aminas primarias o secundarias es la formación de un carbamato, que normalmente ocurre a través de la formación de un zwitterion, y la subsiguiente desprotonación catalizada por la base del zwitterion.

$$
2 \mathrm{R}_{1} \mathrm{R}_{2} \mathrm{NH}+\mathrm{CO}_{2} \rightleftarrows\left[\mathrm{R}_{1} \mathrm{R}_{2} \mathrm{NCO}_{2}^{-}+\mathrm{R}_{1} \mathrm{R}_{2} \mathrm{NH}_{2}^{+}\right]
$$

Después se regenera el $\mathrm{CO}_{2}$ por medio de la conversión del carbamato a bicarbonato, a través de una reacción de hidrólisis y la formación de bicarbonato. 


$$
\begin{gathered}
\mathrm{R}_{1} \mathrm{R}_{2} \mathrm{NCO}_{2}^{-}+\mathrm{H}_{2} \mathrm{O} \rightleftarrows \mathrm{R}_{1} \mathrm{R}_{2} \mathrm{NH}+\mathrm{HCO}_{3}^{-} \\
\mathrm{CO}_{2}+\mathrm{OH}^{-} \rightleftarrows \mathrm{HCO}_{3}^{-}
\end{gathered}
$$

La principal diferencia con las aminas terciarias en comparación con las aminas primarias y secundarias es que el $\mathrm{CO}_{2}$ no reacciona para formar carbamato. Los mecanismos implicados en la absorción de $\mathrm{CO}_{2}$ por soluciones acuosas de aminas terciarias, como la dimetilaminoetanol (DMAE), son diferentes. Estas actúan como base, catalizando la reacción de hidratación del $\mathrm{CO}_{2}$. Así pues, existe un mayor interés en el uso de soluciones acuosas de aminas terciarias:

$$
\mathrm{R}_{1} \mathrm{R}_{2} \mathrm{R}_{3} \mathrm{~N}+\mathrm{CO}_{2}+\mathrm{H}_{2} \mathrm{O} \rightleftarrows \mathrm{R}_{1} \mathrm{R}_{2} \mathrm{R}_{3} \mathrm{NH}^{+}+\mathrm{HCO}_{3}^{-}
$$

\section{PRIMARY AMINES}

Monoethanolamine (MEA)

$\mathrm{C}_{2} \mathrm{H}_{4} \mathrm{OH}-\mathrm{NH}_{2}$
DIGLYCOLAMINE Agent (DGA)

$\mathrm{HOC}_{2} \mathrm{H}_{4} \mathrm{OC}_{2} \mathrm{H}_{4}-\mathrm{NH}_{2}$

\section{SECONDARY AMINES}

Diethanolamine (DEA)<smiles>CCONCCO</smiles>

Diisopropanolamine (DIPA)

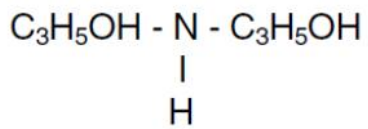

\section{TERTIARY AMINES}

Triethanolamine (TEA)<smiles>CCON(C=O)CO</smiles>

Methyldiethanolamine (MDEA)<smiles>CCON(C)CCO</smiles>

Figura 1.7. Ejemplos de aminas usadas en la captura de $\mathrm{CO}_{2}$.

Las disoluciones típicas a base de aminas que se utilizan en la captura de $\mathrm{CO}_{2}$ son disoluciones acuosas, con unas concentraciones entre el $10 \%$ y $30 \%$ en peso.

Uno de los problemas que surgen, después del proceso de captura, es la eliminación de las impurezas nocivas de la corriente del $\mathrm{CO}_{2}$ como son: los óxidos de azufre, los óxidos de nitrógeno, el oxígeno, el monóxido de carbono y el agua. Si las corrientes de $\mathrm{CO}_{2}$ no se purifican al nivel adecuado, se corren riesgos de corrosión en las tuberías y reacciones secundarias no deseadas con hidrocarburos. El alcance de la purificación variará dependiendo del tipo y nivel de impurezas, que a su vez dependen de la tecnología de captura usada y el tipo de combustible utilizado [14]. Por otra parte, hay que tener en cuenta la degradación de los solventes, la formación de subproductos y los problemas de corrosión que acarrean estos disolventes ricos en $\mathrm{CO}_{2}$. Un aspecto importante y que condiciona la viabilidad del proceso es la enorme energía de regeneración necesaria para recuperar el disolvente, que es proporcional a la cantidad de $\mathrm{CO}_{2}$ a separar [13]. 
La absorción de $\mathrm{CO}_{2}$ con aminas tiene alta eficiencia de captura y alta selectividad del $\mathrm{CO}_{2}$ a bajas presiones parciales de este compuesto.

Además de la necesidad de información sobre la eficiencia de captura y selectividad de $\mathrm{CO}_{2}$ en las aminas en los procesos de absorción química, resulta necesario un mayor conocimiento de las propiedades termofísicas que intervienen en dichos procesos.

Es importante conocer los parámetros de operación de las plantas para determinar la viabilidad técnica de los procesos de captura como, por ejemplo: elección de una amina adecuada por su eficiencia o posibles problemas, la mezcla óptima de esta para generar un disolvente adecuado que tenga una gran capacidad de absorción y regeneración disminuyendo su corrosividad, y sus propiedades como la densidad, necesaria para el cálculo de la solubilidad y otras propiedades fisicoquímicas.

\subsection{Objetivos de la Tesis Doctoral}

El objetivo general de esta Tesis Doctoral es desarrollar técnicas experimentales que permitan la caracterización termodinámica de soluciones acuosas de aminas con $\mathrm{CO}_{2}$ a través de la densidad, viscosidad y solubilidad, así como validar las modificaciones instrumentales efectuadas. Esto conlleva los siguientes objetivos específicos:

\section{Adaptar una técnica para la medida de equilibrios isotermos a altas temperaturas y presiones.}

a. Modificar el equipo para medir equilibrios líquido-vapor a altas temperaturas (hasta $393 \mathrm{~K}$ ) y presiones (hasta $6 \mathrm{MPa}$ ). Esto es necesario, debido a que la técnica original VLE fue diseñada para medir a una temperatura fija de $313 \mathrm{~K}$ y presiones bajas.

b. Validar el equipo con la medida de equilibrios líquido-vapor de mezclas binarias de hidrocarburos, alcoholes y cetonas desde $313 \mathrm{~K}$ hasta $393 \mathrm{~K}$. Ajustar los valores de equilibrio a la ecuación de Margules y a diferentes modelos (Wilson, NRTL y UNIQUAC).

2. Poner a punto un densímetro para la medida de sistemas líquido-gas a altas temperaturas y presiones.

a. Modificar el densímetro de tubo vibrante Anton Paar DMA HPM, disponible en el laboratorio TERMOCAL, para la medida de mezclas de líquidos con $\mathrm{CO}_{2}$.

b. Medir los sistemas de hidrocarburos con $\mathrm{CO}_{2}$ en un rango de temperaturas desde $273 \mathrm{~K}$ hasta $373 \mathrm{~K}$, presiones desde $5 \mathrm{MPa}$ hasta $100 \mathrm{MPa}$ a diferentes concentraciones de $\mathrm{CO}_{2}$. Correlacionar los valores de las densidades con la ecuación Tammann-Tait modificada. 
3. Poner a punto un viscosímetro para la medida de sistemas líquido-gas a altas temperaturas y presiones

a. Modificar el viscosímetro de hilo vibrante, desarrollado en el laboratorio TERMOCAL, para la medida de mezclas de hidrocarburo $+\mathrm{CO}_{2}$.

b. Efectuar las medidas de las viscosidades de dichas mezclas en un rango de temperaturas desde $293 \mathrm{~K}$ hasta $373 \mathrm{~K}$, presiones desde $5 \mathrm{MPa}$ hasta $80 \mathrm{MPa}$ y distintas concentraciones de $\mathrm{CO}_{2}$. Correlacionar las viscosidades dinámicas con la ecuación Vogel-Fulcher-Tammann modificada (VFT).

4. Determinar las propiedades $p, V, T$ de soluciones acuosas de aminas con $\mathrm{CO}_{2}$.

Medir las densidades de soluciones acuosas de aminas con $\mathrm{CO}_{2}$, en amplios rangos de temperatura y de presión (desde $293 \mathrm{~K}$ hasta $373 \mathrm{~K}$ y desde $0.1 \mathrm{MPa}$ hasta 60 MPa) utilizando el densímetro de tubo vibrante Anton Paar DMA HPM. Estas densidades son necesarias para poder calcular la solubilidad de $\mathrm{CO}_{2}$ en mezclas acuosas de amina. Correlacionar los datos de las densidades con la ecuación Tammann-Tait modificada.

5. Determinar la solubilidad de $\mathrm{CO}_{2}$ en soluciones acuosas de aminas.

Medir las solubilidades de $\mathrm{CO}_{2}$ en disoluciones acuosas de amina con la celda de equilibrio isoterma reformada a diferentes temperaturas y una presión máxima de $6 \mathrm{MPa}$. Correlacionar las solubilidades del $\mathrm{CO}_{2}$ en soluciones acuosas de amina con la ecuación de Xu y Rochelle.

\section{Determinar las incertidumbres de medida para todas las propiedades termodinámicas determinadas.}

Esta tesis doctoral se enmarca en los objetivos propuestos en los siguientes proyectos y contratos:

(a) "Biogás renovable y procesos de captura del $\mathrm{CO}_{2}$ de combustión asociados como base a la sostenibilidad energética ambiental: Investigación Termodinámica Experimental (VA391A12-1) ", subvencionado por la Junta de Castilla y León.

(b) "Revalorización de recursos renovables regionales biomásicos y eólicos para la producción de gases energéticos sostenibles (biogás e hidrógeno) y su incorporación en la red de gas natural (VA035U16)", concedido por la Junta de Castilla y León.

(c) "Gases energéticos: biogás y gas natural enriquecido con hidrógeno (ENE2013-47812R)", subvencionado por el Ministerio de Economía y Competitividad de España.

(d) "Caracterización termodinámica de mezclas de cetonas", financiado por la empresa UBE Chemical.

(e) Contratos Pre-doctorales del Fondo Social Europeo (FSE) y Junta de Castilla y León. 


\subsection{Estructura de la Tesis Doctoral.}

La distribución del presente trabajo se describe a continuación:

En el primer capítulo se expone la problemática mundial de los gases que contribuyen al efecto invernadero, en especial las emisiones de $\mathrm{CO}_{2}$, y se describen las diferentes tecnologías de captura para mitigar este problema. Por otra parte, se plantean los objetivos de la investigación, siendo el punto principal la obtención de la densidad y la solubilidad del $\mathrm{CO}_{2}$ en soluciones acuosas de amina para su captura en gases de combustión.

El segundo capítulo describe la técnica experimental para la determinación del equilibrio líquido-vapor y las mejoras para la obtención de medidas a altas temperaturas. Además, se describe el tratamiento de los datos experimentales y su ajuste a diferentes modelos (Margules, Wilson, NRTL y UNIQUAC).

En el tercer capítulo se presentan los datos experimentales de las medidas de equilibrio líquido-vapor de los diferentes sistemas. También se recoge el ajuste de los datos con los modelos anteriores, y el cálculo de la energía de Gibbs de exceso.

El cuarto capítulo describe la modificación del procedimiento experimental y el calibrado de un equipo de equilibrio líquido-vapor basado en una celda isoterma de presión total para la realización de las medidas de solubilidad de $\mathrm{CO}_{2}$ en soluciones acuosas de amina a altas presiones y temperatura. Se describe la técnica experimental del densímetro de tubo vibrante, su calibrado y el cálculo de las incertidumbres asociadas a la medida.

En el quinto capítulo se muestran los datos experimentales de las medidas de densidad y solubilidad de $\mathrm{CO}_{2}$ en disoluciones acuosas de aminas. Además, se recoge el ajuste de los datos de densidad mediante la ecuación de Tammann-Tait modificada y el ajuste de la solubilidad con la ecuación de Xu y Rochelle.

El sexto capítulo se describe la técnica experimental y las ecuaciones que rigen el funcionamiento de un viscosímetro de hilo vibrante. Por otra parte, se muestra la calibración y el cálculo de incertidumbre asociada a la viscosidad.

En el séptimo capítulo se presentan los datos experimentales de las medidas de densidad y viscosidad de las mezclas hidrocarburo $+\mathrm{CO}_{2}$. Por último, se recoge el ajuste de los datos de densidad con la ecuación de Tammann-Tait modificada y el ajuste de los datos de viscosidad dinámica mediante el modelo Vogel-Fulcher-Tammann (VFT).

En el octavo y último capítulo se presentan las conclusiones derivadas de la investigación.

\subsection{Referencias.}

[1] B. Smit, J. R. Reimer, C. M. Oldenburg, I. C. Bourg. "Introduction to carbon capture and sequestration". Imperial College Press (2014).

[2] Intergovernmental Panel on Climate Change (IPCC). "Climate change 2013. The physical science basis". ISBN 978-92-9169-138-8. 
[3] Y. Sanchez-Vicente, W. J. Tay, S. Z. Al Ghafri, J. P. M. Trusler. "Thermodynamics of carbon dioxide-hydrocarbon systems". Applied Energy. Vol. 220, (2018) 629-642.

[4] United Nations. Framework convention on climate change (FCCC). "Adoption of the Paris Agreement". FCCC/CP/2015/L.9/Rev.1.

[5] L. McInnes. " $\mathrm{CO}_{2}$, temperature, and sunspot activity since $1850 "$.

Disponible en: <https://commons.wikimedia.org/wiki/File:Temp-sunspot-co2.svg> [accedido 19/08/2019].

[6] J. M. Abad Liñán, J. Galán. "La emisión de $\mathrm{CO}_{2}$ por países". Disponible en: <https://elpais.com/internacional/2017/11/06/actualidad/1509979154_987066.html> [accedido 10/04/2019].

[7] J. Tollefson. "The $2{ }^{\circ} \mathrm{C}$ dream". Nature. Vol. 527, (2015) 436-438.

[8] D. Koenig. "Carbon tax gets renewed attention but still faces resistance". Industrial Equipment News (IEN) (2018).

Disponible en: <https://www.ien.com/regulation/news/21026336/carbon-tax-getsrenewed-attention-but-still-faces-resistance> [accedido 10/04/2019].

[9] Committee on developing a research agenda for utilization of gaseous carbon waste streams. "Gaseous carbon waste streams utilization: status and research needs". The national academies press (2018).

[10] Committee on developing a research agenda for carbon dioxide removal and reliable sequestration. "Negative emissions technologies and reliable sequestration: a research agenda". The national academies press (2018).

[11] B. Metz, O. Davidson, H. de Coninck, M. Loos, L. Meyer. "IPCC special report on carbon dioxide capture and storage". Cambridge University Press (2005).

[12] N. Florin, P. Fennell. "Carbon capture technology: future fossil fuel use and mitigating climate change". Grantham Institute for Climate Change, Imperial College London (2010).

[13] L. M. Romeo, I. Bolea. "Overview post-combustion $\mathrm{CO}_{2}$ capture". Boletín del grupo Español del Carbón. Vol. 35, (2015) 8-11.

[14] Z. Abbas, T. Mezher, M. R. M. Abu-Zahra. "CO ${ }_{2}$ purification. Part I: Purification requirement review and the selection of impurities deep removal technologies". International Journal of Greenhouse Gas Control. Vol. 16, (2013) 324-334. 



\section{Capítulo 2}

\section{DETERMINACIÓN EXPERIMENTAL DEL EQUILIBRIO LÍQUIDO-VAPOR A DIFERENTES TEMPERATURAS}





\subsection{Introducción.}

La determinación del equilibrio líquido-vapor de una mezcla de fluidos requiere, por un lado, el conocimiento de la temperatura y la presión a la que sucede el equilibrio termodinámico, y por otro, la determinación de la composición de la fase líquida y la fase vapor en equilibrio.

Conocer el equilibrio entre fases permite la determinación directa de la función energía de Gibbs de exceso $\left(G^{E}\right)$, siendo esta propiedad fundamental tanto en la formulación de modelos teóricos de comportamiento de mezclas como, la aplicación directa en el ámbito industrial en procesos de destilación, absorción o extracción.

Existen diversas técnicas experimentales para la determinación del equilibrio líquidovapor. La elección de uno u otro método está determinada principalmente por el tipo de datos de equilibrio que se quieran obtener (isobáricos o isotérmicos), por el rango de presiones y temperaturas de trabajo, y por la propia naturaleza del sistema del que se quiere obtener los datos de equilibrio.

En el método estático, el estado final es un estado de verdadero equilibrio termodinámico. Cantidades conocidas de la mezcla se cargan en un recipiente cerrado al que previamente se le ha hecho vacío. El recipiente se introduce en un baño termostatizado y se agita hasta que se establece el equilibrio entre el líquido y el vapor. De acuerdo con el teorema de Duhem [1], con el conocimiento de las variables intensivas de presión y temperatura, junto con las cantidades totales de los componentes presentes en la mezcla, se pueden calcular el resto de las variables del equilibrio. Por tanto, las únicas medidas necesarias son la temperatura y presión de la celda, y las masas de los compuestos puros inyectadas en la celda para formar la mezcla. Dichas medidas pueden realizarse con una baja incertidumbre por lo que resulta una técnica muy precisa.

\subsection{Descripción del método de medida utilizado para la determinación del equilibrio líquido-vapor.}

La técnica utilizada para la determinación experimental del equilibrio líquido-vapor está basada en un método estático de medida isoterma de la presión total. El dispositivo experimental ha sido construido de acuerdo con el diseño original de Van Ness y sus colaboradores, Gibbs et al. [2] (1972); Dielsi et al. [3] (1978), la puesta a punto del mismo y la determinación de los primeros sistemas binarios se realizó en una tesis doctoral anterior, Montero [4] (1996). La mejora de la técnica y su adaptación para sistemas ternarios dio lugar a otra tesis doctoral Segovia [5] (1997) y fue utilizada en otras dos tesis más Chamorro [6] (1998), Alonso-Tristán [7] (2001). Posteriormente se fueron adoptando una serie de mejoras para la medida de la presión de vapor directamente en la celda, evitando el uso de una celda de presión diferencial y el medidor de presión tipo Bourdon, dando lugar a la tesis doctoral de Moreau [8] (2014). En el presente trabajo, se amplía la capacidad de medida del equipo incrementando el rango de temperatura y presión para la medida de solubilidades. 
La técnica del equilibrio líquido-vapor es de muy alta precisión. Los instrumentos de medida se calibran periódicamente, asegurando la trazabilidad de las medidas a patrones internacionales. El diagrama del equipo original se representa en la Figura 2.1, indicando los elementos de los cuales consta.

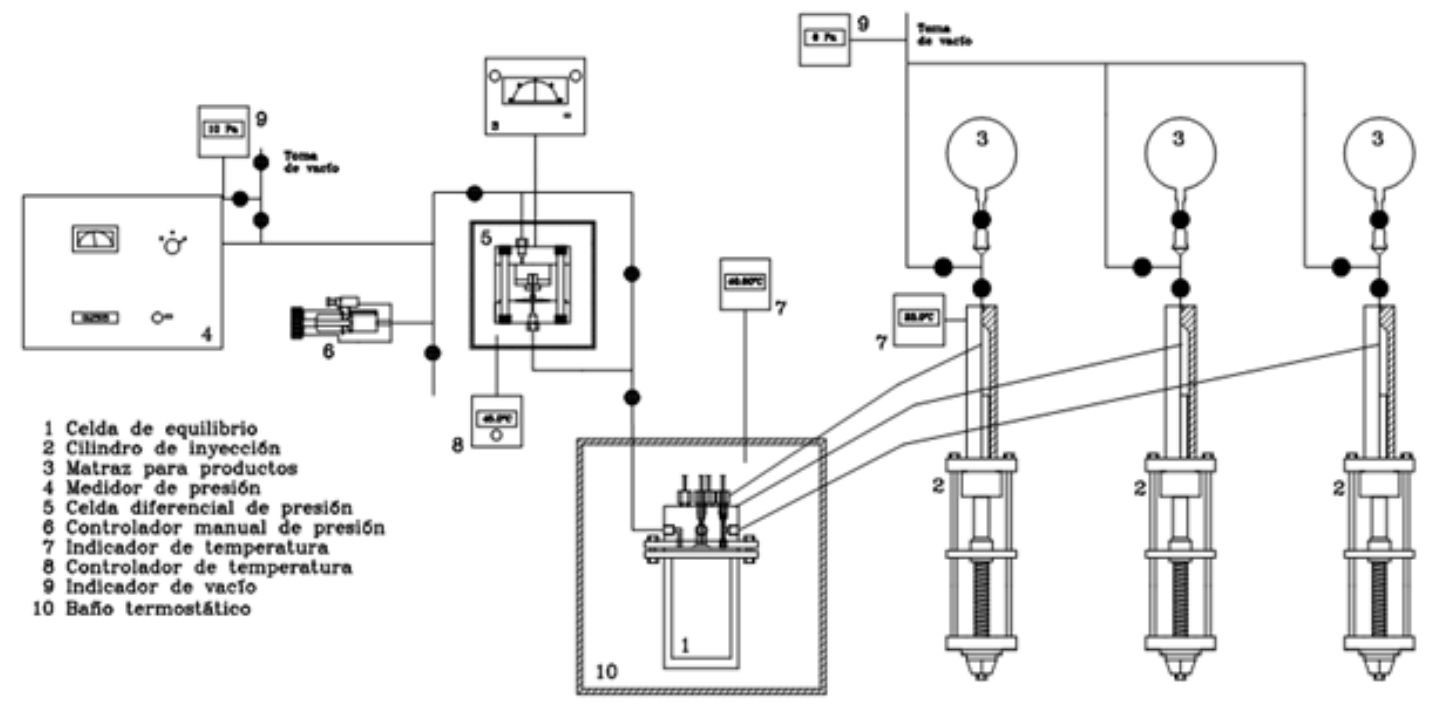

Figura 2.1. Esquema del aparato de medida del equilibrio líquido-vapor (VLE).

El equipo consta de cinco partes las cuales serán descritas a continuación:

- Celda de equilibrio.

- Sistema de inyección.

- Sistema de medida de la presión tipo Bourdon.

- Sistema de medida de presión mediante un transductor de presión.

- Sistema de medida y control de la temperatura.

- Sistema de vacío.

\subsubsection{Celda de equilibrio.}

La celda de equilibrio está formada por una vasija de acero inoxidable, con una capacidad aproximada de $180 \mathrm{~cm}^{3}$, en el interior se encuentra un agitador magnético para asegurar una rápida mezcla de los componentes puros. La tapa de la vasija está unida por medio de una brida y una junta tórica para garantizar la estanquidad.

En la Figura 2.2 se muestra la tapa de la celda de equilibrio. En la tapa se encuentran tres válvulas de aguja que permiten la entrada de los diferentes componentes puros, previamente desgasificados. La tapa también dispone de una válvula de plato que conecta la celda al sistema de vacío. Además, hay una conexión para una válvula de venteo y una tubería que conecta la celda con los diferentes medidores de presión. 

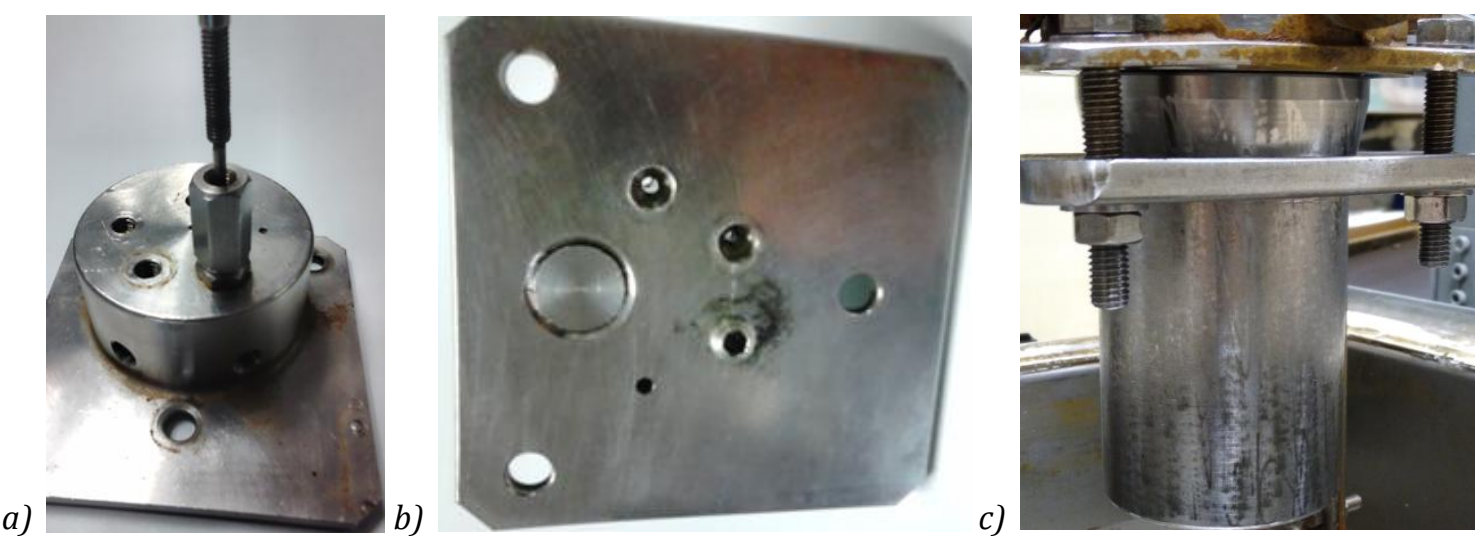

Figura 2.2. a) Parte superior de la tapa de la celda de equilibrio líquido-vapor. b) Parte inferior de la tapa de la celda de equilibrio líquido-vapor. c) Celda de equilibrio.

\subsubsection{Sistema de inyección.}

El procedimiento para barrer las distintas composiciones consiste en inyectar el primer componente en la celda de equilibrio. A continuación, se van añadiendo volúmenes conocidos del segundo componente para obtener las distintas composiciones molares, barriendo de 0.05 en 0.05 molar hasta una composición de 0.6. En el punto final el volumen es de unos $120 \mathrm{~mL}$.

Para la inyección de los componentes, se dispone de tres bombas de desplazamiento positivo que permiten añadir volúmenes conocidos de los componentes puros a medir. Los cilindros utilizados son de la marca Ruska, modelo 2200-801, de $100 \mathrm{~mL}$ de capacidad y graduados en intervalos de $0.01 \mathrm{~mL}$. En la Figura 2.3 se puede observar el sistema de inyección.

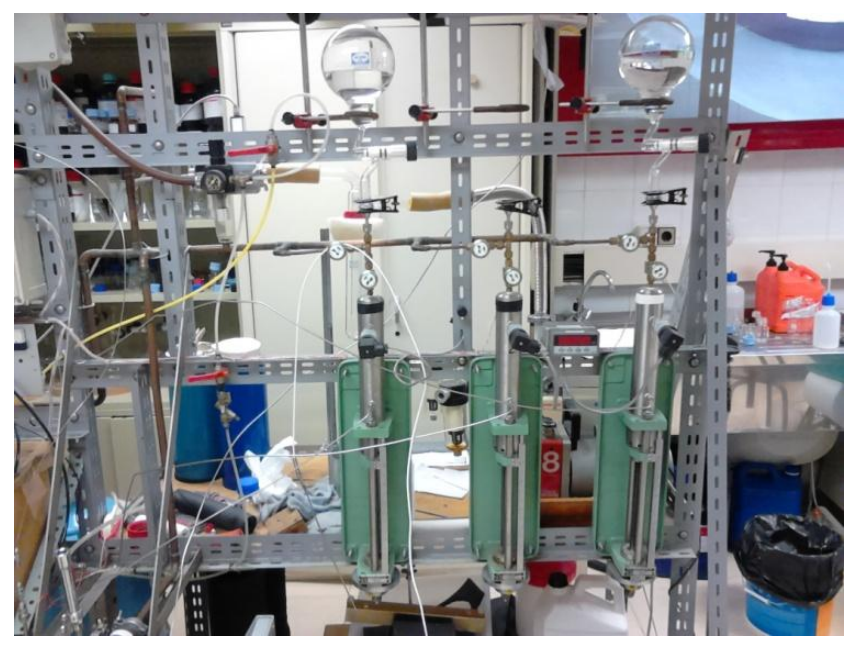

Figura 2.3. Sistema de inyección de los componentes puros.

Cada uno de los cilindros posee un transductor de presión (Druck, modelo PTX-1400), permitiendo la lectura en cada instante de la presión del líquido en el interior del cilindro. 
La temperatura de la bomba se mide con una termorresistencia de platino Pt100, que está conectada a una unidad de adquisición de datos Agilent 34970A. Las masas de los diferentes productos inyectados en la celda de medida se determinan a través de las densidades a la temperatura de inyección de cada componente. Los cilindros no están termostatados, pero al poseer una gran inercia térmica no se ven afectados por cambios rápidos en la temperatura del laboratorio. Los cambios en la temperatura ambiente del laboratorio serán amortiguados al disponer de climatización permanente en la zona de trabajo.

Los compuestos puros, previamente desgasificados, se encuentran en matraces de 1000 $\mathrm{mL}$ conectados a las bombas mediante un sistema de válvulas para el llenado de las mismas. Para poder realizar el llenado de los cilindros, es necesario hacer vacío en todo el sistema. El nivel de vacío que se logra en la instalación está en torno a los 0,5 - 0,6 Pa. Una vez han sido llenados los cilindros, se ajusta la presión del cilindro, haciendo que sea la misma al comienzo y final de las sucesivas cargas. El tiempo entre lecturas es el necesario para que se haya alcanzado el equilibrio térmico. Los volúmenes inyectados se determinan por diferencia.

La celda de medida se encuentra conectada con las bombas de desplazamiento positivo mediante una tubería de acero inoxidable. Las válvulas utilizadas son de tipo aguja para tener una mejor regulación de la apertura y el cierre, encontrándose instaladas en la tapa de la celda de medida.

Los cilindros de inyección se han calibrado en el propio laboratorio, siendo las incertidumbres debidas al volumen inyectado de $0,03 \mathrm{~mL}$.

\subsubsection{Sistemas de medida de presión.}

La medida de presión consta de los equipos necesarios que nos permitan abordar las medidas de los sistemas que forman parte de esta tesis en amplios rangos de presión y temperatura. A continuación, se detallan las características técnicas de los principales elementos que forman cada sistema de medida de la presión.

\subsubsection{Manómetro tipo Bourdon.}

Este sistema es usado solamente para las medidas de equilibrios líquido-vapor a $40^{\circ} \mathrm{C}$. En el presente trabajo se usó para la medida del equilibrio líquido-vapor del sistema 2Pentanol + Ciclohexano a $40^{\circ} \mathrm{C}$.

La medida de la presión se realiza mediante un manómetro diferencial de presión tipo Bourdon. La referencia del medidor de presión está conectada al sistema de vacío por lo que el equipo mide presiones absolutas. El aparato consta de dos partes básicas, una cápsula Bourdon y el sensor de lectura óptico de fotodetectores de silicio. La cápsula permanece a una temperatura constante de $49{ }^{\circ} \mathrm{C}$ para evitar la influencia de las variaciones de la temperatura ambiente. 
El medidor de presión es de la marca Texas Instruments, modelo PPG-149, con una cápsula Bourdon de cuarzo tipo 801. En la tabla 2.1. se resumen las características de este equipo.

Tabla 2.1. Características del medidor de presión diferencial.

Intervalo de medida

Resolución

Repetibilidad mecánica

Incertidumbre debida al control de la temperatura de cápsula
$1 \mathrm{~Pa}-125 \mathrm{kPa}$

$1 \mathrm{~Pa}$

$0.0005 \%$ fondo de escala

$0.0065 \%$ lectura

La calibración del equipo se ha realizado utilizando como patrón primario una balanza neumática de pesos muertos trazable a patrones nacionales. La incertidumbre expandida $(k=2)$ del equipo es mejor que $2 \cdot 10^{-4} \cdot \mathrm{p} / \mathrm{Pa}+5 \mathrm{~Pa}$.

Para utilizar el manómetro tipo Bourdon como medidor de presión absoluto, se ha conectado como instrumento de lectura de la presión de referencia un medidor de vacío marca Leybold Vacuum, modelo TTR 91S, con las características que se indican en la tabla 2.2 .

Tabla 2.2. Características del medidor de vacío.

Intervalo de medida

Incertidumbre de la medida

Repetibilidad
$0.05-100000 \mathrm{~Pa}$

15\% en el rango $0.1-10000 \mathrm{~Pa}$

$2 \%$ en el rango $0.1-10000 \mathrm{~Pa}$

Al existir la posibilidad de que algún compuesto condense en el sensor de cuarzo es necesario aislar el manómetro tipo Bourdon de la celda de equilibrio, para ello se emplea una celda de presión diferencial que tiene como elemento sensible un diafragma. La deformación del diafragma hace variar la inductancia de un circuito magnético que sirve como sistema de detección.

La celda de presión diferencial es de la marca Ruska, modelo 2413-705 y el indicador de presión diferencial o indicador de cero es marca Ruska, modelo 2416-711.

Para lograr la misma presión en los dos lados de la celda de presión diferencial se emplea un controlador manual de presión de volumen variable. La presión máxima de operación es $7 \mathrm{MPa}$ y permite ajustar la presión del circuito con una precisión de $1.75 \mathrm{~Pa}$. Este controlador es de la marca Ruska, modelo 3893-801. 


\subsubsection{Transductores de presión tipo Druck.}

Para la medida de los sistemas de equilibrios a altas temperaturas se han instalado dos transductores de presión tipo Druck acoplados a la celda de equilibrio. Los medidores se sumergen en el baño y quedan termostatizados a la misma temperatura que la celda. En la Figura 2.4. se observan los transductores de presión y su implantación en el equipo de medida.

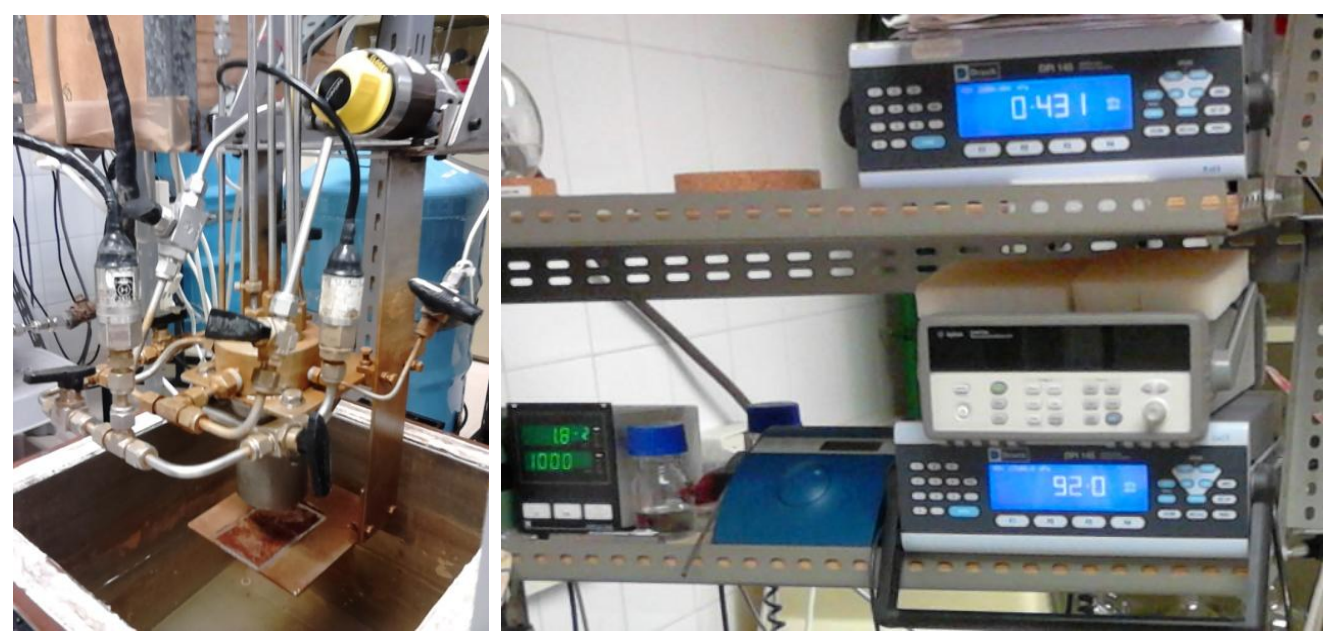

Figura 2.4. Sistema de la medida de presión mediante transductores de presión de medida directa.

El equipo utilizado es un indicador multifunción de presión marca Druck, modelo DPI 145, al que se le conecta un sensor de presión, modelo PDCR-910-1422, calibrado en el rango de presiones de 0.035 a 2 bar. Para presiones de 2 a 20 bar se ha instalado un segundo indicador de la misma marca y modelo. Ambos medidores de presión están calibrados para medidas comprendidas en un rango de temperaturas de $40{ }^{\circ} \mathrm{C}$ a $120{ }^{\circ} \mathrm{C}$. La incertidumbre relativa expandida $(k=2)$ es de $0.05 \%$.

\subsubsection{Manómetro Paroscientific de cuarzo resonante.}

Para la medida de solubilidades de aminas con $\mathrm{CO}_{2}$ a altas presiones se requiere la instalación de un tercer tipo de medidor. Este es un medidor de presión marca Paroscientific, modelo 42K-101 conectado a un indicador de la misma marca, modelo 735. Se conecta externamente mediante un tubo capilar a la celda de medida, midiendo a temperatura ambiente, siendo apto para presiones de hasta 13.8 MPa (Figura 2.5). En estos sistemas, al estar compuesta la fase vapor por $\mathrm{CO}_{2}$, no se presentan problemas de condensación en el medidor.

Como mejora añadida, se monitorizó en continuo este equipo con el fin de observar cuando se alcanza el equilibrio en la medida de la solubilidad del $\mathrm{CO}_{2}$ en solubilidades acuosas de aminas. 


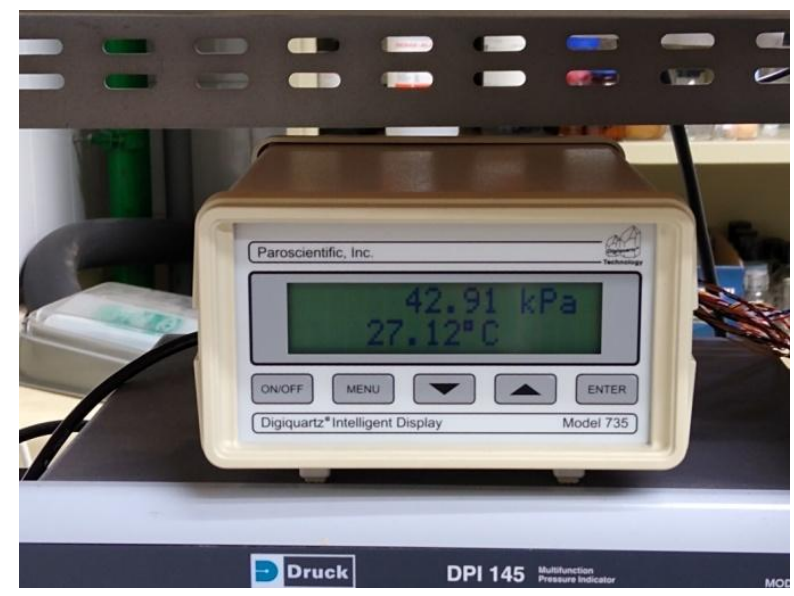

Figura 2.5. Sistema de medida de presión mediante Paroscientific.

\subsubsection{Sistema de medida y control de la temperatura.}

La celda de equilibrio se encuentra inmersa en un baño termostático de gran estabilidad. La temperatura del baño se controla y monitoriza mediante un controlador, que permite fijar el punto de consigna, y una termorresistencia de platino Pt100 para la lectura de la temperatura. La termorresistencia es calibrada y trazable a patrones nacionales con una incertidumbre expandida $(k=2)$ de $20 \mathrm{mK}$. El fluido de trabajo empleado es agua para las temperaturas de $313.15 \mathrm{~K}$ y $333.15 \mathrm{~K}$ ó aceite de silicona para las temperaturas de 353.15 K y $393.15 \mathrm{~K}$.

El baño termostático es de la marca Hart Scientific modelo 6020, en la tabla 2.3. se pueden ver las características principales.

Tabla 2.3. Características del baño termostático.

Volumen del fluido de trabajo

Intervalo de temperatura

Estabilidad con agua a $25^{\circ} \mathrm{C}$

Estabilidad con agua a $60^{\circ} \mathrm{C}$

Gradientes de temperatura
$25 \mathrm{~L}$

$20-200{ }^{\circ} \mathrm{C}$

$\pm 0.0005^{\circ} \mathrm{C}$

$\pm 0.001{ }^{\circ} \mathrm{C}$

$\pm 0.01^{\circ} \mathrm{C} \max$

\subsubsection{Sistema de vacío.}

Para evitar la existencia de otros compuestos en el equipo o gases no condensables como el aire, se hace necesaria la instalación de un sistema de vacío. El sistema evacua todo el equipo hasta niveles de vacío de 0.5 - 0.6 Pa en la entrada de la bomba, antes de proceder al llenado de los cilindros y la celda de equilibrio. El circuito de vacío dispone de una trampa de nitrógeno líquido, donde condensarán todos los residuos que pudiera haber en el circuito y así evitar que lleguen a la bomba, pudiendo llegar en caso contrario a dañarla. 
Otra razón para disponer de este sistema de vacío es la necesidad de una presión de referencia lo suficientemente baja $(\leq 1 \mathrm{~Pa})$ como para poder medir presiones absolutas con el medidor de presión tipo Bourdon.

Se utiliza una bomba de vacío rotativa de dos etapas marca Edwards, modelo E2M8, con un flujo volumétrico nominal de $9.5 \mathrm{~m}^{3} / \mathrm{h}$ y una presión final de $0.025 \mathrm{~Pa}$.

\subsection{Desgasificación de los compuestos puros.}

Una de las fuentes de error más importantes de este método de medida es la debida a una incompleta desgasificación de los compuestos puros, debido a la posible presencia de otros compuestos no deseables. Dado que no existe ningún método general de comprobación de una desgasificación completa, recurriremos a la experiencia, a la repetibilidad de las pruebas y a la comparación de las presiones de vapor de los compuestos puros medidos con los datos disponibles en la bibliografía.

Mediante la desgasificación se consigue la eliminación de los compuestos más volátiles y los gases no condensables de un líquido. Estos compuestos están en muy baja concentración y deben ser eliminados. El proceso consiste en una rectificación a vacío con reflujo total, de modo que existe un enriquecimiento en compuestos volátiles en la cabeza de la columna, siendo eliminados estos a través de una línea de vacío. Este método de desgasificación está basado en el propuesto por Van Ness et al. [9], con algunas modificaciones.

En la Figura 2.6. se puede ver el equipo utilizado para la desgasificación, que consta de tres elementos principales: un calderín, una columna de relleno y un condensador. El calderín es un matraz esférico de $1000 \mathrm{~mL}$ de capacidad con una llave de cierre, conectado a la columna de relleno mediante una unión con junta tórica.

La columna de relleno tiene una longitud de $500 \mathrm{~mm}$, un diámetro interno de $20 \mathrm{~mm}$ y un volumen de relleno de $160 \mathrm{~mL}$. La columna se conecta al resto de elementos de la misma manera que el calderín. El relleno está formado por esferas de vidrio de $3-4 \mathrm{~mm}$ de diámetro.

El condensador, con una longitud de $500 \mathrm{~mm}$, se conecta en la parte superior de la columna de relleno mediante una unión con junta tórica, y está conectado al sistema de vacío a través de un capilar y una válvula. El capilar es de $30 \mathrm{~mm}$ de longitud y $<0.5 \mathrm{~mm}$ de diámetro, limitando el flujo de gases y vapores de la cabeza de la columna. El condensador está también unido al sistema de vacío a través de una segunda línea de mayor diámetro, con una válvula de corte que permite la evacuación completa del condensador y la columna de relleno. 


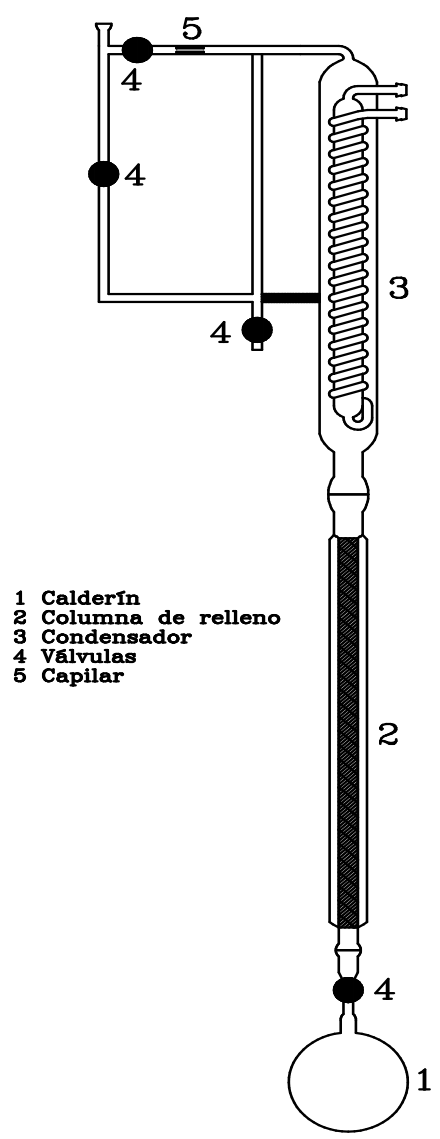

Figura 2.6. Esquema del equipo utilizado para la desgasificación de los compuestos puros.

El procedimiento para la desgasificación de los compuestos puros consiste en sumergir el matraz en un baño de ultrasonidos durante 24 horas.

La bomba de vacío empleada en este equipo es una bomba Edwards, modelo E2M5, con un flujo volumétrico nominal de $5.6 \mathrm{~m}^{3} / \mathrm{h}$ y una presión final de $0.025 \mathrm{~Pa}$.

El baño de ultrasonidos es de la marca Branson 3210, modelo DTH-3210, con una frecuencia de ultrasonidos de $47 \mathrm{kHz}$ y $130 \mathrm{~W}$ de potencia.

\subsection{Reducción termodinámica de los datos de equilibrio líquido-vapor.}

Un sistema aislado está en equilibrio cuando sus propiedades macroscópicas no varían en el tiempo, ni tienen tendencia alguna a variar. En un estado de equilibrio, las propiedades son independientes del tiempo y de la historia previa del sistema. La descripción de este estado se realiza mediante el conocimiento de las variables del sistema, de acuerdo con la regla de las fases y el teorema de Duhem.

Para un sistema aislado donde coexisten varias fases, el sistema está en equilibrio si la temperatura, presión y concentración de cada una de las fases no presentan tendencia al 
cambio. Esto queda matemáticamente representado con la igualdad de los potenciales químicos de cada componente en cada una de las fases.

$$
\mu_{i}^{\alpha}=\mu_{i}^{\beta}=\cdots=\mu_{i}^{\omega}
$$

También podemos decir que varias fases están en equilibrio a la misma temperatura y presión cuando la fugacidad de cada componente es la misma en todo el sistema.

$$
f_{i}^{\alpha}=f_{i}^{\beta}=\cdots=f_{i}^{\omega}
$$

En el caso del equilibrio líquido-vapor de mezclas multicomponentes, la expresión anterior se reduce a:

$$
f_{i}^{l}\left(x_{i}, p, \mathrm{~T}\right)=f_{i}^{v}\left(y_{i}, p, \mathrm{~T}\right)
$$

Donde la fugacidad de cada componente en cada una de las fases es función de la presión, temperatura y de la composición de cada una de las fases $\left(x_{i}\right.$, la fracción molar de la fase líquida e $y_{i}$, la fracción molar de la fase vapor).

La resolución del problema del equilibrio líquido-vapor puede plantearse empleando diversas combinaciones de variables:

1. Punto de burbuja:

- Cálculo de $T$ e $y_{i}$, a partir de $p$ y $x_{i}$.

- Cálculo de $p$ e $y_{i}$, a partir de $T$ y $x_{i}$.

2. Punto de rocío:

- Cálculo de $T$ y $x_{i}$, a partir de $p$ e $y_{i}$.

- Cálculo de $p$ y $x_{i}$, a partir de $T$ e $y_{i}$.

Los procedimientos de cálculo son iterativos y se comienza sustituyendo en la ecuación 2.3, la fugacidad de la fase líquida, $f_{i}^{l}$, por su relación con el coeficiente de actividad, $\gamma_{i}$, y la fugacidad de la fase vapor, $f_{i} v$, se determina en función del coeficiente de fugacidad, $\widehat{\phi}_{i}$.

$$
x_{i} \cdot \gamma_{i} \cdot f_{i}=y_{i} \cdot \widehat{\phi}_{l} \cdot p
$$

Donde $f_{i}$ representa la fugacidad del componente i puro a la presión y temperatura de la mezcla, $\widehat{\phi}_{l}$ es el coeficiente de fugacidad del mismo componente en la fase vapor y $\gamma_{i}$ es el coeficiente de actividad. El cálculo de la fugacidad se realiza a través de la definición de su valor para un componente puro en el estado de vapor.

$$
d G_{i}=\mathrm{RT} d \ln f_{i} \text { ( } \mathrm{T} \text { constante) }
$$

La dependencia con la presión de la fugacidad del líquido se calcula teniendo en cuenta la dependencia de la energía de Gibbs con la presión a través del volumen, dando como resultado: 


$$
d \ln f_{i}=\frac{V_{i}}{\mathrm{RT}} d p
$$

La integración de la expresión anterior, desde el estado de líquido saturado al de líquido comprimido nos da:

$$
\ln \frac{f_{i}}{f_{i}^{\text {sat }}}=\frac{1}{\mathrm{RT}} \int_{p_{i}^{s a t}}^{p} V_{i} d p
$$

Como el volumen molar de la fase líquida, $\mathrm{V}_{\mathrm{i}}$, es una función muy débil de la presión a temperaturas muy por debajo de la temperatura crítica, se obtiene una muy buena aproximación considerando el volumen molar como una constante e igual también al volumen del líquido saturado. Sustituyendo $f_{i}^{\text {sat }}=\phi_{i}^{\text {sat }} p_{i}^{\text {sat }}$ y resolviendo para $f_{i}$, obtenemos:

$$
f_{i}=\phi_{i}^{s a t} p_{i}^{s a t} \exp \frac{\mathrm{V}_{\mathrm{i}}\left(p-p_{i}^{s a t}\right)}{\mathrm{RT}}
$$

Donde esta exponencial se conoce como factor de Poynting.

Sustituyendo en la ecuación 2.4 el valor de $f_{i}$ obtenido en la ecuación 2.8, se obtiene la ecuación de partida para la resolución del problema del equilibrio líquido-vapor.

$$
x_{i} \cdot \gamma_{i} \cdot p_{i}^{s a t}=y_{i} \cdot \Phi_{i} \cdot p
$$

Con

$$
\Phi_{i}=\frac{\widehat{\phi}_{l}}{\phi_{i}^{s a t}} \exp \left[-\frac{\mathrm{V}_{\mathrm{i}}\left(p-p_{i}^{\text {sat }}\right)}{\mathrm{RT}}\right]
$$

De la ecuación 2.9 se obtiene la Ley de Raoult tomándose los coeficientes de actividad y de fugacidad como ideales. Calculando estos coeficientes podremos obtener el comportamiento de las mezclas reales.

\subsubsection{Calculo de los coeficientes de fugacidad y de actividad.}

El cálculo de los coeficientes de fugacidad se realiza mediante ecuaciones de estado que definen el comportamiento real de la fase de vapor, mientras que los coeficientes de actividad se pueden obtener a partir de los datos experimentales.

Para este trabajo, se ha utilizado la ecuación del virial truncada en el segundo término para representar el comportamiento de la fase vapor. De esta manera tenemos que:

$$
\begin{gathered}
\widehat{\phi}_{l}=\exp \frac{p \overline{B_{l}}}{\mathrm{R} T} \\
\phi_{i}^{\text {sat }}=\exp \frac{p_{i}^{s a t} B_{i i}}{\mathrm{R} T}
\end{gathered}
$$

Donde: 


$$
\begin{gathered}
\bar{B}_{l}=B_{i i}+\frac{1}{2} \sum_{k} \sum_{l} y_{k} y_{l}\left(2 \delta_{k i}-\delta_{k l}\right) \\
\delta_{k i}=\delta_{i k}=2 B_{k i}-B_{k k}-B_{i i} \\
\delta_{k l}=\delta_{l k}=2 B_{k l}-B_{k k}-B_{l l}
\end{gathered}
$$

Los coeficientes del virial de los componentes puros, $B_{i i}, B_{k k}, \ldots$ y los coeficientes cruzados, $B_{i k}$, se pueden obtener a través de una correlación (Tsonopoulos) [10]. Los coeficientes del virial se calculan a partir de las propiedades críticas de los compuestos puros $\left(\mathrm{p}_{\mathrm{C}}, \mathrm{T}_{\mathrm{C}}, \mathrm{w}\right)$.

Sustituyendo las ecuaciones 2.11 y 2.12 en la ecuación 2.10 da como resultado:

$$
\Phi_{i}=\exp \frac{B_{i i}\left(p-p_{i}^{s a t}\right)+p_{2}^{\frac{1}{2}} \sum_{k} \sum_{l} y_{k} y_{l}\left(2 \delta_{k i}-\delta_{k l}\right)}{\mathrm{R} T}
$$

Los coeficientes de actividad se calculan a través de modelos de correlación para la energía de Gibbs de exceso, $G^{E} / \mathrm{R} T$, mediante la siguiente ecuación:

$$
\ln \gamma_{i}=\left[\frac{\partial\left(n G^{E} / \mathrm{R} T\right)}{\partial n_{i}}\right]_{p, T, n_{j}}
$$

Considerando que, para mezclas líquidas, $G^{E}$ tiene una débil dependencia con la presión, para el caso de datos a temperatura constante, se pueden proponer expresiones para la energía de Gibbs de exceso solo dependientes de la composición de la mezcla.

$$
\frac{G^{E}}{\mathrm{RT}}=\mathrm{g}\left(x_{1}, x_{2}, \ldots, x_{n}\right)
$$

Una vez conocidos los coeficientes de actividad y las fugacidades, se evalúa la presión total de la mezcla y la composición de la fase vapor, $y_{i}$, mediante un proceso iterativo, a través de las siguientes ecuaciones:

$$
\begin{gathered}
p=\sum_{i} \frac{x_{i} \gamma_{i} p_{i}^{\text {sat }}}{\Phi_{i}} \\
y_{i}=\frac{x_{i} \gamma_{i} p_{i}^{\text {sat }}}{\Phi_{i} p}
\end{gathered}
$$

\subsubsection{Cálculo de la energía de Gibbs de exceso.}

Las expresiones utilizadas como modelo para la función $G^{E} / \mathrm{R} T$ y por extensión al resto de funciones de exceso, son funciones empíricas o semiempíricas que incluyen ciertos parámetros característicos de la naturaleza de los componentes de la mezcla y de las condiciones del entorno. Estos parámetros se determinan a partir de medidas experimentales, que permiten, una vez obtenidos, la correlación de la mezcla en todo el intervalo de composiciones e incluso la predicción en otras condiciones no muy alejadas de las utilizadas durante las medidas experimentales. 
En los siguientes apartados se presentarán los distintos modelos usados para obtener la expresión $G^{E} / \mathrm{R} T$ en función de la composición de la fase líquida, $x_{i}$, a una temperatura determinada a partir de los datos experimentales obtenidos del equilibrio líquido-vapor. Las correlaciones utilizadas en este trabajo y que a continuación se describirán son las siguientes:

- Ecuación de Margules.

- Modelo de Wilson.

- Modelo NRTL.

- Modelo UNIQUAC.

\subsubsection{Ecuación de Margules.}

Es una de las ecuaciones más antiguas y ampliamente utilizadas por su buen comportamiento (Margules 1895) [11]. El trabajo de Margules fue anterior a la introducción de los conceptos de fugacidad y coeficientes de actividad, pero su ecuación propuesta equivale a expresar los coeficientes de actividad, para sistemas binarios o la función $G^{E} / x_{1} X_{2} \mathrm{R} T$ como una serie de potencias de la composición $x_{1}$.

Esta ecuación está basada en el uso de relaciones polinómicas, esto tiene como ventaja una gran flexibilidad para el ajuste de datos de equilibrio líquido-vapor. Para este trabajo, se ha realizado el ajuste de los sistemas binarios mediante la ecuación de Margules de hasta seis parámetros donde solamente fueron necesarios ajustar cuatro parámetros. A continuación, se puede ver la expresión de la función de Gibbs de exceso.

$$
\frac{G^{E}}{x_{1} x_{2} \mathrm{R} T}=\mathrm{A}_{21} x_{1}+\mathrm{A}_{12} x_{2}-\left(\lambda_{21} x_{1}+\lambda_{12} x_{2}\right) x_{1} x_{2}+\left(\eta_{21} x_{1}+\eta_{12} x_{2}\right) x_{1}^{2} x_{2}^{2}
$$

Donde $A_{21}, A_{12}, \lambda_{21}, \lambda_{12}, \eta_{21}$ y $\eta_{12}$ son los parámetros de ajuste de la ecuación de Margules.

En el caso de este tipo de ecuaciones tiene un escaso fundamento teórico, no hay una base racional para su extensión a sistemas multicomponentes.

\subsubsection{Modelo de Wilson.}

Wilson [12] propone una ecuación que combina el modelo desarrollado por Flory [13] y Huggins [14] (1942) con el concepto de composición local. Según este, una mezcla no puede considerarse, desde el punto de vista microscópico, como homogénea, si la composición difiere de unos puntos a otros. En el modelo de Wilson se tiene en cuenta la energía de interacción intermolecular y también las diferencias de tamaño y la distribución espacial de las moléculas presentes en la mezcla. Sustituye las fracciones globales en volumen por fracciones locales en volumen, que tienen lugar debido a las diferentes energías de interacción molecular. Wilson definió empíricamente la fracción local en volumen, $\zeta_{\mathrm{i}}$ como: 


$$
\zeta_{i}=\frac{x_{i} V_{i L} \exp \left(-\frac{\mathrm{a}_{\mathrm{ii}}}{\mathrm{RT}}\right)}{\sum_{j} x_{j} V_{j L} \exp \left(-\frac{\mathrm{a}_{\mathrm{ij}}}{\mathrm{RT}}\right)}
$$

Donde $a_{i j}$ representa la energía de interacción entre moléculas "i-j" $\left(\mathrm{a}_{\mathrm{ij}}=\mathrm{a}_{\mathrm{ji}} ; \mathrm{a}_{\mathrm{ii}} \neq \mathrm{a}_{\mathrm{jij}}\right)$ y $\mathrm{V}_{\mathrm{iL}}$ es el volumen molar del compuesto " $\mathrm{i}$ " en estado líquido. Las fracciones locales en volumen, o concentraciones locales, $\zeta_{\mathrm{i}}$, están definidas en términos probabilísticos utilizando la función de distribución de Boltzmann de energías. Introduciendo el nuevo concepto de composición en el modelo de Flory-Huggins para mezclas atérmicas $\left(\mathrm{H}^{\mathrm{E}}=0\right)$, se obtiene la siguiente expresión generalizada para sistemas multicomponentes de la función de Gibbs molar de exceso:

$$
\frac{G^{E}}{\mathrm{RT}}=-\sum_{i} x_{i} \ln \left(\sum_{j} x_{j} \Lambda_{\mathrm{ij}}\right)
$$

Definiendo los parámetros ajustables como:

$$
\Lambda_{\mathrm{ij}}=\frac{V_{j L}}{V_{i L}} \exp \left(\frac{-\lambda_{\mathrm{ij}}}{\mathrm{RT}}\right)
$$

Siendo $\lambda_{\mathrm{ij}}=\mathrm{a}_{\mathrm{ij}}-\mathrm{a}_{\mathrm{ii}}$ y cumpliéndose que $\Lambda_{\mathrm{ii}}=\Lambda_{\mathrm{ij}}=1$.

Los coeficientes de actividad se obtienen sustituyendo en la ecuación 2.14 la expresión 2.19, quedando de la siguiente manera:

$$
\ln \gamma_{k}=1-\ln \sum_{j=1}^{N} x_{j} \Lambda_{\mathrm{kj}}-\sum_{i=1}^{N} \frac{x_{i} \Lambda_{\mathrm{ik}}}{\sum_{j=1}^{N} x_{j} \Lambda_{\mathrm{ij}}}
$$

El desarrollo del modelo de Wilson para sistemas binarios da la siguiente expresión:

$$
\frac{G^{E}}{\mathrm{R} T}=-x_{1} \ln \left(x_{1}+\Lambda_{12} x_{2}\right)-x_{2} \ln \left(\Lambda_{21} x_{1}+x_{2}\right)
$$

A partir de la cual se pueden obtener los dos coeficientes de actividad:

$$
\begin{aligned}
& \ln \gamma_{1}=-\ln \left(x_{1}+\Lambda_{12} x_{2}\right)+x_{2}\left(\frac{\Lambda_{12}}{x_{1}+\Lambda_{12} x_{2}}-\frac{\Lambda_{21}}{\Lambda_{21} x_{1}+x_{2}}\right) \\
& \ln \gamma_{2}=-\ln \left(\Lambda_{21} x_{1}+x_{2}\right)+x_{1}\left(\frac{\Lambda_{12}}{x_{1}+\Lambda_{12} x_{2}}-\frac{\Lambda_{21}}{\Lambda_{21} x_{1}+x_{2}}\right)
\end{aligned}
$$

Este modelo es capaz de representar el comportamiento de mezclas multicomponentes utilizando únicamente parámetros de interacción binarios $a_{i j}$, que pueden tratarse como independientes de la temperatura. Esta característica, junto con los buenos resultados que se obtienen en la representación del comportamiento de los coeficientes de actividad para mezclas polares y no polares, constituyen sus principales ventajas.

Sin embargo, este modelo no es capaz de predecir la inmiscibilidad, además de no poder representar máximos y mínimos en los coeficientes de actividad frente a la composición. 


\subsubsection{Modelo NRTL.}

Renon y Prausnitz (1968) [15], establecieron un modelo que utiliza el concepto de composición local, similar al de Wilson, y la teoría de dos líquidos de Scott (1956) [16]. El modelo NRTL (Non-Random-Two-Liquid) es aplicable para la correlación de sistemas multicomponentes en equilibrio líquido-líquido, líquido-vapor y líquido-líquido-vapor.

La fracción molar local se calcula como:

$$
x_{i j}=\frac{x_{i} \exp \left(-\alpha_{\mathrm{ji}} \tau_{\mathrm{ji}}\right)}{\sum_{k} x_{k} \exp \left(-\alpha_{\mathrm{ki}} \tau_{\mathrm{ki}}\right)}
$$

En donde $\tau_{\mathrm{ji}}=\left(g_{j i}-g_{i i}\right) / \mathrm{R} T$ y $g_{i i}$ representa la energía de interacción entre el par de moléculas i-j (semejante al parámetro $\mathrm{a}_{\mathrm{ij}}$ en la ecuación de Wilson).

La expresión para la energía de Gibbs de exceso para una mezcla líquida es:

$$
\frac{G^{E}}{\mathrm{RT}}=\sum_{i} x_{i} \frac{\sum_{j} \tau_{\mathrm{ji}} G_{j i} x_{j}}{\sum_{k} G_{k i} x_{k}}
$$

Donde $\mathrm{G}_{\mathrm{ji}}=\exp \left(-\alpha_{\mathrm{ji}} \tau_{\mathrm{ji}}\right)$.

Este modelo introduce el parámetro $\alpha_{\mathrm{ij}}=\alpha_{\mathrm{ji}}$ o parámetro de no distribución al azar, el cual se le asigna un valor a priori siguiendo unas reglas en función de las especies i y j, de acuerdo con la polaridad y el grado de asociación de los componentes de la mezcla, o bien se puede utilizar como un tercer parámetro de ajuste. Sin embargo, este parámetro es una constante empírica y no puede atribuírsele un significado físico.

La expresión de los coeficientes de actividad para el caso general de un sistema multicomponentes resulta:

$$
\ln \gamma_{i}=\frac{\sum_{j=1}^{N} \mathrm{\tau}_{\mathrm{ji}} \mathrm{G}_{\mathrm{ji}} x_{j}}{\sum_{i=1}^{N} \mathrm{G}_{\mathrm{li}} x_{l}}+\sum_{j=1}^{N} \frac{x_{j} \mathrm{G}_{\mathrm{ij}}}{\sum_{j=1}^{N} \mathrm{G}_{\mathrm{lj}} x_{l}}\left(\tau_{i j}-\frac{\sum_{n=1}^{N} x_{n} \tau_{\mathrm{nj}} \mathrm{G}_{\mathrm{nj}}}{\sum_{l=1}^{N} \mathrm{G}_{\mathrm{lj}} x_{l}}\right)
$$

La particularización de la expresión general de $G^{E} / \mathrm{R} T$ para el caso de un sistema binario proporciona la siguiente ecuación:

$$
\frac{G^{E}}{\mathrm{R} T}=x_{1} x_{2}\left(\frac{\tau_{21} \mathrm{G}_{21}}{x_{1}+x_{2} \mathrm{G}_{21}}+\frac{\tau_{12} \mathrm{G}_{12}}{x_{1} \mathrm{G}_{12}+x_{2}}\right)
$$

Que, también particularizando para el binario, permite obtener los coeficientes de actividad:

$$
\begin{aligned}
& \ln \gamma_{1}=x_{2}^{2}\left[\tau_{21}\left(\frac{\mathrm{G}_{21}}{x_{1}+x_{2} \mathrm{G}_{21}}\right)^{2}+\frac{\tau_{12} \mathrm{G}_{12}}{\left(x_{1} \mathrm{G}_{12}+x_{2}\right)^{2}}\right] \\
& \ln \gamma_{2}=x_{1}^{2}\left[\tau_{12}\left(\frac{\mathrm{G}_{12}}{x_{1} \mathrm{G}_{12}+x_{2}}\right)^{2}+\frac{\tau_{21} \mathrm{G}_{21}}{\left(x_{1}+x_{2} \mathrm{G}_{21}\right)^{2}}\right]
\end{aligned}
$$

Su principal desventaja con respecto a los modelos de Wilson y UNIQUAC estriba en que se trata de una ecuación con tres parámetros y no dos como los citados anteriormente. 
Respecto al modelo de Margules presenta la ventaja de poder ser aplicado a sistemas multicomponentes por medio, únicamente, de parámetros binarios.

\subsubsection{Modelo UNIQUAC.}

Abrams y Prausnitz (1975) [17] adoptaron la teoría de dos líquidos y el concepto de composición local para desarrollar la ecuación UNIQUAC (UNIversal QUAsi-Chemical) de carácter simétrico. Este modelo establece que la energía de Gibbs de exceso se puede dividir en dos contribuciones: una que tiene en cuenta las diferencias en el tamaño y forma de las moléculas (parte combinatoria), y una segunda en la que se consideran las interacciones energéticas entre ellas (parte residual).

La expresión que proponen para la función de Gibbs molar de exceso generalizada para sistemas multicomponentes es de la forma:

$$
\frac{G^{E}}{\mathrm{RT}}=\sum_{i} x_{i} \frac{\varphi_{i}}{x_{i}}+\frac{\mathrm{z}}{2} \sum_{i} \mathrm{q}_{\mathrm{i}} x_{i} \ln \frac{\vartheta_{i}}{\varphi_{i}}-\sum_{i} \mathrm{q}_{\mathrm{i}} x_{i} \ln \left(\sum_{j} \vartheta_{j} \tau_{\mathrm{ji}}\right)
$$

Donde:

$$
\begin{gathered}
\vartheta_{i}=\frac{\mathrm{q}_{\mathrm{i}} x_{i}}{\sum_{j} \mathrm{q}_{\mathrm{j}} x_{j}} \text { Es la fracción superficial del componente i. } \\
\varphi_{i}=\frac{\mathrm{r}_{\mathrm{i}} x_{i}}{\sum_{j} \mathrm{r}_{\mathrm{j}} x_{j}} \text { Es la fracción volumétrica del componente i. } \\
\mathrm{Z}=10 \text { Es el número de coordinación. } \\
-\tau_{\mathrm{ji}}=\exp \left(-\frac{\mathrm{u}_{\mathrm{ij}}-\mathrm{u}_{\mathrm{ii}}}{\mathrm{R} T}\right) \text { Son los parámetros ajustables del modelo, siendo - } \mathrm{u}_{\mathrm{ij}} \text { el parámetro } \\
\text { de interacción entre los componentes } \mathrm{j}-\mathrm{i} .\left(\mathrm{u}_{\mathrm{ji}}=\mathrm{u}_{\mathrm{ij}}\right)
\end{gathered}
$$

Los parámetros $r_{i}$ y $q_{i}$ de los compuestos puros son, respectivamente, medidas de los volúmenes moleculares de van der Waals y áreas de la superficie molecular. Estos parámetros se calculan como suma de las contribuciones de los grupos funcionales que forman la molécula del compuesto.

$$
\mathrm{r}_{\mathrm{i}}=\sum_{k} v_{k}^{(i)} \mathrm{R}_{\mathrm{k}} \quad \mathrm{q}_{\mathrm{i}}=\sum_{k} v_{k}^{(i)} \mathrm{Q}_{\mathrm{k}}
$$

Donde $v_{k}^{(i)}$ es el número de grupos del tipo k en la molécula i.

Los coeficientes de actividad para sistemas multicomponentes se expresan teniendo en cuenta la parte combinatoria y la residual.

$$
\ln \gamma_{i}=\ln \gamma_{i}^{C}+\ln \gamma_{i}^{R}
$$

Donde la parte combinatorial viene dada por:

$$
\ln \gamma_{i}^{C}=\ln \frac{\varphi_{i}}{x_{i}}+\frac{\mathrm{z}}{2} \mathrm{q}_{\mathrm{i}} \ln \frac{\vartheta_{i}}{\varphi_{i}}+\mathrm{l}_{\mathrm{i}}-\frac{\varphi_{i}}{x_{i}} \sum_{j} x_{j} \mathrm{l}_{\mathrm{j}}
$$




$$
\mathrm{l}_{\mathrm{i}}=\frac{\mathrm{z}}{2}\left(\mathrm{r}_{\mathrm{i}}-\mathrm{q}_{\mathrm{i}}\right)-\left(\mathrm{r}_{\mathrm{i}}-1\right)
$$

Y la parte residual se expresa como:

$$
\ln \gamma_{i}^{R}=\mathrm{q}_{\mathrm{i}}\left[1-\ln \left(\sum_{j=1}^{m} \vartheta_{j} \tau_{\mathrm{ji}}\right)-\sum_{j=1}^{m} \frac{\vartheta_{j} \tau_{\mathrm{ij}}}{\sum_{k=1}^{m} \vartheta_{k} \tau_{\mathrm{kj}}}\right]
$$

Para el caso particular de sistemas binarios la expresión $G^{E} / \mathrm{R} T$ se queda de la siguiente manera:

$$
\frac{G^{E}}{\mathrm{R} T}=x_{1}\left[\ln \frac{\varphi_{1}}{x_{1}}+\frac{\mathrm{q}_{1} \mathrm{z}}{2} \ln \frac{\vartheta_{1}}{\varphi_{1}}-\mathrm{q}_{1} \ln \left(\vartheta_{1}+\vartheta_{2} \tau_{21}\right)\right]+x_{2}\left[\ln \frac{\varphi_{2}}{x_{2}}+\frac{\mathrm{q}_{2} \mathrm{z}}{2} \ln \frac{\vartheta_{2}}{\varphi_{2}}-\mathrm{q}_{2} \ln \left(\vartheta_{1} \tau_{12}+\vartheta_{2}\right)\right](2
$$

Entre las características destacables de este modelo para sistemas multicomponentes indicar que utiliza únicamente parámetros binarios, también tiene una gran capacidad para describir el equilibrio líquido-líquido y la posibilidad de extender los resultados obtenidos para un intervalo moderado de la temperatura. Este modelo sirve de base para el desarrollo del método predictivo UNIFAC [18], en el que se calculan los coeficientes de actividad mediante las contribuciones de los diferentes grupos que constituyen las moléculas en una disolución.

Su principal inconveniente es la complejidad de su expresión, así como la limitación que presenta en la representación de los datos, puesto que a menudo los resultados obtenidos son peores que los derivados de ecuaciones más sencillas.

\subsubsection{Método de Barker.}

El objetivo principal del estudio experimental del equilibrio líquido-vapor de sistemas multicomponentes es el cálculo de la energía de Gibbs molar de exceso a partir de los datos experimentales de presión, temperatura y composición de la fase líquida. Este proceso recibe el nombre de "reducción de datos", y su resultado final es la obtención de una expresión matemática que sea capaz de modelar la dependencia de $G^{E}$ con la composición de la fase líquida. Una vez obtenida la expresión de $G^{E}$ en función $x_{i}$, mediante la ecuación 2.14 podemos calcular los coeficientes de actividad.

Como consecuencia del teorema de Duhem, para la determinación del equilibrio líquidovapor no es necesario el muestreo de las dos fases. Con los datos de presión $(p)$, temperatura $(T)$, y composición de la fase líquida $\left(x_{i}\right)$, en la celda de equilibrio, podemos calcular los valores de la composición de la fase vapor $\left(y_{i}\right)$.

La técnica de medida empleada en este trabajo, al ser un método estático, proporciona un conjunto de datos $\left(x_{i}, p\right)$ para una temperatura determinada. Con esta serie de datos isotermos sólo hay un posible camino para su reducción. La simplicidad de las medidas necesarias para obtener los datos $x_{i}$ y $p$ con una celda de equilibrio estática, hace posible que estos valores puedan ser conocidos con una gran exactitud, con lo que es posible calcular los valores de $y_{i}$. 
El procesamiento de los datos $p$-x es sencillo. Se elige un modelo para $G^{E} / \mathrm{R} T$, y se estiman los parámetros que conducen a valores de la presión calculada lo más cercanos posibles a los valores de presión experimental. Este método se conoce como método de Barker [19]. Los cálculos son iterativos y requieren de un algoritmo de ajuste no lineal, cuya función objetivo (F.O.) es minimizar la suma de las diferencias entre la presión calculada ( $p_{\text {cal }}$ y la determinada experimentalmente $\left(p_{\text {exp }}\right)$ al cuadrado.

$$
\text { F.O. }=\sum(\Delta p)^{2}=\sum\left(p_{\text {cal }}-p_{\text {exp }}\right)^{2}
$$

El proceso de cálculo se inicia con la estimación de la composición de la fase líquida en la celda de equilibrio, sin tener en cuenta la masa de los compuestos que se encuentran en la fase vapor y que posteriormente se calculará. Conociendo para cada componente el volumen inyectado en la celda de equilibrio $\left(V_{i}\right)$, su densidad $\left(\rho_{i}\right)$ y su peso molecular (PM), se puede calcular el número de moles y la fracción molar de cada componente puro.

$$
n_{i}=\frac{V_{i} \rho_{i}}{\mathrm{PM}_{(\mathrm{i})}} \quad x_{i}=\frac{n_{i}}{\sum n_{i}}
$$

De este modo se pueden evaluar los coeficientes de actividad mediante la ecuación 2.14, y la presión con la ecuación 2.16. Se observa una dependencia exponencial de la presión con la composición.

El programa de cálculo está realizado en una hoja Excel, que incorpora un programa de optimización para la resolución de problemas lineales y no lineales, el programa Microsoft Excel Solver. El algoritmo de cálculo empleado corresponde al método de Newton, y la estimación de las derivadas parciales de la función objeto se realiza mediante diferenciaciones progresivas.

El esquema de iteración para la reducción de los datos experimentales se muestra en la Figura 2.7. El procedimiento de cálculo se inicia leyendo la temperatura ( $T$ ), todos los datos de las presiones experimentales $\left(p_{\text {exp }}\right)$, las composiciones de la fase líquida $\left(x_{i}\right)$ y las constantes necesarias: volumen molar de los líquidos $\left(V_{i}^{L}\right)$, coeficientes del virial $\left(B_{i i}, B_{i j}\right)$ y la presión de saturación de los compuestos puros $\left(p_{i}^{\text {sat }}\right)$ o las constantes de la ecuación de Antoine para calcularla. Con todos estos datos y a través del modelo de correlación elegido ya es posible calcular los coeficientes de actividad $\left(\gamma_{i}\right)$ para cada punto experimental, a partir de una estimación inicial de los parámetros del modelo. El cálculo de $\left(\Phi_{i}\right)$, para cada punto experimental, no puede realizarse al no conocer la composición de la fase vapor $\left(y_{i}\right)$, por lo que inicialmente se le asignan un valor de $\Phi=1$. Con estos valores y a través de las ecuaciones 2.14 y 2.16 ya podemos estimar la presión calculada ( $p_{c a l}$ y la composición de la fase vapor $\left(y_{i}\right)$ y utilizarlos para el cálculo de $\Phi_{i}$ con la ecuación 2.9.

A continuación, se evalúa nuevamente $p_{c a l}$ e $y_{i}$. El cálculo iterativo se realiza hasta que la diferencia de las presiones entre dos iteraciones sucesivas esté por debajo de un valor de tolerancia, $\delta p<\varepsilon$, donde $\varepsilon$ puede ser del orden de $0.01 \mathrm{~Pa}$. Después de esto podemos obtener la función objetivo (F.O.) a minimizar (ecuación 2.36). La minimización de esta F.O. se realiza mediante el programa Solver, estimando los parámetros de los modelos que conducen a la mejor solución de la F.O. 


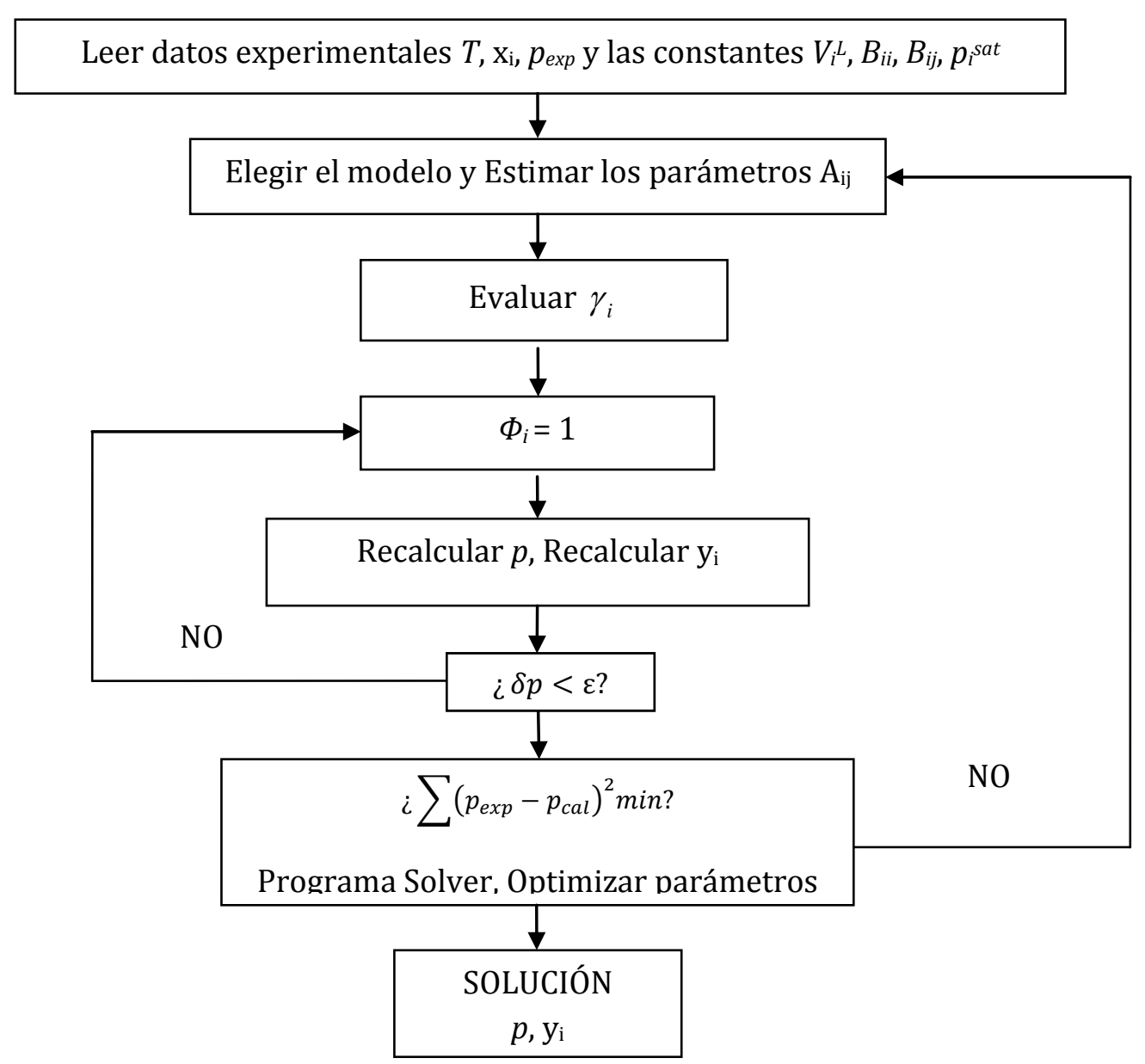

Figura 2.7. Diagrama de bloques para la reducción de datos.

En este diagrama de flujo se ha supuesto que la composición de la fase líquida es inicialmente conocida, como primera aproximación, se supone que la fase líquida tiene como composición la relación existente entre las masas totales inyectadas. Una vez concluido el proceso de cálculo, se obtiene la composición de la fase vapor $\left(y_{i}\right)$ para cada punto experimental.

Con los valores calculados de $y_{i}$ y el volumen total de la celda de equilibrio, corregimos la composición de la fase líquida, inicialmente calculada como si toda la masa inyectada estuviera en la fase líquida. A continuación, se corregirá la masa introducida de los componentes para las medidas realizadas con el medidor de presión diferencial tipo Bourdon, teniendo en cuenta que después de cada medida se evacúa el conducto de unión de la celda de equilibrio con la celda de presión diferencial. No siendo necesario para las medidas con los nuevos transductores.

El volumen molar de la fase vapor para una mezcla gaseosa $\left(V^{V}\right)$, se determina la ecuación de estado del Virial: 


$$
\begin{gathered}
V^{V}=\frac{\mathrm{R} T}{p}+B \\
B=\sum_{i} \sum_{j} y_{i} y_{j} B_{i j}
\end{gathered}
$$

Una vez calculadas las composiciones de fase líquida corregida, se procede a reducir nuevamente los datos experimentales mediante el proceso anteriormente descrito. De la reducción de los datos obtenemos los parámetros del modelo escogido en cada caso, la presión calculada $\left(p_{c a l}\right)$, la composición de la fase líquida corregida $\left(x_{i}\right)$ y la composición de la fase vapor $\left(y_{i}\right)$.

Para finalizar, calculamos los parámetros estadísticos de la desviación cuadrática media $(r m s)$ y máxima diferencia entre la presión medida y la presión calculada, max $\left|p_{\text {exp }}-p_{\text {cal }}\right|$. Dándonos una idea de la bondad del ajuste realizado.

$$
r m s(\Delta p)=\sqrt{\frac{\sum\left(p_{\text {exp }}-p_{\text {cal }}\right)^{2}}{n-m}}
$$

Donde $n$ es el número de puntos experimentales y $m$ el número de parámetros de la ecuación de ajuste. A partir de estos datos es posible evaluar la calidad de la reducción de los datos y elegir el modelo más adecuado para el cálculo de la energía de Gibbs de exceso.

\subsection{Referencias.}

[1] H. C. Van Ness, M. M. Abbott. "Classical thermodynamics of nonelectrolyte solutions with applications to phase equilibria". Mc-Graw Hill, New York (1982).

[2] R. E. Gibbs and H. C. Van Ness. "Vapor-liquid equilibria from total-pressure measurements. A new apparatus". Industrial and Engineering Chemistry Fundamentals. Vol. 11, (1972) 410- 413.

[3] D. P. Dielsi, R. B. Patel, M. M. Abbott, H. C. Van Ness. "Excess thermodynamic functions for ternary systems: 3. Total pressure data and GE for acetone-acetonitrile-methyl acetate at $50{ }^{\circ} \mathrm{C}$. Journal of Chemical \& Engineering Data. Vol. 23, (1978) 242-245.

[4] E. A. Montero. "Bases termodinámicas para el desarrollo de nuevas mezclas combustibles líquidas conteniendo compuestos oxigenados". Universidad de Valladolid. Tesis doctoral (1996).

[5] J. J. Segovia. "Investigación termodinámica del equilibrio de fases fluidas de mezclas ternarias constituidas por aditivos oxigenados MTBE y metanol con hidrocarburos de sustitución para el desarrollo de nuevas gasolinas sin plomo". Universidad de Valladolid. Tesis doctoral (1997).

[6] C. R. Chamorro. "Investigación experimental de las propiedades termodinámicas del equilibrio de fases fluidas de mezclas ternarias de los aditivos oxigenados para gasolinas sin plomo DIPE y TAME con hidrocarburos tipo y con los alcoholes metanol e isopropanol". Universidad de Valladolid. Tesis doctoral (1998). 
[7] C. Alonso. "Investigación experimental de propiedades termodinámicas de aditivos oxigenados tipo éter y alcohol en mezclas líquidas binarias y ternarias de hidrocarburos de sustitución para el desarrollo de nuevas gasolinas sin plomo". Universidad de Valladolid. Tesis doctoral (2001).

[8] A. Moreau. "Caracterización termodinámica de combustibles ambientalmente sostenibles: determinación experimental del equilibrio líquido-vapor y entalpías de exceso de mezclas de interés para la formulación de biogasolinas". Universidad de Valladolid. Tesis doctoral (2014).

[9] H. C. Van Ness, M. M. Abbott. "A procedure for rapid degassing of liquids". Industrial and Engineering Chemistry Fundamentals. Vol. 17, (1978) 66.

[10] C. Tsonopoulos. "An empirical correlation of second virial coefficients". AIChE Journal. Vol. 20, (1974) 263-272.

[11] M. Margules. "Über die zusammensetzung der gesättigten dämpfe von mischungen. sitzber". Kais. Akad. Wiss. Wien, Math.-Naturwiss. Klasse II. Vol. 104, (1895) 1243-1278.

[12] G. M. Wilson. "Vapor-liquid equilibrium XI. A new expression for the excess free energy of mixing". J. Amer. Chem. Soc. Vol. 86, (1964) 127-130.

[13] P. J. Flory. "Thermodynamics of high polymer solutions". Journal of Chemical and Physics. Vol. 10, (1942) 51.

[14] M. L. Huggins. "Thermodynamic properties of solution of long-chain compounds". Annals of the New York Academy of Sciences. Vol. 42, (1942) 1.

[15] H. Renon, J. M. Prausnitz. "Local compositions in thermodynamic excess functions for liquid mixtures". AIChE Journal. Vol. 14, (1968) 135-144.

[16] R. L. Scott. "Solutions of nonelectrolytes". Annual Review of Physical Chemistry. Vol. 7, (1956) 43-66.

[17] D. S. Abrams, J. M. Prausnitz. "Statistical thermodynamics of liquid mixtures: A new expression for the excess gibbs energy of partly or completely miscible systems". AIChE Journal. Vol. 21, (1975) 116-128.

[18] A. Fredenslund, R. L. Jones, J.M. Prausnitz. "Group-contribution estimation of activity coefficients in nonideal liquid mixtures". AIChE Journal. Vol. 21, (1975) 1086-1098.

[19] J. A. Barker. "Determination of activity coefficients from total pressure measurements". Australian Journal of Chemistry. Vol. 6, (1953) 207-210. 

Capítulo 3

\section{RESULTADOS EXPERIMENTALES DEL EQUILIBRIO LÍQUIDO-VAPOR ISOTERMO}





\subsection{Introducción.}

En este capítulo se presentan, en tablas y gráficas, los resultados experimentales del equilibrio líquido-vapor (VLE) obtenidos mediante una técnica estática e isoterma descrita anteriormente. En ellas se presentan la presión $p$, la composición de la fase líquida $x_{i}$, la composición de la fase vapor $y_{i}$, y la energía de Gibbs de exceso $G^{E}$ obtenida con los diferentes modelos.

Este estudio se ha realizado para la caracterización del comportamiento termodinámico de los subproductos de la producción de la ciclohexanona. Este es un producto intermedio en la producción de la caprolactana y el ácido adípico. Siendo la caprolactama un producto químico ampliamente utilizado para la producción de Nylon-6. Además, el ácido adípico es un precursor importante para la fabricación del Nylon-6,6 [1, 2].

El nylon es la materia prima para la producción de fibras, con aplicaciones textiles e industriales. El nylon es un compuesto altamente elástico, resistente a la abrasión y autolubricante. La mayoría de las aplicaciones del nylon son en fibras para ropa, alfombras, muebles y cuerdas. Además, el nylon es un sustituto de los materiales metálicos en rodamientos, engranajes y piezas donde se necesita resistencia y baja fricción.

La 2-heptanona y el hexanal son subproductos de la producción de ciclohexanona, que afectan a la calidad del producto. La 2-heptanona tiene un punto de ebullición similar a la ciclohexanona, por lo que la extracción de sus trazas se convierte en un gran problema de separación en los procesos de destilación convencionales [3]. El objetivo principal de este trabajo es explorar el comportamiento termodinámico de las mezclas (Ciclohexanona +2 Heptanona y Ciclohexanona + Hexanal) con el fin de resolver el problema de su separación.

El conocimiento termodinámico del equilibrio líquido-vapor (presión, temperatura y composición de las fases) para estas mezclas se realiza mediante una técnica de equilibrio líquido-vapor isoterma. Midiendo varias isotermas (de $313.15 \mathrm{~K}$ a $393.15 \mathrm{~K}$ ), podemos averiguar cuál es la mejor temperatura para aumentar el rendimiento del proceso de purificación de la ciclohexanona. Además, si la mezcla presenta un comportamiento azeotrópico, se puede estudiar el efecto de la temperatura en el azeótropo.

Para la puesta a punto del equipo y la validación de las medidas, se midió el sistema binario 2-Pentanol + Ciclohexano a $313.15 \mathrm{~K}$.

Los sistemas medidos han sido:

Binario B0: 2-Pentanol (1) + Ciclohexano (2) a 313.15 K.

Binario B1: Ciclohexanona (1) + 2-Heptanona (2) a 313.15 K, 333.15 K, 353.15 K, 393.15 K.

Binario B2: Ciclohexanona (1) + Hexanal (2) a 313.15 K, 333.15 K, 353.15 K, 393.15 K. 
Para medir estos sistemas binarios se realizaron dos ramas, primero se parte de uno de los compuestos puros i, y se va añadiendo volúmenes conocidos del segundo compuesto j, para barrer las distintas composiciones hasta una $x_{j}=0.6$. La segunda rama se mide en sentido inverso, se parte del segundo compuesto puro $\mathrm{j}$, y se añade el primer compuesto, hasta una $x_{i}=0.6$, solapándose las ramas entre las composiciones de $x=0.4$ a $x=0.6$, comprobando que son coincidentes y asegurando la calidad de las medidas.

\subsection{Relación de los productos utilizados.}

Los productos utilizados tienen una alta pureza para la obtención de unos resultados fiables. En la Tabla 3.1. se muestran los productos utilizados, la procedencia y la pureza dada por los certificados de análisis. No se ha utilizado ningún método de purificación adicional.

Los compuestos puros son desgasificados previamente mediante una columna de rectificación a vacío empleando un baño de ultrasonidos para la agitación.

Tabla 3.1. Características de los productos utilizados.

\begin{tabular}{llll}
\hline Compuesto & Fórmula & Marca comercial & Pureza (GC\%) \\
\hline 2-Pentanol & $\mathrm{C}_{5} \mathrm{H}_{12} \mathrm{O}$ & Sigma-Aldrich & $\geq 98 \%$ \\
Ciclohexano & $\mathrm{C}_{6} \mathrm{H}_{12}$ & Sigma-Aldrich & $\geq 99.5 \%$ \\
2-Heptanona & $\mathrm{C}_{7} \mathrm{H}_{14} \mathrm{O}$ & Sigma-Aldrich & $\geq 99.6 \%$ \\
Ciclohexanona & $\mathrm{C}_{6} \mathrm{H}_{10}(=\mathrm{O})$ & Sigma-Aldrich & $\geq 99.99 \%$ \\
Hexanal & $\mathrm{CH}_{3}\left(\mathrm{CH}_{2}\right)_{4} \mathrm{CHO}$ & Sigma-Aldrich & $\geq 99.8 \%$ \\
\hline
\end{tabular}

Una de las formas de comprobar el funcionamiento del equipo y la correcta desgasificación de los compuestos, es la medida de las presiones de vapor de los compuestos puros a las diferentes temperaturas. La comparación de los valores experimentales con la bibliografía se muestra en la tabla 3.2 . 
Tabla 3.2. Comparación de los resultados de la presión de vapor de los compuestos puros a las diferentes temperaturas obtenidos experimentalmente, $p^{\text {sat }}{ }_{(m e d)}$ y los valores de la literatura $p^{\text {sat }}{ }_{(i i t)}$.

\begin{tabular}{|c|c|c|c|}
\hline Compuesto & $p^{s a t}($ med) $/ \mathrm{kPa}$ & Temperatura $\left({ }^{\circ} \mathrm{C}\right)$ & $p^{\text {sat }}($ lit) $/ \mathbf{k P a}$ \\
\hline \multirow{7}{*}{ 2-Heptanona } & 1.281 & 40 & $1.268[4]$ \\
\hline & 3.706 & 60 & $3.672^{[4]}$ \\
\hline & & & $3.676^{[5]}$ \\
\hline & 9.288 & 80 & $9.144[4]$ \\
\hline & & & $9.153^{[5]}$ \\
\hline & 40.990 & 120 & $40.357[4]$ \\
\hline & & & $40.385^{[5]}$ \\
\hline \multirow{6}{*}{ Ciclohexanona } & 1.307 & 40 & $1.290[4]$ \\
\hline & 3.583 & 60 & $3.591[4]$ \\
\hline & 8.692 & 80 & $8.677[4]$ \\
\hline & & & $8.60[6]$ \\
\hline & 36.563 & 120 & $36.753^{[4]}$ \\
\hline & & & $36.753[7]$ \\
\hline \multirow{8}{*}{ Hexanal } & 3.604 & 40 & $3.502^{[8]}$ \\
\hline & & & $3.281^{[9]}$ \\
\hline & 9.119 & 60 & $9.082^{[8]}$ \\
\hline & & & $8.558[9]$ \\
\hline & 20.643 & 80 & $20.643[8]$ \\
\hline & & & $19.613[9]$ \\
\hline & 79.036 & 120 & $79.035^{[8]}$ \\
\hline & & & $76.614[9]$ \\
\hline \multirow{2}{*}{ 2-Pentanol } & 2.294 & 40 & $2.260[10]$ \\
\hline & & & $2.281[11]$ \\
\hline \multirow{4}{*}{ Ciclohexano } & 24.575 & 40 & $24.670^{[12]}$ \\
\hline & & & $24.630[13]$ \\
\hline & & & $24.635[14]$ \\
\hline & & & $24.650[15]$ \\
\hline
\end{tabular}




\subsection{Presentación de los resultados experimentales del equilibrio líquido-vapor.}

A continuación, se muestran los datos experimentales obtenidos del equilibrio líquidovapor isotermo para los tres sistemas a las temperaturas de $313.15 \mathrm{~K}, 333.15 \mathrm{~K}, 353.15 \mathrm{~K}$ y $393.15 \mathrm{~K}$. En cada uno de estos sistemas binarios se presentan los datos experimentales de presión total $p$, en función de las composiciones de la fase líquida $x_{i}$, y la fase vapor $y_{i}$ calculada, junto a la función termodinámica de la energía de Gibbs de exceso calculada con el modelo de NRTL.

La información se completa con los datos de los componentes puros, presión de vapor $p_{i}^{\text {sat }}$, volúmenes molares $V_{i}^{L}$ (obtenidos experimentalmente), y los coeficientes del virial de los componentes puros $B_{i i}$ y de la mezcla $B_{i j}$, obtenidos mediante la correlación de Tsonopoulos [16], usando las constantes críticas para cada compuesto puro y su factor de acentricidad [4].

Los datos se ajustan mediante la ecuación de Margules (hasta cuatro parámetros) [17], además de los modelos de Wilson [18], NRTL [19] y UNIQUAC [20], utilizando el método de Barker [21] haciendo mínima la diferencia cuadrática entre los valores experimentales y los calculados. Junto a los valores de los parámetros para los diferentes modelos, se incluye también la desviación cuadrática media de la presión residual (ecuación 2.41) y la máxima desviación entre la presión experimental y la presión calculada, max $\left|p_{\text {exp }}-p_{\text {cal }}\right|$. Por otra parte, se muestra la composición del azeótropo y su presión de vapor para cada uno de los modelos en los sistemas binarios donde este aparece. 


\subsubsection{Binario B0: 2-Pentanol (1) + Ciclohexano (2) a 313.15 K.}

Tabla 3.3. Datos experimentales $p$-x-y y energía de Gibbs de exceso calculada mediante la ecuación de Margules para el sistema binario B0: 2-Pentanol (1) + Ciclohexano (2) a $313.15 \mathrm{~K}$.

\begin{tabular}{cccccccc}
$\mathbf{x}_{\mathbf{1}}$ & $\mathbf{\mathbf { y } _ { 1 } , \mathbf { c a l c }}$ & $\mathbf{p} / \mathbf{k P a}$ & $\begin{array}{c}\mathbf{G} \\
\mathbf{J} \cdot \mathbf{m o l}^{\mathbf{1}}\end{array}$ & $\mathbf{x}_{\mathbf{1}}$ & $\mathbf{\mathbf { y } _ { 1 } , \mathbf { c a l c }}$ & $\mathbf{p} / \mathbf{k P a}$ & $\begin{array}{c}\mathbf{G} / \\
\text { J·mol-1 }\end{array}$ \\
\hline 0.0000 & 0.0000 & 24.575 & 0.00 & 0.5010 & 0.0730 & 20.565 & 909.54 \\
0.0497 & 0.0293 & 24.563 & 277.08 & 0.5510 & 0.0790 & 19.753 & 878.11 \\
0.1000 & 0.0377 & 24.330 & 480.17 & 0.5511 & 0.0790 & 19.765 & 877.97 \\
0.1502 & 0.0421 & 24.036 & 628.10 & 0.6008 & 0.0862 & 18.832 & 832.13 \\
0.2004 & 0.0460 & 23.708 & 737.20 & 0.6013 & 0.0863 & 18.834 & 831.61 \\
0.2507 & 0.0501 & 23.328 & 816.88 & 0.6513 & 0.0953 & 17.759 & 771.52 \\
0.3006 & 0.0543 & 22.899 & 872.47 & 0.7013 & 0.1070 & 16.497 & 698.11 \\
0.3489 & 0.0585 & 22.421 & 907.15 & 0.7513 & 0.1225 & 15.008 & 611.88 \\
0.4007 & 0.0631 & 21.879 & 925.43 & 0.8016 & 0.1448 & 13.234 & 512.27 \\
0.4007 & 0.0631 & 21.875 & 925.43 & 0.8513 & 0.1788 & 11.140 & 401.28 \\
0.4508 & 0.0678 & 21.263 & 925.63 & 0.9007 & 0.2391 & 8.672 & 278.77 \\
0.4509 & 0.0678 & 21.259 & 925.62 & 0.9498 & 0.3765 & 5.820 & 145.49 \\
0.5009 & 0.0730 & 20.558 & 909.59 & 1.0000 & 1.0000 & 2.294 & 0.00 \\
\hline
\end{tabular}

Tabla 3.4. Valor experimental de la presión de vapor de los compuestos puros $p_{i}^{\text {sat }}$, volúmenes molares $V_{i}^{L}$, y coeficientes del virial de los puros $B_{i i} y$ de la mezcla $B_{i j}$, para el sistema binario B0: 2-Pentanol (1) + Ciclohexano (2) a $313.15 \mathrm{~K}$.

\begin{tabular}{ccccccc}
\hline $\begin{array}{c}\mathbf{p}_{1}^{\text {sat }} \\
\mathbf{k P a}\end{array}$ & $\begin{array}{c}\mathbf{p}_{2}^{\text {sat }} \\
\mathbf{k P a}\end{array}$ & $\begin{array}{c}\mathbf{V}_{\mathbf{1}}^{\mathrm{L}} \\
\mathbf{c m}^{\mathbf{3}} \cdot \mathbf{m o l}^{-\mathbf{1}}\end{array}$ & $\begin{array}{c}\mathbf{V}_{\mathbf{2}}^{\mathrm{L}} \\
\mathbf{c m}^{\mathbf{3}} \cdot \mathbf{m o l}^{-\mathbf{1}}\end{array}$ & $\begin{array}{c}\mathbf{B}_{\mathbf{1 1}} \\
\mathbf{c m}^{\mathbf{3}} \cdot \mathbf{m o l} \mathbf{- 1}\end{array}$ & $\begin{array}{c}\mathbf{B}_{22} \\
\mathbf{c m}^{\mathbf{3}} \cdot \mathbf{m o l} \mathbf{- 1}\end{array}$ & $\begin{array}{c}\mathbf{B}_{\mathbf{1 2}} \\
\mathbf{c m}^{\mathbf{3}} \cdot \mathbf{m o l}^{-\mathbf{1}}\end{array}$ \\
\hline 2.294 & 24.575 & 111.20 & 111.08 & -2560 & -1508 & -1794 \\
\hline
\end{tabular}

Tabla 3.5. Parámetros de ajuste del sistema binario B0: 2-Pentanol (1) + Ciclohexano (2) $313.15 \mathrm{~K}$.

\begin{tabular}{lcccc}
\hline & Margules & Wilson & NRTL & UNIQUAC \\
\hline $\mathrm{A}_{12}$ & 2.5113 & 0.1210 & 0.6698 & 1.8150 \\
$\mathrm{~A}_{21}$ & 1.1411 & 0.7783 & 1.9306 & 0.2097 \\
$\lambda_{12}$ & 4.4992 & & & \\
$\lambda_{21}$ & 0.6424 & & & \\
$\eta_{12}$ & 5.9819 & & & \\
$\eta_{21}$ & 0.8839 & & & \\
$\alpha$ & & & 0.6372 & \\
$\mathrm{rms} \Delta \mathrm{p} / \mathrm{kPa}$ & 0.031 & 0.137 & 0.092 & 0.264 \\
$\max |\Delta \mathrm{p}| / \mathrm{kPa}$ & 0.057 & 0.263 & 0.194 & 0.521 \\
\hline
\end{tabular}




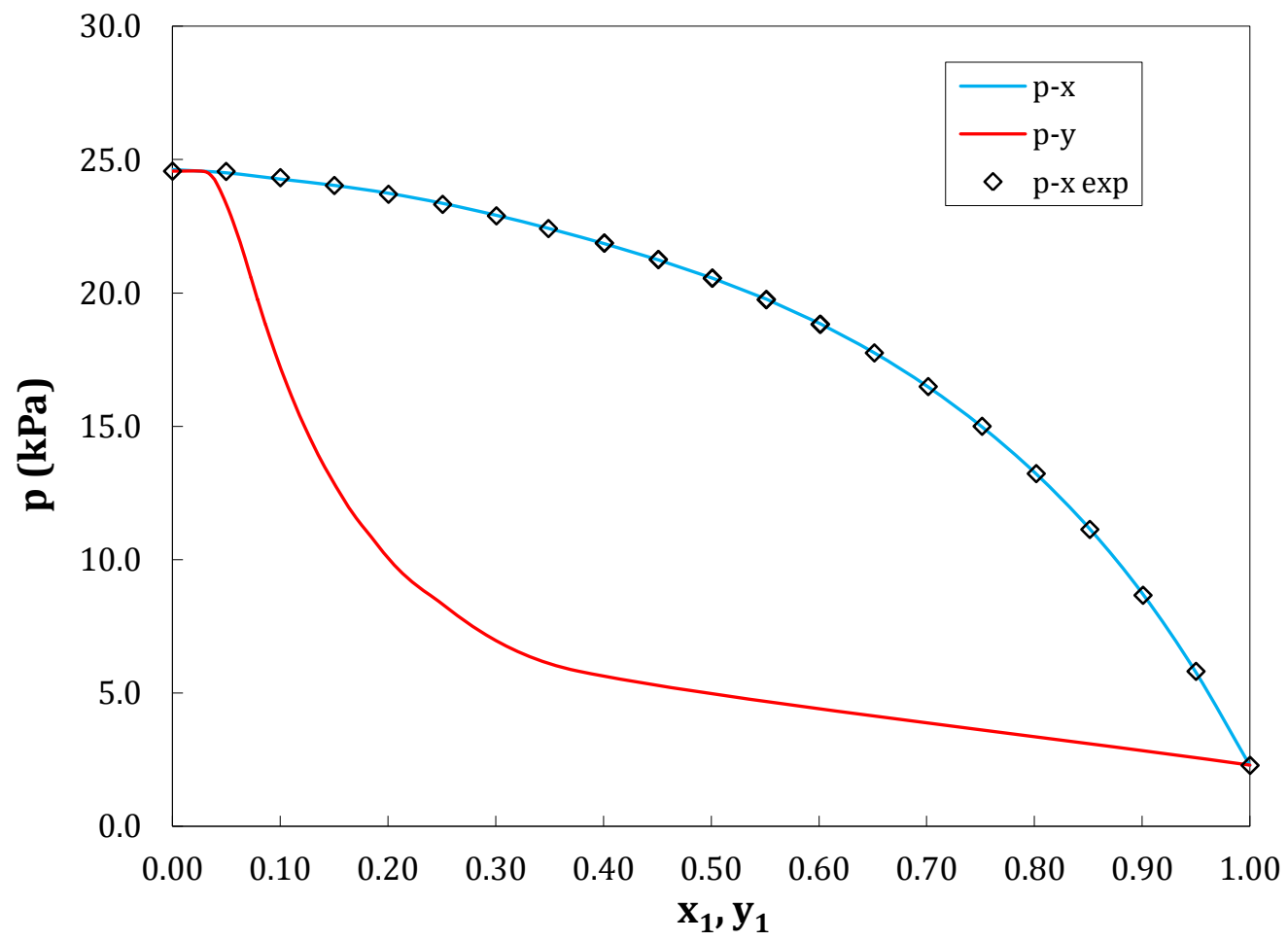

Figura 3.1. Presión de vapor ( $p$ ) en función de las fracciones molares de la fase líquida $x_{1}$ y la fase vapor $y_{1}$, para el sistema binario B0: 2-Pentanol (1) + Ciclohexano (2) a 313.15 K. $(\diamond)$ Datos experimentales. (-) Ajuste mediante la ecuación de Margules.

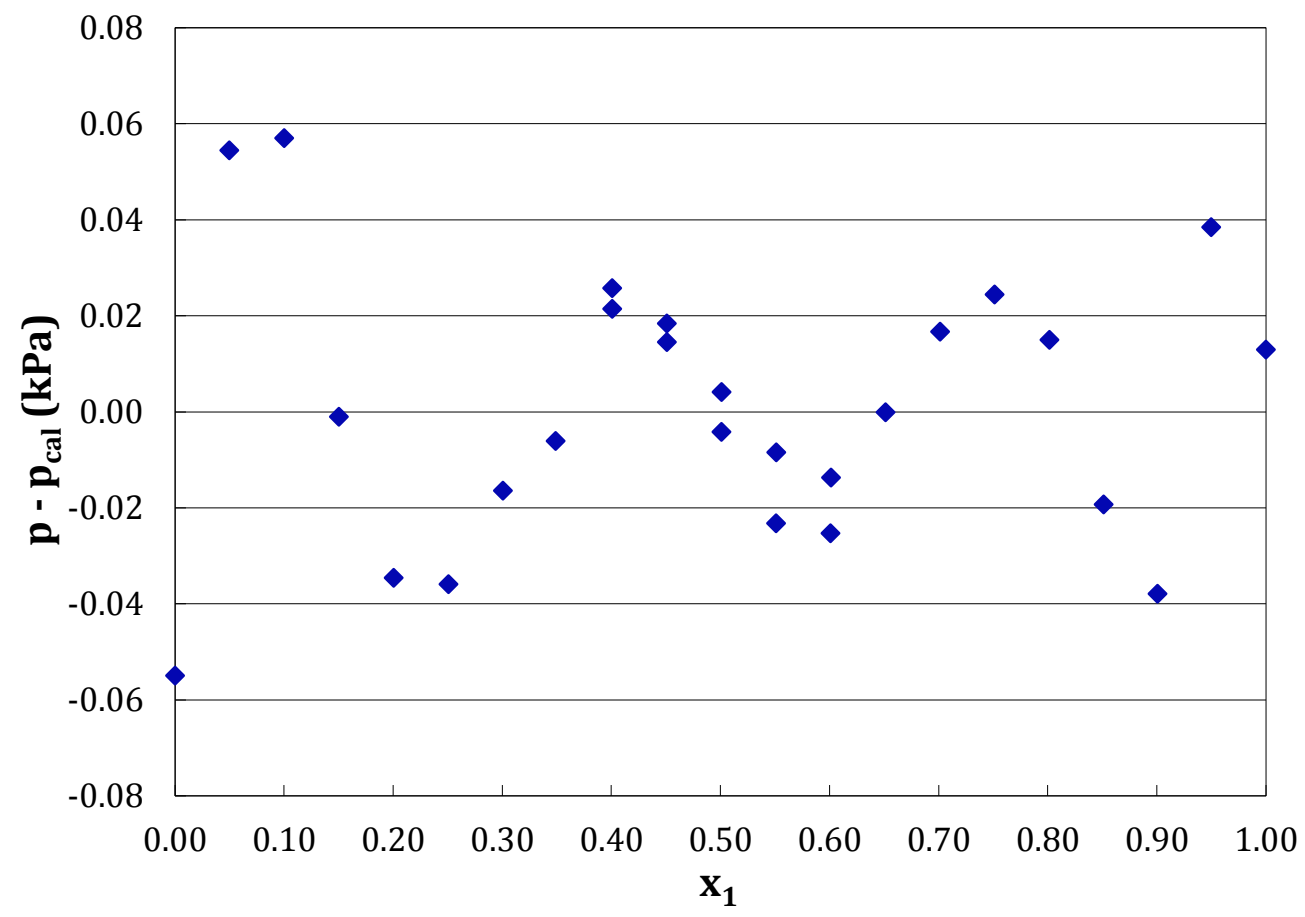

Figura 3.2. Comparación de las diferencias entre la presión experimental (p) y la presión calculada $\left(p_{\text {cal }}\right)$ mediante la ecuación de Margules ( $\bullet$ ), para el sistema binario B0: 2-Pentanol (1) + Ciclohexano (2) $a 313.15 \mathrm{~K}$. 


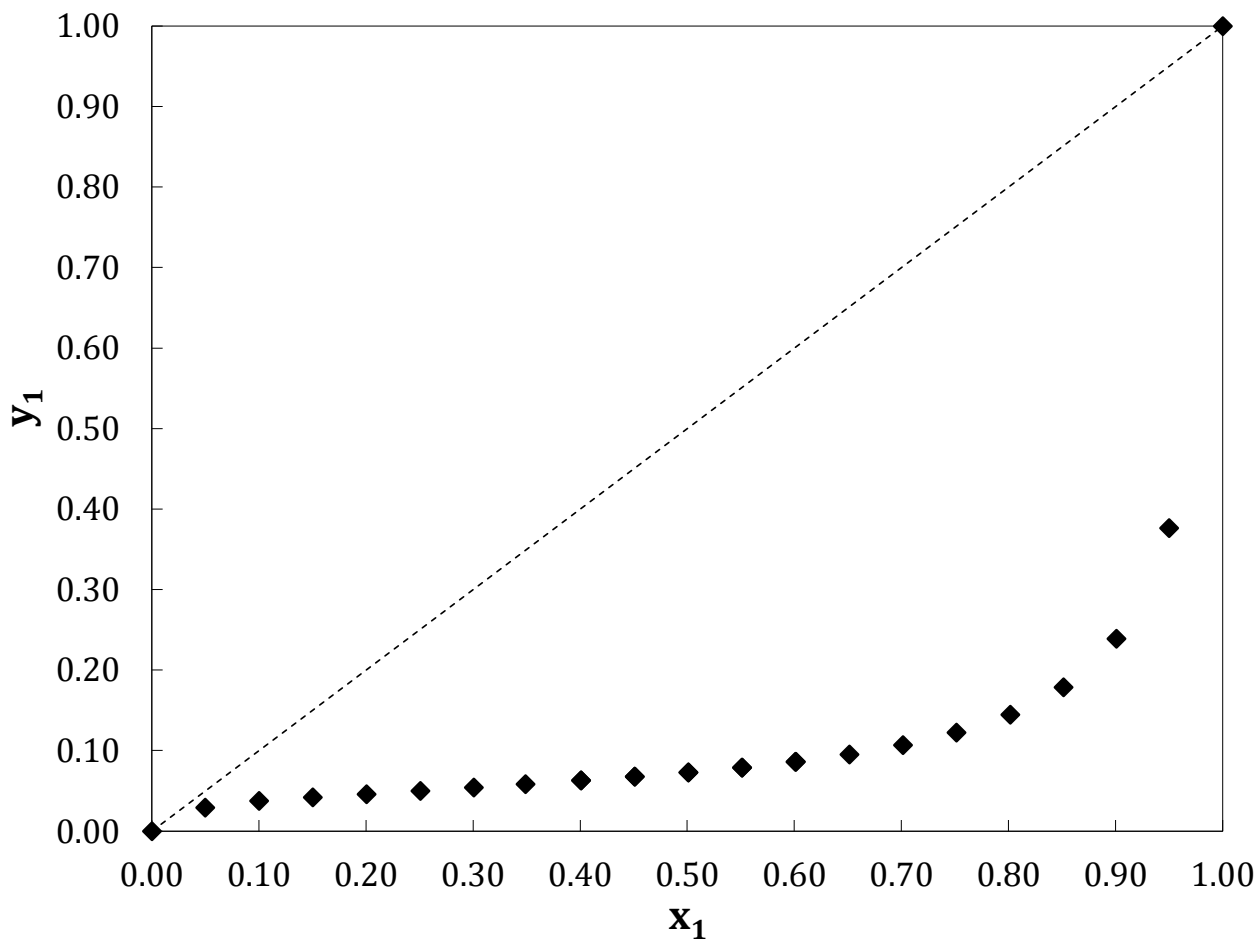

Figura 3.3. Fracción molar de la fase vapor del $\left(y_{1}\right)$ en función de la fracción molar de la fase líquida $\left(x_{1}\right)$ para el sistema binario B0: 2-Pentanol (1) + Ciclohexano (2) a 313.15 K. Datos calculados mediante la ecuación de Margules ( $\bullet$ ).

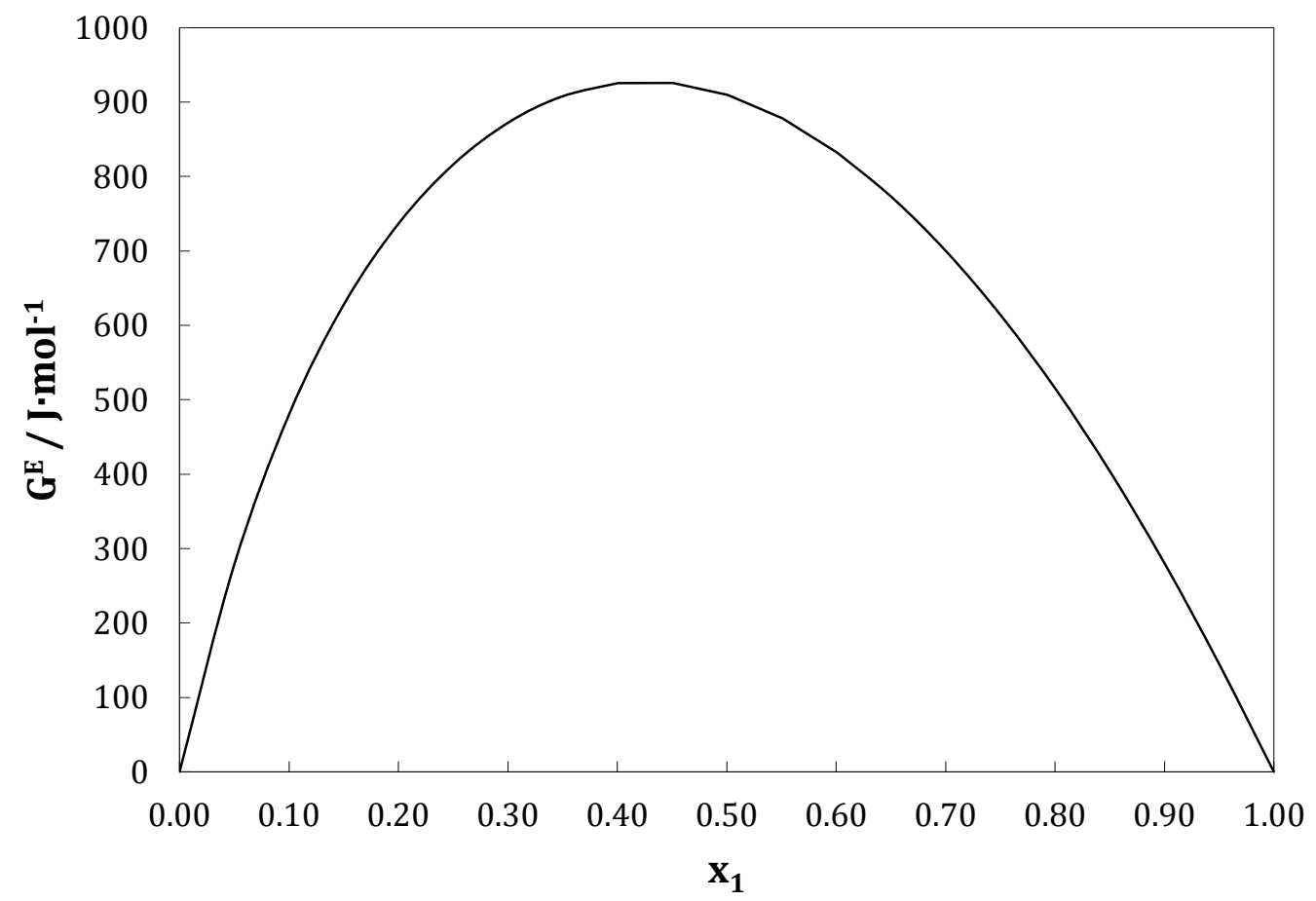

Figura 3.4. Energía de Gibbs de exceso $\left(G^{E}\right)$ en función de la fracción molar de la fase líquida $\left(x_{1}\right)$ para el sistema binario B0: 2-Pentanol (1) + Ciclohexano (2) a $313.15 \mathrm{~K}$. 


\subsubsection{Binario B1: Ciclohexanona (1) + 2-Heptanona (2) a $313.15 \mathrm{~K}$.}

Tabla 3.6. Datos experimentales $p-x-y$ calculados mediante la ecuación de Margules y energía de Gibbs de exceso calculada mediante el modelo NRTL para el sistema binario B1: Ciclohexanona (1) + 2Heptanona (2) a $313.15 \mathrm{~K}$.

\begin{tabular}{cccccccc}
\hline $\mathbf{x}_{\mathbf{1}}$ & $\mathbf{y}_{\mathbf{1}, \text { calc }}$ & $\mathbf{p} / \mathbf{k P a}$ & $\begin{array}{c}\mathbf{G}^{\mathbf{E}} / \\
\mathbf{J} \cdot \mathbf{m o l}-\mathbf{1}\end{array}$ & $\mathbf{x}_{\mathbf{1}}$ & $\mathbf{y}_{\mathbf{1}, \text { calc }}$ & $\mathbf{p} / \mathbf{k P a}$ & $\begin{array}{c}\mathbf{G}^{\mathbf{E}} / \\
\mathbf{J} \cdot \mathbf{m o l}-\mathbf{1}\end{array}$ \\
\hline 0.0000 & 0.0000 & 1.281 & 0.00 & 0.5007 & 0.5042 & 1.324 & 56.81 \\
0.0428 & 0.0484 & 1.289 & 11.70 & 0.5501 & 0.5523 & 1.328 & 54.96 \\
0.0965 & 0.1058 & 1.304 & 24.20 & 0.6002 & 0.6008 & 1.324 & 52.08 \\
0.1415 & 0.1521 & 1.303 & 32.94 & 0.6501 & 0.6490 & 1.320 & 48.27 \\
0.1966 & 0.2072 & 1.306 & 41.62 & 0.6967 & 0.6939 & 1.318 & 43.91 \\
0.2477 & 0.2576 & 1.310 & 47.86 & 0.7504 & 0.7457 & 1.320 & 38.00 \\
0.2981 & 0.3069 & 1.313 & 52.41 & 0.8009 & 0.7945 & 1.320 & 31.65 \\
0.3479 & 0.3555 & 1.316 & 55.46 & 0.8530 & 0.8455 & 1.316 & 24.34 \\
0.3977 & 0.4039 & 1.318 & 57.16 & 0.9045 & 0.8972 & 1.314 & 16.41 \\
0.4486 & 0.4536 & 1.325 & 57.61 & 0.9582 & 0.9535 & 1.306 & 7.44 \\
0.4502 & 0.4551 & 1.321 & 57.60 & 1.0000 & 1.0000 & 1.298 & 0.00 \\
\hline
\end{tabular}

Tabla 3.7. Valor experimental de la presión de vapor de los compuestos puros $p_{i}^{\text {sat }}$, volúmenes molares $V_{i}^{L}, y$ coeficientes del virial de los puros $B_{i i} y$ de la mezcla $B_{i j}$, para el sistema binario B1: Ciclohexanona (1) + 2-Heptanona (2) a 313.15 K.

\begin{tabular}{|c|c|c|c|c|c|c|}
\hline $\begin{array}{l}p_{1}^{\text {sat }} \\
\text { kPa }\end{array}$ & $\begin{array}{l}\mathbf{p}_{2}^{\text {sat }} \\
\mathbf{k P a}\end{array}$ & $\begin{array}{c}\mathrm{V}_{1}^{\mathrm{L}} \\
\mathrm{cm}^{3} \cdot \mathrm{mol}^{-1}\end{array}$ & $\begin{array}{c}\mathrm{V}_{2}^{\mathrm{L}} \\
\mathrm{cm}^{3} \cdot \mathrm{mol}^{-1}\end{array}$ & $\begin{array}{c}\mathrm{B}_{11} \\
\mathrm{~cm}^{3} \cdot \mathrm{mol}^{-1} \\
\end{array}$ & $\begin{array}{c}\mathrm{B}_{22} \\
\mathbf{c m}^{3} \cdot \mathrm{mol}^{-1}\end{array}$ & $\begin{array}{c}\mathrm{B}_{12} \\
\mathrm{~cm}^{3 \cdot} \cdot \mathrm{mol}^{-1} \\
\end{array}$ \\
\hline 1.298 & 1.281 & 105.61 & 142.97 & -2796 & -3571 & -2980 \\
\hline
\end{tabular}

Tabla 3.8. Parámetros de ajuste del sistema binario B1: Ciclohexanona (1) + 2-Heptanona (2) a $313.15 \mathrm{~K}$

\begin{tabular}{lcccc}
\hline & Margules & Wilson & NRTL & UNIQUAC \\
\hline $\mathrm{A}_{12}$ & 0.1532 & 0.6281 & -0.0461 & 1.9409 \\
$\mathrm{~A}_{21}$ & 0.0398 & 1.3520 & 0.1629 & 0.4166 \\
$\lambda_{12}$ & 0.2567 & & & \\
$\lambda_{21}$ & -0.1776 & & & \\
$\alpha$ & & & 2.0778 & \\
$\mathrm{rms} \Delta \mathrm{p} / \mathrm{kPa}$ & 0.003 & 0.003 & 0.004 & 0.003 \\
$\mathrm{max}|\Delta \mathrm{p}| / \mathrm{kPa}$ & 0.009 & 0.009 & 0.009 & 0.009 \\
$\mathrm{x}_{1, \text { azeótropo }}$ & 0.601 & 0.560 & 0.560 & 0.574 \\
$\mathrm{p}_{\text {azeótropo }} / \mathrm{kPa}$ & 1.324 & 1.323 & 1.323 & 1.323 \\
\hline
\end{tabular}




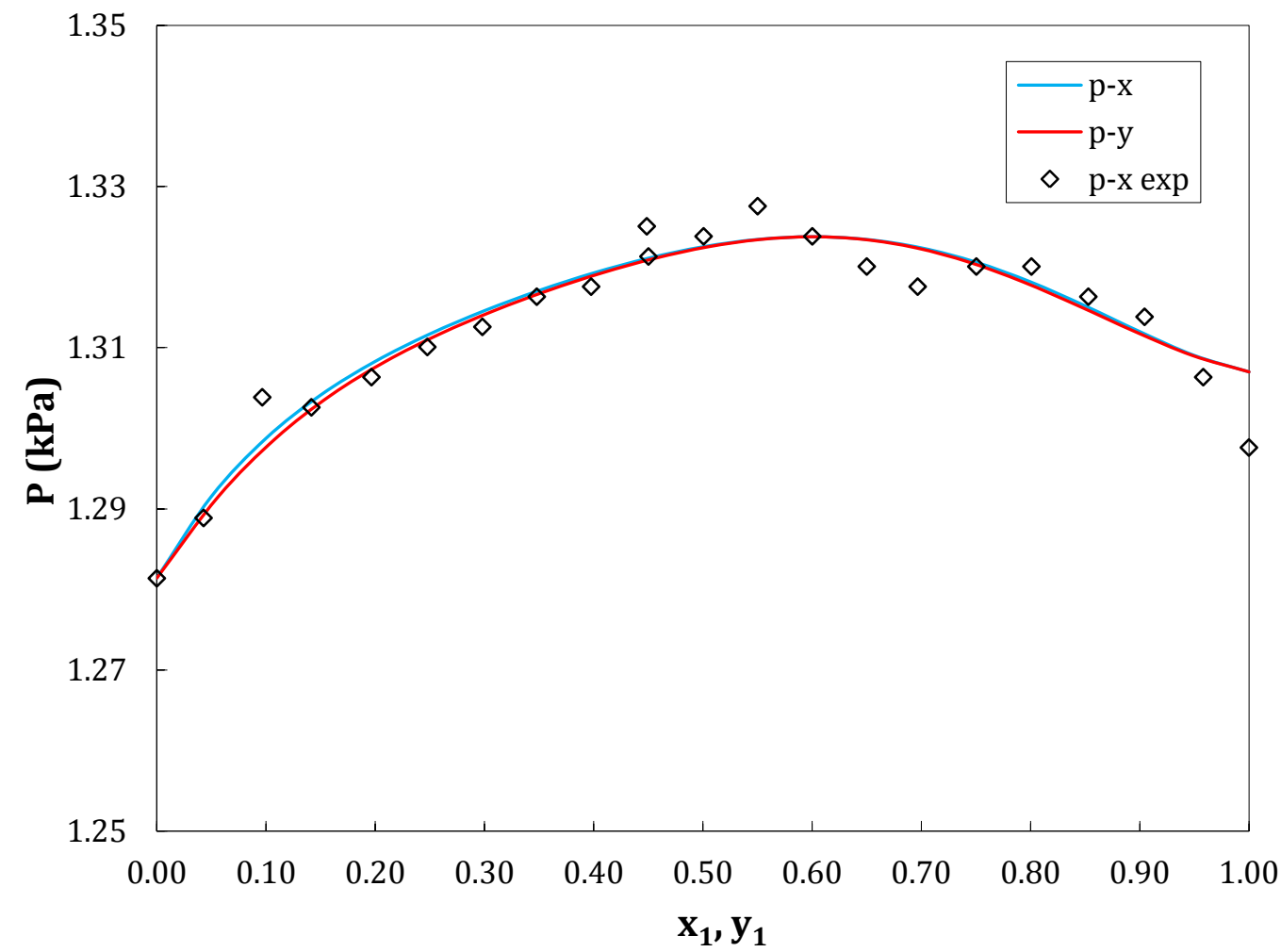

Figura 3.5. Presión de vapor $(p)$ en función de las fracciones molares de la fase líquida $x_{1}$ y la fase vapor $y_{1}$, para el sistema binario B1: Ciclohexanona (1) + 2-Heptanona (2) a 313.15 K. $(\diamond)$ Datos experimentales. (-) Ajuste mediante la ecuación de Margules.

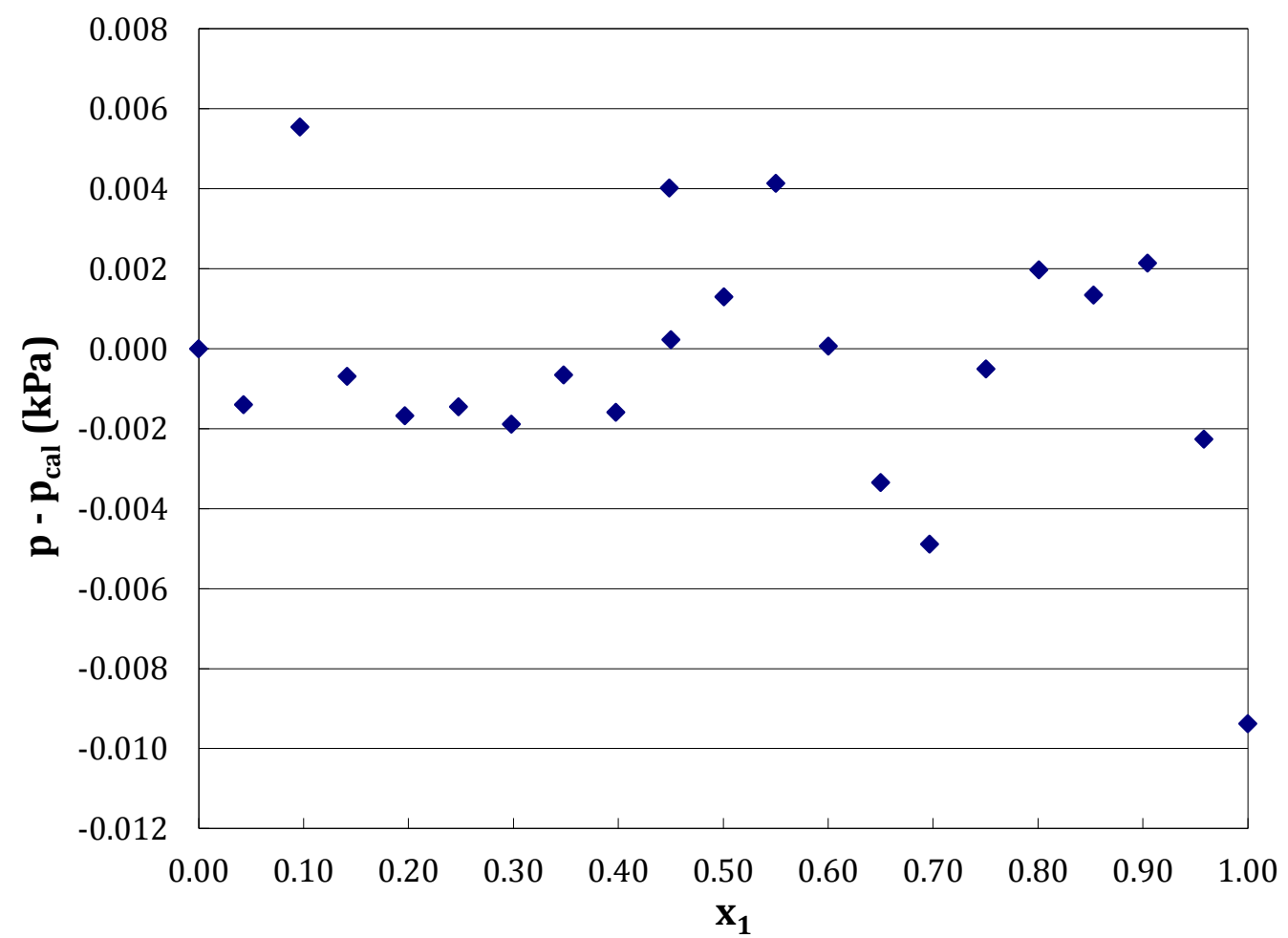

Figura 3.6. Comparación de las diferencias entre la presión experimental (p) y la presión calculada (pal) mediante la ecuación de Margules ( $\diamond$ ), para el sistema binario B1: Ciclohexanona (1) + 2Heptanona (2) a $313.15 \mathrm{~K}$. 


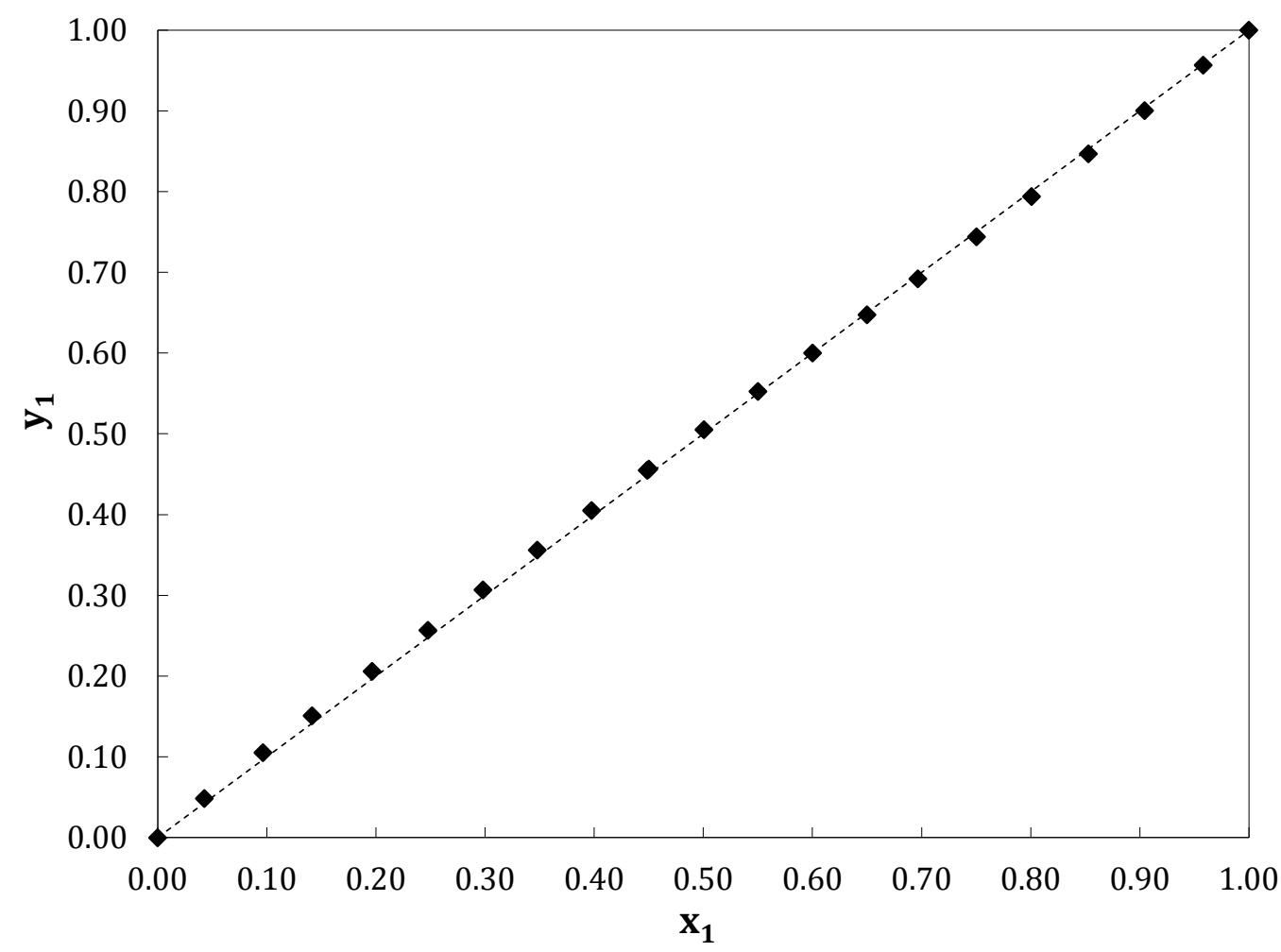

Figura 3.7. Fracción molar de la fase vapor del $\left(y_{1}\right)$ en función de la fracción molar de la fase líquida $\left(x_{1}\right)$ para el sistema binario B1: Ciclohexanona (1) + 2-Heptanona (2) a 313.15 K. Datos calculados mediante la ecuación de Margules ( $\bullet$ ).

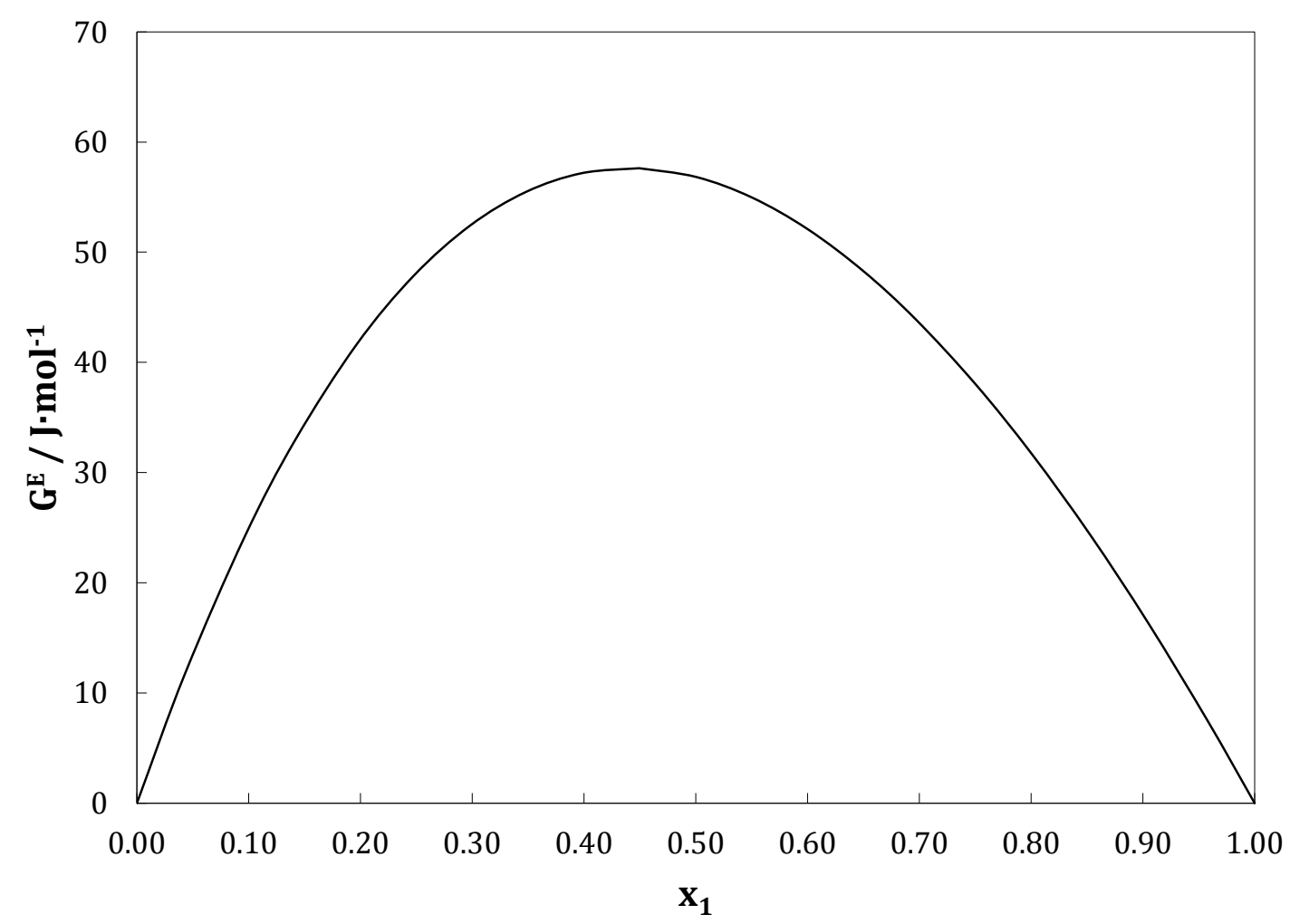

Figura 3.8. Energía de Gibbs de exceso $\left(G^{E}\right)$ en función de la fracción molar de la fase líquida $\left(x_{1}\right)$ para el sistema binario B1: Ciclohexanona (1) + 2-Heptanona (2) a $313.15 \mathrm{~K}$. 


\subsubsection{Binario B1: Ciclohexanona (1) + 2-Heptanona (2) a 333.15 K.}

Tabla 3.9. Datos experimentales $p-x-y$ calculados mediante la ecuación de Margules y energía de Gibbs de exceso calculada mediante el modelo NRTL para el sistema binario B1: Ciclohexanona (1) + 2Heptanona (2) a $333.15 \mathrm{~K}$.

\begin{tabular}{cccccccc}
\hline $\mathbf{x}_{\mathbf{1}}$ & $\mathbf{y}_{\mathbf{1}, \text { calc }}$ & $\mathbf{p} / \mathbf{k P a}$ & $\begin{array}{c}\mathbf{G}^{\mathbf{E}} / \\
\mathbf{J} \cdot \mathbf{m o l}-\mathbf{1}\end{array}$ & $\mathbf{x}_{\mathbf{1}}$ & $\mathbf{y}_{\mathbf{1}, \text { calc }}$ & $\mathbf{p} / \mathbf{k P a}$ & $\begin{array}{c}\mathbf{G}^{\mathbf{E}} / \\
\mathbf{J} \cdot \mathbf{m o l}-\mathbf{1}\end{array}$ \\
\hline 0.0000 & 0.0000 & 3.697 & 0.00 & 0.5503 & 0.5334 & 3.715 & 64.41 \\
0.0472 & 0.0508 & 3.712 & 17.88 & 0.6003 & 0.5825 & 3.707 & 59.70 \\
0.0989 & 0.1054 & 3.726 & 33.96 & 0.6499 & 0.6321 & 3.694 & 54.09 \\
0.1467 & 0.1544 & 3.735 & 45.92 & 0.7001 & 0.6830 & 3.683 & 47.64 \\
0.1988 & 0.2057 & 3.743 & 55.98 & 0.7503 & 0.7344 & 3.670 & 40.54 \\
0.2495 & 0.2539 & 3.751 & 63.11 & 0.8008 & 0.7864 & 3.655 & 32.88 \\
0.2998 & 0.3006 & 3.756 & 67.85 & 0.8503 & 0.8376 & 3.631 & 24.98 \\
0.3495 & 0.3460 & 3.759 & 70.47 & 0.9006 & 0.8902 & 3.613 & 16.70 \\
0.3995 & 0.3916 & 3.763 & 71.25 & 0.9502 & 0.9435 & 3.583 & 8.39 \\
0.4606 & 0.4482 & 3.738 & 69.99 & 1.0000 & 1.0000 & 3.563 & 0.00 \\
0.5000 & 0.4852 & 3.728 & 68.04 & & & & \\
\hline
\end{tabular}

Tabla 3.10. Valor experimental de la presión de vapor de los compuestos puros $p_{i}^{\text {sat }}$, volúmenes molares $V_{i}^{L}$, y coeficientes del virial de los puros $B_{i i} y$ de la mezcla $B_{i j}$, para el sistema binario B1: Ciclohexanona (1) + 2-Heptanona (2) a $333.15 \mathrm{~K}$.

\begin{tabular}{ccccccc}
\hline $\begin{array}{c}\mathbf{p}_{\mathbf{1}}^{\text {sat }} \\
\mathbf{k P a}\end{array}$ & $\begin{array}{c}\mathbf{p}_{\mathbf{2}}^{\text {sat }} \\
\mathbf{k P a}\end{array}$ & $\begin{array}{c}\mathbf{V}_{\mathbf{1}}^{\mathrm{L}} \\
\mathbf{c m}^{\mathbf{3}} \cdot \mathbf{m o l}^{\mathbf{1}}\end{array}$ & $\begin{array}{c}\mathbf{V}_{\mathbf{2}}^{\mathrm{L}} \\
\mathbf{c m}^{\mathbf{3}} \mathbf{\mathbf { m o l } ^ { - 1 }}\end{array}$ & $\begin{array}{c}\mathbf{B}_{\mathbf{1 1}} \\
\mathbf{c m}^{\mathbf{3}} \cdot \mathbf{m o l}^{\mathbf{1}}\end{array}$ & $\begin{array}{c}\mathbf{B}_{22} \\
\mathbf{c m}^{\mathbf{3}} \cdot \mathbf{m o l}^{\mathbf{- 1}}\end{array}$ & $\begin{array}{c}\mathbf{B}_{\mathbf{1 2}} \\
\mathbf{c m}^{\mathbf{3}} \cdot \mathbf{m o l}^{-\mathbf{1}}\end{array}$ \\
\hline 3.563 & 3.697 & 107.70 & 146.22 & -2340 & -2954 & -2484 \\
\hline
\end{tabular}

Tabla 3.11. Parámetros de ajuste del sistema binario B1: Ciclohexanona (1) +2 -Heptanona (2) a $333.15 \mathrm{~K}$.

\begin{tabular}{lcccc}
\hline & Margules & Wilson & NRTL & UNIQUAC \\
\hline $\mathrm{A}_{12}$ & 0.1175 & 0.4928 & -1.7954 & 2.0158 \\
$\mathrm{~A}_{21}$ & 0.0510 & 1.5521 & 2.2306 & 0.3800 \\
$\lambda_{12}$ & -0.1393 & & & \\
$\lambda_{21}$ & 0.0010 & & & \\
$\alpha$ & & & 0.0824 & \\
$\mathrm{rms} \Delta \mathrm{p} / \mathrm{kPa}$ & 0.008 & 0.009 & 0.009 & 0.009 \\
$\mathrm{max}|\Delta \mathrm{p}| / \mathrm{kPa}$ & 0.020 & 0.020 & 0.020 & 0.020 \\
$\mathrm{x}_{1, \text { azeótropo }}$ & 0.311 & 0.276 & 0.283 & 0.266 \\
$\mathrm{p}_{\text {azeótropo } / \mathrm{kPa}}$ & 3.754 & 3.753 & 3.753 & 3.753 \\
\hline
\end{tabular}




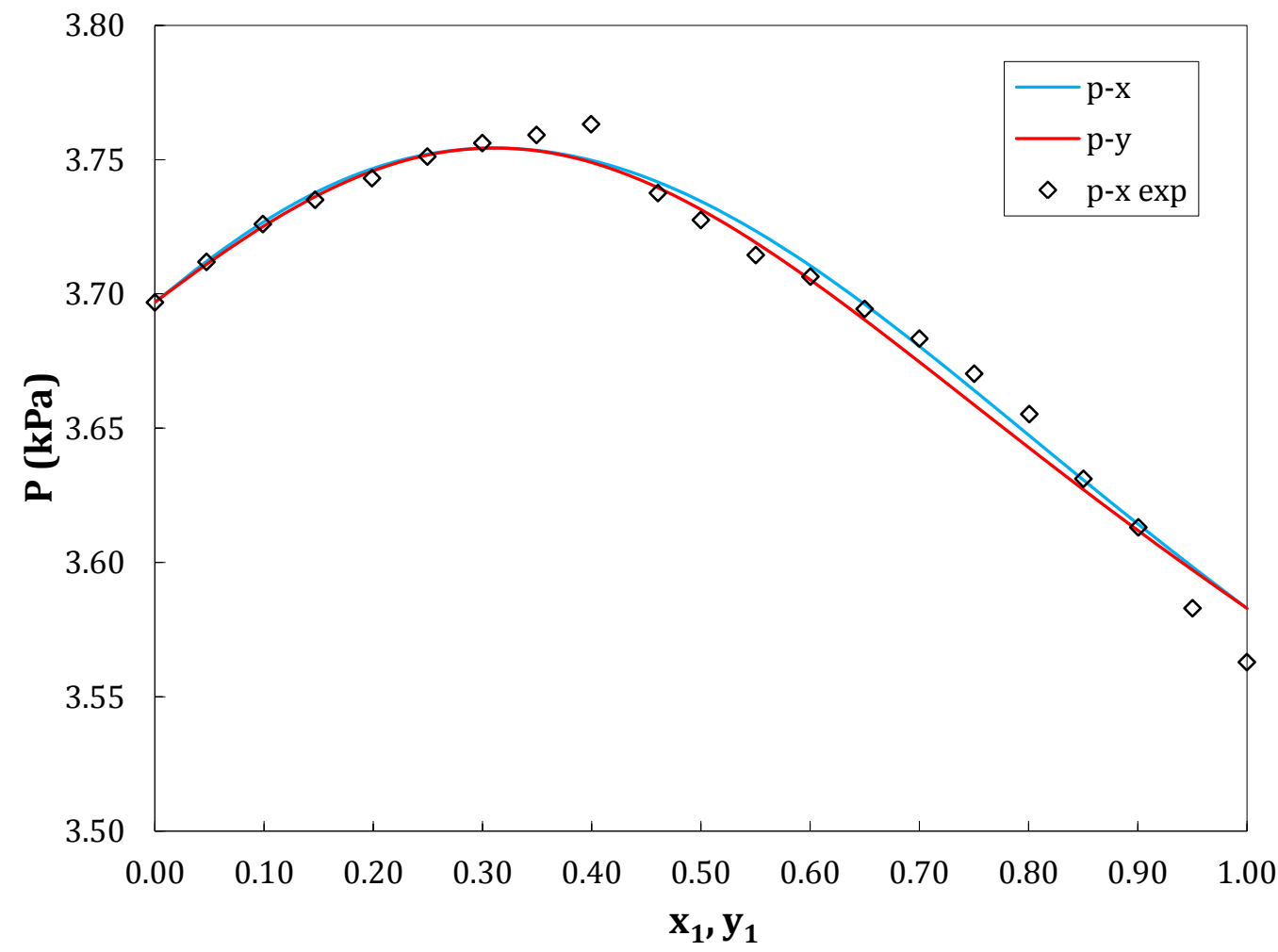

Figura 3.9. Presión de vapor $(p)$ en función de las fracciones molares de la fase líquida $x_{1} y$ la fase vapor $y_{1}$, para el sistema binario B1: Ciclohexanona (1) + 2-Heptanona (2) a 333.15K. ( $\left.\diamond\right)$ Datos experimentales. (-) Ajuste mediante la ecuación de Margules.

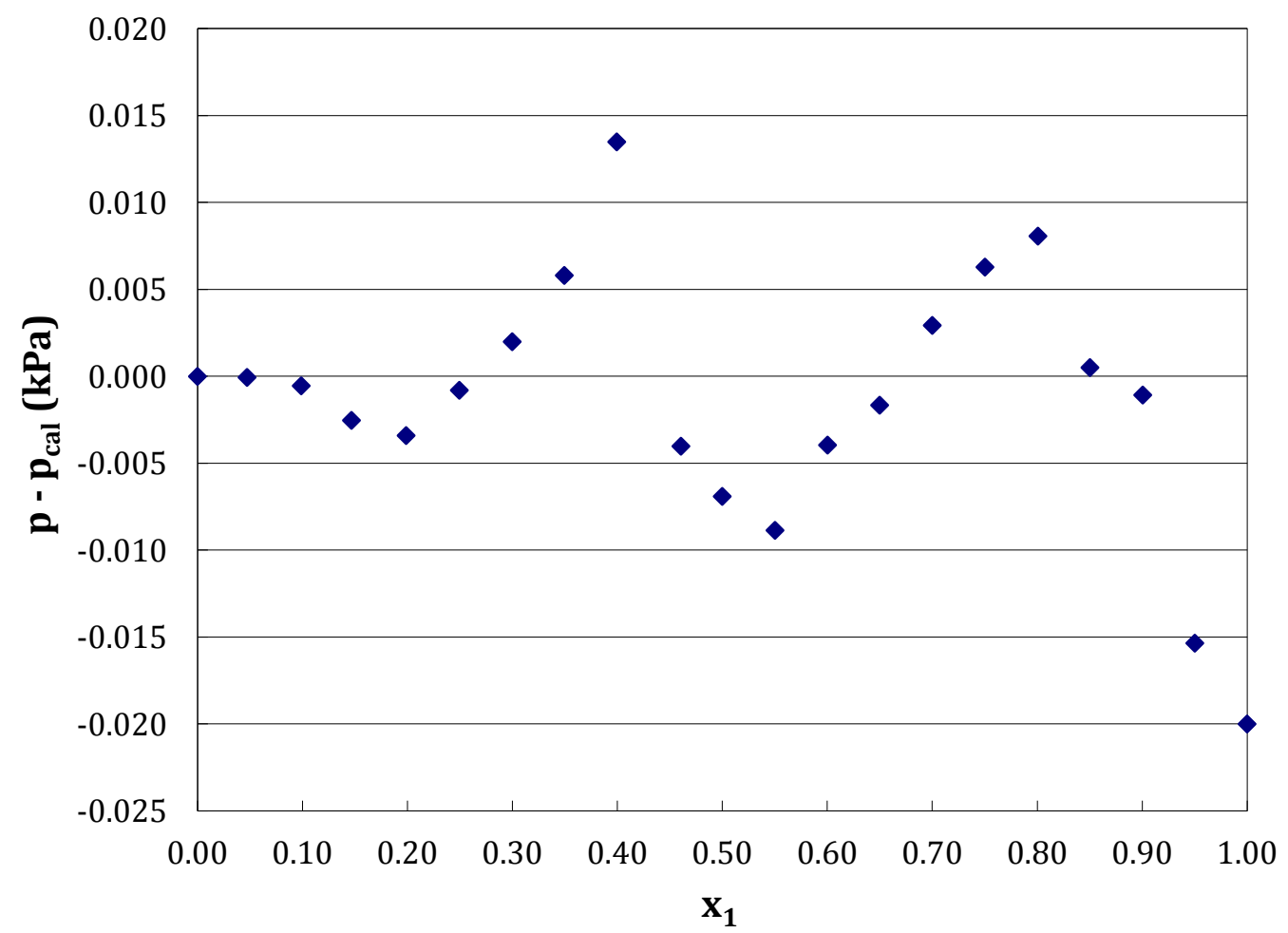

Figura 3.10. Comparación de las diferencias entre la presión experimental ( $p$ ) y la presión calculada $\left(p_{\text {cal }}\right)$ mediante la ecuación de Margules ( $\diamond$ ), para el sistema binario B1: Ciclohexanona (1) + 2Heptanona (2) a $333.15 \mathrm{~K}$. 


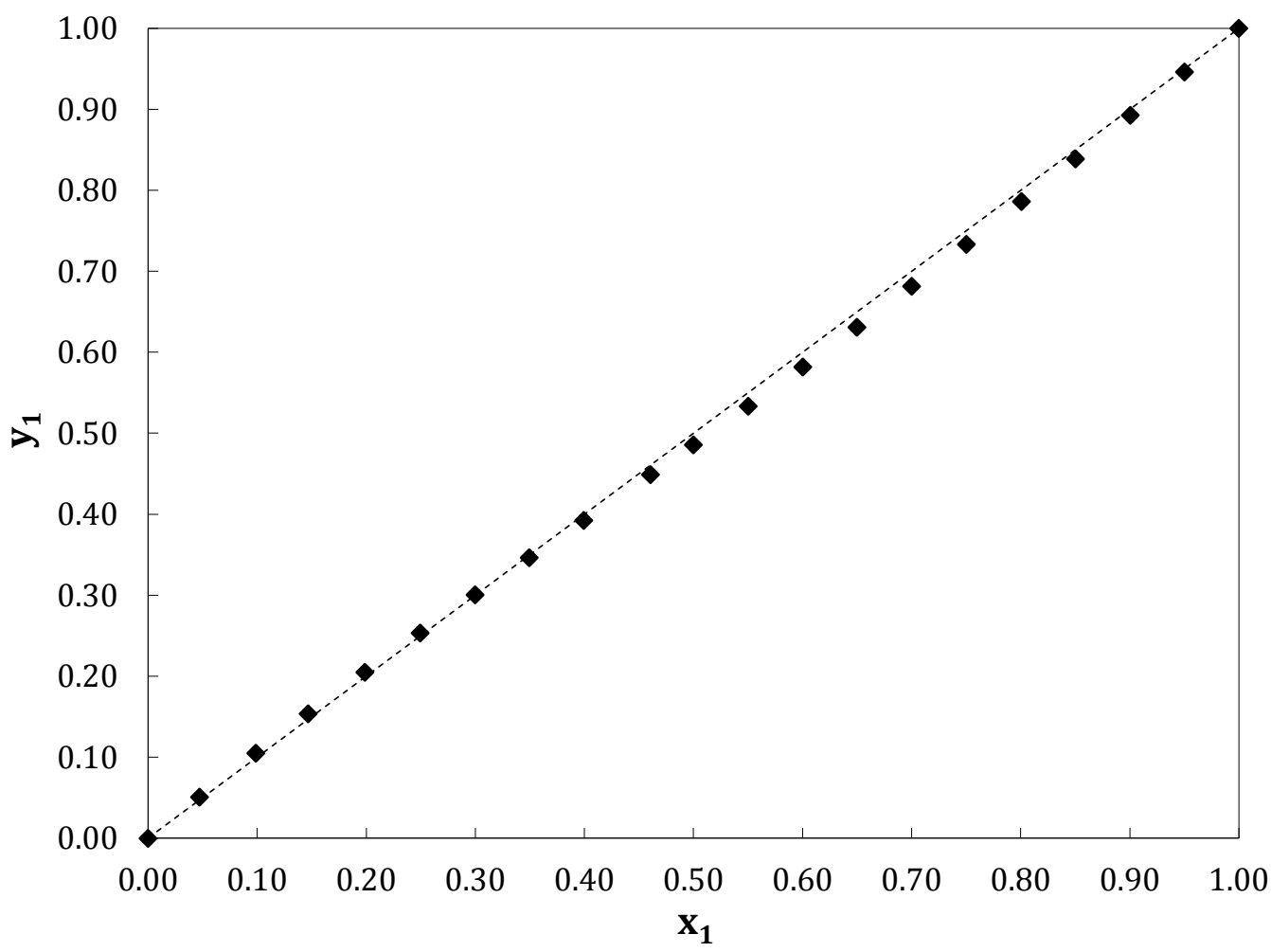

Figura 3.11. Fracción molar de la fase vapor del $\left(y_{1}\right)$ en función de la fracción molar de la fase líquida $\left(x_{1}\right)$ para el sistema binario B1: Ciclohexanona (1) + 2-Heptanona (2) a 333.15 K. Datos calculados mediante la ecuación de Margules ( $\bullet$ ).

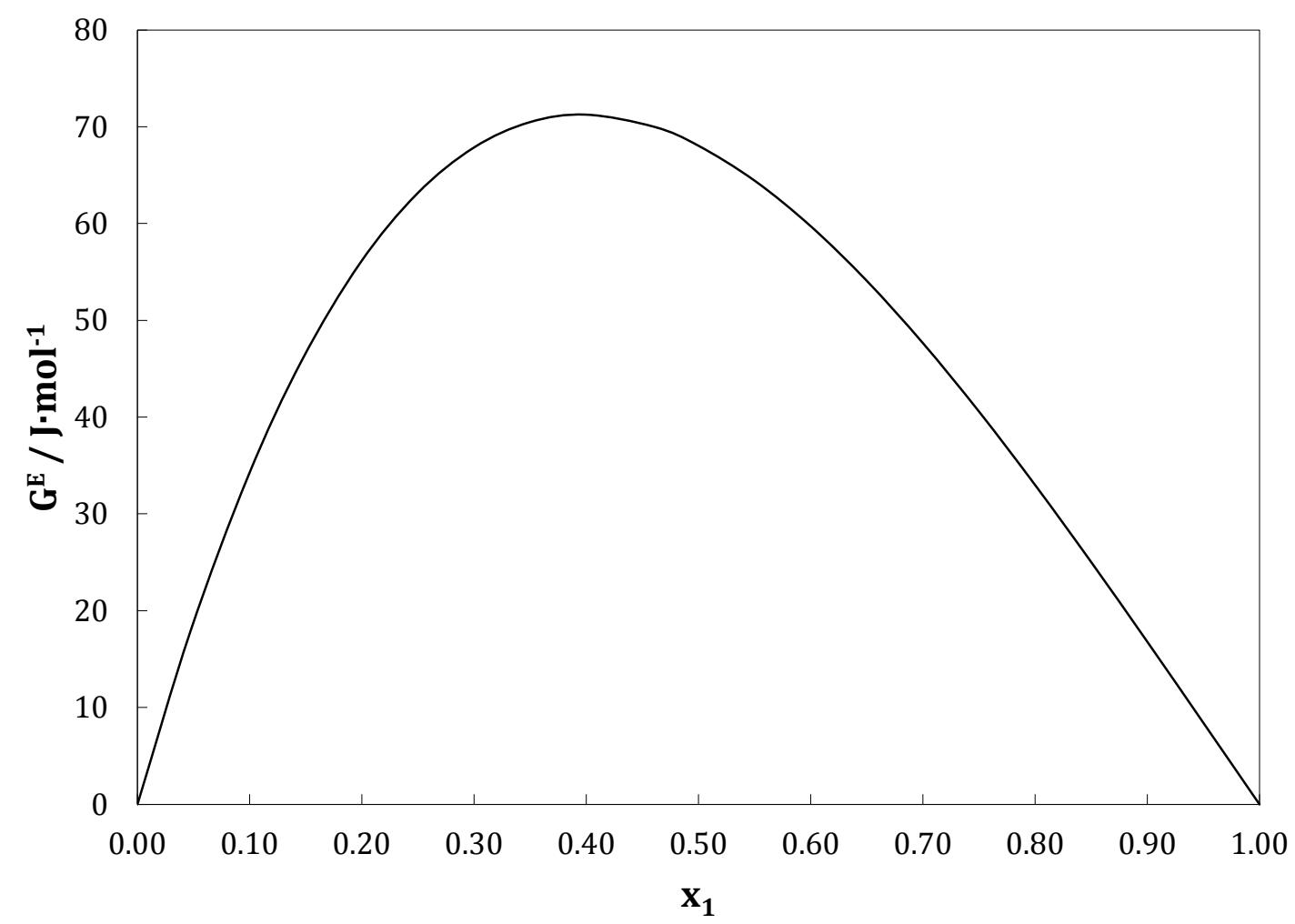

Figura 3.12. Energía de Gibbs de exceso $\left(G^{E}\right)$ en función de la fracción molar de la fase líquida $\left(x_{1}\right)$ para el sistema binario B1: Ciclohexanona (1) + 2-Heptanona (2) a $333.15 \mathrm{~K}$. 


\subsubsection{Binario B1: Ciclohexanona (1) + 2-Heptanona (2) a $353.15 \mathrm{~K}$.}

Tabla 3.12. Datos experimentales $p-x-y$ calculados mediante la ecuación de Margules y energía de Gibbs de exceso calculada mediante el modelo NRTL para el sistema binario B1: Ciclohexanona (1) + 2Heptanona (2) a $353.15 \mathrm{~K}$.

\begin{tabular}{cccccccc}
\hline $\mathbf{x}_{\mathbf{1}}$ & $\mathbf{y}_{\mathbf{1}, \mathbf{c a l}}$ & $\mathbf{p} / \mathbf{k P a}$ & $\begin{array}{c}\mathbf{G}^{\mathbf{E}} / \\
\mathbf{J} \cdot \mathbf{m o l}-\mathbf{1}\end{array}$ & $\mathbf{x}_{\mathbf{1}}$ & $\mathbf{y}_{\mathbf{1}, \text { calc }}$ & $\mathbf{p} / \mathbf{k P a}$ & $\begin{array}{c}\mathbf{G}^{\mathbf{E}} / \\
\mathbf{J} \cdot \mathbf{m o l}-\mathbf{1}\end{array}$ \\
\hline 0.0000 & 0.0000 & 9.288 & 0.00 & 0.5004 & 0.4750 & 9.243 & 89.66 \\
0.0487 & 0.0498 & 9.306 & 18.06 & 0.5505 & 0.5224 & 9.200 & 87.05 \\
0.0990 & 0.1036 & 9.319 & 34.68 & 0.6009 & 0.5721 & 9.160 & 82.48 \\
0.1492 & 0.1563 & 9.333 & 49.10 & 0.6508 & 0.6233 & 9.109 & 76.15 \\
0.1994 & 0.2063 & 9.346 & 61.36 & 0.7008 & 0.6759 & 9.065 & 68.15 \\
0.2492 & 0.2532 & 9.352 & 71.40 & 0.7512 & 0.7296 & 9.017 & 58.60 \\
0.2995 & 0.2983 & 9.357 & 79.36 & 0.8010 & 0.7826 & 8.964 & 47.92 \\
0.3496 & 0.3418 & 9.363 & 85.13 & 0.8511 & 0.8356 & 8.907 & 36.24 \\
0.3998 & 0.3852 & 9.367 & 88.75 & 0.9006 & 0.8878 & 8.847 & 24.14 \\
0.4497 & 0.4290 & 9.341 & 90.25 & 0.9509 & 0.9423 & 8.779 & 11.72 \\
0.4503 & 0.4295 & 9.293 & 90.25 & 1.0000 & 1.0000 & 8.689 & 0.00 \\
0.4998 & 0.4744 & 9.343 & 89.68 & & & & \\
\hline
\end{tabular}

Tabla 3.13. Valor experimental de la presión de vapor de los compuestos puros $p_{i}^{\text {sat }}$, volúmenes molares $V_{i}^{L}, y$ coeficientes del virial de los puros $B_{i i} y$ de la mezcla $B_{i j}$, para el sistema binario B1: Ciclohexanona (1) + 2-Heptanona (2) a 353.15K.

\begin{tabular}{|c|c|c|c|c|c|c|}
\hline $\begin{array}{l}\mathbf{p}_{1}^{\text {sat }} \\
\mathbf{k P a} \\
\end{array}$ & $\begin{array}{l}\mathbf{p}_{2}^{\text {sat }} \\
\mathbf{k P a}\end{array}$ & $\begin{array}{c}\mathrm{V}_{1}^{\mathrm{L}} \\
\mathrm{cm}^{3 \cdot \mathrm{mol}^{-1}}\end{array}$ & $\begin{array}{c}\mathrm{V}_{2}^{\mathrm{L}} \\
\mathrm{cm}^{3} \cdot \mathrm{mol}^{-1}\end{array}$ & $\begin{array}{c}\mathrm{B}_{11} \\
\mathrm{~cm}^{3 \cdot \mathrm{mol}^{-1}} \\
\end{array}$ & $\begin{array}{c}\quad B_{22} \\
\mathbf{c m}^{3} \cdot \mathbf{m o l}^{-1} \\
\end{array}$ & $\begin{array}{c}\mathrm{B}_{12} \\
\mathrm{~cm}^{3 \cdot \mathrm{mol}^{-1}} \\
\end{array}$ \\
\hline 8.689 & 9.288 & 109.93 & 149.66 & -1987 & -2480 & -2101 \\
\hline
\end{tabular}

Tabla 3.14. Parámetros de ajuste del sistema binario B1: Ciclohexanona (1) + 2-Heptanona (2) a $353.15 \mathrm{~K}$.

\begin{tabular}{lcccc}
\hline & Margules & Wilson & NRTL & UNIQUAC \\
\hline $\mathrm{A}_{12}$ & 0.0441 & 0.6762 & -0.9746 & 0.6883 \\
$\mathrm{~A}_{21}$ & 0.1508 & 1.2472 & 0.7282 & 1.4534 \\
$\lambda_{12}$ & -0.5504 & & & \\
$\lambda_{21}$ & 0.3479 & & & \\
$\alpha$ & & & -0.5064 & \\
$\mathrm{rms} \Delta \mathrm{p} / \mathrm{kPa}$ & 0.019 & 0.025 & 0.024 & 0.024 \\
$\mathrm{max}|\Delta \mathrm{p}| / \mathrm{kPa}$ & 0.060 & 0.070 & 0.065 & 0.066 \\
$\mathrm{x}_{1, \text { azeótropo }}$ & 0.292 & 0.224 & 0.238 & 0.238 \\
$\mathrm{p}_{\text {azeótropo }} / \mathrm{kPa}$ & 9.356 & 9.352 & 9.349 & 9.346 \\
\hline
\end{tabular}




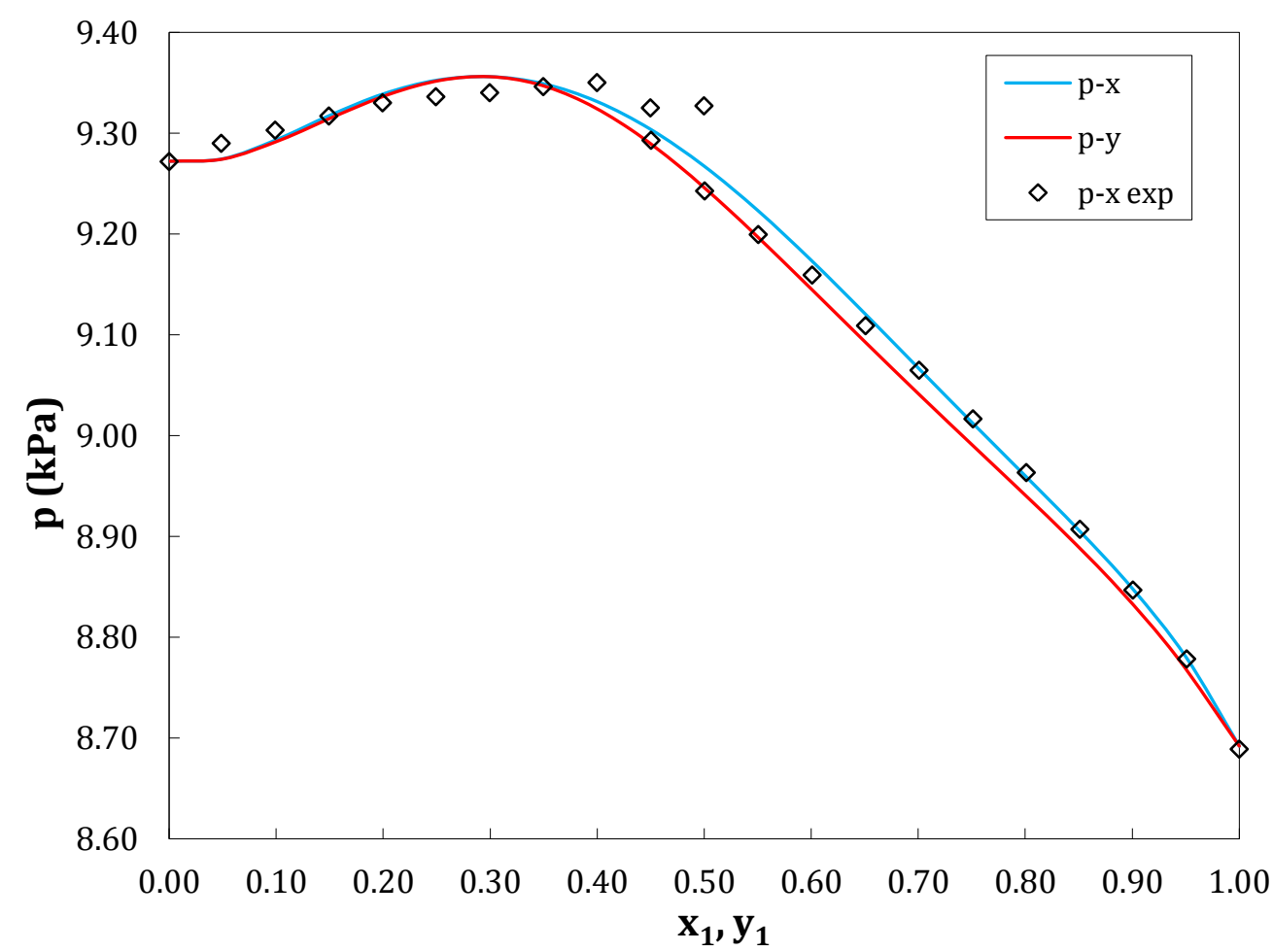

Figura 3.13. Presión de vapor ( $p$ ) en función de las fracciones molares de la fase líquida $x_{1}$ y la fase vapor $y_{1}$, para el sistema binario B1: Ciclohexanona (1) + 2-Heptanona (2) a $353.15 \mathrm{~K}$. $\diamond$ ) Datos experimentales. (-) Ajuste mediante la ecuación de Margules.

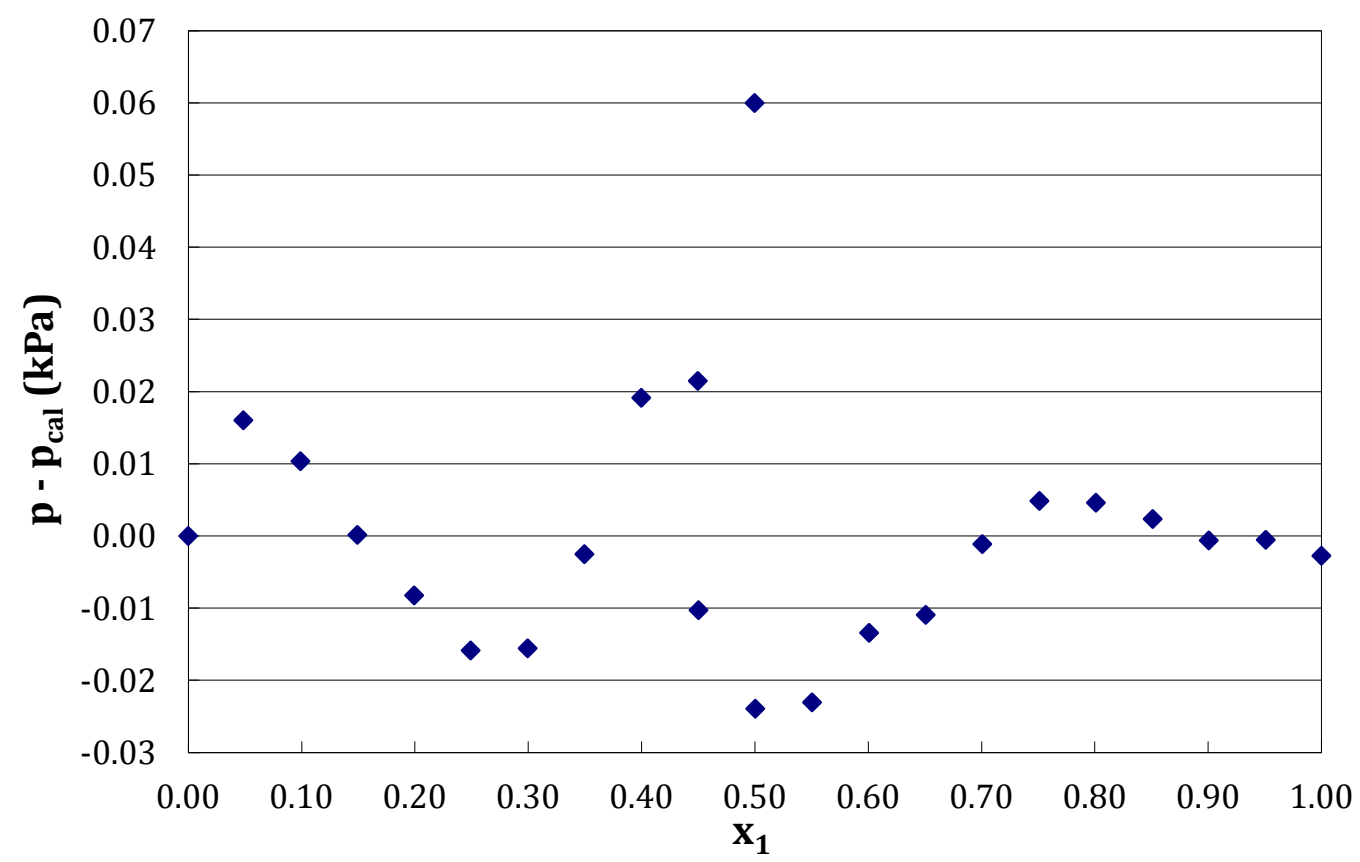

Figura 3.14. Comparación de las diferencias entre la presión experimental ( $p$ ) y la presión calculada $\left(p_{\text {cal }}\right)$ mediante la ecuación de Margules ( $)$, para el sistema binario B1: Ciclohexanona (1) + 2Heptanona (2) a $353.15 \mathrm{~K}$. 


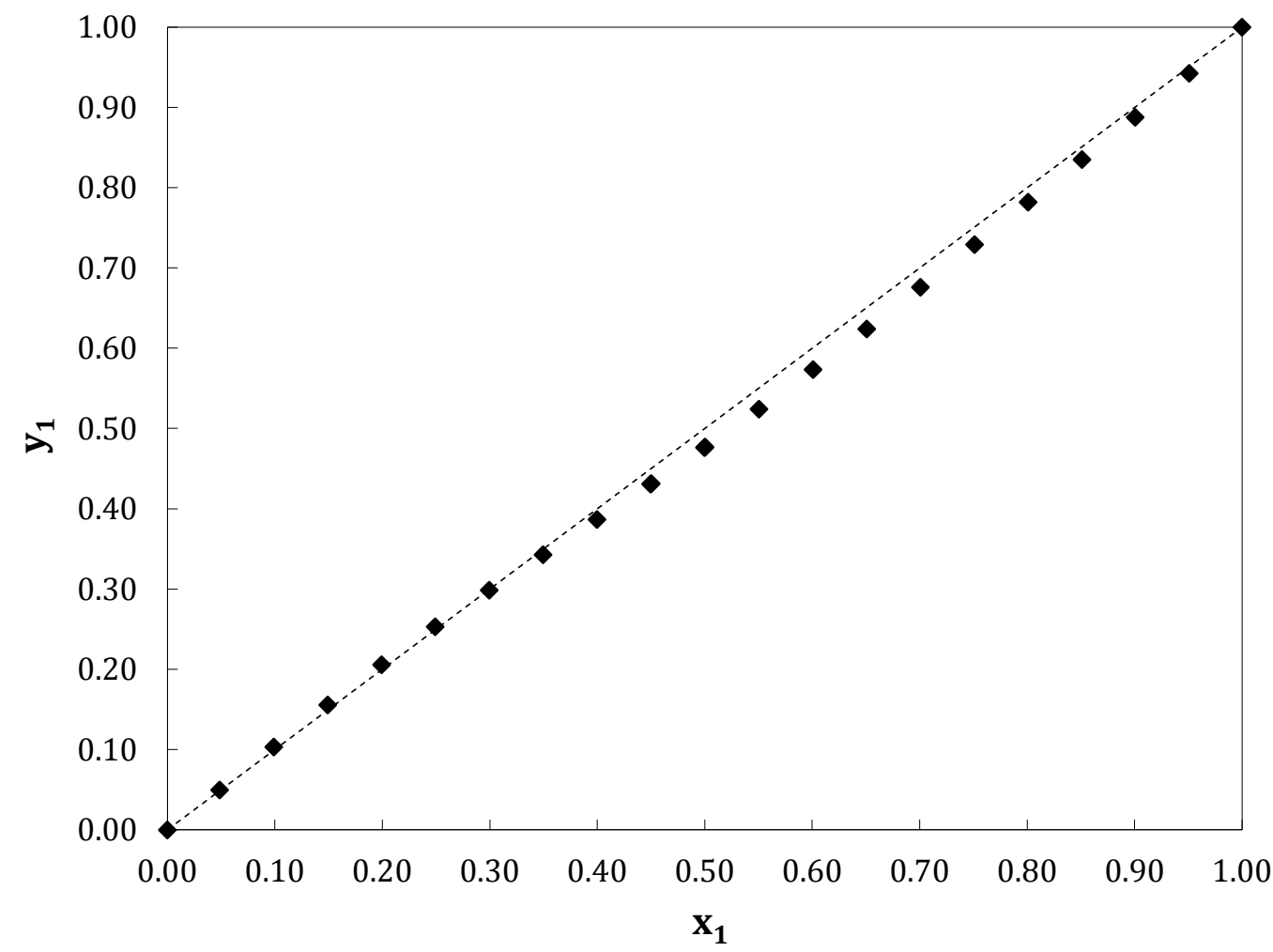

Figura 3.15. Fracción molar de la fase vapor del $\left(y_{1}\right)$ en función de la fracción molar de la fase líquida $\left(x_{1}\right)$ para el sistema binario B1: Ciclohexanona (1) + 2-Heptanona (2) a 353.15 K. Datos calculados mediante la ecuación de Margules ( $\bullet$ ).

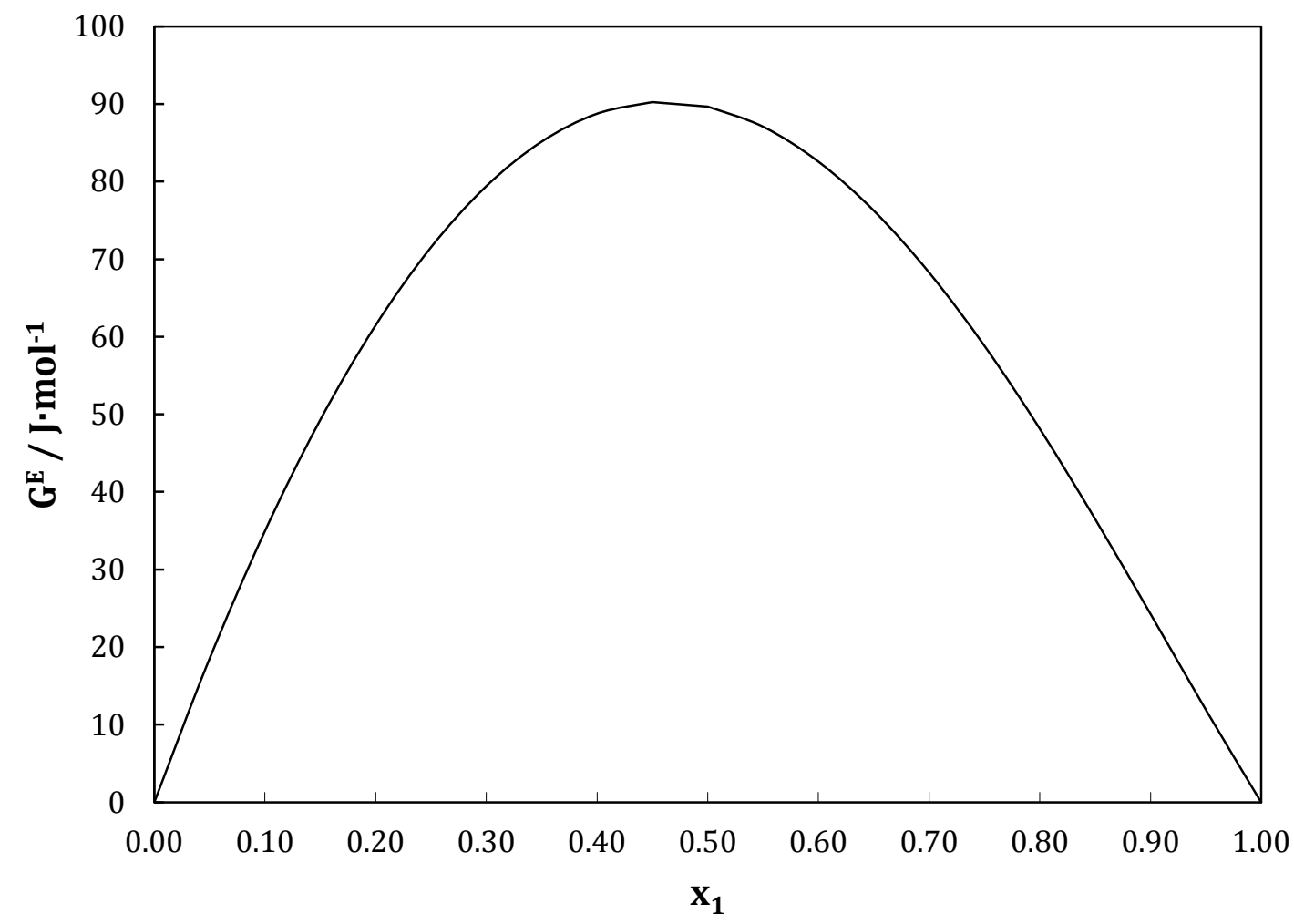

Figura 3.16. Energía de Gibbs de exceso $\left(G^{E}\right)$ en función de la fracción molar de la fase líquida $\left(x_{1}\right)$ para el sistema binario B1: Ciclohexanona (1) + 2-Heptanona (2) a 353.15K. 


\subsubsection{Binario B1: Ciclohexanona (1) + 2-Heptanona (2) a $393.15 \mathrm{~K}$.}

Tabla 3.15. Datos experimentales $p-x-y$ calculados mediante la ecuación de Margules y energía de Gibbs de exceso calculada mediante el modelo NRTL para el sistema binario B1: Ciclohexanona (1) + 2Heptanona (2) a $393.15 \mathrm{~K}$.

\begin{tabular}{cccccccc}
\hline $\mathbf{x}_{\mathbf{1}}$ & $\mathbf{y}_{\mathbf{1}, \text { calc }}$ & $\mathbf{p} / \mathbf{k P a}$ & $\begin{array}{c}\mathbf{G}^{\mathbf{E}} / \\
\mathbf{J} \cdot \mathbf{m o l}-\mathbf{1}\end{array}$ & $\mathbf{x}_{\mathbf{1}}$ & $\mathbf{y}_{\mathbf{1}, \text { calc }}$ & $\mathbf{p} / \mathbf{k P a}$ & $\begin{array}{c}\mathbf{G}^{\mathbf{E}} / \\
\mathbf{J} \cdot \mathbf{m o l}^{-\mathbf{1}}\end{array}$ \\
\hline 0.0000 & 0.0000 & 40.990 & 0.00 & 0.5510 & 0.5191 & 39.234 & 58.79 \\
0.0473 & 0.0464 & 40.960 & 16.60 & 0.6010 & 0.5688 & 38.935 & 56.03 \\
0.0987 & 0.0953 & 40.866 & 30.68 & 0.6513 & 0.6199 & 38.718 & 52.56 \\
0.1488 & 0.1419 & 40.763 & 41.17 & 0.7003 & 0.6704 & 38.467 & 48.59 \\
0.1988 & 0.1879 & 40.648 & 49.05 & 0.7516 & 0.7242 & 38.170 & 43.87 \\
0.2491 & 0.2339 & 40.525 & 54.79 & 0.8021 & 0.7781 & 37.832 & 38.70 \\
0.2992 & 0.2799 & 40.399 & 58.69 & 0.8522 & 0.8326 & 37.650 & 33.05 \\
0.3496 & 0.3265 & 40.249 & 61.05 & 0.9012 & 0.8869 & 37.331 & 26.98 \\
0.3997 & 0.3734 & 40.082 & 62.07 & 0.9517 & 0.9441 & 37.069 & 19.71 \\
0.4508 & 0.4219 & 39.762 & 61.93 & 1.0000 & 1.0000 & 36.687 & 0.00 \\
0.5008 & 0.4699 & 39.435 & 60.80 & & & & \\
\hline
\end{tabular}

Tabla 3.16. Valor experimental de la presión de vapor de los compuestos puros $p_{i}^{\text {sat }}$, volúmenes molares $V_{i}^{L}$, y coeficientes del virial de los puros $B_{i i} y$ de la mezcla $B_{i j}$, para el sistema binario B1: Ciclohexanona (1) + 2-Heptanona (2) a 393.15 K.

\begin{tabular}{|c|c|c|c|c|c|c|}
\hline $\begin{array}{l}p_{1}^{\text {sat }} \\
\mathrm{kPa}\end{array}$ & $\begin{array}{l}p_{2}^{\text {sat }} \\
\mathbf{k P a}\end{array}$ & $\begin{array}{c}\mathrm{V}_{1}^{\mathrm{L}} \\
\mathrm{cm}^{3} \cdot \mathrm{mol}^{-1}\end{array}$ & $\begin{array}{c}\mathrm{V}_{2}^{\mathrm{L}} \\
\mathrm{cm}^{3 \cdot} \cdot \mathrm{mol}^{-1}\end{array}$ & $\begin{array}{c}\mathrm{B}_{11} \\
\mathrm{~cm}^{3} \cdot \mathrm{mol}^{-1} \\
\end{array}$ & $\begin{array}{c}\quad \mathrm{B}_{22} \\
\mathrm{~cm}^{3} \cdot \mathrm{mol}^{-1}\end{array}$ & $\begin{array}{c}\mathrm{B}_{12} \\
\mathrm{~cm}^{3} \cdot \mathrm{mol}^{-1}\end{array}$ \\
\hline 36.687 & 40.990 & 114.63 & 157.15 & -1486 & -1814 & -1557 \\
\hline
\end{tabular}

Tabla 3.17. Parámetros de ajuste del sistema binario B1: Ciclohexanona (1) + 2-Heptanona (2) a $393.15 \mathrm{~K}$.

\begin{tabular}{lcccc}
\hline & Margules & Wilson & NRTL & UNIQUAC \\
\hline $\mathrm{A}_{12}$ & 0.0687 & 0.6151 & 0.5970 & 0.9391 \\
$\mathrm{~A}_{21}$ & 0.1435 & 1.3826 & 0.1156 & 1.1505 \\
$\lambda_{12}$ & -0.1978 & & & \\
$\lambda_{21}$ & 0.4585 & & & \\
$\alpha$ & & & 8.1085 & \\
$\operatorname{rms} \Delta \mathrm{p} / \mathrm{kPa}$ & 0.051 & 0.075 & 0.061 & 0.089 \\
$\max |\Delta \mathrm{p}| / \mathrm{kPa}$ & 0.124 & 0.175 & 0.124 & 0.187 \\
\hline
\end{tabular}




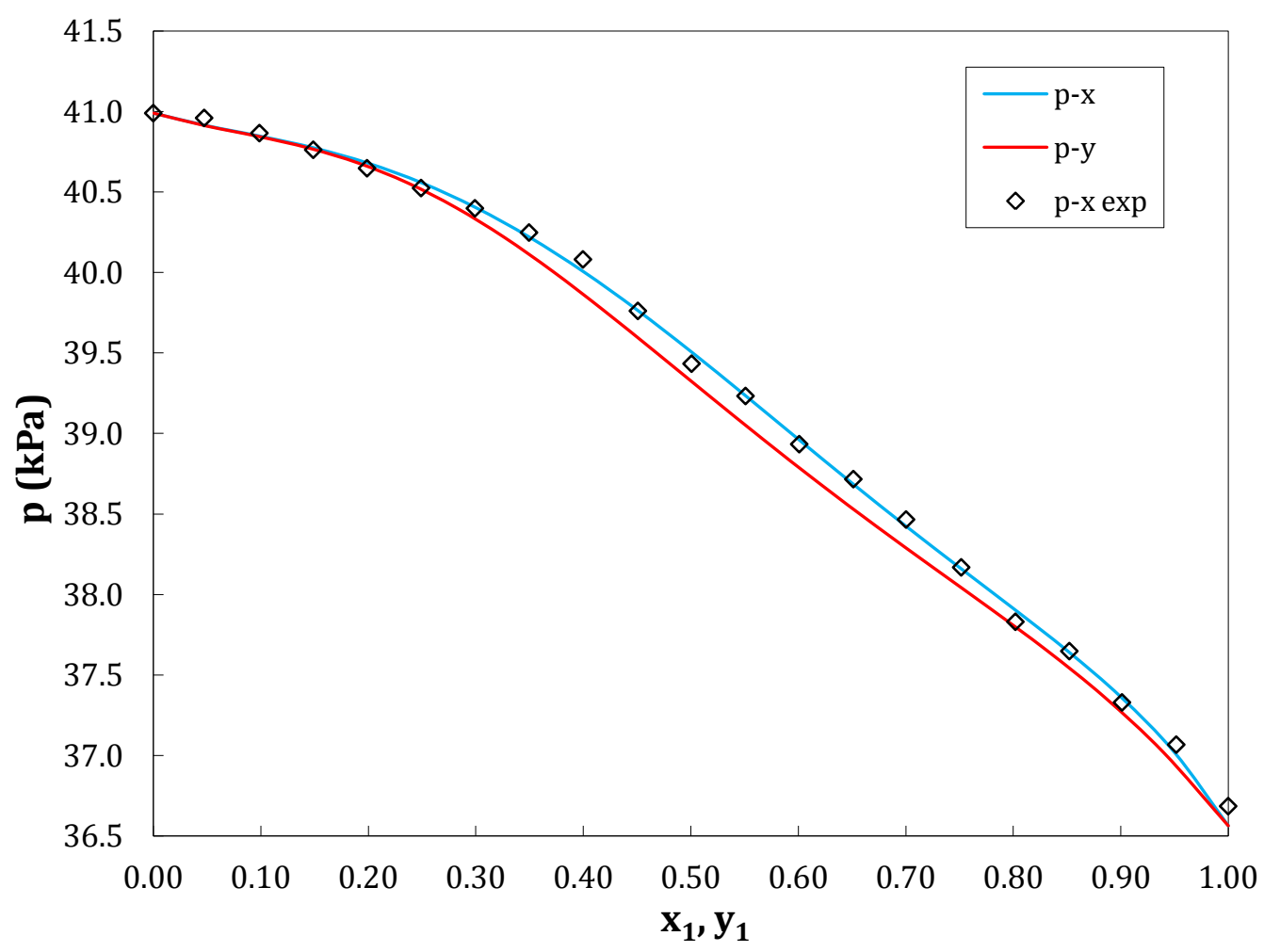

Figura 3.17. Presión de vapor $(p)$ en función de las fracciones molares de la fase líquida $x_{1} y$ la fase vapor $y_{1}$, para el sistema binario B1: Ciclohexanona (1) + 2-Heptanona (2) a 393.15 K. $(\diamond)$ Datos experimentales. (-) Ajuste mediante la ecuación de Margules.

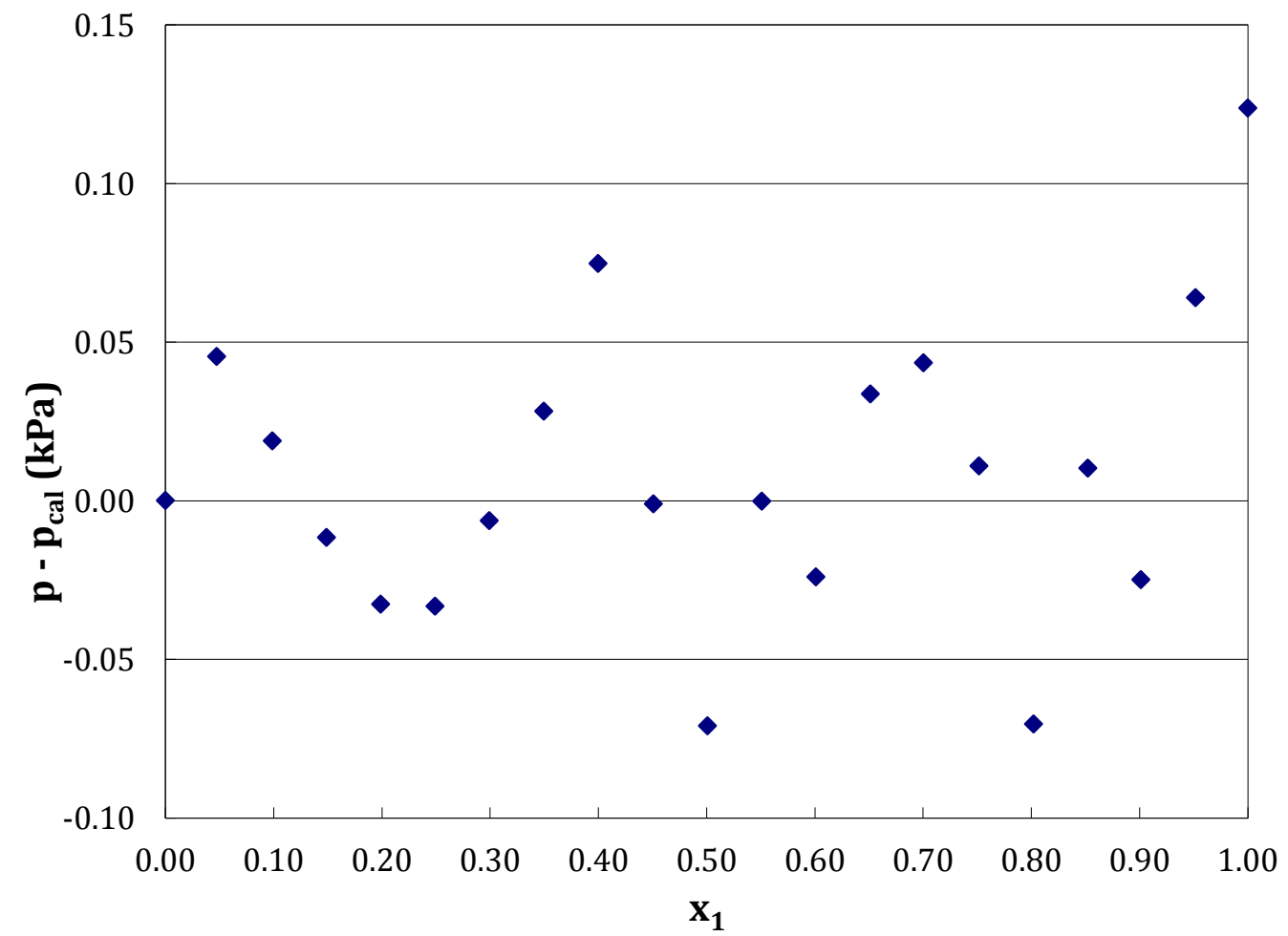

Figura 3.18. Comparación de las diferencias entre la presión experimental ( $p$ ) y la presión calculada $\left(p_{\text {cal }}\right)$ mediante la ecuación de Margules ( $\diamond$ ), para el sistema binario B1: Ciclohexanona (1) + 2Heptanona (2) a $393.15 \mathrm{~K}$. 


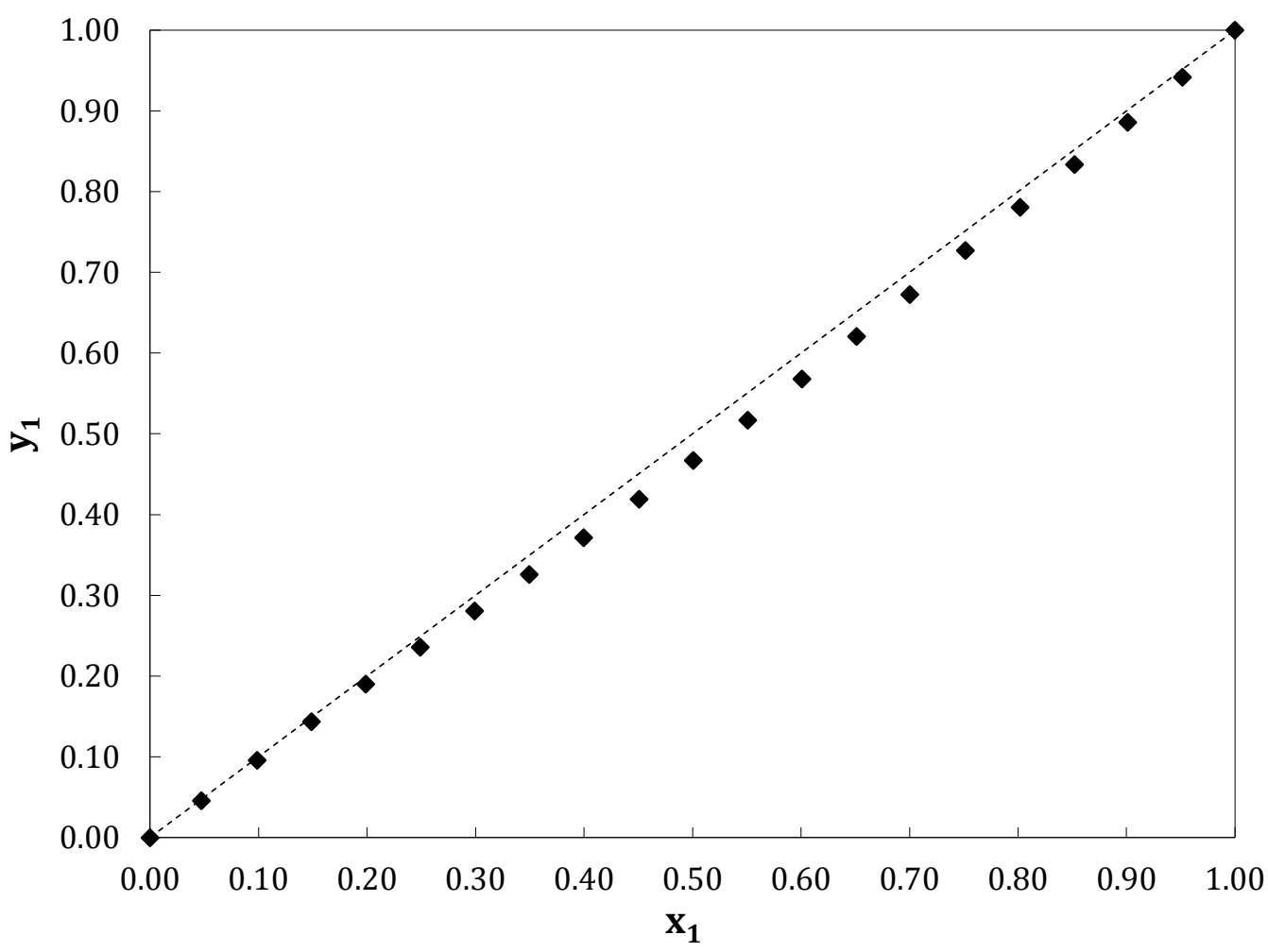

Figura 3.19. Fracción molar de la fase vapor del $\left(y_{1}\right)$ en función de la fracción molar de la fase líquida $\left(x_{1}\right)$ para el sistema binario B1: Ciclohexanona (1) + 2-Heptanona (2) a 393.15 K. Datos calculados mediante la ecuación de Margules ( $\bullet$ ).

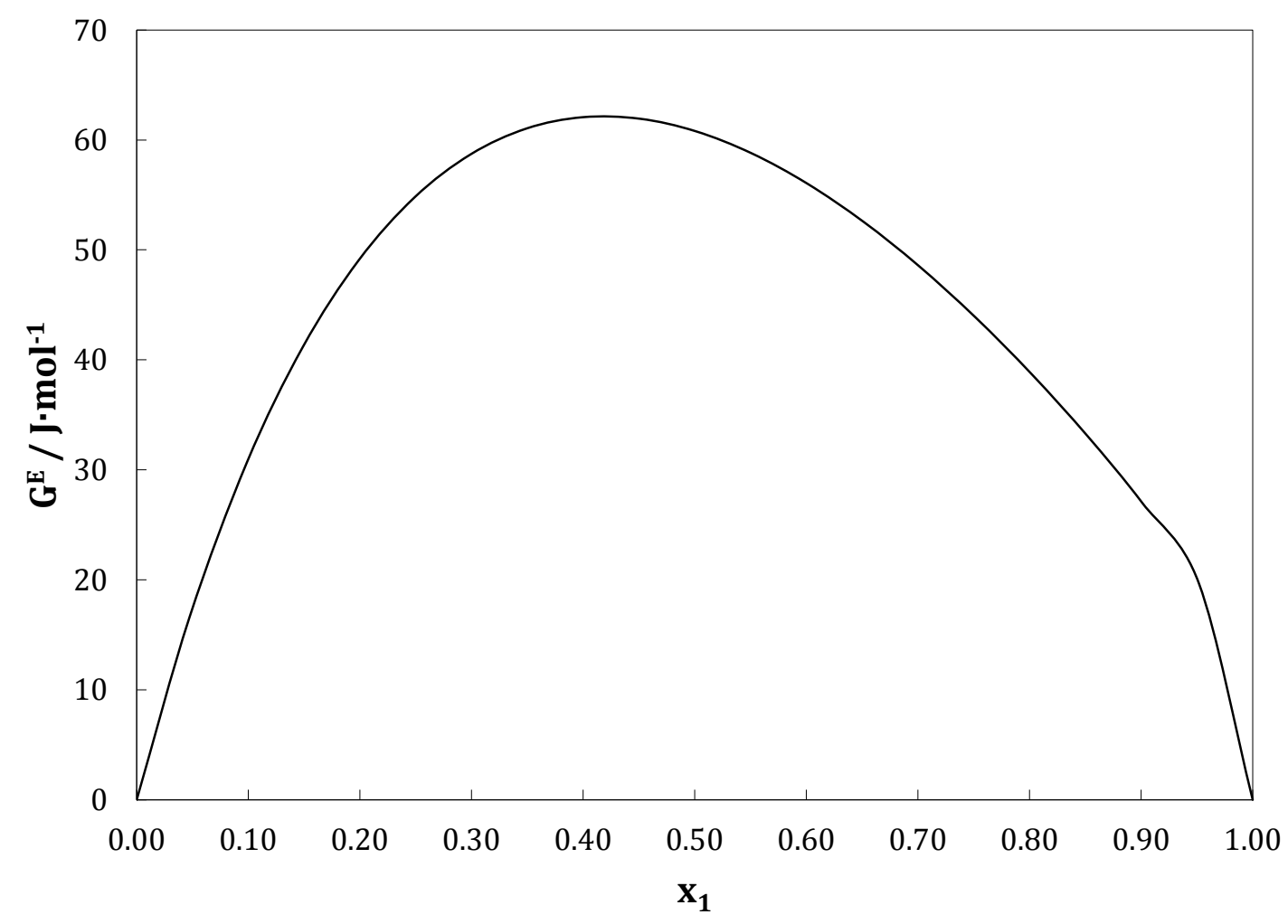

Figura 3.20. Energía de Gibbs de exceso $\left(G^{E}\right)$ en función de la fracción molar de la fase líquida $\left(x_{1}\right)$ para el sistema binario B1: Ciclohexanona (1) + 2-Heptanona (2) a $393.15 \mathrm{~K}$. 


\subsubsection{Binario B2: Ciclohexanona (1) + Hexanal (2) a $313.15 \mathrm{~K}$.}

Tabla 3.18. Datos experimentales $p-x-y$ calculados mediante la ecuación de Margules y energía de Gibbs de exceso calculada mediante el modelo NRTL para el sistema binario B2: Ciclohexanona (1) + Hexanal (2) a $313.15 \mathrm{~K}$.

\begin{tabular}{cccccccc}
\hline $\mathbf{x}_{\mathbf{1}}$ & $\mathbf{y}_{\mathbf{1}, \text { calc }}$ & $\mathbf{p} / \mathbf{k P a}$ & $\begin{array}{c}\mathbf{G}^{\mathbf{E}} / \\
\mathbf{J} \cdot \mathbf{m o l}-\mathbf{1}\end{array}$ & $\mathbf{x}_{\mathbf{1}}$ & $\mathbf{y}_{\mathbf{1}, \text { calc }}$ & $\mathbf{p} / \mathbf{k P a}$ & $\begin{array}{c}\mathbf{G}^{\mathbf{E}} / \\
\mathbf{J} \cdot \mathbf{m o l}^{-\mathbf{1}}\end{array}$ \\
\hline 0.0000 & 0.0000 & 3.604 & 0.00 & 0.5012 & 0.2701 & 2.466 & 3.45 \\
0.0489 & 0.0169 & 3.498 & 0.64 & 0.5513 & 0.3113 & 2.335 & 3.41 \\
0.0992 & 0.0383 & 3.351 & 1.23 & 0.5514 & 0.3114 & 2.338 & 3.41 \\
0.1502 & 0.0626 & 3.221 & 1.76 & 0.5990 & 0.3554 & 2.224 & 3.31 \\
0.2005 & 0.0881 & 3.095 & 2.21 & 0.6511 & 0.4096 & 2.113 & 3.13 \\
0.2504 & 0.1143 & 2.982 & 2.59 & 0.7011 & 0.4677 & 2.004 & 2.89 \\
0.3007 & 0.1416 & 2.885 & 2.90 & 0.7514 & 0.5321 & 1.891 & 2.58 \\
0.3506 & 0.1699 & 2.791 & 3.14 & 0.8017 & 0.6027 & 1.782 & 2.19 \\
0.4010 & 0.2004 & 2.691 & 3.31 & 0.8526 & 0.6810 & 1.667 & 1.73 \\
0.4510 & 0.2334 & 2.577 & 3.41 & 0.9016 & 0.7655 & 1.551 & 1.22 \\
0.4511 & 0.2335 & 2.583 & 3.41 & 0.9494 & 0.8629 & 1.446 & 0.66 \\
0.5011 & 0.2701 & 2.452 & 3.45 & 1.0000 & 1.0000 & 1.315 & 0.00 \\
\hline
\end{tabular}

Tabla 3.19. Valor experimental de la presión de vapor de los compuestos puros $p_{i}^{\text {sat }}$, volúmenes molares $V_{i}^{L}$, y coeficientes del virial de los puros $B_{i i} y$ de la mezcla $B_{i j}$, para el sistema binario B2: Ciclohexanona (1) + Hexanal (2) a $313.15 \mathrm{~K}$.

\begin{tabular}{|c|c|c|c|c|c|c|}
\hline $\begin{array}{l}\mathbf{p}_{1}^{\text {sat }} \\
\mathrm{kPa} \\
\end{array}$ & $\begin{array}{l}p_{2}^{\text {sat }} \\
\text { kPa }\end{array}$ & $\begin{array}{c}\mathrm{V}_{1}^{\mathrm{L}} \\
\mathrm{cm}^{3} \cdot \mathrm{mol}^{-1} \\
\end{array}$ & $\begin{array}{c}\mathrm{V}_{2}^{\mathrm{L}} \\
\mathrm{cm}^{3} \cdot \mathrm{mol}^{-1} \\
\end{array}$ & $\begin{array}{c}\mathrm{B}_{11} \\
\mathrm{~cm}^{3} \cdot \mathrm{mol}^{-1} \\
\end{array}$ & $\begin{array}{c}\mathrm{B}_{22} \\
\mathrm{~cm}^{3} \cdot \mathrm{mol}^{-1} \\
\end{array}$ & $\begin{array}{c}\mathrm{B}_{12} \\
\mathrm{~cm}^{3} \cdot \mathrm{mol}^{-1} \\
\end{array}$ \\
\hline 1.315 & 3.604 & 105.70 & 125.00 & -2796 & -3100 & -2841 \\
\hline
\end{tabular}

Tabla 3.20. Parámetros de ajuste del sistema binario B2: Ciclohexanona (1) + Hexanal (2) a 313.15 K.

\begin{tabular}{lcccc} 
& Margules & Wilson & NRTL & UNIQUAC \\
\hline $\mathrm{A}_{12}$ & -0.2001 & 2.0127 & 8.0329 & 1.5751 \\
$\mathrm{~A}_{21}$ & 0.2296 & 0.3140 & -7.0168 & 0.6349 \\
$\lambda_{12}$ & -0.8125 & & & \\
$\lambda_{21}$ & 0.7227 & & & \\
$\alpha$ & & & 0.0175 & \\
$\mathrm{rms} \Delta \mathrm{p} / \mathrm{kPa}$ & 0.010 & 0.018 & 0.017 & 0.019 \\
$\max |\Delta \mathrm{p}| / \mathrm{kPa}$ & 0.021 & 0.043 & 0.037 & 0.044 \\
\hline
\end{tabular}




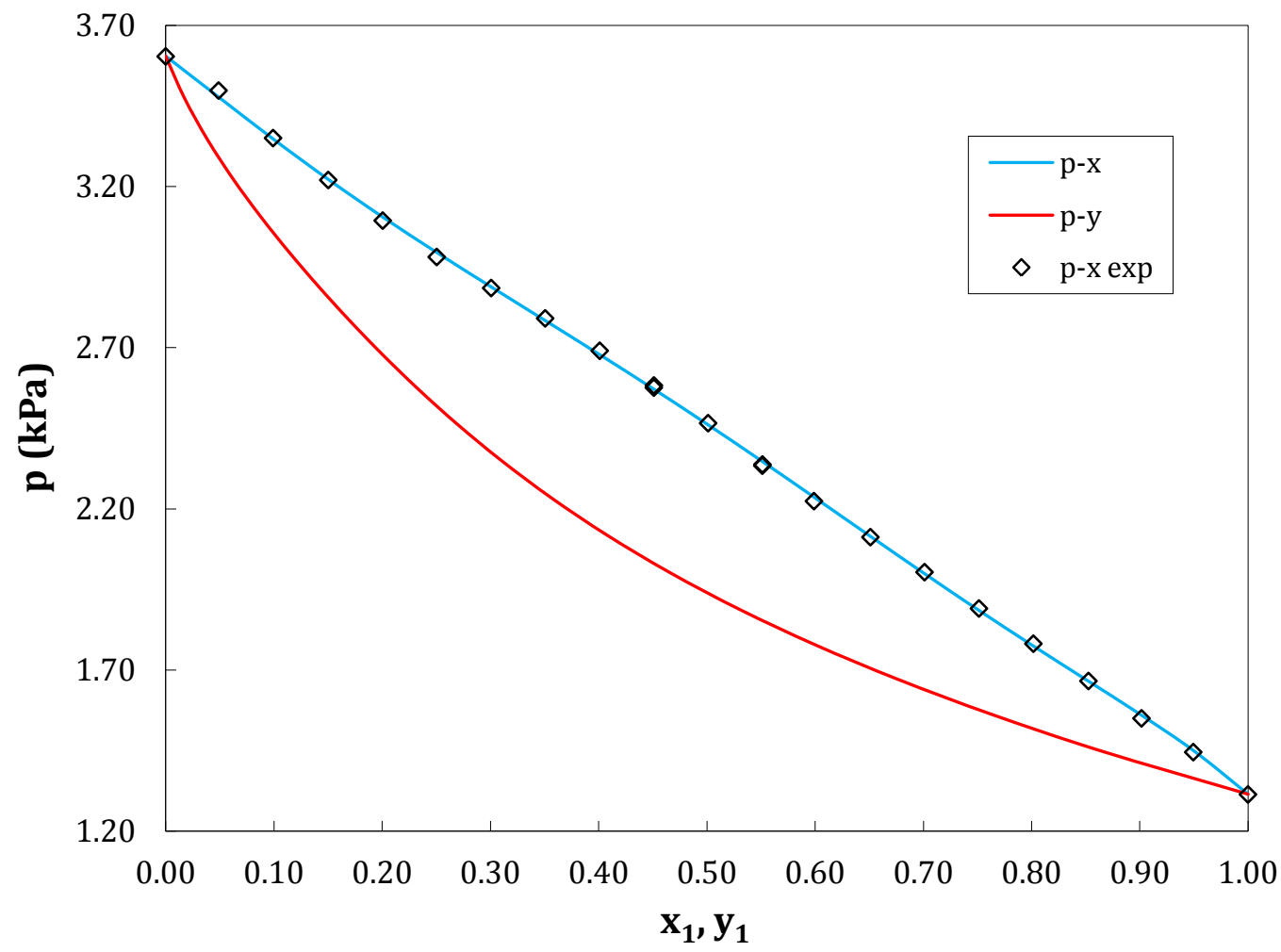

Figura 3.21. Presión de vapor $(p)$ en función de las fracciones molares de la fase líquida $x_{1} y$ la fase vapor $y_{1}$, para el sistema binario B2: Ciclohexanona (1) + Hexanal (2) a 313.15 K. ( $\left.\diamond\right)$ Datos experimentales. (-) Ajuste mediante la ecuación de Margules.

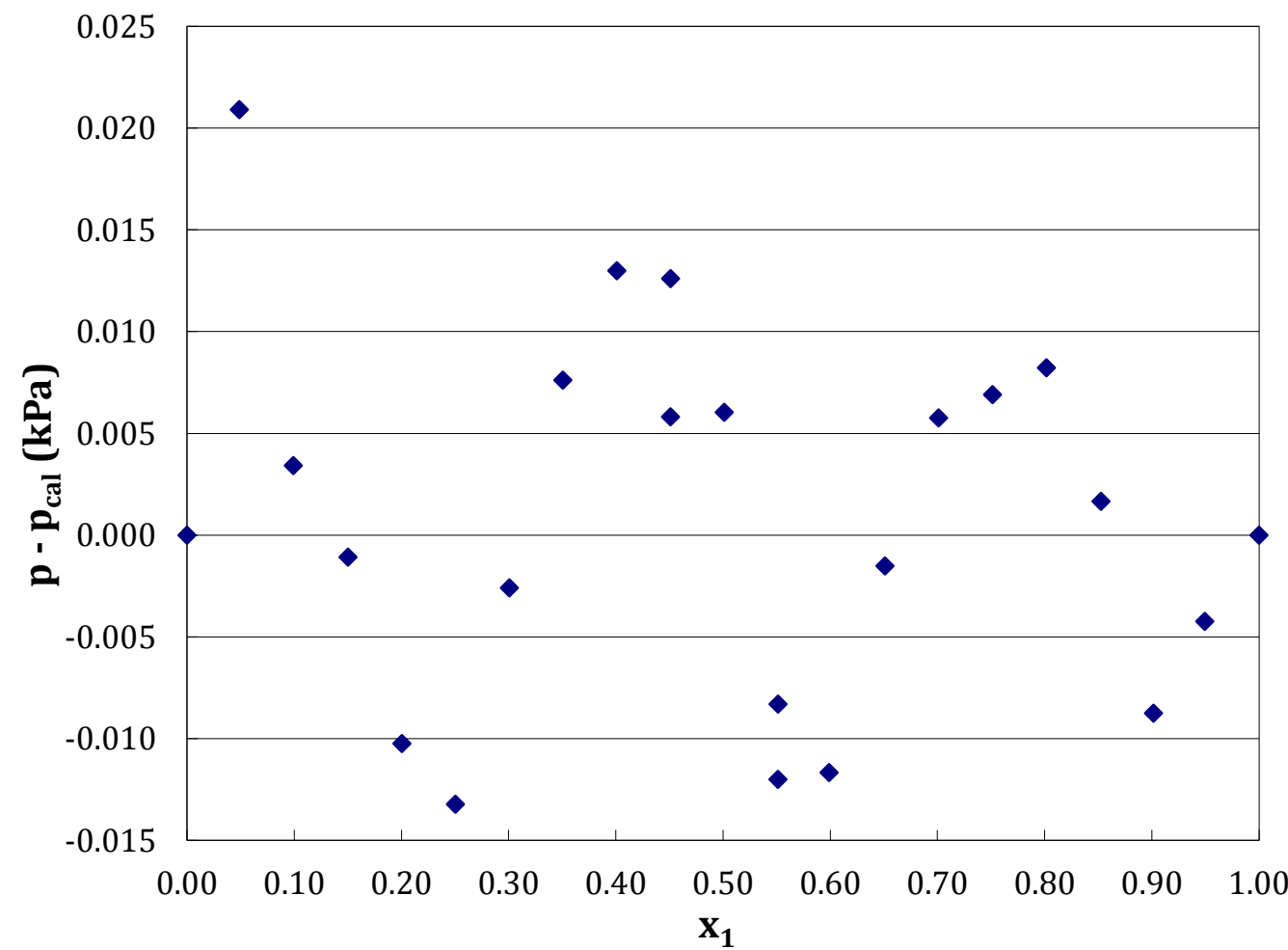

Figura 3.22. Comparación de las diferencias entre la presión experimental ( $p$ ) y la presión calculada (pal) mediante la ecuación de Margules ( $\bullet$ ), para el sistema binario B2: Ciclohexanona (1) + Hexanal (2) $a 313.15 \mathrm{~K}$. 


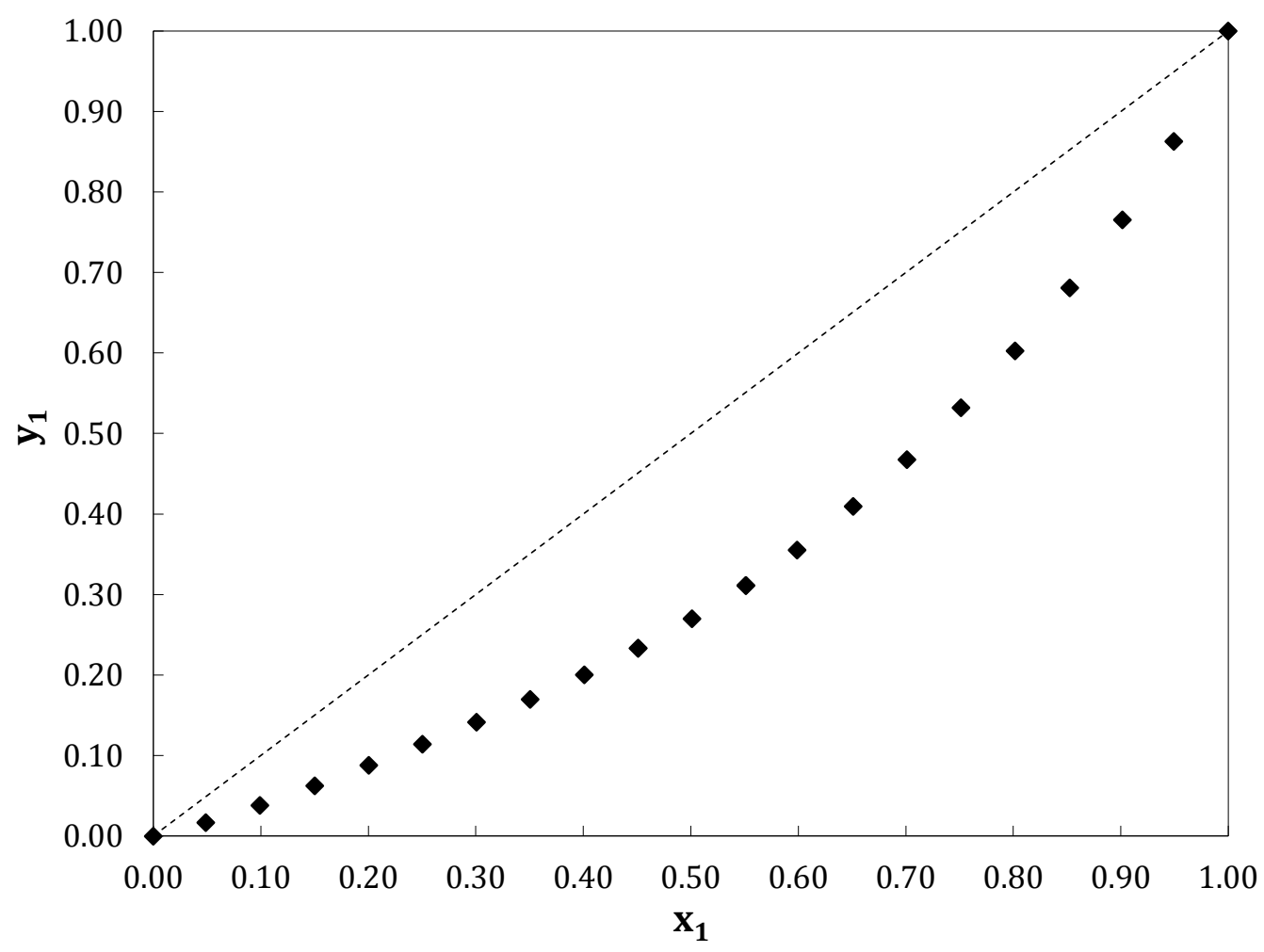

Figura 3.23. Fracción molar de la fase vapor del $\left(y_{1}\right)$ en función de la fracción molar de la fase líquida $\left(x_{1}\right)$ para el sistema binario B2: Ciclohexanona (1) + Hexanal (2) a 313.15 K. Datos calculados mediante la ecuación de Margules ( $\bullet$ ).

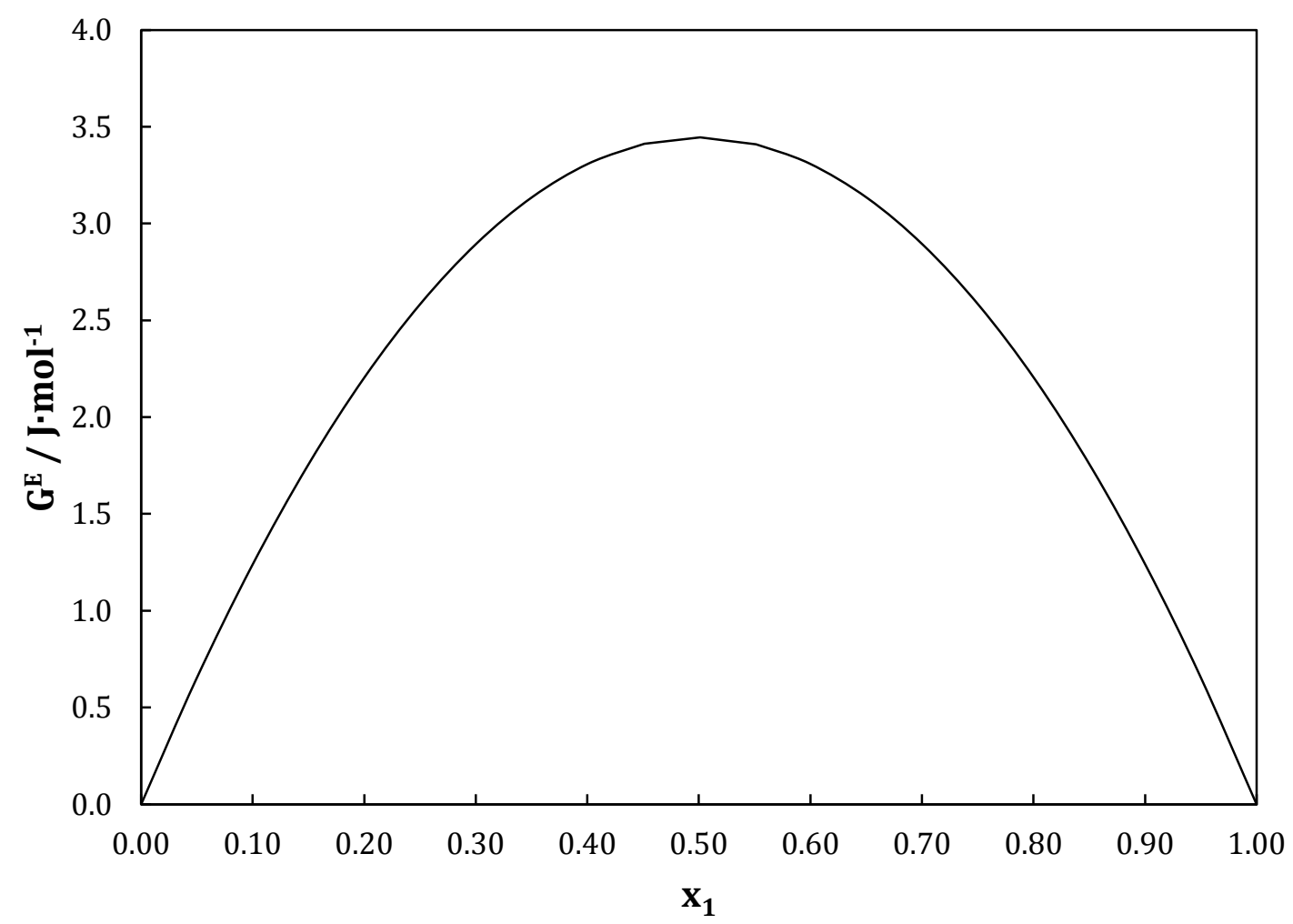

Figura 3.24. Energía de Gibbs de exceso $\left(G^{E}\right)$ en función de la fracción molar de la fase líquida $\left(x_{1}\right)$ para el sistema binario B2: Ciclohexanona (1) + Hexanal (2) a $313.15 \mathrm{~K}$. 


\subsubsection{Binario B2: Ciclohexanona (1) + Hexanal (2) a $333.15 \mathrm{~K}$.}

Tabla 3.21. Datos experimentales $p-x-y$ calculados mediante la ecuación de Margules y energía de Gibbs de exceso calculada mediante el modelo NRTL para el sistema binario B2: Ciclohexanona (1) + Hexanal (2) a $333.15 \mathrm{~K}$.

\begin{tabular}{cccccccc}
\hline $\mathbf{x}_{\mathbf{1}}$ & $\mathbf{y}_{\mathbf{1}, \text { calc }}$ & $\mathbf{p} / \mathbf{k P a}$ & $\begin{array}{c}\mathbf{G}^{\mathbf{E}} / \\
\mathbf{J} \cdot \mathbf{m o l}-\mathbf{1}\end{array}$ & $\mathbf{x}_{\mathbf{1}}$ & $\mathbf{y}_{\mathbf{1}, \text { calc }}$ & $\mathbf{p} / \mathbf{k P a}$ & $\begin{array}{c}\mathbf{G}^{\mathbf{E}} / \\
\mathbf{J} \cdot \mathbf{m o l}-\mathbf{1}\end{array}$ \\
\hline 0.0000 & 0.0000 & 9.119 & 0.00 & 0.5010 & 0.2792 & 6.424 & 23.72 \\
0.0481 & 0.0200 & 8.837 & 7.39 & 0.5523 & 0.3238 & 6.073 & 21.87 \\
0.0993 & 0.0438 & 8.550 & 13.68 & 0.6011 & 0.3715 & 5.799 & 19.78 \\
0.1494 & 0.0684 & 8.297 & 18.42 & 0.6510 & 0.4261 & 5.527 & 17.40 \\
0.1999 & 0.0938 & 8.030 & 21.92 & 0.7020 & 0.4877 & 5.252 & 14.79 \\
0.2499 & 0.1197 & 7.766 & 24.28 & 0.7527 & 0.5543 & 4.981 & 12.11 \\
0.2999 & 0.1465 & 7.502 & 25.65 & 0.8038 & 0.6266 & 4.707 & 9.38 \\
0.3502 & 0.1752 & 7.238 & 26.18 & 0.8452 & 0.6890 & 4.479 & 7.22 \\
0.4002 & 0.2060 & 7.002 & 25.96 & 0.8993 & 0.7781 & 4.182 & 4.49 \\
0.4504 & 0.2403 & 6.772 & 25.11 & 0.9423 & 0.8590 & 3.941 & 2.46 \\
0.4511 & 0.2409 & 6.741 & 25.09 & 1.0000 & 1.0000 & 3.604 & 0.00 \\
\hline
\end{tabular}

Tabla 3.22. Valor experimental de la presión de vapor de los compuestos puros $p_{i}^{\text {sat }}$, volúmenes molares $V_{i}^{L}$, y coeficientes del virial de los puros $B_{i i} y$ de la mezcla $B_{i j}$, para el sistema binario B2: Ciclohexanona (1) + Hexanal (2) a 333.15 K.

\begin{tabular}{|c|c|c|c|c|c|c|}
\hline $\begin{array}{l}p_{1}^{\text {sat }} \\
\mathrm{kPa}\end{array}$ & $\begin{array}{l}p_{2}^{\text {sat }} \\
\mathrm{kPa}\end{array}$ & $\begin{array}{c}\mathrm{V}_{1}^{\mathrm{L}} \\
\mathrm{cm}^{3} \cdot \mathrm{mol}^{-1}\end{array}$ & $\begin{array}{c}\mathrm{V}_{2}^{\mathrm{L}} \\
\mathrm{cm}^{3 \cdot} \cdot \mathrm{mol}^{-1}\end{array}$ & $\begin{array}{c}\mathrm{B}_{11} \\
\mathrm{~cm}^{3} \cdot \mathrm{mol}^{-1} \\
\end{array}$ & $\begin{array}{c}\quad \mathrm{B}_{22} \\
\mathrm{~cm}^{3} \cdot \mathrm{mol}^{-1}\end{array}$ & $\begin{array}{c}\mathrm{B}_{12} \\
\mathrm{~cm}^{3} \cdot \mathrm{mol}^{-1}\end{array}$ \\
\hline 3.606 & 9.119 & 107.76 & 128.01 & -2340 & -2567 & -2370 \\
\hline
\end{tabular}

Tabla 3.23. Parámetros de ajuste del sistema binario B2: Ciclohexanona (1) + Hexanal (2) a $333.15 \mathrm{~K}$.

\begin{tabular}{lcccc} 
& Margules & Wilson & NRTL & UNIQUAC \\
\hline $\mathrm{A}_{12}$ & -0.0270 & 0.5398 & -1.2652 & 1.9861 \\
$\mathrm{~A}_{21}$ & 0.2126 & 1.5529 & 1.5243 & 0.4070 \\
$\lambda_{12}$ & -0.4413 & & & \\
$\lambda_{21}$ & 0.9152 & & & \\
$\alpha$ & & & 0.1148 & \\
$\mathrm{rms} \Delta \mathrm{p} / \mathrm{kPa}$ & 0.025 & 0.035 & 0.036 & 0.035 \\
$\max |\Delta \mathrm{p}| / \mathrm{kPa}$ & 0.056 & 0.084 & 0.083 & 0.085 \\
\hline
\end{tabular}




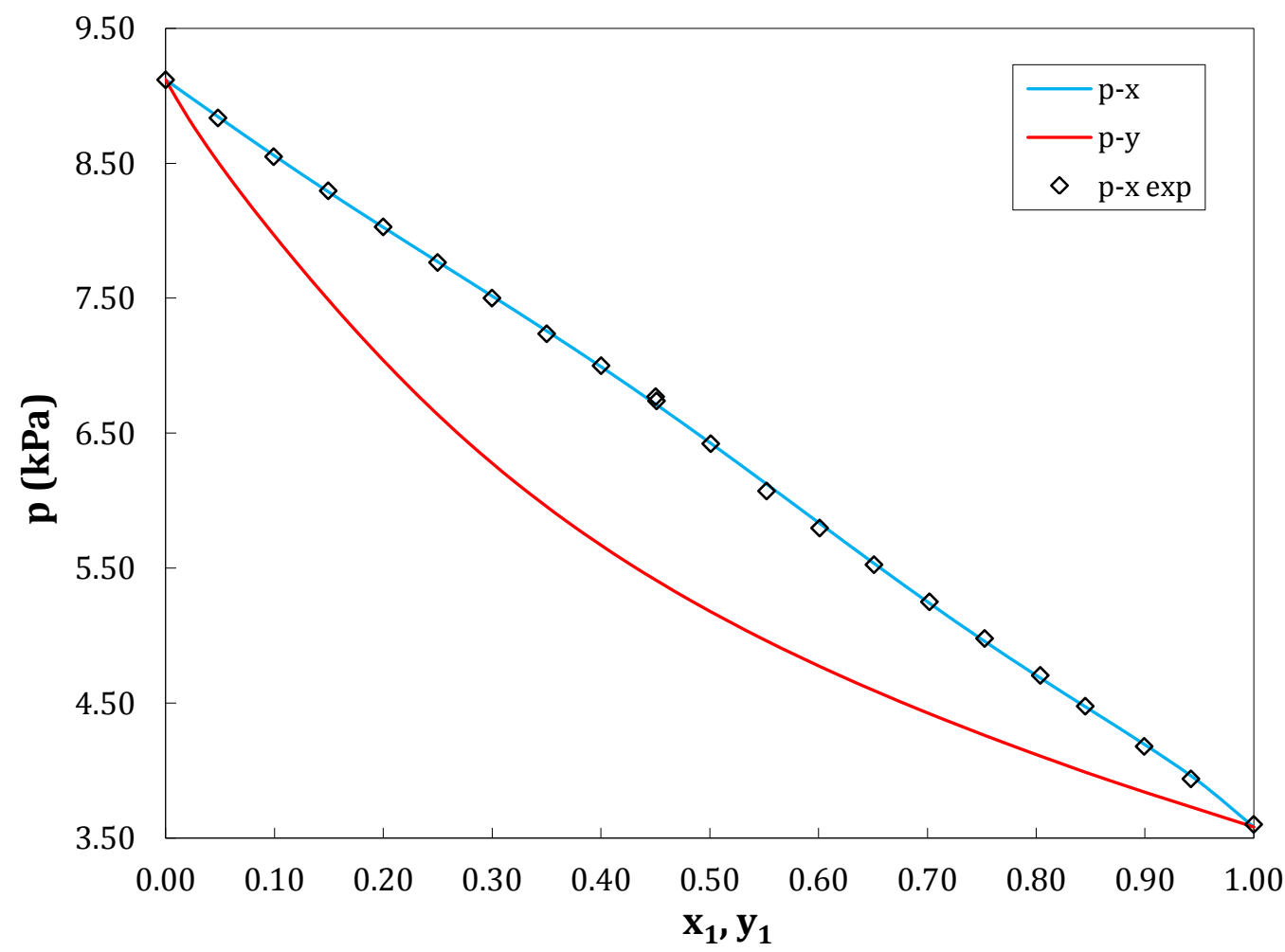

Figura 3.25. Presión de vapor $(p)$ en función de las fracciones molares de la fase líquida $x_{1} y$ la fase vapor $y_{1}$, para el sistema binario B2: Ciclohexanona (1) + Hexanal (2) a 333.15 K. ( $\left.\diamond\right)$ Datos experimentales. (-) Ajuste mediante la ecuación de Margules.

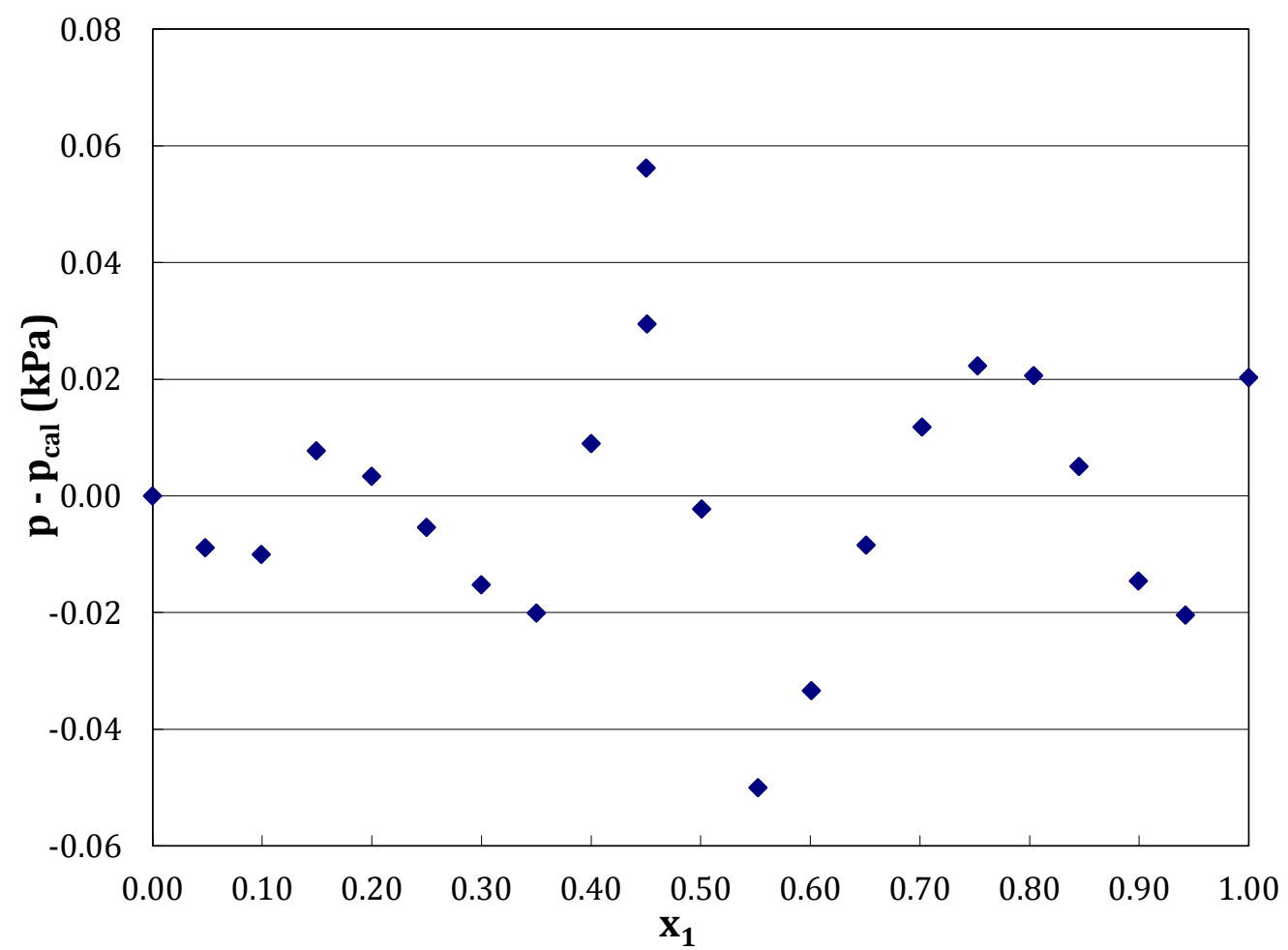

Figura 3.26. Comparación de las diferencias entre la presión experimental ( $p$ ) y la presión calculada (pal) mediante la ecuación de Margules ( $\bullet$ ), para el sistema binario B2: Ciclohexanona (1) + Hexanal (2) $a 333.15 \mathrm{~K}$. 


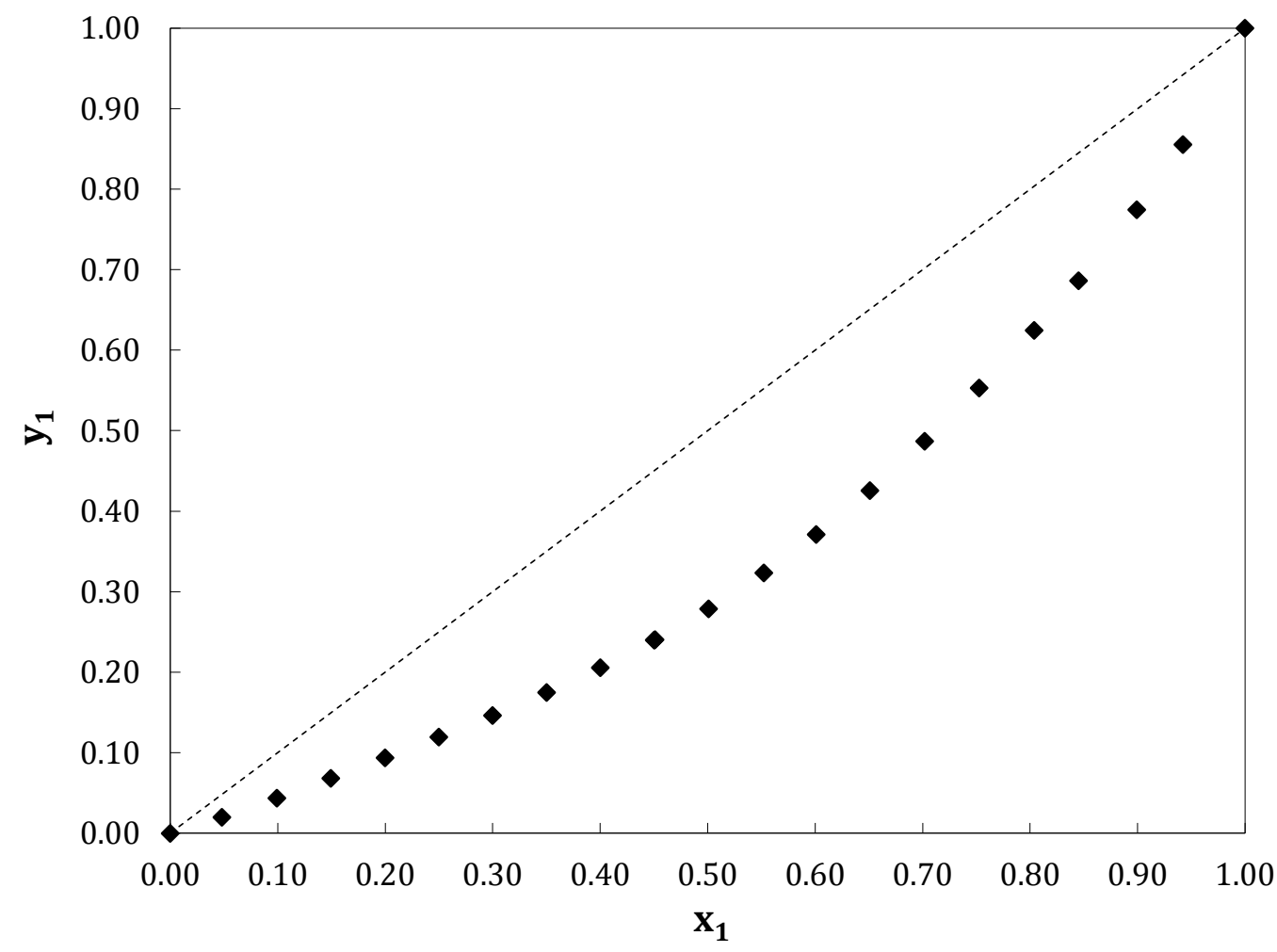

Figura 3.27. Fracción molar de la fase vapor del $\left(y_{1}\right)$ en función de la fracción molar de la fase líquida $\left(x_{1}\right)$ para el sistema binario B2: Ciclohexanona (1) + Hexanal (2) a 333.15 K. Datos calculados mediante la ecuación de Margules ( $\bullet$ ).

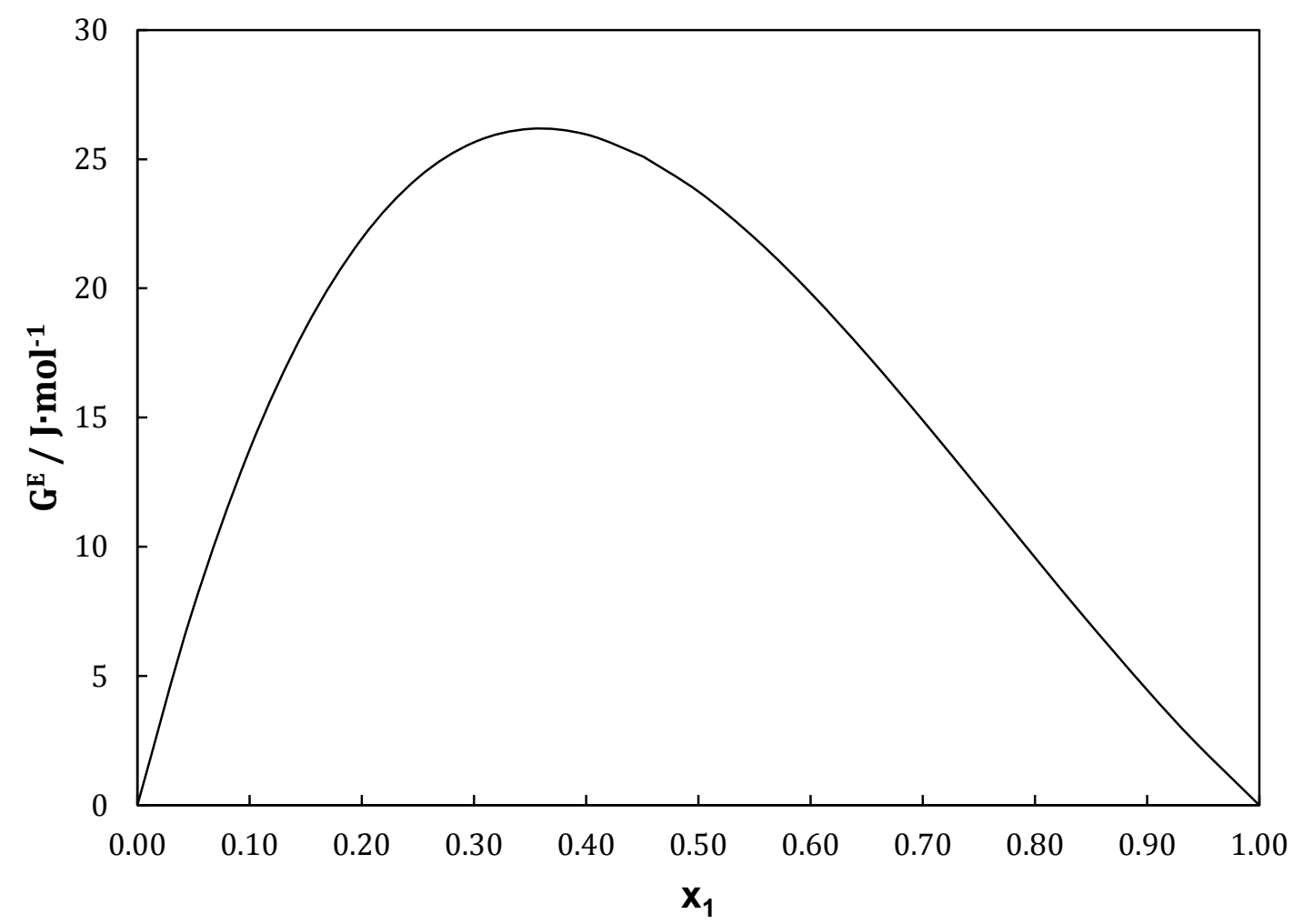

Figura 3.28. Energía de Gibbs de exceso $\left(G^{E}\right)$ en función de la fracción molar de la fase líquida $\left(x_{1}\right)$ para el sistema binario B2: Ciclohexanona (1) + Hexanal (2) a $333.15 \mathrm{~K}$. 


\subsubsection{Binario B2: Ciclohexanona (1) + Hexanal (2) a $353.15 \mathrm{~K}$.}

Tabla 3.24. Datos experimentales $p-x-y$ calculados mediante la ecuación de Margules y energía de Gibbs de exceso calculada mediante el modelo NRTL para el sistema binario B2: Ciclohexanona (1) + Hexanal (2) a $353.15 \mathrm{~K}$.

\begin{tabular}{cccccccc}
\hline $\mathbf{x}_{\mathbf{1}}$ & $\mathbf{y}_{\mathbf{1}, \text { calc }}$ & $\mathbf{p} / \mathbf{k P a}$ & $\begin{array}{c}\mathbf{G}^{\mathbf{E}} / \\
\mathbf{J} \cdot \mathbf{m o l}-\mathbf{1}\end{array}$ & $\mathbf{x}_{\mathbf{1}}$ & $\mathbf{y}_{\mathbf{1}, \text { calc }}$ & $\mathbf{p} / \mathbf{k P a}$ & $\begin{array}{c}\mathbf{G}^{\mathbf{E}} / \\
\mathbf{J} \cdot \mathbf{m o l}-\mathbf{1}\end{array}$ \\
\hline 0.0000 & 0.0000 & 20.643 & 0.00 & 0.5009 & 0.2866 & 14.722 & 66.42 \\
0.0493 & 0.0224 & 20.057 & 15.01 & 0.5512 & 0.3313 & 14.040 & 64.40 \\
0.0998 & 0.0473 & 19.486 & 28.18 & 0.5997 & 0.3795 & 13.422 & 61.27 \\
0.1499 & 0.0727 & 18.907 & 39.14 & 0.6497 & 0.4341 & 12.817 & 56.93 \\
0.2002 & 0.0985 & 18.324 & 48.16 & 0.7002 & 0.4936 & 12.215 & 51.46 \\
0.2501 & 0.1245 & 17.745 & 55.25 & 0.7518 & 0.5584 & 11.610 & 44.80 \\
0.3002 & 0.1516 & 17.183 & 60.61 & 0.8018 & 0.6241 & 11.017 & 37.40 \\
0.3504 & 0.1804 & 16.633 & 64.32 & 0.8514 & 0.6930 & 10.420 & 29.19 \\
0.4004 & 0.2118 & 16.102 & 66.46 & 0.8970 & 0.7624 & 9.867 & 20.94 \\
0.4505 & 0.2469 & 15.584 & 67.14 & 0.9414 & 0.8426 & 9.319 & 12.29 \\
0.4508 & 0.2471 & 15.511 & 67.14 & 1.0000 & 1.0000 & 8.295 & 0.00 \\
\hline
\end{tabular}

Tabla 3.25. Valor experimental de la presión de vapor de los compuestos puros $p_{i}^{\text {sat }}$, volúmenes molares $V_{i}^{L}$, y coeficientes del virial de los puros $B_{i i} y$ de la mezcla $B_{i j}$, para el sistema binario B2: Ciclohexanona (1) + Hexanal (2) a $353.15 \mathrm{~K}$.

\begin{tabular}{|c|c|c|c|c|c|c|}
\hline $\begin{array}{l}p_{1}^{\text {sat }} \\
\mathrm{kPa}\end{array}$ & $\begin{array}{l}p_{2}^{\text {sat }} \\
\mathbf{k P a}\end{array}$ & $\begin{array}{c}\mathrm{V}_{1}^{\mathrm{L}} \\
\mathrm{cm}^{3} \cdot \mathrm{mol}^{-1}\end{array}$ & $\begin{array}{c}\mathrm{V}_{2}^{\mathrm{L}} \\
\mathrm{cm}^{3} \cdot \mathrm{mol}^{-1}\end{array}$ & $\begin{array}{c}\mathrm{B}_{11} \\
\mathrm{~cm}^{3} \cdot \mathrm{mol}^{-1} \\
\end{array}$ & $\begin{array}{c}\quad \mathrm{B}_{22} \\
\mathrm{~cm}^{3} \cdot \mathrm{mol}^{-1}\end{array}$ & $\begin{array}{c}\mathrm{B}_{12} \\
\mathrm{~cm}^{3} \cdot \mathrm{mol}^{-1}\end{array}$ \\
\hline 8.295 & 20.643 & 109.94 & 131.24 & -1987 & -2158 & -2006 \\
\hline
\end{tabular}

Tabla 3.26. Parámetros de ajuste del sistema binario B2: Ciclohexanona (1) + Hexanal (2) $353.15 \mathrm{~K}$.

\begin{tabular}{lcccc}
\hline & Margules & Wilson & NRTL & UNIQUAC \\
\hline $\mathrm{A}_{12}$ & 0.05302 & 0.63482 & -0.3966 & 1.8821 \\
$\mathrm{~A}_{21}$ & 0.39338 & 1.33941 & 0.5600 & 0.4431 \\
$\lambda_{12}$ & -0.24618 & & & \\
$\lambda_{21}$ & 1.31833 & & & \\
$\alpha$ & & & 0.3100 & \\
$\operatorname{rms} \Delta \mathrm{p} / \mathrm{kPa}$ & 0.049 & 0.094 & 0.092 & 0.099 \\
$\max |\Delta \mathrm{p}| / \mathrm{kPa}$ & 0.142 & 0.223 & 0.222 & 0.201 \\
\hline
\end{tabular}




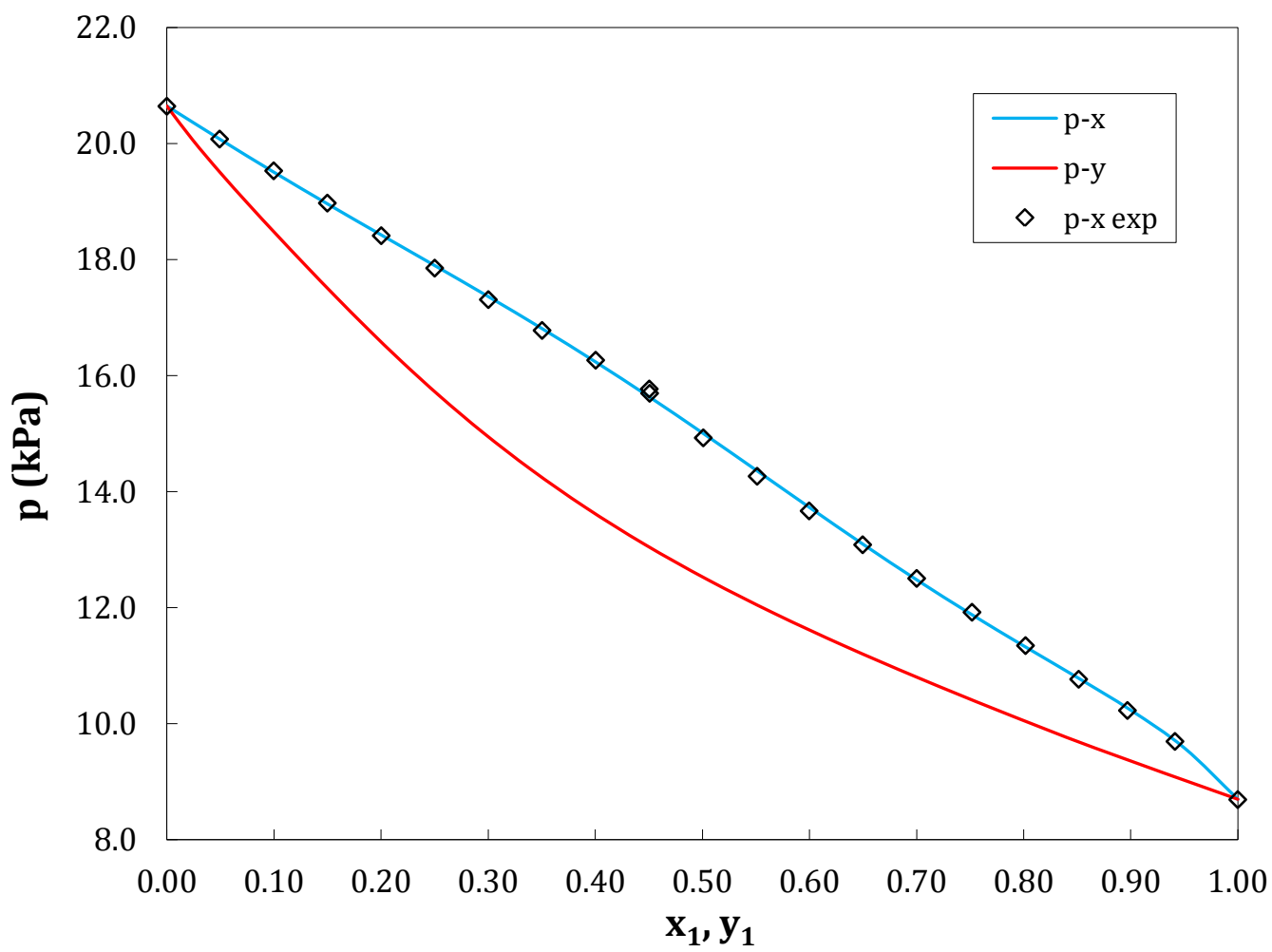

Figura 3.29. Presión de vapor $(p)$ en función de las fracciones molares de la fase líquida $x_{1} y$ la fase vapor $y_{1}$, para el sistema binario B2: Ciclohexanona (1) + Hexanal (2) a 353.15 K. ( $\left.\diamond\right)$ Datos experimentales. (-) Ajuste mediante la ecuación de Margules.

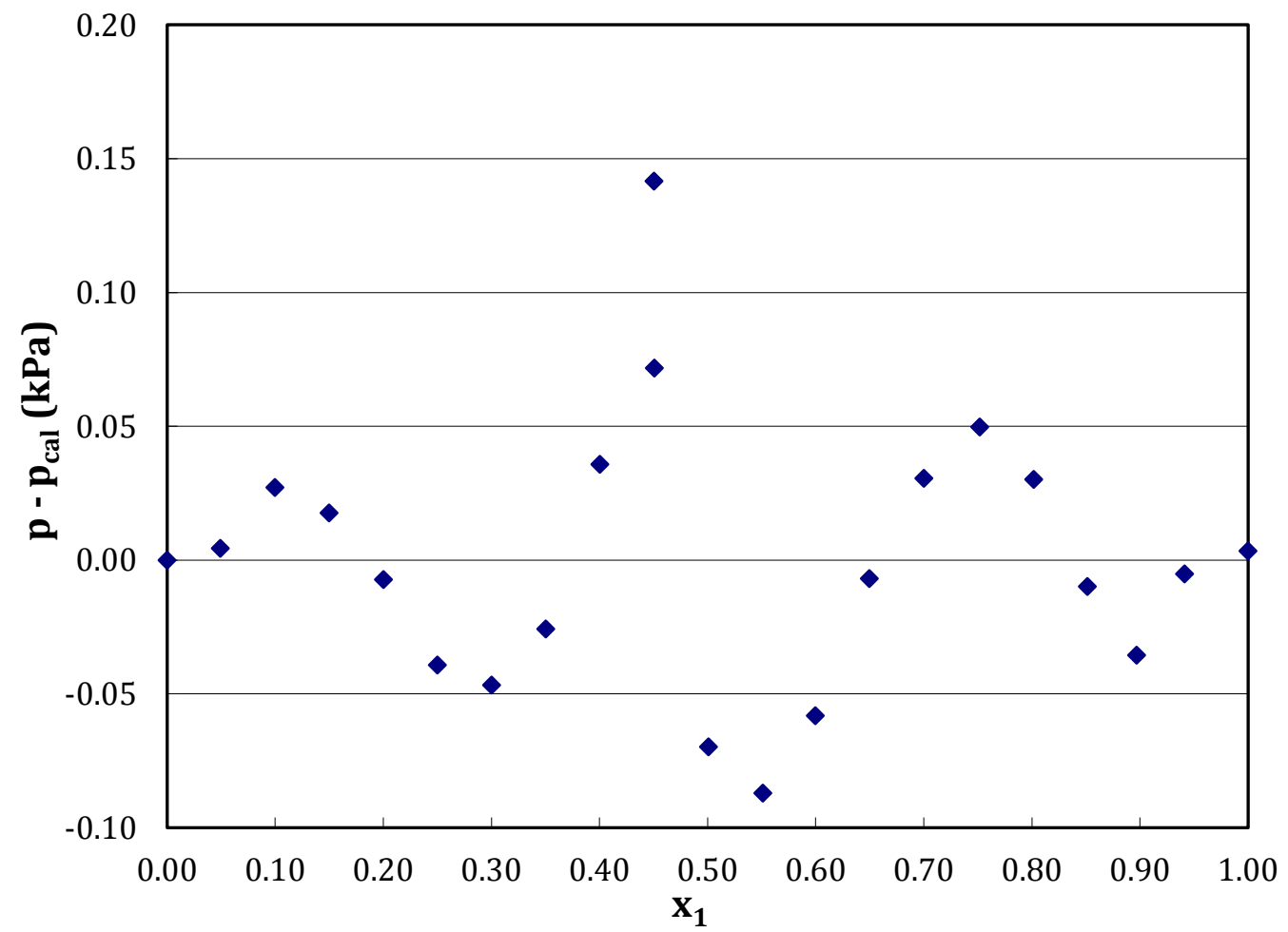

Figura 3.30. Comparación de las diferencias entre la presión experimental ( $p$ ) y la presión calculada (pal) mediante la ecuación de Margules ( $\bullet$ ), para el sistema binario B2: Ciclohexanona (1) + Hexanal (2) $a 353.15 \mathrm{~K}$. 


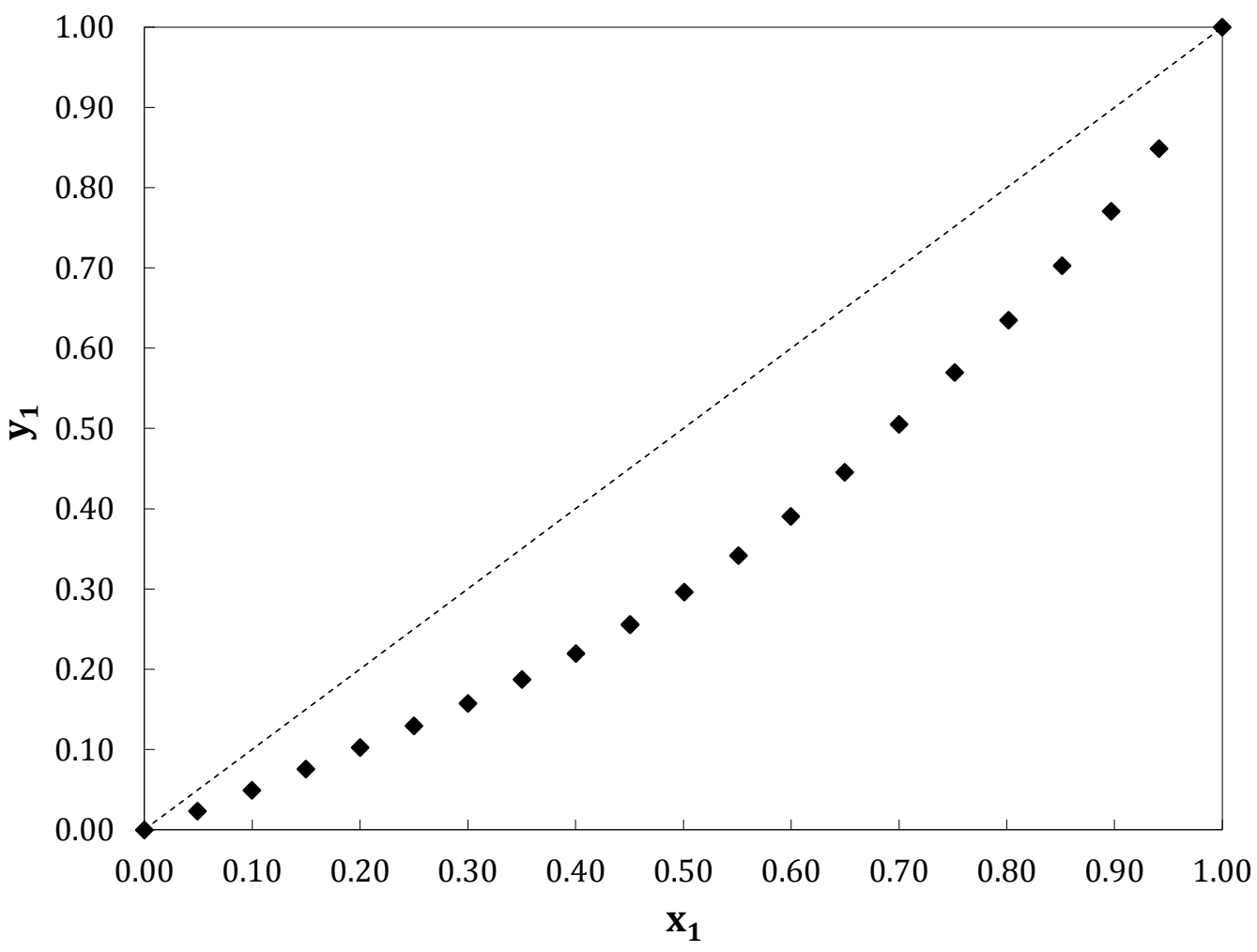

Figura 3.31. Fracción molar de la fase vapor del $\left(y_{1}\right)$ en función de la fracción molar de la fase líquida $\left(x_{1}\right)$ para el sistema binario B2: Ciclohexanona (1) + Hexanal (2) a 353.15 K. Datos calculados mediante la ecuación de Margules ( $\bullet$ ).

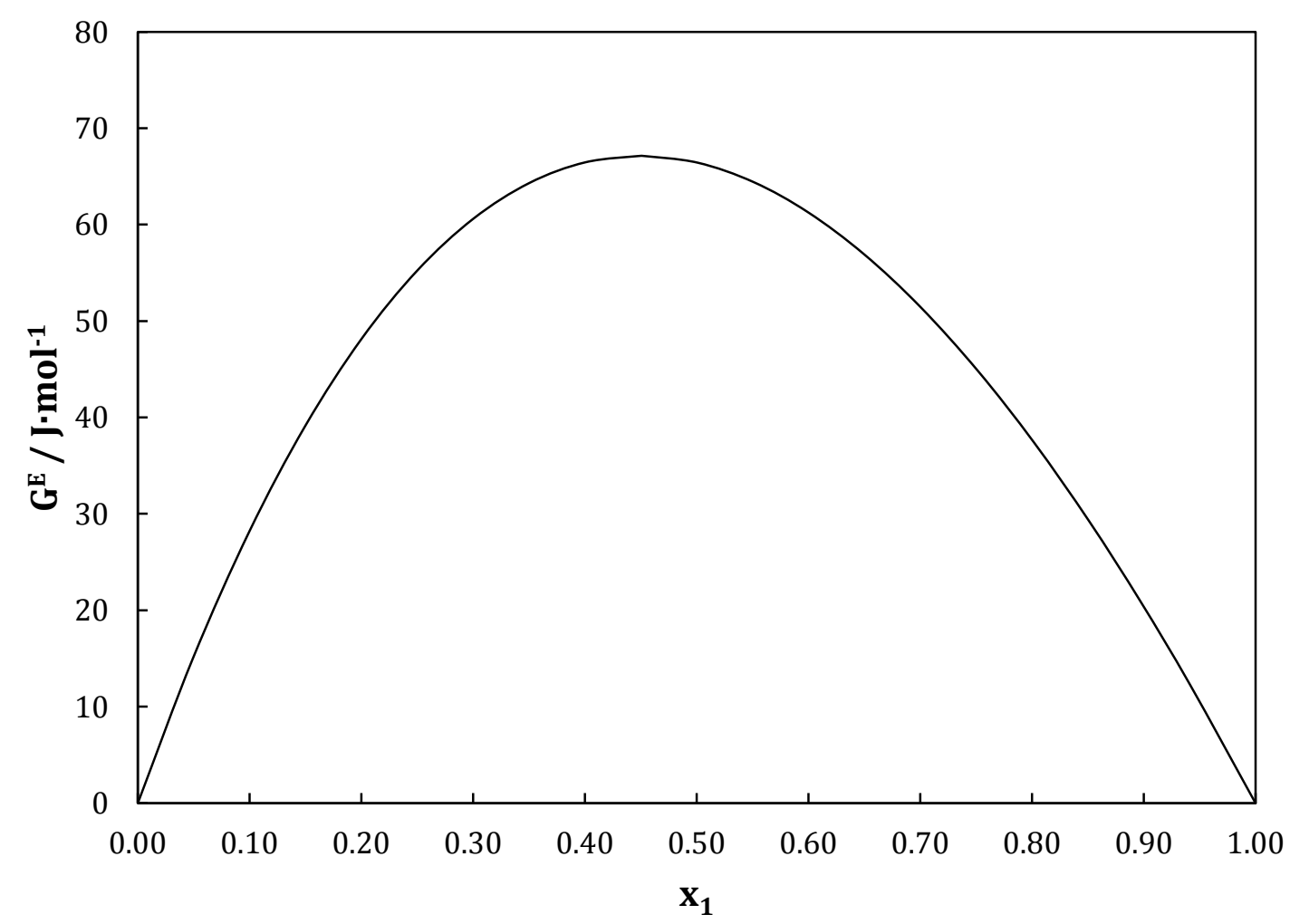

Figura 3.32. Energía de Gibbs de exceso $\left(G^{E}\right)$ en función de la fracción molar de la fase líquida $\left(x_{1}\right)$ para el sistema binario B2: Ciclohexanona (1) + Hexanal (2) a $353.15 \mathrm{~K}$. 


\subsubsection{Binario B2: Ciclohexanona (1) + Hexanal (2) a $393.15 \mathrm{~K}$.}

Tabla 3.27. Datos experimentales $p-x-y$ calculados mediante la ecuación de Margules y energía de Gibbs de exceso calculada mediante el modelo NRTL para el sistema binario B2: Ciclohexanona (1) + Hexanal (2) a $393.15 \mathrm{~K}$.

\begin{tabular}{cccccccc}
\hline $\mathbf{x}_{\mathbf{1}}$ & $\mathbf{y}_{\mathbf{1}, \text { calc }}$ & $\mathbf{p} / \mathbf{k P a}$ & $\begin{array}{c}\mathbf{G}^{\mathbf{E}} / \\
\mathbf{J} \cdot \mathbf{m o l}-\mathbf{1}\end{array}$ & $\mathbf{x}_{\mathbf{1}}$ & $\mathbf{y}_{\mathbf{1}, \text { calc }}$ & $\mathbf{p} / \mathbf{k P a}$ & $\begin{array}{c}\mathbf{G}^{\mathbf{E}} / \\
\mathbf{J} \cdot \mathbf{m o l}^{-\mathbf{1}}\end{array}$ \\
\hline 0.0000 & 0.0000 & 79.036 & 0.00 & 0.5511 & 0.3547 & 56.575 & 50.02 \\
0.0478 & 0.0273 & 77.525 & 33.30 & 0.6014 & 0.4054 & 54.241 & 40.93 \\
0.1002 & 0.0562 & 75.419 & 58.61 & 0.6511 & 0.4604 & 51.990 & 32.26 \\
0.1504 & 0.0833 & 73.563 & 74.08 & 0.7011 & 0.5202 & 49.756 & 24.18 \\
0.2005 & 0.1102 & 71.799 & 82.75 & 0.7514 & 0.5849 & 47.528 & 16.92 \\
0.2509 & 0.1378 & 70.144 & 86.10 & 0.8010 & 0.6529 & 45.364 & 10.83 \\
0.3023 & 0.1681 & 67.992 & 85.31 & 0.8510 & 0.7260 & 43.142 & 5.94 \\
0.3507 & 0.1974 & 66.035 & 81.43 & 0.8995 & 0.8028 & 41.032 & 2.51 \\
0.4009 & 0.2303 & 63.901 & 75.32 & 0.9510 & 0.8945 & 38.681 & 0.42 \\
0.4510 & 0.2679 & 61.601 & 67.66 & 1.0000 & 1.0000 & 36.439 & 0.00 \\
0.5011 & 0.3091 & 59.014 & 59.05 & & & & \\
\hline
\end{tabular}

Tabla 3.28. Valor experimental de la presión de vapor de los compuestos puros $p_{i}^{\text {sat }}$, volúmenes molares $V_{i}^{L}$, y coeficientes del virial de los puros $B_{i i} y$ de la mezcla $B_{i j}$, para el sistema binario B2: Ciclohexanona (1) + Hexanal (2) a 393.15 K.

\begin{tabular}{|c|c|c|c|c|c|c|}
\hline $\begin{array}{l}p_{1}^{\text {sat }} \\
\mathrm{kPa}\end{array}$ & $\begin{array}{l}p_{2}^{\text {sat }} \\
\mathbf{k P a}\end{array}$ & $\begin{array}{c}\mathrm{V}_{1}^{\mathrm{L}} \\
\mathrm{cm}^{3} \cdot \mathrm{mol}^{-1}\end{array}$ & $\begin{array}{c}\mathrm{V}_{2}^{\mathrm{L}} \\
\mathrm{cm}^{3} \cdot \mathrm{mol}^{-1}\end{array}$ & $\begin{array}{c}\mathrm{B}_{11} \\
\mathrm{~cm}^{3} \cdot \mathrm{mol}^{-1} \\
\end{array}$ & $\begin{array}{c}\quad \mathrm{B}_{22} \\
\mathrm{~cm}^{3} \cdot \mathrm{mol}^{-1}\end{array}$ & $\begin{array}{c}\mathrm{B}_{12} \\
\mathrm{~cm}^{3} \cdot \mathrm{mol}^{-1}\end{array}$ \\
\hline 36.439 & 79.036 & 114.53 & 139.13 & -1486 & -1582 & -1489 \\
\hline
\end{tabular}

Tabla 3.29. Parámetros de ajuste del sistema binario B2: Ciclohexanona (1) + Hexanal (2) a $393.15 \mathrm{~K}$.

\begin{tabular}{lcccc} 
& Margules & Wilson & NRTL & UNIQUAC \\
\hline $\mathrm{A}_{12}$ & 0.2123 & 0.2634 & -0.6058 & 2.2363 \\
$\mathrm{~A}_{21}$ & 0.1096 & 2.0503 & 1.0903 & 0.2937 \\
$\lambda_{12}$ & 0.1011 & & & \\
$\lambda_{21}$ & 0.6242 & & & \\
$\alpha$ & & & 0.5425 & \\
$\operatorname{rms~} \Delta \mathrm{p} / \mathrm{kPa}$ & 0.133 & 0.197 & 0.181 & 0.165 \\
$\max |\Delta \mathrm{p}| / \mathrm{kPa}$ & 0.217 & 0.371 & 0.280 & 0.292 \\
\hline
\end{tabular}




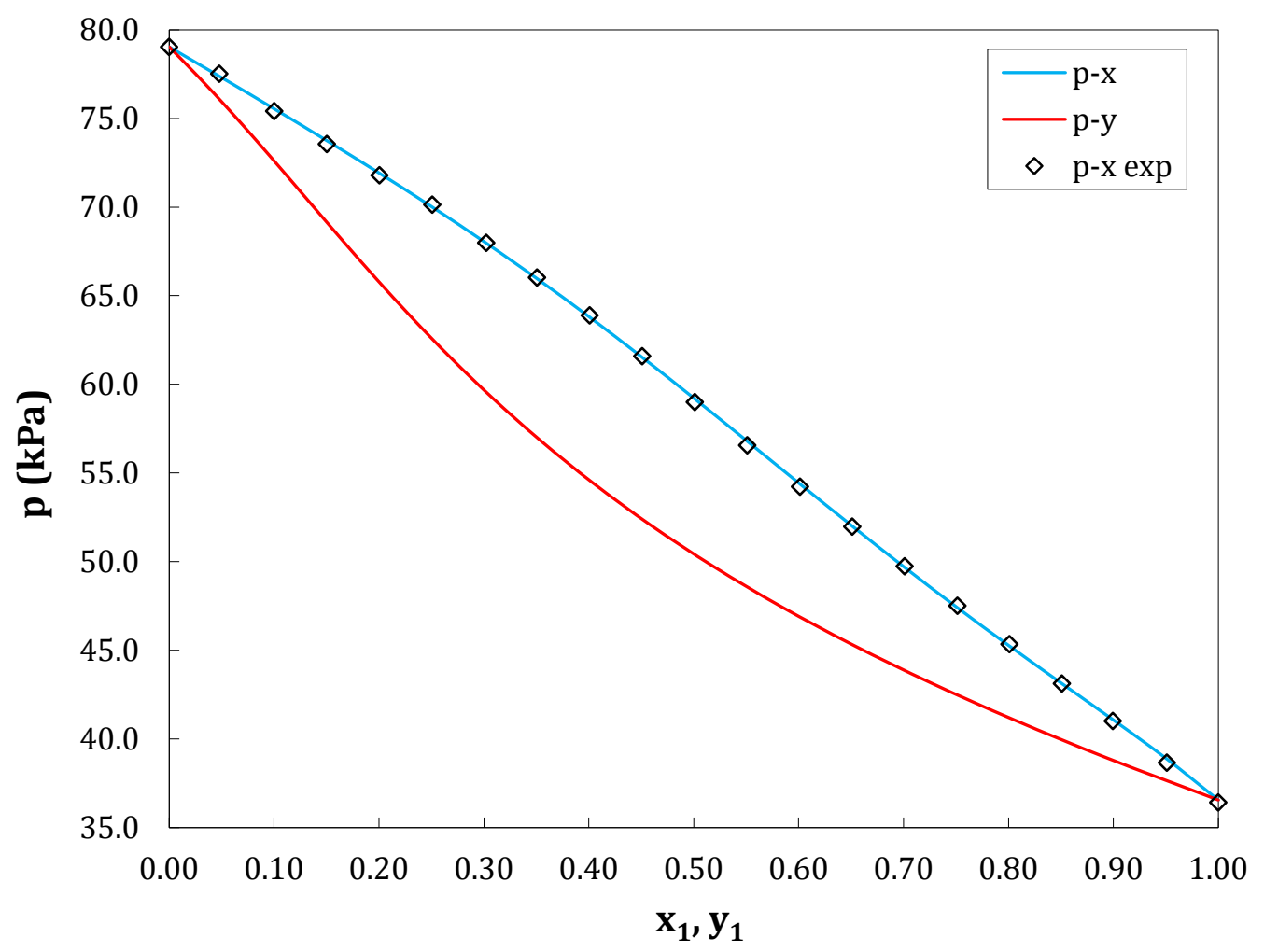

Figura 3.33. Presión de vapor $(p)$ en función de las fracciones molares de la fase líquida $x_{1} y$ la fase vapor $y_{1}$, para el sistema binario B2: Ciclohexanona (1) + Hexanal (2) a 393.15 K. ( $\left.\diamond\right)$ Datos experimentales. (-) Ajuste mediante la ecuación de Margules.

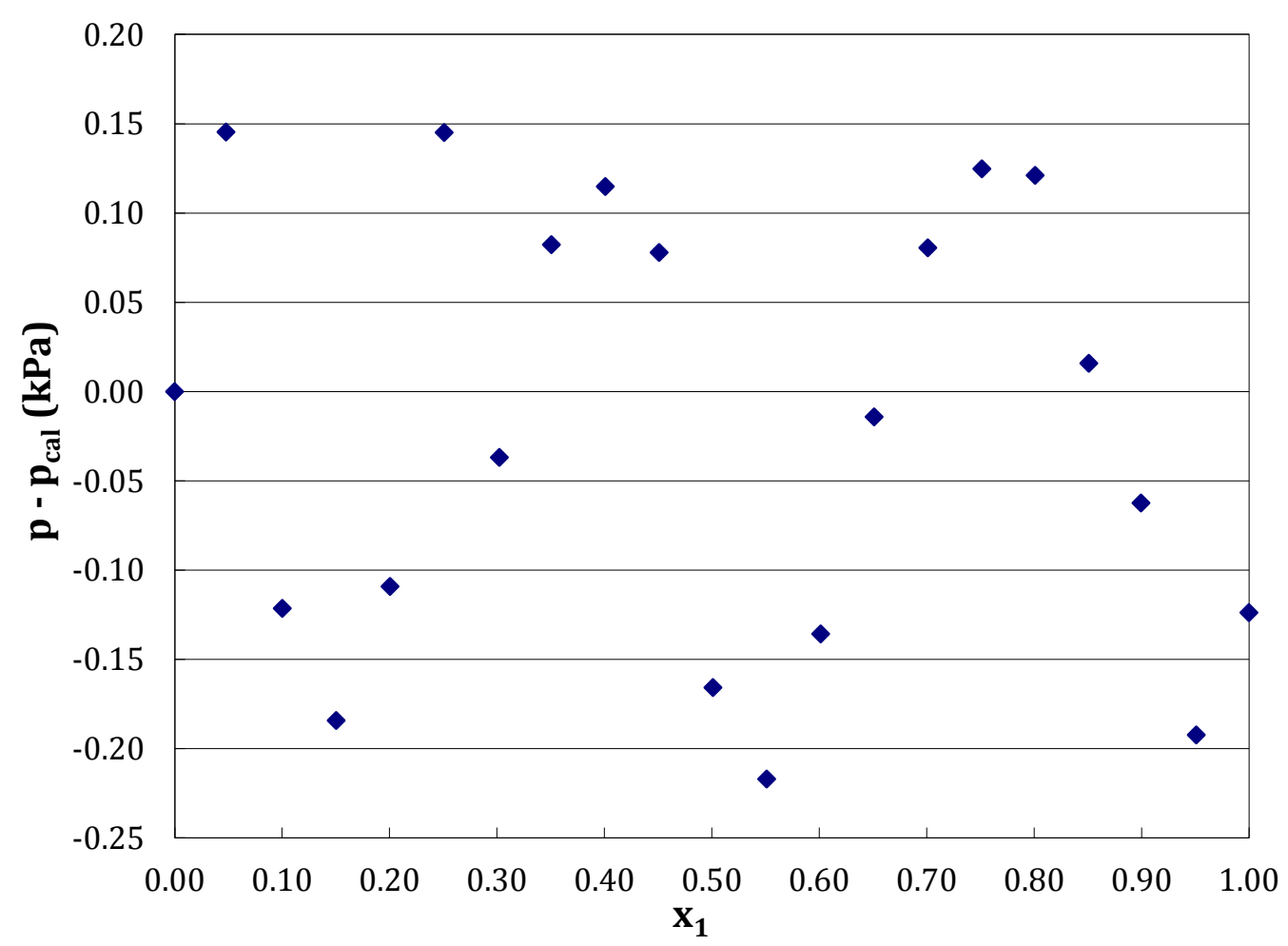

Figura 3.34. Comparación de las diferencias entre la presión experimental ( $p$ ) y la presión calculada (pal) mediante la ecuación de Margules ( $\bullet$ ), para el sistema binario B2: Ciclohexanona (1) + Hexanal (2) $a 393.15 \mathrm{~K}$. 


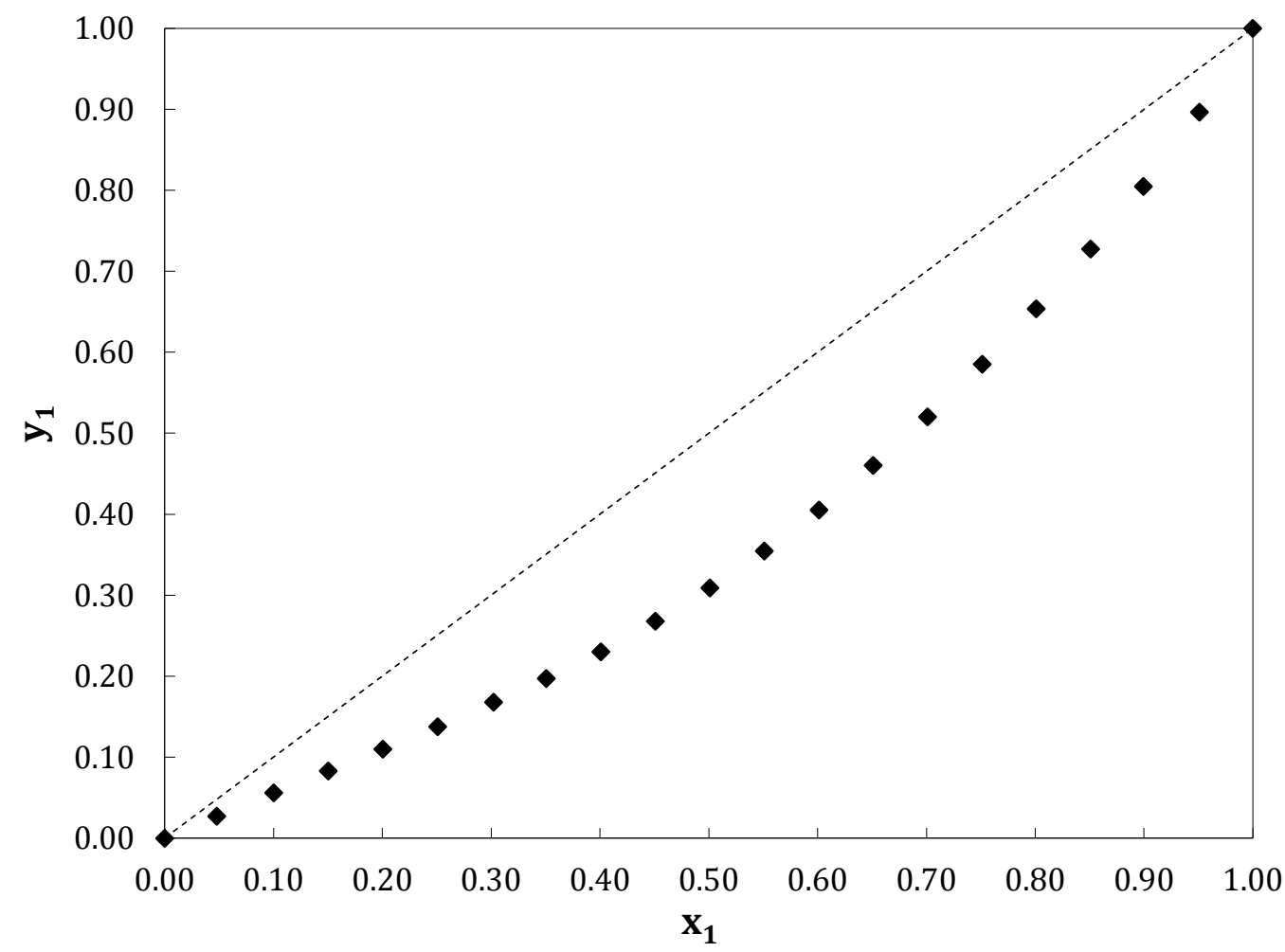

Figura 3.35. Fracción molar de la fase vapor del $\left(y_{1}\right)$ en función de la fracción molar de la fase líquida $\left(x_{1}\right)$ para el sistema binario B2: Ciclohexanona (1) + Hexanal (2) a 393.15 K. Datos calculados mediante la ecuación de Margules ( $\bullet$ ).

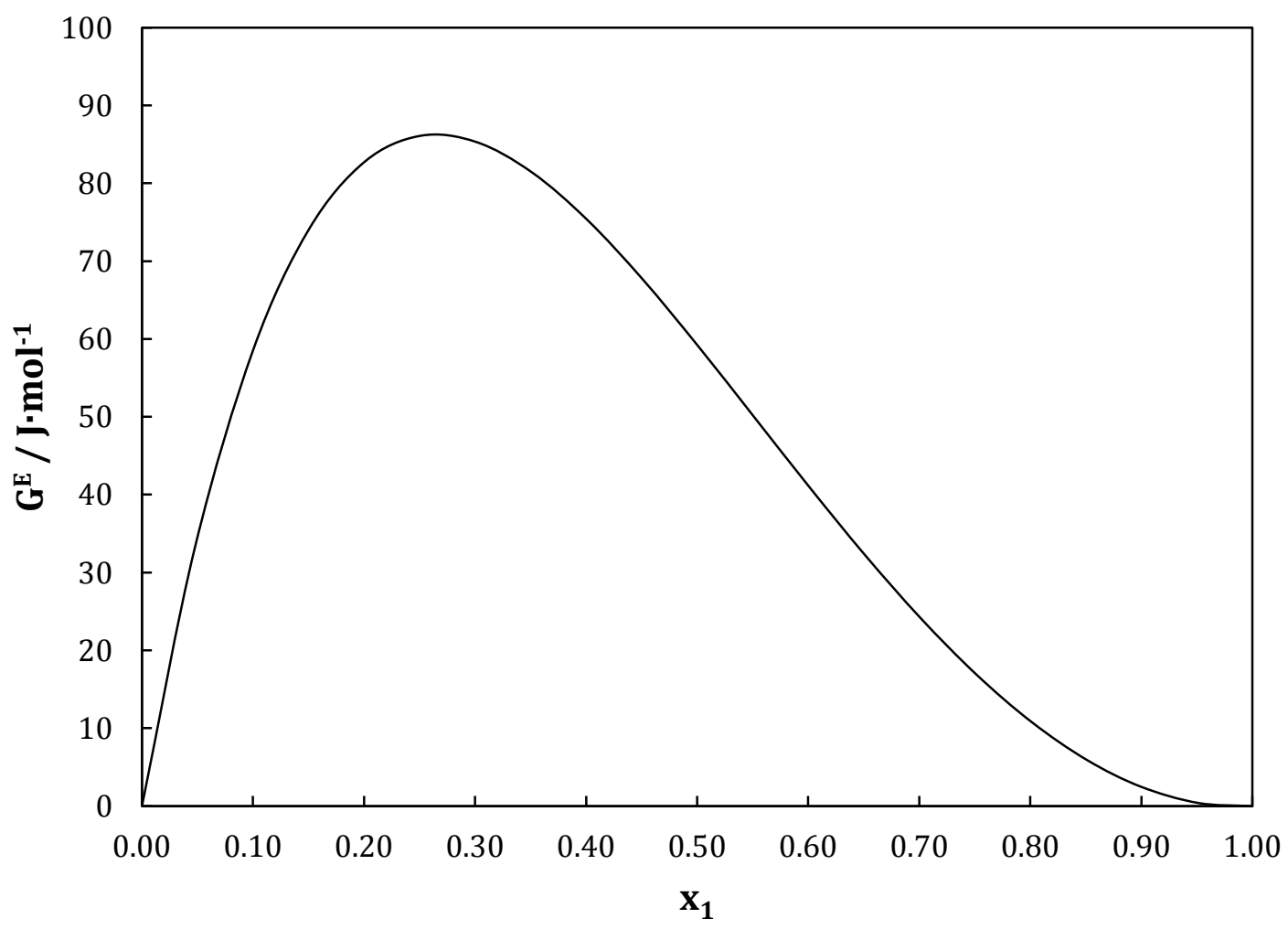

Figura 3.36. Energía de Gibbs de exceso $\left(G^{E}\right)$ en función de la fracción molar de la fase líquida $\left(x_{1}\right)$ para el sistema binario B2: Ciclohexanona (1) + Hexanal (2) a 393.15 K. 


\subsection{Discusión de los resultados.}

El sistema binario 2-Pentanol (1) + Ciclohexano (2) a $313.15 \mathrm{~K}$ ha sido medido como puesta a punto y validación del equipo. Los datos fueron publicados en el trabajo de Moreau et al. [22].

En la tabla 3.30. se resumen los valores del $r m s(\Delta p)$ de todos los sistemas binarios estudiados, donde $\Delta p$ es la diferencia entre la presión calculada y la presión experimental. Estando siempre en un rango de desviación de $0.003 \mathrm{kPa}$ a $0.051 \mathrm{kPa}$ para la ecuación de Margules en los sistemas binarios formados por el 2-Heptanona. En el caso de los sistemas binarios formados por el Hexanal, esta desviación cuadrática aumenta ligeramente, estando en el rango de $0.010 \mathrm{kPa}$ a $0.133 \mathrm{kPa}$. En todos los casos las desviaciones siempre fueron menores al $0.90 \%$. Para el resto de los modelos los valores del $r m s(\Delta p)$ aumentan ligeramente.

El promedio de las desviaciones relativas obtenidas por la ecuación de Margules fueron $0.14 \%, 0.09 \%, 0.12 \%$ y $0.07 \%$ para el sistema con 2-heptanone a $313.15 \mathrm{~K}, 333.15 \mathrm{~K}$, $353.15 \mathrm{~K}$ y $393.15 \mathrm{~K}$ respectivamente. En el sistema con hexanal fue $0.30 \%, 0.28 \%, 0.25 \%$ y $0.17 \%$ para $313.15 \mathrm{~K}, 333.15 \mathrm{~K}, 353.15 \mathrm{~K}$ y $393.15 \mathrm{~K}$. En el sistema 2-pentanol + ciclohexano fue de $0.12 \%$.

Tabla 3.30. Desviación cuadrática media de los tres sistemas binarios calculada con la ecuación de Margules y los modelos de Wilson, NRTL y UNIQUAC.

\begin{tabular}{ccccc}
\hline \multicolumn{5}{c}{ rms $\Delta$ p / kPa } \\
Temperatura & Margules & Wilson & NRTL & UNIQUAC \\
\hline \multirow{3}{*}{$313.15 \mathrm{~K}$} & 0.031 & 2-Pentanol (1) + Ciclohexano (2) \\
& \multicolumn{4}{c}{ Ciclohexanona (1) + 2-Heptanona (2) } \\
$313.15 \mathrm{~K}$ & 0.003 & 0.003 & 0.004 & 0.264 \\
$333.15 \mathrm{~K}$ & 0.008 & 0.009 & 0.009 & 0.003 \\
$353.15 \mathrm{~K}$ & 0.019 & 0.025 & 0.024 & 0.009 \\
$393.15 \mathrm{~K}$ & 0.051 & 0.075 & 0.061 & 0.024 \\
& & Ciclohexanona (1) + Hexanal (2) & 0.089 \\
$313.15 \mathrm{~K}$ & 0.010 & 0.018 & 0.017 & 0.019 \\
$333.15 \mathrm{~K}$ & 0.025 & 0.035 & 0.036 & 0.035 \\
$353.15 \mathrm{~K}$ & 0.049 & 0.094 & 0.092 & 0.099 \\
$393.15 \mathrm{~K}$ & 0.133 & 0.197 & 0.181 & 0.165 \\
\hline
\end{tabular}

Sólo se encontraron azeótropos en el sistema ciclohexanona + 2-heptanona para tres de las cuatro temperaturas estudiadas. Los datos del azeótropo (fracción molar y presión) están resumidos en la tabla 3.31 . 
Tabla 3.31. Datos de los azeótropos para el sistema ciclohexanona + 2-heptanona calculados usando diferentes modelos.

\begin{tabular}{|c|c|c|c|c|c|c|c|}
\hline \multicolumn{2}{|c|}{ Margules } & \multicolumn{2}{|c|}{ Wilson } & \multicolumn{2}{|c|}{ NRTL } & \multicolumn{2}{|c|}{ UNIQUAC } \\
\hline $\begin{array}{c}\mathbf{x}_{1} \\
\text { azeótropo }\end{array}$ & $\begin{array}{c}\mathbf{p}_{\text {azeótropo }} \\
\mathbf{k P a}\end{array}$ & $\begin{array}{c}\mathbf{x}_{\mathbf{1}} \\
\text { azeótropo }\end{array}$ & $\begin{array}{c}\mathbf{p}_{\text {azeótropo }} \\
\mathbf{k P a} \\
\end{array}$ & $\begin{array}{c}\mathbf{X}_{1}, \\
\text { azeótropo }\end{array}$ & $\begin{array}{c}\mathbf{p}_{\text {azeótropo }} \\
\mathbf{k P a}\end{array}$ & $\begin{array}{c}\mathbf{x}_{1} \\
\text { azeótropo }\end{array}$ & $\begin{array}{c}\mathbf{p}_{\text {azeótropo }} \\
\mathbf{k P a} \\
\end{array}$ \\
\hline \multicolumn{8}{|c|}{ Temperatura a $313.15 \mathrm{~K}$} \\
\hline 0.601 & 1.324 & 0.560 & 1.323 & 0.560 & 1.323 & 0.574 & 1.323 \\
\hline \multicolumn{8}{|c|}{ Temperatura a $333.15 \mathrm{~K}$} \\
\hline 0.311 & 3.754 & 0.276 & 3.753 & 0.283 & 3.753 & 0.266 & 3.753 \\
\hline \multicolumn{8}{|c|}{ Temperatura a $353.15 \mathrm{~K}$} \\
\hline 0.292 & 9.356 & 0.224 & 9.352 & 0.238 & 9.349 & 0.238 & 9.346 \\
\hline
\end{tabular}

Puede verse que el azeótropo aparece a bajas composiciones de ciclohexanona cuando la temperatura incrementa, y desaparece a $393.15 \mathrm{~K}$.

Los sistemas estudiados no presentan grandes desviaciones de la idealidad en términos de energía de Gibbs en exceso, siendo el sistema menos ideal el formado por la 2-heptanona, donde los valores máximos de $G^{E}$ están comprendidos entre $57.62 \mathrm{~J} / \mathrm{mol}$ y $90.30 \mathrm{~J} / \mathrm{mol}$, mientras que el sistema formado por el hexanal tiene unos valores máximos de $G^{E}$ comprendidos entre $3.45 \mathrm{~J} / \mathrm{mol}$ y $86.24 \mathrm{~J} / \mathrm{mol}$.

\subsection{Referencias.}

[1] U. Schuchardt, W. A. Carvalho, E. V. Spinacé. "Why is it interesting to study cyclohexane oxidation?". Synlett. Vol. 10, (1993) 713-718.

[2] J. Xie, Y. Wang, Y. Li, Y. Wei. "Self-assembly preparation of $\mathrm{Au} / \mathrm{SiO}_{2}$ catalyst and its catalysis for cyclohexane oxidation with air". Reac. Kinet. Mech. Cat. Vol. 102, (2011) 143154.

[3] Q. Zhao, Y. Huang, K. Tang, J. Li, Y. He. "Adsorption of HZSM-5 zeolite on hexanal and 2heptanone in cyclohexanone". Journal of the Chinese Ceramic Society. Vol. 39, (2011) 18621866.

[4] J. A. Riddick, W. B. Bunger, T. K. Sakano. "Organic solvents, physical properties and methods of purification, techniques of chemistry, vol. II". Wiley/Interscience (1986).

[5] D. Ambrose, J. H. Ellender, E. B. Lees, C. H. S. Sprake, R. Townsend. "Thermodynamic properties of organic oxygen compounds". J. Chem. Thermodyn. Vol. 7, (1975) 453-472.

[6] M. Teodorescu, A. Barhala, D. Dragoescu. "Isothermal (vapor + liquid) equilibria for the binary (cyclopentanone or cyclohexanone with 1,1,2,2-tetrachloroethane) systems at temperatures of $(343.15,353.15$, and 363.15)". K. J. Chem. Thermodynamics. Vol. 38, (2006) 1432-1437. 
[7] E. F. Meyer, R. D. Hotz. "High-precision vapor-pressure data for eight organic compounds". Journal of Chemical and Engineering Data. Vol. 18, (1973) 359-362.

[8] D. A. Meneses, A. Bejarano, J. C. de la Fuente. "Vapor pressure data for ethyl-2methylbutyrate, hexanal and (E)-2-hexenal at a pressure range of (25 to 190) $\mathrm{KPa}^{\prime}$. The Journal of Chemical Thermodynamics. Vol. 74, (2014) 16-21.

[9] M. Palczewska-Tulińska, P. Oracz. "Vapor pressures of hexanal, 2-methylcyclohexanone, and 2-cyclohexen-1-one". Journal of Chemical and Engineering Data. Vol. 51, (2006) 639641.

[10] J. M. Rhodes, V. B. Bhethanabotla, S. W. Campbell. "Total vapor pressure measurements for heptane + 1-pentanol, + 2-pentanol, + 3-pentanol, + 2-methyl-1-butanol, + 2-methyl-2butanol, + 3-methyl-1-butanol, and + 3-methyl-2-butanol at $313.15 \mathrm{~K}$ ". Journal of Chemical and Engineering Data. Vol. 42, (1997) 731-734.

[11] D. P. Barton, V. R. Bhethanabotla, S. W. Campbell. "Binary total pressure measurements for methanol with 1-pentanol, 2-pentanol, 3-pentanol, 2-methyl-1-butanol, 2-methyl-2butanol, 3-methyl-1-butanol, and 3-methyl-2-butanol at 313.15 K". Journal of Chemical and Engineering Data. Vol. 41, (1996) 1138-1140.

[12] R. C. Reid, J.M. Prausnitz, B.E. Poling, "The properties of gases and liquids". Mc-Graw Hill, New York (1987).

[13] C. R. Chamorro, M. C. Martín, M. A. Villamañán, J. J. Segovia. "Characterization and modelling of a gasoline containing 1,1-dimethylethyl methyl ether (MTBE), diisopropyl ether (DIPE) or 1,1-dimethylpropyl methyl ether (TAME) as fuel oxygenate based on new isothermal binary vapour-liquid data". Fluid Phase Equilibria. Vol. 220, (2004) 105-112.

[14] J. J. Segovia. "Investigación termodinámica del equilibrio de fases fluidas de mezclas ternarias constituidas por aditivos oxigenados MTBE y metanol con hidrocarburos de sustitución para el desarrollo de nuevas gasolinas sin plomo". Universidad de Valladolid. Tesis doctoral (1997).

[15] C. R. Chamorro, J. J. Segovia, M. C. Martín, E. A. Montero, M. A. Villamanán. "Phase equilibrium properties of binary and ternary systems containing tert-amylmethyl ether TAME. As oxygenate additive and gasoline substitution hydrocarbons at $313.15 \mathrm{~K}^{\prime \prime}$. Fluid Phase Equilibria. Vol. 156, (1999) 73-87.

[16] C. Tsonopoulos. "An empirical correlation of second virial coefficients". AIChE Journal. Vol. 20, (1974) 263-272.

[17] M. Margules. "Über die zusammensetzung der gesättigten dämpfe von mischungen. sitzber". Kais. Akad. Wiss. Wien, Math.-Naturwiss. Klasse II. Vol. 104, (1895) 1243-1278.

[18] G. M. Wilson. "Vapor-liquid equilibrium XI. A new expression for the excess free energy of mixing". J. Amer. Chem. Soc. Vol. 86, (1964) 127-130.

[19] H. Renon, J. M. Prausnitz. "Local compositions in thermodynamic excess functions for liquid mixtures". AIChE Journal. Vol. 14, (1968) 135-144. 
[20] D. S. Abrams, J. M. Prausnitz. "Statistical thermodynamics of liquid mixtures: A new expression for the excess gibbs energy of partly or completely miscible systems". AIChE Journal. Vol. 21, (1975) 116-128.

[21] J. A. Barker. "Determination of activity coefficients from total pressure measurements". Australian Journal of Chemistry. Vol. 6, (1953) 207-210.

[22] A. Moreau, J. J. Segovia, J. Rubio, M. C. Martín. "Thermodynamics properties, VLE and $H^{E}$, of the systems 2-pentanol and cyclohexane or methylbenzene for contributing to the knowledge of new biofuels". Fluid Phase Equilibria. Vol. 409, (2016) 92-97. 



\section{Capítulo 4}

\section{DETERMINACIÓN DE MEDIDAS DE LAS DENSIDADES Y SOLUBILIDADES DE $\mathrm{CO}_{2}$ EN SOLUCIONES ACUOSAS DE AMINAS}





\subsection{Medidas de Solubilidad. Introducción.}

Los métodos experimentales para la investigación del equilibrio de fases a alta presión pueden clasificarse en dos tipos dependiendo de cómo se determine la composición: métodos analíticos (o métodos de muestreo directo) y métodos sintéticos (o métodos indirectos). Aunque esta clasificación engloba la mayor parte de los métodos utilizados, puede haber métodos que no encajen en ninguno de los anteriores [1].

Los métodos analíticos implican la determinación experimental de la composición de las fases. Esto se puede hacer tomando muestras de cada fase y analizándolas a presión atmosférica fuera de la celda o bien a presión en el interior. Un factor importante es el muestreo, puesto que si se retiran grandes cantidades de muestra se altera el equilibrio de fases.

Los métodos sintéticos se basan en preparar una mezcla de composición conocida y observar el comportamiento de fases en una celda de equilibrio. Conocida la cantidad de cada componente en la celda, se ajustan los valores de presión y temperatura. Cada experimento proporciona un punto $p T x$ de la superficie de coexistencia.

La medida de las solubilidades de $\mathrm{CO}_{2}$, se realiza con un equipo para la medida de equilibrios liquido-vapor [2] a diferentes temperaturas. Es una técnica isocórica dado que opera a volumen constante y está catalogada dentro de los métodos sintéticos. Esta técnica es novedosa dado que en la mayor parte de la bibliografía se realizan las medidas de solubilidad de $\mathrm{CO}_{2}$ en aminas mediante métodos analíticos con cromatografía [3] y [4].

\subsection{Descripción del método de medida utilizado para la determinación de la solubilidad del $\mathrm{CO}_{2}$ en mezclas acuosas de amina.}

Para la medida de las solubilidades de $\mathrm{CO}_{2}$ en mezclas acuosas de amina, se hizo mediante un equipo inicialmente construido para la medida de equilibrios liquido-vapor [2], este equipo fue descrito en el apartado 2.2 .

Para poder realizar las medidas de solubilidades de $\mathrm{CO}_{2}$, fue preciso la realización de unas reformas a la técnica inicial, como se ve en la Figura 4.1. La principal modificación se realizó para la inyección de $\mathrm{CO}_{2}$ en la celda, descrita en el apartado 2.2.3 del Capítulo 2 . Estas modificaciones se resumen en: 


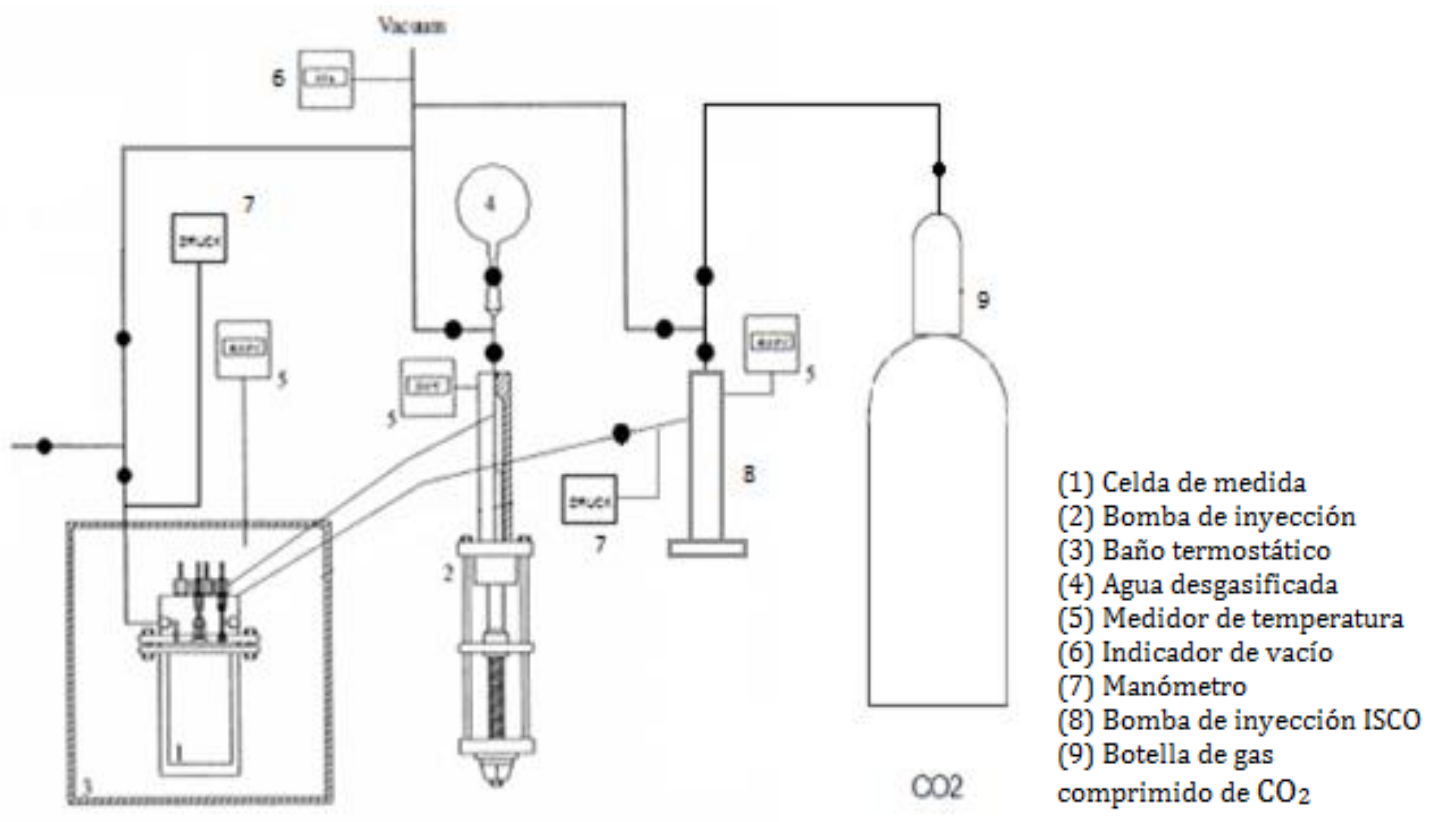

Figura 4.1. Esquema del aparato modificado de medida del equilibrio líquido-vapor para la medida de solubilidades de $\mathrm{CO}_{2}$ en aminas.

\section{Sistema de inyección de $\mathrm{CO}_{2}$ en la celda:}

El sistema consta de una bomba de desplazamiento positivo modelo ISCO. Esta se carga a través de una botella de $\mathrm{CO}_{2}$ de alta pureza en condiciones de $\mathrm{CO}_{2}$ líquido, encontrándose a una temperatura y presión constante $\left(0{ }^{\circ} \mathrm{C}\right.$ y 55 bar $)$. La bomba de $\mathrm{CO}_{2}$ está unida a nuestra celda de medida directamente a través de una válvula de bola. La bomba dispone de un manómetro Druck 104 para la medida de la presión de la rama de inyección.

\section{El sistema de medición de presión:}

Tal como se indicó en el apartado 2.2.3, se realizaron una serie de reformas al equipo usando tres sensores para la medida de presión a diferentes rangos. Para un rango de medidas de 0 a 2 bar se instaló un sensor de presión marca Druck, modelo PDCR-9101422. Para medidas comprendidas entre 2 y 20 bar, se instaló un sensor adicional de la misma marca y modelo. Finalmente, para medidas superiores a 20 bar se instaló un tercer medidor de presión marca Paroscientific, modelo 42K-101 hasta 138 bar. Estos también fueron calibrados en el laboratorio TERMOCAL en la Universidad de Valladolid.

\subsection{Procedimiento experimental para la medida de la solubilidad.}

Antes de la realización de las medidas se procedió a una calibración del volumen de la celda de equilibrio teniendo en cuenta los diferentes tramos de los diferentes medidores de presión que se encuentran instalados en el equipo. 
La calibración del volumen de la celda de equilibrio se hizo mediante un llenado progresivo con $\mathrm{CO}_{2}$ (agitador incluido) en un rango de temperaturas desde $40{ }^{\circ} \mathrm{C}$ hasta 80 ${ }^{\circ} \mathrm{C}$. El $\mathrm{CO}_{2}$ se suministra con una bomba ISCO. A partir de la temperatura, la presión de la bomba y con el volumen desplazado se determina la masa añadida a la celda. Cuando en la celda se alcanza el equilibrio, se determina la presión y a través de la temperatura y la masa de $\mathrm{CO}_{2}$ calculamos el volumen. Siendo las ecuaciones de la calibración del volumen total de la celda $(\mathrm{mL})$ en función de la temperatura $\left({ }^{\circ} \mathrm{C}\right)$ para cada uno de los medidores:

- Medidor Druck de 2 bar: El valor del volumen de calibrado fue de $197.88 \mathrm{~mL}$ a $40{ }^{\circ} \mathrm{C}$.

- Medidor Druck de 20 bar: $V_{\text {total }}=0.1047 \cdot T+194.3$

- Medidor Paroscientific hasta 138 bar: $V_{\text {total }}=0.1047 \cdot T+190.9$

Los compuestos puros fueron desgasificados previamente en la propia celda de equilibrio mediante una serie de ciclos (solidificación-fundición) como se puede observar en la Figura 4.2. Primero se solidifica el compuesto por medio de un enfriamiento en un baño de nitrógeno líquido, y después se funde por medio de un calentamiento a temperatura ambiente. Estos ciclos se repiten hasta que la presión de vapor del compuesto a temperatura ambiente es la misma entre dos ciclos consecutivos, dando por desgasificado el compuesto. La amina introducida en la celda se obtiene pesando la cantidad requerida de amina para un volumen final de unos $120 \mathrm{~mL}$, a la concentración en $\%$ en peso a estudiar. En el caso del agua, se desgasificó según el método empleado en el apartado 2.3.
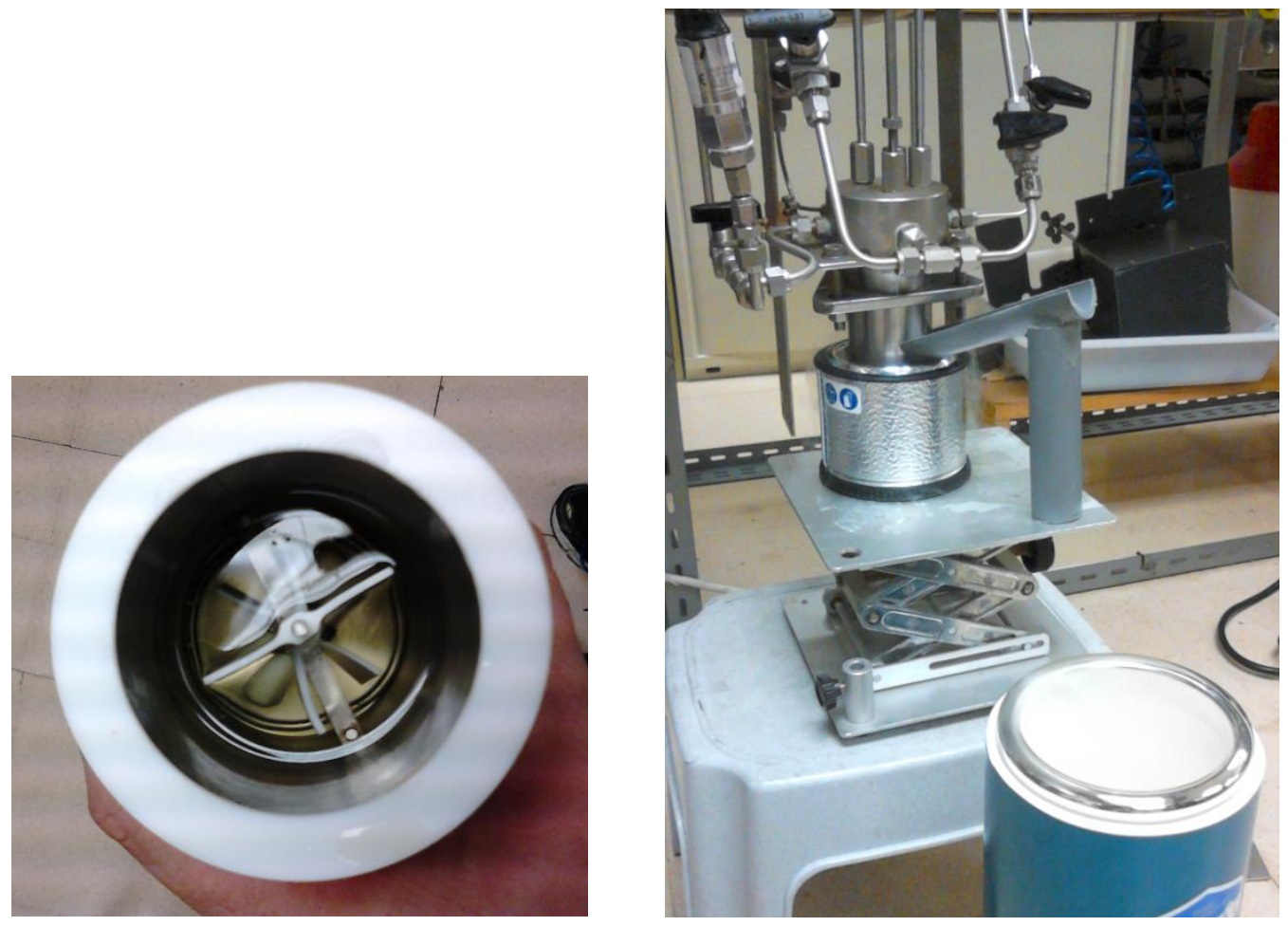

4.2. Figura Sistema para la desgasificación de la amina.

El $\mathrm{CO}_{2}$ puro fue cargado directamente desde la botella a la bomba de desplazamiento positivo, donde se almacena en unas condiciones de presión y temperatura establecidas 
(75 bar y $0^{\circ} \mathrm{C}$ ). Encontrándose en estas condiciones en estado líquido, es decir, apto para la carga en la celda.

Una vez desgasificada la amina, sumergimos la celda en el baño termostático agitando la amina para una perfecta homogeneización.

Cuando la temperatura y presión de la celda sea estable, se determina el valor de la presión de vapor de la amina. A continuación, introducimos en la celda el agua desgasificada mediante el cilindro de inyección hasta la concentración a estudiar, anotando el volumen de agua introducida, la temperatura y presión de inyección del cilindro. Una vez que la presión en la celda es estable determinamos la presión de vapor de la mezcla.

Alcanzada la estabilidad en temperatura y presión de la bomba de $\mathrm{CO}_{2}$, inyectamos el $\mathrm{CO}_{2}$ en la celda. Determinando la masa de $\mathrm{CO}_{2}$ inyectada mediante la temperatura, y la presión de la bomba y el volumen desplazado en cada carga.

Una vez alcanzando el equilibrio, se toma la presión total de la celda mediante el manómetro adecuado según el rango de presión de trabajo.

Al finalizar toda una serie de medidas se desmonta la celda de equilibrio para su posterior limpieza.

\subsection{Cálculo de la concentración en la mezcla.}

El cálculo de la concentración de la mezcla se expresa mediante el parámetro $\alpha$ (índice de carga), definido como los moles de $\mathrm{CO}_{2}\left(n_{\mathrm{CO} 2}\right)$ que se encuentran en la fase líquida entre los moles de amina ( $\left.n_{\text {amina }}\right)$ en la fase líquida:

$$
\alpha=\frac{n_{\mathrm{CO}_{2}} \text { (fase líquida) }}{\left.n_{\text {amina }} \text { (fase líquida }\right)}
$$

Suponiendo que la presión de vapor de la mezcla amina $+\mathrm{H}_{2} \mathrm{O}$ permanece constante a la temperatura dada, obtenemos la presión parcial del $\mathrm{CO}_{2}\left(p_{\mathrm{CO} 2}\right)$ restando a la presión total $\left(p_{\text {total }}\right)$ la presión parcial de la solución acuosa de amina $\left(p_{\text {amina aq. }}\right)$ :

$$
p_{\mathrm{CO}_{2}}=p_{\text {total }}-p_{\text {amina aq }} .
$$

A continuación, se calcula la masa del $\mathrm{CO}_{2}\left(m_{\mathrm{CO} 2}\right)$ en la fase vapor según la ecuación 4.3. El cálculo de la densidad del $\mathrm{CO}_{2}$ en la fase vapor $\left(\rho_{\mathrm{CO} 2}\right)$ se hace a través de una ecuación de estado utilizando el programa REFPROP. Como volumen de la fase vapor ( $V_{\text {fase }}$ vapor $)$ se toma el volumen total menos el ocupado por la fase líquida.

$$
m_{\mathrm{CO}_{2} \text { fase vapor }}=\rho_{\mathrm{CO}_{2}}\left(p_{\mathrm{CO}_{2}}, T\right) \cdot V_{\text {fase vapor }}
$$

Recalculamos los moles de $\mathrm{CO}_{2}$ en la fase líquida según la ecuación 4.4. Siendo $\mathrm{PM}_{\mathrm{CO} 2}$ el peso molecular del $\mathrm{CO}_{2}$. 


$$
n_{\mathrm{CO}_{2} \text { fase líquida }}=\frac{\left(m_{\mathrm{CO}_{2}} \text { total }-m_{\mathrm{CO}_{2}} \text { fase vapor }\right)}{\mathrm{PM}_{\mathrm{CO}_{2}}}
$$

El cálculo de la densidad de la mezcla $\left(\rho_{\text {mezcla }}\right)$ se realiza mediante la ecuación de ajuste de las densidades de las mezclas (4.5).

$$
\rho_{\text {mezcla }}=\mathrm{a}+\frac{\mathrm{b}}{\propto}+\frac{\mathrm{c}}{\alpha^{2}}+\mathrm{d} \cdot p_{\text {total }}
$$

Donde a, b, c y d son los coeficientes de ajuste de la densidad a la temperatura de trabajo, y $\alpha$ es el índice de carga calculado con la ecuación 4.1. Esta ecuación se ha desarrollado para la obtención de la densidad de mezcla en función del índice de carga y la presión total. Los parámetros de ajuste para cada sistema se encuentran calculados en el capítulo 5 de la presente tesis.

Finalmente, el volumen ocupado por la mezcla $\left(V_{\text {mezcla }}\right)$ se calcula según la ecuación 4.6. Definiendo la masa total de la fase líquida como la masa introducida menos la masa de la fase vapor.

$$
V_{\text {mezcla }}=m_{\text {total líquido }} / \rho_{\text {mezcla }}
$$

Para obtener el volumen de la fase vapor ( $V_{\text {fase vapor }}$ ), se resta el volumen total calibrado $\left(V_{\text {total }}\right)$ y el volumen de la mezcla de la fase líquida $\left(V_{\text {mezcla }}\right)$.

$$
V_{\text {fase vapor }}=V_{\text {total }}-V_{\text {mezcla }}
$$

\subsection{Medidas de densidad. Introducción.}

La masa y el volumen son magnitudes extensivas. Una magnitud extensiva depende del tamaño de la muestra observada. Sin embargo, si se divide la masa de una sustancia por su volumen, se obtiene la densidad, una magnitud intensiva. Una magnitud intensiva es independiente del tamaño de la muestra observada [5].

$$
\text { densidad }(\rho)=\frac{\operatorname{masa}(m)}{\text { volumen }(V)}
$$

La densidad es una magnitud física usada para la caracterización de sustancias puras y de sus mezclas, y es un factor determinante para el cálculo de otras magnitudes físicas derivadas. La densidad depende de la temperatura y la presión, afectando la variación de estas condiciones al valor de dicha magnitud. Para la obtención de la máxima exactitud en las mediciones de densidad se han desarrollado nuevas técnicas, especialmente cuando se requiere realizar medidas a altas temperaturas y presiones. A partir de los datos generados por estas técnicas podremos desarrollar modelos con amplios intervalos de presión y temperatura, para una mejor descripción del comportamiento de estas sustancias. Existen diferentes métodos para la determinación experimental de la densidad. Se puede establecer una clasificación en base a dos grandes familias de técnicas que miden esta propiedad, métodos directos y métodos indirectos [6]. 
Los métodos directos se basan en la comparación de la masa de un determinado volumen de una sustancia con la masa del mismo volumen de otra sustancia tomada como término de comparación. Las técnicas experimentales que aplican el método directo son: picnometría, densimetría de flotador, basada en el equilibrio entre los efectos opuestos de la gravedad, empuje y campo magnético, y la balanza hidrostática que se fundamenta en el principio de Arquímedes [7].

Los métodos indirectos relacionan propiedades de la materia con la densidad, con lo que se requiere de sustancias patrones para el calibrado de los mismos. Un ejemplo típico son los refractómetros, basados en un método óptico para la determinación de la velocidad de propagación de la luz en un medio, relacionado directamente con la densidad de este medio. Otros son los densímetros de oscilación mecánica, los cuales se basan en fenómenos de resonancia.

La densimetría de oscilación mecánica es la técnica más utilizada, siendo uno de los métodos más precisos para la determinación de densidades a altas presiones. Son relativamente rápidos y evitan complicados procedimientos de llenado y pesada. Existen numerosos trabajos donde es utilizado este método con éxito para la determinación de densidades de disoluciones acuosas de aminas y mezclas ternarias amina $+\mathrm{H}_{2} \mathrm{O}+\mathrm{CO}_{2}$ $[8][9][10][11]$.

\subsection{Principio de funcionamiento del densímetro de tubo vibrante.}

El densímetro de tubo vibrante permite medir densidades a altas temperaturas y presiones mediante un diseño sencillo. Este equipo fue diseñado y desarrollado por Stabinger et al. [12], y es capaz de determinar la densidad por medición de la frecuencia natural de resonancia de un tubo de vidrio oscilante en forma de U lleno de la sustancia a medir.

Un densímetro de este tipo comercializado por la casa Anton Paar está disponible en el Laboratorio Termocal. Presenta las ventajas de requerir poco volumen de muestra, amplios intervalos de operación de temperatura y presión, alta precisión en la medida, fácil manejo, rapidez en la medida, visualización digital del periodo y escaso mantenimiento. Este densímetro ya ha sido descrito en anteriores tesis como E. Concepción [6] y J. Zambrano [13].

Gracias a su principio de funcionamiento, donde la vibración del tubo se puede asociar a un oscilador armónico simple, podremos llegar matemáticamente a una expresión en la cual se relaciona la densidad $(\rho)$ con el período $(\tau)$ según la ecuación $(4.9)$.

$$
\rho=\mathrm{A} \tau^{2}-\mathrm{B}
$$

Siendo A y B:

$$
\mathrm{A}=\frac{k}{4 \pi^{2} V} \quad \mathrm{~B}=\frac{m_{0}}{V}
$$


Donde $k$ es el módulo de elasticidad del sistema, $V$ es el volumen de la celda y $m_{0}$ es la masa del tubo vacío. Teniendo en cuenta que las principales variables de trabajo son la presión $(p)$ y la temperatura $(T)$, la expresión anterior se describirá como:

$$
\rho(T, p)=\mathrm{A}(T, p) \tau^{2}-\mathrm{B}(T, p)
$$

De esta forma es posible obtener la densidad de un líquido partiendo de la medida del periodo cuando el sistema está en resonancia conociendo los parámetros $\mathrm{A}(T, p)$ y $\mathrm{B}(T, p)$.

El conocimiento de estos dos parámetros se realiza mediante un calibrado por lo que la técnica de tubo vibrante es considerada un método indirecto de medición.

\subsection{Descripción del método de medida utilizado para la determinación de la densidad.}

La técnica experimental utilizada para la determinación de la densidad a alta presión de los fluidos puros y de sus mezclas, es un densímetro de tubo vibrante automatizado. El sistema requiere de un conjunto de elementos que permitan el llenado de la celda con las muestras, su vaciado y limpieza, el control y medida de la presión y la temperatura, y el registro de los datos obtenidos.

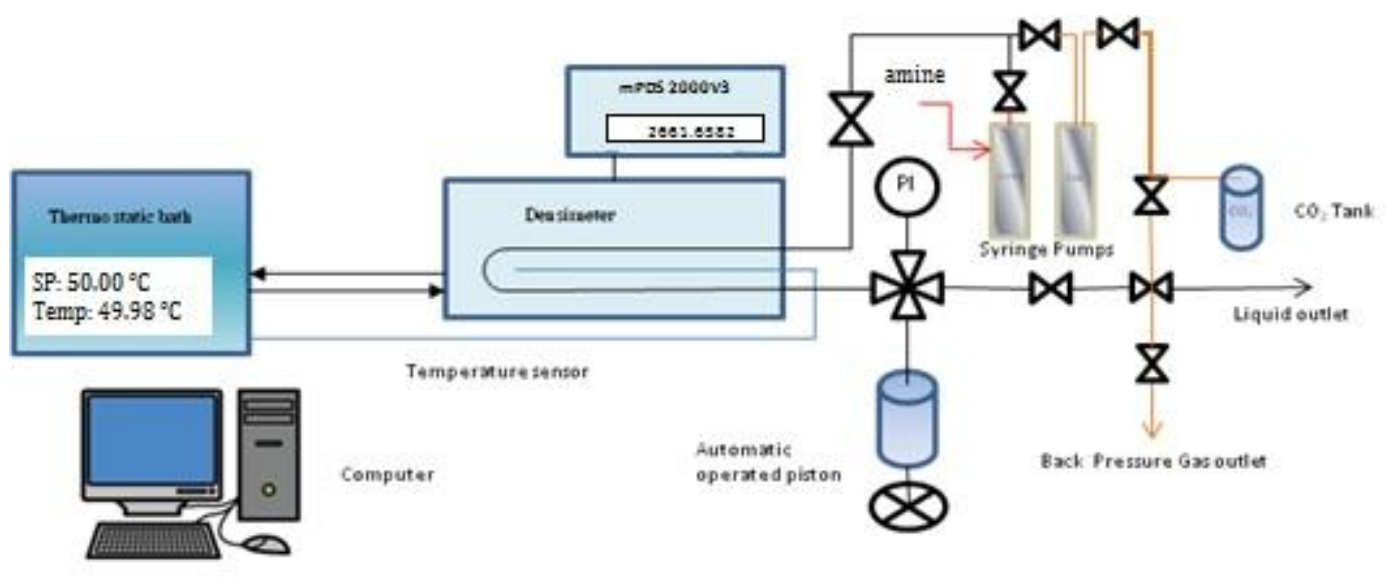

Figura 4.3. Esquema del equipo de medida de la densidad.

El densímetro de tubo vibrante es de la marca Anton Paar modelo HPM, con un rango de operación de $0-3 \mathrm{~g} \cdot \mathrm{cm}^{-3}$ y una resolución de $1 \cdot 10^{-5} \mathrm{~g} \cdot \mathrm{cm}^{-3}$. El rango de temperatura de medida es de $263 \mathrm{~K}$ a $473 \mathrm{~K}$ y una presión máxima de $140 \mathrm{MPa}$.

El módulo DMA HPM consta de un tubo oscilador en forma de "U" hecho de Hastelloy. Este tubo se halla dispuesto en el centro de un cilindro, de pared doble. El espacio comprendido entre el oscilador y la pared interior del cilindro está lleno de un gas que posee alta conductividad térmica y una densidad baja para no ocasionar amortiguamiento en las oscilaciones. Esto facilita el equilibrio térmico a través de la doble pared con el líquido procedente de un termostato que fluye alrededor del cilindro. La celda de medida está conectada por cables coaxiales teniendo la misión de excitar el tubo en $\mathrm{U}$, hasta alcanzar la 
resonancia a su frecuencia natural de excitación, en una dirección perpendicular al plano que contiene el tubo. Los cables coaxiales suministran la excitación electrónica al oscilador mediante dos bobinas y un circuito electrónico que mantiene constante la amplitud. La vibración es transmitida a la celda por medio de una lámina metálica pegada en el tubo y es detectada ópticamente por la interrupción del rayo de luz entre el emisor y el receptor, que están colocados perpendicularmente al plano definido por el tubo. Mediante su propia unidad de proceso, este módulo interpreta cualquier señal de salida de la celda de medida reproduciendo el valor del periodo de oscilación del tubo, que contiene la muestra objeto de estudio. La unidad de evaluación mPDS 2000V3 mide el periodo de vibración $\tau$ con una incertidumbre de $1 \cdot 10^{-6} \mu \mathrm{s}$, teniendo periodos en torno a $2.6 \mu \mathrm{s}$, y calcula la densidad con una ecuación de 9 coeficientes obtenidos de su calibración, también mide la temperatura interior de la celda de medida y permite la comunicación con un PC mediante conexión RS232.

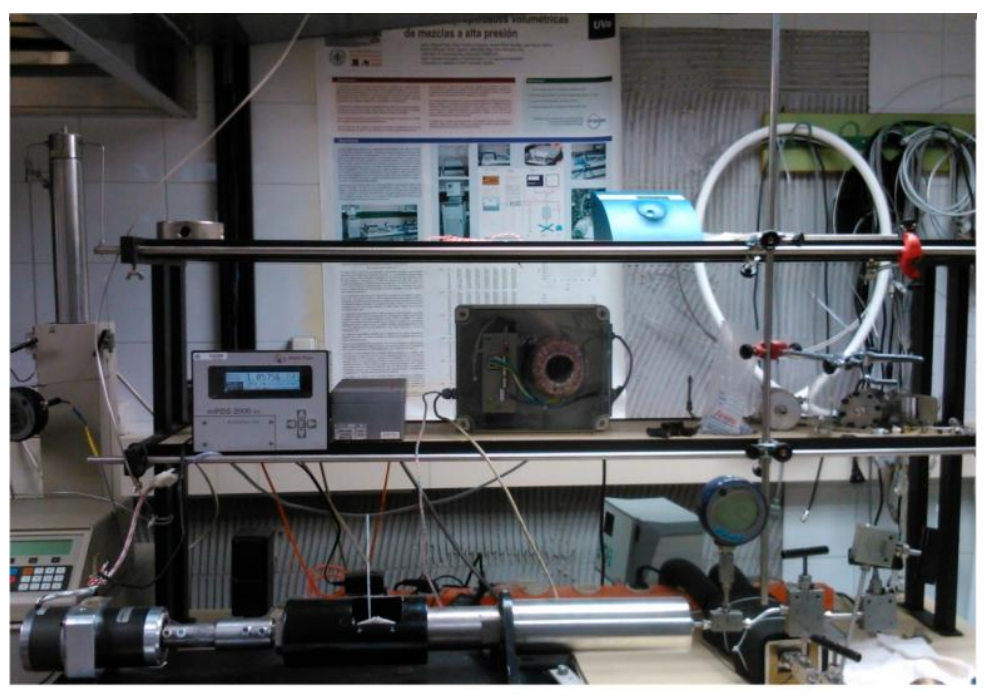

Figura 4.4. Imagen del densímetro y de sus componentes.

Exceptuando los procedimientos de llenado, vaciado y limpieza del equipo, todos los procesos de control de temperatura, presión del sistema y la adquisición de datos se realizan de modo totalmente automatizado, con un programa desarrollado en el entorno de programación Agilent VEE-Pro 7.0.

El sistema de control de temperatura está formado por un baño externo JULABO F25 HE, que mantiene constante la temperatura de la celda de medida haciendo circular un fluido (aceite de silicona) por la camisa interna de la célula de medida. Los tubos de este circuito están protegidos por un aislante térmico, para reducir la transmisión de calor con el ambiente del laboratorio.

El baño utiliza como sonda de temperatura interna de control una Pt100 a cuatro hilos. La sonda se encuentra insertada en el interior de la unidad DMA HPM en una cavidad situada para tal efecto. Esta sonda ha sido calibrada en el laboratorio TERMOCAL. El baño termostático posee comunicación al PC mediante RS232. 
El sistema de control de presión está compuesto por un sistema de medida de presión, un generador de presión y un motor paso a paso encargado de accionar el generador de presión. Como sistema de medida de presión se utiliza un medidor de presión Druck DPI 104 con un rango de operación de $0.1-140 \mathrm{MPa}$, una resolución de $0.01 \mathrm{MPa}$, calibrado con una incertidumbre expandida relativa de $0.02 \%$ (factor de cobertura $k=2$ ) y conectado mediante USB al PC. Este conjunto fue calibrado en veinte puntos de su rango de medida de presión. El generador de presión usado en este trabajo es un pistón modelo 68-5.75-15 de la marca HiP, con un volumen máximo de $35 \mathrm{~cm}^{3}$ y una presión máxima de trabajo de $140 \mathrm{MPa}$. Las empaquetaduras de sellado del pistón son de teflón, siendo este química, mecánica y térmicamente estable dado el rango de trabajo y el tipo de fluidos. No existe separación entre ambos circuitos, siendo el propio fluido de medida el fluido portador del circuito hidráulico. El émbolo del pistón es movido por un motor paso a paso ACP\&D 6530-R211.

Todos los conductos del circuito de presión son de tubo de 1/4" y 1/8" de alta presión. El acoplamiento es por anillo de presión roscado sobre el tubo y contra-tuerca roscada al cuerpo de las válvulas y accesorios. Las válvulas montadas en el sistema para las funciones de llenado, aireación y conexión al vacío son válvulas de aguja para alta presión HiP, hasta $200 \mathrm{MPa}$ y con conexiones a tubo 1/4", y válvulas de bola Ham-Let hasta $20 \mathrm{MPa}$ para las conexiones de llenado a baja presión.

El sistema de llenado de las muestras se realiza por medio de dos bombas ISCO de desplazamiento positivo, en una de ellas se introduce el $\mathrm{CO}_{2}$ puro en estado líquido. Y en la otra se introduce una mezcla de amina + agua a una concentración conocida. Para la preparación de la disolución de amina, se hace por medio de pesada en una balanza RADWAG PS750/C/2, con una incertidumbre expandida de $0.007 \mathrm{~g}(k=2)$. La disolución es desgasificada antes de introducirla en la bomba mediante un baño de ultrasonidos marca Branson, modelo DTH-3210, con una frecuencia de $47 \mathrm{kHz}$ y una potencia de 335 W. La bomba de $\mathrm{CO}_{2}$ se encuentra a una temperatura y presión constante de $0{ }^{\circ} \mathrm{C}$ y 55 bar, y la bomba del segundo componente a una temperatura y presión constante de $20{ }^{\circ} \mathrm{C}$ y 55 bar. Ambas bombas se conectan por medio de válvulas de bola a una $\mathrm{T}$ de mezcla y esta al densímetro por una válvula de aguja. Para asegurar el llenado a presión constante, se encuentra instalada una válvula de contrapresión Mity-Mite modelo S-91XW situada en la salida del circuito. La contrapresión aplicada es de 50 bar a través de una válvula que comunica con la botella de $\mathrm{CO}_{2}$.

La evacuación de la muestra se realiza a través de una válvula conectada a la válvula de contrapresión, desplazando el pistón a la posición final de su recorrido. También se dispone de una válvula de aguja HiP para la función de aireación poniendo a presión atmosférica todo el sistema de manera rápida. Además, esta válvula permite conectar el circuito de presión a una línea de vacío. Antes de su conexión a vacío, se introduce agua destilada para disolver y arrastrar toda la amina y facilitar su posterior limpieza mediante vacío. El sistema de vacío está compuesto por un vacuómetro Leybold Vakuum Thermovac, una trampa fría con nitrógeno líquido y una bomba rotativa de vacío Leybold Trivac, alcanzando un vacío de $2.5 \mathrm{~Pa}$. 


\subsection{Procedimiento experimental para medida de la densidad.}

Para llenar el equipo procedemos a programar la bomba que contiene el $\mathrm{CO}_{2}$ a flujo constante y abrimos la válvula que la comunica con la T de mezcla. Después conectamos la segunda bomba a flujo constante y manteniendo una contrapresión procederemos a una carga en dinámico. La relación de flujos volumétricos nos permite calcular las composiciones de la mezcla inyectada.

Se deja fluir un volumen suficiente para asegurar la homogeneización de los dos compuestos y que la concentración de la mezcla sea la deseada. Desplazamos paulatinamente el pistón del generador de presión hacia atrás hasta llenarlo. Una vez lleno, paramos el pistón y de nuevo dejamos fluir un volumen suficiente que nos permita asegurar la correcta concentración de la mezcla amina $+\mathrm{H}_{2} \mathrm{O}+\mathrm{CO}_{2}$.

Terminada la carga del equipo es importante esperar el tiempo suficiente no solo para llegar a un equilibrio mecánico y térmico, sino que al ser compuestos que reaccionan entre sí, es preciso alcanzar un equilibrio material de la muestra.

El programa desarrollado en lenguaje Agilent VEE controla todo el proceso de medida mediante tres lazos de control jerárquicamente en serie: de temperatura, de presión y de período. La temperatura se registra cuando se alcanza la condición de estabilidad, siendo tal que la temperatura se encuentre $\mathrm{a} \pm 0.05^{\circ} \mathrm{C}$ del punto de consigna.

El generador de presión se controla a través del programa mediante una estrategia de control todo-nada con zona muerta y control adaptativo de la reduen el lazo de presiónctora. El lazo se cierra con la medida de la presión mediante el transductor Druck 104. El criterio de estabilidad en el lazo de presión se establece cuando la desviación estándar, $\sigma$, de las diez últimas medidas en el período es menor que $1 \cdot 10^{-3} \mu$ s del valor medio. Registrado éste, el programa salta al siguiente punto en presión para esa isoterma, o comienza una nueva isoterma, aumentando la presión o temperatura en cada punto según corresponda. Al finalizar se procede a la evacuación y limpieza del equipo.

\subsection{Calibración del densímetro.}

Es necesario conocer los parámetros característicos del oscilador para conocer la relación entre el periodo de vibración $(\tau)$ y la densidad $(\rho)$ en un densímetro de tubo vibrante. Según la ecuación (4.11) la densidad $(\rho)$ se puede expresar en función de los parámetros $(\mathrm{A}(T, p)$ y $\mathrm{B}(T, p))$ y el período de vibración. Para la calibración del equipo será necesario medir el periodo de vibración de dos fluidos de referencia, de densidades conocidas en función de la temperatura y presión, para la obtención de las constantes $\mathrm{A}(T, p)$ y $\mathrm{B}(T, p)$.

$$
\rho_{1}(T, p)=\mathrm{A}(T, p) \tau_{1}^{2}-\mathrm{B}(T, p) \quad \rho_{2}(T, p)=\mathrm{A}(T, p) \tau_{2}^{2}-\mathrm{B}(T, p)
$$

Donde $\rho_{1}$ y $\rho_{2}$ son las densidades conocidas del fluido 1 y 2 respectivamente a las temperaturas y presiones de trabajo, para las cuales serán medidos sus periodos $\tau_{1}$ y $\tau_{2}$. De esta forma una vez medidos los períodos de estas sustancias obtendremos los parámetros característicos de nuestro densímetro. 


$$
\mathrm{A}(T, p)=\frac{\rho_{1}(T, p)-\rho_{2}(T, p)}{\tau_{1}^{2}(T, p)-\tau_{2}^{2}(T, p)} \quad \mathrm{B}(T, p)=\mathrm{A}(T, p) \tau_{2}^{2}(T, p)-\rho_{2}(T, p)
$$

Una vez obtenidos los parámetros $\mathrm{A}(T, p)$ y $\mathrm{B}(T, p)$, podremos determinar la densidad de cualquier fluido mediante la ecuación (4.11), dentro del rango de presiones y temperaturas en las cuales se determinaron.

Han sido desarrollados diferentes métodos para la calibración del densímetro de tubo vibrante como son los trabajos presentados por Holcomb et al. [14] y Vega [15]. Se han escogido como fluidos de calibrado el vacío y el agua, tal y como se describe en el procedimiento desarrollado por Lagourette et al. [16]. En este procedimiento se considera que el coeficiente A $(T)$ solamente será función de la temperatura y asumimos que el coeficiente B $(T, p)$ varia significantemente con la presión. Esto implica que la constante $k$ (módulo de elasticidad del sistema) y que $V$ (volumen de la celda) variarán con la presión de la misma forma. De manera que tendremos que crear un vacio adecuado, menor de 100 $\mathrm{Pa}$, donde el valor de la densidad del aire sea menor de $10^{-6} \mathrm{~g} \cdot \mathrm{cm}^{-3}$.

Asumiendo la propuesta de Lagourette et al. [16], para la calibración del densímetro la segunda sustancia de referencia será el agua, dada su disponibilidad y su alto grado de pureza. Además, se conoce su densidad con unos valores de incertidumbre muy bajos en los rangos de temperatura y presión en los que se va a trabajar, siendo este uno de los requisitos para minimizar la incertidumbre de la medida.

En la Figura 4.5 se muestra la relación $\mathrm{A}(T) / \mathrm{B}(T, p)$ frente a la presión, y el coeficiente $\mathrm{A}(T)$ frente a la temperatura en la Figura 4.6. Ambas se obtienen de los datos de calibrado realizados en el agua y vacío para este trabajo.

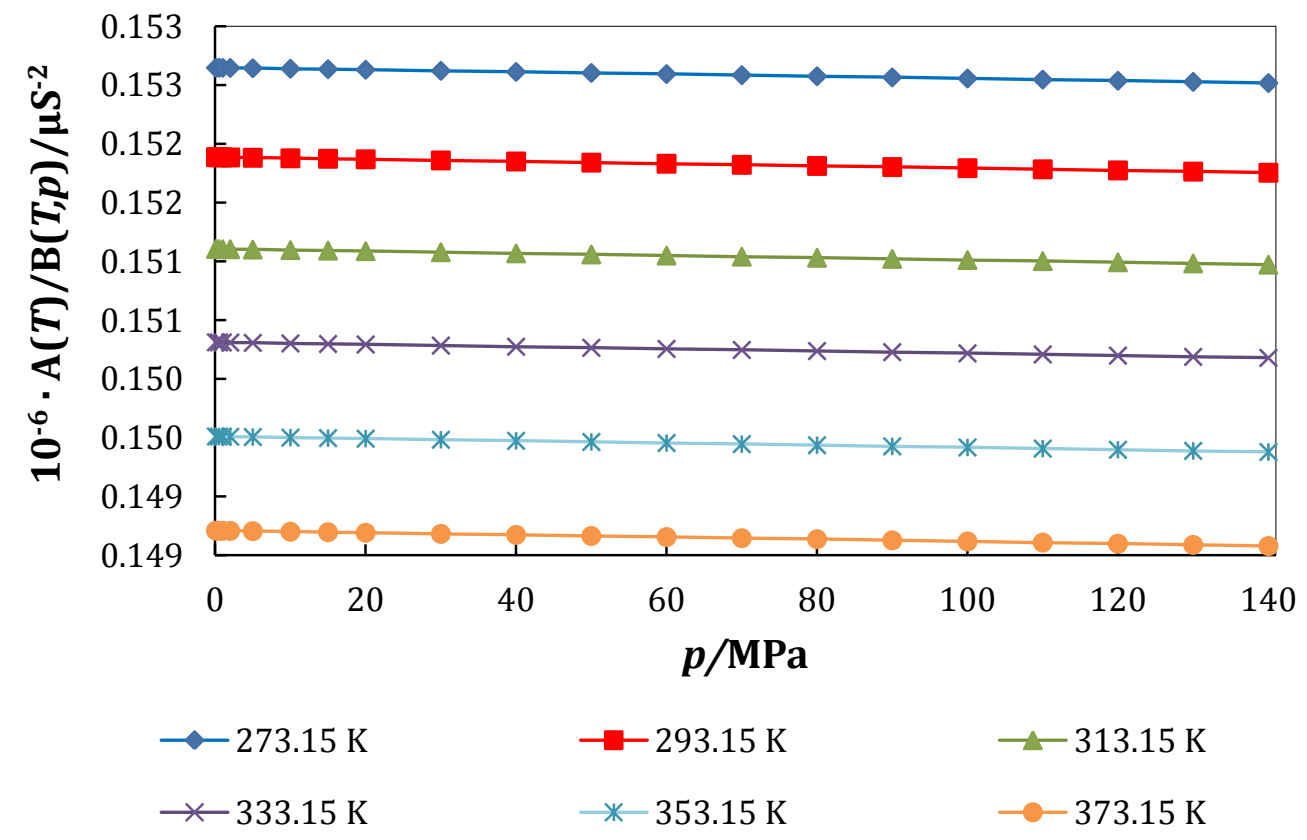

Figura 4.5. Cociente de los parámetros característicos $\mathrm{A}(T) / \mathrm{B}(T, p)$ del tubo vibrante frente a la presión. 
Se aprecia que $\mathrm{A}(T) / \mathrm{B}(T, p)$ varía ligeramente con la presión y que $\mathrm{A}(T)$ decrece ligeramente con la temperatura. Ambos comportamientos repiten tendencias halladas tanto por Lagourette et al. [16], como por Vega [15].

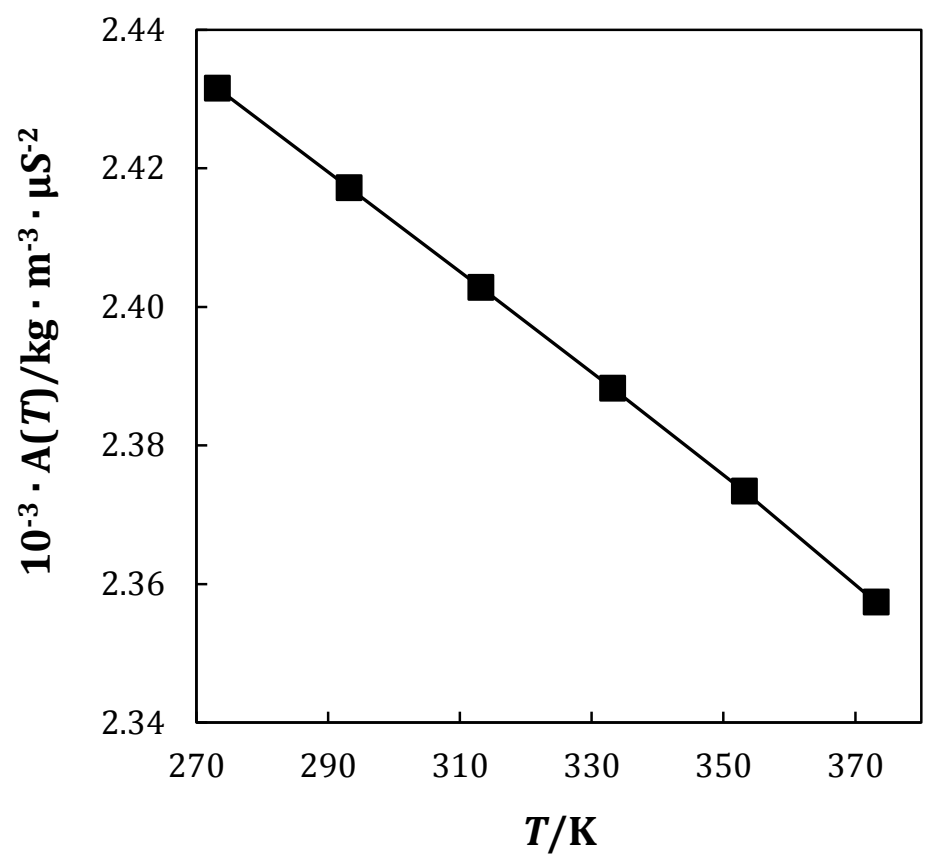

Figura 4.6. Parámetro característico de la calibración del tubo vibrante A(T) frente a la temperatura.

Teniendo en cuenta lo expuesto anteriormente según las ecuaciones (4.12) y (4.13), obtenemos que la relación de la densidad con el periodo y los parámetros de calibración se expresan como:

$$
\begin{gathered}
\rho(T, p)=\rho_{w}(T, p)+\frac{\rho_{w}(T, 0.1 \mathrm{MPa})}{\rho_{\mathrm{w}}^{2}(T, 0.1 \mathrm{MPa})-\rho_{v}(T)}\left(\tau^{2}(T, p)-\tau_{w}^{2}(T, p)\right) \\
\mathrm{A}(T)=\frac{\rho_{\mathrm{W}}(T, 0.1 \mathrm{MPa})}{\tau_{w}^{2}(T, 0.1 \mathrm{MPa})-\tau_{v}^{2}(T)} \\
\mathrm{B}(T, p)=\frac{\rho_{w}(T, 0.1 \mathrm{MPa})}{\tau_{w}^{2}(T, 0.1 \mathrm{MPa})-\tau_{\mathrm{V}}^{2}(T)} \tau_{w}^{2}(T, p)-\rho_{w}(T, p)
\end{gathered}
$$

Donde $\rho_{w}(T, p)$ es la densidad del agua a la misma temperatura $T$ y presión $p$ del fluido contenido en la celda y $\rho_{w}(T, 0.1 \mathrm{MPa})$ es la densidad del agua a la misma temperatura $T$ y a presión atmosférica; $\tau_{w}(T, p)$ y $\tau_{w}(T, 0.1 \mathrm{MPa})$ son los períodos experimentales del fluido de referencia a las condiciones mencionadas, $\tau_{v}(T)$ es el período experimental a la temperatura $T$ del tubo vibrante al vacío, $\tau^{2}(T, p)$ es el periodo experimental del fluido que deseamos medir. Las densidades del agua como fluido de calibración fueron obtenidas con la ecuación de estado de Wagner y Pruss [17] debido a su baja incertidumbre. 


\subsection{Incertidumbre en la medida.}

El cálculo de la incertidumbre se realizó en base al documento GUM 2008 [18], que es la guía para el cálculo de incertidumbres de medida de la Oficina Internacional de Pesas y Medidas (BIMP).

La incertidumbre de la composición en $\mathrm{CO}_{2}$ de la disolución acuosa de amina acuosa está asociada a la balanza con la que se realizó la mezcla binaria amina $+\mathrm{H}_{2} \mathrm{O}$, a las densidades de los compuestos de cada bomba y los caudales de dichas bombas. Siendo la incertidumbre en caudal para las bombas ISCO del $0.5 \%$.

La incertidumbre de la balanza se presenta en la Tabla 4.1, y las incertidumbres de las densidades de los compuestos en la Tabla 4.2.

Tabla 4.1. Cálculo de la incertidumbre de la balanza para la mezcla de la amina acuosa en la bomba.

\begin{tabular}{|l|l|l|l|l|l|}
\hline & & Unidad & Estimación & Divisor & $\mathrm{u}(x)$ \\
\hline & & & & & $\mathrm{g}$ \\
\hline \multirow{3}{*}{$\mathrm{u}(m)$} & Linealidad & \multirow{3}{*}{$\mathrm{g}$} & 0.003 & 1 & \\
\cline { 2 - 4 } & Resolución & & 0.001 & 1 & 0.0035 \\
\cline { 2 - 5 } & Repetibilidad & & 0.0015 & 1 & \\
\hline \multicolumn{3}{|c|}{$\mathrm{g}(m)$} & $\mathrm{g}$ & $\mathrm{k}=1$ & 0.0035 \\
\hline $\mathrm{U}(m)$ & $\mathrm{k}=2$ & 0.007 \\
\hline
\end{tabular}

Tabla 4.2. Cálculo de la incertidumbre asociada a la densidad de cada compuesto en las bombas en las condiciones de inyección de la mezcla.

\begin{tabular}{|c|l|l|l|l|l|}
\hline & & Unidad & Estimación & Divisor & \multicolumn{1}{c|}{$\mathrm{u}(x) \mathrm{CO}_{2}$} \\
\hline & & & & & $\mathrm{kg} / \mathrm{m}^{3}$ \\
\hline \multirow{2}{*}{$\mathrm{u}(T) \mathrm{CO}_{2}$} & Calibración & \multirow{2}{*}{${ }^{\circ} \mathrm{C}$} & 0.020 & 2 & \multirow{2}{*}{0.064} \\
\cline { 2 - 4 } & Resolución & & 0.010 & $2 \sqrt{3}$ & \\
\hline \multirow{2}{*}{$\mathrm{u}(p) \mathrm{CO}_{2}$} & Calibración & \multirow{2}{*}{$\mathrm{MPa}$} & 0.02 & 2 & \multirow{2}{*}{0.000047} \\
\cline { 2 - 5 } & Resolución & & 0.01 & $2 \sqrt{3}$ & \\
\cline { 1 - 2 } $\mathrm{u}(\rho(T, p)) \mathrm{CO}_{2}$ & $\mathrm{~kg} / \mathrm{m}^{3}$ & $\mathrm{k}=1$ & 0.06 \\
\hline $\mathrm{U}(\rho(T, p)) \mathrm{CO}_{2}$ & $\mathrm{~kg} / \mathrm{m}^{3}$ & $\mathrm{k}=2$ & 0.13 \\
\hline
\end{tabular}

\begin{tabular}{|l|l|l|l|l|l|}
\hline & & Unidad & Estimación & Divisor & $\mathrm{u}(x)$ Amina aq \\
\hline & & & & & $\mathrm{kg} / \mathrm{m}^{3}$ \\
\hline \multirow{2}{*}{$\mathrm{u}(T)$ Amina aq } & Calibración & \multirow{2}{*}{${ }^{\circ} \mathrm{C}$} & 0.020 & 2 & \multirow{2}{*}{0.007} \\
\cline { 4 - 6 } & Resolución & & 0.010 & $2 \sqrt{3}$ & \\
\hline \multirow{2}{*}{$\mathrm{u}(p)$ Amina aq } & Calibración & \multirow{2}{*}{$\mathrm{MPa}$} & 0.02 & 2 & \multirow{2}{*}{0.000004} \\
\cline { 4 - 5 } & Resolución & & 0.01 & $2 \sqrt{3}$ & \\
\hline \multirow{2}{*}{$\mathrm{u}(\rho(T, p))$ Amina aq } & $\mathrm{kg} / \mathrm{m}^{3}$ & $\mathrm{k}=1$ & 0.007 \\
\hline $\mathrm{U}(\rho(T, p))$ Amina aq & $\mathrm{kg} / \mathrm{m}^{3}$ & $\mathrm{k}=2$ & 0.01 \\
\hline
\end{tabular}


Representándose en este caso en la Tabla 4.3. las incertidumbres asociadas a la composición de la mezcla. La incertidumbre expandida en la fracción molar de $\mathrm{CO}_{2}$ de la mezcla con un factor de cobertura $k=2$ (95\% de confianza) es de 0.0009 .

Tabla 4.3. Cálculo de la incertidumbre asociada a la composición de la mezcla.

\begin{tabular}{|c|c|l|l|l|l|}
\hline & & Unidad & Estimación & Divisor & $\mathrm{u}(x)$ \\
\hline & & & & & $\mathrm{mol} / \mathrm{mol}$ \\
\hline $\mathrm{u}(\dot{n})$ Amina aq. & - & $\mathrm{mol} / \mathrm{min}$ & 0.0017 & 1 & 0.0004 \\
\hline $\mathrm{u}(\dot{m}) \mathrm{CO}_{2}$ & - & $\mathrm{g} / \mathrm{min}$ & 0.0022 & 1 & 0.0002 \\
\hline $\mathrm{u}(x) \mathrm{CO}_{2}$ & $\mathrm{~mol} / \mathrm{mol}$ & $\mathrm{k}=1$ & 0.0004 \\
\hline $\mathrm{U}(x) \mathrm{CO}_{2}$ & $\mathrm{~mol} / \mathrm{mol}$ & $\mathrm{k}=2$ & 0.0009 \\
\hline
\end{tabular}

Para el cálculo de las incertidumbres de la densidad asociadas al equipo partiremos de la ecuación de la base de cálculo (4.14), que establece la relación entre la densidad y el período de oscilación de la celda de medida mediante los parámetros de calibración definidos en (4.15) y (4.16). La ley de propagación de incertidumbres aplicada en base a estas expresiones permite calcular la incertidumbre de los dos parámetros y la densidad de la siguiente manera:

Para el coeficiente $A(T)$, expresado en la ecuación (4.15), la incertidumbre expandida $(k=2)$ es:

$$
\begin{gathered}
\mathrm{U}(\mathrm{A}(T))=2\left[\left(\frac{\partial \mathrm{A}(T)}{\partial \rho_{W}}\right)^{2} \mathrm{u}^{2}\left(\rho_{W}\right)+\left(\frac{\partial \mathrm{A}(T)}{\partial \tau_{\mathrm{W}}}\right)^{2} \mathrm{u}^{2}\left(\tau_{W}\right)+\left(\frac{\partial \mathrm{A}(T)}{\partial \tau_{v}}\right)^{2} \mathrm{u}^{2}\left(\tau_{v}\right)\right]^{\frac{1}{2}} \\
\mathrm{U}(\mathrm{A}(T))=2\left[\left(\frac{\mathrm{A}(T)}{\rho_{W}}\right)^{2} \mathrm{u}^{2}\left(\rho_{W}\right)+\left(\frac{-2 \mathrm{~A}^{2}(T) \tau_{W}}{\rho_{W}}\right)^{2} \mathrm{u}^{2}\left(\tau_{W}\right)+\left(\frac{2 \mathrm{~A}^{2}(T) \tau_{v}}{\rho_{W}}\right)^{2} \mathrm{u}^{2}\left(\tau_{v}\right)\right]^{\frac{1}{2}}
\end{gathered}
$$

Para el coeficiente $B(T, p)$, expresado en la ecuación (4.16), la incertidumbre expandida $(k=2)$ es:

$$
\mathrm{U}(\mathrm{B}(T, p))=2\left[\left(\frac{\partial \mathrm{B}(T, p)}{\partial \rho_{W}}\right)^{2} \mathrm{u}^{2}\left(\rho_{W}\right)+\left(\frac{\partial \mathrm{B}(T, p)}{\partial \tau_{W}}\right)^{2} \mathrm{u}^{2}\left(\tau_{W}\right)+\left(\frac{\partial \mathrm{B}(T, p)}{\partial \tau_{v}}\right)^{2} \mathrm{u}^{2}\left(\tau_{v}\right)\right]^{\frac{1}{2}}
$$

donde:

$$
\frac{\partial \mathrm{B}(T, p)}{\partial \rho_{w}}=\frac{\mathrm{A}(T) \cdot \tau_{W}^{2}(T, p)}{\rho_{w}(T, 0.1 \mathrm{MPa})}-1
$$


Determinación de medidas de las densidades y solubilidades de $\mathrm{CO}_{2}$ en soluciones acuosas de aminas

$$
\begin{gathered}
\frac{\partial \mathrm{B}(T, p)}{\partial \tau_{w}}=2 \mathrm{~A}(T) \cdot \tau_{w}(T, p)\left[1-\mathrm{A}(T) \frac{\tau_{w}(T, 0.1 \mathrm{MPa}) \cdot \tau_{w}(T, p)}{\rho_{w}(T, 0.1 \mathrm{MPa})}\right] \\
\frac{\partial \mathrm{B}(T, p)}{\partial \tau_{v}}=2 \frac{\tau_{v}(T) \cdot \mathrm{A}^{2}(T)}{\rho_{w}(T, 0.1 \mathrm{MPa})} \cdot \tau_{w}^{2}(T, p)
\end{gathered}
$$

La incertidumbre expandida $(k=2)$ de la densidad se calcula de la forma siguiente:

$$
\mathrm{U}(\rho(T, p))=2\left[\left(\tau^{2}(T, p)\right)^{2} \mathrm{u}^{2}(\mathrm{~A}(T))+(2 \mathrm{~A}(T) \cdot \tau(T, p))^{2} \mathrm{u}^{2}(\tau(T, p))+\mathrm{u}^{2}(\mathrm{~B}(T, p))\right]^{\frac{1}{2}}
$$

$$
\mathrm{U}(\rho(T, p))=2\left[\left(\frac{\partial \rho(T, p)}{\partial \mathrm{A}(T)}\right)^{2} \mathrm{u}^{2}(\mathrm{~A}(T))+\left(\frac{\partial \rho(T, p)}{\partial \tau(T, p)}\right)^{2} \mathrm{u}^{2}(\tau(T, p))+\left(\frac{\partial \rho(T, p)}{\partial \mathrm{B}(T, p)}\right)^{2} \mathrm{u}^{2}(\mathrm{~B}(T, p))\right]^{\frac{1}{2}}
$$

En base a los cálculos anteriores, en la Tabla 4.4 se presentan las incertidumbres asociadas

\begin{tabular}{|c|c|c|c|c|c|}
\hline & & Unidad & Estimación & Divisor & $\mathrm{u}(x)$ \\
\hline & & & & & $\mathrm{kg} / \mathrm{m}^{3}$ \\
\hline $\mathrm{u}\left(\rho_{w}\right)$ & $\begin{array}{c}\text { Referencia } \\
\text { material }\end{array}$ & $\mathrm{kg} / \mathrm{m}^{3}$ & 0.01 & $\sqrt{3}$ & 0.0058 \\
\hline \multirow{3}{*}{$\mathrm{u}(T)$} & Calibración & \multirow{3}{*}{${ }^{\circ} \mathrm{C}$} & 0.020 & 2 & \multirow{3}{*}{0.0046} \\
\hline & Resolución & & 0.010 & $2 \sqrt{3}$ & \\
\hline & Repetibilidad & & 0.0008 & 1 & \\
\hline \multirow{3}{*}{$\mathrm{u}(p)$} & Calibración & \multirow{3}{*}{$\mathrm{MPa}$} & 0.02 & 2 & \multirow{3}{*}{0.0041} \\
\hline & Resolución & & 0.01 & $2 \sqrt{3}$ & \\
\hline & Repetibilidad & & 0.002 & 1 & \\
\hline \multirow{2}{*}{$\mathrm{u}(\tau)$} & Repetibilidad & \multirow[b]{2}{*}{$\mu \mathrm{S}$} & 0.0006 & 1 & \multirow{2}{*}{$8.5 \mathrm{E}-03$} \\
\hline & Resolución & & \begin{tabular}{|l|}
$1.00 \mathrm{E}-03$ \\
\end{tabular} & $2 \sqrt{3}$ & \\
\hline $\mathrm{U}(\mathrm{A}(T))$ & & $\mathrm{kg} / \mathrm{m}^{3} \mu \mathrm{s}^{2}$ & & $\mathrm{k}=2$ & $7.6 \mathrm{E}-08$ \\
\hline $\mathrm{U}(\mathrm{B}(T, p))$ & & $\mathrm{kg} / \mathrm{m}^{3}$ & & $\mathrm{k}=2$ & 0.43 \\
\hline
\end{tabular}
a los parámetros de calibración a nuestro intervalo de temperatura.

Tabla 4.4. Cálculo de la incertidumbre asociada a los parámetros de calibración en un intervalo de temperaturas de (273.15-373.15) Ky presiones 0.1-140 MPa.

En la Tabla 4.5. se representan las incertidumbres asociadas a la medida de densidad a diferentes rangos de temperatura. La incertidumbre expandida con un factor de cobertura $k=2$ (95\% de confianza) en la medida de densidad para un intervalo de temperaturas de 293.15-373.15 Ky presiones de 0.1-60 MPa es de $0.7 \mathrm{~kg} / \mathrm{m}^{3}$. 
Determinación de medidas de las densidades y solubilidades de $\mathrm{CO}_{2}$ en soluciones acuosas de aminas

Tabla 4.5. Cálculo de la incertidumbre asociada a la densidad experimental en un intervalo de temperaturas de (293.15-373.15) Ky presiones 0.1-60 MPa.

\begin{tabular}{|c|c|c|c|c|c|}
\hline & & Unidad & Estimación & Divisor & $\mathrm{u}(x)$ \\
\hline & & & & & $\mathrm{kg} / \mathrm{m}^{3}$ \\
\hline \multirow{2}{*}{$\mathrm{u}(\tau)$} & Repetibilidad & \multirow{2}{*}{$\mu \mathrm{s}$} & 0.0006 & 1 & \multirow{2}{*}{$8.5 \mathrm{E}-03$} \\
\hline & Resolución & & $1.00 \mathrm{E}-03$ & $2 \sqrt{3}$ & \\
\hline \multirow{3}{*}{$\mathrm{u}(T)$} & Calibración & \multirow{3}{*}{${ }^{\circ} \mathrm{C}$} & 0.020 & 2 & \multirow{3}{*}{0.0046} \\
\hline & Resolución & & 0.010 & $2 \sqrt{3}$ & \\
\hline & Repetibilidad & & 0.0008 & 1 & \\
\hline \multirow{3}{*}{$\mathrm{u}(p)$} & Calibración & \multirow{3}{*}{$\mathrm{MPa}$} & 0.02 & 2 & \multirow{3}{*}{0.0041} \\
\hline & Resolución & & 0.01 & $2 \sqrt{3}$ & \\
\hline & Repetibilidad & & 0.002 & 1 & \\
\hline $\mathrm{u}(\mathrm{A}(T))$ & $\mathrm{kg} / \mathrm{m}^{3} \mu \mathrm{S}^{2}$ & & $7.6 \mathrm{E}-08$ & 2 & 0.26 \\
\hline $\mathrm{u}(\mathrm{B}(T, p))$ & $\mathrm{kg} / \mathrm{m}^{3}$ & & 0.43 & 2 & 0.21 \\
\hline $\mathrm{u}(\rho)$ & \multicolumn{3}{|c|}{$\mathrm{kg} / \mathrm{m}^{3}$} & $\mathrm{k}=1$ & 0.34 \\
\hline $\mathrm{U}(\rho)$ & \multicolumn{3}{|c|}{$\mathrm{kg} / \mathrm{m}^{3}$} & $\mathrm{k}=2$ & 0.7 \\
\hline $\mathrm{U}_{\mathrm{r}}(\rho)$ & \multicolumn{3}{|c|}{$100 \cdot\left(\mathrm{kg} / \mathrm{m}^{3} / \mathrm{kg} / \mathrm{m}^{3}\right)$} & $\mathrm{k}=2$ & $0.07 \%$ \\
\hline
\end{tabular}

En la Tabla 4.5. se observa que la mayor contribución a la incertidumbre proviene de los parámetros de calibrado A $(T)$ y B $(T, p)$. Se deduce entonces la importancia de disponer de buenos datos de densidad a diferentes rangos de presiones y temperaturas de los fluidos de referencia. La incertidumbre relativa expandida de $0.07 \%$ se calculó para la menor densidad dentro de nuestro rango de medidas.

\subsection{Validación del equipo.}

Para la validación del equipo se realizó con una medida de tolueno en un rango de temperaturas de $273.15 \mathrm{~K}$ a $373.15 \mathrm{~K}$ y en un rango de presiones desde $0.1 \mathrm{MPa}$ hasta 140 MPa. Las máximas desviaciones respecto al REFPROP fueron de $0.07 \%$ el cual utiliza la ecuación de estado descrita en [19]. Las incertidumbres en densidad para esa ecuación de estado son de $0.05 \%$ en la fase líquida (hasta $540 \mathrm{~K}$ y $100 \mathrm{MPa}$ ) y de $0.5 \%$ en presiones comprendidas entre 100 a $500 \mathrm{MPa}$. Encontrándose las desviaciones en consonancia con nuestra incertidumbre de medida.

\subsection{Referencias.}

[1] R. Dohrn. G. Brunner. "High-pressure fluid-phase equilibria: Experimental methods and systems investigated (1988-1993)". Fluid Phase Equilibria. Vol. 106, (1995) 213-282.

[2] A. Moreau. "Caracterización termodinámica de combustibles ambientalmente sostenibles: determinación experimental del equilibrio líquido-vapor y entalpías de exceso de mezclas de interés para la formulación de biogasolinas". Universidad de Valladolid. Tesis doctoral (2014). 
[3] F. Y. Jou, A. E. Mather, F. D. Otto. "The solubility of $\mathrm{CO}_{2}$ in a 30 mass percent monoethanolamine solution". Can. J. Chem. Eng. Vol. 73, (1995) 140-147.

[4] D. Tong, J. P. M. Trusler, G. C. Maitland, J. Gibbins, P. S. Fennell. "Solubility of carbon dioxide in aqueous solution of monoethanolamine or 2-amino-2-methyl-1-propanol: Experimental measurements and modelling". International Journal of Greenhouse Gas Control. Vol. 6, (2012) 37-47.

[5] R. H. Petrucci, F. G. Herring, J. D. Madura, C. Bissonnette. "Química general, principios y aplicaciones modernas". Pearson (2011).

[6] E. I. Concepción (2017). "Caracterización termodinámica de mezclas acuosas de aminas". Universidad de Valladolid. Tesis doctoral.

[7] F. Aguilar. "Puesta en marcha de una técnica de equilibrio líquido vapor isóbaro e investigación termodinámica de equilibrios binarios y ternarios de aditivos oxigenados en hidrocarburos de sustitución de gasolinas sin plomo". Universidad de Burgos. Tesis Doctoral (2010).

[8] M. Sobrino, E. I. Concepción, A. Gómez-Hernández, M. C. Martín, J. J. Segovia. "Viscosity and density measurements of aqueous amines at high pressures: MDEA-water and MEAwater mixtures for $\mathrm{CO}_{2}$ capture". J. Chem. Thermodynamics. Vol. 98, (2016) 231-241.

[9] S. Chen, L. Zhang, Y. Zhang, S. Chen, J. Chen. "Density and viscosity of monoethylethanolamine $+\mathrm{H}_{2} \mathrm{O}$ and monoethylethanolamine + diethylethanolamine solutions for $\mathrm{CO}_{2}$ capture". Thermochim. Acta. Vol. 642, (2016) 52-58.

[10] J. Han, J. Jin, D. A. Eimer, M. C. Melaaen. "Density of water (1) + monoethanolamine (2) + $\mathrm{CO}_{2}$ (3) from (298.15 to 413.15) $\mathrm{K}$ and surface tension of water (1) + monoethanolamine (2) from (303.15 to 333.15) K". J. Chem. Eng. Data. Vol. 57, (2012) 1095-1103.

[11] J. Zhang, P. S. Fennell, J. P. M. Trusler. "Density and viscosity of partially carbonated aqueous ternary alkanolamine solutions at temperatures between (298.15 and 353.15) K". J. Chem. Eng. Data. Vol. 60, (2015) 2392-2399.

[12] H. Stabinger, H. Leopold, O. Kratky. "Eine neue methode zur Präzisionsmessung der dichte von flüssigkeiten". Monatshefte fur chemie. Vol. 98, (1967) 436-438.

[13] J. R. Zambrano. "Desarrollo de un viscosímetro de hilo vibrante para la caracterización termofísica a alta presión de nuevos biocombustibles". Universidad de Valladolid. Tesis Doctoral (2014).

[14] C. D. Holcomb, S. L. Outcalt. "A theoretically-based calibration and evaluation procedure for vibrating-tube densimeters". Fluid Phase Equilibria. Vol. 150, (1998) 815827.

[15] D. Vega. "Caracterización termodinámica de combustibles líquidos de nueva generación con componentes renovables mediante un nuevo calorímetro isobárico de alta presión y medidas densitométricas". Universidad de Valladolid. Tesis doctoral (2009). 
Determinación de medidas de las densidades y solubilidades de $\mathrm{CO}_{2}$ en soluciones acuosas de aminas

[16] B. Lagourette, C. Boned, H. Saint-Guirons, P. Xans, H. Zhou. "Densimeter calibration method versus temperature and pressure". Meas. Sci. Technol. Vol. 3, (1992) 699-703.

[17] W. Wagner, A. Pruss. "The IAPWS formulation 1995 for the thermodynamic properties of ordinary water substance for general and scientific use". J. Phys. Chem. Vol. 31, (2002) 387-535.

[18] JCGM, "Evaluation of measurement data-Guide to the expression of uncertainty in measurement". BIPM (2008).

[19] E. W. Lemmon and R. Span. "Short fundamental equations of state for 20 industrial fluids". J. Chem. Eng. Data. Vol. 51, (2006) 785-850. 
Capítulo 5

RESULTADOS DE MEDIDAS

EXPERIMENTALES DE LAS DENSIDADES

Y SOLUBILIDADES DE $\mathrm{CO}_{2}$ EN

SOLUCIONES ACUOSAS DE AMINAS 



\subsection{Introducción.}

En este capítulo se presentan los resultados experimentales de las medidas de densidad en mezclas ternarias de soluciones acuosas de aminas $+\mathrm{CO}_{2}$ en el rango de temperaturas de $293.15 \mathrm{~K}$ a $373.15 \mathrm{~K}$ y presiones desde $0.1 \mathrm{MPa}$ hasta $60 \mathrm{MPa}$. Estos resultados se obtuvieron mediante la técnica del densímetro de tubo vibrante descrito en el capítulo anterior. Por otra parte, se presentan los resultados experimentales de las solubilidades de $\mathrm{CO}_{2}$ en disoluciones acuosas de aminas mediante el equipo de equilibrio líquido-vapor a dos temperaturas $313.15 \mathrm{~K}$ y $353.15 \mathrm{~K}$. Las diferentes aminas a estudiar fueron la Monoetanolamina (MEA), la dimetilaminoetanol (DMAE) y la Trietanolamina (TEA).

Este estudio se realizó para el conocimiento de los parámetros de operación, que son determinantes en la viabilidad técnica y económica de los procesos de captura de $\mathrm{CO}_{2}$. Son etapas importantes la elección de la amina y la mezcla óptima de esta para producir un solvente con una excelente capacidad de absorción y regeneración, disminuyendo su corrosividad.

En el presente capítulo, además de los datos experimentales, se presentan los ajustes a funciones que describan su comportamiento. Se utilizaron las ecuaciones de densidades de mezcla (ecuación 4.5) y de Tammann-Tait para describir las densidades, y la ecuación de Xu y Rochelle para las solubilidades. Se describe también el procedimiento de ajuste de los datos experimentales de densidad y solubilidad de las distintas mezclas a estudiar.

Los resultados experimentales se muestran en forma de tablas y gráficas. En ellas se presentan la presión $p$, la temperatura $T$ y la densidad $\rho$. En el caso de las solubilidades se muestra la presión parcial del $\mathrm{CO}_{2}\left(p_{\mathrm{CO} 2}\right)$ en función del índice de carga $\alpha$ (moles de $\mathrm{CO}_{2}$ por mol de amina) a diferentes temperaturas para las mezclas estudiadas.

Para el cálculo de las solubilidades de las mezclas de $\mathrm{CO}_{2}$ con soluciones acuosas de aminas a partir de las medidas experimentales, es preciso conocer la densidad de las mezclas, y de esta manera conocer el volumen ocupado por la mezcla en la fase líquida dentro de la celda de medida.

Las mezclas realizadas para la medida de la densidad en este trabajo son disoluciones acuosas al $10 \%$ y al $30 \%$ en peso de MEA a diferentes concentraciones de $\mathrm{CO}_{2}$ para la validación de la técnica. Además, se midieron dos sistemas diferentes de disoluciones acuosas al 30\% en peso con $\mathrm{CO}_{2}$, una con DMAE y otra con TEA. Todas las medidas se realizan en un amplio rango de temperatura (desde $293.15 \mathrm{~K}$ hasta $373.15 \mathrm{~K}$ ), de presión (de $0.1 \mathrm{MPa}$ a $60 \mathrm{MPa}$ ) y a diferentes composiciones de $\mathrm{CO}_{2}$ en función de $\alpha$ desde 0.2 hasta 1.5 .

Se han medido las densidades de tres mezclas acuosas de aminas $+\mathrm{CO}_{2}$ a diferentes concentraciones:

MEA $10 \% \mathrm{w}+\mathrm{CO}_{2}$ a $\alpha=0.8$ y 1.

MEA $30 \% \mathrm{w}+\mathrm{CO}_{2}$ a $\alpha=0.2,0.44,0.67$ y 1.

DMAE $30 \% \mathrm{w}+\mathrm{CO}_{2}$ a $\alpha=0.3,0.7,1.1$ y 1.5 . 
TEA $30 \% \mathrm{w}+\mathrm{CO}_{2}$ a $\alpha=0.3,0.6,0.9$ y 1.2 .

Las solubilidades que se midieron en este trabajo son disoluciones acuosas al $10 \% \mathrm{y}$ al $30 \%$ en peso de MEA con $\mathrm{CO}_{2}$, y dos sistemas de $\mathrm{CO}_{2}$ con disoluciones acuosas al $30 \%$ en peso, una con DMAE y otra con TEA. Todos los sistemas a excepción de la MEA al 10\% en peso se realizaron a dos temperaturas, $313.15 \mathrm{~K}$ y $353.15 \mathrm{~K}$, con presiones desde la presión de vapor de la solución acuosa de la amina hasta presiones de $6 \mathrm{MPa}$. Estas medidas se realizaron con la celda de equilibrio isoterma.

En este trabajo se han medido cuatro sistemas ternarios a dos temperaturas diferentes:

MEA $10 \% \mathrm{w}+\mathrm{CO}_{2}$ a $313.15 \mathrm{~K}$.

MEA $30 \% \mathrm{w}+\mathrm{CO}_{2}$ a $313.15 \mathrm{Ky} 353.15 \mathrm{~K}$.

DMAE $30 \% \mathrm{w}+\mathrm{CO}_{2}$ a $313.15 \mathrm{~K}$ y $353.15 \mathrm{~K}$.

TEA $30 \% \mathrm{w}+\mathrm{CO}_{2}$ a $313.15 \mathrm{~K} \mathrm{y} 353.15 \mathrm{~K}$.

\subsection{Ajuste de los datos de densidad (ecuación de Tammann-Tait modificada).}

Los datos de densidad a diferentes temperaturas y presiones han sido ajustados mediante la ecuación de Tammann-Tait [1] modificada para cada concentración. Esta ecuación se ha estado utilizando desde finales del siglo XIX para el ajuste de datos de densidad a alta presión para todo tipo de líquidos (Wohl 1921 [2], Hayward [3]), hasta polímeros de alto peso molecular e incluso datos de compresibilidad de sólidos y sales fundidas.

En 1888 para describir la compresibilidad del agua P.G. Tait [4] propone la siguiente ecuación:

$$
\frac{v^{0}-v}{v^{0}}=\frac{\mathrm{A}}{\mathrm{B}+p}
$$

donde $v$, es el volumen molar, $v^{0}$ es el volumen molar a presión atmosférica, $p$ es la presión y A y B son dos parámetros independientes de la presión que dependen de la temperatura.

Después en 1985, G. Tammann [5] propuso la siguiente modificación:

$$
\frac{v^{0}-v}{v^{0}}=\frac{\mathrm{C} \cdot \ln (\mathrm{B}+p)}{\mathrm{B}}
$$

donde B y C son dos parámetros independientes de la presión.

En otros trabajos como en el realizado por Comuñas et al. [6] propone la ecuación Tammann-Tait modificada según muestra la ecuación 5.3. Este tipo de ajuste también ha sido usado en anteriores trabajos del grupo TERMOCAL como el presentado por Sobrino et al. [7].

$$
\rho(T, p)=\frac{\rho\left(T, p_{r e f}\right)}{1-\mathrm{C} \ln \left[\frac{\mathrm{B}(T)+p}{\mathrm{~B}(T)+p_{\mathrm{ref}}}\right]}
$$


siendo $\rho$ la densidad, $T$ la temperatura, $C$ es un parámetro el cual le consideramos independiente de la temperatura y $\rho\left(T, p_{\text {ref }}\right)$ es la dependencia de la densidad con la temperatura a la presión de referencia, siendo por lo general en nuestro caso la presión de $5 \mathrm{MPa}$. Esta función toma la forma expresada en la ecuación 5.4.

$$
\rho\left(T, p_{\text {ref }}\right)=\sum_{\mathrm{i}=0} \mathrm{~A}_{\mathrm{i}} T^{\mathrm{i}}=\mathrm{A}_{0}+\mathrm{A}_{1} T+\mathrm{A}_{2} T^{2}
$$

Donde los valores de $A_{i}$ se determinan a partir de los valores experimentales de la densidad a la presión de referencia. Y la función $\mathrm{B}(T)$ tiene una forma polinómica según la ecuación 5.5 .

$$
\mathrm{B}(T)=\sum_{\mathrm{i}=0} \mathrm{~B}_{\mathrm{i}} T^{\mathrm{i}}=\mathrm{B}_{0}+\mathrm{B}_{1} T+\mathrm{B}_{2} T^{2}
$$

Los valores de $B_{i}$ se determinan a partir de los valores experimentales. De esta forma, a partir de la ecuación 5.3, podremos representar los valores de la densidad para un amplio rango de temperatura y presión mediante los parámetros ajustables $\mathrm{A}_{\mathrm{i}}, \mathrm{B}_{\mathrm{i}} \mathrm{y} \mathrm{C}$.

Para evaluar el ajuste realizado se hizo por medio de la desviación estándar $\sigma$ y la máxima desviación $M D \%$ entre datos experimentales y el modelo utilizado siendo estas las ecuaciones 5.6 y 5.7 respectivamente.

$$
\begin{aligned}
& \sigma=\sqrt{\left[\frac{1}{N-m}\right] \sum_{i=0}^{N}\left(x_{\text {exp }}-x_{c a l}\right)^{2}} \\
& M D \%=\operatorname{Max} \cdot\left|\frac{\left(x_{c a l}-x_{\text {exp }}\right)}{x_{\text {exp }}} \cdot 100\right|
\end{aligned}
$$

donde, $N$ es el número de datos experimentales, $m$ es el número de parámetros ajustados, $x_{\text {exp }}$ es el valor experimental y $x_{c a l}$ el valor calculado mediante la ecuación de TammannTait modificada 5.3.

También se evaluó la bondad del ajuste de la densidad de mezcla (ecuación 4.5) por medio de la desviación estándar $\sigma$ entre datos experimentales y el modelo utilizado, mediante la ecuación 5.6.

$$
\rho_{\text {mezcla }}=\mathrm{a}+\frac{\mathrm{b}}{\propto}+\frac{\mathrm{c}}{\alpha^{2}}+\mathrm{d} \cdot p_{\text {total }}
$$

\subsection{Ajuste de los datos de solubilidad.}

El ajuste de los datos del índice de carga $\alpha$ frente a la presión parcial del $\mathrm{CO}_{2}\left(p_{\mathrm{CO}}\right)$, se realizó siguiendo la ecuación de Xu y Rochelle [8], donde se relaciona $\alpha$ y la temperatura de trabajo mediante 6 parámetros, con la presión parcial del $\mathrm{CO}_{2}$. Siendo esta ecuación de ajuste:

$$
\ln p_{\mathrm{CO}_{2}}(\mathrm{~Pa})=\mathrm{A}_{1}-\mathrm{A}_{2} / T+\mathrm{A}_{3} \cdot \alpha+\mathrm{A}_{4} \cdot \alpha^{2}+\mathrm{A}_{5} \cdot \alpha / T+\mathrm{A}_{6} \cdot \alpha^{2} / T
$$


siendo $p_{\mathrm{CO}_{2}}$ la presión parcial del $\mathrm{CO}_{2}, T$ la temperatura y $\alpha$ índice de carga en moles de $\mathrm{CO}_{2}$ por mol de amina. Los parámetros ajustables son $\mathrm{A}_{1}, \mathrm{~A}_{2}, \mathrm{~A}_{3}, \mathrm{~A}_{4}, \mathrm{~A}_{5}$ y $\mathrm{A}_{6}$. Para evaluar el ajuste realizado se hizo mediante la desviación estándar $\sigma$ (ecuación 5.6) entre datos experimentales y la ecuación utilizada.

\subsection{Relación de los productos utilizados.}

En la Tabla 5.1. se muestran los productos utilizados, la procedencia y la pureza dada por los certificados de análisis. En nuestro caso no se utilizó ningún método de purificación adicional, a excepción del agua para la medida de las solubilidades. El agua se desgasificó previamente mediante una columna de rectificación a vacío empleando un baño de ultrasonidos para la agitación.

Las aminas se desgasificaron en la celda de equilibrio mediante una serie de ciclos, primero solidificando el compuesto por medio de un enfriamiento en un baño de nitrógeno líquido y después fundiéndolo por medio de un calentamiento a temperatura ambiente.

Las mezclas acuosas de aminas utilizadas para las medidas de densidades se desgasificaron mediante un baño de ultrasonidos marca Branson, modelo DTH-3210, con una frecuencia de $47 \mathrm{kHz}$ y una potencia de $335 \mathrm{~W}$. El tiempo que se aplicó para la desgasificación fue de un mínimo de 5 horas.

Tabla 5.1. Características de los productos utilizados.

\begin{tabular}{llll}
\hline Sustancia & Fórmula & Marca comercial & Pureza \\
\hline MEA & $\mathrm{C}_{2} \mathrm{H}_{7} \mathrm{NO}$ & Sigma-Aldrich & $\geq 99.0 \%$ \\
DMAE & $\mathrm{C}_{4} \mathrm{H}_{11} \mathrm{NO}$ & Sigma-Aldrich & $\geq 99.5 \%$ \\
TEA & $\mathrm{C}_{6} \mathrm{H}_{15} \mathrm{NO}_{3}$ & Sigma-Aldrich & $\geq 99.0 \%$ \\
Agua (ultrapura) & $\mathrm{H}_{2} \mathrm{O}$ & Sigma-Aldrich y Fluka & Conductividad $\leq 2$ \\
& & & $\mu \mathrm{ohm}^{-1} \cdot \mathrm{cm}^{-1} \mathrm{a} 25^{\circ} \mathrm{C}$ \\
Dióxido de Carbono & $\mathrm{CO}_{2}$ & Carburos metálicos & $\mathrm{CO}_{2} \geq 99.995 \%$ \\
(ultrapuro) & & & $\mathrm{O}_{2}<10 \mathrm{ppm}$ \\
& & & $\mathrm{H}_{2} \mathrm{O}<7 \mathrm{ppm}$ \\
& & & $\mathrm{CO}<2 \mathrm{ppm}$ \\
& & & $\mathrm{THC}<5 \mathrm{ppm}$ \\
& & & $\mathrm{N}_{2}<25 \mathrm{ppm}$ \\
\hline
\end{tabular}




\subsection{Presentación de los resultados experimentales de las medidas de densidad.}

A continuación, se muestran los datos experimentales obtenidos de la densidad para las mezclas estudiadas, tanto en forma de tablas, como en gráficas. Se presentan los resultados del ajuste de los datos experimentales al modelo Tammann-Tait obtenidos mediante el método descrito en el apartado 5.2. Este ajuste se realizó para cada una de las composiciones estudiadas, en función de la temperatura y la presión. Además, se muestra tanto la desviación estándar del ajuste $\sigma$ (ecuación 5.6) como la máxima desviación $M D \%$ entre datos experimentales y el modelo utilizado (ecuación 5.7). También se representan los parámetros del ajuste de los datos experimentales de las densidades de mezcla en función de $\alpha$, la presión total y la desviación estándar de la ecuación 4.5. 
Resultado de medidas experimentales de las densidades y solubilidades de $\mathrm{CO}_{2}$ en soluciones acuosas de aminas

\subsubsection{Sistema MEA $10 \% w+\mathrm{CO}_{2}$.}

Tabla 5.2. Datos experimentales de densidad, $\rho\left(\mathrm{kg}^{-\mathrm{m}^{-3}}\right)$, para la mezcla $\mathrm{MEA} 10.003 \% \mathrm{w}+\mathrm{CO}_{2}$ a $\alpha=$ 0.7989 .

\begin{tabular}{ccc}
\hline \multicolumn{3}{c}{$\rho / \mathrm{kg} \cdot \mathrm{m}^{-3}$} \\
\hline \multicolumn{3}{c}{$T / \mathrm{K}$} \\
\hline$p / \mathrm{MPa}$ & 293.15 & 313.15 \\
\hline 5 & 1057.10 & 1052.54 \\
10 & 1059.10 & 1054.65 \\
15 & 1061.10 & 1056.71 \\
20 & 1063.03 & 1058.74 \\
30 & 1066.85 & 1062.72 \\
40 & 1070.65 & 1066.69 \\
50 & 1074.33 & 1070.46 \\
60 & 1077.96 & 1074.29 \\
\hline
\end{tabular}

Tabla 5.3. Coeficientes y estadística de la ecuación Tammann-Tait modificada para la mezcla MEA $10.003 \% w+\mathrm{CO}_{2}$ a $\alpha=0.7989, p_{\text {ref }}=5 \mathrm{MPa}$.

Parámetros de la ecuación Tammann-Tait modificada

\begin{tabular}{ccccccccc}
\hline $\begin{array}{c}\mathrm{A}_{0} \\
\mathrm{~kg} \cdot \mathrm{m}^{-3}\end{array}$ & $\begin{array}{c}\mathrm{A}_{1} \\
\mathrm{~kg} \cdot \mathrm{m}^{-3} \cdot \mathrm{K}^{-1}\end{array}$ & $\begin{array}{c}\mathrm{A}_{2} \\
\mathrm{~kg} \cdot \mathrm{m}^{-3} \cdot \mathrm{K}^{-2}\end{array}$ & $\begin{array}{c}\mathrm{B}_{0} \\
\mathrm{MPa}\end{array}$ & $\begin{array}{c}\mathrm{B}_{1} \\
\mathrm{MPa} \cdot \mathrm{K}^{-1}\end{array}$ & $\begin{array}{c}\mathrm{B}_{2} \\
\mathrm{MPa} \cdot \mathrm{K}^{-2}\end{array}$ & $\mathrm{C}$ & $\begin{array}{c}\sigma \\
\mathrm{kg} \cdot \mathrm{m}^{-3}\end{array}$ & $\begin{array}{c}M D \\
(\%)\end{array}$ \\
\hline 7691.0 & -43.60 & $7.15 \mathrm{E}-02$ & 312.0 & 0.965 & $-2.9 \mathrm{E}-03$ & 0.131 & 0.03 & 0.004 \\
\hline
\end{tabular}




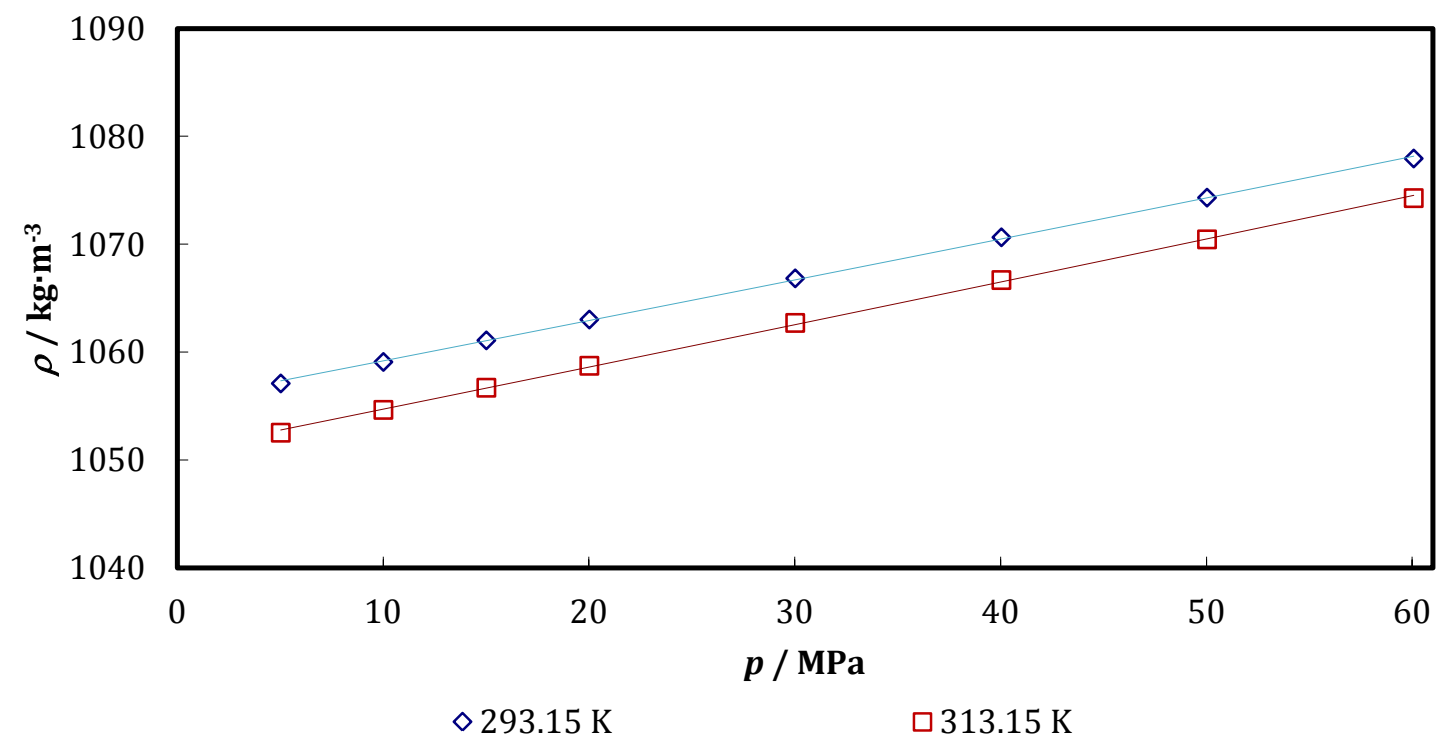

Figura 5.1. Valores experimentales de la densidad para la mezcla $\mathrm{MEA} 10.003 \% w+\mathrm{CO}_{2}$ a $\alpha=0.7989$ a diferentes temperaturas en función de la presión. La línea continua ecuación Tammann-Tait modificada, puntos valores experimentales.

Tabla 5.4. Datos experimentales de densidad, $\rho\left(\mathrm{kg}^{\prime} \mathrm{m}^{-3}\right)$, para la mezcla MEA $10.003 \% \mathrm{w}+\mathrm{CO}_{2} a \alpha=$ 0.9984.

\begin{tabular}{cccc}
\hline \multicolumn{4}{c}{$\rho / \mathrm{kg} \cdot \mathrm{m}^{-3}$} \\
\hline \multicolumn{4}{c}{$T / \mathrm{K}$} \\
\hline$p / \mathrm{MPa}$ & 293.15 & 313.15 & 333.15 \\
\hline 5 & 1064.54 & 1057.01 \\
10 & 1066.56 & 1058.99 & 1048.37 \\
15 & 1068.55 & 1060.94 & 1050.39 \\
20 & 1070.50 & 1062.87 & 1052.39 \\
30 & 1074.34 & 1066.62 & 1056.20 \\
40 & 1078.08 & 1070.30 & 1059.98 \\
50 & 1081.66 & 1073.91 & 1063.63 \\
60 & 1085.25 & 1077.45 & 1067.21 \\
\hline
\end{tabular}


Resultado de medidas experimentales de las densidades y solubilidades de $\mathrm{CO}_{2}$ en soluciones acuosas de aminas

Tabla 5.5. Coeficientes y estadística de la ecuación Tammann-Tait modificada para la mezcla MEA $10.003 \% \mathrm{w}+\mathrm{CO}_{2}$ a $\alpha=0.9984, p_{\text {ref }}=10 \mathrm{MPa}$.

Parámetros de la ecuación Tammann-Tait modificada

\begin{tabular}{|c|c|c|c|c|c|c|c|c|}
\hline $\begin{array}{c}\mathrm{A}_{0} \\
\mathrm{~kg} \cdot \mathrm{m}^{-3}\end{array}$ & $\begin{array}{c}\mathrm{A}_{1} \\
\mathrm{~kg} \cdot \mathrm{m}^{-3} \cdot \mathrm{K}^{-1}\end{array}$ & $\begin{array}{c}\mathrm{A}_{2} \\
\mathrm{~kg} \cdot \mathrm{m}^{-3} \cdot \mathrm{K}^{-2}\end{array}$ & $\begin{array}{c}\mathrm{B}_{0} \\
\mathrm{MPa}\end{array}$ & $\begin{array}{c}\mathrm{B}_{1} \\
\mathrm{MPa} \cdot \mathrm{K}^{-1}\end{array}$ & $\begin{array}{c}\mathrm{B}_{2} \\
\mathrm{MPa} \cdot \mathrm{K}^{-2}\end{array}$ & C & $\begin{array}{c}\sigma \\
\mathrm{kg} \cdot \mathrm{m}^{-3}\end{array}$ & $\begin{array}{l}M D \\
(\%)\end{array}$ \\
\hline 834.6 & 1.888 & $-3.74 \mathrm{E}-03$ & 137.7 & 1.0303 & $-1.93 \mathrm{E}-03$ & 0.105 & 0.08 & 0.01 \\
\hline
\end{tabular}

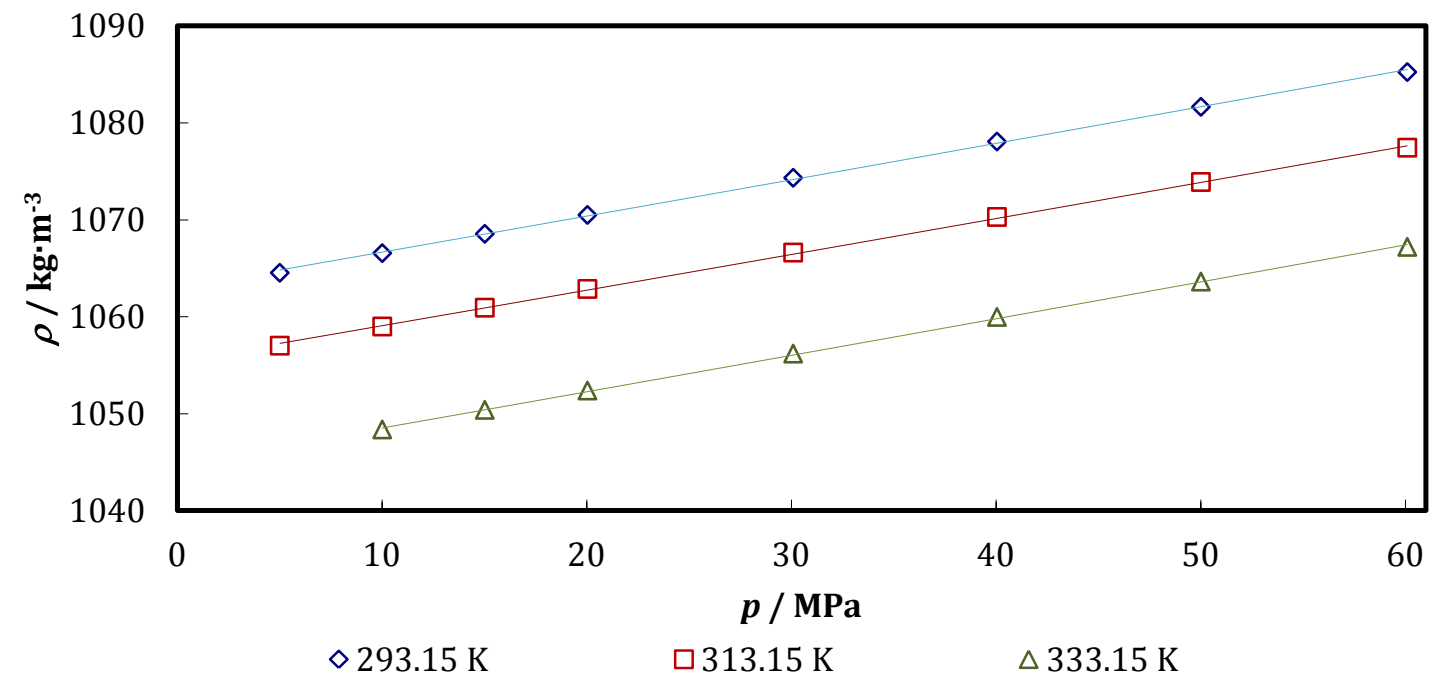

Figura 5.2. Valores experimentales de la densidad para la mezcla $\mathrm{MEA} 10.003 \% w+\mathrm{CO}_{2}$ a $\alpha=0.9984$ a diferentes temperaturas en función de la presión. La línea continua ecuación Tammann-Tait modificada, puntos valores experimentales.

Tabla 5.6. Parámetros de la ecuación de ajuste de la densidad con a y $p_{\text {total }}$ (4.5) para la mezcla MEA $10.003 \% \mathrm{w}+\mathrm{CO}_{2}$.

\begin{tabular}{cccccc}
\hline \multicolumn{5}{c}{ Parámetros de la ecuación 4.5. } \\
\hline $\begin{array}{c}\text { Temperatura } \\
\mathrm{K}\end{array}$ & $\mathrm{a}$ & $\mathrm{b}$ & $\mathrm{c}$ & $\mathrm{d}$ & $\sigma$ \\
$\mathrm{kg} \cdot \mathrm{m}^{-3}$ & $\mathrm{~kg} \cdot \mathrm{m}^{-3}$ & $\mathrm{~kg} \cdot \mathrm{m}^{-3}$ & $\mathrm{~kg} \cdot \mathrm{m}^{-3} \cdot \mathrm{bar}^{-1}$ & $\mathrm{~kg} \cdot \mathrm{m}^{-3}$ \\
\hline 313.15 & 893.61 & 302.54 & -141.20 & 0.0383 & 0.29 \\
\hline
\end{tabular}


Resultado de medidas experimentales de las densidades y solubilidades de $\mathrm{CO}_{2}$ en soluciones acuosas de aminas

\subsubsection{Sistema MEA 30\%w + $\mathrm{CO}_{2}$.}

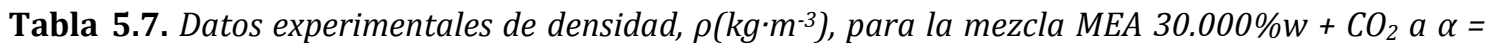
0.1999.

\begin{tabular}{cccccc}
\hline \multicolumn{5}{c}{$\rho / \mathrm{kg} \cdot m^{-3}$} \\
\hline$p / \mathrm{MPa}$ & 293.15 & 313.15 & 333.15 & 353.15 & 373.15 \\
\hline 0.1 & 1060.4 & 1051.9 & 1041.0 & 1029.2 & 1016.1 \\
0.5 & 1060.5 & 1051.9 & 1041.2 & 1029.3 & 1016.3 \\
1 & 1060.7 & 1052.1 & 1041.3 & 1029.5 & 1016.5 \\
5 & 1062.1 & 1053.6 & 1042.8 & 1031.1 & 1018.3 \\
10 & 1063.9 & 1055.5 & 1044.7 & 1033.1 & 1020.4 \\
15 & 1065.7 & 1057.3 & 1046.6 & 1035.1 & 1022.4 \\
20 & 1067.5 & 1059.1 & 1048.5 & 1037.0 & 1024.5 \\
30 & 1070.9 & 1062.6 & 1052.1 & 1040.8 & 1028.5 \\
40 & 1074.3 & 1066.0 & 1055.7 & 1044.5 & 1032.4 \\
50 & 1077.5 & 1069.3 & 1059.2 & 1048.1 & 1036.1 \\
60 & 1080.7 & 1072.7 & 1062.6 & 1051.7 & 1039.9 \\
\hline
\end{tabular}

Tabla 5.8. Coeficientes y estadística de la ecuación Tammann-Tait modificada para la mezcla MEA $30.000 \% \mathrm{w}+\mathrm{CO}_{2}$ a $\alpha=0.1999, p_{\text {ref }}=0.1 \mathrm{MPa}$.

Parámetros de la ecuación Tammann-Tait modificada

\begin{tabular}{ccccccccc}
\hline $\begin{array}{c}\mathrm{A}_{0} \\
\mathrm{~kg} \cdot \mathrm{m}^{-3}\end{array}$ & $\begin{array}{c}\mathrm{A}_{1} \\
\mathrm{~kg} \cdot \mathrm{m}^{-3} \cdot \mathrm{K}^{-1}\end{array}$ & $\begin{array}{c}\mathrm{A}_{2} \\
\mathrm{~kg} \cdot \mathrm{m}^{-3} \cdot \mathrm{K}^{-2}\end{array}$ & $\begin{array}{c}\mathrm{B}_{0} \\
\mathrm{MPa}\end{array}$ & $\begin{array}{c}\mathrm{B}_{1} \\
\mathrm{MPa} \cdot \mathrm{K}^{-1}\end{array}$ & $\begin{array}{c}\mathrm{B}_{2} \\
\mathrm{MPa} \cdot \mathrm{K}^{-2}\end{array}$ & $\mathrm{C}$ & $\begin{array}{c}\sigma \\
\mathrm{kg} \cdot \mathrm{m}^{-3}\end{array}$ & $\begin{array}{c}M D \\
(\%)\end{array}$ \\
\hline 1025.4 & 0.6509 & $-1.81 \mathrm{E}-03$ & 497.6 & 0.285 & $-1.93 \mathrm{E}-03$ & 0.139 & 0.21 & 0.03 \\
\hline
\end{tabular}




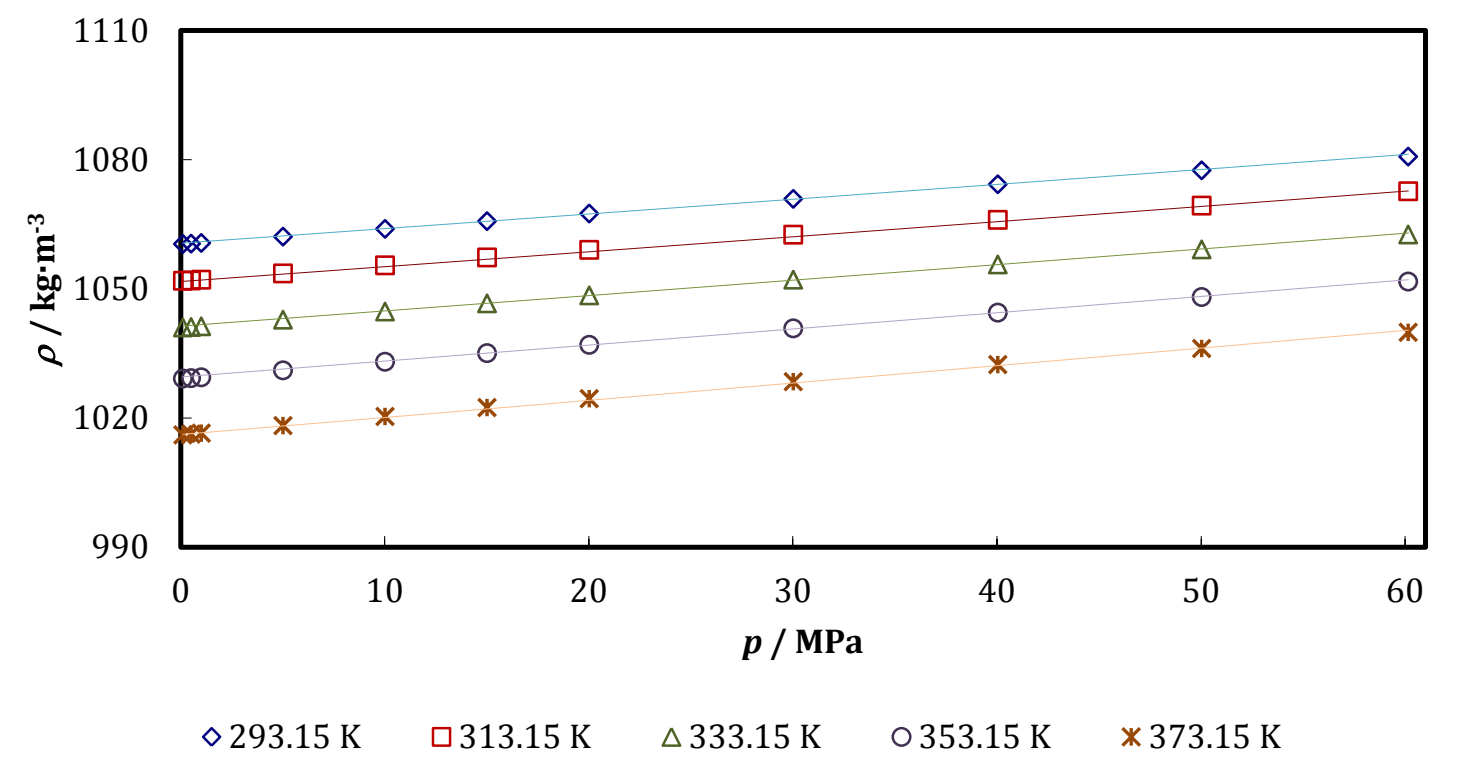

Figura 5.3. Valores experimentales de la densidad para la mezcla $\mathrm{MEA} 30.000 \% w+\mathrm{CO}_{2}$ a $\alpha=0.1999$ a diferentes temperaturas en función de la presión. La línea continua ecuación Tammann-Tait modificada, puntos valores experimentales.

Tabla 5.9. Datos experimentales de densidad, $\rho\left(\mathrm{kg}^{-\mathrm{m}^{-3}}\right)$, para la mezcla $\mathrm{MEA} 30.001 \% \mathrm{w}+\mathrm{CO}_{2}$ a $\alpha=$ 0.4399 .

\begin{tabular}{cccccc}
\hline \multicolumn{5}{c}{$\rho / \mathrm{kg} \cdot \mathrm{m}^{-3}$} \\
\hline \multicolumn{5}{c}{$T / \mathrm{K}$} \\
\hline$p / \mathrm{MPa}$ & 293.15 & 313.15 & 333.15 & 353.15 & 373.15 \\
\hline 0.5 & 1109.1 & 1107.9 & 1104.0 & 1092.0 & 1078.7 \\
1 & 1109.6 & 1108.2 & 1104.2 & 1092.2 & 1079.0 \\
5 & 1111.3 & 1109.9 & 1105.9 & 1093.9 & 1080.8 \\
10 & 1113.5 & 1112.1 & 1107.9 & 1096.0 & 1082.9 \\
15 & 1115.6 & 1114.2 & 1109.9 & 1098.1 & 1085.0 \\
20 & 1117.6 & 1116.2 & 1111.9 & 1100.1 & 1087.1 \\
30 & 1121.5 & 1120.2 & 1115.6 & 1104.0 & 1091.2 \\
40 & 1125.3 & 1124.0 & 1119.3 & 1107.8 & 1095.1 \\
50 & 1129.0 & 1127.7 & 1122.9 & 1111.5 & 1098.9 \\
60 & 1132.5 & 1131.3 & 1126.4 & 1115.0 & 1102.7 \\
\hline
\end{tabular}


Resultado de medidas experimentales de las densidades y solubilidades de $\mathrm{CO}_{2}$ en soluciones acuosas de aminas

Tabla 5.10. Coeficientes y estadística de la ecuación Tammann-Tait modificada para la mezcla MEA $30.001 \% \mathrm{w}+\mathrm{CO}_{2}$ a $\alpha=0.4399, p_{\text {ref }}=0.5 \mathrm{MPa}$.

Parámetros de la ecuación Tammann-Tait modificada

\begin{tabular}{ccccccccc}
\hline $\begin{array}{c}\mathrm{A}_{0} \\
\mathrm{~kg} \cdot \mathrm{m}^{-3}\end{array}$ & $\begin{array}{c}\mathrm{A}_{1} \\
\mathrm{~kg} \cdot \mathrm{m}^{-3} \cdot \mathrm{K}^{-1}\end{array}$ & $\begin{array}{c}\mathrm{A}_{2} \\
\mathrm{~kg} \cdot \mathrm{m}^{-3} \cdot \mathrm{K}^{-2}\end{array}$ & $\begin{array}{c}\mathrm{B}_{0} \\
\mathrm{MPa}\end{array}$ & $\begin{array}{c}\mathrm{B}_{1} \\
\mathrm{MPa} \cdot \mathrm{K}^{-1}\end{array}$ & $\begin{array}{c}\mathrm{B}_{2} \\
\mathrm{MPa} \cdot \mathrm{K}^{-2}\end{array}$ & $\mathrm{C}$ & $\begin{array}{c}\sigma \\
\mathrm{kg} \cdot \mathrm{m}^{-3}\end{array}$ & $\begin{array}{c}M D \\
(\%)\end{array}$ \\
\hline 610.5 & 3.343 & $-5.60 \mathrm{E}-03$ & -471.3 & 4.45 & $-6.9 \mathrm{E}-03$ & 0.0930 & 0.68 & 0.10 \\
\hline
\end{tabular}

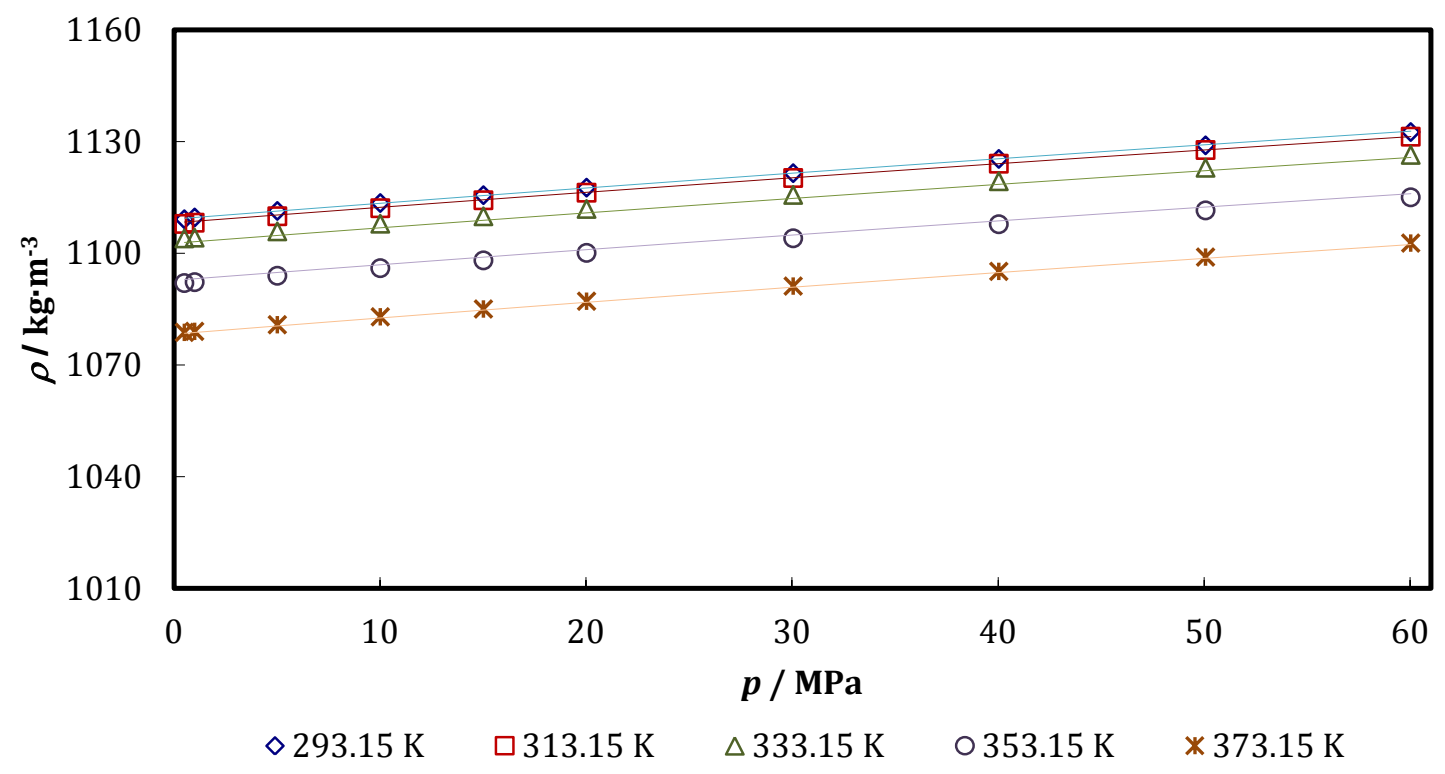

Figura 5.4. Valores experimentales de la densidad para la mezcla $\mathrm{MEA} 30.001 \% w+\mathrm{CO}_{2}$ a $\alpha=0.4399$ a diferentes temperaturas en función de la presión. La línea continua ecuación Tammann-Tait modificada, puntos valores experimentales. 
Resultado de medidas experimentales de las densidades y solubilidades de $\mathrm{CO}_{2}$ en soluciones acuosas de aminas

Tabla 5.11. Datos experimentales de densidad, $\rho\left(\mathrm{kg} \cdot \mathrm{m}^{-3}\right)$, para la mezcla $M E A 30.000 \% \mathrm{w}+\mathrm{CO}_{2}$ a $\alpha=$ 0.6661 .

\begin{tabular}{cccccc}
\hline \multicolumn{5}{c}{$\rho / \mathrm{kg} \cdot \mathrm{m}^{-3}$} \\
\hline \multicolumn{5}{c}{$T / \mathrm{K}$} \\
\hline$p / \mathrm{MPa}$ & 293.15 & 313.15 & 333.15 & 353.15 & 373.15 \\
\hline 0.1 & 1132.3 & 1123.5 & & & \\
0.5 & 1132.3 & 1123.5 & 1113.2 & 1100.8 & \\
1 & 1132.5 & 1123.7 & 1113.4 & 1101.0 & 1086.9 \\
5 & 1133.8 & 1125.0 & 1114.8 & 1102.5 & 1088.5 \\
10 & 1135.4 & 1126.7 & 1116.5 & 1104.3 & 1090.5 \\
15 & 1137.1 & 1128.3 & 1118.2 & 1106.1 & 1092.4 \\
20 & 1138.7 & 1129.9 & 1119.9 & 1107.8 & 1094.3 \\
30 & 1141.8 & 1133.1 & 1123.2 & 1111.3 & 1097.9 \\
40 & 1145.0 & 1136.2 & 1126.4 & 1114.7 & 1101.6 \\
50 & 1147.9 & 1139.3 & 1129.6 & 1118.0 & 1105.1 \\
60 & 1151.0 & 1142.3 & 1132.8 & 1121.3 & 1108.6 \\
\hline
\end{tabular}

Tabla 5.12. Coeficientes y estadística de la ecuación Tammann-Tait modificada para la mezcla MEA $30.000 \% \mathrm{w}+\mathrm{CO}_{2}$ a $\alpha=0.6661, p_{\text {ref }}=1 \mathrm{MPa}$.

Parámetros de la ecuación Tammann-Tait modificada

\begin{tabular}{ccccccccc}
\hline $\begin{array}{c}\mathrm{A}_{0} \\
\mathrm{~kg} \cdot \mathrm{m}^{-3}\end{array}$ & $\begin{array}{c}\mathrm{A}_{1} \\
\mathrm{~kg} \cdot \mathrm{m}^{-3} \cdot \mathrm{K}^{-1}\end{array}$ & $\begin{array}{c}\mathrm{A}_{2} \\
\mathrm{~kg} \cdot \mathrm{m}^{-3} \cdot \mathrm{K}^{-2}\end{array}$ & $\begin{array}{c}\mathrm{B}_{0} \\
\mathrm{MPa}\end{array}$ & $\begin{array}{c}\mathrm{B}_{1} \\
\mathrm{MPa} \cdot \mathrm{K}^{-1}\end{array}$ & $\begin{array}{c}\mathrm{B}_{2} \\
\mathrm{MPa} \cdot \mathrm{K}^{-2}\end{array}$ & $\mathrm{C}$ & $\begin{array}{c}\sigma \\
\mathrm{kg} \cdot \mathrm{m}^{-3}\end{array}$ & $\begin{array}{c}M D \\
(\%)\end{array}$ \\
\hline 1064.8 & 0.860 & $-2.14 \mathrm{E}-03$ & 596.8 & 0.1450 & $-1.93 \mathrm{E}-03$ & 0.1345 & 0.12 & 0.02 \\
\hline
\end{tabular}




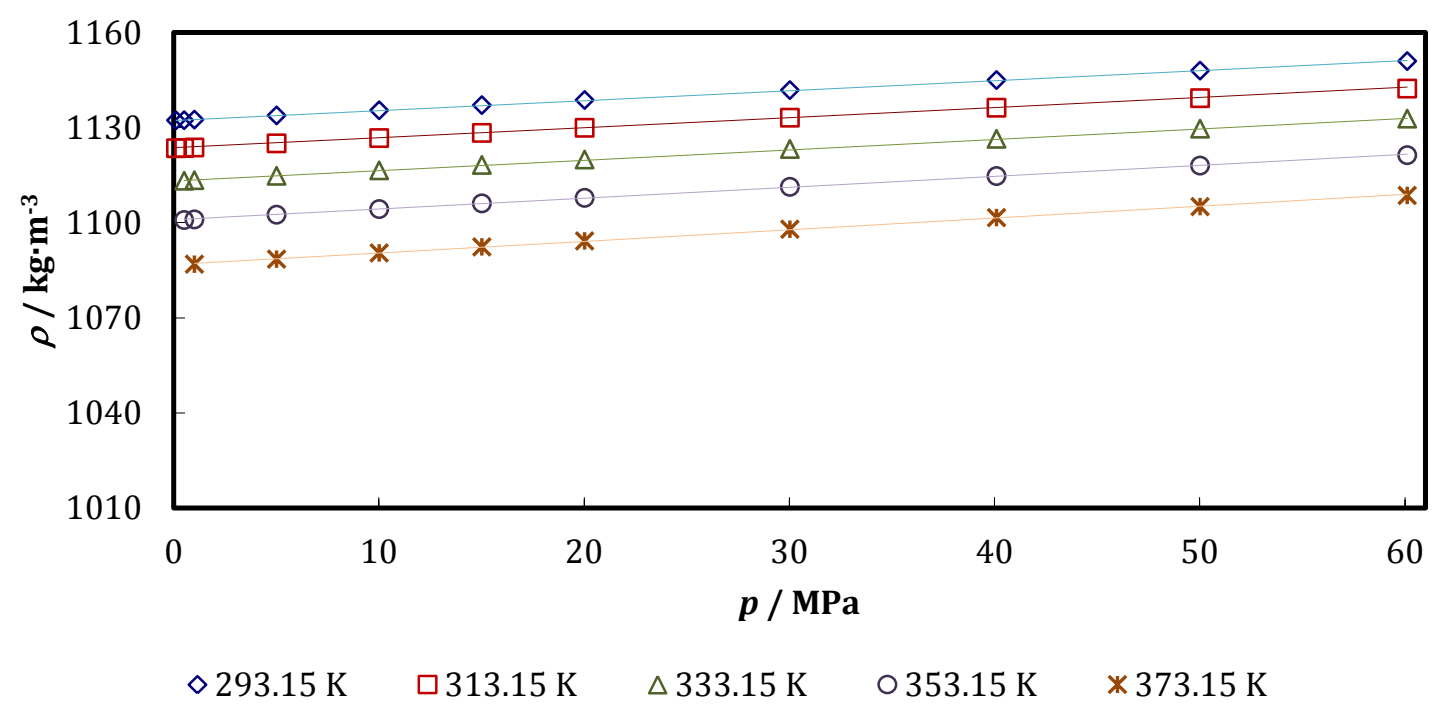

Figura 5.5. Valores experimentales de la densidad para la mezcla $\mathrm{MEA} 30.000 \% w+\mathrm{CO}_{2}$ a $\alpha=0.6661$ a diferentes temperaturas en función de la presión. La línea continua ecuación Tammann-Tait modificada, puntos valores experimentales.

Tabla 5.13. Datos experimentales de densidad, $\rho\left(\mathrm{kg} \cdot \mathrm{m}^{-3}\right)$, para la mezcla $M E A 30.000 \% \mathrm{w}+\mathrm{CO}_{2}$ a $\alpha=$ 0.9997.

\begin{tabular}{cccccc}
\hline \multicolumn{5}{c}{$\rho / \mathrm{kg} \cdot \mathrm{m}^{-3}$} \\
\hline \multicolumn{5}{c}{$T / \mathrm{K}$} \\
\hline$p / \mathrm{MPa}$ & 293.15 & 313.15 & 333.15 & 353.15 & 373.15 \\
\hline 5 & 1142.4 & 1134.7 & 1126.0 & & \\
10 & 1143.8 & 1136.4 & 1127.6 & 1115.4 & \\
15 & 1145.4 & 1137.9 & 1129.3 & 1117.2 & 1102.0 \\
20 & 1147.0 & 1139.5 & 1130.9 & 1119.1 & 1103.3 \\
30 & 1150.1 & 1142.6 & 1134.0 & 1122.7 & 1106.8 \\
40 & 1153.1 & 1145.6 & 1137.1 & 1126.3 & 1110.6 \\
50 & 1156.1 & 1148.5 & 1140.0 & 1129.7 & 1114.3 \\
60 & 1159.0 & 1151.5 & 1143.0 & 1133.1 & 1117.9 \\
\hline
\end{tabular}


Resultado de medidas experimentales de las densidades y solubilidades de $\mathrm{CO}_{2}$ en soluciones acuosas de aminas

Tabla 5.14. Coeficientes y estadística de la ecuación Tammann-Tait modificada para la mezcla MEA $30.000 \% \mathrm{w}+\mathrm{CO}_{2}$ a $\alpha=0.9997, p_{\text {ref }}=15 \mathrm{MPa}$.

Parámetros de la ecuación Tammann-Tait modificada

\begin{tabular}{|c|c|c|c|c|c|c|c|c|}
\hline $\begin{array}{c}\mathrm{A}_{0} \\
\mathrm{~kg} \cdot \mathrm{m}^{-3}\end{array}$ & $\begin{array}{c}\mathrm{A}_{1} \\
\mathrm{~kg} \cdot \mathrm{m}^{-3} \cdot \mathrm{K}^{-1}\end{array}$ & $\begin{array}{c}\quad \mathrm{A}_{2} \\
\mathrm{~kg} \cdot \mathrm{m}^{-3} \cdot \mathrm{K}^{-2}\end{array}$ & $\begin{array}{l}\mathrm{B}_{0} \\
\mathrm{MPa}\end{array}$ & $\begin{array}{c}\mathrm{B}_{1} \\
\mathrm{MPa} \cdot \mathrm{K}^{-1}\end{array}$ & $\begin{array}{c}\mathrm{B}_{2} \\
\mathrm{MPa} \cdot \mathrm{K}^{-2}\end{array}$ & $\mathrm{C}$ & $\begin{array}{c}\sigma \\
\mathrm{kg} \cdot \mathrm{m}^{-3}\end{array}$ & $\begin{array}{l}M D \\
(\%)\end{array}$ \\
\hline 937.1 & 1.691 & $-3.35 \mathrm{E}-03$ & 1257.5 & -0.875 & $-1.93 \mathrm{E}-03$ & 0.222 & 0.53 & 0.09 \\
\hline
\end{tabular}

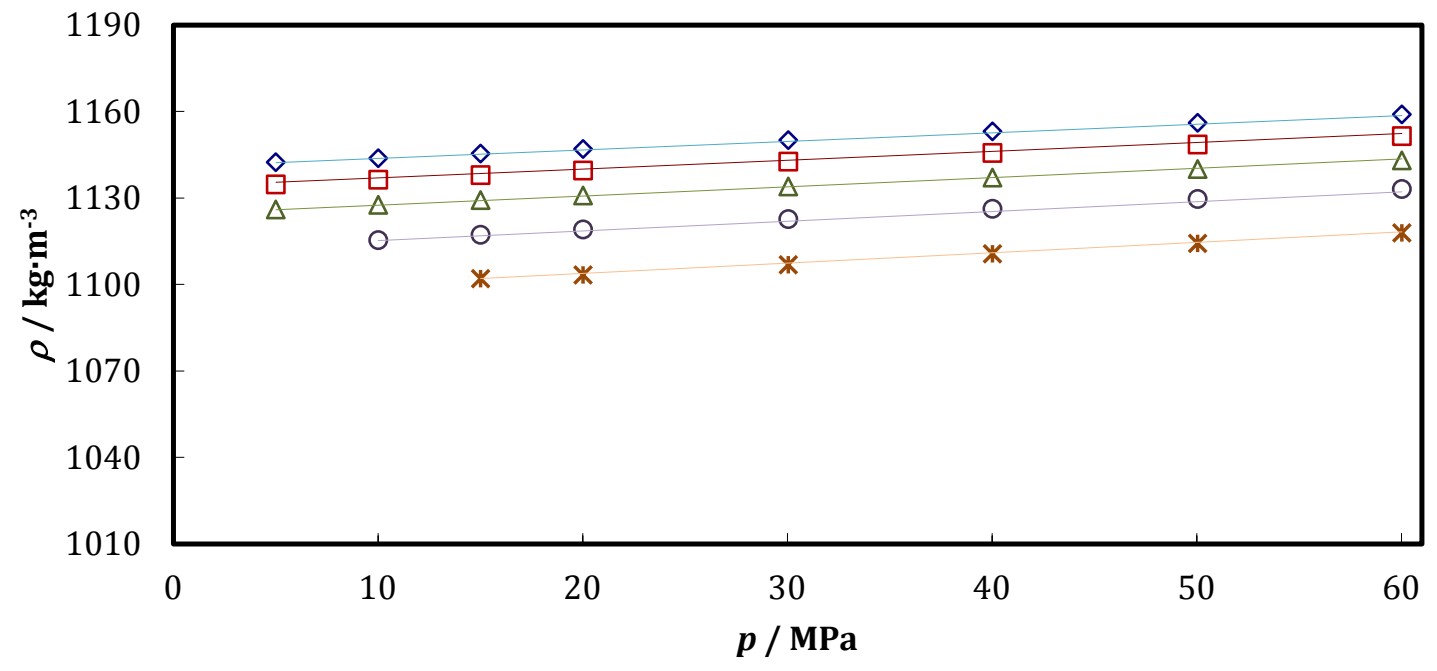

$\diamond 293.15 \mathrm{~K} \quad \square 313.15 \mathrm{~K} \quad \Delta 333.15 \mathrm{~K} \quad 0353.15 \mathrm{~K} \quad * 373.15 \mathrm{~K}$

Figura 5.6. Valores experimentales de la densidad para la mezcla $\mathrm{MEA} 30.000 \% w+\mathrm{CO}_{2}$ a $\alpha=0.9997$ a diferentes temperaturas en función de la presión. La línea continua ecuación Tammann-Tait modificada, puntos valores experimentales.

Tabla 5.15. Parámetros de la ecuación de ajuste de la densidad con $\alpha$ y $p_{\text {total }}$ (4.5) para la mezcla MEA $30.000 \% \mathrm{w}+\mathrm{CO}_{2}$.

\begin{tabular}{cccccc}
\hline \multicolumn{5}{c}{ Parámetros de la ecuación 4.5. } \\
\hline $\begin{array}{c}\text { Temperatura } \\
\mathrm{K}\end{array}$ & $\mathrm{a}$ & $\mathrm{b}$ & $\mathrm{c}$ & $\mathrm{d}$ & $\sigma$ \\
\hline 313.15 & 1148.38 & -15.71 & -0.71 & 0.0345 & 0.76 \\
353.15 & 1118.8 & -7.6 & -2.06 & 0.0377 & 1.95 \\
\hline
\end{tabular}


Los resultados de la densidad obtenidos para las mezclas de MEA 30\%w $+\mathrm{CO}_{2}$ se compararon con los encontrados en la literatura [9], [10] y [11]. Para la comparación de los datos experimentales a las presiones y concentraciones de este trabajo, se interpolaron los datos de densidad a las concentraciones de la literatura.

En la figura 5.7 se han representado las desviaciones relativas obtenidas entre los datos experimentales y los de la literatura a $313.15 \mathrm{~K}$. La desviación media obtenida (AAD\%) para la mezcla a $313.15 \mathrm{~K}$ fue de $1.08 \%$ con [9], $1.57 \%$ con [10] y $1.24 \%$ con [11].

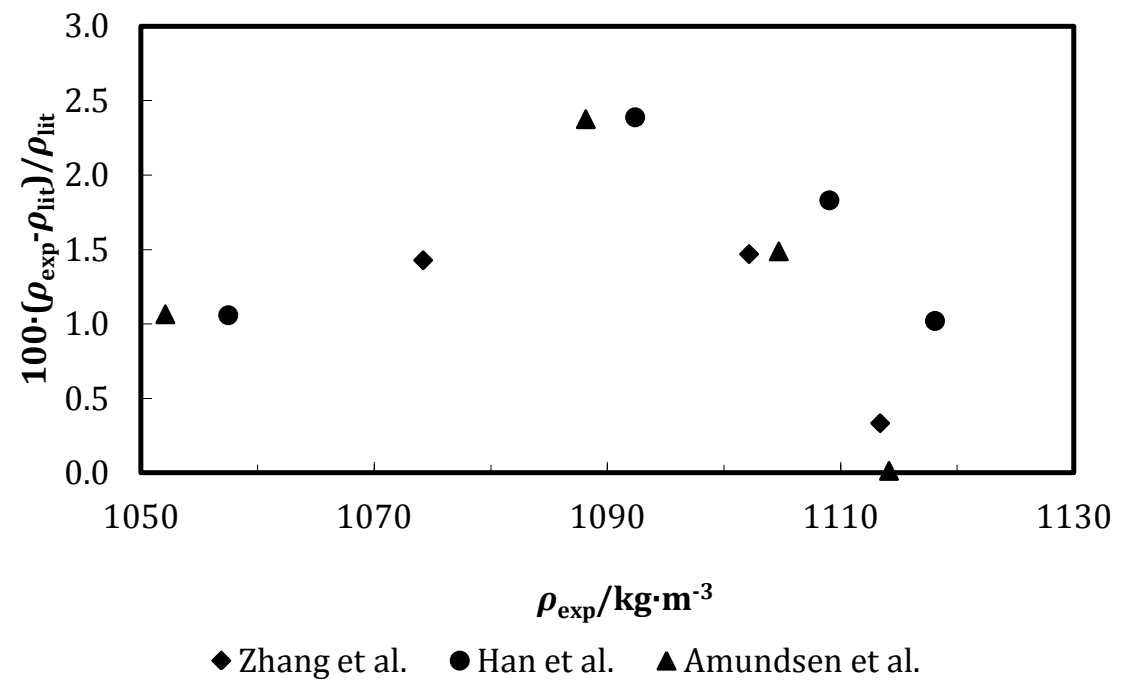

Figura 5.7. Desviación relativa porcentual de la $\rho$ experimental a $313.15 \mathrm{~K}$ respecto a los datos de la bibliografía de Zhang et al. [9], Han et al. [10] y Amundsen et al. [11].

En la figura 5.8 se han representado las desviaciones relativas obtenidas entre los datos experimentales y los de la literatura a $333.15 \mathrm{~K}$. La desviación media obtenida ( $A A D \%$ ) para la mezcla a $313.15 \mathrm{~K}$ fue de $1.95 \%$ con [9], $1.99 \%$ con [10] y $1.64 \%$ con [11].

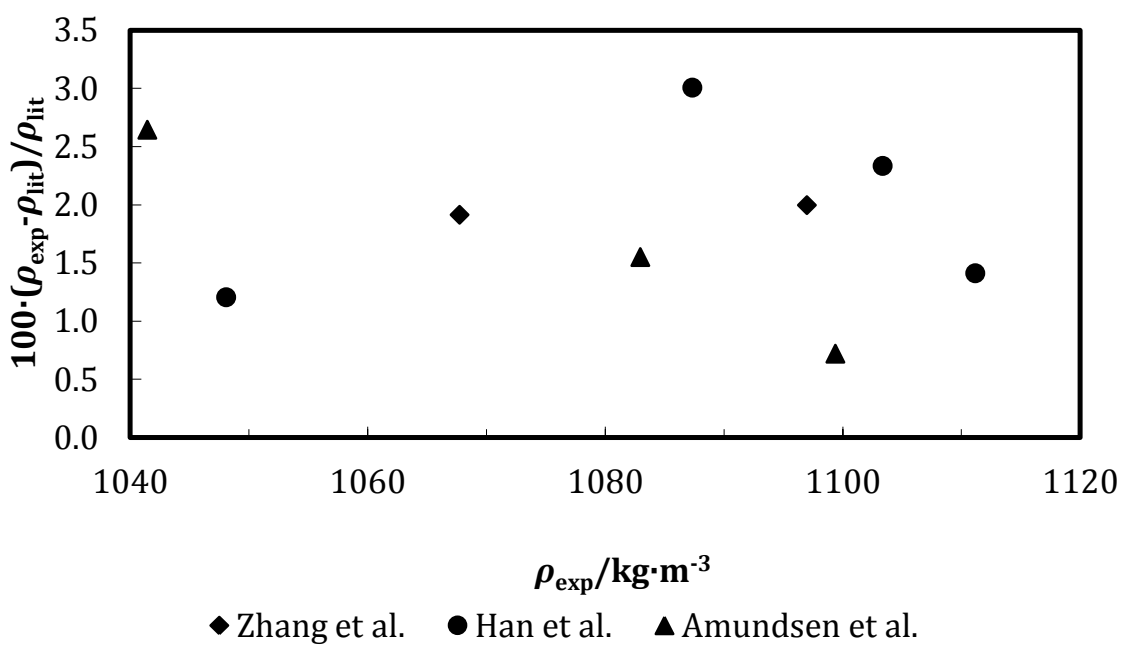

Figura 5.8. Desviación relativa porcentual de la $\rho$ experimental a $333.15 \mathrm{~K}$ respecto a los datos de la bibliografía de Zhang et al. [9], Han et al. [10] y Amundsen et al. [11]. 
Resultado de medidas experimentales de las densidades y solubilidades de $\mathrm{CO}_{2}$ en soluciones acuosas de aminas

En la figura 5.9 se han representado las desviaciones relativas obtenidas entre los datos experimentales y los de la literatura a $353.15 \mathrm{~K}$. La desviación media obtenida (AAD\%) para la mezcla a $313.15 \mathrm{~K}$ fue de $1.96 \%$ con [9], $2.05 \%$ con [10] y $2.02 \%$ con [11].

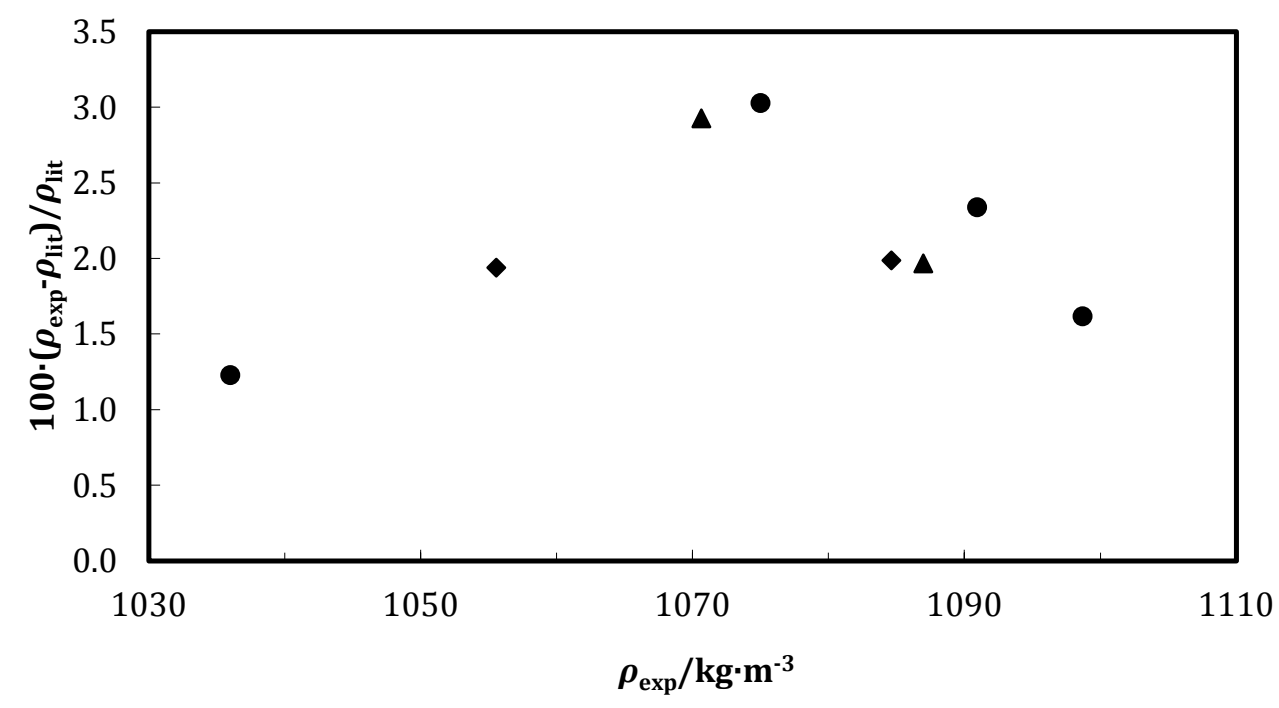

- Zhang et al. • Han et al. $\Delta$ Amundsen et al.

Figura 5.9. Desviación relativa porcentual de la $\rho$ experimental a $353.15 \mathrm{~K}$ respecto a los datos de la bibliografía de Zhang et al. [9], Han et al. [10] y Amundsen et al. [11]. 
Resultado de medidas experimentales de las densidades y solubilidades de $\mathrm{CO}_{2}$ en soluciones acuosas de aminas

\subsubsection{Sistema DMAE $30 \% \mathrm{w}+\mathrm{CO}_{2}$.}

Tabla 5.16. Datos experimentales de densidad, $\rho\left(\mathrm{kg} \cdot \mathrm{m}^{-3}\right)$, para la mezcla DMAE $29.978 \% \mathrm{w}+\mathrm{CO}_{2}$ a $\alpha=$ 0.3002 .

\begin{tabular}{cccc}
\hline \multicolumn{4}{c}{$\rho / \mathrm{kg} \cdot \mathrm{m}^{-3}$} \\
\hline \multicolumn{4}{c}{$T / \mathrm{K}$} \\
\hline$p / \mathrm{MPa}$ & 313.15 & 333.15 & 353.15 \\
\hline 0.5 & 1041.98 & 1029.17 & 1015.03 \\
1 & 1042.25 & 1029.42 & 1015.41 \\
5 & 1044.07 & 1031.36 & 1017.39 \\
10 & 1046.38 & 1033.68 & 1019.75 \\
15 & 1048.58 & 1035.97 & 1022.11 \\
20 & 1050.74 & 1038.22 & 1024.40 \\
30 & 1054.99 & 1042.53 & 1028.84 \\
40 & 1059.01 & 1046.62 & 1033.03 \\
50 & 1062.84 & 1050.61 & 1037.09 \\
60 & 1066.60 & 1054.49 & 1041.04 \\
\hline
\end{tabular}

Tabla 5.17. Coeficientes y estadística de la ecuación Tammann-Tait modificada para la mezcla DMAE $29.978 \% \mathrm{w}+\mathrm{CO}_{2}$ a $\alpha=0.3002, p_{\text {ref }}=0.5 \mathrm{MPa}$.

Parámetros de la ecuación Tammann-Tait modificada

\begin{tabular}{ccccccccc}
\hline $\begin{array}{c}\mathrm{A}_{0} \\
\mathrm{~kg} \cdot \mathrm{m}^{-3}\end{array}$ & $\begin{array}{c}\mathrm{A}_{1} \\
\mathrm{~kg} \cdot \mathrm{m}^{-3} \cdot \mathrm{K}^{-1}\end{array}$ & $\begin{array}{c}\mathrm{A}_{2} \\
\mathrm{~kg} \cdot \mathrm{m}^{-3} \cdot \mathrm{K}^{-2}\end{array}$ & $\begin{array}{c}\mathrm{B}_{0} \\
\mathrm{MPa}\end{array}$ & $\begin{array}{c}\mathrm{B}_{1} \\
\mathrm{MPa} \cdot \mathrm{K}^{-1}\end{array}$ & $\begin{array}{c}\mathrm{B}_{2} \\
\mathrm{MPa} \cdot \mathrm{K}^{-2}\end{array}$ & $\mathrm{C}$ & $\begin{array}{c}\sigma \\
\mathrm{kg} \cdot \mathrm{m}^{-3}\end{array}$ & $\begin{array}{c}M D \\
(\%)\end{array}$ \\
\hline 1068.0 & 0.4387 & $-1.67 \mathrm{E}-03$ & 80.6 & 0.920 & $-1.94 \mathrm{E}-03$ & 0.0807 & 0.04 & 0.009 \\
\hline
\end{tabular}




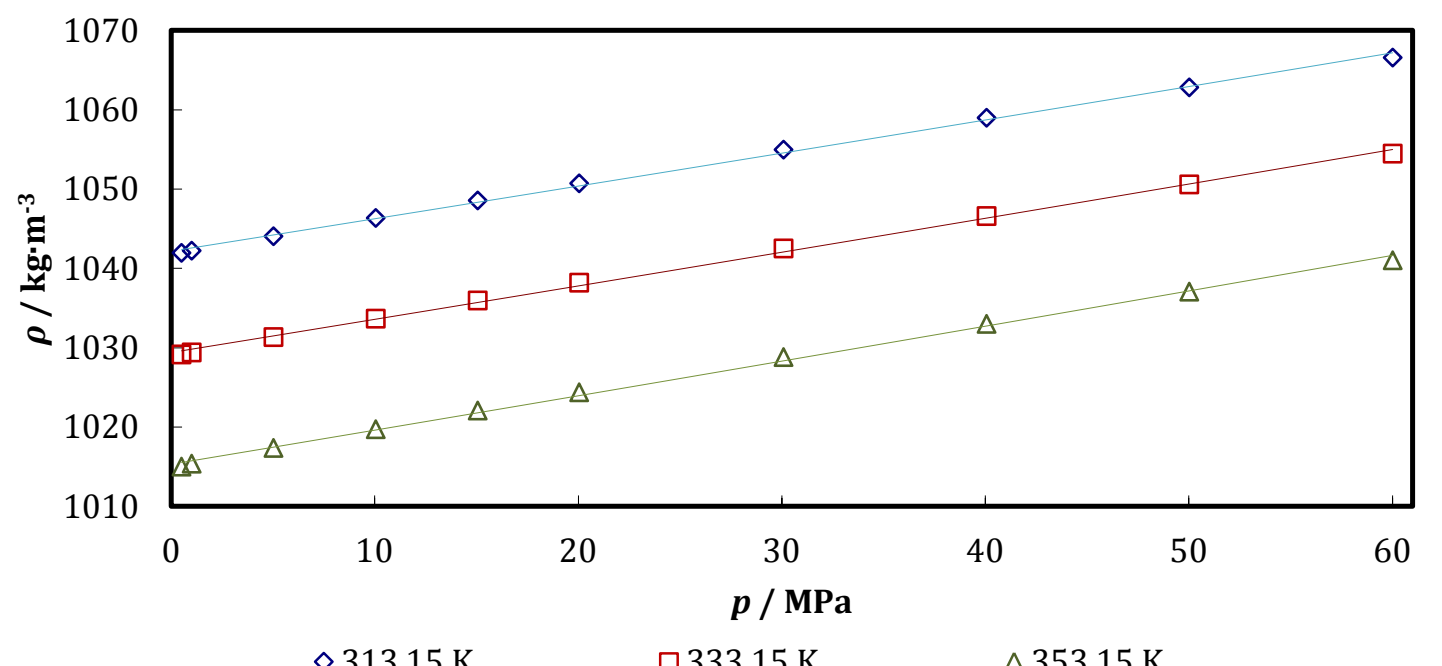

Figura 5.10. Valores experimentales de la densidad para la mezcla DMAE $29.978 \% w+\mathrm{CO}_{2}$ a $\alpha=$ 0.3002 a diferentes temperaturas en función de la presión. La línea continua ecuación Tammann-Tait modificada, puntos valores experimentales.

Tabla 5.18. Datos experimentales de densidad, $\rho\left(\mathrm{kg} \cdot \mathrm{m}^{-3}\right)$, para la mezcla DMAE $29.978 \% \mathrm{w}+\mathrm{CO}_{2}$ a $\alpha=$ 0.7001 .

\begin{tabular}{cccc}
\hline \multicolumn{4}{c}{$\rho\left(\mathrm{kg} / \mathrm{m}^{3}\right)$} \\
\hline \multicolumn{4}{c}{$T / \mathrm{K}$} \\
\hline$p / \mathrm{MPa}$ & 313.15 & 333.15 & 353.15 \\
\hline 5 & 1082.69 & 1071.73 & 1059.07 \\
10 & 1084.69 & 1073.79 & 1061.28 \\
15 & 1086.59 & 1075.76 & 1063.36 \\
20 & 1088.45 & 1077.69 & 1065.29 \\
30 & 1092.00 & 1081.43 & 1069.24 \\
40 & 1095.51 & 1084.99 & 1072.99 \\
50 & 1098.90 & 1088.51 & 1076.65 \\
60 & 1102.23 & 1091.96 & 1080.22 \\
\hline
\end{tabular}


Resultado de medidas experimentales de las densidades y solubilidades de $\mathrm{CO}_{2}$ en soluciones acuosas de aminas

Tabla 5.19. Coeficientes y estadística de la ecuación Tammann-Tait modificada para la mezcla DMAE $29.978 \% \mathrm{w}+\mathrm{CO}_{2}$ a $\alpha=0.7001, p_{\text {ref }}=5 \mathrm{MPa}$.

Parámetros de la ecuación Tammann-Tait modificada

\begin{tabular}{|c|c|c|c|c|c|c|c|c|}
\hline $\mathrm{A}_{0}$ & $\mathrm{~A}_{1}$ & $\mathrm{~A}_{2}$ & $\mathrm{~B}_{0}$ & $\mathrm{~B}_{1}$ & $\mathrm{~B}_{2}$ & $\mathrm{C}$ & $\sigma$ & $M D$ \\
\hline $\mathrm{kg} \cdot \mathrm{m}^{-3}$ & $\mathrm{~kg} \cdot \mathrm{m}^{-3} \cdot \mathrm{K}^{-1}$ & $\mathrm{~kg} \cdot \mathrm{m}^{-3} \cdot \mathrm{K}^{-2}$ & $\mathrm{MPa}$ & $\mathrm{MPa} \cdot \mathrm{K}^{-1}$ & $\mathrm{MPa} \cdot \mathrm{K}^{-2}$ & & $\mathrm{~kg} \cdot \mathrm{m}^{-3}$ & $(\%)$ \\
\hline 1031.6 & 0.8310 & $-2.13 \mathrm{E}-03$ & 198.4 & 0.690 & $-1.94 \mathrm{E}-03$ & 0.0822 & 0.04 & 0.006 \\
\hline
\end{tabular}

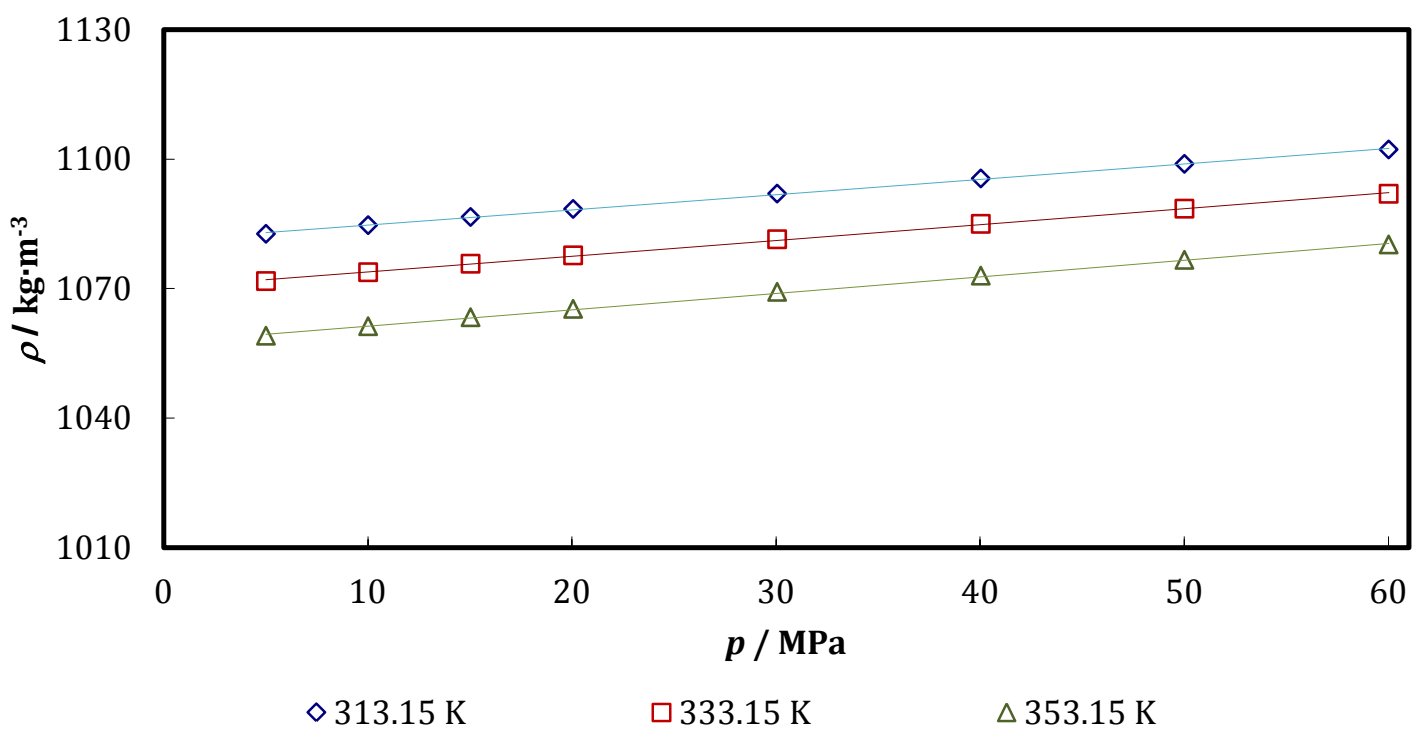

Figura 5.11. Valores experimentales de la densidad para la mezcla DMAE 29.978\%w+CO $a \alpha=$ 0.7001 a diferentes temperaturas en función de la presión. La línea continua ecuación Tammann-Tait modificada, puntos valores experimentales. 
Resultado de medidas experimentales de las densidades y solubilidades de $\mathrm{CO}_{2}$ en soluciones acuosas de aminas

Tabla 5.20. Datos experimentales de densidad, $\rho\left(\mathrm{kg} \cdot \mathrm{m}^{-3}\right)$, para la mezcla DMAE $29.978 \% \mathrm{w}+\mathrm{CO}_{2}$ a $\alpha=$ 1.1001.

\begin{tabular}{cccc}
\hline \multicolumn{4}{c}{$\rho / \mathrm{kg} \cdot \mathrm{m}^{-3}$} \\
\hline \multicolumn{4}{c}{$T / \mathrm{K}$} \\
\hline$p / \mathrm{MPa}$ & 313.15 & 333.15 & 353.15 \\
\hline 5 & 1101.54 & 1090.41 & 1077.44 \\
10 & 1103.28 & 1092.21 & 1079.23 \\
15 & 1104.90 & 1093.87 & 1081.18 \\
20 & 1106.46 & 1095.55 & 1082.95 \\
30 & 1109.51 & 1098.71 & 1086.36 \\
40 & 1112.58 & 1101.89 & 1089.77 \\
50 & 1115.54 & 1105.02 & 1093.12 \\
60 & 1118.48 & 1108.04 & 1096.32 \\
\hline
\end{tabular}

Tabla 5.21. Coeficientes y estadística de la ecuación Tammann-Tait modificada para la mezcla DMAE $29.978 \% w+\mathrm{CO}_{2} a \alpha=1.1001, p_{\text {ref }}=5 \mathrm{MPa}$.

Parámetros de la ecuación Tammann-Tait modificada

\begin{tabular}{|c|c|c|c|c|c|c|c|c|}
\hline$A_{0}$ & $A_{1}$ & $\mathrm{~A}_{2}$ & $\mathrm{~B}_{0}$ & $\mathrm{~B}_{1}$ & $\mathrm{~B}_{2}$ & $\mathrm{C}$ & $\sigma$ & $M D$ \\
\hline $\mathrm{kg} \cdot \mathrm{m}^{-3}$ & $\mathrm{~kg} \cdot \mathrm{m}^{-3} \cdot \mathrm{K}^{-1}$ & $\mathrm{~kg} \cdot \mathrm{m}^{-3} \cdot \mathrm{K}^{-2}$ & $\mathrm{MPa}$ & $\mathrm{MPa} \cdot \mathrm{K}^{-1}$ & $\mathrm{MPa} \cdot \mathrm{K}^{-2}$ & & $\mathrm{~kg} \cdot \mathrm{m}^{-3}$ & $(\%)$ \\
\hline 1036.2 & 0.929 & $-2.30 \mathrm{E}-03$ & 335.8 & 0.406 & $-2.00 \mathrm{E}-03$ & 0.0812 & 0.07 & 0.01 \\
\hline
\end{tabular}




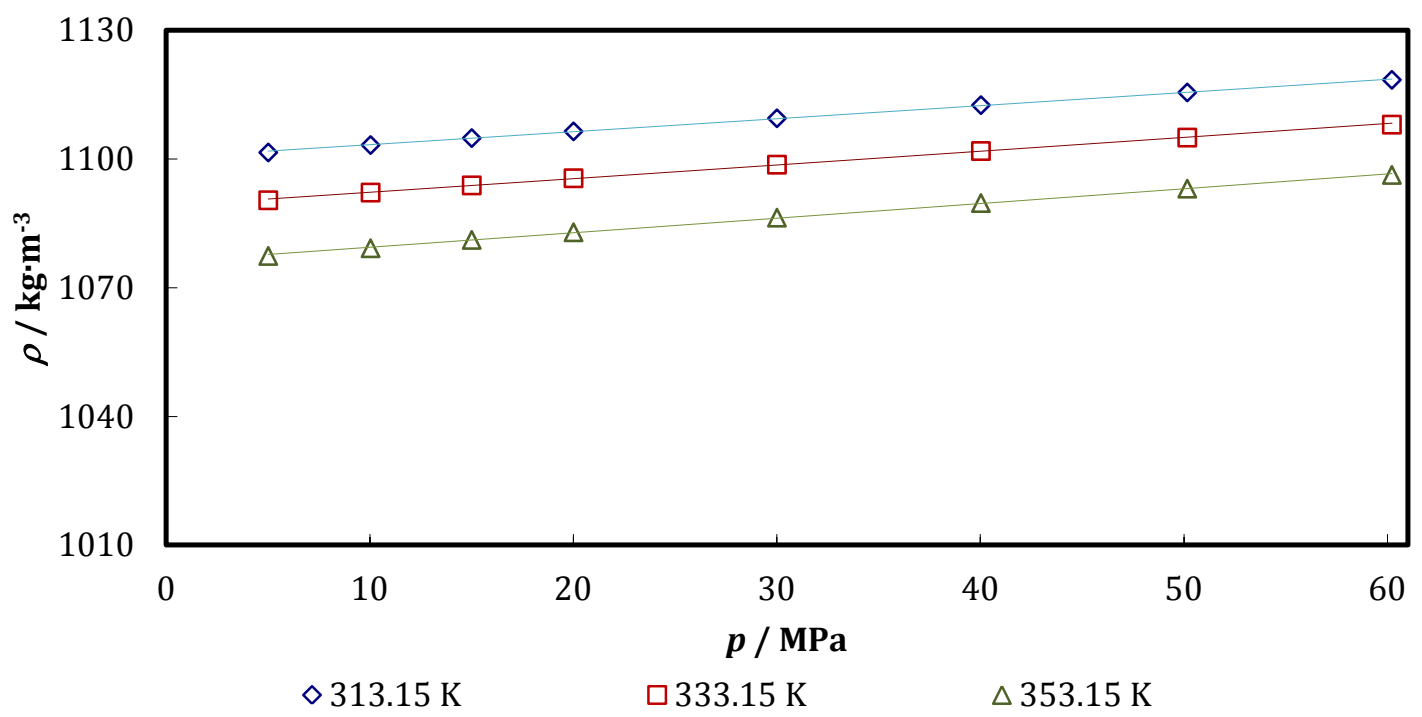

Figura 5.12. Valores experimentales de la densidad para la mezcla DMAE $29.978 \% w+\mathrm{CO}_{2}$ a $\alpha=$ 1.1001 a diferentes temperaturas en función de la presión. La línea continua ecuación Tammann-Tait modificada, puntos valores experimentales.

Tabla 5.22. Datos experimentales de densidad, $\rho\left(\mathrm{kg} \cdot \mathrm{m}^{-3}\right)$, para la mezcla DMAE $29.978 \% \mathrm{w}+\mathrm{CO}_{2}$ a $\alpha=$ 1.5008.

\begin{tabular}{cccc}
\hline \multicolumn{4}{c}{$\rho / \mathrm{kg} \cdot \mathrm{m}^{-3}$} \\
\hline \multicolumn{4}{c}{$T / \mathrm{K}$} \\
\hline$p / \mathrm{MPa}$ & 313.15 & 333.15 & 353.15 \\
\hline 10 & 1105.6 & 1094.4 & 1078.9 \\
15 & 1107.2 & 1095.7 & 1081.5 \\
20 & 1108.8 & 1097.1 & 1083.0 \\
30 & 1111.8 & 1100.0 & 1086.2 \\
40 & 1114.8 & 1102.9 & 1089.4 \\
50 & 1117.7 & 1105.9 & 1092.6 \\
60 & 1120.5 & 1108.7 & 1095.7 \\
\hline
\end{tabular}


Resultado de medidas experimentales de las densidades y solubilidades de $\mathrm{CO}_{2}$ en soluciones acuosas de aminas

Tabla 5.23. Coeficientes y estadística de la ecuación Tammann-Tait modificada para la mezcla DMAE $29.978 \% \mathrm{w}+\mathrm{CO}_{2}$ a $\alpha=1.5008, p_{\text {ref }}=10 \mathrm{MPa}$.

Parámetros de la ecuación Tammann-Tait modificada

\begin{tabular}{|c|c|c|c|c|c|c|c|c|}
\hline $\mathrm{A}_{0}$ & $\mathrm{~A}_{1}$ & $\mathrm{~A}_{2}$ & $\mathrm{~B}_{0}$ & $\mathrm{~B}_{1}$ & $\mathrm{~B}_{2}$ & $\mathrm{C}$ & $\sigma$ & $M D$ \\
\hline $\mathrm{kg} \cdot \mathrm{m}^{-3}$ & $\mathrm{~kg} \cdot \mathrm{m}^{-3} \cdot \mathrm{K}^{-1}$ & $\mathrm{~kg} \cdot \mathrm{m}^{-3} \cdot \mathrm{K}^{-2}$ & $\mathrm{MPa}$ & $\mathrm{MPa} \cdot \mathrm{K}^{-1}$ & $\mathrm{MPa} \cdot \mathrm{K}^{-2}$ & & $\mathrm{~kg} \cdot \mathrm{m}^{-3}$ & $(\%)$ \\
\hline 969.8 & 1.402 & $-3.09 \mathrm{E}-03$ & 313.8 & 0.481 & $-2.00 \mathrm{E}-03$ & 0.0783 & 0.30 & 0.05 \\
\hline
\end{tabular}

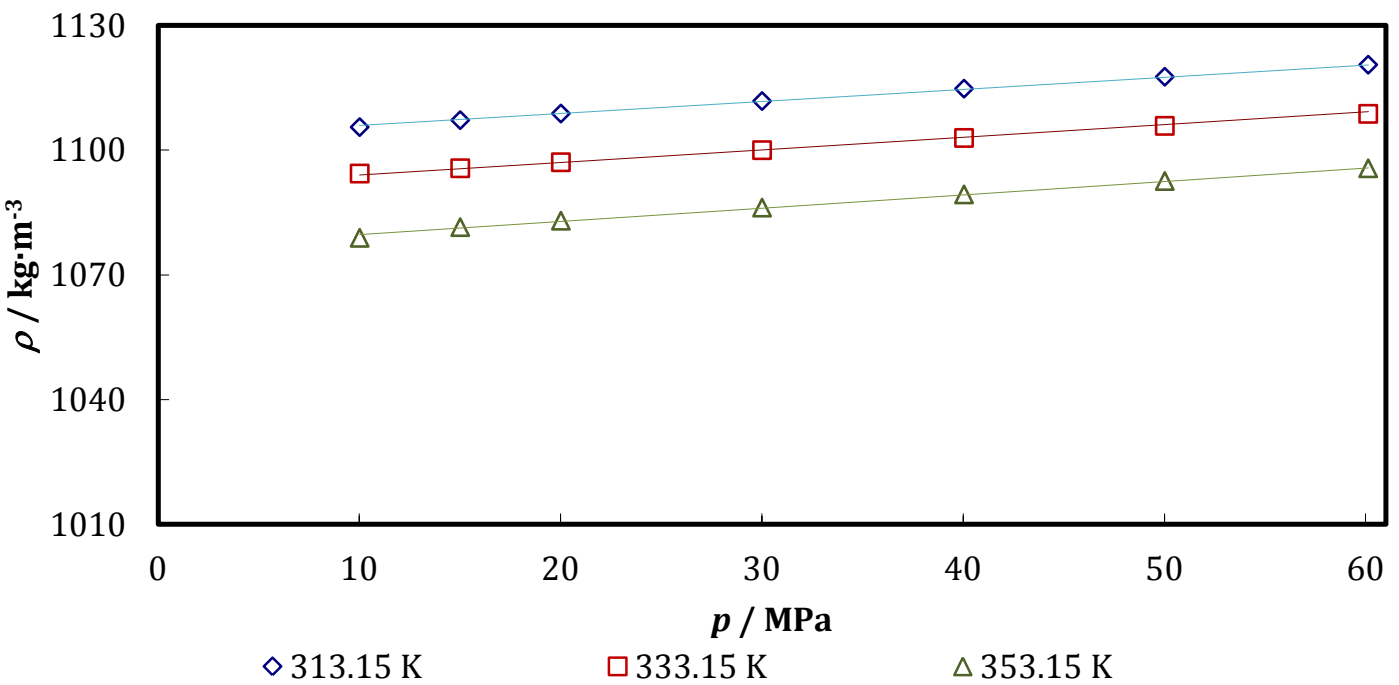

Figura 5.13. Valores experimentales de la densidad para la mezcla DMAE 29.978\% $\mathrm{w}+\mathrm{CO}_{2}$ a $\alpha=$ 1.5008 a diferentes temperaturas en función de la presión. La línea continua ecuación Tammann-Tait modificada, puntos valores experimentales.

Tabla 5.24. Parámetros de la ecuación de ajuste de la densidad con $\alpha$ y $p_{\text {total }}$ (4.5) para la mezcla DMAE 29.978\%w+ $\mathrm{CO}_{2}$.

\begin{tabular}{|c|c|c|c|c|c|}
\hline \multicolumn{6}{|c|}{ Parámetros de la ecuación 4.5.} \\
\hline Temperatura & $\mathrm{a}$ & $\mathrm{b}$ & $\mathrm{c}$ & $\mathrm{d}$ & $\sigma$ \\
\hline $\mathrm{K}$ & $\mathrm{kg} \cdot \mathrm{m}^{-3}$ & $\mathrm{~kg} \cdot \mathrm{m}^{-3}$ & $\mathrm{~kg} \cdot \mathrm{m}^{-3}$ & $\mathrm{~kg} \cdot \mathrm{m}^{-3} \cdot \mathrm{bar}^{-1}$ & $\mathrm{~kg} \cdot \mathrm{m}^{-3}$ \\
\hline 313.15 & 1123.5 & -32.3 & 2.49 & 0.0349 & 2.11 \\
\hline 353.15 & 1093.5 & -24.8 & -0.51 & 0.0377 & 2.57 \\
\hline
\end{tabular}


Resultado de medidas experimentales de las densidades y solubilidades de $\mathrm{CO}_{2}$ en soluciones acuosas de aminas

\subsubsection{Sistema TEA $30 \% \mathrm{w}+\mathrm{CO}_{2}$.}

Tabla 5.25. Datos experimentales de densidad, $\rho\left(\mathrm{kg} \cdot \mathrm{m}^{-3}\right)$, para la mezcla TEA $29.999 \% \mathrm{w}+\mathrm{CO}_{2}$ a $\alpha=$ 0.3006 .

\begin{tabular}{cccc}
\hline \multicolumn{4}{c}{$\rho / \mathrm{kg} \cdot \mathrm{m}^{-3}$} \\
\hline \multicolumn{4}{c}{$T / \mathrm{K}$} \\
\hline$p / \mathrm{MPa}$ & 313.15 & 333.15 & 353.15 \\
\hline 1 & 1074.61 & 1064.86 & 1051.87 \\
5 & 1076.40 & 1066.70 & 1053.87 \\
10 & 1078.59 & 1068.95 & 1056.13 \\
15 & 1080.73 & 1071.13 & 1058.37 \\
20 & 1082.87 & 1073.28 & 1060.62 \\
30 & 1086.95 & 1077.42 & 1064.91 \\
40 & 1090.85 & 1081.36 & 1069.05 \\
50 & 1094.63 & 1085.24 & 1073.03 \\
60 & 1098.34 & 1088.97 & 1077.00 \\
\hline
\end{tabular}

Tabla 5.26. Coeficientes y estadística de la ecuación Tammann-Tait modificada para la mezcla TEA $29.999 \% w+\mathrm{CO}_{2}$ a $\alpha=0.3006, p_{\text {ref }}=1 \mathrm{MPa}$.

Parámetros de la ecuación Tammann-Tait modificada

\begin{tabular}{ccccccccc}
\hline $\begin{array}{c}\mathrm{A}_{0} \\
\mathrm{~kg} \cdot \mathrm{m}^{-3}\end{array}$ & $\begin{array}{c}\mathrm{A}_{1} \\
\mathrm{~kg} \cdot \mathrm{m}^{-3} \cdot \mathrm{K}^{-1}\end{array}$ & $\begin{array}{c}\mathrm{A}_{2} \\
\mathrm{~kg} \cdot \mathrm{m}^{-3} \cdot \mathrm{K}^{-2}\end{array}$ & $\begin{array}{c}\mathrm{B}_{0} \\
\mathrm{MPa}\end{array}$ & $\begin{array}{c}\mathrm{B}_{1} \\
\mathrm{MPa} \cdot \mathrm{K}^{-1}\end{array}$ & $\begin{array}{c}\mathrm{B}_{2} \\
\mathrm{MPa} \cdot \mathrm{K}^{-2}\end{array}$ & $\mathrm{C}$ & $\begin{array}{c}\sigma \\
\mathrm{kg} \cdot \mathrm{m}^{-3}\end{array}$ & $\begin{array}{c}M D \\
(\%)\end{array}$ \\
\hline 828.1 & 1.989 & $-3.84 \mathrm{E}-03$ & 108.4 & 0.890 & $-1.94 \mathrm{E}-03$ & 0.0825 & 0.05 & 0.01 \\
\hline
\end{tabular}




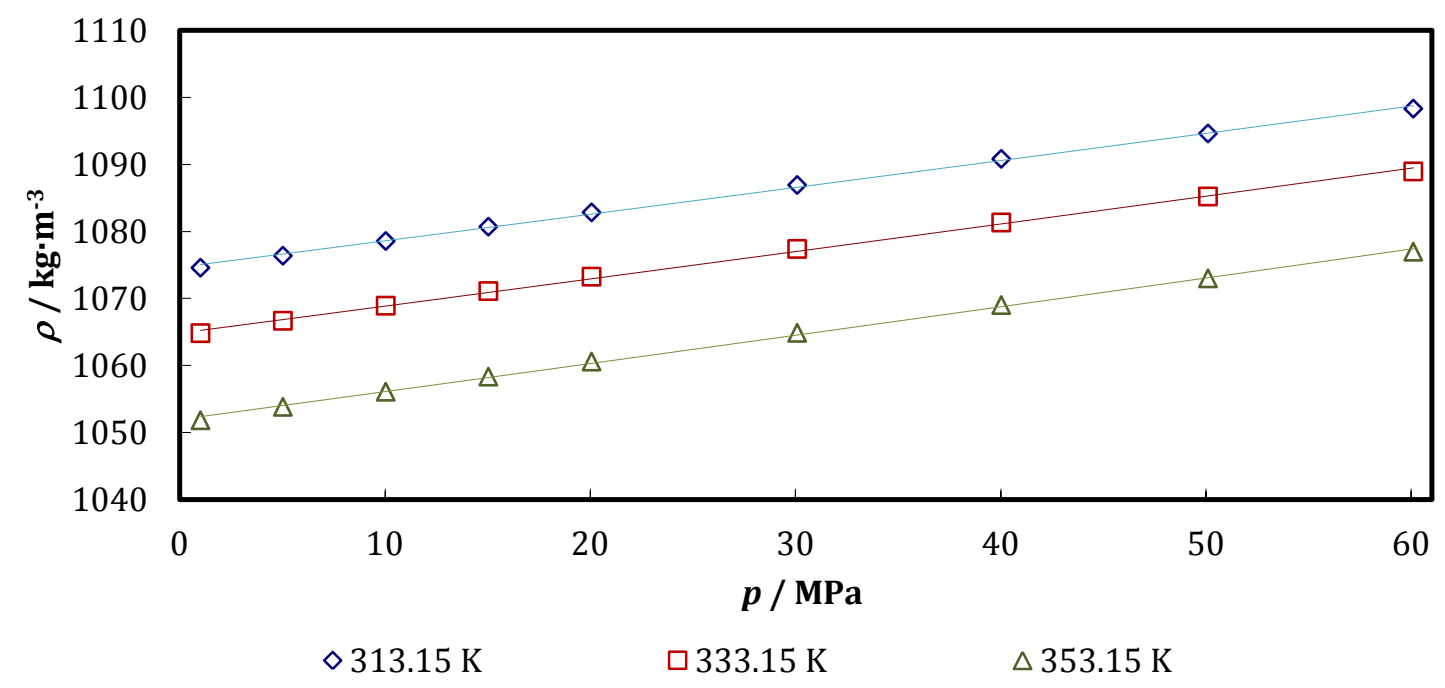

Figura 5.14. Valores experimentales de la densidad para la mezcla TEA 29.999\%w+ $\mathrm{CO}_{2}$ a $\alpha=0.3006$ a diferentes temperaturas en función de la presión. La línea continua ecuación Tammann-Tait modificada, puntos valores experimentales.

Tabla 5.27. Datos experimentales de densidad, $\rho\left(\mathrm{kg} \cdot \mathrm{m}^{-3}\right)$, para la mezcla TEA $29.999 \% \mathrm{w}+\mathrm{CO}_{2}$ a $\alpha=$ 0.6011 .

\begin{tabular}{cccc}
\hline \multicolumn{4}{c}{$\rho / \mathrm{kg} \cdot \mathrm{m}^{-3}$} \\
\hline \multicolumn{4}{c}{$T / \mathrm{K}$} \\
\hline$p / \mathrm{MPa}$ & 313.15 & 333.15 & 353.15 \\
\hline 5 & 1092.26 & 1082.37 & 1068.22 \\
10 & 1094.30 & 1084.51 & 1070.53 \\
15 & 1096.30 & 1086.61 & 1072.73 \\
20 & 1098.20 & 1088.58 & 1074.91 \\
30 & 1101.96 & 1092.57 & 1079.15 \\
40 & 1105.65 & 1096.34 & 1083.24 \\
50 & 1109.22 & 1100.08 & 1087.20 \\
60 & 1112.73 & 1103.74 & 1091.07 \\
\hline
\end{tabular}


Resultado de medidas experimentales de las densidades y solubilidades de $\mathrm{CO}_{2}$ en soluciones acuosas de aminas

Tabla 5.28. Coeficientes y estadística de la ecuación Tammann-Tait modificada para la mezcla TEA $29.999 \% w+\mathrm{CO}_{2}$ a $\alpha=0.6011, p_{\text {ref }}=5 \mathrm{MPa}$.

Parámetros de la ecuación Tammann-Tait modificada

\begin{tabular}{ccccccccc}
\hline $\mathrm{A}_{0}$ & $\mathrm{~A}_{1}$ & $\mathrm{~A}_{2}$ & $\mathrm{~B}_{0}$ & $\mathrm{~B}_{1}$ & $\mathrm{~B}_{2}$ & $\mathrm{C}$ & $\sigma$ & $M D$ \\
$\mathrm{~kg} \cdot \mathrm{m}^{-3}$ & $\mathrm{~kg} \cdot \mathrm{m}^{-3} \cdot \mathrm{K}^{-1}$ & $\mathrm{~kg} \cdot \mathrm{m}^{-3} \cdot \mathrm{K}^{-2}$ & $\mathrm{MPa}$ & $\mathrm{MPa} \cdot \mathrm{K}^{-1}$ & $\mathrm{MPa} \cdot \mathrm{K}^{-2}$ & & $\mathrm{~kg} \cdot \mathrm{m}^{-3}$ & \begin{tabular}{c}
$(\%)$ \\
\hline 720.1
\end{tabular} \\
\hline 2.776 & $-5.07 \mathrm{E}-03$ & 284.0 & 0.462 & $-1.94 \mathrm{E}-03$ & 0.0898 & 0.05 & 0.009 \\
\hline
\end{tabular}

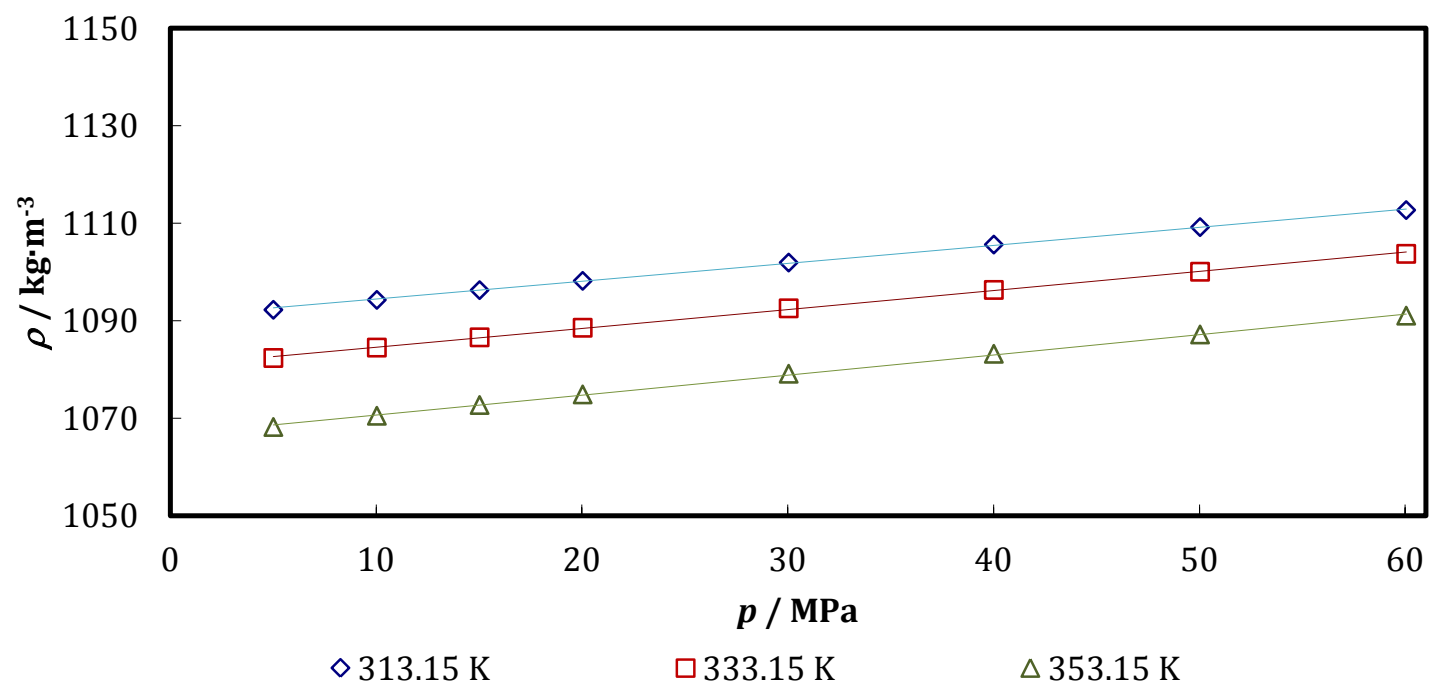

Figura 5.15. Valores experimentales de la densidad para la mezcla TEA $29.999 \% w+\mathrm{CO}_{2}$ a $\alpha=0.6011$ a diferentes temperaturas en función de la presión. La línea continua ecuación Tammann-Tait modificada, puntos valores experimentales. 
Resultado de medidas experimentales de las densidades y solubilidades de $\mathrm{CO}_{2}$ en soluciones acuosas de aminas

Tabla 5.29. Datos experimentales de densidad, $\rho\left(\mathrm{kg}^{-3} \mathrm{~m}^{-3}\right)$, para la mezcla TEA $29.999 \% \mathrm{w}+\mathrm{CO}_{2}$ a $\alpha=$ 0.8982 .

\begin{tabular}{cccc}
\hline \multicolumn{4}{c}{$\rho / \mathrm{kg} \cdot \mathrm{m}^{-3}$} \\
\hline \multicolumn{4}{c}{$T / \mathrm{K}$} \\
\hline$p / \mathrm{MPa}$ & 313.15 & 333.15 & 353.15 \\
\hline 5 & 1106.13 & 1093.87 & 1078.53 \\
10 & 1108.12 & 1095.85 & 1080.82 \\
15 & 1109.94 & 1097.82 & 1083.01 \\
20 & 1111.71 & 1099.78 & 1085.13 \\
30 & 1115.24 & 1103.55 & 1089.39 \\
40 & 1118.70 & 1107.16 & 1093.44 \\
50 & 1122.11 & 1110.76 & 1097.30 \\
60 & 1125.41 & 1114.29 & 1101.14 \\
\hline
\end{tabular}

Tabla 5.30. Coeficientes y estadística de la ecuación Tammann-Tait modificada para la mezcla TEA $29.999 \% w+\mathrm{CO}_{2} a \alpha=0.8982, p_{\text {ref }}=5 \mathrm{MPa}$.

Parámetros de la ecuación Tammann-Tait modificada

\begin{tabular}{ccccccccc}
\hline $\begin{array}{c}\mathrm{A}_{0} \\
\mathrm{~kg} \cdot \mathrm{m}^{-3}\end{array}$ & $\begin{array}{c}\mathrm{A}_{1} \\
\mathrm{~kg} \cdot \mathrm{m}^{-3} \cdot \mathrm{K}^{-1}\end{array}$ & $\begin{array}{c}\mathrm{A}_{2} \\
\mathrm{~kg} \cdot \mathrm{m}^{-3} \cdot \mathrm{K}^{-2}\end{array}$ & $\begin{array}{c}\mathrm{B}_{0} \\
\mathrm{MPa}\end{array}$ & $\begin{array}{c}\mathrm{B}_{1} \\
\mathrm{MPa} \cdot \mathrm{K}^{-1}\end{array}$ & $\begin{array}{c}\mathrm{B}_{2} \\
\mathrm{MPa} \cdot \mathrm{K}^{-2}\end{array}$ & $\begin{array}{c}\mathrm{C} \\
\mathrm{kg} \cdot \mathrm{m}^{-3}\end{array}$ & $\begin{array}{c}M D \\
(\%)\end{array}$ \\
\hline 935.3 & 1.643 & $-3.50 \mathrm{E}-03$ & 434.2 & 0.0497 & $-1.94 \mathrm{E}-03$ & 0.0898 & 0.08 & 0.01 \\
\hline
\end{tabular}




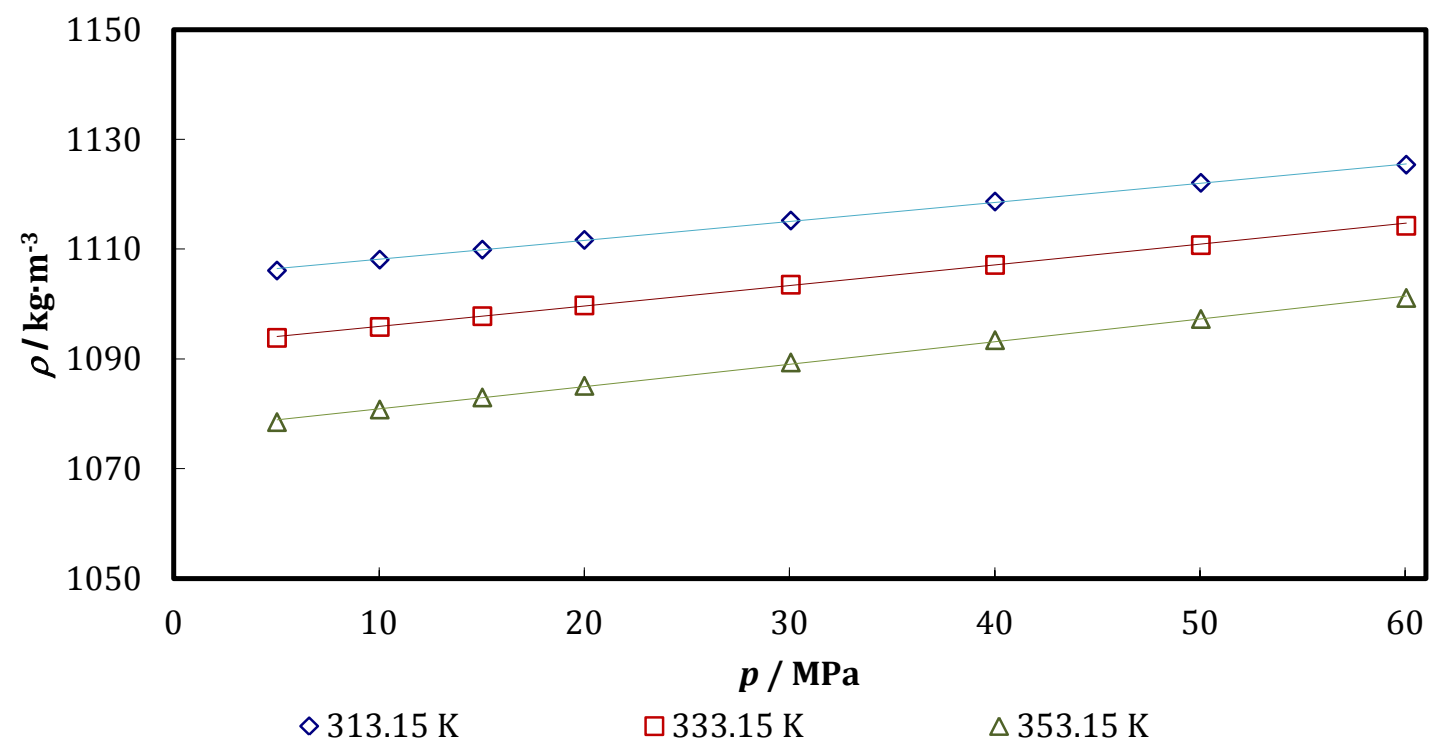

Figura 5.16. Valores experimentales de la densidad para la mezcla TEA $29.999 \% w+\mathrm{CO}_{2}$ a $\alpha=0.8982$ a diferentes temperaturas en función de la presión. La línea continua ecuación Tammann-Tait modificada, puntos valores experimentales.

Tabla 5.31. Datos experimentales de densidad, $\rho\left(\mathrm{kg} \cdot \mathrm{m}^{-3}\right)$, para la mezcla TEA $30.000 \% \mathrm{w}+\mathrm{CO}_{2}$ a $\alpha=$ 1.2010.

\begin{tabular}{cccc}
\hline \multicolumn{4}{c}{$\rho / \mathrm{kg} \cdot \mathrm{m}^{-3}$} \\
\hline \multicolumn{4}{c}{$T / \mathrm{K}$} \\
\hline$p / \mathrm{MPa}$ & 313.15 & 333.15 & 353.15 \\
\hline 5 & 1116.4 & 1103.6 & \\
10 & 1118.3 & 1105.6 & 1088.4 \\
15 & 1120.1 & 1107.5 & 1090.9 \\
20 & 1121.8 & 1109.4 & 1093.0 \\
30 & 1125.2 & 1113.0 & 1097.1 \\
40 & 1128.5 & 1116.6 & 1101.1 \\
50 & 1131.8 & 1120.1 & 1105.0 \\
60 & 1135.0 & 1123.4 & 1108.7 \\
\hline
\end{tabular}


Resultado de medidas experimentales de las densidades y solubilidades de $\mathrm{CO}_{2}$ en soluciones acuosas de aminas

Tabla 5.32. Coeficientes y estadística de la ecuación Tammann-Tait modificada para la mezcla TEA $30.000 \% \mathrm{w}+\mathrm{CO}_{2}$ a $\alpha=1.2010, p_{\text {ref }}=10 \mathrm{MPa}$.

Parámetros de la ecuación Tammann-Tait modificada

\begin{tabular}{ccccccccc}
\hline $\begin{array}{c}\mathrm{A}_{0} \\
\mathrm{~kg} \cdot \mathrm{m}^{-3}\end{array}$ & $\begin{array}{c}\mathrm{A}_{1} \\
\mathrm{~kg} \cdot \mathrm{m}^{-3} \cdot \mathrm{K}^{-1}\end{array}$ & $\begin{array}{c}\mathrm{A}_{2} \\
\mathrm{~kg} \cdot \mathrm{m}^{-3} \cdot \mathrm{K}^{-2}\end{array}$ & $\begin{array}{c}\mathrm{B}_{0} \\
\mathrm{MPa}\end{array}$ & $\begin{array}{c}\mathrm{B}_{1} \\
\mathrm{MPa} \cdot \mathrm{K}^{-1}\end{array}$ & $\begin{array}{c}\mathrm{B}_{2} \\
\mathrm{MPa} \cdot \mathrm{K}^{-2}\end{array}$ & $\mathrm{C}$ & $\begin{array}{c}\sigma \\
\mathrm{kg} \cdot \mathrm{m}^{-3}\end{array}$ & $\begin{array}{c}M D \\
(\%)\end{array}$ \\
\hline 802.8 & 2.559 & $-4.95 \mathrm{E}-03$ & 455.4 & -0.0530 & $-1.94 \mathrm{E}-03$ & 0.0827 & 0.11 & 0.02 \\
\hline
\end{tabular}

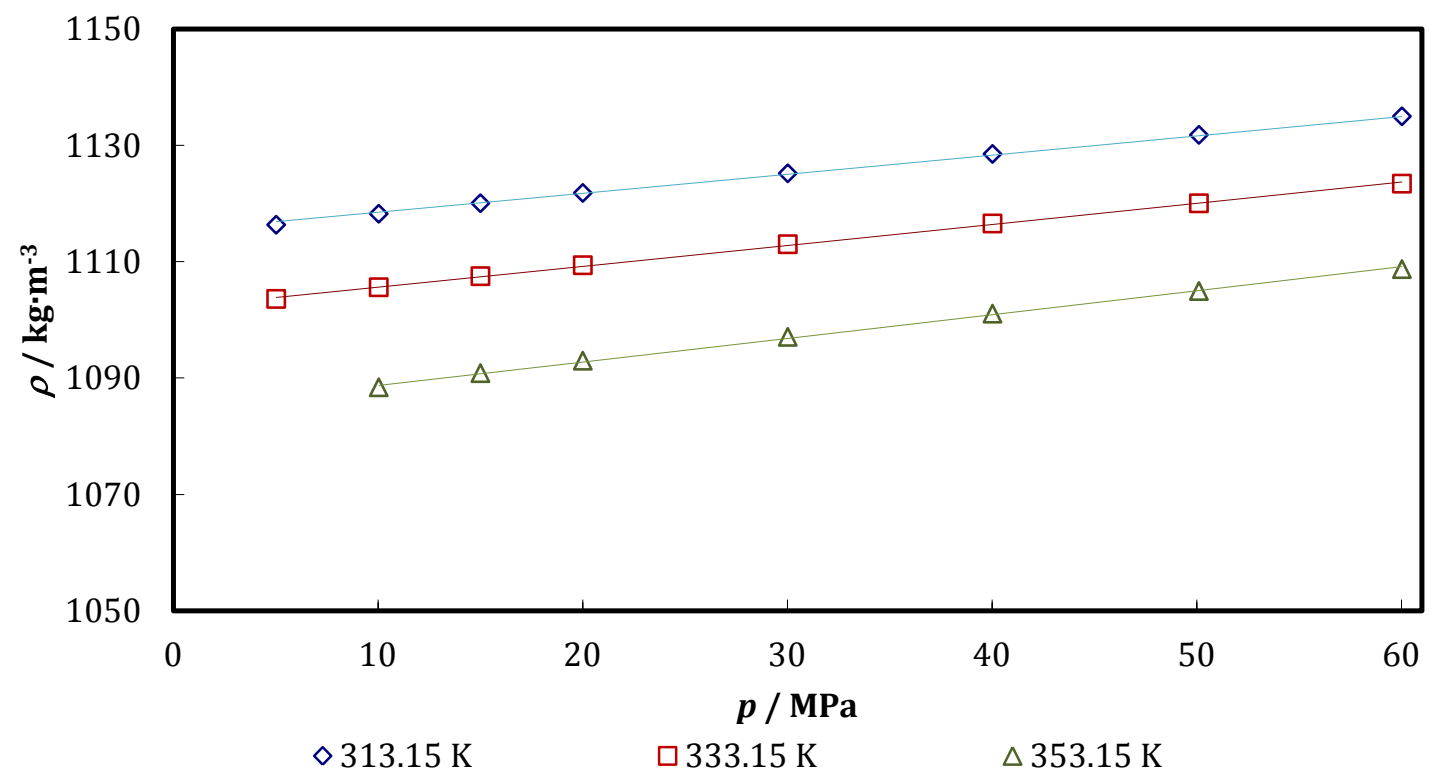

Figura 5.17. Valores experimentales de la densidad para la mezcla TEA $30.000 \% w+\mathrm{CO}_{2}$ a $\alpha=1.2010$ a diferentes temperaturas en función de la presión. La línea continua ecuación Tammann-Tait modificada, puntos valores experimentales.

Tabla 5.33. Parámetros de la ecuación de ajuste de la densidad con $\alpha$ y ptotal (4.5) para la mezcla TEA $30.000 \% \mathrm{w}+\mathrm{CO}_{2}$.

\begin{tabular}{cccccc}
\hline \multicolumn{5}{c}{ Parámetros de la ecuación 4.5. } \\
\hline $\begin{array}{c}\text { Temperatura } \\
\mathrm{K}\end{array}$ & $\mathrm{a}$ & $\mathrm{b}$ & $\mathrm{c}$ & $\mathrm{d}$ & $\sigma$ \\
\hline 313.15 & 1147.52 & -46.91 & 7.59 & 0.0367 & 0.66 \\
353.15 & 1107.28 & -32.72 & 4.85 & 0.0417 & 0.31 \\
\hline
\end{tabular}




\subsection{Discusión de resultados de la densidad experimental.}

En este trabajo se determinaron las densidades de tres mezclas ternarias de soluciones acuosas de aminas con $\mathrm{CO}_{2}$ realizadas en un rango de temperatura de $293.15 \mathrm{~K}$ a $373.15 \mathrm{~K}$ para las mezclas con MEA. Siendo el rango de temperatura de $313.15 \mathrm{~K}$ a $353.15 \mathrm{~K}$ para las mezclas con DMAE y TEA. Todas ellas a rango de presiones de $0.1 \mathrm{MPa}$ hasta $60 \mathrm{MPa}$.

Los ajustes con la ecuación Tammann-Tait modificada fueron buenos teniendo en cuenta la complejidad de las mezclas. Esto es debido a la reacción del $\mathrm{CO}_{2}$ con la amina, necesitando tiempos muy largos hasta alcanzar el equilibrio. La desviación máxima porcentual $(M D \%)$ es inferior al $0.12 \%$ en todos los casos y las desviaciones estándar $(\sigma)$ varían entre $0.03 \mathrm{~kg} \cdot \mathrm{m}^{-3}$ y $0.70 \mathrm{~kg} \cdot \mathrm{m}^{-3}$ para el conjunto de las mezclas estudiadas.

El mejor ajuste de la ecuación Tammann-Tait modificada presenta una desviación estándar de $0.03 \mathrm{~kg} \cdot \mathrm{m}^{-3} \mathrm{y}$ una máxima desviación porcentual de $0.004 \%$ para el sistema compuesto por MEA $10 \% \mathrm{w}+\mathrm{CO}_{2}$ a $\alpha=0.8$.

Los valores experimentales obtenidos muestran una tendencia creciente de la densidad con la presión y decreciente al aumentar la temperatura. En cuanto a la concentración de $\mathrm{CO}_{2}$, la densidad siempre aumenta a medida que aumenta la concentración para todas las mezclas.

La densidad también aumenta en función de la amina, a las mismas condiciones de presión, temperatura y concentración de $\mathrm{CO}_{2}$.

Comparando las diferentes aminas a una presión, temperatura y composición de $\mathrm{CO}_{2}$, la mayor densidad la presenta la MEA:

$$
\rho_{D M A E 30 \% W}<\rho_{\text {TEA } 30 \% W}<\rho_{M E A 30 \% W}
$$

Se observa para todas las temperaturas que la densidad obtenida es mayor a los valores de la bibliografía. Por ello se realizó un estudio de la estabilidad de las medidas con el tiempo para determinar el final de la reacción. Se observó que no se alcanza el equilibrio en tiempos inferiores a 24 horas, al contrario que en otros trabajos, en los cuales el tiempo de espera fue inferior a 6 horas. En el ejemplo que se muestra en la figura 5.18, se ve como a la espera de las 42 horas la diferencia respecto a otros autores se reduce a la mitad.

En el ejemplo se observa la variación de la densidad con el tiempo para una mezcla de MEA al 30\%w y con una concentración de 0.2 moles de $\mathrm{CO}_{2}$ por mol de MEA, tomándose el valor del periodo en tiempos de 0.5 horas. Se observa que al cabo de $35.5 \mathrm{~h}$ aún no se había alcanzado el equilibrio en la reacción, dado que todavía sigue aumentando la densidad. En nuestro caso se estableció como criterio que, la pendiente entre medidas tomadas en intervalos de 5 horas sea inferior al $10 \%$ de la incertidumbre de medida del equipo para $k=1$, llegando a un valor de incertidumbre inferior a $0.36 \mathrm{~kg} / \mathrm{m}^{3}$ para tiempos de espera comprendidos entre 24 y 48 horas, dependiendo del tipo de amina, concentración en $\mathrm{CO}_{2}$ y temperatura de trabajo. 


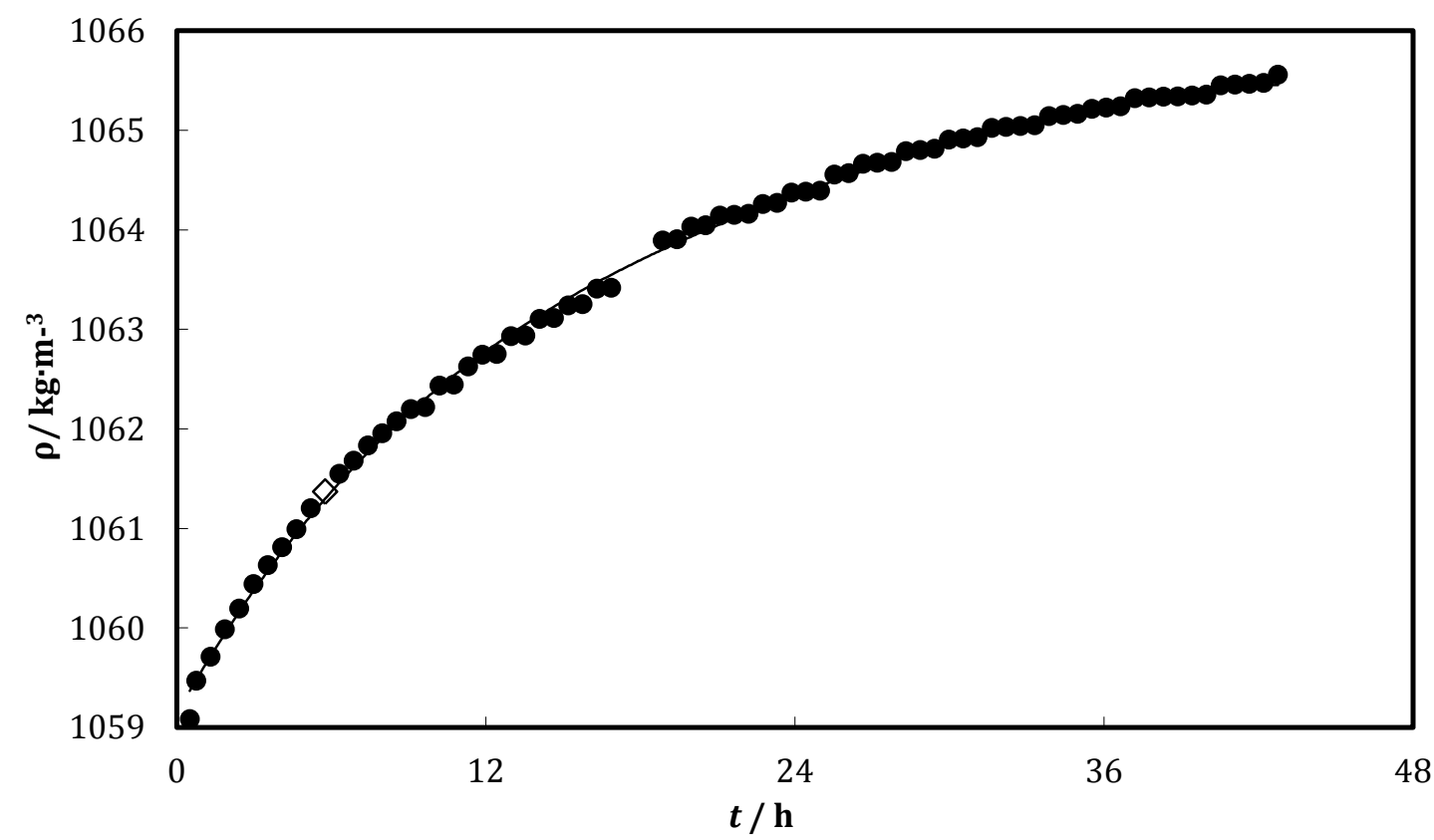

Figura 5.18. Representación de la densidad en función del tiempo para la mezcla MEA 30\%w y 0.2 moles de $\mathrm{CO}_{2}$ por mol de MEA a $313.15 \mathrm{Ky} 50$ bar. $(\diamond)$ Representa la densidad medida para un tiempo cercano a las 6 horas.

\subsection{Presentación de los resultados experimentales de las medidas de solubilidad.}

A continuación, se muestran los datos experimentales de solubilidad obtenidos para las mezclas estudiadas, tanto en forma de tablas, como en gráficas. También se presentan los resultados del ajuste de los datos experimentales a la ecuación de Xu y Rochelle calculados por el método descrito en el apartado 5.3. Este ajuste se realizó para cada sistema estudiado, en función de la temperatura y el índice de carga de $\mathrm{CO}_{2}(\alpha)$. Además, se muestra la desviación estándar $\sigma$ (ecuación 5.6) entre datos experimentales y el modelo utilizado. 
Resultado de medidas experimentales de las densidades y solubilidades de $\mathrm{CO}_{2}$ en soluciones acuosas de aminas

\subsubsection{Sistema MEA $10 \% \mathrm{w}+\mathrm{CO}_{2}$ a $313.15 \mathrm{~K}$.}

Tabla 5.34. Datos experimentales de solubilidad para la mezcla $\mathrm{MEA} 10.000 \% \mathrm{w}+\mathrm{CO}_{2}$ a $313.15 \mathrm{~K}$.

\begin{tabular}{cc}
\hline \multicolumn{2}{c}{$T / \mathrm{K}$} \\
\hline$p_{\mathrm{CO}_{2}} / \mathrm{kPa}$ & $\alpha_{\mathrm{CO}_{2}}$ \\
\hline 30.310 & 0.877 \\
953.9 & 1.021 \\
1552.5 & 1.111 \\
2012.4 & 1.193 \\
2540.7 & 1.255 \\
3069.8 & 1.321 \\
3564.9 & 1.382 \\
4050.3 & 1.440 \\
4497.8 & 1.496 \\
4998.4 & 1.547 \\
5866.7 & 1.674 \\
\hline
\end{tabular}

Tabla 5.35. Coeficientes y estadística de la ecuación de Xu y Rochelle para la mezcla MEA 10.000\%w $+\mathrm{CO}_{2}$ a $313.15 \mathrm{~K}$.

Parámetros de la ecuación Xu y Rochelle

\begin{tabular}{ccccccc}
\hline $\mathrm{A}_{1}$ & $\mathrm{~A}_{2}$ & $\mathrm{~A}_{3}$ & $\mathrm{~A}_{4}$ & $\mathrm{~A}_{5}$ & $\mathrm{~A}_{6}$ & $\sigma$ \\
$\mathrm{kg} \cdot \mathrm{m}^{-3}$ & $\mathrm{~kg} \cdot \mathrm{m}^{-3} \cdot \mathrm{K}^{-1}$ & $\mathrm{~kg} \cdot \mathrm{m}^{-3} \cdot \mathrm{K}^{-2}$ & $\mathrm{MPa}$ & $\mathrm{MPa} \cdot \mathrm{K}^{-1}$ & $\mathrm{MPa} \cdot \mathrm{K}^{-2}$ & \\
\hline 0.989 & 1308.28 & 1.019 & 0.993 & 4067.06 & -1613.06 & 0.09 \\
\hline
\end{tabular}




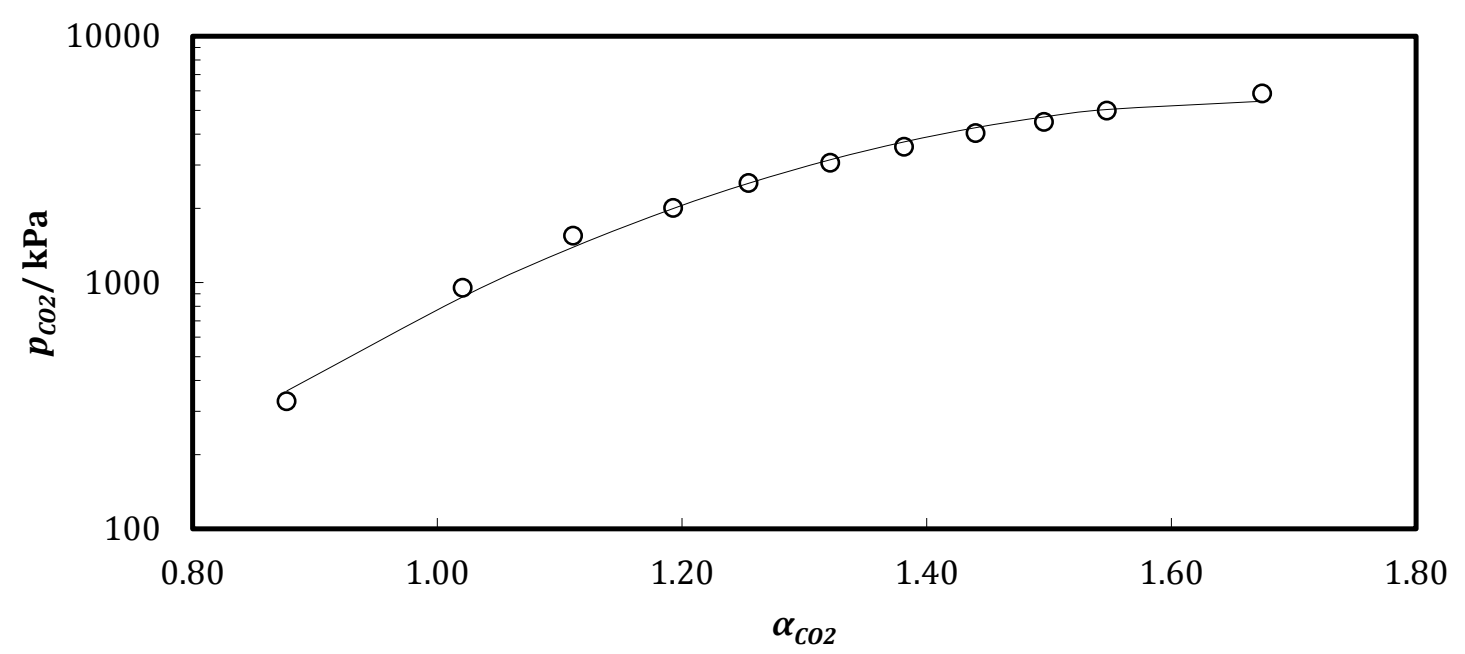

Figura 5.19. Valores experimentales de la solubilidad (O) para la mezcla $\mathrm{MEA} 10.000 \% \mathrm{w}+\mathrm{CO}_{2}$ a 313.15 K. Las líneas continuas muestran los valores calculados con los coeficientes de la ecuación de Xu y Rochelle y los puntos muestran los valores experimentales para cada índice de carga $\alpha$.

\subsubsection{Sistema MEA $30 \% \mathrm{w}+\mathrm{CO}_{2}$ a $313.15 \mathrm{~K}$ y $353.15 \mathrm{~K}$.}

Tabla 5.36. Datos experimentales de solubilidad para la mezcla $\mathrm{MEA} 30.015 \% \mathrm{w}+\mathrm{CO}_{2}$ a $313.15 \mathrm{Ky}$ MEA 29.999\%w $+\mathrm{CO}_{2}$ a $353.15 \mathrm{~K}$.

\begin{tabular}{cccc}
\hline \multicolumn{3}{c}{$T / \mathrm{K}$} \\
\hline$p_{\mathrm{CO}_{2}} / \mathrm{kPa}$ & $\alpha_{\mathrm{CO}_{2}}$ & $p_{\mathrm{CO}_{2}} / \mathrm{kPa}$ & $\alpha_{\mathrm{CO}_{2}}$ \\
\hline 0.232 & 0.462 & 22.031 & 0.462 \\
4.891 & 0.529 & 76.793 & 0.516 \\
29.938 & 0.583 & 236.9 & 0.567 \\
82.310 & 0.630 & 362.5 & 0.590 \\
91.632 & 0.636 & 547.1 & 0.615 \\
146.1 & 0.664 & 1061.4 & 0.662 \\
185.0 & 0.679 & 1736.2 & 0.704 \\
284.4 & 0.710 & 4207.2 & 0.804 \\
452.0 & 0.748 & & \\
734.9 & 0.792 & & \\
1232.4 & 0.846 & & \\
\hline
\end{tabular}


Tabla 5.37. Coeficientes y estadística de la ecuación de Xu y Rochelle para la mezcla MEA 30.015\%w + $\mathrm{CO}_{2}$ a $313.15 \mathrm{Ky} \mathrm{MEA} 29.999 \% w+\mathrm{CO}_{2}$ a $353.15 \mathrm{~K}$.

Parámetros de la ecuación Xu y Rochelle

\begin{tabular}{ccccccc}
\hline $\mathrm{A}_{1}$ & $\mathrm{~A}_{2}$ & $\mathrm{~A}_{3}$ & $\mathrm{~A}_{4}$ & $\mathrm{~A}_{5}$ & $\mathrm{~A}_{6}$ & $\sigma$ \\
$\mathrm{kg} \cdot \mathrm{m}^{-3}$ & $\mathrm{~kg} \cdot \mathrm{m}^{-3} \cdot \mathrm{K}^{-1}$ & $\mathrm{~kg} \cdot \mathrm{m}^{-3} \cdot \mathrm{K}^{-2}$ & $\mathrm{MPa}$ & $\mathrm{MPa} \cdot \mathrm{K}^{-1}$ & $\mathrm{MPa} \cdot \mathrm{K}^{-2}$ & \\
\hline 22.13 & 15563.50 & 49.07 & -67.19 & 9119.34 & 7247.48 & 0.27 \\
\hline
\end{tabular}

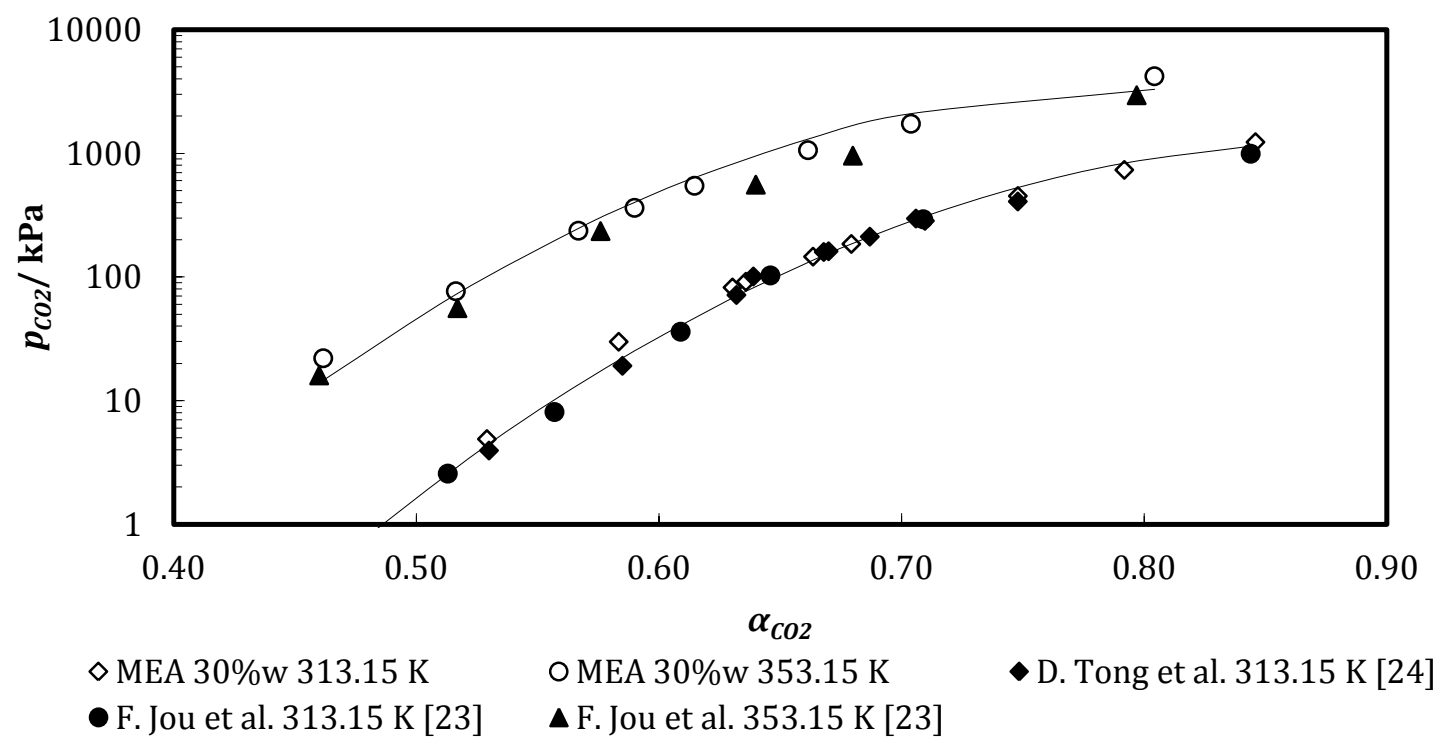

Figura 5.20. Valores experimentales y bibliográficos de la solubilidad para la mezcla MEA 30.015\%w + $\mathrm{CO}_{2}$ a $313.15 \mathrm{~K}$ y $\mathrm{MEA} 29.999 \% \mathrm{w}+\mathrm{CO}_{2}$ a $353.15 \mathrm{~K}$. Las líneas continuas muestran los valores calculados con los coeficientes de la ecuación de Xu y Rochelle y los puntos muestran los valores experimentales para cada temperatura y índice de carga $\alpha[12,13]$.

Los resultados de la solubilidad obtenidos para las mezclas de MEA 30\%w $+\mathrm{CO}_{2}$ se compararon con los encontrados en la literatura [12] y [13], a $313.15 \mathrm{~K}$ y $353.15 \mathrm{~K}$. Para su comparación, se ajustaron los datos experimentales a un polinomio y se interpolaron a las concentraciones de cada autor, comparando las presiones parciales de $\mathrm{CO}_{2}$ obtenidas a una misma concentración.

En la figura 5.21 se han representado las desviaciones relativas obtenidas entre los datos experimentales y los de la literatura a $313.15 \mathrm{~K}$ y $353.15 \mathrm{~K}$. La desviación media obtenida $(A A D \%)$ para la mezcla a $313.15 \mathrm{~K}$ fue de $17.2 \%$ con [12] y $11.2 \%$ con [13]. La desviación media obtenida $(A A D \%)$ para la mezcla a $353.15 \mathrm{~K}$ fue de $25.9 \%$ con [12]. Se puede observar que las diferencias entre los autores son del mismo orden de magnitud que las obtenidas en este trabajo. 


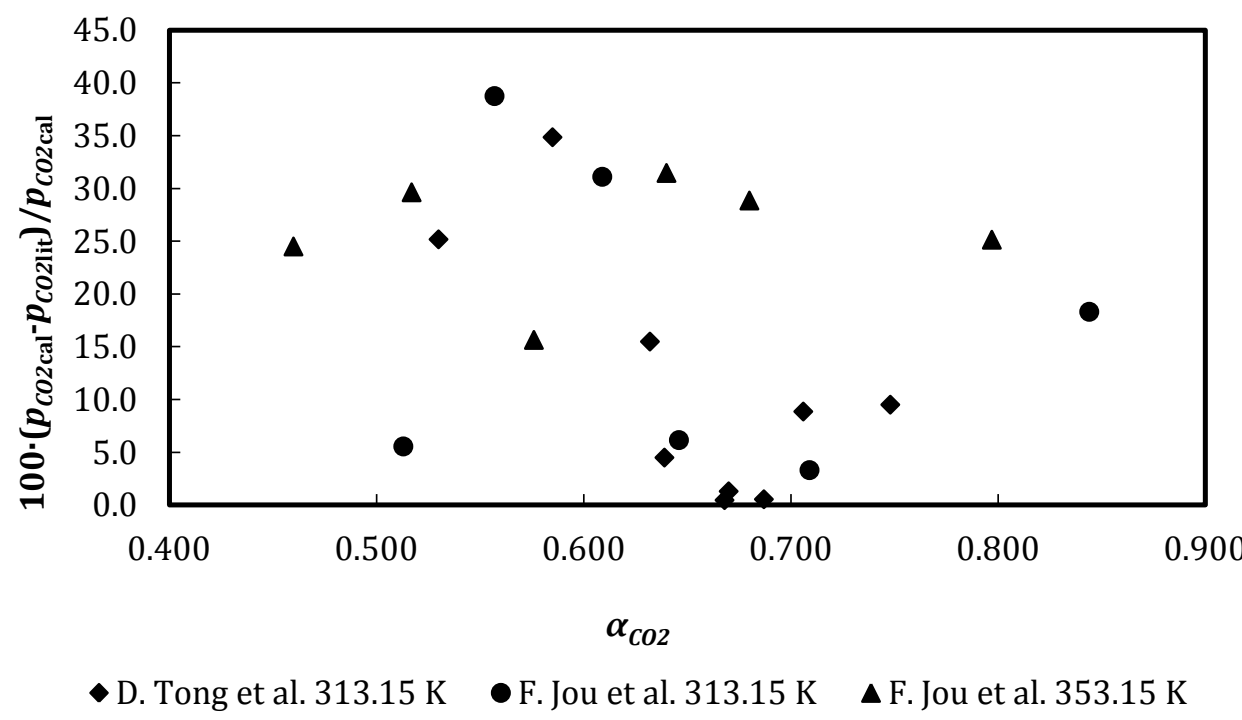

Figura 5.21. Desviación relativa porcentual de la solubilidad experimental para la mezcla MEA $30.015 \% \mathrm{w}+\mathrm{CO}_{2}$ respecto a los datos de la bibliografía de Jou et al. $313.15 \mathrm{~K}$ y $353.15 \mathrm{~K}$ [12], y Tong et al. $313.15 K$ [13].

\subsubsection{Sistema DMAE $30 \% \mathrm{w}+\mathrm{CO}_{2}$ a $313.15 \mathrm{~K}$ y $353.15 \mathrm{~K}$.}

Tabla 5.38. Datos experimentales de solubilidad para la mezcla DMAE $30.000 \% \mathrm{w}+\mathrm{CO}_{2}$ a $313.15 \mathrm{Ky}$ DMAE $30.000 \% w+\mathrm{CO}_{2}$ a $353.15 \mathrm{~K}$.

\begin{tabular}{cccc}
\hline \multicolumn{3}{c}{$T / \mathrm{K}$} \\
\hline & 313.15 & & 353.15 \\
\hline$p_{\mathrm{CO}_{2}} / \mathrm{kPa}$ & $\alpha_{\mathrm{CO}_{2}}$ & $p_{\mathrm{CO}_{2}} / \mathrm{kPa}$ & $\alpha_{\mathrm{CO}_{2}}$ \\
\hline 3.404 & 0.378 & 43.790 & 0.350 \\
5.975 & 0.476 & 58.376 & 0.410 \\
10.306 & 0.576 & 79.031 & 0.475 \\
16.033 & 0.652 & 118.1 & 0.563 \\
55.698 & 0.823 & 200.1 & 0.675 \\
99.351 & 0.885 & 449.8 & 0.822 \\
935.2 & 1.024 & 1052.2 & 0.936 \\
1987.2 & 1.081 & 1996.8 & 1.009 \\
& & 2894.9 & 1.052 \\
& & 4050.6 & 1.095 \\
& & 5840.0 & 1.156 \\
\hline
\end{tabular}


Resultado de medidas experimentales de las densidades y solubilidades de $\mathrm{CO}_{2}$ en soluciones acuosas de aminas

Tabla 5.39. Coeficientes y estadística de la ecuación de Xu y Rochelle para la mezcla DMAE 30.000\%w + $\mathrm{CO}_{2}$ a $313.15 \mathrm{Ky}$ DMAE $30.000 \% \mathrm{w}+\mathrm{CO}_{2}$ a $353.15 \mathrm{~K}$.

Parámetros de la ecuación Xu y Rochelle

\begin{tabular}{ccccccc}
\hline $\begin{array}{c}\mathrm{A}_{1} \\
\mathrm{~kg} \cdot \mathrm{m}^{-3}\end{array}$ & $\begin{array}{c}\mathrm{A}_{2} \\
\mathrm{~kg} \cdot \mathrm{m}^{-3} \cdot \mathrm{K}^{-1}\end{array}$ & $\begin{array}{c}\mathrm{A}_{3} \\
\mathrm{~kg} \cdot \mathrm{m}^{-3} \cdot \mathrm{K}^{-2}\end{array}$ & $\begin{array}{c}\mathrm{A}_{4} \\
\mathrm{MPa}\end{array}$ & $\begin{array}{c}\mathrm{A}_{5} \\
\mathrm{MPa} \cdot \mathrm{K}^{-1}\end{array}$ & $\begin{array}{c}\mathrm{A}_{6} \\
\mathrm{MPa} \cdot \mathrm{K}^{-2}\end{array}$ & $\sigma$ \\
\hline 19.30 & 5622.52 & 27.23 & -30.25 & -9572.38 & 12083.66 & 0.15 \\
\hline
\end{tabular}

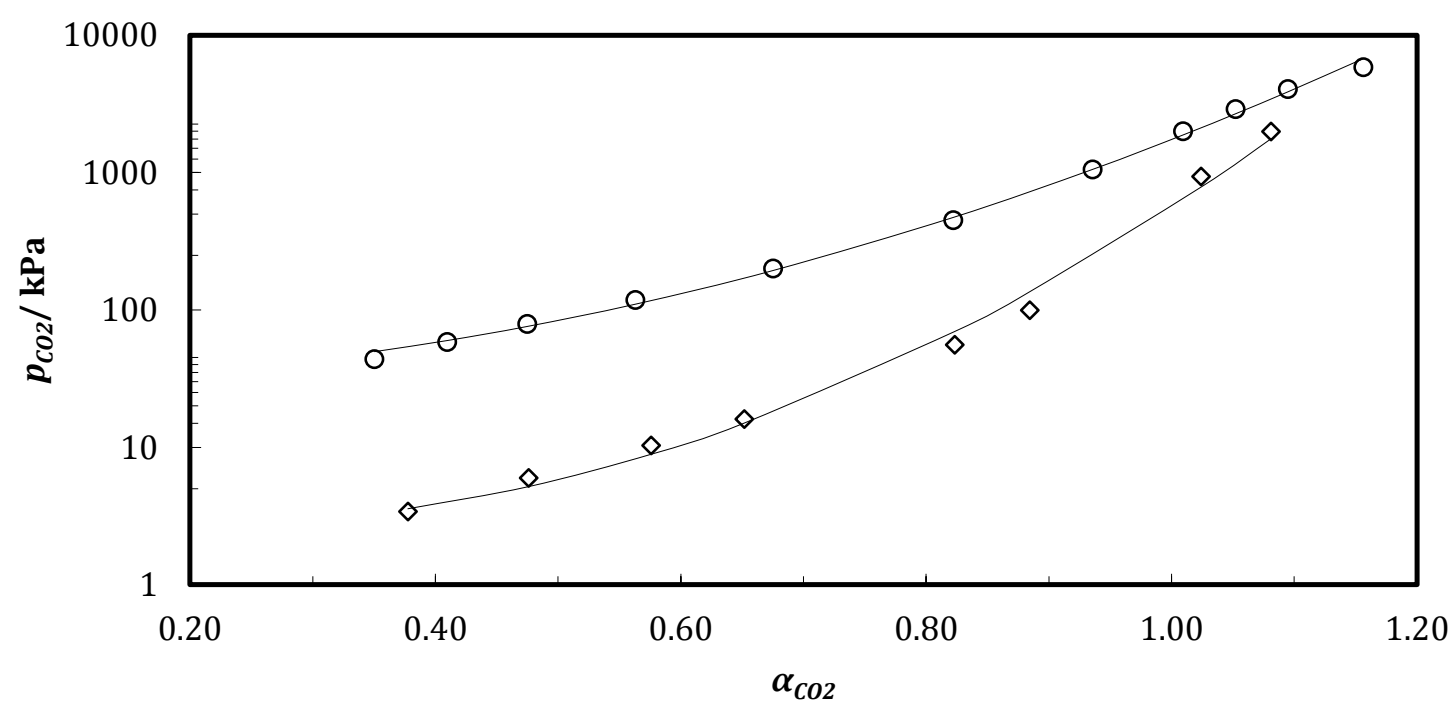

$\diamond$ DMAE $30 \% 313.15 \mathrm{~K}$

O DMAE $30 \% 353.15 \mathrm{~K}$

Figura 5.22. Valores experimentales de la solubilidad para la mezcla DMAE $30.000 \% \mathrm{w}+\mathrm{CO}_{2}$ a 313.15 $\mathrm{K}$ y DMAE $30.000 \% w+\mathrm{CO}_{2}$ a $353.15 \mathrm{~K}$. Las líneas continuas muestran los valores calculados con los coeficientes de la ecuación de Xu y Rochelle y los puntos muestran los valores experimentales para cada índice de carga $\alpha$. 
Resultado de medidas experimentales de las densidades y solubilidades de $\mathrm{CO}_{2}$ en soluciones acuosas de aminas

\subsubsection{Sistema TEA $30 \% \mathrm{w}+\mathrm{CO}_{2}$ a $313.15 \mathrm{~K}$ y $353.15 \mathrm{~K}$.}

Tabla 5.40. Datos experimentales de solubilidad para la mezcla TEA $30.010 \% w+\mathrm{CO}_{2}$ a $313.15 \mathrm{Ky}$ TEA $29.825 \% \mathrm{w}+\mathrm{CO}_{2}$ a $353.15 \mathrm{~K}$.

\begin{tabular}{cccc}
\hline & \multicolumn{3}{c}{$T / \mathrm{K}$} \\
\hline$p_{\mathrm{CO}_{2}} / \mathrm{kPa}$ & $\alpha_{\mathrm{CO}_{2}}$ & $p_{\mathrm{CO}_{2}} / \mathrm{kPa}$ & $\alpha_{\mathrm{CO}_{2}}$ \\
\hline 39.135 & 0.332 & 264.7 & 0.278 \\
90.152 & 0.496 & 392.5 & 0.350 \\
139.5 & 0.590 & 553.0 & 0.421 \\
288.8 & 0.754 & 747.7 & 0.489 \\
960.0 & 0.976 & 974.5 & 0.556 \\
1509.8 & 1.062 & 1310.5 & 0.635 \\
2178.2 & 1.140 & 1873.9 & 0.733 \\
3005.6 & 1.213 & 2999.4 & 0.883 \\
4041.0 & 1.292 & 4114.0 & 0.985 \\
5999.9 & 1.422 & 5838.6 & 1.103 \\
\hline
\end{tabular}

Tabla 5.41. Coeficientes y estadística de la ecuación de Xu y Rochelle para la mezcla TEA 30.010\%w+ $\mathrm{CO}_{2}$ a $313.15 \mathrm{Ky}$ TEA $29.825 \% w+\mathrm{CO}_{2}$ a $353.15 \mathrm{~K}$.

Parámetros de la ecuación Xu y Rochelle

\begin{tabular}{ccccccc}
\hline $\begin{array}{c}\mathrm{A}_{1} \\
\mathrm{~kg} \cdot \mathrm{m}^{-3}\end{array}$ & $\begin{array}{c}\mathrm{A}_{2} \\
\mathrm{~kg} \cdot \mathrm{m}^{-3} \cdot \mathrm{K}^{-1}\end{array}$ & $\begin{array}{c}\mathrm{A}_{3} \\
\mathrm{~kg} \cdot \mathrm{m}^{-3} \cdot \mathrm{K}^{-2}\end{array}$ & $\begin{array}{c}\mathrm{A}_{4} \\
\mathrm{MPa}\end{array}$ & $\begin{array}{c}\mathrm{A}_{5} \\
\mathrm{MPa} \cdot \mathrm{K}^{-1}\end{array}$ & $\begin{array}{c}\mathrm{A}_{6} \\
\mathrm{MPa} \cdot \mathrm{K}^{-2}\end{array}$ & $\sigma$ \\
\hline 21.17 & 6063.76 & 10.27 & -12.04 & -1439.22 & 3608.63 & 0.07 \\
\hline
\end{tabular}




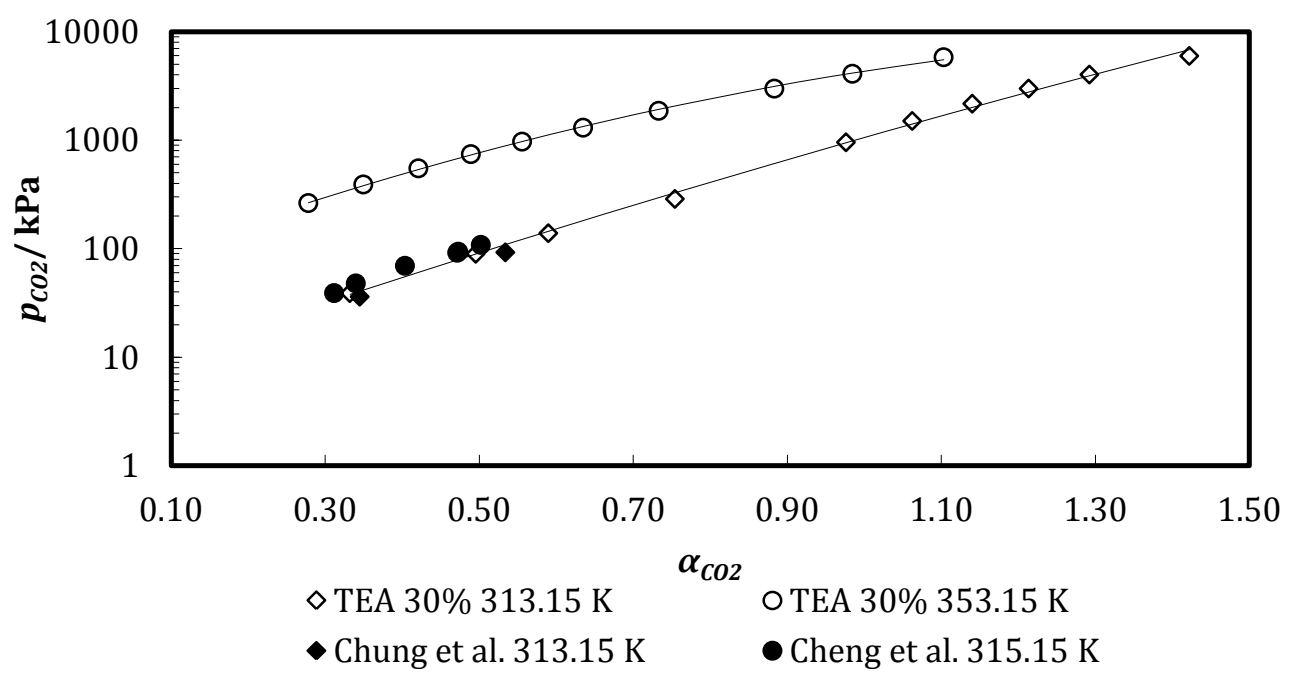

Figura 5.23. Valores experimentales y bibliográficos de la solubilidad para la mezcla TEA $30.010 \% \mathrm{w}$ + $\mathrm{CO}_{2}$ a $313.15 \mathrm{~K}$ y TEA $29.825 \% \mathrm{w}+\mathrm{CO}_{2}$ a $353.15 \mathrm{~K}$. Las líneas continuas muestran los valores calculados con los coeficientes de la ecuación de Xu y Rochelle y los puntos muestran los valores experimentales para cada temperatura y índice de carga $\alpha$.

Los resultados de la solubilidad obtenidos para las mezclas de TEA $30 \% \mathrm{w}+\mathrm{CO}_{2}$ se compararon con los encontrados en la literatura [14] y [15], a $313.15 \mathrm{~K}$. En la figura 5.24 se han representado las desviaciones relativas obtenidas entre los datos experimentales y los de la literatura a $313.15 \mathrm{~K}$. La desviación media obtenida $(A A D \%)$ para la mezcla a $313.15 \mathrm{~K}$ fue de $14.0 \%$ con [14] y $16.8 \%$ con [15]. Como resultado de dichas desviaciones se puede concluir que estos valores son coherentes con la literatura para la medida de la solubilidad, estando los valores experimentales comprendidos entre los valores de la literatura.

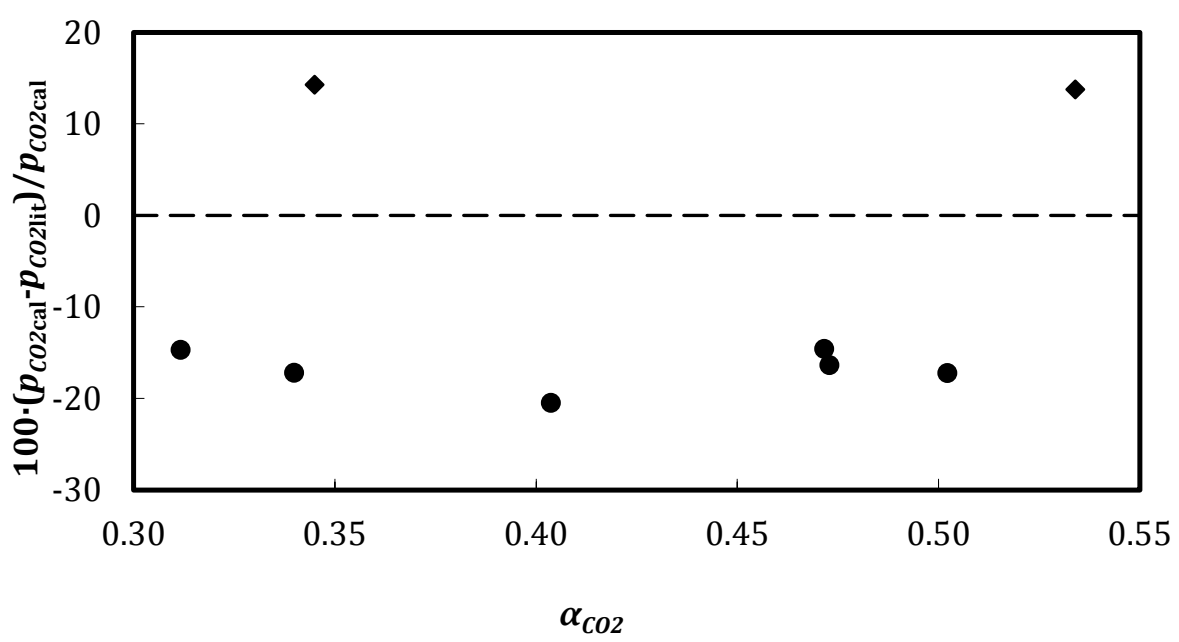

$\bullet$ Chung et al. $\bullet$ Cheng et al.

Figura 5.24. Desviación relativa porcentual de la solubilidad experimental para la mezcla TEA $30.010 \% w+\mathrm{CO}_{2}$ a $313.15 \mathrm{~K}$ respecto a los datos de la bibliografía de Chung et al. [14] y Cheng et al. [15]. 


\subsection{Discusión de resultados de la solubilidad experimental.}

En este trabajo se determinaron las solubilidades de tres mezclas ternarias de soluciones acuosas de aminas con $\mathrm{CO}_{2}$ (MEA 10\%w, MEA 30\%w, DMAE 30\%w y TEA 30\%w) realizadas a $313.15 \mathrm{~K}$ y $353.15 \mathrm{~K}$. El rango de presión varió desde la presión de vapor de la mezcla amina-agua hasta $6 \mathrm{MPa}$, e índices de carga hasta 1.7 moles de $\mathrm{CO}_{2}$ por mol de amina.

Los ajustes con la ecuación de Xu y Rochelle fueron buenos, ajustándose peor a presiones superiores a $5 \mathrm{MPa}$ y a las presiones cercanas a la presión de vapor de la mezcla amina + $\mathrm{H}_{2} \mathrm{O}$. La desviación estándar $(\sigma)$ en todos los casos es inferior a 0.27 para el conjunto de mezclas estudiadas.

El mejor ajuste de la ecuación de Xu y Rochelle obtuvo una desviación estándar $(\sigma)$ de 0.07 para el sistema TEA $30 \% \mathrm{w}+\mathrm{CO}_{2}$.

Los valores experimentales muestran una tendencia de la solubilidad creciente con la presión y decreciente al aumentar la temperatura. Siendo el aumento de solubilidad más marcado a bajas presiones para todas las mezclas a cualquier temperatura.

En la figura 5.25 se compara el comportamiento de las tres aminas al $30 \% \mathrm{w}$ a la temperatura de $313.15 \mathrm{~K}$.

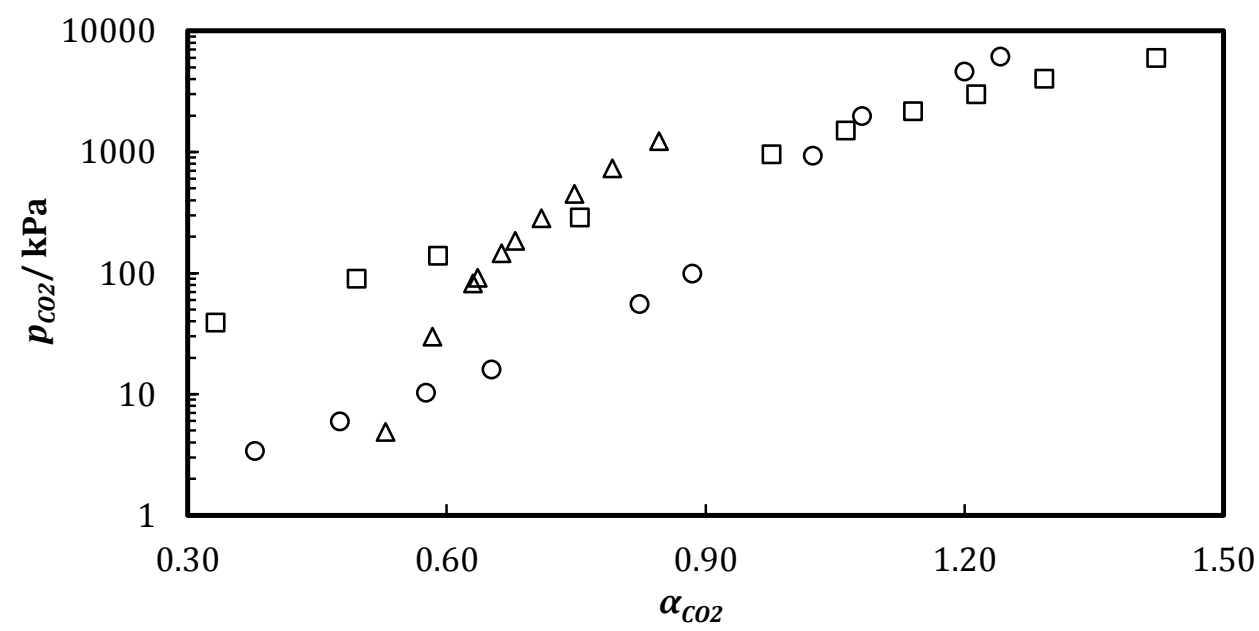

Figura 5.25. Valores experimentales de la solubilidad a $313.15 \mathrm{~K}$ para la mezcla MEA $30 \% \mathrm{w}(\triangle)$, DMAE 30\%w (०) y TEA 30\%w (口).

En la Figura 5.25 se puede observar que a bajas presiones menores de $150 \mathrm{kPa}$ la TEA tiene menor solubilidad que MEA y la DMAE. A partir de $150 \mathrm{kPa}$ la MEA es la que tiene menor solubilidad. Este comportamiento hace que sea más eficiente la utilización de TEA, a presiones por encima de $2000 \mathrm{kPa}$, y por debajo de estas presiones la DMAE. 


\subsection{Referencias.}

[1] J. H. Dymond, R. Malhotra. "The Tait equation: 100 years on". Int. J. Thermophys. Vol. 9, (1988) 941-951.

[2] A. Wohl. "Studies pertaining to the equation of state, Part 4: Equation of compression for fluids". Z. Phys. Chem. Vol. 99, (1921) 226-233.

[3] A. T. J. Hayward. "Compressibility equations for liquids: A comparative study". Brit. J. Appl. Phys. Vol. 18, (1967) 965-977.

[4] P.G. Tait. "Report on some of the physical properties of fresh water and sea water". Phys. Chem. (1888) 1-76.

[5] G. Tammann. "Tammann compressibility equation for liquids". Z. Phys. Chem. Vol. 17, (1895) 620.

[6] M. J. P. Comuñas, A. Baylaucq, C. Boned, J. Fernández. "High-pressure measurements of the viscosity and density of two polyethers and two dialkyl carbonates at high pressures". Int. J. Thermophys. Vol. 22, (2001) 749-768.

[7] M. Sobrino, E. I. Concepción, A. Gómez-Hernández, M. C. Martín, J. J. Segovia. "Viscosity and density measurements of aqueous amines at high pressures: MDEA-water and MEAwater mixtures for $\mathrm{CO}_{2}$ capture". J. Chem. Thermodynamics. Vol. 98, (2016) 231-241.

[8] Q. Xu, G. Rochelle. "Total pressure and $\mathrm{CO}_{2}$ solubility at high temperature in aqueous amines". Energy Procedia. Vol. 4, (2011) 117-124.

[9] J. Zhang, P. S. Fennell, J. P. Trusler. "Density and viscosity of partially carbonated aqueous tertiary alkanolamine solutions at temperatures between (298.15 and 353.15) K". J. Chem. Eng. Data. Vol. 60, (2015) 2392-2399.

[10] J. Han, J. Jin, D. A. Eimer, M. C. Melaaen. "Density of water (1) + monoethanolamine (2) $+\mathrm{CO}_{2}$ (3) from (298.15 to 413.15) K and surface tension of water (1) + monoethanolamine (2) from (303.15 to 333.15) K". J. Chem. Eng. Data. Vol. 57, (2012) 1095-1103.

[11] T. G. Amundsen, L. E. Øi, D. A. Eimer. "Density and viscosity of monoethanolamine + water + carbon dioxide from (25 to 80) ${ }^{\circ} \mathrm{C}^{\prime}$. J. Chem. Eng. Data. Vol. 54, (2009) 3096-3100.

[12] F. Y. Jou, A. E. Mather, F. D. Otto. "The solubility of $\mathrm{CO}_{2}$ in a 30 mass percent monoethanolamine solution". Can. J. Chem. Eng. Vol. 73, (1995) 140-147.

[13] D. Tong, J. P. M. Trusler, G. C. Maitland, J. Gibbins, P. S. Fennell. "Solubility of carbon dioxide in aqueous solution of monoethanolamine or 2-amino-2-methyl-1-propanol: Experimental measurements and modelling". International Journal of Greenhouse Gas Control. Vol. 6, (2012) 37-47.

[14] P. Chung, A. N. Soriano, R. B. Leron, M. Li. "Equilibrium solubility of carbon dioxide in the amine solvent system of (triethanolamine + piperazine + water)". J. Chem. Thermodynamics. Vol. 42, (2010) 802-807. 
Resultado de medidas experimentales de las densidades y solubilidades de $\mathrm{CO}_{2}$ en soluciones acuosas de aminas

[15] M. Cheng, A. R. Caparanga, A. N. Soriano, M. Li. "Solubility of $\mathrm{CO}_{2}$ in the solvent system (water + monoethanolamine + triethanolamine)". J. Chem. Thermodynamics. Vol. 42, (2010) 342-347. 
Capítulo 6

\section{DETERMINACIÓN DE MEDIDAS DE DENSIDADES Y VISCOSIDADES EN MEZCLAS DE $\mathrm{CO}_{2}+$ HIDROCARBURO}





\subsection{Introducción.}

La viscosidad se define como la propiedad que tienen los fluidos de ofrecer resistencia al movimiento relativo de sus moléculas. También se suele definir la viscosidad como una propiedad de los fluidos que causa fricción, esto da origen a la perdida de energía en el flujo.

La viscosidad es una de las propiedades termofísicas más importantes para muchas aplicaciones tecnológicas y científicas, por lo que se ha realizado un enorme esfuerzo para su medición, correlación e interpretación. Pero, por otro lado, este esfuerzo es completamente inconsistente con el hecho de que, probablemente, sea la propiedad con menos valores de referencia independientes, que puedan ser usados como herramienta de orientación, calibración o de diseño [1].

Los estudios sobre la viscosidad tienen gran interés para los investigadores. Primero, la construcción de equipos capaces de determinar el comportamiento viscoso de líquidos de manera absoluta en amplios rangos de temperatura, presión y viscosidad. Segundo, la búsqueda de líquidos que puedan servir de referencia para calibrar los viscosímetros relativos. Y por último, la modelización del comportamiento en presión y temperatura de esta propiedad de transporte con ecuaciones precisas y con sentido físico [1].

La viscosidad dinámica ( $\boldsymbol{\eta}$ ) es la propiedad de los fluidos que se caracteriza por su resistencia a fluir, debida al rozamiento entre sus moléculas. En el Sistema Internacional se mide en Pascales segundo (Pa·s), pero la unidad más utilizada es el centipoise (cps), equivalente a $1 \mathrm{mPa} \cdot \mathrm{s}$.

La viscosidad cinemática ( $v$ ) es el cociente entre viscosidad dinámica y densidad, y se mide en el Sistema Internacional en $\left(\mathrm{m}^{2} / \mathrm{s}\right)$ o centistokes $\left(1 \mathrm{cts}=1 \mathrm{~mm}^{2} / \mathrm{s}\right)$. Esta relación viene dada por la ecuación 6.1.

$$
\eta=\rho \cdot v
$$

De ahora en adelante, la viscosidad siempre se expresará como viscosidad dinámica, asignada por la letra griega $\eta$. Los fluidos a tratar no tendrán viscosidades excesivamente elevadas, con lo cual las unidades a utilizar para expresar esta propiedad serán $\mathrm{mPa} \cdot \mathrm{s}$.

La medida de la viscosidad de mezclas de hidrocarburos es especialmente importante en los procesos de extracción de petróleo asistido con $\mathrm{CO}_{2}$. Al inyectar el $\mathrm{CO}_{2}$, éste desplaza al petróleo contenido en los poros de la roca del yacimiento, permitiendo una recuperación adicional de hidrocarburos.

La principal ventaja de esta tecnología es que el $\mathrm{CO}_{2}$ usado en la extracción queda automáticamente capturado en la misma roca que contenía el petróleo. También evita el uso del agua para la extracción y mejora la recuperación de petróleo al disminuir la viscosidad de este. Es importante la correlación de datos experimentales a una ecuación que permita el conocimiento del comportamiento de mezclas de hidrocarburos con $\mathrm{CO}_{2}$ en las condiciones de recuperación de crudo de los pozos petrolíferos.

El aumento de la composición de $\mathrm{CO}_{2}$ da lugar a una disminución significativa de la viscosidad y un aumento de la densidad de las mezclas. Se estudiaron la densidad y la 
viscosidad dinámica para sistemas binarios de $\mathrm{CO}_{2}+\mathrm{n}$-pentano y $\mathrm{CO}_{2}+\mathrm{n}$-hexano, a diferentes concentraciones $x_{C O 2}=[0.1,0.2,0.3,0.4$ y 0.6$]$, temperaturas de $0^{\circ} \mathrm{C}, 20^{\circ} \mathrm{C}, 40^{\circ} \mathrm{C}$, $60^{\circ} \mathrm{C}, 80^{\circ}$ y $100^{\circ} \mathrm{C}$ y presiones de hasta $100 \mathrm{MPa}$.

\subsection{Técnicas experimentales para la medida de la viscosidad.}

Los métodos experimentales para medir la viscosidad pueden ser clasificados como absolutos y relativos, como puede verse en Assael et al. [2] y Castro [3]. Un método relativo no tiene una teoría completa y las ecuaciones de trabajo contienen parámetros empíricos cuyos valores se determinan utilizando sustancias de referencia, en las mismas condiciones que las mediciones.

Un método absoluto se describe mediante ecuaciones precisas con un sentido físico riguroso, y variables accesibles de forma independiente. Si es necesaria la calibración para la obtención de uno o varios parámetros utilizando muestras y condiciones estándar, este método se denomina cuasi-absoluto. Siendo la precisión igual a la de un método absoluto.

Los métodos desarrollados para medir la viscosidad de un fluido se han agrupado en cuatro tipos: viscosímetros de cuerpo oscilante, capilares, de caída de cuerpo y de vibración; se clasifican como métodos primarios si son capaces de trabajar en modo absoluto.

Cuerpo oscilante (rotacionales): Dentro de estos se encuentran los del tipo Brookfield, de cono y plato, el viscosímetro Stormer y el Stabinger. En todos ellos se emplea la idea de que la fuerza requerida para rotar un objeto inmerso en un fluido puede indicar la viscosidad del fluido, diferenciándose en la forma del tipo del rotor. Algunos de ellos permiten medir a alta presión. Un ejemplo de los que solo permiten medir a presión atmosférica es el viscosímetro Stabinger SVM 3000, de geometría cilíndrica.

Capilares: La determinación de la viscosidad empleando este dispositivo o instrumento, se basa en la medida del tiempo necesario para que un volumen determinado de líquido fluya a través de un capilar. Ejemplos de estos viscosímetros son los de Ostwald, CannonFenske y Ubbelohde.

Caída de cuerpo: La determinación de la viscosidad mediante viscosimetría de caída de cuerpo, o método de Stokes, se basa en la medida del tiempo que tarda en caer un cuerpo, esfera o cilindro en velocidad límite a través de un líquido cuya viscosidad se quiere determinar.

Vibración: La pieza activa del sensor es un elemento vibrante. La amplitud de la vibración varía según la viscosidad del líquido en el cual se sumerge el sensor. Estos son convenientes para medir fluidos hasta viscosidades altas (hasta 1.000.000 cP). Ejemplos de estos es el viscosímetro de hilo vibrante y el de torsión de cristal.

Los viscosímetros capilares y de caída de cuerpo se apoyan en ecuaciones de trabajo, pero requieren de calibración con un líquido de viscosidad conocida, a una determinada temperatura y presión, con el fin de establecer los factores de corrección incluidos en las ecuaciones. Estos factores corrigen los efectos que podrían contribuir a errores 
sistemáticos de las medidas. Por otra parte, los viscosímetros de cuerpo oscilante y de vibración no requieren factores de corrección al considerar restricciones específicas en las ecuaciones de trabajo, pero se debe realizar una medida en el vacío para determinar la auto-amortiguación de los mismos.

En nuestro caso, los viscosímetros de cuerpo vibrante son de construcción sencilla y con ecuaciones de trabajo menos complejas que los viscosímetros de cuerpo oscilante, y permiten realizar mediciones con una baja incertidumbre, menor del 1\%, para una extensa gama de líquidos y gases, en un amplio rango de temperatura y presión [1,4].

El viscosímetro de hilo vibrante es un medidor absoluto, siempre que se tenga especial cuidado en la fabricación y caracterización de sus componentes. El radio del hilo es el parámetro más difícil de determinar de forma independiente. Los radios pueden ser de 10 $\mu \mathrm{m}$ para gases, y de $50 \mu \mathrm{m}$ a $200 \mu \mathrm{m}$ para líquidos, dependiendo de la viscosidad. La uniformidad y la forma de la sección transversal del hilo son cuestiones importantes, al igual que la calidad de la superficie. El hilo utilizado es de tungsteno debido a su alta densidad, el módulo y la resistencia a la tracción de Young, la baja expansión térmica y la inercia química [2]. Hasta hace poco la mayoría de los viscosímetros habían sido construidos con hilos de alta pureza, pero con radios poco caracterizados, por lo que se utilizaban de forma casi absoluta, determinando el radio a partir de un punto de calibración. Para operar en un amplio rango de condiciones es importante tener en cuenta los efectos de la temperatura y la presión sobre el hilo.

\subsection{Principio de funcionamiento del Viscosímetro de Hilo Vibrante.}

El movimiento de un hilo oscilante en un líquido denso fue descrito por primera vez en 1828 por Bessel [5], cuando introdujo la necesidad de una corrección del incremento de la masa al mover un péndulo en el aire. El efecto de la masa añadida fue descubierto de forma independiente por Du Buat en 1786 [6]. Y en 1850, Stokes derivó la dependencia del incremento de masa con la viscosidad del líquido que se encuentra alrededor [6].

El primer viscosímetro de hilo vibrante fue presentado por Tough et al. [7] en 1963 como un nuevo método de medida de viscosidad.

Al aplicar un campo magnético externo, se produce una distorsión en el hilo, el cual se encuentra sumergido en un fluido. Este es de sección circular de radio $R$, una longitud $L$ mucho mayor que su radio $(L>>R$ ) y densidad conocida. Para la operación del hilo vibrante se pueden emplear dos modos de oscilación: el modo forzado (FR) y el modo transitorio (TR). En nuestro caso funciona en modo forzado, donde las características de las curvas de resonancia para las oscilaciones transversales del hilo están determinadas por la viscosidad y densidad del fluido. Este modo opera en el dominio de la frecuencia, pasando una corriente alterna AC constante por el hilo, sobre un rango de frecuencia suficiente para cubrir su primer armónico. La viscosidad se calcula a partir del ancho de la señal de resonancia. En el caso del modo transitorio, opera en el dominio del tiempo conduciendo una corriente por el hilo por un tiempo corto a una frecuencia cercana a su resonancia, se desconecta la fuente de corriente y se calcula la viscosidad a partir del tiempo de amortiguación [1]. 
El valor del radio $R$ es el radio promedio del hilo obtenido mediante calibración. Para ello se utiliza como referencia estándar la viscosidad del agua a $20{ }^{\circ} \mathrm{C}$ y un segundo fluido alternativo como patrón, por lo general el tolueno [8].

En este trabajo se midió la viscosidad dinámica de mezclas de $\mathrm{CO}_{2}+$ hidrocarburo de hasta aproximadamente $0.53 \mathrm{mPa} \cdot \mathrm{s}$. El hilo es de tungsteno y está sujeto por ambos extremos en un mecanismo eléctricamente aislado. El hilo se encuentra situado entre los polos de un imán, el cual se encuentra sumergido en un baño termostatado, de tal forma que el imán requerido para proporcionar el flujo magnético en el hilo es de pequeño tamaño. Reduciendo de esta forma la dimensión del equipo de medida y la muestra requerida, que es aproximadamente de $50 \mathrm{~cm}^{3}$, incluyendo todo el sistema de tuberías y el circuito de presión.

El rango de viscosidades de las mezclas de hidrocarburo $+\mathrm{CO}_{2}$ seleccionada está comprendida entre $0.10 \mathrm{mPa} \cdot \mathrm{s}$ y $0.53 \mathrm{mPa} \cdot \mathrm{s}$. Los resultados obtenidos tienen una incertidumbre relativa expandida menor al $1.5 \%$.

\subsection{Ecuaciones de trabajo.}

Las ecuaciones de trabajo utilizadas y su desarrollo matemático, se encuentran a continuación tal y como se presentaron en la Tesis de Johnny Zambrano [1].

El sensor está compuesto por un hilo tensado y fijado en los extremos mediante dos abrazaderas acopladas en el cuerpo del sensor, el cual se coloca dentro de un recipiente a presión, y se le aplica un campo magnético externo constante. La circulación de una corriente sinusoidal constante a través del hilo, en combinación con el campo magnético, generan la vibración del hilo estable y de forma transversal. Este movimiento induce un voltaje a través del hilo debido a la ley de Faraday.

En los terminales del hilo mediremos mediante un amplificador lock-in de dos fases, la fuerza electromotriz (FEM) generada a través del hilo vibrante. La FEM está compuesta por dos términos complejos $V_{1}$ y $V_{2}$, representada por la ecuación 6.2.

$$
V=V_{1}+V_{2}
$$

Siendo: $V$ la tensión (voltaje) a través del hilo, $V_{1}$ el voltaje resultante debida a la impedancia eléctrica del hilo fijo y $V_{2}$ el voltaje inducido por el movimiento del hilo.

Donde $V_{1}$ se puede expresar como:

$$
V_{1}=\mathrm{a}+\mathrm{ib}+\mathrm{ic} f
$$

Donde $f$ es la frecuencia del movimiento del hilo, i es la parte imaginaria ya, b, c son constantes que representan la impedancia eléctrica del hilo y absorben los desplazamientos en el amplificador lock-in para asegurar que la señal de voltaje se detectada en el rango más sensible, los cuales son parámetros ajustables.

El voltaje $V_{2}$ inducido es proporcional a la velocidad del hilo y a la fuerza del campo magnético $B$; lo cual proviene de su movimiento y se expresa en la ecuación 6.4. 


$$
V_{2}=\frac{i f \Lambda}{f_{0}^{2}-(1+\beta) f^{2}+\left(\beta^{\prime}+2 \Delta_{0}\right) f^{2} i}
$$

Donde $\Lambda$ corresponde a la amplitud, $f_{o}$ es la frecuencia de resonancia en el vacío, $\Delta_{\mathrm{o}}$ es la amortiguación natural del hilo en vacío, $\beta$ es la masa adicional del fluido y $\beta^{\prime}$ la amortiguación debido a la viscosidad del fluido. Siendo definidas $\beta$ y $\beta^{\prime}$ por las ecuaciones 6.5. y 6.6. respectivamente.

$$
\begin{aligned}
& \beta=k \frac{\rho}{\rho_{s}} \\
& \beta^{\prime}=k^{\prime} \frac{\rho}{\rho_{s}}
\end{aligned}
$$

Donde $\rho_{s}$ es la densidad del hilo, siendo $k$ y $k^{\prime}$ funciones de $\Omega$ dada por la ecuación 6.7. La cual es una relación de la viscosidad y expresada en función de la frecuencia $f$.

$$
\Omega=\frac{2 \pi f \rho R^{2}}{\eta}
$$

Siendo el voltaje total a través del hilo la suma de estas dos componentes:

$$
V=\frac{i f \Lambda}{f_{0}^{2}-(1+\beta) f^{2}+\left(\beta^{\prime}+2 \Delta_{0}\right) f^{2} i}+\mathrm{a}+\mathrm{ib}+\mathrm{ic} f
$$

Para la estimación de la viscosidad se realiza la siguiente aproximación que relaciona los valores de la frecuencia de resonancia $f_{\mathrm{r}}$ y el ancho de banda de la señal obtenida $f_{\mathrm{b}}$, esta misma aproximación será empleada para el cálculo de incertidumbres debido a la imposibilidad de aplicación a una función compleja:

$$
\eta=\frac{\pi f_{r} R_{W}^{2} \rho}{6}\left(\frac{f_{b}}{f_{r}}\right)^{2}\left(1+\frac{\rho_{s}}{\rho}\right)^{2}
$$

El ancho de banda $f_{\mathrm{b}}$ se encuentra de la siguiente forma:

$$
f_{b}=f_{+}-f_{-}
$$

donde $f_{+}$y $f$ - son las frecuencias donde la amplitud de $V_{2}$ es $\frac{1}{\sqrt{2}}$ de la resonancia.

Para la estimación de la frecuencia de resonancia en el vacío $f_{0}^{2}$ a una densidad $\rho$ conocida se aproxima en la ecuación 6.11 .

$$
f_{0}^{2} \approx(1+\beta) f_{r}^{2}
$$

en la cual el valor de $\beta$ se calcula con una densidad conocida y una viscosidad estimada.

El procedimiento de ajuste de los datos se realiza con la técnica de minimización Chi cuadrado y de la mejora como método de Marquardt [9] dentro del programa de tratamiento de datos. 


\subsection{Descripción del equipo.}

Para la obtención de los datos experimentales de la viscosidad dinámica de compuestos puros o mezclas, se utilizó un viscosímetro de hilo vibrante, el cual emplea como sensor un hilo de tungsteno de longitud $L=50 \mathrm{~mm}$ y radio nominal $R=75 \mu \mathrm{m}$.

Este hilo se encuentra sujeto y tensionado por medio de un par de abrazaderas que forman parte de la estructura del sensor, estando acopladas en los extremos de este. A su vez, el sensor se encuentra alojado en el interior de una celda de presión, de tal modo que este centrado y orientado en un campo magnético constante. El campo magnético se genera por medio de un imán en forma de $\mathrm{C}$, de esta forma se hará pasar una corriente alterna constante a través del hilo, generando una oscilación transversal, y el voltaje inducido producido en el hilo se medirá mediante un amplificador lock-in. La respuesta de frecuencia de estado estacionario del hilo oscilante se mide en la proximidad de la resonancia del modo transversal fundamental.

\subsubsection{Sensor de viscosidad.}

El desarrollo inicial de este sensor de hilo vibrante se detalla en [10] y [11]. El sensor utilizado en nuestro caso pertenece a una cuarta generación [11]. Las principales partes y materiales que lo componen son:

Cuerpo del sensor: En él se aloja el hilo vibrante. Está fabricado por un tubo de una cerámica mecanizable Shapal-M [12]. Este material cerámico de nitruro de aluminio tiene un coeficiente de dilatación térmica de $4.4 \times 10^{-6} \mathrm{~K}^{-1}$ entre $0{ }^{\circ} \mathrm{C}$ y $400{ }^{\circ} \mathrm{C}$, siendo similar al del hilo de tungsteno, y es resistente a altas presiones y temperaturas. Las dimensiones del cuerpo son de $48 \mathrm{~mm}$ de longitud, con un diámetro exterior de $10.2 \mathrm{~mm}$ y de $8 \mathrm{~mm}$ de diámetro interior.

Hilo de tungsteno: Este hilo se encuentra recubierto con oro y fue seleccionado debido a su buen comportamiento y resistencia en hidrocarburos para un amplio rango de presiones y temperaturas. Las propiedades nominales de este son de un radio $R=75 \mu \mathrm{m}$, longitud $L=50 \mathrm{~mm}$, densidad $\rho_{\mathrm{s}}=19300 \mathrm{~kg} / \mathrm{m}^{3}$, modulo de elasticidad $410 \mathrm{kN} / \mathrm{mm}^{2} \approx \approx$ $411 \mathrm{GPa}$ ) y coeficiente de expansión térmica de $4.5 \times 10^{-6} \mathrm{~K}^{-1}[13]$.

Abrazaderas de sujeción: Están fabricadas en acero inoxidable del tipo 316L [14]. Fue seleccionado por ser un material no magnético que no influye en las medidas. Los tornillos de sujeción de las abrazaderas y el pasador de guía son del mismo material. 


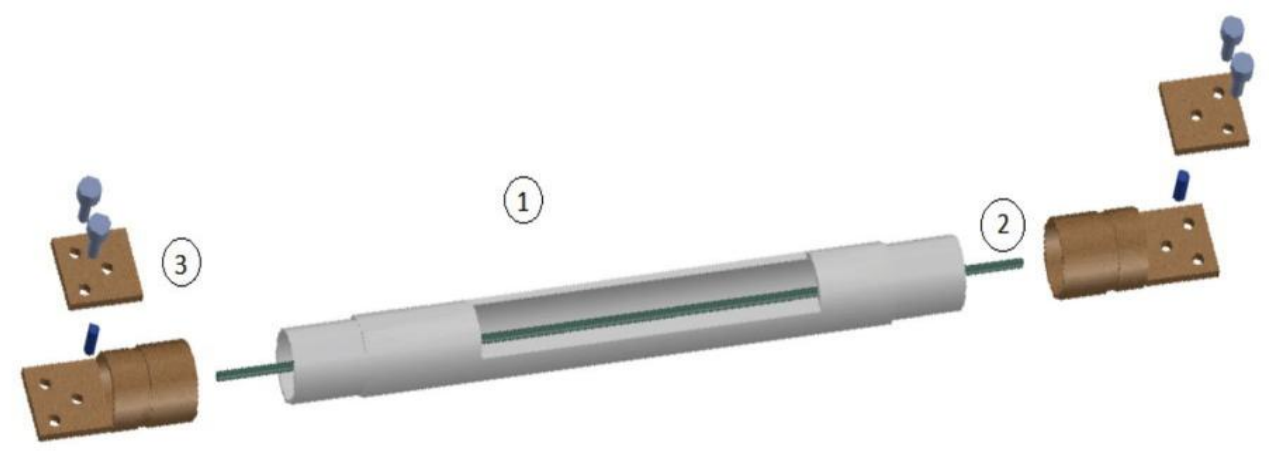

Figura 6.1. Sensor de hilo vibrante: tubo cerámico (1), hilo de tungsteno (2), abrazaderas de sujeción y pasador de guía (3).

A fin de evitar el desplazamiento del sensor de hilo vibrante dentro de la celda de presión, se adaptaron dos camisas de teflón para mantenerlo centrado en el campo magnético.

\subsubsection{Campo magnético.}

Para generar el campo magnético se hará por medio de un imán en forma de $\mathrm{C}$ de la marca Eclipse Magnetics Figura 6.2, el material de este es una aleación de aluminio, níquel, cobalto y hierro tipo 862 [15]. Las características de este tipo de imán favorecen los requerimientos para la construcción del equipo, tanto en temperatura de operación como en la longitud del campo magnético, teniendo este una longitud del campo magnético $L_{m}$ de $50 \mathrm{~mm}$, un ancho de $103.5 \mathrm{~mm}$, el alto de $111 \mathrm{~mm}$, un peso de $2.9 \mathrm{~kg}$, material Alcomax III, un valor magnético 2000 Gauss y una temperatura máxima de operación de $550{ }^{\circ} \mathrm{C}$.

a)

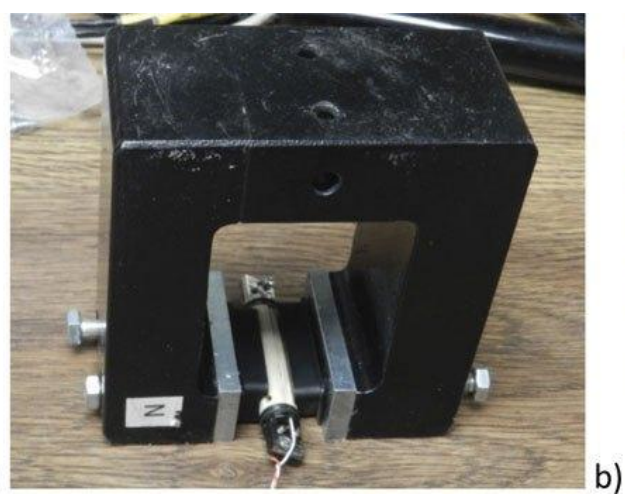

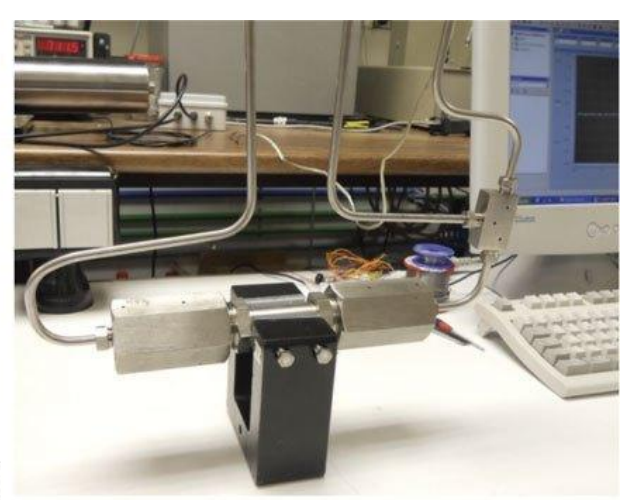

Figura. 6.2. Campo magnético: a) imán de dos polos, b) centrado y orientación de la celda de presión.

\subsubsection{Conexión eléctrica.}

El circuito eléctrico del viscosímetro de hilo vibrante está compuesto por cuatro hilos conductores; siendo un par de hilos los que suministran la corriente de excitación sinusoidal constante y los otros dos los que miden el voltaje inducido por el hilo vibrante. En su colocación se evita su paso por el interior del tubo cerámico. Tanto las conexiones eléctricas a la fuente de alimentación como las de medición, salen al exterior de la celda del circuito de presión a través de conectores de alta presión. 
Fuente de alimentación del sensor e interfaz. El voltaje de alimentación del circuito eléctrico del viscosímetro es suministrado, controlado y visualizado por un amplificador Lock-in marca Stanford Research Systems modelo SR380 DPS Figura 6.3, el cual además permite seleccionar los diferentes parámetros de intensidad de corriente alterna suministrada, el ángulo de la fase de incidencia, el tiempo de exposición y espera antes del cambio de la frecuencia y la sensibilidad de medición. Por otra parte, nos permite una visualización simultanea del voltaje inducido tanto en la componente real como la imaginaria y el barrido de frecuencias de oscilación.

a)
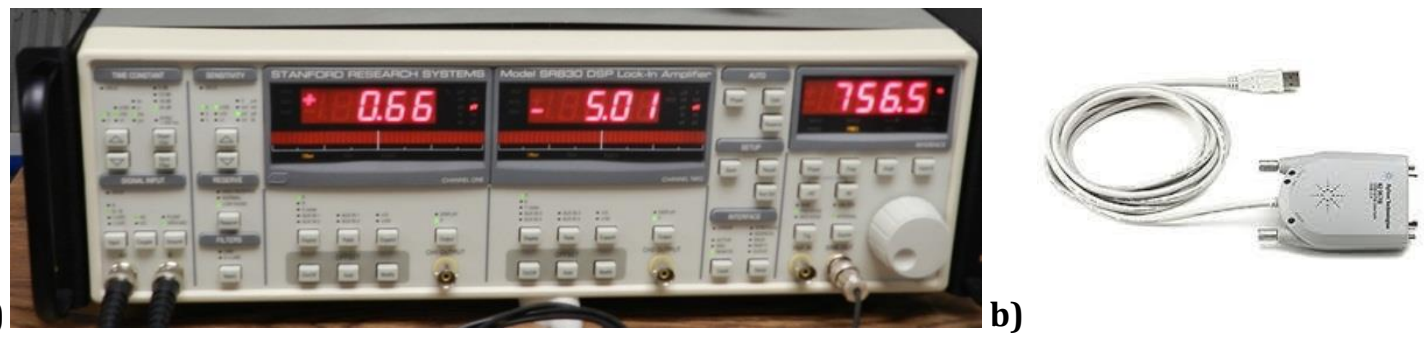

Figura 6.3. Fuente de alimentación: a) amplificador lock-in, b) interfaz USB-GPIB.

La fuente suministra una salida máxima de $5 \mathrm{~V}_{\mathrm{AC}}$. En este estudio por lo general se uso una sensibilidad de medición de $500 \mu \mathrm{V}$, una amplitud de $0.8 \mathrm{~Hz}$ con una constante de tiempo de 300 ms. Estos parámetros fueron elegidos en función de la mezcla a medir.

La interfaz con el ordenador se hace por medio de un adaptador USB-GPIB Agilent modelo 82357A Figura 6.3. b), permitiendo la adquisición de los datos medidos y la asignación de valores referenciales iniciales. La interfaz gráfica de control y procesamiento de datos se realiza con software Agilent VEE-Pro V7.0.

El amplificador lock-in dispone de una fuente de tensión constante alterna $V$ que a través de una resistencia en serie $R$ de $1 \mathrm{k} \Omega$ excita el hilo vibrante, que tiene una resistencia $R s$ de menos de $1 \Omega$. La corriente que circula por el hilo es determinada por la entrada diferencial B-A del amplificador lock-in.

a)

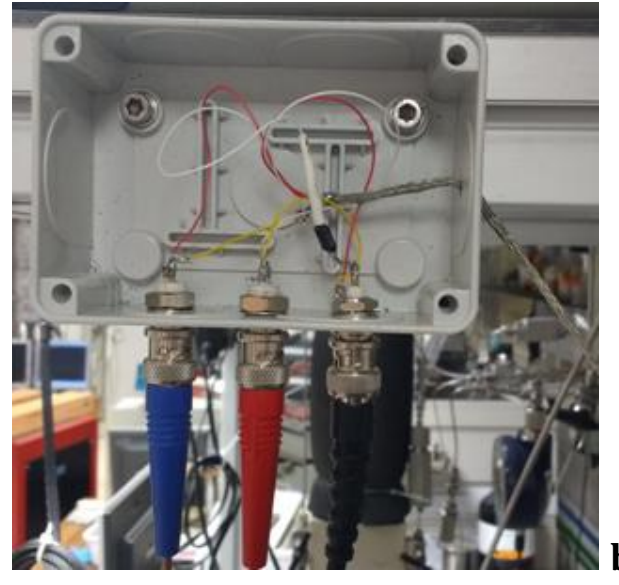

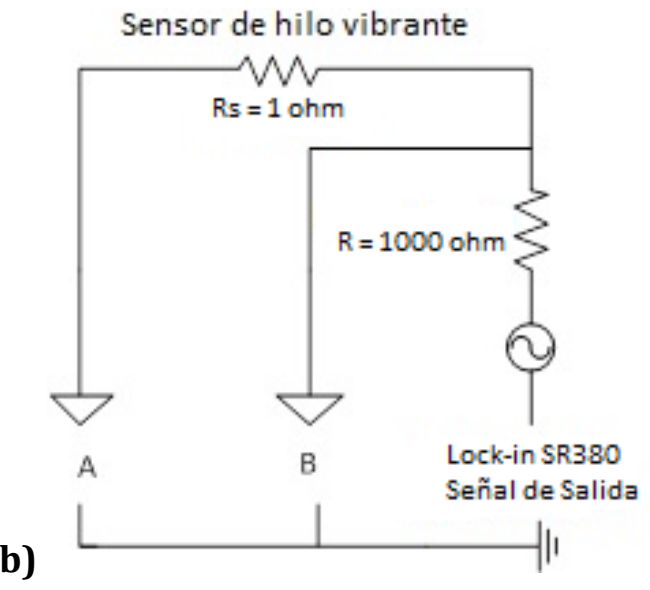

Figura 6.4. Circuito eléctrico y conexión del hilo vibrante: a) resistencia de $1 \mathrm{k} \Omega$, b) esquema de conexión. 
Detección de señal. El hilo alimentado con una corriente alterna y bajo la acción de un campo magnético genera una oscilación. De esta forma actuará una fuerza de Lorentz en dirección alterna sobre el hilo provocando su oscilación forzada. Este movimiento genera un voltaje inducido de acuerdo con la Ley de Faraday que se puede detectar a través del amplificador lock-in, Figura 6.3, capaz de medir señales pequeñas de corriente alterna. Este amplificador produce una señal continua amplificando la entrada y mezclándola con una señal de referencia. Esta técnica proporcionará un ancho de banda reducido con una amplificación de las señales pequeñas, además de la eliminación del ruido y señales de otras frecuencias.

\subsubsection{Control de temperatura.}

La celda de presión junto con el imán se encuentran sumergidos en un baño termostático, marca Hart Scientific modelo 5004 iso-therm, con un rango de temperatura de $20^{\circ} \mathrm{C}$ a 300 ${ }^{\circ} \mathrm{C}$ y una estabilidad de $\pm 0.01{ }^{\circ} \mathrm{C}$. El fluido de trabajo del baño puede ser mezcla agua-glicol hasta $100^{\circ} \mathrm{C}$ o aceites con base de silicona para mayores temperaturas.

Para la mejora de la estabilidad y la posibilidad de trabajar a temperaturas menores o iguales a $40{ }^{\circ} \mathrm{C}$, este baño lleva acoplada una refrigeración a través de un segundo baño externo marca JULABO modelo F25 HE.

La medida de la temperatura se realiza con un equipo ASL F-100 y dos sondas termométricas de platino Pt100, dentro del baño, calibradas en el Laboratorio Termocal. La incertidumbre obtenida es de $0.02 \mathrm{~K}$ (factor de cobertura $k=2$ ), en el rango de $-40{ }^{\circ} \mathrm{C} \mathrm{a}$ $230{ }^{\circ} \mathrm{C}$.

\subsubsection{Circuito de presión.}

El sensor de hilo vibrante se encuentra alojado en el interior de una celda de presión. La celda tiene unas dimensiones de $110 \mathrm{~mm}$ de longitud, un diámetro exterior de $18 \mathrm{~mm}$ y uno interno de $11.8 \mathrm{~mm}$. Está fabricada en acero inoxidable tipo 316L.

Todo el circuito de presión está construido con tubería de 1/4" de acero inoxidable HiP 209M4-316SS. El generador de presión es un pistón modelo 68-5.75-10 de la marca HiP, con un volumen máximo de $35 \mathrm{~cm}^{3}$ y una presión máxima de trabajo de $140 \mathrm{MPa}$. Figura 6.5.

El sensor de presión del sistema es un manómetro digital marca General Electric DRUCK modelo DPI 104, con resolución de 0.1 bar en todo el rango de medición (de 1.0 a 1400.0 bar). Este manómetro fue calibrado en el Laboratorio Termocal con una incertidumbre de $0.02 \%$ (factor de cobertura $k=2$ ) Figura 6.5. 
a)

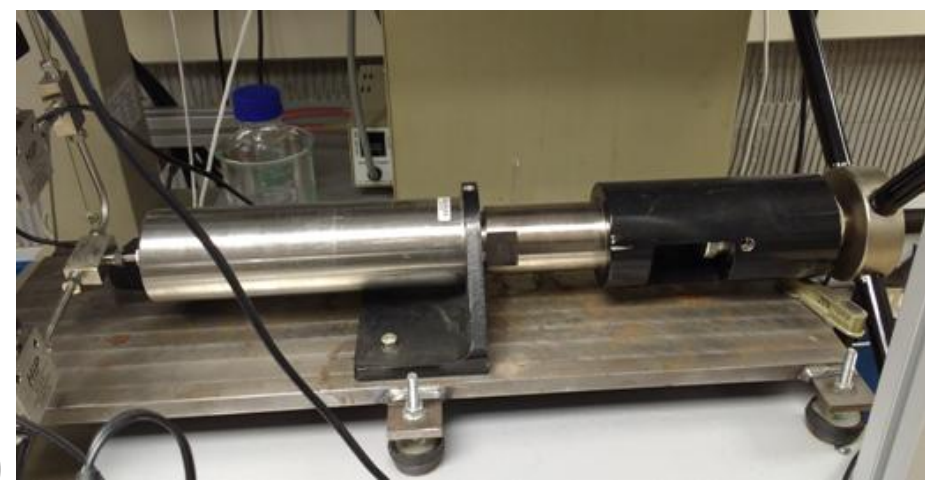

b)

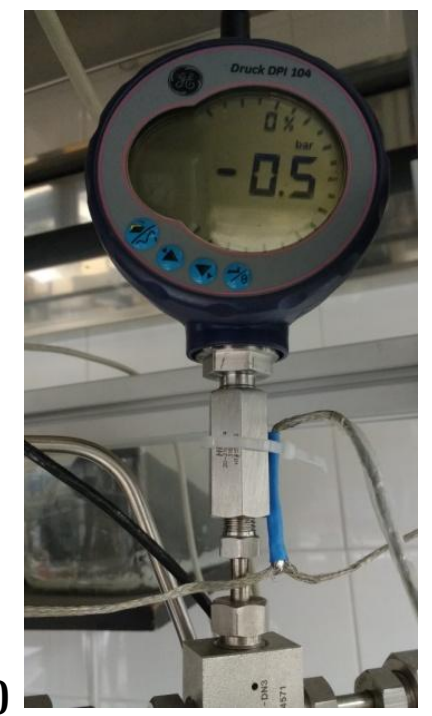

Figura 6.5. a) Pistón de presión, b) sensor de presión.

El circuito se encuentra en forma de U para facilitar su introducción en el baño termostatizado Figura 6.6.

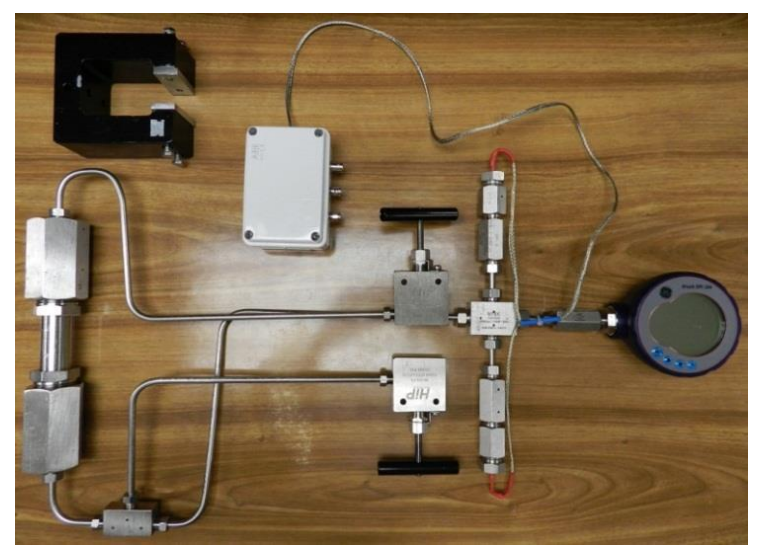

Figura 6.6. Circuito de presión armado en forma de $U$.

Para asegurar el llenado a una presión constante, se encuentra instalada una válvula de contrapresión Mity-Mite modelo S-91XW situada en la parte final del circuito. La contrapresión aplicada es de 50 bar. Figura 6.7.

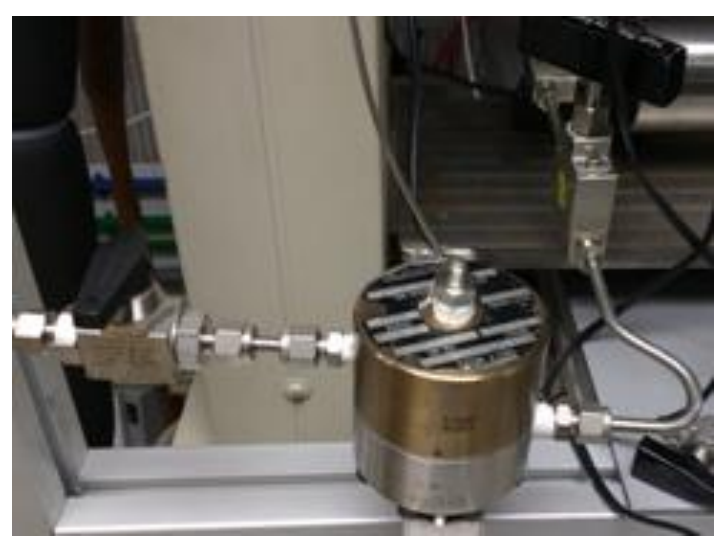

Figura 6.7. Válvula de contrapresión Mity-Mite modelo S-91XW. 


\section{Sistema de llenado, limpieza y vacío.}

En el caso de la medida de viscosidad de los compuestos puros, el llenado de la muestra se realiza por succión. La muestra se encuentra desgasificada a presión atmosférica en un embudo de decantación Pirex, que dispone de una adaptación formada por un tubo de teflón acoplado a un tubo HiP 1/4" con rosca para conectarse a las válvulas HiP. Previamente al succionado de la muestra, se ha de realizar una limpieza del circuito de presión y vacío en el sistema.

El sistema de vacío está compuesto por un vacuómetro Leybold Vakuum Thermovac, una trampa fría con nitrógeno líquido y una bomba rotativa de vacío Leybold Trivac. El sistema permite alcanzar un vacío de $2.5 \mathrm{~Pa}$.

\section{Bombas de inyección.}

El circuito de presión dispone de una conexión adicional para realizar mezclas multicomponentes, como es el caso de los hidrocarburos con $\mathrm{CO}_{2}$. Esto se realiza mediante dos bombas de jeringa marca ISCO Syringe Pump 260D (Figura 6.8), con cilindros de 500 mL cada una y rango de presión de 0 a 500 bar. La bomba de $\mathrm{CO}_{2}$ se encuentra a una temperatura y presión constante de $0{ }^{\circ} \mathrm{C}$ y 55 bar, y la bomba del segundo componente (el hidrocarburo puro previamente desgasificado) se encuentra a una temperatura y presión constante de $10{ }^{\circ} \mathrm{C}$ y 55 bar. En la Figura 6.9 se presenta el esquema general de las conexiones de la técnica del viscosímetro de hilo vibrante y los equipos que la integran.

a)

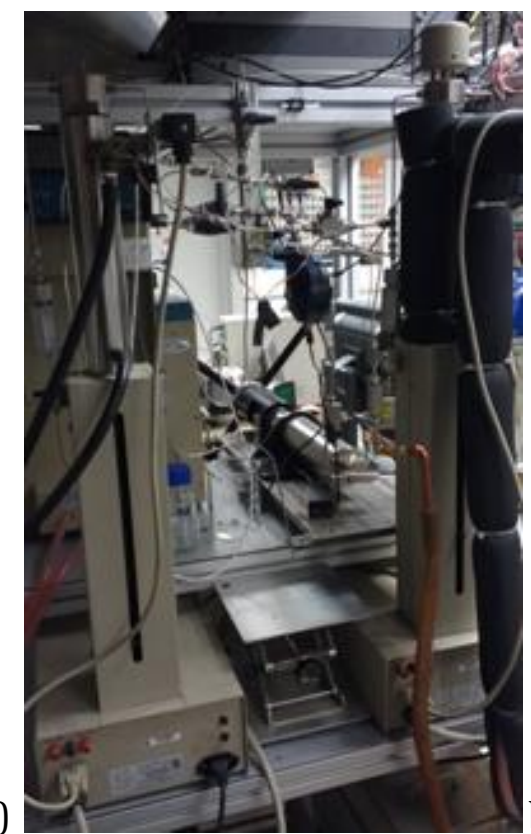

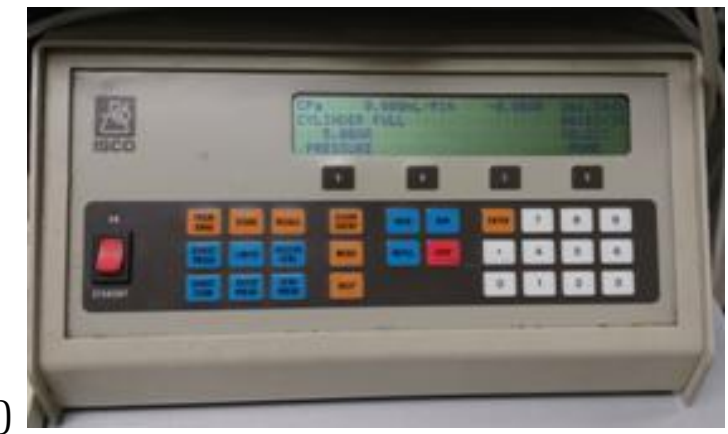

Figura 6.8. Bombas de jeringa ISCO para mezclas de fluidos y su controlador. 


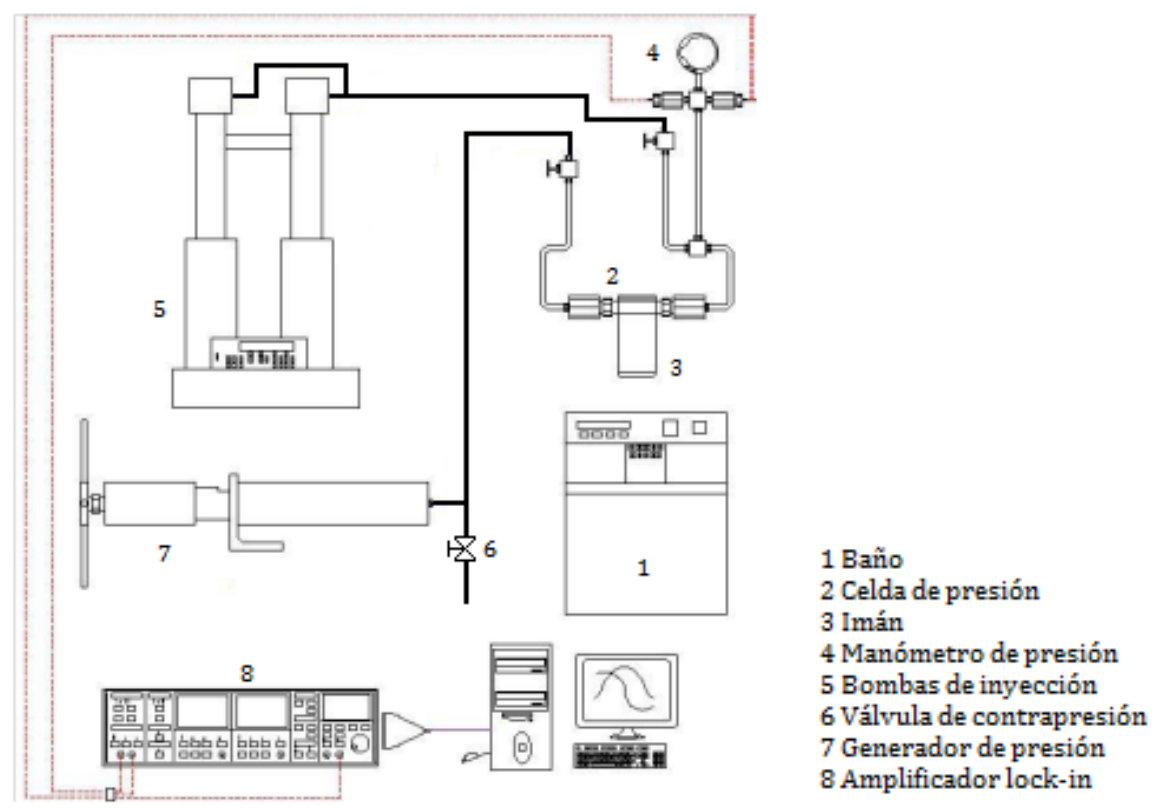

Figura 6.9. Esquema de conexiones de la técnica del viscosímetro de hilo vibrante para mezclas de fluidos.

\subsection{Procedimiento de medida de la viscosidad de mezclas complejas.}

\section{Ajuste (Tuning) de la señal.}

Es necesario verificar la alineación del hilo con el campo magnético y su tensión, de esta forma tendremos una señal de respuesta (voltaje inducido frente a frecuencia de oscilación) simétrica respecto al "eje $\mathrm{Y}$ " en la frecuencia de resonancia $f_{r}$. Los voltajes reales inducidos $\mathrm{X}$, así como simétricamente opuesta respecto al "eje X" en la $f_{r}$ para los voltajes imaginarios inducidos Y, Figura 6.10. También se puede observar el ancho de banda $f_{b}$. La componente imaginaria se ajusta al perfil de cambio de pendiente en tres puntos (los puntos extremos marcan el ancho de banda $f_{b}$ ideal y en el punto medio, para $f_{r}$, el voltaje imaginario inducido es cero).

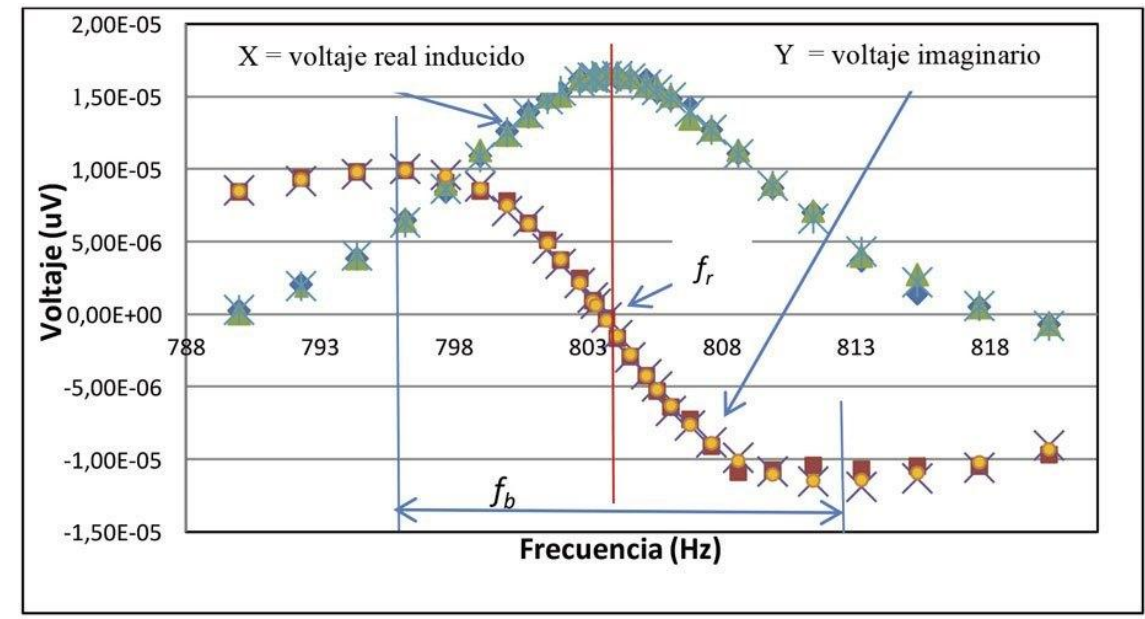

Figura 6.10. Gráfica de los voltajes reales e imaginarios inducidos respecto a la frecuencia de oscilación del hilo vibrante. 


\section{Preparación de muestras, llenado del equipo y vaciado.}

Los compuestos puros se desgasificaron previamente en un baño de ultrasonidos marca Branson, modelo DTH-3210, con una frecuencia de $47 \mathrm{kHz}$ y una potencia de $335 \mathrm{~W}$. El tiempo en desgasificar nunca es inferior a 5 horas. El compuesto seleccionado una vez desgasificado, es cargado en la bomba de jeringa, y fijando unas condiciones de presión y temperatura adecuadas para la carga del viscosímetro. El $\mathrm{CO}_{2}$ puro es cargado directamente desde la botella a la bomba de jeringa, donde se encuentra a unas condiciones de presión y temperatura de 50 bar y $0{ }^{\circ} \mathrm{C}$ (estado líquido).

Una vez que se alcanza un vacío en el equipo inferior a $5 \mathrm{~Pa}$ y la temperatura y presión de las bombas de jeringa permanecen estables, se procede al llenado del circuito de presión. Se conecta, a flujo constante la bomba que contiene el $\mathrm{CO}_{2}$ y se abre la válvula que comunica con la $\mathrm{T}$ de mezcla.

Una vez lleno el circuito con $\mathrm{CO}_{2}$, se procede a poner en marcha la segunda bomba de jeringa, enviando el otro compuesto a la $\mathrm{T}$ de mezcla. Se espera que pase a través del equipo un volumen lo suficientemente grande que asegure la homogeneización de los compuestos.

\section{Software de medida.}

La automatización del viscosímetro se ha desarrollado en el software Agilent VEE-Pro V7.0. El programa "Quick Scan modified" sirve para explorar de manera rápida la frecuencia de resonancia $\left(f_{r}\right)$ aproximada. El programa "Fit for know density $(T, p)$ " sirve para calcular la viscosidad dinámica y el programa "Find Radius" para encontrar el "verdadero" radio del hilo como parte de la calibración de la técnica (Figuras 6.11, 6.12 y 6.13 , respectivamente).

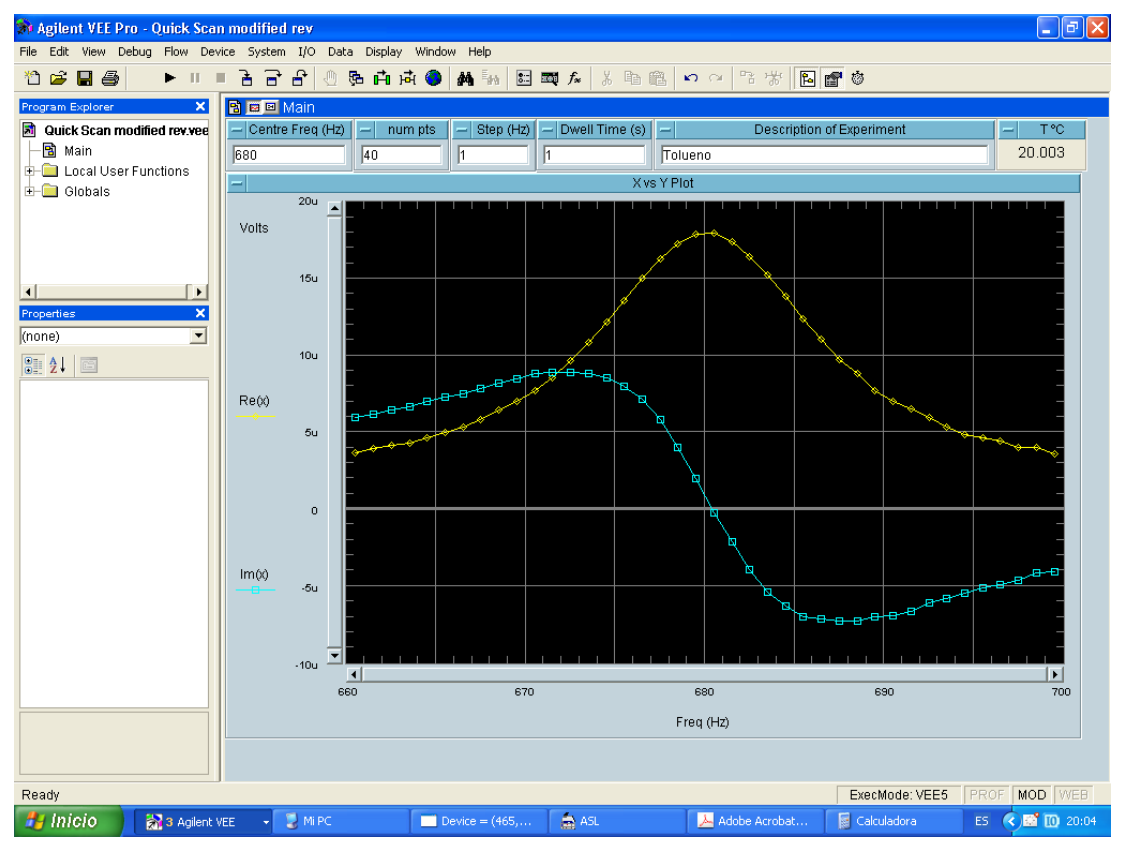

Figura 6.11. Programa Quick Scan modified; explora la frecuencia de resonancia $\left(f_{r}\right)$ aproximada. 


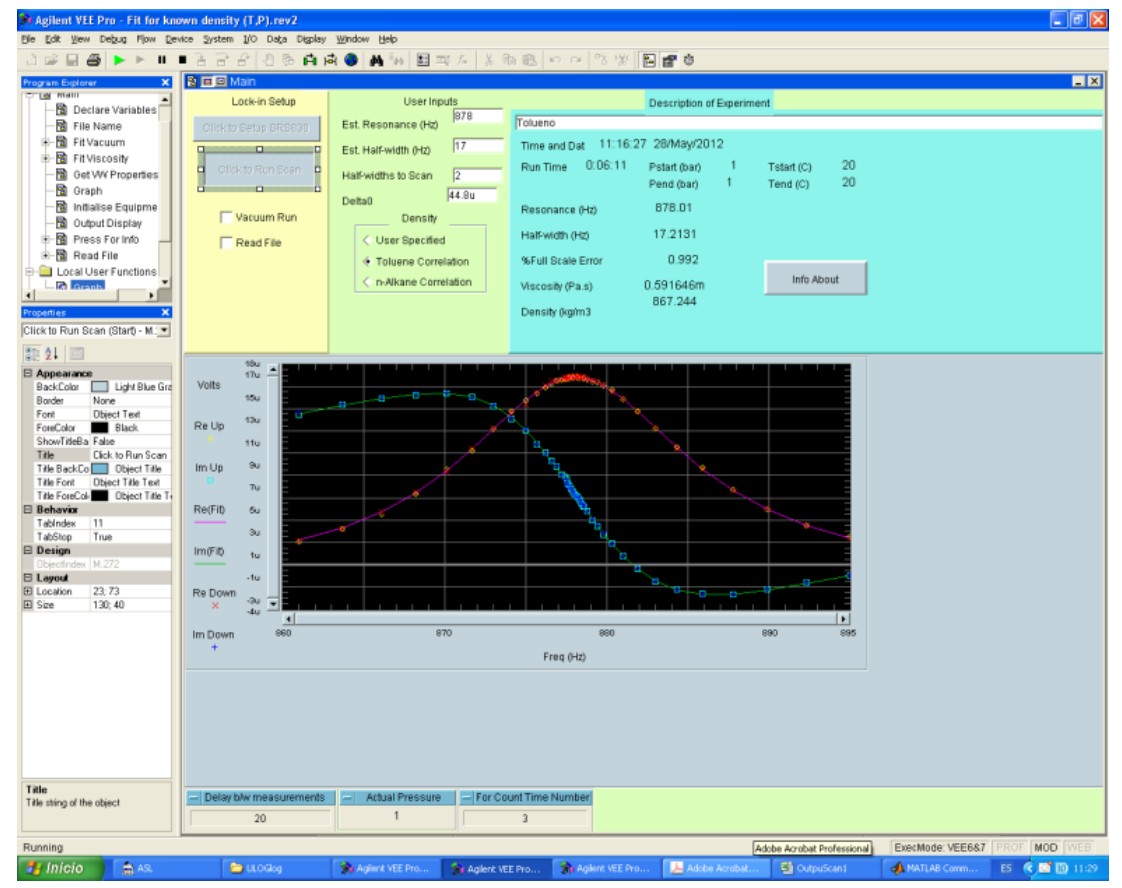

Figura 6.12. Programa Fit for know density $(T, p)$; calcula la viscosidad dinámica.

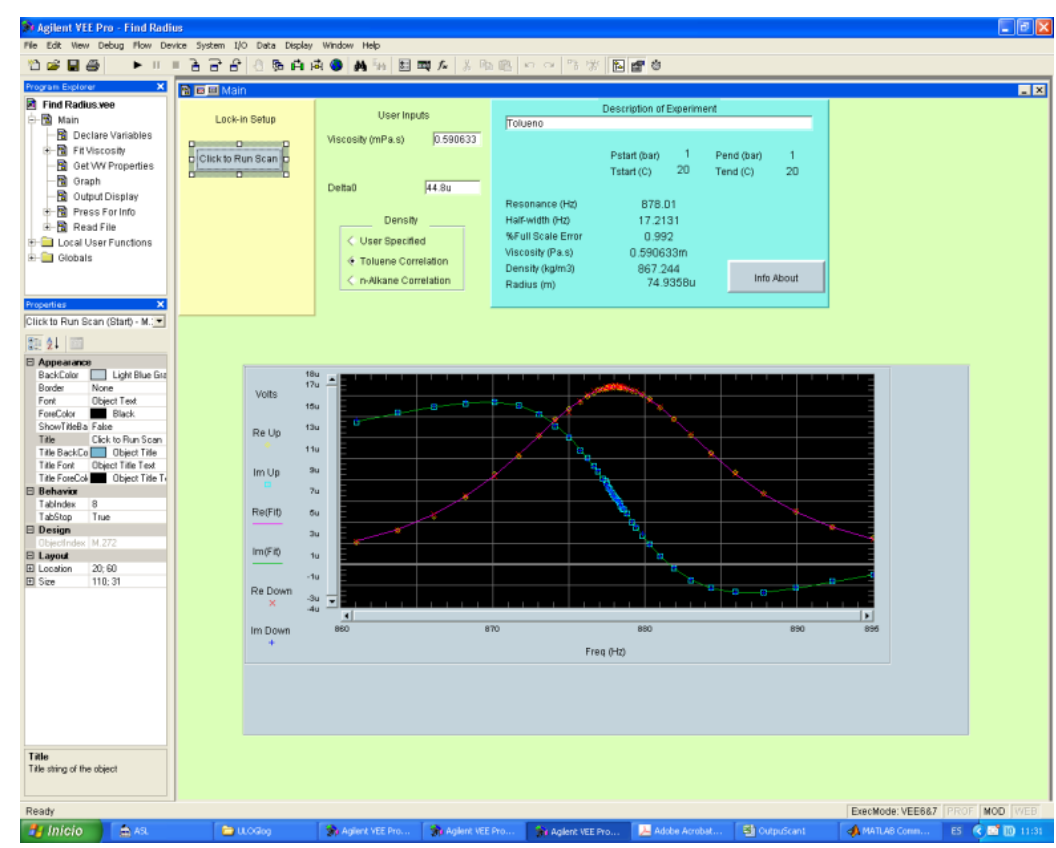

Figura 6.13. Programa Find Radius; para encontrar el radio del hilo como parte de la calibración de la técnica.

El programa "Quick Scan modified" requiere del conocimiento de la frecuencia de resonancia estimada, calculada conociendo la fuerza aplicada en el sensor o a partir de la $f_{o}$ y después de realizar un barrido de frecuencia, proporciona la frecuencia de resonancia $\left(f_{r}\right)$ aproximada. A una determinada temperatura o isoterma, la frecuencia de resonancia será menor que la $f_{o}$ según se incremente la presión en el sistema. Para una misma presión o isóbara, la frecuencia de resonancia será mayor que la $f_{o}$ según se incremente la 
temperatura en el sistema. Con el barrido de frecuencia se estima el ancho de banda $\left(f_{b}\right)$, definido como las frecuencias donde la amplitud del voltaje inducido es $\frac{1}{\sqrt{2}}$ de la frecuencia de resonancia.

El programa "Fit for know density $(T, p)$ ", para calcular la $\eta$ requiere de cuatro entradas: (1) la frecuencia de resonancia $\left(f_{r}\right)$ obtenida con el programa de exploración rápida; (2) el ancho de banda medio $\left(f_{b}\right)$ estimado de la exploración rápida, después de calcular la $\eta$ el programa proporciona un valor preciso de $f_{b}$; (3) el número de anchos de banda medio a escanear ( 4 anchos de banda medio son analizados en forma normal porque no hay señal más allá de este límite), y (4) la densidad del fluido objeto de medición (se introduce manualmente o el programa la proporciona mediante ecuaciones de estado para un determinado número de compuestos). El software realiza dos barridas completas, una con frecuencia creciente y otra con frecuencia decreciente, registrando los voltajes inducidos, reales e imaginarios, en el hilo vibrante. Como se observa en la Figura 6.12, se ajustan los valores a una Lorentziana del voltaje inducido. Cuando la desviación estándar $\sigma$, de cinco medidas en viscosidad, es menor que el $1 \%$, se toma el valor medio.

La determinación de la viscosidad dinámica de los compuestos puros y sus mezclas, con la técnica del viscosímetro de hilo vibrante, requiere del conocimiento de la densidad de los fluidos seleccionados para el desarrollo de la investigación. Siendo necesario la realización de las medidas tanto de densidad para la completa caracterización de las mezclas con $\mathrm{CO}_{2}$. Las medidas de densidad se realizaron con el densímetro de tubo vibrante Anton Paar HPM descrito anteriormente en el apartado 4.7.

\section{Calibración del sensor de hilo vibrante.}

El viscosímetro de hilo vibrante es un dispositivo absoluto, que en teoría no requiere calibración a partir de fluidos de referencia. Sin embargo, en la práctica, el radio del hilo $R$ y el amortiguamiento interno (o decremento logarítmico natural en vacío) $\Delta_{o}$ no se pueden establecer con suficiente precisión por métodos independientes, de ahí que estos dos parámetros se fijan mediante calibración. Esta calibración inicial y la obtención del verdadero radio del hilo, fue realizada previamente en la tesis de J. Zambrano [1].

En este trabajo se realizó una nueva calibración de la constante de amortiguamiento $\left(\Delta_{o}\right)$, realizando un buen vacio en el equipo. Mediante la opción Vacuum Run del programa "Fit for know density", se obtuvo la nueva $\Delta_{o}$ que será la utilizada en nuestro estudio. En nuestro caso la constante aumentó, pasando de $44.8 \cdot 10^{-6}$ a $106.8 \cdot 10^{-6}$.

Para la nueva calibración del radio del hilo $R_{w}$ se utilizó tolueno, ya que sus propiedades son conocidas. La medida se realiza a una determinada temperatura y presión con valores de viscosidad y densidad del tolueno reportadas por Assael et al. [8]. En nuestro caso se midió a $293.15 \mathrm{~K}$ y $0.1 \mathrm{MPa}$. Como resultado se ha comprobado que el radio del hilo no ha cambiado respecto al valor dado en [1].

Los resultados de la calibración se encuentran en la Tabla 6.1. Siendo $\Delta_{o}$ la nueva constante de amortiguamiento natural del hilo obtenida en este estudio. No variando el resto de los parámetros dados para la calibración del hilo. 


\begin{tabular}{|l|l|r|}
\hline Radio nominal del hilo & $R(\mu \mathrm{m})$ & 75 \\
\hline Radio medio del hilo calibrado & $R_{W}(\mu \mathrm{m})$ & 74.86 \\
\hline Longitud del hilo & $L(\mathrm{~mm})$ & 50 \\
\hline $\begin{array}{l}\text { Frecuencia de resonancia en } \\
\text { vacío }\end{array}$ & $f_{o}(\mathrm{~Hz})$ & 829.093 \\
\hline $\begin{array}{l}\text { Constante de amortiguamiento } \\
\text { natural del hilo }\end{array}$ & $\Delta_{o} \times 10^{-6}$ & 106.8 \\
\hline Densidad del hilo & $\rho_{s}\left(\mathrm{~kg} / \mathrm{m}^{3}\right)$ & 19300 \\
\hline Radio del hilo calibrado $\left(20^{\circ} \mathrm{C}\right)$ & $R_{w}(\mu \mathrm{m})$ & 75.08 \\
\hline $\begin{array}{l}\text { Frecuencia de resonancia en } \\
\text { fluido de calibración (tolueno) }\end{array}$ & $f_{r}(\mathrm{~Hz})$ & 803.121 \\
\hline Ancho de banda & $f_{b}(\mathrm{~Hz})$ & 18.513 \\
\hline $\begin{array}{l}\text { Densidad del fluido de } \\
\text { calibración (tolueno) }\end{array}$ & $\rho(\mathrm{kg} \mathrm{m}-3)$ & 867.24 \\
\hline $\begin{array}{l}\text { Viscosidad dinámica del } \\
\text { tolueno }\end{array}$ & \multicolumn{1}{|c|}{$\eta(m P a \cdot s)=\frac{\pi f_{r} R_{W}^{2} \rho}{6}\left(\frac{f_{b}}{f_{r}}\right)^{2}\left(1+\frac{\rho_{s}}{\rho}\right)^{2}$} & 0.5907 \\
\hline
\end{tabular}

Tabla 6.1. Resultados de calibración del hilo vibrante y cálculo de $\eta$ del tolueno a 293.15 Ky 0.1 MPa.

Finalizada la calibración se midió la viscosidad del tolueno a una presión de 10 bar y una temperatura de $25{ }^{\circ} \mathrm{C}$, comparando los resultados con REFPROP y comprobando que la desviación de los datos experimentales no excedía del 1.5\%. El tolueno utilizado es de la marca Sigma Aldrich con una pureza $\geq 99.8 \%$. Las propiedades de referencia, tanto de la $\rho$ como de la $\eta$, se tomaron del REFPROP. Las máximas desviaciones respecto al REFPROP fueron de $1.17 \%$ y la desviación media obtenida ( $A A D \%$ ) fue de $0.75 \%$.

\section{Cálculo de la incertidumbre del equipo.}

Se ha realizado el cálculo de la incertidumbre del viscosímetro de hilo vibrante desarrollado en el Laboratorio Termocal en base al documento GUM 2008 [16]; guía para el cálculo de incertidumbres de medida del Bureau Internacional de Pesas y Medidas (BIPM).

La incertidumbre en la composición de $\mathrm{CO}_{2}$ de la mezcla con el hidrocarburo, está asociada a las densidades de los compuestos de cada bomba y los caudales de dichas bombas, siendo la incertidumbre del caudal de las bombas un $0.5 \%$.

Las incertidumbres de las densidades de los compuestos asociadas a las condiciones de temperatura y presión de cada bomba se representan en la Tabla 6.2. 
Tabla 6.2. Cálculo de la incertidumbre asociada a la densidad de cada compuesto en las bombas en las condiciones de inyección de la mezcla.

\begin{tabular}{|c|c|c|c|c|c|}
\hline & & Unidad & Estimación & Divisor & $\mathrm{u}(x)$ \\
\hline & & & & & $\mathrm{kg} \cdot \mathrm{m}^{-3}$ \\
\hline \multirow{2}{*}{$\mathrm{u}(T) \mathrm{CO}_{2}$} & Calibración & \multirow{2}{*}{${ }^{\circ} \mathrm{C}$} & 0.02 & 2 & \multirow{2}{*}{0.064} \\
\hline & Resolución & & 0.01 & $2 \sqrt{3}$ & \\
\hline \multirow{2}{*}{$\mathrm{u}(p) \mathrm{CO}_{2}$} & Calibración & \multirow{2}{*}{$\mathrm{MPa}$} & 0.02 & 2 & \multirow{2}{*}{0.00005} \\
\hline & Resolución & & 0.01 & $2 \sqrt{3}$ & \\
\hline $\mathrm{u}(\rho) \mathrm{CO}_{2}$ & & \multicolumn{2}{|l|}{$\mathrm{kg} \cdot \mathrm{m}^{-3}$} & $\mathrm{k}=1$ & 0.06 \\
\hline $\mathrm{U}(\rho) \mathrm{CO}_{2}$ & & \multicolumn{2}{|l|}{$\mathrm{kg} \cdot \mathrm{m}^{-3}$} & $\mathrm{k}=2$ & 0.13 \\
\hline
\end{tabular}

\begin{tabular}{|c|c|c|c|c|c|}
\hline & & Unidad & Estimación & Divisor & $\mathrm{u}(x)$ \\
\hline & & & & & $\mathrm{kg} \cdot \mathrm{m}^{-3}$ \\
\hline \multirow{2}{*}{ u(T) Hidrocarburo } & Calibración & \multirow{2}{*}{${ }^{\circ} \mathrm{C}$} & 0.02 & 2 & \multirow{2}{*}{0.009} \\
\hline & Resolución & & 0.01 & $2 \sqrt{3}$ & \\
\hline \multirow{2}{*}{$\mathrm{u}(p)$ Hidrocarburo } & Calibración & \multirow{2}{*}{$\mathrm{MPa}$} & 0.02 & 2 & \multirow{2}{*}{0.000009} \\
\hline & Resolución & & 0.01 & $2 \sqrt{3}$ & \\
\hline \multicolumn{2}{|l|}{$\mathrm{u}(\rho)$ Hidrocarburo } & \multicolumn{2}{|l|}{$\mathrm{kg} \cdot \mathrm{m}^{-3}$} & $\mathrm{k}=1$ & 0.009 \\
\hline \multicolumn{2}{|l|}{$\mathrm{U}(\rho)$ Hidrocarburo } & $\mathrm{kg} \cdot \mathrm{m}^{-3}$ & & $\mathrm{k}=2$ & 0.02 \\
\hline
\end{tabular}

En la Tabla 6.3. se representan las incertidumbres asociadas a la composición de la mezcla. La incertidumbre expandida con un factor de cobertura $k=2$ (95\% de confianza) en la fracción molar del $\mathrm{CO}_{2}$ de la mezcla es de 0.0013 .

Tabla 6.3. Cálculo de la incertidumbre asociada a la composición de la mezcla.

\begin{tabular}{|c|c|l|l|l|l|}
\hline & & Unidad & Estimación & Divisor & $\mathrm{u}(x)$ \\
\hline & & & & & $\mathrm{mol} / \mathrm{mol}$ \\
\hline $\mathrm{u}(\dot{m})$ Hidrocarburo & - & $\mathrm{g} / \mathrm{min}$ & 0.005 & 1 & 0.0004 \\
\hline $\mathrm{u}(\dot{m}) \mathrm{CO}_{2}$ & - & $\mathrm{g} / \mathrm{min}$ & 0.014 & 1 & 0.0004 \\
\hline $\mathrm{u}(x) \mathrm{CO}_{2}$ & $\mathrm{~mol} / \mathrm{mol}$ & $\mathrm{k}=1$ & 0.0006 \\
\hline $\mathrm{U}(x) \mathrm{CO}_{2}$ & $\mathrm{~mol} / \mathrm{mol}$ & $\mathrm{k}=2$ & 0.0013 \\
\hline
\end{tabular}

La ecuación base del cálculo de incertidumbre en la viscosidad es la función (6.9), que permite abordar la ley de propagación de varianzas con funciones explícitas:

$$
\eta \approx \frac{\pi f_{r} R_{w}^{2} \rho}{6}\left(\frac{f_{b}}{f_{r}}\right)^{2}\left(1+\frac{\rho_{s}}{\rho}\right)^{2}
$$

Esta función establece la relación entre la viscosidad del fluido dentro del sensor y la frecuencia de oscilación del hilo vibrante, en función de las variables: $f_{r}, f_{b}, R_{w}, \rho$ y $\rho_{s}$ (frecuencia de resonancia, ancho medio de banda, radio del hilo, densidad del fluido objeto de medición y densidad del hilo respectivamente). La ley de propagación de incertidumbres aplicada a (6.9) permite calcular la incertidumbre de la viscosidad a partir de las cinco variables. 
Para la viscosidad dinámica $\eta$, expresada como (6.9) en las condiciones experimentales $(T$, $p)$, la incertidumbre expandida $(k=2)$ es:

$$
\begin{aligned}
& U(\eta(T, p))=2\left[\left(\frac{\partial \eta(T, p)}{\partial f_{r}}\right)^{2} u^{2}\left(f_{r}\right)+\left(\frac{\partial \eta(T, p)}{\partial R_{W}}\right)^{2} u^{2}\left(R_{W}\right)+\left(\frac{\partial \eta(T, p)}{\partial \rho}\right)^{2} u^{2}(\rho)+\right. \\
& \left.\left(\frac{\partial \eta(T, p)}{\partial f_{b}}\right)^{2} u^{2}\left(f_{b}\right)+\left(\frac{\partial \eta(T, p)}{\partial \rho_{s}}\right)^{2} u^{2}\left(\rho_{s}\right)\right]^{0.5}
\end{aligned}
$$

Para facilitar la diferenciación de (6.9) respecto de cada una de las variables que depende y reemplazar en (6.12), se la expresará como:

$$
\eta(T, p)=\frac{\pi R_{w}^{2}}{6} \frac{f_{b}^{2}}{f_{r}} \frac{\left(\rho+\rho_{S}\right)^{2}}{\rho}
$$

Realizando la diferenciación de (6.13) respecto de las variables que depende, se tiene:

$$
\begin{gathered}
\frac{\partial \eta(T, p)}{\partial f_{r}}=-\frac{\pi R_{W}^{2}}{6} \frac{f_{b}^{2}}{\rho} \frac{\left(\rho+\rho_{s}\right)^{2}}{f_{r}^{2}}=-\frac{\eta}{f_{r}} \\
\frac{\partial \eta(T, p)}{\partial R_{W}}=\frac{2 \pi}{6} \frac{f_{b}^{2}}{f_{r}} \frac{\left(\rho+\rho_{s}\right)^{2} R_{W}}{\rho} \frac{R_{W}}{R_{w}}=-\frac{2 \eta}{R_{w}} \\
\frac{\partial \eta(T, p)}{\partial \rho}=\frac{\pi}{6} \frac{R_{w}^{2} f_{b}^{2}}{f_{r}}\left(1-\frac{\rho_{s}^{2}}{\rho^{2}}\right)=\frac{\eta\left(\rho-\rho_{S}\right)}{\rho\left(\rho+\rho_{s}\right)} \\
\frac{\partial \eta(T, p)}{\partial f_{b}}=\frac{2 \pi}{6} \frac{R_{W}{ }^{2}}{f_{r}} \frac{\left(\rho+\rho_{s}\right)^{2} f_{b}}{\rho} \frac{f_{b}}{f_{b}}=\frac{2 \eta}{f_{b}} \\
\frac{\partial \eta(T, p)}{\partial \rho_{s}}=\frac{2 \pi}{6} \frac{R_{W}^{2} f_{b}{ }^{2}}{f_{r}}\left(1+\frac{\rho_{s}}{\rho}\right)=\frac{2 \eta}{\left(\rho+\rho_{s}\right)}
\end{gathered}
$$

Reemplazando en (6.12), se tiene la expresión (6.19):

$$
\begin{aligned}
& U(\eta(T, p))=2 \eta\left[\left(-\frac{1}{f_{r}}\right)^{2} u^{2}\left(f_{r}\right)+\left(\frac{2}{R_{W}}\right)^{2} u^{2}\left(R_{W}\right)+\left(\frac{\left(\rho-\rho_{s}\right)}{\rho\left(\rho+\rho_{s}\right)}\right)^{2} u^{2}(\rho)+\right. \\
& \left.\left(\frac{2}{f_{b}}\right)^{2} u^{2}\left(f_{b}\right)+\left(\frac{2}{\left(\rho+\rho_{s}\right)}\right)^{2} u^{2}\left(\rho_{s}\right)\right]^{0.5}
\end{aligned}
$$

En la Tabla 6.4. se representan las incertidumbres asociadas a la medida de viscosidad a diferentes rangos de temperatura. La incertidumbre expandida con un factor de cobertura $k=2$ (95\% de confianza) en la medida de viscosidad para un intervalo de temperaturas de 293.15-373.15 K y presiones de 0.1-100 MPa es de 0.0032 mPa.s. 
Tabla 6.4. Cálculo de la incertidumbre asociada a la viscosidad experimental en un intervalo de temperaturas de (293.15-353.15) Ky presiones 0.1-100 MPa.

\begin{tabular}{|c|c|c|c|c|c|}
\hline & & Unidad & Estimación & Divisor & $\mathrm{u}(x)$ \\
\hline & & & & & $\mathrm{mPa} \cdot \mathrm{s}$ \\
\hline \multirow{3}{*}{$\mathrm{u}\left(f_{\mathrm{r}}\right)$} & Calibración & \multirow{3}{*}{$\mathrm{Hz}$} & 0.010 & 2 & \multirow{3}{*}{0.000006} \\
\hline & Repetibilidad & & 0.0010 & $2 \sqrt{3}$ & \\
\hline & Resolución & & 0.04 & 1 & \\
\hline \multirow{3}{*}{$\mathrm{u}(T)$} & Calibración & \multirow{3}{*}{${ }^{\circ} \mathrm{C}$} & 0.020 & 2 & \multirow{3}{*}{0.000048} \\
\hline & Resolución & & 0.010 & $2 \sqrt{3}$ & \\
\hline & Repetibilidad & & 0.03 & 1 & \\
\hline \multirow{3}{*}{$\mathrm{u}(p)$} & Calibración & \multirow{3}{*}{$\mathrm{MPa}$} & 0.02 & 2 & \multirow{3}{*}{0.000025} \\
\hline & Resolución & & 0.01 & $2 \sqrt{3}$ & \\
\hline & Repetibilidad & & 0.00 & 1 & \\
\hline \multirow{2}{*}{$\mathrm{u}(\rho)$} & Sólido & $\mathrm{kg} \cdot \mathrm{m}^{-3}$ & 19.0 & 2 & 0.0003 \\
\hline & Fluido & $\mathrm{kg} \cdot \mathrm{m}^{-3}$ & 4.7 & 2 & 0.0011 \\
\hline $\begin{array}{c}\text { Calibración } \\
\text { del radio }\end{array}$ & & $\mathrm{mm}$ & 0.08 & 2 & 0.0004 \\
\hline \multirow{3}{*}{$\mathrm{u}\left(f_{\mathrm{b}}\right)$} & Calibración & \multirow{3}{*}{$\mathrm{Hz}$} & 0.01 & 2 & \multirow{3}{*}{0.001} \\
\hline & Repetibilidad & & 0.018 & 1 & \\
\hline & Resolución & & 0.001 & $2 \sqrt{3}$ & \\
\hline \multicolumn{2}{|l|}{$\mathrm{u}(\eta)$} & \multicolumn{2}{|l|}{$\mathrm{mPa} \cdot \mathrm{s}$} & $\mathrm{k}=1$ & 0.0016 \\
\hline \multicolumn{2}{|l|}{$\mathrm{U}(\eta)$} & \multicolumn{2}{|l|}{$\mathrm{mPa} \cdot \mathrm{s}$} & $\mathrm{k}=2$ & 0.0032 \\
\hline \multicolumn{2}{|l|}{$\mathrm{U}_{\mathrm{r}}(\eta)$} & \multicolumn{2}{|c|}{$100 \cdot(\mathrm{mPa} \cdot \mathrm{s} / \mathrm{mPa} \cdot \mathrm{s})$} & $\mathrm{k}=2$ & $1.5 \%$ \\
\hline
\end{tabular}

En la Tabla 6.4. se observa que la mayor contribución a la incertidumbre proviene de la densidad del fluido de calibración y la frecuencia del ancho de banda.

\subsection{Descripción del método de medida utilizado para la determinación de la densidad de mezclas $\mathrm{CO}_{2}+$ hidrocarburo.}

La medida de las densidades de mezclas $\mathrm{CO}_{2}+$ hidrocarburo se hizo con un densímetro de tubo vibrante inicialmente descrito en el apartado 4.7. Este equipo no tuvo que ser reformado para estas medidas y consta de las partes anteriormente descritas.

\subsection{Incertidumbre en la medida.}

El cálculo de la incertidumbre se realizó en base al documento GUM 2008 [16], que es la guía para el cálculo de incertidumbres de medida del Oficina Internacional de Pesas y Medidas (BIMP).

La incertidumbre de la composición de $\mathrm{CO}_{2}$ de la mezcla con el hidrocarburo, está asociada a las densidades de los compuestos de cada bomba y los caudales de dichas bombas. Siendo la incertidumbre del caudal para las bombas de $0.5 \%$. 
Las incertidumbres de las densidades de los compuestos asociadas a las condiciones de temperatura y presión de cada bomba se presentan en la Tabla 6.2.

La incertidumbre expandida de la composición de la mezcla con un factor de cobertura $k=2$ (95\% de confianza) en la fracción molar del $\mathrm{CO}_{2}$ de la mezcla es de 0.0034 y se presenta en la Tabla 6.5.

Tabla 6.5. Cálculo de la incertidumbre asociada a la composición de la mezcla.

\begin{tabular}{|c|c|l|l|l|l|}
\hline & & Unidad & Estimación & Divisor & $\mathrm{u}(x)$ \\
\hline & & & & & $\mathrm{mol} / \mathrm{mol}$ \\
\hline $\mathrm{u}(\dot{m})$ Hidrocarburo & - & $\mathrm{g} / \mathrm{min}$ & 0.005 & 1 & 0.0012 \\
\hline $\mathrm{u}(\dot{m}) \mathrm{CO}_{2}$ & - & $\mathrm{g} / \mathrm{min}$ & 0.014 & 1 & 0.0012 \\
\hline $\mathrm{u}(x) \mathrm{CO}_{2}$ & $\mathrm{~mol} / \mathrm{mol}$ & $\mathrm{k}=1$ & 0.0017 \\
\hline $\mathrm{U}(x) \mathrm{CO}_{2}$ & $\mathrm{~mol} / \mathrm{mol}$ & $\mathrm{k}=2$ & 0.0034 \\
\hline
\end{tabular}

Las tablas 6.6. y 6.7. presentan las incertidumbres de medida de la densidad. La incertidumbre expandida con un factor de cobertura $k=2$ (95\% de confianza) para un intervalo de temperaturas de 273.15-373.15 K y presiones de 0.1-140 MPa es de 0.7 $\mathrm{kg} / \mathrm{m}^{3}$.

Tabla 6.6. Cálculo de la incertidumbre asociada a los parámetros de calibración en un intervalo de temperaturas de (273.15-373.15) Ky presiones 0.1-140 MPa.

\begin{tabular}{|c|c|c|c|c|c|}
\hline & & Unidad & Estimación & Divisor & $\mathrm{u}(x)$ \\
\hline & & & & & $\mathrm{kg} \cdot \mathrm{m}^{-3}$ \\
\hline $\mathrm{u}\left(\rho_{\mathrm{w}}\right)$ & $\begin{array}{c}\text { Referencia } \\
\text { material }\end{array}$ & $\mathrm{kg} \cdot \mathrm{m}^{-3}$ & 0.01 & $\sqrt{3}$ & 0.0058 \\
\hline \multirow{3}{*}{$\mathrm{u}(T)$} & Calibración & \multirow{3}{*}{${ }^{\circ} \mathrm{C}$} & 0.020 & 2 & \multirow{3}{*}{0.0046} \\
\hline & Resolución & & 0.010 & $2 \sqrt{3}$ & \\
\hline & Repetibilidad & & 0.0008 & 1 & \\
\hline \multirow{3}{*}{$\mathrm{u}(p)$} & Calibración & \multirow{3}{*}{$\mathrm{MPa}$} & 0.02 & 2 & \multirow{3}{*}{0.0041} \\
\hline & Resolución & & 0.01 & $2 \sqrt{3}$ & \\
\hline & Repetibilidad & & 0.002 & 1 & \\
\hline \multirow{2}{*}{$\mathrm{u}(\tau)$} & Repetibilidad & \multirow{2}{*}{$\mu \mathrm{s}$} & 0.0006 & 1 & \multirow{2}{*}{$8.45 \mathrm{E}-03$} \\
\hline & Resolución & & $1.00 \mathrm{E}-03$ & $2 \sqrt{3}$ & \\
\hline $\mathrm{U}(\mathrm{A}(T))$ & & $\mathrm{kg} \cdot \mathrm{m}^{-3} \cdot \mu \mathrm{s}^{-2}$ & & $\mathrm{k}=2$ & $7.6 \mathrm{E}-08$ \\
\hline $\mathrm{U}(\mathrm{B}(T, p))$ & & $\mathrm{kg} \cdot \mathrm{m}^{-3}$ & & $\mathrm{k}=2$ & 0.43 \\
\hline
\end{tabular}


Tabla 6.7. Cálculo de la incertidumbre asociada a la densidad experimental en un intervalo de temperaturas de (273.15-373.15) Ky presiones 5-100 MPa.

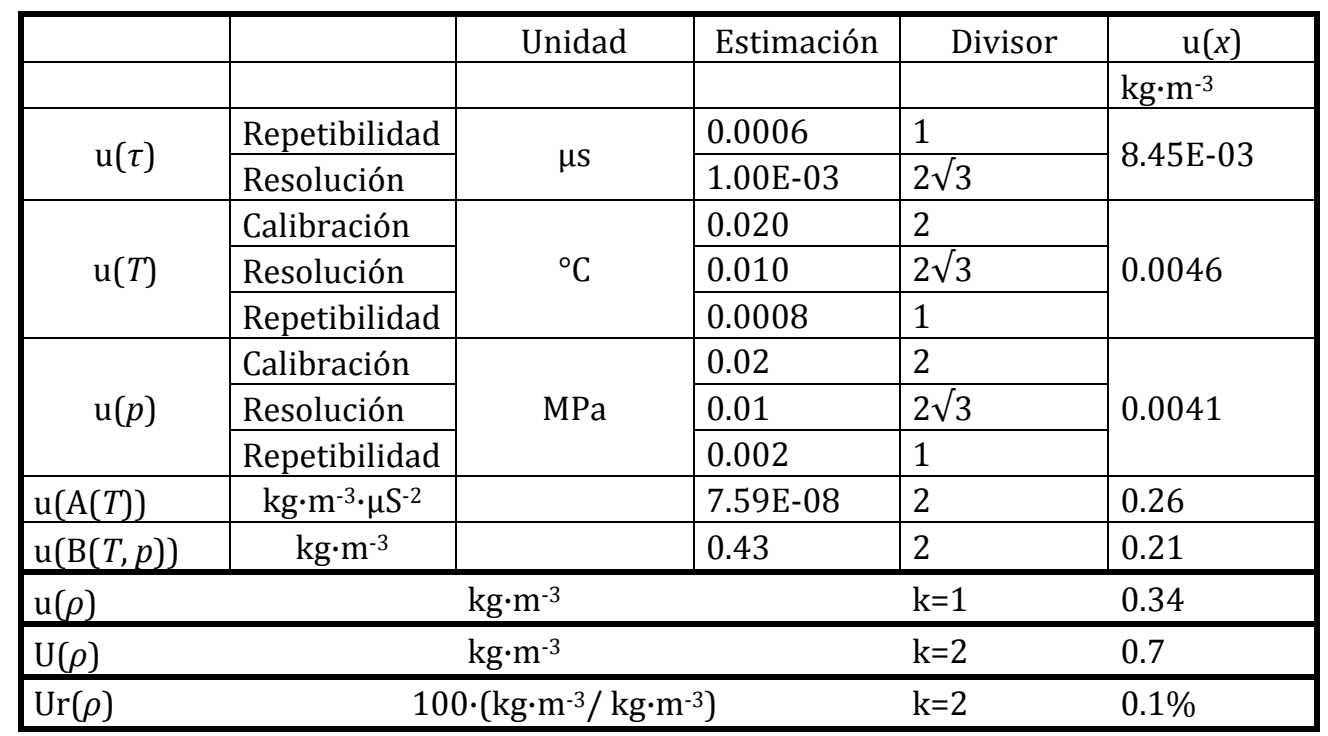

En la Tabla 6.7. se observa que la mayor contribución a la incertidumbre proviene de los parámetros de calibrado $\mathrm{A}(T)$ y $\mathrm{B}(T, p)$.

\subsection{Referencias.}

[1] J. R. Zambrano. "Desarrollo de un Viscosímetro de Hilo Vibrante para la Caracterización Termofísica a Alta Presión de Nuevos Biocombustibles". Universidad de Valladolid. Tesis Doctoral (2014).

[2] R. H. Assael, M. Goodwin. "Experimental thermodynamics volume IX: Advances in transport properties of fluids". R. Soc. Chem. Journals (2004).

[3] C. Nieto de Castro, F. J. V. Santos, J. M. N. A. Fareleira, W. A. Wakeham. "Metrology of viscosity: Have we learned enough?". J. Chem. Eng. Data Vol. 54 (2), (2009) 171-178.

[4] M. Sobrino. "Development of a viscometer for the characterization at high pressure of new biofuels and aqueous mixtures of amines for the capture of $\mathrm{CO}_{2}$ ". University of Valladolid. Doctoral Thesis (2015).

[5] D. Candela, K. M. Martini, R. V. Krotkov, K. H. Langley. "Bessel's improved Kater pendulum in the teaching lab.". Am. J. Phys. Vol. 69(6), (2001) 714-720.

[6] R. Nelson, M. G. Olsson. "The pendulum - Rich physics from a simple system". Am. J. Phys. Vol. 54(2), (1986) 112-121.

[7] J. T. Tough, W. D. McCormick, J. G. Dash. "Vibrating wire viscosimeter". Rev. Sci. Instrum. Vol. 35 (10), (1964) 1345-1348. 
[8] M. J. Assael, H. M. T. Avelino, N. K. Dalaouti, J. M. N. A. Fareleira, K. R. Harris. "Reference correlation for the viscosity of liquid toluene from 213 to $373 \mathrm{~K}$ at pressures to $250 \mathrm{MPa}$ ". Int. J. Thermophys. Vol. 22 (3), (2001) 789-799.

[9] P. R. Bevington, D. K. Robinson. "Data reduction and error analysis for the physical sciences, fitting composite curves". McGraw-Hill (2003) 161-163.

[10] F. Peleties. "Advanced fluid property measurement for oilfield applications". University of London / Imperial College London. Doctoral Thesis (2007).

[11] F. Peleties, J. P. M. Trusler. "Viscosity of liquid di-isodecyl phthalate at temperatures between (274 and 373) $\mathrm{K}$ and at pressures up to $140 \mathrm{MPa}$ ". Journal of Chemical and Engineering Data Vol. 56, (2011) 2236-2241.

[12] Goodfellow Cambridge Limited, Shapal $M$ product data - disponible en: <http://www.goodfellow.com/pdf/3328_1111010.pdf> [accedido 17.09.2019].

[13] Goodfellow Cambridge Limited, material information - disponible en: <http://www.goodfellow.com/S/Tungsteno.html > [accedido 17.09.2019].

[14] AK steel product data sheet UNS 316/316L stainLess steel - disponible en: <http://www.aksteel.com/pdf/markets_products/stainless/austenitic/316_316L_Data_Sh eet.pdf> [accedido 17.09.2019].

[15] Alnico major magnet Eclipse Magnetics data sheet - disponible en: <http://www.eclipse-magnetics.co.uk/product/magnet-materials/cast-alnico/major> [accedido 17.09.2019].

[16] JCGM, "Evaluation of measurement data-guide to the expression of uncertainty in measurement". BIPM (2008). 
Capítulo 7

RESULTADOS EXPERIMENTALES DE LAS MEDIDAS DE DENSIDADES Y VISCOSIDADES EN MEZCLAS DE $\mathrm{CO}_{2}+$ HIDROCARBURO 



\subsection{Introducción.}

En este capítulo se presentan los resultados experimentales de las medidas de densidad y viscosidad en mezclas binarias de hidrocarburos $+\mathrm{CO}_{2}$. Los rangos de temperaturas son de $273.15 \mathrm{~K}$ a $373.15 \mathrm{~K}$ y rangos de presiones desde $5 \mathrm{MPa}$ hasta $100 \mathrm{MPa}$. Estos se obtuvieron mediante las técnicas del densímetro de tubo vibrante y el viscosímetro de hilo vibrante respectivamente, descritos en el capítulo anterior.

El aumento de la composición de $\mathrm{CO}_{2}$ da lugar a una disminución significativa de la viscosidad y aumento de la densidad de la mezcla.

En este trabajo se han determinado las densidades de dos mezclas de hidrocarburo $+\mathrm{CO}_{2}$ en un rango de temperaturas desde $273.15 \mathrm{~K}$ a $373.15 \mathrm{~K}$, dentro del rango de presiones desde $5 \mathrm{MPa}$ hasta $100 \mathrm{MPa}$ y a diferentes composiciones de 0.1 hasta 0.6 en fracción molar del $\mathrm{CO}_{2}\left(x_{\mathrm{CO}}\right)$ :

Mezcla: Pentano $+\mathrm{CO}_{2}$ a $x_{\mathrm{CO} 2}=0.1,0.2,0.3,0.4$ y 0.6 .

Mezcla: Hexano $+\mathrm{CO}_{2}$ a $x_{\mathrm{CO} 2}=0.1,0.2,0.3,0.4$ y 0.6.

También se han medido las viscosidades de estas dos mezclas en las mismas condiciones de presión y temperatura a composiciones de 0.1 y 0.3 en fracción molar del $\mathrm{CO}_{2}\left(x_{\mathrm{CO} 2}\right)$ :

Mezcla: Pentano $+\mathrm{CO}_{2}$ a $x_{C O 2}=0.1$ y 0.3 .

Mezcla: Hexano $+\mathrm{CO}_{2}$ a $x_{\mathrm{CO} 2}=0.1$ y 0.3 .

\subsection{Ajuste de los datos de densidad (ecuación de Tammann-Tait modificada).}

Los datos de densidad a diferentes temperaturas y presiones se ajustaron mediante la ecuación de Tammann-Tait modificada para cada concentración. Este tipo de ajuste también es usado en el capítulo 5. La ecuación usada es la propuesta por Comuñas et al. [1]:

$$
\rho(T, p)=\frac{\rho\left(T, p_{\mathrm{ref}}(T)\right)}{1-\mathrm{C} \ln \left[\frac{\mathrm{B}(T)+p}{\mathrm{~B}(T)+p_{r e f}(T)}\right]}
$$

siendo $\rho$ la densidad, $T$ la temperatura, $C$ es un parámetro el cual le consideramos independiente de la temperatura y $\rho\left(T, p_{\text {ref }}(T)\right)$ es la dependencia de la densidad con la temperatura a la presión de referencia, siendo en nuestro caso como norma general la presión de 5 MPa. Esta función toma la forma expresada en la ecuación 7.2.

$$
\rho\left(T, p_{\text {ref }}(T)\right)=\sum_{i=0} \mathrm{~A}_{\mathrm{i}} T^{i}=\mathrm{A}_{0}+\mathrm{A}_{1} T+\mathrm{A}_{2} T^{2}
$$

Donde los valores de $\mathrm{A}_{i}$ se determinan a partir de los valores experimentales de la densidad a la presión de referencia, normalmente $0.1 \mathrm{MPa}$, y la función $\mathrm{B}(T)$ tiene una forma polinómica según la ecuación 7.3. 


$$
\mathrm{B}(T)=\sum_{i=0} \mathrm{~B}_{i} T^{i}=\mathrm{B}_{0}+\mathrm{B}_{1} T+\mathrm{B}_{2} T^{2}
$$

Los valores de $B_{i}$ se determinan a partir de los valores experimentales. De esta forma, a partir de la ecuación 7.1, podremos representar los valores de la densidad para un amplio rango de temperatura y presión mediante los parámetros ajustables $\mathrm{A}_{i}, \mathrm{~B}_{i}$ y C.

Para evaluar la bondad del ajuste se realiza por medio de la desviación estándar $\sigma$ y la máxima desviación $M D \%$ entre datos experimentales y el modelo utilizado, estas se describieron en las ecuaciones 5.6 y 5.7.

\subsection{Ajuste de los datos de viscosidad (modelo VFT).}

Una de las ecuaciones más empleadas para correlacionar datos de viscosidad dinámica a presión atmosférica es la del modelo VFT (Vogel [2], Fulcher [3] y Tammann [4]) ecuación 7.4. La ecuación se obtiene realizando algunas suposiciones $[5,6]$ a partir de la teoría de Adam-Gibbs de entropía configuracional [7].

$$
\eta(T)=\mathrm{A} \cdot \exp \left(\frac{\mathrm{B}}{T-\mathrm{C}}\right)=\mathrm{A} \cdot \exp \left(\frac{\mathrm{D} \cdot T_{0}}{T-T_{0}}\right)
$$

D es el índice de fragilidad (o parámetro de Angell) y $T_{o}$ la temperatura de Vogel. Esta ecuación ha servido para amplios rangos de temperatura y en condiciones isobáricas. Para tener la posibilidad de establecer una relación de la viscosidad con la presión, algunos autores han llegado a extender dicha ecuación. Una de las modificaciones propuestas es la publicada por Comuñas et al. [1] y Paredes et al. [8]:

$$
\eta(T, p)=\mathrm{A} \cdot \exp \left(\frac{\mathrm{B}}{T-\mathrm{C}}\right) \cdot\left(\frac{p+\mathrm{E}(T)}{p_{r e f}+\mathrm{E}(T)}\right)^{\mathrm{F}}
$$

donde:

$$
\mathrm{E}(T)=\mathrm{E}_{0}+\mathrm{E}_{1} T+\mathrm{E}_{2} T^{2}
$$

donde $\mathrm{A}, \mathrm{B}$ y $\mathrm{C}$ se determinan mediante un ajuste preliminar de la viscosidad en función de la temperatura a la presión de referencia en este trabajo $5 \mathrm{MPa}$, y los coeficientes $\mathrm{F}, \mathrm{E}_{0}, \mathrm{E}_{1} \mathrm{y}$ $\mathrm{E}_{2}$ se ajustan a los valores de viscosidad a presión diferente de la de referencia. En la ecuación 7.5, los parámetros $\mathrm{C}=T_{0}, \mathrm{~B}=\mathrm{D} \cdot T_{0}$ son independientes de la presión. Este modelo también se usó en anteriores trabajos del grupo TERMOCAL como el presentado por Sobrino et al. [9].

Los coeficientes A, B, C, F, E $E_{0}, E_{1}$ y $E_{2}$ de la ecuación 7.5 se determinan ajustando los datos experimentales de viscosidad en función de la temperatura mediante un algoritmo para estimación de mínimos cuadrados de parámetros no lineales [10].

Para evaluar el ajuste se realizó por medio de la desviación estándar $\sigma$ entre datos experimentales y el modelo utilizado siendo esta descrita en la ecuación 5.6. 


\subsection{Relación de los productos utilizados.}

En la Tabla 7.1. se muestran los productos utilizados, la procedencia y la pureza dada por los certificados de análisis. No se ha utilizado ningún método de purificación adicional.

Los compuestos puros fueron desgasificados previamente mediante un baño de ultrasonidos marca Branson, modelo DTH-3210, con una frecuencia de $47 \mathrm{kHz}$ y una potencia de $335 \mathrm{~W}$.

Tabla 7.1. Características de los productos utilizados.

\begin{tabular}{llll}
\hline Sustancia & Fórmula & Marca comercial & Pureza \\
\hline Pentano & $\mathrm{C}_{5} \mathrm{H}_{12}$ & Fluka & $\geq 99.0 \%(\mathrm{GC} \%)$ \\
Hexano & $\mathrm{C}_{6} \mathrm{H}_{14}$ & Sigma-Aldrich & $\geq 99.0 \%(\mathrm{GC} \%)$ \\
Dióxido de Carbono & $\mathrm{CO}_{2}$ & Carburos metálicos & $\mathrm{CO}_{2} \geq 99.995 \%$ \\
(ultrapuro) & & $\mathrm{O}_{2}<10 \mathrm{ppm}$ \\
& & $\mathrm{H}_{2} \mathrm{O}<7 \mathrm{ppm}$ \\
& & $\mathrm{CO}<2 \mathrm{ppm}$ \\
& & $\mathrm{THC}<5 \mathrm{ppm}$ \\
& & $\mathrm{N}_{2}<25 \mathrm{ppm}$ \\
\hline
\end{tabular}

\subsection{Presentación de los resultados experimentales de las medidas de densidad.}

A continuación, se muestran los datos experimentales obtenidos de la densidad para las mezclas estudiadas en forma de tablas y gráficas, así como los resultados del ajuste de los datos experimentales al modelo Tammann-Tait. Este ajuste se realizó para cada una de las composiciones estudiadas, en función de la temperatura y la presión. Además, se muestra tanto la desviación estándar del ajuste $\sigma$ (ecuación 5.6), la máxima desviación $M D \%$ entre datos experimentales y el modelo utilizado (ecuación 5.7). 
Resultados experimentales de las medidas de densidades y viscosidades en mezclas de $\mathrm{CO}_{2}+$ hidrocarburo

7.5.1. Mezcla: Pentano $+\mathrm{CO}_{2}$.

Tabla 7.2. Datos experimentales de densidad, $\rho\left(\mathrm{kg} \cdot \mathrm{m}^{-3}\right)$, para la mezcla Pentano $+\mathrm{CO}_{2}$ a $x_{\mathrm{CO} 2}=0.1004$.

\begin{tabular}{ccccccc}
\hline \multicolumn{7}{c}{$\rho\left(\mathrm{kg} / \mathrm{m}^{3}\right)$} \\
\hline \multicolumn{7}{c}{$T / \mathrm{K}$} \\
\hline$p / \mathrm{MPa}$ & 273.15 & 293.15 & 313.15 & 333.15 & 353.15 & 373.15 \\
\hline 5 & 661.1 & 642.9 & 622.7 & 600.6 & 576.8 & 551.1 \\
10 & 666.3 & 649.1 & 630.5 & 609.9 & 588.2 & 565.7 \\
15 & 671.3 & 655.0 & 637.2 & 618.0 & 597.9 & 577.4 \\
20 & 675.8 & 660.3 & 643.4 & 625.2 & 606.4 & 587.4 \\
30 & 684.2 & 669.8 & 654.3 & 637.7 & 620.7 & 603.8 \\
40 & 691.8 & 678.4 & 663.9 & 648.4 & 632.7 & 617.4 \\
50 & 698.7 & 686.0 & 672.3 & 657.8 & 643.1 & 628.8 \\
60 & 705.1 & 693.0 & 680.0 & 666.3 & 652.4 & 638.8 \\
70 & 711.0 & 699.5 & 687.1 & 673.9 & 660.6 & 647.8 \\
80 & 716.6 & 705.6 & 693.6 & 681.1 & 668.2 & 656.1 \\
90 & 721.9 & 711.2 & 699.8 & 687.6 & 675.3 & 663.7 \\
100 & 726.8 & 716.5 & 705.4 & 693.7 & 681.8 & 670.6 \\
\hline
\end{tabular}

Tabla 7.3. Coeficientes y estadística de la ecuación Tammann-Tait modificada para la mezcla Pentano $+\mathrm{CO}_{2}$ a $x_{\mathrm{CO} 2}=0.1004, p_{\text {ref }}=5 \mathrm{MPa}$.

Parámetros de la ecuación Tammann-Tait modificada

\begin{tabular}{ccccccccc}
\hline $\begin{array}{c}\mathrm{A}_{0} \\
\mathrm{~kg} \cdot \mathrm{m}^{-3}\end{array}$ & $\begin{array}{c}\mathrm{A}_{1} \\
\mathrm{~kg} \cdot \mathrm{m}^{-3} \cdot \mathrm{K}^{-1}\end{array}$ & $\begin{array}{c}\mathrm{A}_{2} \\
\mathrm{~kg} \cdot \mathrm{m}^{-3} \cdot \mathrm{K}^{-2}\end{array}$ & $\begin{array}{c}\mathrm{B}_{0} \\
\mathrm{MPa}\end{array}$ & $\begin{array}{c}\mathrm{B}_{1} \\
\mathrm{MPa} \cdot \mathrm{K}^{-1}\end{array}$ & $\begin{array}{c}\mathrm{B}_{2} \\
\mathrm{MPa} \cdot \mathrm{K}^{-2}\end{array}$ & $\mathrm{C}$ & $\begin{array}{c}\sigma \\
\mathrm{kg} \cdot \mathrm{m}^{-3}\end{array}$ & $\begin{array}{c}M D \\
(\%)\end{array}$ \\
\hline 723.06 & 0.413 & $-2.34 \mathrm{E}-03$ & 302.84 & -1.32 & $1.42 \mathrm{E}-03$ & 0.090 & 0.15 & 0.05 \\
\hline
\end{tabular}


Resultados experimentales de las medidas de densidades y viscosidades en mezclas de $\mathrm{CO}_{2}+$ hidrocarburo

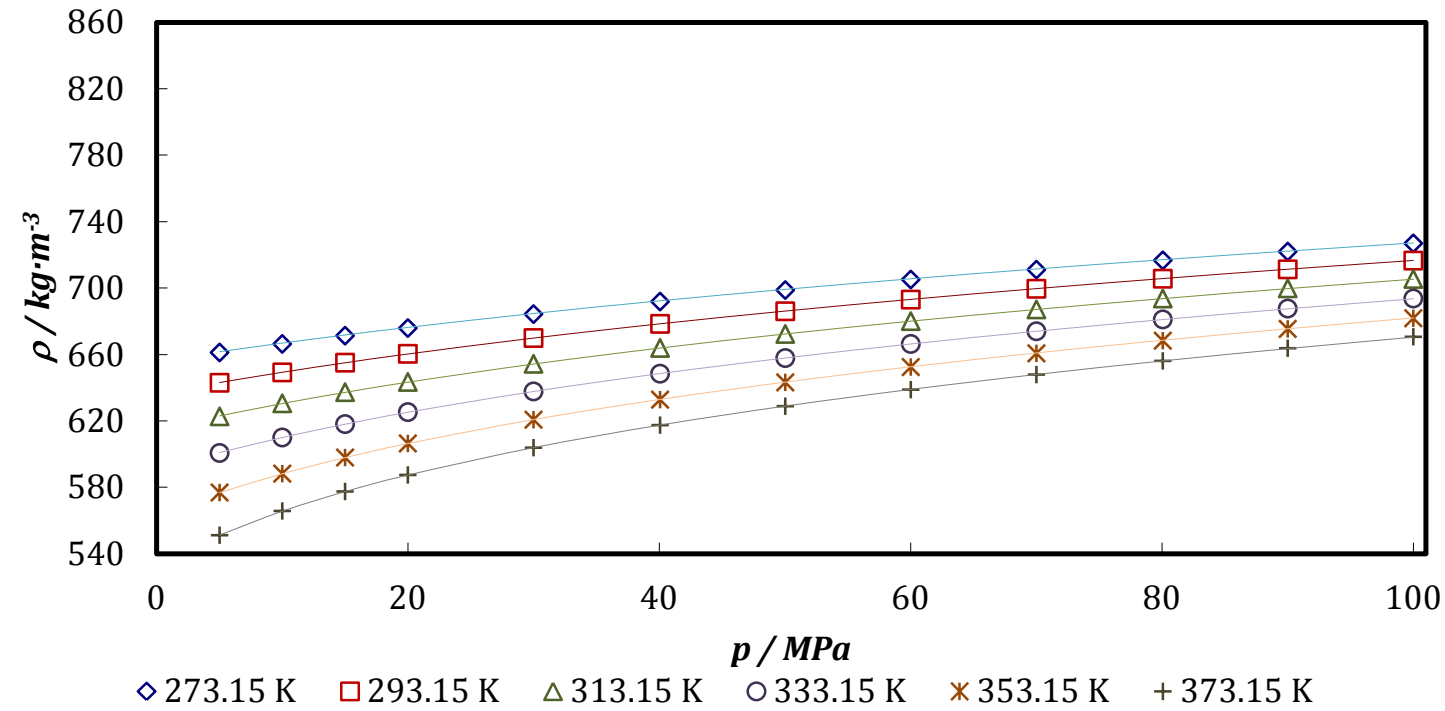

Figura 7.1. Valores experimentales de la densidad para la mezcla Pentano $+\mathrm{CO}_{2}$ a $\mathrm{x}_{\mathrm{CO} 2}=0.1004 a$ diferentes temperaturas en función de la presión. Líneas continuas valores ajustados con la ecuación Tammann-Tait modificada y los puntos valores experimentales.

Tabla 7.4. Datos experimentales de densidad, $\rho\left(\mathrm{kg} \cdot \mathrm{m}^{-3}\right)$, para la mezcla Pentano $+\mathrm{CO}_{2}$ a $x_{\mathrm{CO} 2}=0.2006$.

\begin{tabular}{ccccccc}
\hline \multicolumn{7}{c}{$\rho\left(\mathrm{kg} / \mathrm{m}^{3}\right)$} \\
\hline \multicolumn{7}{c}{$T / \mathrm{K}$} \\
\hline$p / \mathrm{MPa}$ & 273.15 & 293.15 & 313.15 & 333.15 & 353.15 & 373.15 \\
\hline 5 & 674.9 & 654.2 & 631.4 & 606.1 & 578.5 & 548.2 \\
10 & 681.0 & 661.7 & 640.3 & 617.3 & 592.7 & 566.8 \\
15 & 686.8 & 668.4 & 648.2 & 626.8 & 604.3 & 581.1 \\
20 & 692.1 & 674.5 & 655.3 & 635.1 & 614.3 & 593.1 \\
30 & 701.6 & 685.5 & 667.8 & 649.5 & 631.2 & 612.4 \\
40 & 710.3 & 695.2 & 678.6 & 661.7 & 645.1 & 628.2 \\
50 & 718.1 & 703.9 & 688.2 & 672.4 & 657.1 & 641.4 \\
60 & 725.3 & 711.8 & 696.9 & 681.8 & 667.6 & 653.0 \\
70 & 732.0 & 719.1 & 704.8 & 690.5 & 677.1 & 663.2 \\
80 & 738.2 & 725.8 & 712.1 & 698.4 & 685.6 & 672.5 \\
90 & 744.1 & 732.1 & 718.8 & 705.7 & 693.6 & 680.8 \\
100 & 749.6 & 738.0 & 725.2 & 712.4 & 700.8 & 688.6 \\
\hline
\end{tabular}


Resultados experimentales de las medidas de densidades y viscosidades en mezclas de $\mathrm{CO}_{2}+$ hidrocarburo

Tabla 7.5. Coeficientes y estadística de la ecuación Tammann-Tait modificada para la mezcla Pentano $+\mathrm{CO}_{2} a x_{\mathrm{CO} 2}=0.2006, p_{\text {ref }}=5 \mathrm{MPa}$.

Parámetros de la ecuación Tammann-Tait modificada

\begin{tabular}{ccccccccc}
\hline $\mathrm{A}_{0}$ & $\mathrm{~A}_{1}$ & $\mathrm{~A}_{2}$ & $\mathrm{~B}_{0}$ & $\mathrm{~B}_{1}$ & $\mathrm{~B}_{2}$ & $\mathrm{C}$ & $\sigma$ & $M D$ \\
$\mathrm{~kg} \cdot \mathrm{m}^{-3}$ & $\mathrm{~kg} \cdot \mathrm{m}^{-3} \cdot \mathrm{K}^{-1}$ & $\mathrm{~kg} \cdot \mathrm{m}^{-3} \cdot \mathrm{K}^{-2}$ & $\mathrm{MPa}$ & $\mathrm{MPa} \cdot \mathrm{K}^{-1}$ & $\mathrm{MPa} \cdot \mathrm{K}^{-2}$ & & $\mathrm{~kg} \cdot \mathrm{m}^{-3}$ & $(\%)$ \\
\hline 712.60 & 0.692 & $-3.04 \mathrm{E}-03$ & 293.25 & -1.30 & $1.42 \mathrm{E}-03$ & 0.093 & 0.27 & 0.09 \\
\hline
\end{tabular}

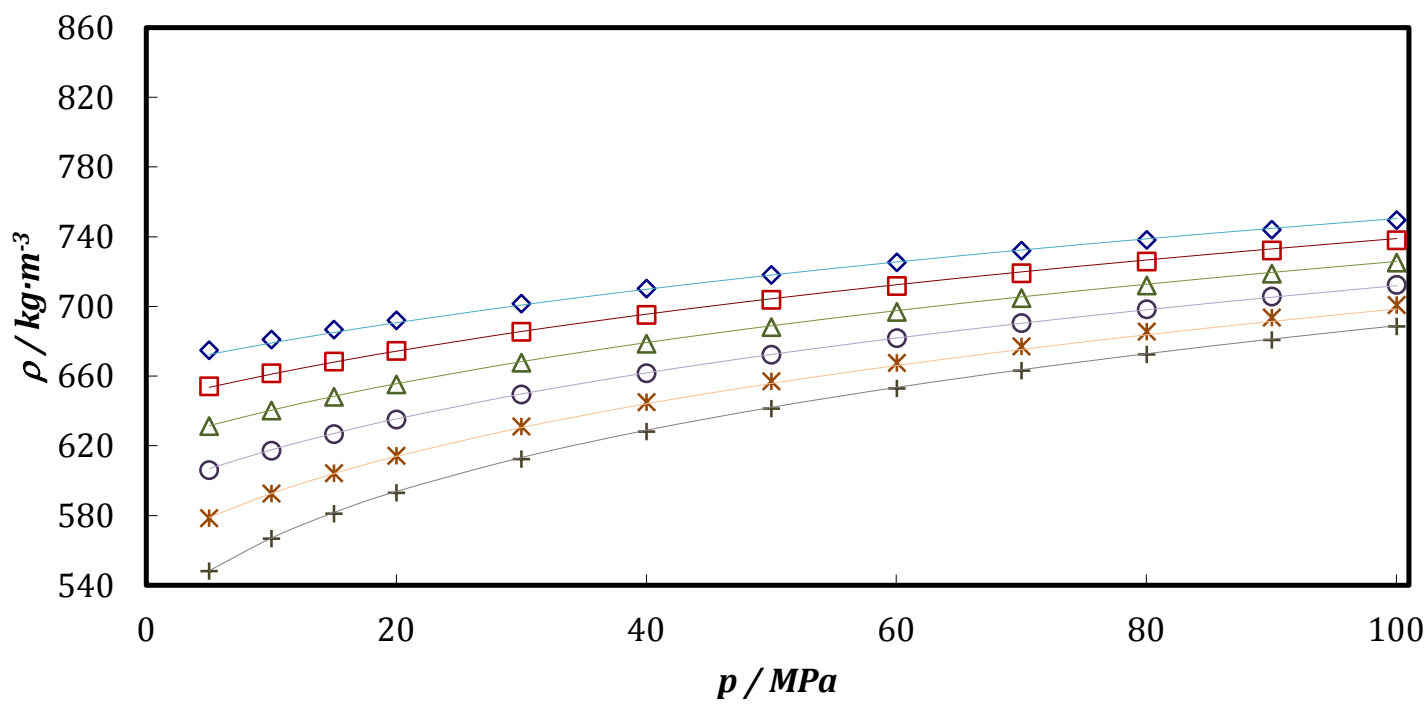

$\diamond 273.15 \mathrm{~K} \quad \square 293.15 \mathrm{~K} \quad \triangle 313.15 \mathrm{~K} \quad 0333.15 \mathrm{~K} \quad * 353.15 \mathrm{~K} \quad+373.15 \mathrm{~K}$

Figura 7.2. Valores experimentales de la densidad para la mezcla Pentano $+\mathrm{CO}_{2} a x_{\mathrm{CO} 2}=0.2006 a$ diferentes temperaturas en función de la presión. Líneas continúas valores ajustados con la ecuación Tammann-Tait modificada y los puntos valores experimentales. 
Resultados experimentales de las medidas de densidades y viscosidades en mezclas de $\mathrm{CO}_{2}+$ hidrocarburo

Tabla 7.6. Datos experimentales de densidad, $\rho\left(\mathrm{kg} \cdot \mathrm{m}^{-3}\right)$, para la mezcla Pentano $+\mathrm{CO}_{2}$ a $x_{\mathrm{CO} 2}=0.2994$.

\begin{tabular}{ccccccc}
\hline \multicolumn{7}{c}{$\rho\left(\mathrm{kg} / \mathrm{m}^{3}\right)$} \\
\hline \multicolumn{7}{c}{$T / \mathrm{K}$} \\
\hline$p / \mathrm{MPa}$ & 273.15 & 293.15 & 313.15 & 333.15 & 353.15 & 373.15 \\
\hline 5 & 691.1 & 667.6 & 643.8 & 612.3 & 576.0 & \\
10 & 698.0 & 676.3 & 655.6 & 627.4 & 596.8 & 563.5 \\
15 & 704.5 & 684.0 & 665.8 & 639.9 & 612.9 & 583.8 \\
20 & 710.4 & 691.0 & 674.8 & 650.8 & 626.3 & 600.1 \\
30 & 721.1 & 703.5 & 690.3 & 669.3 & 648.1 & 625.6 \\
40 & 730.9 & 714.5 & 703.6 & 684.5 & 665.8 & 646.0 \\
50 & 739.9 & 724.4 & 715.1 & 697.5 & 680.4 & 662.3 \\
60 & 748.2 & 733.4 & 725.4 & 709.0 & 693.1 & 676.5 \\
70 & 755.9 & 741.9 & 734.8 & 719.4 & 704.4 & 689.0 \\
80 & 763.1 & 749.7 & 743.3 & 728.7 & 714.5 & 700.0 \\
90 & 769.9 & 757.0 & 751.2 & 737.3 & 723.7 & 709.9 \\
100 & 776.2 & 763.9 & 758.4 & 745.2 & 732.2 & 719.1 \\
\hline
\end{tabular}

Tabla 7.7. Coeficientes y estadística de la ecuación Tammann-Tait modificada para la mezcla Pentano $+\mathrm{CO}_{2}$ a $x_{\mathrm{CO} 2}=0.2994, p_{\text {ref }}=100 \mathrm{MPa}$.

\begin{tabular}{ccccccccc}
\hline \multicolumn{7}{c}{ Parámetros de la ecuación Tammann-Tait modificada } \\
\hline $\mathrm{A}_{0}$ & $\mathrm{~A}_{1}$ & $\mathrm{~A}_{2}$ & $\mathrm{~B}_{0}$ & $\mathrm{~B}_{1}$ & $\mathrm{~B}_{2}$ & $\mathrm{C}$ & $\sigma$ & $\mathrm{MD}$ \\
$\mathrm{kg} \cdot \mathrm{m}^{-3}$ & $\mathrm{~kg} \cdot \mathrm{m}^{-3} \cdot \mathrm{K}^{-1}$ & $\mathrm{~kg} \cdot \mathrm{m}^{-3} \cdot \mathrm{K}^{-2}$ & $\mathrm{MPa}$ & $\mathrm{MPa} \cdot \mathrm{K}^{-1}$ & $\mathrm{MPa} \cdot \mathrm{K}^{-2}$ & & $\mathrm{~kg} \cdot \mathrm{m}^{-3}$ & $(\%)$ \\
\hline 735.01 & 0.671 & $-1.92 \mathrm{E}-03$ & 424.60 & -2.00 & $2.32 \mathrm{E}-03$ & 0.122 & 1.37 & 0.41 \\
\hline
\end{tabular}


Resultados experimentales de las medidas de densidades y viscosidades en mezclas de $\mathrm{CO}_{2}+$ hidrocarburo

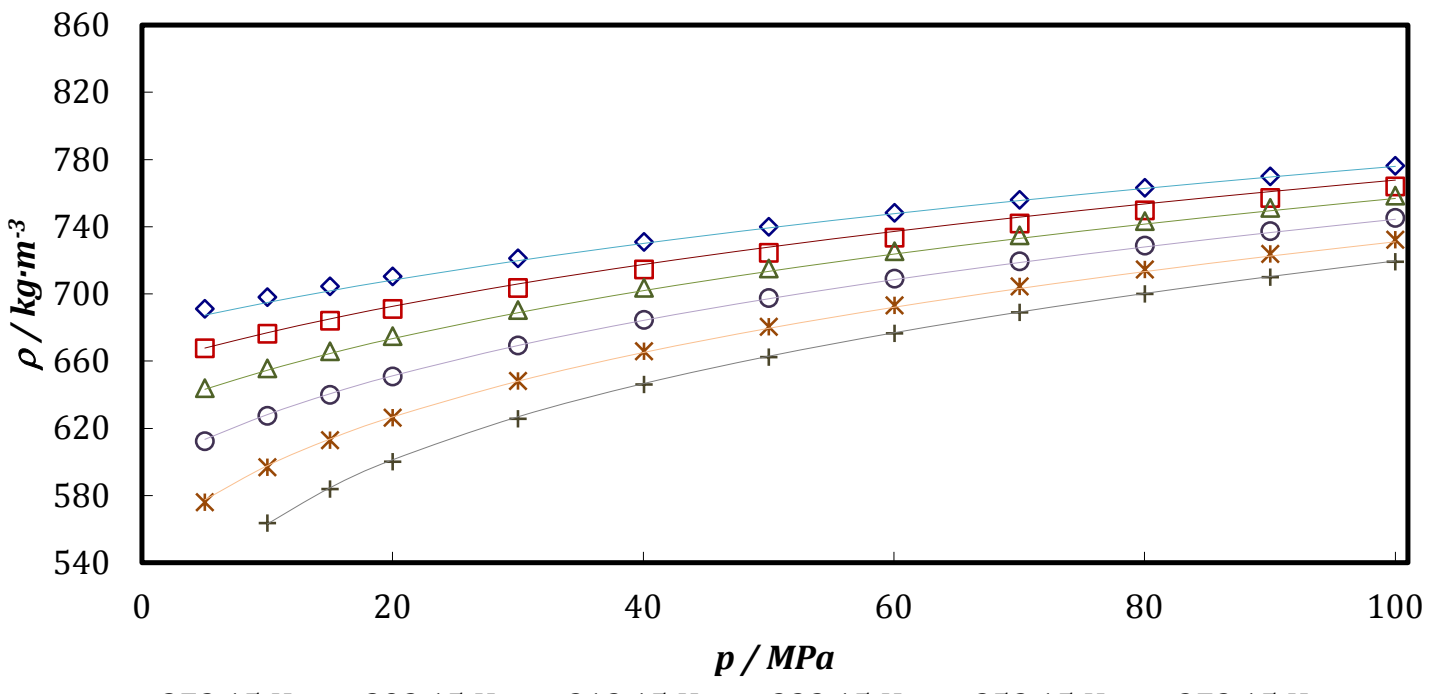

$\diamond 273.15 \mathrm{~K} \quad \square 293.15 \mathrm{~K} \quad \triangle 313.15 \mathrm{~K} \quad$ ○333.15K $\quad * 353.15 \mathrm{~K} \quad+373.15 \mathrm{~K}$

Figura 7.3. Valores experimentales de la densidad para la mezcla Pentano $+\mathrm{CO}_{2}$ a $\mathrm{x}_{\mathrm{CO} 2}=0.2994$ a diferentes temperaturas en función de la presión. Líneas continúas valores ajustados con la ecuación Tammann-Tait modificada y los puntos valores experimentales.

Tabla 7.8. Datos experimentales de densidad, $\rho\left(\mathrm{kg} \cdot \mathrm{m}^{-3}\right)$, para la mezcla Pentano $+\mathrm{CO}_{2}$ a $x_{\mathrm{CO} 2}=0.3994$.

\begin{tabular}{ccccccc}
\hline \multicolumn{5}{c}{$\rho\left(\mathrm{kg} / \mathrm{m}^{3}\right)$} \\
\hline \multicolumn{5}{c}{$T / \mathrm{K}$} \\
\hline$p / \mathrm{MPa}$ & 273.15 & 293.15 & 313.15 & 333.15 & 353.15 & 373.15 \\
\hline 5 & 705.3 & 678.8 & 648.6 & 613.7 & & \\
10 & 713.0 & 688.7 & 661.5 & 631.3 & 596.9 & 558.4 \\
15 & 720.1 & 697.5 & 672.9 & 645.7 & 616.2 & 583.5 \\
20 & 726.6 & 705.4 & 683.0 & 658.2 & 631.8 & 602.7 \\
30 & 738.7 & 719.3 & 700.4 & 679.0 & 656.8 & 632.3 \\
40 & 749.6 & 731.5 & 715.2 & 696.1 & 676.5 & 655.1 \\
50 & 759.5 & 742.4 & 728.0 & 710.6 & 692.8 & 673.6 \\
60 & 768.5 & 752.4 & 739.4 & 723.4 & 706.8 & 689.3 \\
70 & 776.8 & 761.7 & 749.6 & 734.6 & 719.2 & 702.8 \\
80 & 784.5 & 770.1 & 759.0 & 744.8 & 730.3 & 714.9 \\
90 & 791.6 & 778.0 & 767.6 & 754.2 & 740.3 & 725.7 \\
100 & 798.4 & 785.5 & 775.6 & 762.9 & 749.6 & 735.7 \\
\hline
\end{tabular}


Resultados experimentales de las medidas de densidades y viscosidades en mezclas de $\mathrm{CO}_{2}+$ hidrocarburo

Tabla 7.9. Coeficientes y estadística de la ecuación Tammann-Tait modificada para la mezcla Pentano $+\mathrm{CO}_{2}$ a $x_{\mathrm{CO} 2}=0.3994, p_{\text {ref }}=100 \mathrm{MPa}$.

Parámetros de la ecuación Tammann-Tait modificada

\begin{tabular}{ccccccccc}
\hline $\begin{array}{c}\mathrm{A}_{0} \\
\mathrm{~kg} \cdot \mathrm{m}^{-3}\end{array}$ & $\begin{array}{c}\mathrm{A}_{1} \\
\mathrm{~kg} \cdot \mathrm{m}^{-3} \cdot \mathrm{K}^{-1}\end{array}$ & $\begin{array}{c}\mathrm{A}_{2} \\
\mathrm{~kg} \cdot \mathrm{m}^{-3} \cdot \mathrm{K}^{-2}\end{array}$ & $\begin{array}{c}\mathrm{B}_{0} \\
\mathrm{MPa}\end{array}$ & $\begin{array}{c}\mathrm{B}_{1} \\
\mathrm{MPa} \cdot \mathrm{K}^{-1}\end{array}$ & $\begin{array}{c}\mathrm{B}_{2} \\
\mathrm{MPa} \cdot \mathrm{K}^{-2}\end{array}$ & $\mathrm{C}$ & $\begin{array}{c}\sigma \\
\mathrm{kg} \cdot \mathrm{m}^{-3}\end{array}$ & $\begin{array}{c}M D \\
(\%)\end{array}$ \\
\hline 853.55 & 0.112 & $-1.15 \mathrm{E}-03$ & 441.19 & -2.14 & $2.54 \mathrm{E}-03$ & 0.127 & 0.88 & 0.34 \\
\hline
\end{tabular}

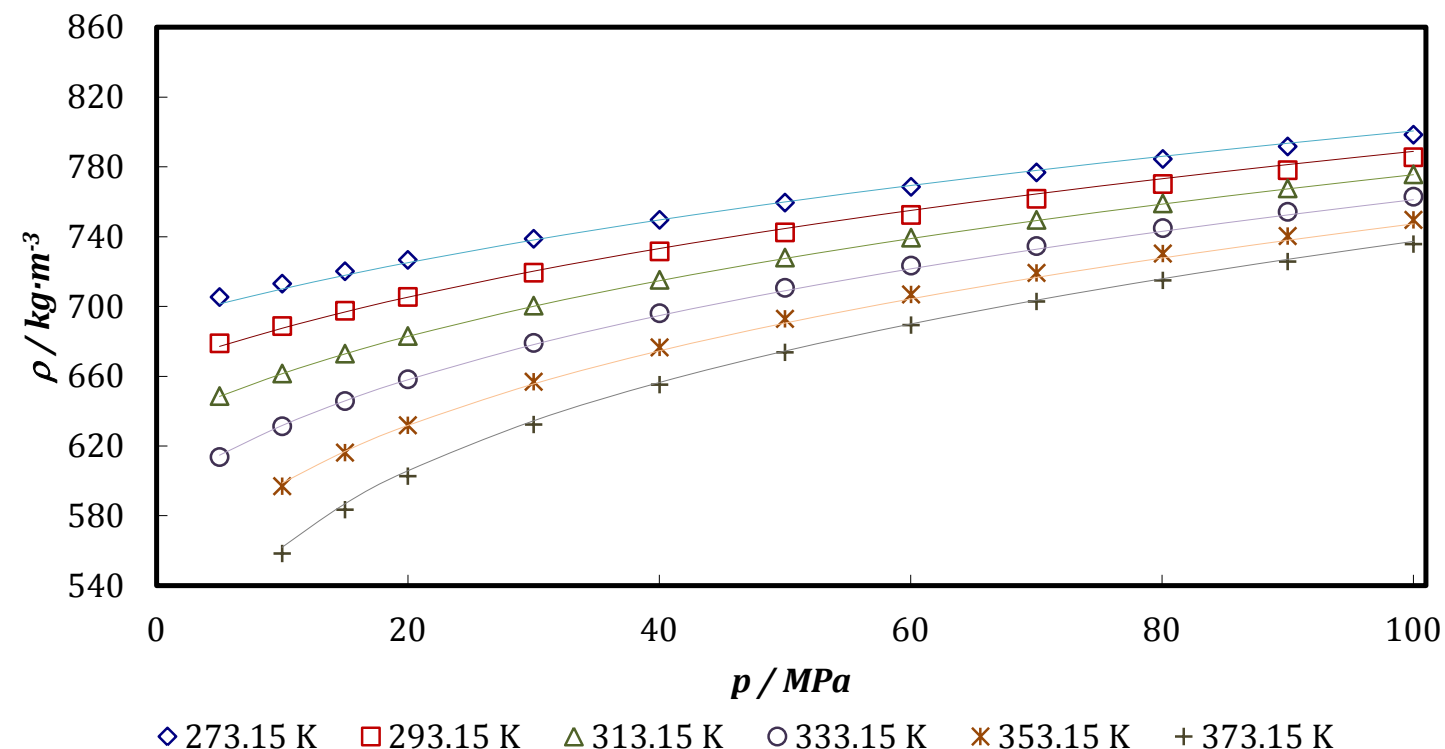

Figura 7.4. Valores experimentales de la densidad para la mezcla Pentano $+\mathrm{CO}_{2} a \mathrm{x}_{\mathrm{CO} 2}=0.3994 a$ diferentes temperaturas en función de la presión. Líneas continuas valores ajustados con la ecuación Tammann-Tait modificada y los puntos valores experimentales. 
Resultados experimentales de las medidas de densidades y viscosidades en mezclas de $\mathrm{CO}_{2}+$ hidrocarburo

Tabla 7.10. Datos experimentales de densidad, $\rho\left(\mathrm{kg} \cdot \mathrm{m}^{-3}\right)$, para la mezcla Pentano $+\mathrm{CO}_{2}$ a $x_{\mathrm{CO} 2}=$ 0.5996.

\begin{tabular}{cccccc}
\hline \multicolumn{5}{c}{$\rho\left(\mathrm{kg} / \mathrm{m}^{3}\right)$} \\
\hline$p / \mathrm{MPa}$ & 273.15 & 293.15 & 313.15 & 333.15 & 353.15 \\
\hline 10 & 756.3 & 722.6 & 682.8 & 639.2 & \\
15 & 765.5 & 734.4 & 698.5 & 662.6 & \\
20 & 773.7 & 744.6 & 711.7 & 680.7 & \\
30 & 788.1 & 762.0 & 733.3 & 708.0 & 643.2 \\
40 & 800.6 & 776.8 & 750.9 & 728.9 & 676.3 \\
50 & 811.6 & 789.5 & 765.9 & 745.9 & 700.5 \\
60 & 821.6 & 800.9 & 779.0 & 760.4 & 719.8 \\
70 & 830.6 & 811.3 & 790.6 & 773.2 & 736.3 \\
80 & 839.0 & 820.6 & 801.2 & 784.7 & 750.6 \\
90 & 846.8 & 829.4 & 810.8 & 795.2 & 763.3 \\
100 & 854.1 & 837.5 & 819.7 & 804.7 & 774.8 \\
\hline
\end{tabular}

Tabla 7.11. Coeficientes y estadística de la ecuación Tammann-Tait modificada para la mezcla Pentano $+\mathrm{CO}_{2}$ a $x_{\mathrm{CO} 2}=0.5996, p_{\text {ref }}=10 \mathrm{MPa}$.

Parámetros de la ecuación Tammann-Tait modificada

\begin{tabular}{ccccccccc}
\hline $\begin{array}{c}\mathrm{A}_{0} \\
\mathrm{~kg} \cdot \mathrm{m}^{-3}\end{array}$ & $\begin{array}{c}\mathrm{A}_{1} \\
\mathrm{~kg} \cdot \mathrm{m}^{-3} \cdot \mathrm{K}^{-1}\end{array}$ & $\begin{array}{c}\mathrm{A}_{2} \\
\mathrm{~kg} \cdot \mathrm{m}^{-3} \cdot \mathrm{K}^{-2}\end{array}$ & $\begin{array}{c}\mathrm{B}_{0} \\
\mathrm{MPa}\end{array}$ & $\begin{array}{c}\mathrm{B}_{1} \\
\mathrm{MPa} \cdot \mathrm{K}^{-1}\end{array}$ & $\begin{array}{c}\mathrm{B}_{2} \\
\mathrm{MPa} \cdot \mathrm{K}^{-2}\end{array}$ & $\mathrm{C}$ & $\begin{array}{c}\sigma \\
\mathrm{kg} \cdot \mathrm{m}^{-3}\end{array}$ & $\begin{array}{c}M D \\
(\%)\end{array}$ \\
\hline 846.50 & 1.15 & $-5.38 \mathrm{E}-03$ & 514.28 & -2.87 & $3.96 \mathrm{E}-03$ & 0.086 & 2.30 & 0.97 \\
\hline
\end{tabular}


Resultados experimentales de las medidas de densidades y viscosidades en mezclas de $\mathrm{CO}_{2}+$ hidrocarburo

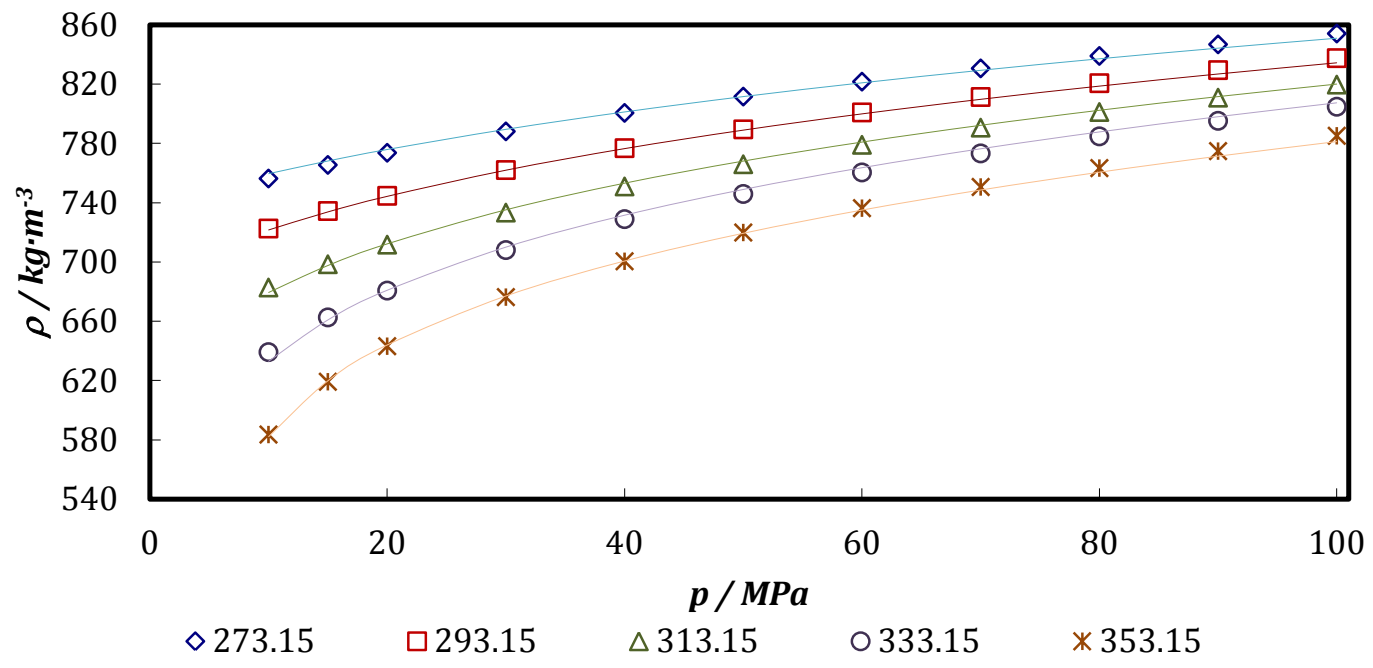

Figura 7.5. Valores experimentales de la densidad para la mezcla Pentano $+\mathrm{CO}_{2} a x_{\mathrm{CO} 2}=0.5996 a$ diferentes temperaturas en función de la presión. Líneas continuas valores ajustados con la ecuación Tammann-Tait modificada y los puntos valores experimentales.

Los resultados de la densidad obtenidos para las mezclas de Pentano $+\mathrm{CO}_{2}$ se compararon con los encontrados en la literatura [11], donde se encuentra el sistema de Pentano $+\mathrm{CO}_{2}$ a $373.15 \mathrm{~K}$, con concentración al $20 \% \mathrm{w}$ de $\mathrm{CO}_{2}$, que equivale a la $x_{C O 2}=0.3$ de nuestros datos.

En la figura 7.6 se han representado las desviaciones relativas obtenidas entre los datos experimentales y los de la literatura. La desviación media obtenida $(A A D \%)$ para la mezcla con concentración en $20 \% \mathrm{w}$ de $\mathrm{CO}_{2}$ fue de $1.2 \%$. Como resultado de dichas desviaciones se puede concluir que estos valores son coherentes con la literatura para la medida de la densidad de las mezclas hidrocarburo $+\mathrm{CO}_{2}$.

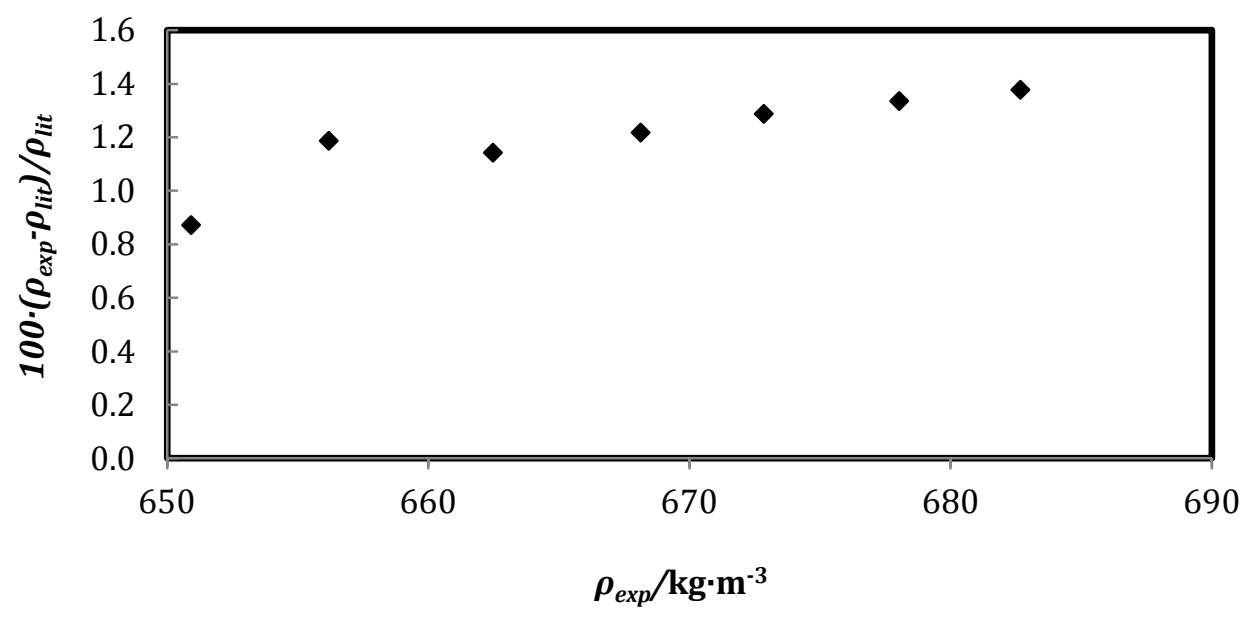

- $20 \%$ w CO2 Kiran et al.

Figura 7.6. Desviación relativa porcentual de la $\rho$ experimental respecto a los datos de la bibliografía de Kiran et al. [11]. 
Resultados experimentales de las medidas de densidades y viscosidades en mezclas de $\mathrm{CO}_{2}+$ hidrocarburo

\subsubsection{Mezcla: Hexano + $\mathrm{CO}_{2}$}

Tabla 7.12. Datos experimentales de densidad, $\rho\left(\mathrm{kg} \cdot \mathrm{m}^{-3}\right)$, para la mezcla Hexano $+\mathrm{CO}_{2}$ a $x_{\mathrm{CO} 2}=$ 0.1000 .

\begin{tabular}{ccccccc}
\hline \multicolumn{7}{c}{$\rho\left(\mathrm{kg} / \mathrm{m}^{3}\right)$} \\
\hline \multicolumn{7}{c}{$T / \mathrm{K}$} \\
\hline$p / \mathrm{MPa}$ & 273.15 & 293.15 & 313.15 & 333.15 & 353.15 & 373.15 \\
\hline 5 & 685.9 & 670.0 & 652.7 & 634.9 & 614.6 & 593.4 \\
10 & 690.2 & 675.2 & 658.8 & 642.5 & 623.5 & 604.0 \\
15 & 694.4 & 680.0 & 664.3 & 649.3 & 631.3 & 613.2 \\
20 & 698.2 & 684.4 & 669.3 & 655.6 & 638.4 & 621.4 \\
30 & 705.5 & 692.5 & 678.4 & 666.9 & 651.3 & 635.8 \\
40 & 712.1 & 699.9 & 686.7 & 677.0 & 662.7 & 648.5 \\
50 & 718.2 & 706.6 & 694.4 & 686.1 & 672.7 & 659.6 \\
60 & 724.0 & 712.8 & 701.7 & 694.4 & 682.0 & 669.7 \\
70 & 729.4 & 718.7 & 708.6 & 702.0 & 690.3 & 678.8 \\
80 & 734.4 & 724.2 & 715.1 & 709.1 & 698.0 & 687.2 \\
90 & 739.3 & 729.4 & 721.2 & 715.6 & 705.2 & 694.8 \\
100 & 744.0 & 734.4 & 727.0 & 721.8 & 711.8 & 702.0 \\
\hline
\end{tabular}

Tabla 7.13. Coeficientes y estadística de la ecuación Tammann-Tait modificada para la mezcla Hexano $+\mathrm{CO}_{2}$ a $_{\mathrm{CO}}=0.1000, p_{\text {ref }}=5 \mathrm{MPa}$.

Parámetros de la ecuación Tammann-Tait modificada

\begin{tabular}{ccccccccc}
\hline $\mathrm{A}_{0}$ & $\mathrm{~A}_{1}$ & $\mathrm{~A}_{2}$ & $\mathrm{~B}_{0}$ & $\mathrm{~B}_{1}$ & $\mathrm{~B}_{2}$ & $\mathrm{C}$ & $\begin{array}{c}\sigma \\
\mathrm{kg} \cdot \mathrm{m}^{-3}\end{array}$ & $\begin{array}{c}\mathrm{MD} \\
(\%)\end{array}$ \\
\hline 761.97 & 0.203 & $-1.76 \mathrm{E}-03$ & 533.51 & -2.47 & $2.93 \mathrm{E}-03$ & 0.100 & 0.82 & 0.23 \\
\hline
\end{tabular}


Resultados experimentales de las medidas de densidades y viscosidades en mezclas de $\mathrm{CO}_{2}+$ hidrocarburo

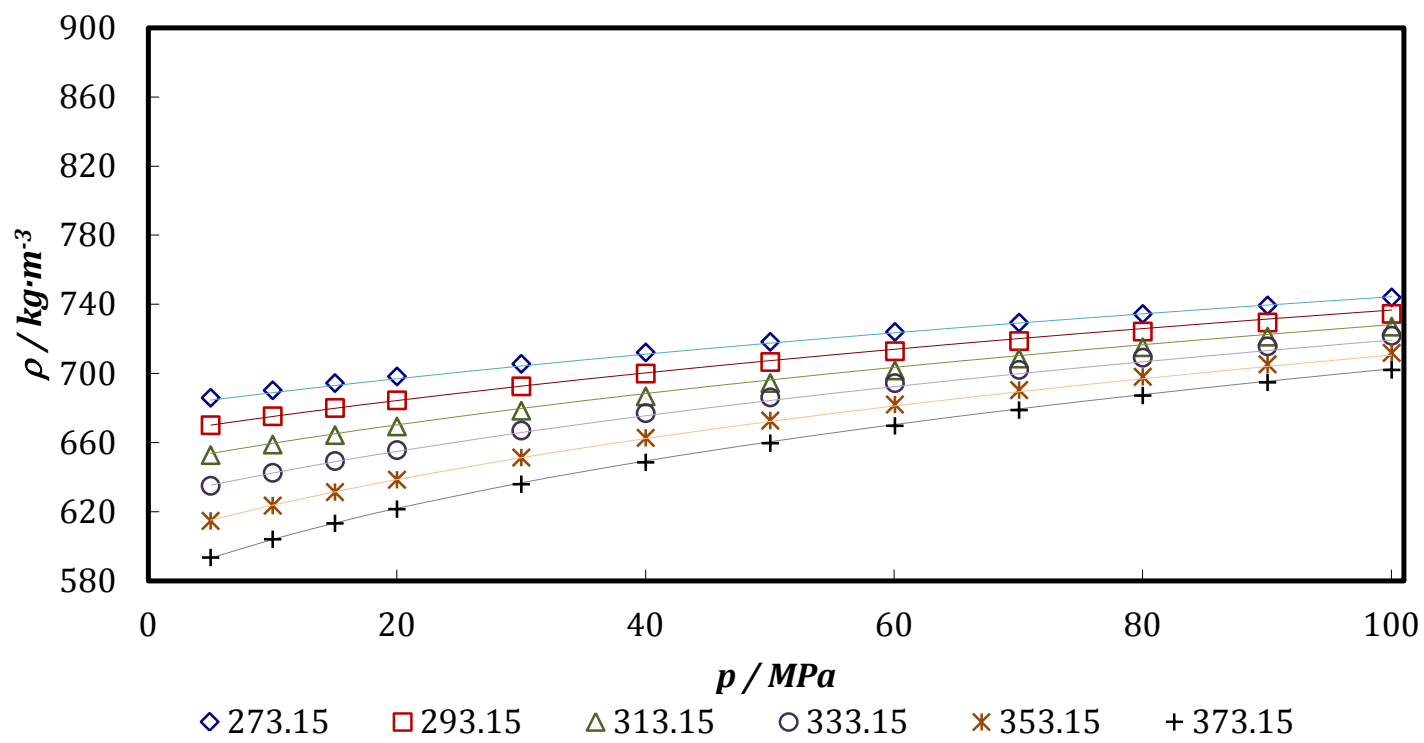

Figura 7.7. Valores experimentales de la densidad para la mezcla Hexano $+\mathrm{CO}_{2}$ a $\mathrm{x}_{\mathrm{CO} 2}=0.1000$ a diferentes temperaturas en función de la presión. Líneas continuas valores ajustados con la ecuación Tammann-Tait modificada y los puntos valores experimentales.

Tabla 7.14. Datos experimentales de densidad, $\rho\left(\mathrm{kg} \cdot \mathrm{m}^{-3}\right)$, para la mezcla Hexano $+\mathrm{CO}_{2}$ a $x_{\mathrm{CO} 2}=$ 0.2003 .

\begin{tabular}{ccccccc}
\hline \multicolumn{7}{c}{$\rho\left(\mathrm{kg} / \mathrm{m}^{3}\right)$} \\
\hline \multicolumn{7}{c}{$T / \mathrm{K}$} \\
\hline$p / \mathrm{MPa}$ & 273.15 & 293.15 & 313.15 & 333.15 & 353.15 & 373.15 \\
\hline 5 & 700.1 & 682.2 & 662.3 & 641.8 & 618.0 & 592.5 \\
10 & 705.2 & 688.2 & 669.5 & 650.9 & 629.1 & 606.3 \\
15 & 709.9 & 693.7 & 676.0 & 659.2 & 638.7 & 617.9 \\
20 & 714.4 & 698.9 & 681.9 & 666.6 & 647.2 & 628.1 \\
30 & 722.6 & 708.1 & 692.7 & 679.9 & 662.4 & 645.7 \\
40 & 730.0 & 716.6 & 702.5 & 691.4 & 675.4 & 660.8 \\
50 & 737.0 & 724.2 & 711.5 & 701.7 & 686.9 & 673.5 \\
60 & 743.4 & 731.3 & 719.8 & 710.9 & 697.2 & 684.9 \\
70 & 749.6 & 737.9 & 727.5 & 719.4 & 706.6 & 695.1 \\
80 & 755.4 & 744.2 & 734.8 & 727.2 & 715.1 & 704.4 \\
90 & 761.0 & 750.1 & 741.5 & 734.5 & 723.0 & 712.8 \\
100 & 766.3 & 755.7 & 747.9 & 741.2 & 730.3 & 720.6 \\
\hline
\end{tabular}


Resultados experimentales de las medidas de densidades y viscosidades en mezclas de $\mathrm{CO}_{2}+$ hidrocarburo

Tabla 7.15. Coeficientes y estadística de la ecuación Tammann-Tait modificada para la mezcla Hexano $+\mathrm{CO}_{2}$ a $x_{\mathrm{CO} 2}=0.2003, p_{\text {ref }}=5 \mathrm{MPa}$.

Parámetros de la ecuación Tammann-Tait modificada

\begin{tabular}{ccccccccc}
\hline $\begin{array}{c}\mathrm{A}_{0} \\
\mathrm{~kg} \cdot \mathrm{m}^{-3}\end{array}$ & $\begin{array}{c}\mathrm{A}_{1} \\
\mathrm{~kg} \cdot \mathrm{m}^{-3} \cdot \mathrm{K}^{-1}\end{array}$ & $\begin{array}{c}\mathrm{A}_{2} \\
\mathrm{~kg} \cdot \mathrm{m}^{-3} \cdot \mathrm{K}^{-2}\end{array}$ & $\begin{array}{c}\mathrm{B}_{0} \\
\mathrm{MPa}\end{array}$ & $\begin{array}{c}\mathrm{B}_{1} \\
\mathrm{MPa} \cdot \mathrm{K}^{-1}\end{array}$ & $\begin{array}{c}\mathrm{B}_{2} \\
\mathrm{MPa} \cdot \mathrm{K}^{-2}\end{array}$ & $\begin{array}{c}\sigma \\
\mathrm{kg} \cdot \mathrm{m}^{-3}\end{array}$ & $\begin{array}{c}M D \\
(\%)\end{array}$ \\
\hline 749.24 & 0.481 & $-2.42 \mathrm{E}-03$ & 465.16 & -2.15 & $2.52 \mathrm{E}-03$ & 0.100 & 0.81 & 0.20 \\
\hline
\end{tabular}

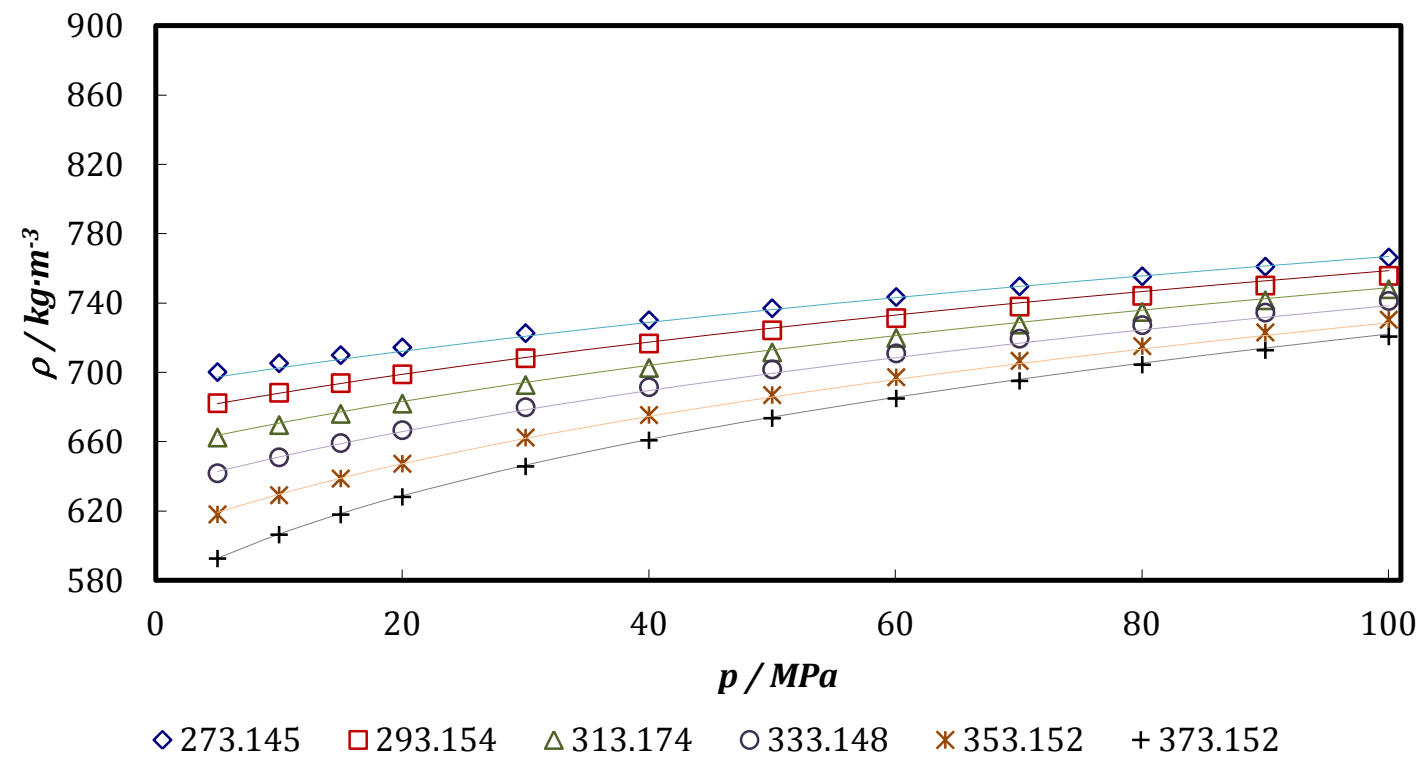

Figura 7.8. Valores experimentales de la densidad para la mezcla Hexano $+\mathrm{CO}_{2}$ a $\mathrm{x}_{\mathrm{CO} 2}=0.2003 a$ diferentes temperaturas en función de la presión. Líneas continuas valores ajustados con la ecuación Tammann-Tait modificada y los puntos valores experimentales. 
Resultados experimentales de las medidas de densidades y viscosidades en mezclas de $\mathrm{CO}_{2}+$ hidrocarburo

Tabla 7.16. Datos experimentales de densidad, $\rho\left(\mathrm{kg}^{\prime} \mathrm{m}^{-3}\right)$, para la mezcla Hexano $+\mathrm{CO}_{2} a x_{\mathrm{CO} 2}=$ 0.2996.

\begin{tabular}{ccccccc}
\hline \multicolumn{7}{c}{$\rho\left(\mathrm{kg} / \mathrm{m}^{3}\right)$} \\
\hline \multicolumn{7}{c}{$T / \mathrm{K}$} \\
\hline$p / \mathrm{MPa}$ & 273.15 & 293.15 & 313.15 & 333.15 & 353.15 & 373.15 \\
\hline 5 & 712.8 & 692.6 & 670.2 & 647.5 & 618.7 & 586.7 \\
10 & 718.4 & 699.4 & 678.5 & 659.1 & 633.0 & 605.3 \\
15 & 723.6 & 705.7 & 686.3 & 669.3 & 645.1 & 620.6 \\
20 & 728.6 & 711.4 & 693.6 & 678.4 & 655.7 & 633.7 \\
30 & 737.6 & 721.8 & 706.8 & 694.2 & 674.2 & 655.6 \\
40 & 745.7 & 731.2 & 718.6 & 707.7 & 690.1 & 673.7 \\
50 & 753.5 & 739.6 & 729.1 & 719.4 & 703.6 & 688.8 \\
60 & 760.7 & 747.7 & 738.7 & 730.1 & 715.6 & 702.0 \\
70 & 767.5 & 755.2 & 747.6 & 739.6 & 726.6 & 713.6 \\
80 & 773.9 & 762.4 & 755.8 & 748.4 & 736.3 & 724.2 \\
90 & 780.0 & 769.2 & 763.4 & 756.5 & 745.3 & 733.6 \\
100 & 785.7 & 775.6 & 770.6 & 764.1 & 753.7 & 742.5 \\
\hline
\end{tabular}

Tabla 7.17. Coeficientes y estadística de la ecuación Tammann-Tait modificada para la mezcla Hexano $+\mathrm{CO}_{2}$ a $x_{\mathrm{CO} 2}=0.2996, p_{\text {ref }}=5 \mathrm{MPa}$.

Parámetros de la ecuación Tammann-Tait modificada

\begin{tabular}{ccccccccc}
\hline $\begin{array}{c}\mathrm{A}_{0} \\
\mathrm{~kg} \cdot \mathrm{m}^{-3}\end{array}$ & $\begin{array}{c}\mathrm{A}_{1} \\
\mathrm{~kg} \cdot \mathrm{m}^{-3} \cdot \mathrm{K}^{-1}\end{array}$ & $\begin{array}{c}\mathrm{A}_{2} \\
\mathrm{~kg} \cdot \mathrm{m}^{-3} \cdot \mathrm{K}^{-2}\end{array}$ & $\begin{array}{c}\mathrm{B}_{0} \\
\mathrm{MPa}\end{array}$ & $\begin{array}{c}\mathrm{B}_{1} \\
\mathrm{MPa} \cdot \mathrm{K}^{-1}\end{array}$ & $\begin{array}{c}\mathrm{B}_{2} \\
\mathrm{MPa} \cdot \mathrm{K}^{-2}\end{array}$ & $\begin{array}{c}\sigma \\
\mathrm{kg} \cdot \mathrm{m}^{-3}\end{array}$ & $\begin{array}{c}\mathrm{MD} \\
(\%)\end{array}$ \\
\hline 712.80 & 0.918 & $-3.33 \mathrm{E}-03$ & 505.13 & -2.42 & $2.93 \mathrm{E}-03$ & 0.103 & 0.95 & 0.26 \\
\hline
\end{tabular}


Resultados experimentales de las medidas de densidades y viscosidades en mezclas de $\mathrm{CO}_{2}+$ hidrocarburo

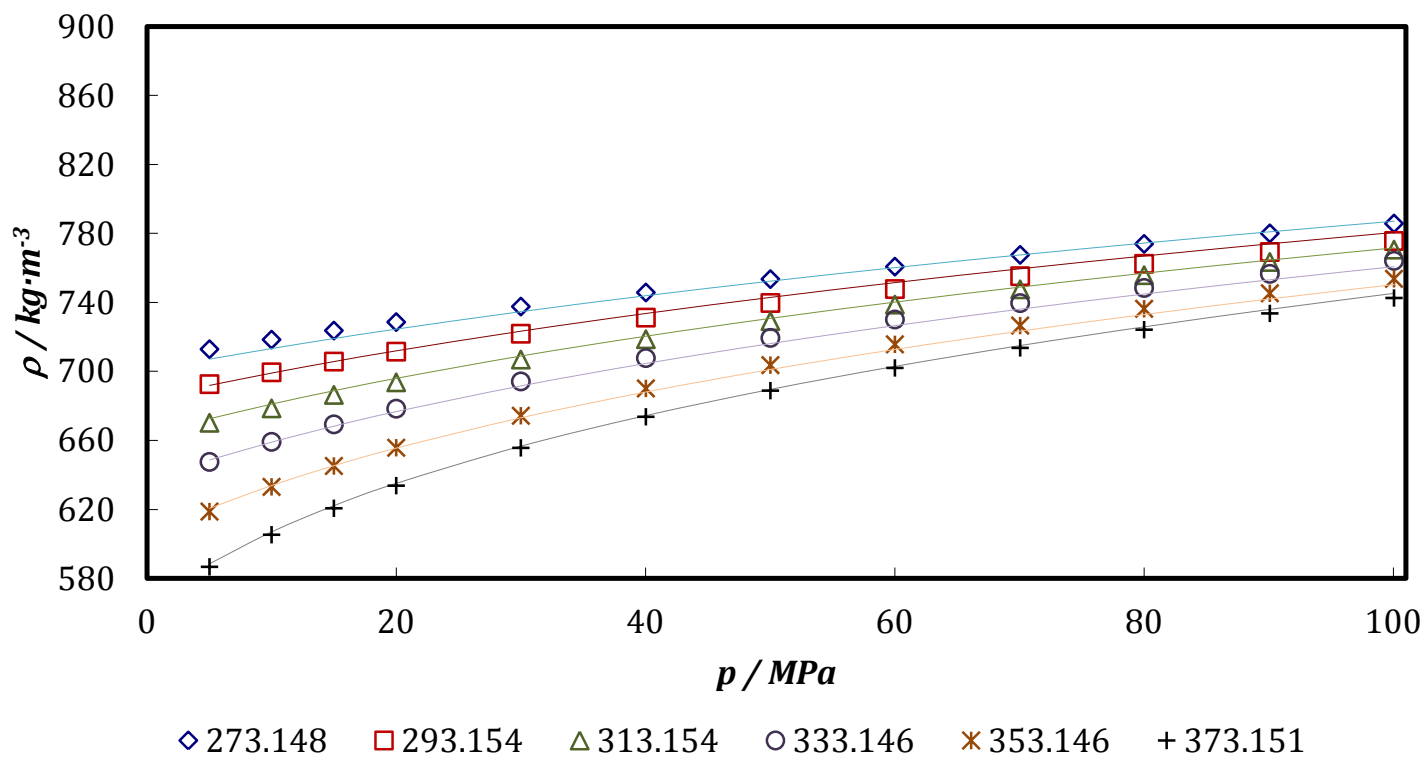

Figura 7.9. Valores experimentales de la densidad para la mezcla Hexano $+\mathrm{CO}_{2}$ a $\mathrm{x}_{\mathrm{CO} 2}=0.2996$ a diferentes temperaturas en función de la presión. Líneas continuas valores ajustados con la ecuación Tammann-Tait modificada y los puntos valores experimentales.

Tabla 7.18. Datos experimentales de densidad, $\rho\left(\mathrm{kg}^{\prime} \mathrm{m}^{-3}\right)$, para la mezcla Hexano $+\mathrm{CO}_{2}$ a $\mathrm{x}_{\mathrm{CO} 2}=$ 0.3998.

\begin{tabular}{ccccccc}
\hline \multicolumn{7}{c}{$\rho\left(\mathrm{kg} / \mathrm{m}^{3}\right)$} \\
\hline \multicolumn{7}{c}{$T / \mathrm{K}$} \\
\hline$p / \mathrm{MPa}$ & 273.15 & 293.15 & 313.15 & 333.15 & 353.15 & 373.15 \\
\hline 5 & 739.2 & 716.6 & 691.6 & 663.3 & & \\
10 & 745.2 & 724.4 & 701.3 & 675.7 & 648.3 & 618.3 \\
15 & 751.2 & 731.5 & 709.7 & 686.2 & 661.4 & 635.0 \\
20 & 756.7 & 737.9 & 717.3 & 695.5 & 672.6 & 648.6 \\
30 & 766.5 & 749.4 & 730.9 & 711.7 & 691.4 & 670.6 \\
40 & 775.6 & 759.7 & 743.0 & 725.6 & 707.2 & 688.5 \\
50 & 784.0 & 768.9 & 753.8 & 737.8 & 720.8 & 705.1 \\
60 & 791.9 & 777.4 & 763.6 & 748.7 & 733.0 & 718.1 \\
70 & 799.2 & 785.2 & 772.5 & 758.6 & 743.8 & 729.7 \\
80 & 806.0 & 792.6 & 780.8 & 767.5 & 753.5 & 740.2 \\
90 & 812.5 & 799.5 & 788.4 & 775.8 & 762.4 & 749.7 \\
100 & 818.5 & 806.0 & 795.6 & 783.5 & 770.7 & 758.5 \\
\hline
\end{tabular}


Resultados experimentales de las medidas de densidades y viscosidades en mezclas de $\mathrm{CO}_{2}+$ hidrocarburo

Tabla 7.19. Coeficientes y estadística de la ecuación Tammann-Tait modificada para la mezcla Hexano $+\mathrm{CO}_{2}$ a $x_{\mathrm{CO} 2}=0.3998, p_{\text {ref }}=100 \mathrm{MPa}$.

Parámetros de la ecuación Tammann-Tait modificada

\begin{tabular}{ccccccccc}
\hline $\mathrm{A}_{0}$ & $\mathrm{~A}_{1}$ & $\mathrm{~A}_{2}$ & $\mathrm{~B}_{0}$ & $\mathrm{~B}_{1}$ & $\mathrm{~B}_{2}$ & $\mathrm{C}$ & $\sigma$ & $M D$ \\
$\mathrm{~kg} \cdot \mathrm{m}^{-3}$ & $\mathrm{~kg} \cdot \mathrm{m}^{-3} \cdot \mathrm{K}^{-1}$ & $\mathrm{~kg} \cdot \mathrm{m}^{-3} \cdot \mathrm{K}^{-2}$ & $\mathrm{MPa}$ & $\mathrm{MPa} \cdot \mathrm{K}^{-1}$ & $\mathrm{MPa} \cdot \mathrm{K}^{-2}$ & & $\mathrm{~kg} \cdot \mathrm{m}^{-3}$ & $(\%)$ \\
\hline 939.72 & -0.327 & $-4.26 \mathrm{E}-04$ & 440.27 & -2.02 & $2.28 \mathrm{E}-03$ & 0.118 & 0.44 & 0.18 \\
\hline
\end{tabular}

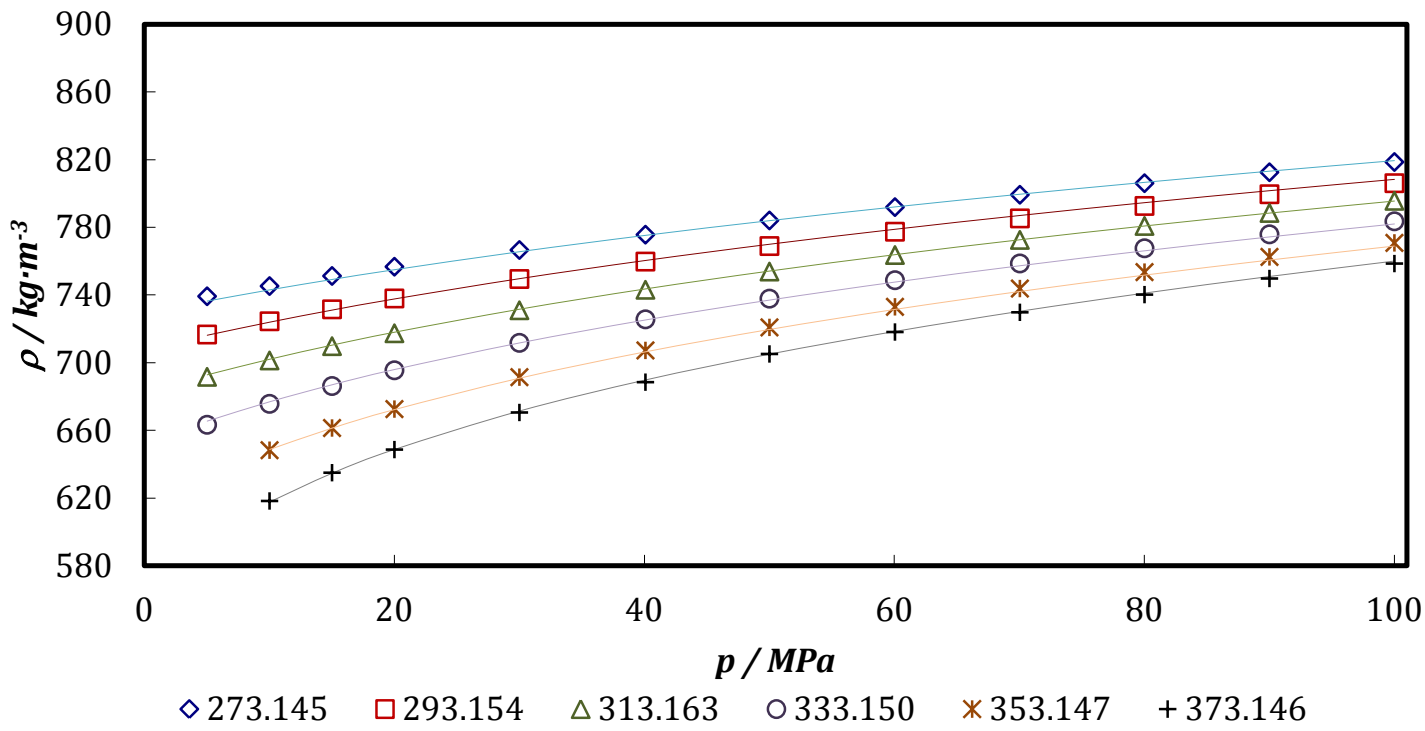

Figura 7.10. Valores experimentales de la densidad para la mezcla Hexano $+\mathrm{CO}_{2}$ a $x_{\mathrm{CO} 2}=0.3998$ a diferentes temperaturas en función de la presión. Líneas continuas valores ajustados con la ecuación Tammann-Tait modificada y los puntos valores experimentales. 
Resultados experimentales de las medidas de densidades y viscosidades en mezclas de $\mathrm{CO}_{2}+$ hidrocarburo

Tabla 7.20. Datos experimentales de densidad, $\rho\left(\mathrm{kg} \cdot \mathrm{m}^{-3}\right)$, para la mezcla Hexano $+\mathrm{CO}_{2}$ a $x_{\mathrm{CO} 2}=$ 0.5994.

\begin{tabular}{ccccccc}
\hline \multicolumn{7}{c}{$\rho\left(\mathrm{kg} / \mathrm{m}^{3}\right)$} \\
\hline \multicolumn{7}{c}{$T / \mathrm{K}$} \\
\hline$p / \mathrm{MPa}$ & 273.15 & 293.15 & 313.15 & 333.15 & 353.15 & 373.15 \\
\hline 15 & 815.8 & 780.8 & 743.7 & 698.4 & 647.2 & 590.8 \\
20 & 823.1 & 791.2 & 758.6 & 719.4 & 675.5 & 631.4 \\
30 & 836.1 & 808.2 & 781.4 & 749.4 & 712.2 & 677.7 \\
40 & 848.0 & 822.4 & 798.8 & 771.5 & 734.3 & 708.2 \\
50 & 858.5 & 834.7 & 813.3 & 789.8 & 757.1 & 731.6 \\
60 & 867.8 & 845.7 & 828.0 & 804.7 & 776.1 & 751.1 \\
70 & 876.4 & 855.8 & 838.6 & 817.8 & 790.3 & 767.8 \\
80 & 884.5 & 865.0 & 848.7 & 829.5 & 803.1 & 782.4 \\
90 & 891.8 & 873.7 & 858.0 & 840.7 & 814.7 & 795.5 \\
100 & 898.8 & 881.8 & 866.9 & 850.1 & 824.5 & 807.5 \\
\hline
\end{tabular}

Tabla 7.21. Coeficientes y estadística de la ecuación Tammann-Tait modificada para la mezcla Hexano $+\mathrm{CO}_{2}$ a $\mathrm{x}_{\mathrm{CO} 2}=0.5994, p_{\text {ref }}=100 \mathrm{MPa}$.

Parámetros de la ecuación Tammann-Tait modificada

\begin{tabular}{ccccccccc}
\hline $\mathrm{A}_{0}$ & $\mathrm{~A}_{1}$ & $\mathrm{~A}_{2}$ & $\mathrm{~B}_{0}$ & $\mathrm{~B}_{1}$ & $\mathrm{~B}_{2}$ & $\mathrm{C}$ & $\begin{array}{c}\sigma \\
\mathrm{kg} \cdot \mathrm{m}^{-3}\end{array}$ & $\begin{array}{c}\text { MD } \\
(\%)\end{array}$ \\
\hline $\mathrm{kg} \cdot \mathrm{m}^{-3}$ & $\mathrm{~kg} \cdot \mathrm{m}^{-3} \cdot \mathrm{K}^{-1}$ & $\mathrm{~kg} \cdot \mathrm{m}^{-3} \cdot \mathrm{K}^{-2}$ & $\mathrm{MPa}$ & $\mathrm{MPa} \cdot \mathrm{K}^{-1}$ & $\mathrm{MPa} \cdot \mathrm{K}^{-2}$ & & 2.43 & 1.16 \\
\hline 877.24 & 0.838 & $-2.77 \mathrm{E}-03$ & 642.19 & -3.35 & $4.30 \mathrm{E}-03$ & 0.121 & 2.35 \\
\hline
\end{tabular}




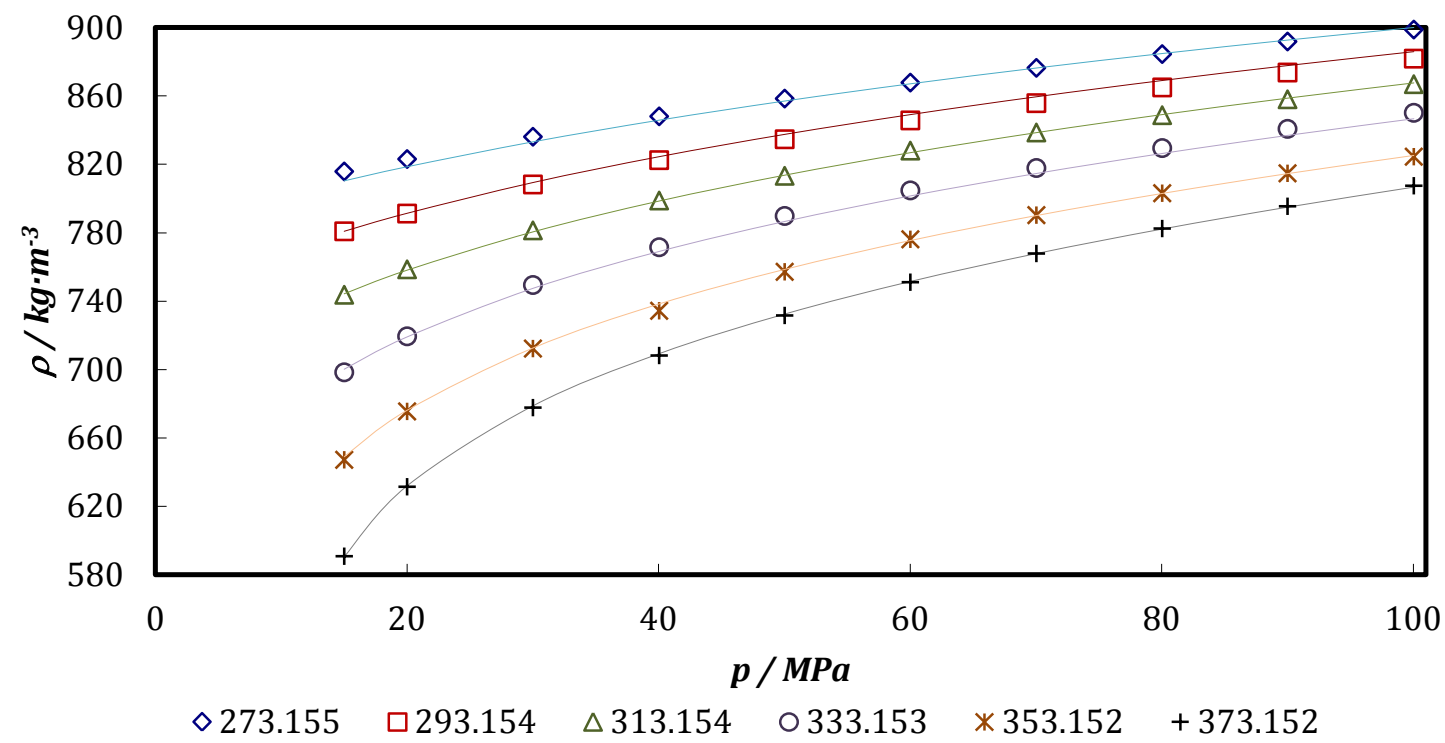

Figura 7.11. Valores experimentales de la densidad para la mezcla Hexano $+\mathrm{CO}_{2}$ a $\mathrm{x}_{\mathrm{CO} 2}=0.5994$ a diferentes temperaturas en función de la presión. Líneas continuas valores ajustados con la ecuación Tammann-Tait modificada y los puntos valores experimentales.

\subsection{Discusión de resultados de la densidad experimental.}

En este trabajo se han determinado las densidades de dos mezclas binarias de hidrocarburos con $\mathrm{CO}_{2}$ realizadas en un rango de temperatura de $273.15 \mathrm{~K}$ a $373.15 \mathrm{~K}$ y en un rango de presiones de $5 \mathrm{MPa}$ hasta $100 \mathrm{MPa}$.

Los ajustes con la ecuación Tammann-Tait modificada fueron buenos teniendo en cuenta la complejidad de las mezclas al ser hidrocarburos con $\mathrm{CO}_{2}$. La máxima desviación porcentual $(M D \%)$ es inferior al $0.41 \%$ en todos los casos y las desviaciones estándar $(\sigma)$ variaron entre $0.15 \mathrm{~kg} \cdot \mathrm{m}^{-3}$ y $1.37 \mathrm{~kg} \cdot \mathrm{m}^{-3}$, para el conjunto de las mezclas estudiadas a excepción de las mezclas a $x_{C O 2}=0.6$.

En el caso del Pentano $+\mathrm{CO}_{2}$ a $x_{C O 2}=0.6$ se obtuvo una desviación estándar de $2.30 \mathrm{~kg} \cdot \mathrm{m}^{-3}$ y una máxima desviación porcentual de $0.97 \%$ con una desviación media porcentual del $0.23 \%$. Para el Hexano $+\mathrm{CO}_{2}$ a $x_{\mathrm{CO} 2}=0.6$ se obtuvo una desviación estándar de $2.43 \mathrm{~kg} \cdot \mathrm{m}^{-3}$ y una máxima desviación porcentual de $1.16 \%$ con una desviación media porcentual del $0.26 \%$.

Los valores experimentales obtenidos muestran una tendencia creciente de la densidad con la presión y decreciente al aumentar la temperatura.

La densidad incrementa con el aumento de la fracción molar de $\mathrm{CO}_{2}$ para ambas mezclas de hidrocarburo $+\mathrm{CO}_{2}$. Siendo el orden de los valores de densidad a una temperatura y presión constante el siguiente, Figura 7.12. 
Resultados experimentales de las medidas de densidades y viscosidades en mezclas de $\mathrm{CO}_{2}+$ hidrocarburo

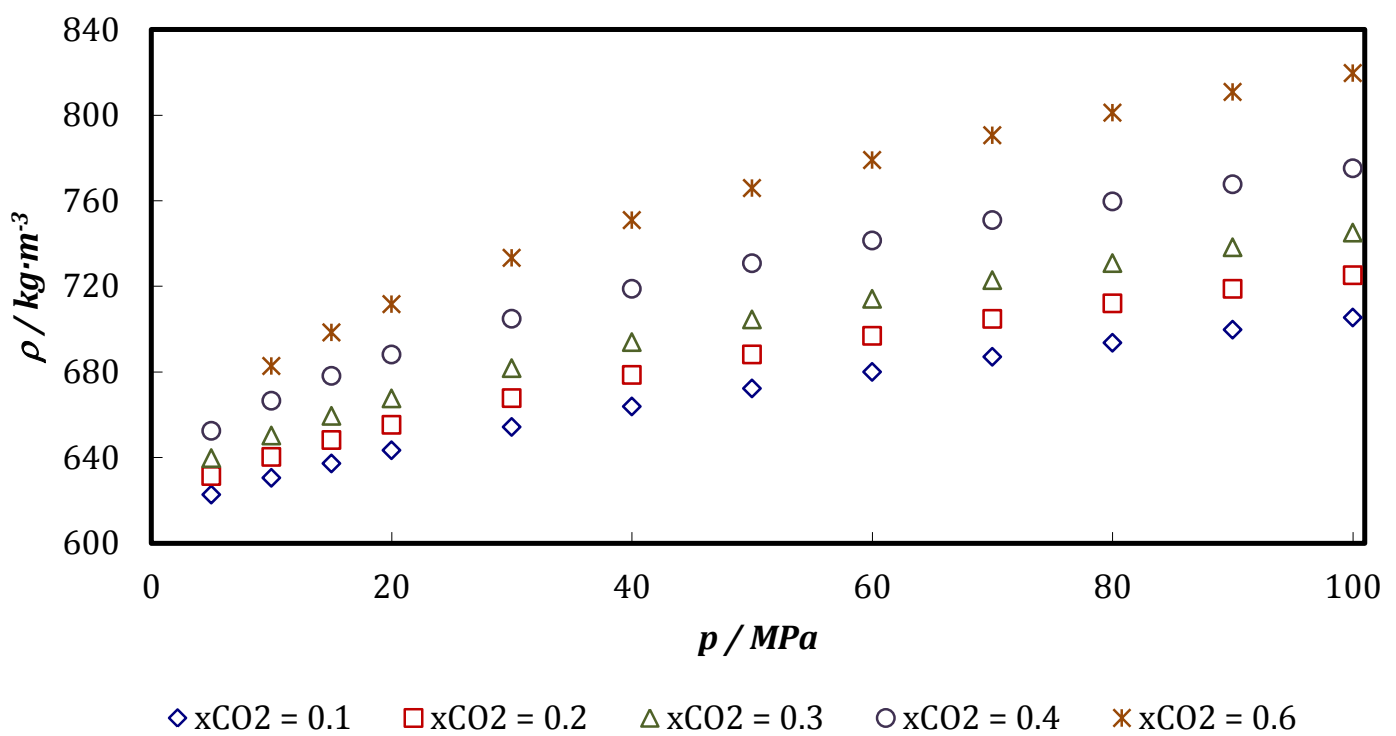

Figura 7.12. Valores experimentales de la densidad para la mezcla Pentano $+\mathrm{CO}_{2}$ a $313.15 \mathrm{~K}$ con diferentes concentraciones en $\mathrm{CO}_{2}$ en función de la presión.

\subsection{Presentación de los resultados experimentales de las medidas de viscosidad.}

A continuación, se muestran los datos experimentales obtenidos de la viscosidad para las mezclas estudiadas, tanto en forma de tablas, como en gráficas. Así como los resultados del ajuste de los datos experimentales al modelo VFT descrito en el apartado 7.3. Este ajuste se realizó para cada una de las composiciones estudiadas, en función de la temperatura y la presión. Además, se muestra la desviación estándar del ajuste $\sigma$ (ecuación 5.6) entre datos experimentales y el modelo utilizado. 
Resultados experimentales de las medidas de densidades y viscosidades en mezclas de $\mathrm{CO}_{2}+$ hidrocarburo

\subsubsection{Pentano puro.}

Tabla 7.22. Datos experimentales de viscosidad, $\eta(\mathrm{mPa} \cdot \mathrm{s})$, para Pentano.

\begin{tabular}{ccccccc}
\hline \multicolumn{7}{c}{$\eta(m P a \cdot s)$} \\
\hline \multicolumn{7}{c}{$T / \mathrm{K}$} \\
\hline$p / \mathrm{MPa}$ & 293.15 & 303.15 & 313.15 & 333.15 & 353.15 & 373.15 \\
\hline 0.1 & 0.2270 & 0.2067 & & & \\
1 & 0.2279 & 0.2098 & 0.1965 & 0.1623 & 0.1386 & \\
2.5 & 0.2316 & 0.2120 & 0.1997 & 0.1672 & 0.1413 & 0.1249 \\
5 & 0.2380 & 0.2190 & 0.2055 & 0.1728 & 0.1463 & 0.1301 \\
10 & 0.2498 & 0.2316 & 0.2165 & 0.1823 & 0.1569 & 0.1382 \\
20 & 0.2772 & 0.2576 & 0.2381 & 0.2037 & 0.1772 & 0.1561 \\
30 & 0.3032 & 0.2807 & 0.2605 & 0.2259 & 0.1944 & 0.1768 \\
40 & 0.3288 & 0.3030 & 0.2814 & 0.2443 & 0.2165 & 0.1919 \\
50 & 0.3520 & 0.3268 & 0.3033 & 0.2674 & 0.2360 & 0.2082 \\
60 & 0.3786 & 0.3420 & 0.3255 & 0.2844 & 0.2504 & 0.2261 \\
70 & 0.4001 & 0.3722 & 0.3466 & 0.3079 & 0.2711 & 0.2437 \\
80 & 0.4256 & 0.3928 & 0.3657 & 0.3238 & 0.2888 & 0.2625 \\
\hline
\end{tabular}

Tabla 7.23. Coeficientes y estadística de la ecuación VFT modificada para Pentano, $p_{r e f}=2.5 M P a$.

\begin{tabular}{cccccccc}
\hline \multicolumn{7}{c}{ Parámetros de la ecuación VFT modificada } \\
\hline $\mathrm{A}$ & $\mathrm{B}$ & $\mathrm{C}$ & $\mathrm{E}_{0}$ & $\mathrm{E}_{1}$ & $\mathrm{E}_{2}$ & $\mathrm{~F}$ & $\sigma$ \\
$\mathrm{mPa} \cdot \mathrm{s}$ & $\mathrm{K}$ & $\mathrm{K}$ & $\mathrm{MPa}$ & $\mathrm{MPa} \cdot \mathrm{K}^{-1}$ & $\mathrm{MPa} \cdot \mathrm{K}^{-2}$ & & $\mathrm{mPa} \cdot \mathrm{s}$ \\
\hline 0.0098 & 1047.66 & -37.07 & 14.02 & 0.58 & -0.001 & 0.899 & 0.0030 \\
\hline
\end{tabular}


Resultados experimentales de las medidas de densidades y viscosidades en mezclas de $\mathrm{CO}_{2}+$ hidrocarburo

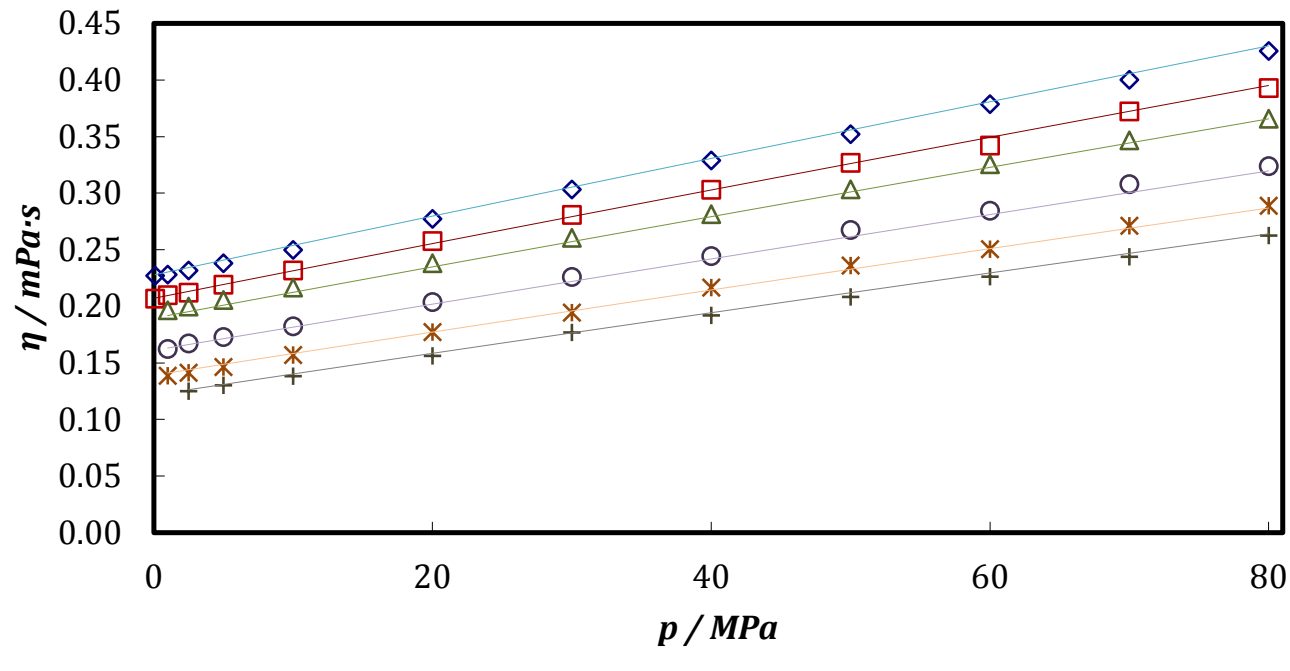

$\diamond 293.15 \mathrm{~K} \quad \square 303.15 \mathrm{~K} \quad \Delta 313.15 \mathrm{~K} \quad 0333.15 \mathrm{~K} * 353.15 \mathrm{~K}+373.15 \mathrm{~K}$

Figura 7.13. Valores experimentales de la viscosidad para el Pentano a diferentes temperaturas en función de la presión. Líneas continuas valores ajustados con la ecuación VFT modificada y los puntos valores experimentales.

\subsubsection{Mezcla: Pentano $+\mathrm{CO}_{2}$.}

Tabla 7.24. Datos experimentales de viscosidad, $\eta(\mathrm{mPa} \cdot \mathrm{s})$, para la mezcla Pentano $+\mathrm{CO}_{2}$ a $x_{\mathrm{CO} 2}=$ 0.0950 .

\begin{tabular}{cccccc}
\hline \multicolumn{5}{c}{$\eta(m P a \cdot s)$} \\
\hline$p / \mathrm{MPa}$ & 293.15 & 313.15 & 333.15 & 353.15 & 373.15 \\
\hline 5 & 0.2049 & 0.1727 & 0.1436 & 0.1240 & 0.1111 \\
10 & 0.2169 & 0.1827 & 0.1539 & 0.1341 & 0.1197 \\
15 & 0.2282 & 0.1927 & 0.1637 & 0.1434 & 0.1296 \\
20 & 0.2400 & 0.2027 & 0.1740 & 0.1519 & 0.1370 \\
30 & 0.2610 & 0.2253 & 0.1931 & 0.1695 & 0.1539 \\
40 & 0.2835 & 0.2419 & 0.2110 & 0.1868 & 0.1691 \\
50 & 0.3040 & 0.2630 & 0.2292 & 0.2028 & 0.1840 \\
60 & 0.3249 & 0.2818 & 0.2479 & 0.2193 & 0.1982 \\
70 & 0.3469 & 0.3018 & 0.2652 & 0.2341 & 0.2120 \\
80 & 0.3686 & 0.3204 & 0.2818 & 0.2518 & 0.2273 \\
\hline
\end{tabular}


Resultados experimentales de las medidas de densidades y viscosidades en mezclas de $\mathrm{CO}_{2}+$ hidrocarburo

Tabla 7.25. Coeficientes y estadística de la ecuación VFT modificada para la mezcla Pentano $+\mathrm{CO}_{2}$ a $x_{C O 2}=0.0950, p_{r e f}=5 M P a$.

\begin{tabular}{cccccccc}
\hline \multicolumn{7}{c}{ Parámetros de la ecuación VFT modificada } \\
\hline $\mathrm{A}$ & $\mathrm{B}$ & $\mathrm{C}$ & $\mathrm{E}_{0}$ & $\mathrm{E}_{1}$ & $\mathrm{E}_{2}$ & $\mathrm{~F}$ & $\sigma$ \\
$\mathrm{mPa} \cdot \mathrm{s}$ & $\mathrm{K}$ & $\mathrm{K}$ & $\mathrm{MPa}$ & $\mathrm{MPa} \cdot \mathrm{K}^{-1}$ & $\mathrm{MPa} \cdot \mathrm{K}^{-2}$ & & $\mathrm{mPa} \cdot \mathrm{s}$ \\
\hline 0.009 & 1051.69 & -38.17 & 6.27 & 0.56 & -0.001 & 0.84 & 0.0024 \\
\hline
\end{tabular}

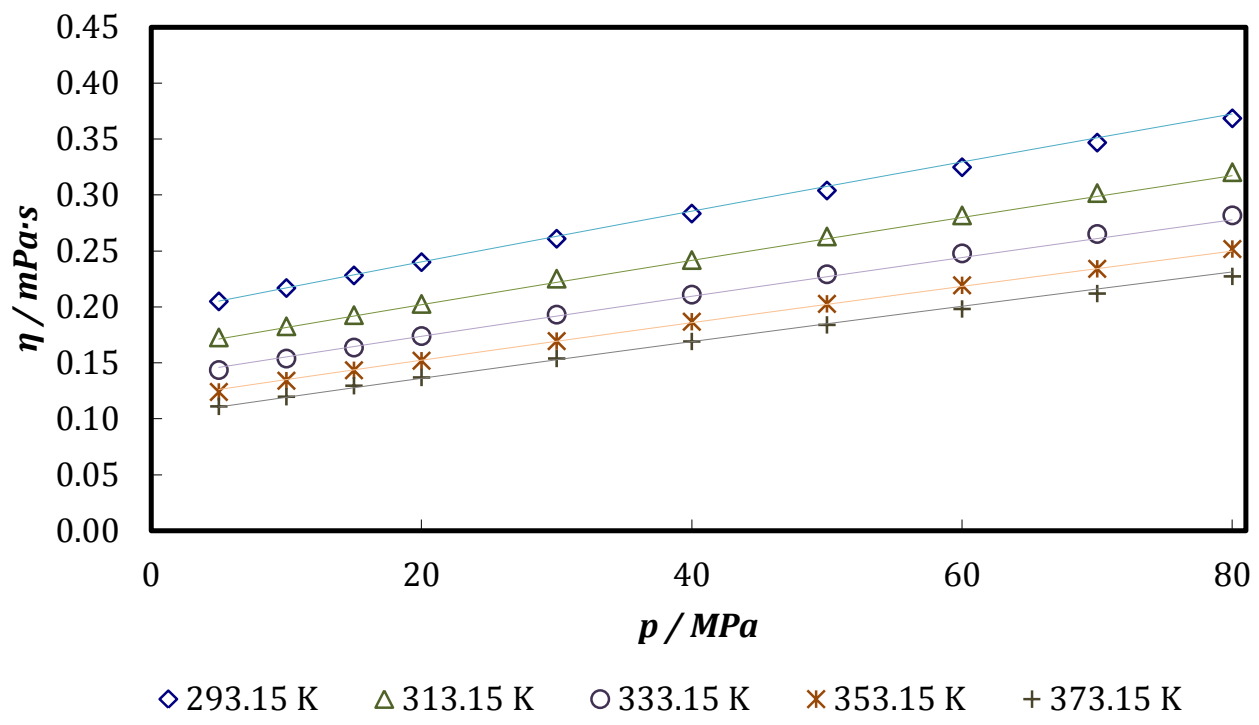

Figura 7.14. Valores experimentales de la viscosidad para Pentano $+\mathrm{CO}_{2}$ a $\mathrm{x}_{\mathrm{CO} 2}=0.0950$ a diferentes temperaturas en función de la presión. Líneas continuas valores ajustados con la ecuación VFT modificada y los puntos valores experimentales. 
Resultados experimentales de las medidas de densidades y viscosidades en mezclas de $\mathrm{CO}_{2}+$ hidrocarburo

Tabla 7.26. Datos experimentales de viscosidad, $\eta(\mathrm{mPa} \cdot \mathrm{s})$, para la mezcla Pentano $+\mathrm{CO}_{2}$ a $\mathrm{x}_{\mathrm{CO} 2}=$ 0.3003 .

\begin{tabular}{cccccc}
\hline \multicolumn{5}{c}{$\eta(\mathrm{mPa} \cdot \mathrm{s})$} \\
\hline \multicolumn{5}{c}{$T / \mathrm{K}$} \\
\hline 5 & 0.1722 & 0.1429 & 0.1205 & 0.1036 & \\
10 & 0.1826 & 0.1534 & 0.1300 & 0.1138 & 0.1056 \\
15 & 0.1928 & 0.1631 & 0.1397 & 0.1234 & 0.1156 \\
20 & 0.2029 & 0.1722 & 0.1487 & 0.1318 & 0.1241 \\
30 & 0.2221 & 0.1896 & 0.1655 & 0.1477 & 0.1377 \\
40 & 0.2411 & 0.2068 & 0.1818 & 0.1627 & 0.1497 \\
50 & 0.2592 & 0.2244 & 0.1970 & 0.1766 & 0.1622 \\
60 & 0.2771 & 0.2408 & 0.2124 & 0.1905 & 0.1757 \\
70 & 0.2959 & 0.2579 & 0.2278 & 0.2034 & 0.1879 \\
80 & 0.3137 & 0.2746 & 0.2423 & 0.2189 & 0.2005 \\
\hline
\end{tabular}

Tabla 7.27. Coeficientes y estadística de la ecuación VFT modificada para la mezcla Pentano $+\mathrm{CO}_{2}$ a $x_{C O 2}=0.3003, p_{\text {ref }}=10 \mathrm{MPa}$.

\begin{tabular}{cccccccc}
\hline \multicolumn{7}{c}{ Parámetros de la ecuación VFT modificada } \\
\hline $\mathrm{A}$ & $\mathrm{B}$ & $\mathrm{C}$ & $\mathrm{E}_{0}$ & $\mathrm{E}_{1}$ & $\mathrm{E}_{2}$ & $\mathrm{~F}$ & $\sigma$ \\
$\mathrm{mPa} \cdot \mathrm{s}$ & $\mathrm{K}$ & $\mathrm{K}$ & $\mathrm{MPa}$ & $\mathrm{MPa} \cdot \mathrm{K}^{-1}$ & $\mathrm{MPa} \cdot \mathrm{K}^{-2}$ & & $\mathrm{mPa} \cdot \mathrm{s}$ \\
\hline 0.009 & 1048.50 & -52.93 & -12.36 & 0.47 & -0.001 & 0.64 & 0.0020 \\
\hline
\end{tabular}


Resultados experimentales de las medidas de densidades y viscosidades en mezclas de $\mathrm{CO}_{2}+$ hidrocarburo

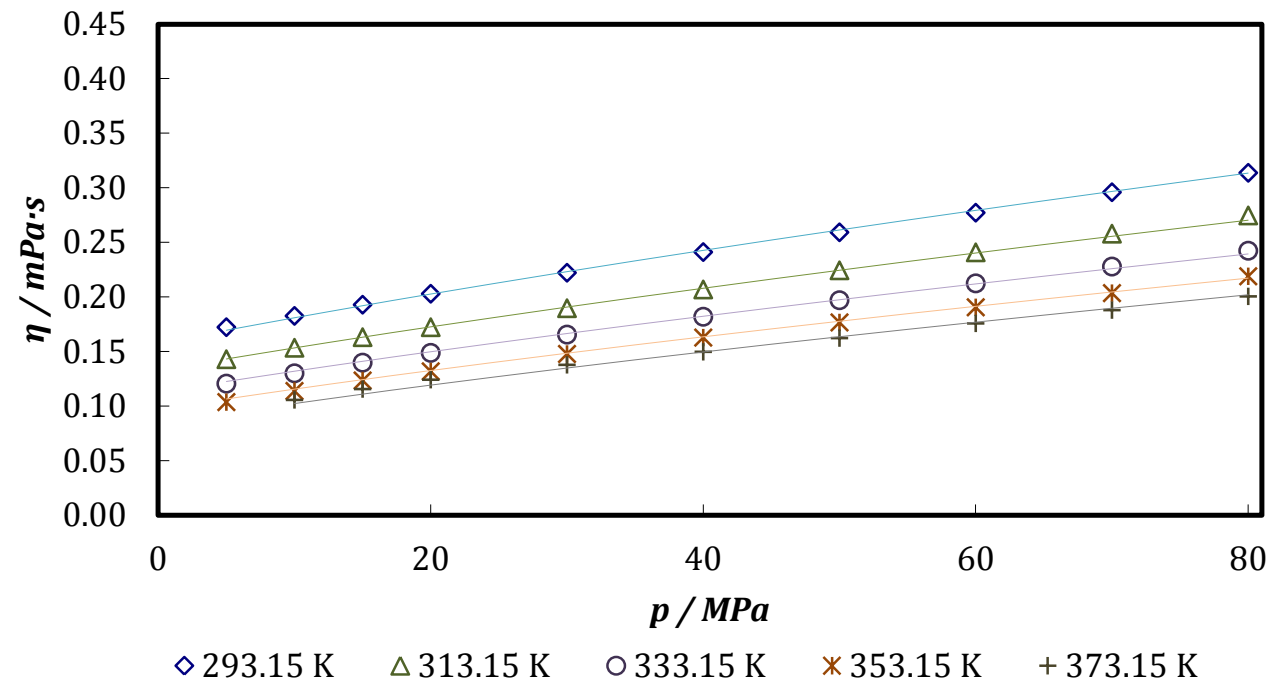

Figura 7.15. Valores experimentales de la viscosidad para Pentano $+\mathrm{CO}_{2}$ a $\mathrm{x}_{\mathrm{CO} 2}=0.3003$ a diferentes temperaturas en función de la presión. Líneas continuas valores ajustados con la ecuación VFT modificada y los puntos valores experimentales.

Para el cálculo de las viscosidades del pentano se utilizaron los datos de densidad reportados en la Tesis de J. Zambrano [12] y para las mezclas se utilizaron las densidades medidas en esta tesis.

Los resultados de la viscosidad obtenidos para el pentano se han comparado con los encontrados en la literatura [13], donde se encuentran viscosidades a dos temperaturas $303.15 \mathrm{~K}$ y $353.15 \mathrm{~K}$.

En la figura 7.16 se han representado las desviaciones relativas obtenidas entre los datos experimentales y los de la literatura. La desviación media obtenida ( $A A D \%$ ) para el pentano a $303.15 \mathrm{~K}$ fue de $1.2 \%$. Siendo para el pentano a $353.15 \mathrm{~K}$ de $2.6 \%$.

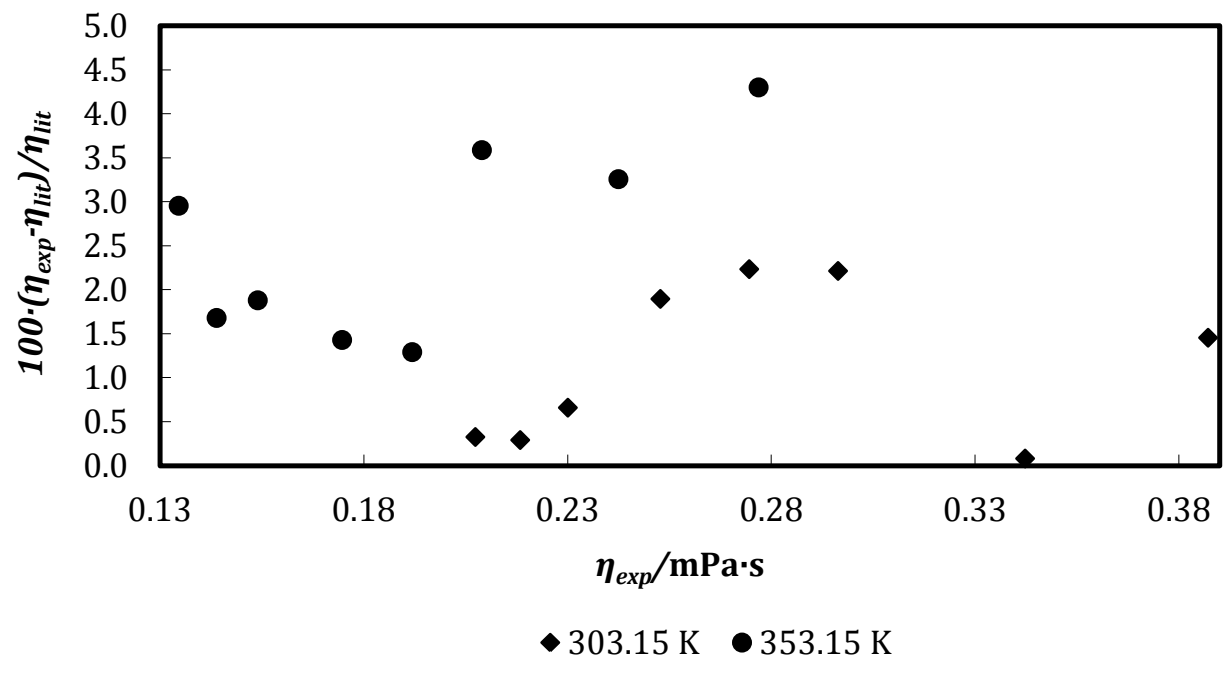

Figura 7.16. Desviación relativa porcentual de la $\eta$ experimental del pentano respecto a los datos de la bibliografía de Audonnet y Pádua [13]. 
Resultados experimentales de las medidas de densidades y viscosidades en mezclas de $\mathrm{CO}_{2}+$ hidrocarburo

\subsubsection{Mezcla: Hexano $+\mathrm{CO}_{2}$.}

Tabla 7.28. Datos experimentales de viscosidad, $\eta(\mathrm{mPa} \cdot \mathrm{s})$, para la mezcla Hexano $+\mathrm{CO}_{2}$ a $\mathrm{x}_{\mathrm{CO} 2}=$ 0.1001.

\begin{tabular}{cccccc}
\hline \multicolumn{5}{c}{$\eta(\mathrm{mPa} \cdot s)$} \\
\hline$p / \mathrm{MPa}$ & 293.15 & 313.15 & 333.15 & 353.15 & 373.15 \\
\hline 5 & 0.2626 & 0.2165 & 0.1832 & 0.1609 & 0.1513 \\
10 & 0.2774 & 0.2298 & 0.1965 & 0.1741 & 0.1646 \\
15 & 0.2918 & 0.2427 & 0.2083 & 0.1838 & 0.1753 \\
20 & 0.3063 & 0.2552 & 0.2204 & 0.1951 & 0.1861 \\
30 & 0.3308 & 0.2806 & 0.2417 & 0.2146 & 0.2045 \\
40 & 0.3575 & 0.3052 & 0.2640 & 0.2343 & 0.2217 \\
50 & 0.3882 & 0.3286 & 0.2845 & 0.2534 & 0.2385 \\
60 & 0.4144 & 0.3537 & 0.3054 & 0.2708 & 0.2542 \\
70 & 0.4465 & 0.3773 & 0.3264 & 0.2898 & 0.2705 \\
80 & 0.4722 & 0.4008 & 0.3477 & 0.3104 & 0.2854 \\
90 & 0.5010 & 0.4256 & 0.3684 & 0.3273 & 0.3038 \\
100 & 0.5346 & 0.4491 & 0.3901 & 0.3468 & 0.3194 \\
\hline
\end{tabular}

Tabla 7.29. Coeficientes y estadística de la ecuación VFT modificada para la mezcla Hexano $+\mathrm{CO}_{2}$ a $x_{C O 2}=0.1001, p_{r e f}=5 M P a$.

\begin{tabular}{cccccccc}
\hline \multicolumn{7}{c}{ Parámetros de la ecuación VFT modificada } \\
\hline $\mathrm{A}$ & $\mathrm{B}$ & $\mathrm{C}$ & $\mathrm{E}_{0}$ & $\mathrm{E}_{1}$ & $\mathrm{E}_{2}$ & $\mathrm{~F}$ & $\sigma$ \\
$\mathrm{mPa} \cdot \mathrm{s}$ & $\mathrm{K}$ & $\mathrm{K}$ & $\mathrm{MPa}$ & $\mathrm{MPa} \cdot \mathrm{K}^{-1}$ & $\mathrm{MPa} \cdot \mathrm{K}^{-2}$ & & $\mathrm{mPa} \cdot \mathrm{s}$ \\
\hline 0.012 & 1048.60 & -50.34 & -16.68 & 0.65 & -0.001 & 0.87 & 0.0044 \\
\hline
\end{tabular}


Resultados experimentales de las medidas de densidades y viscosidades en mezclas de $\mathrm{CO}_{2}+$ hidrocarburo

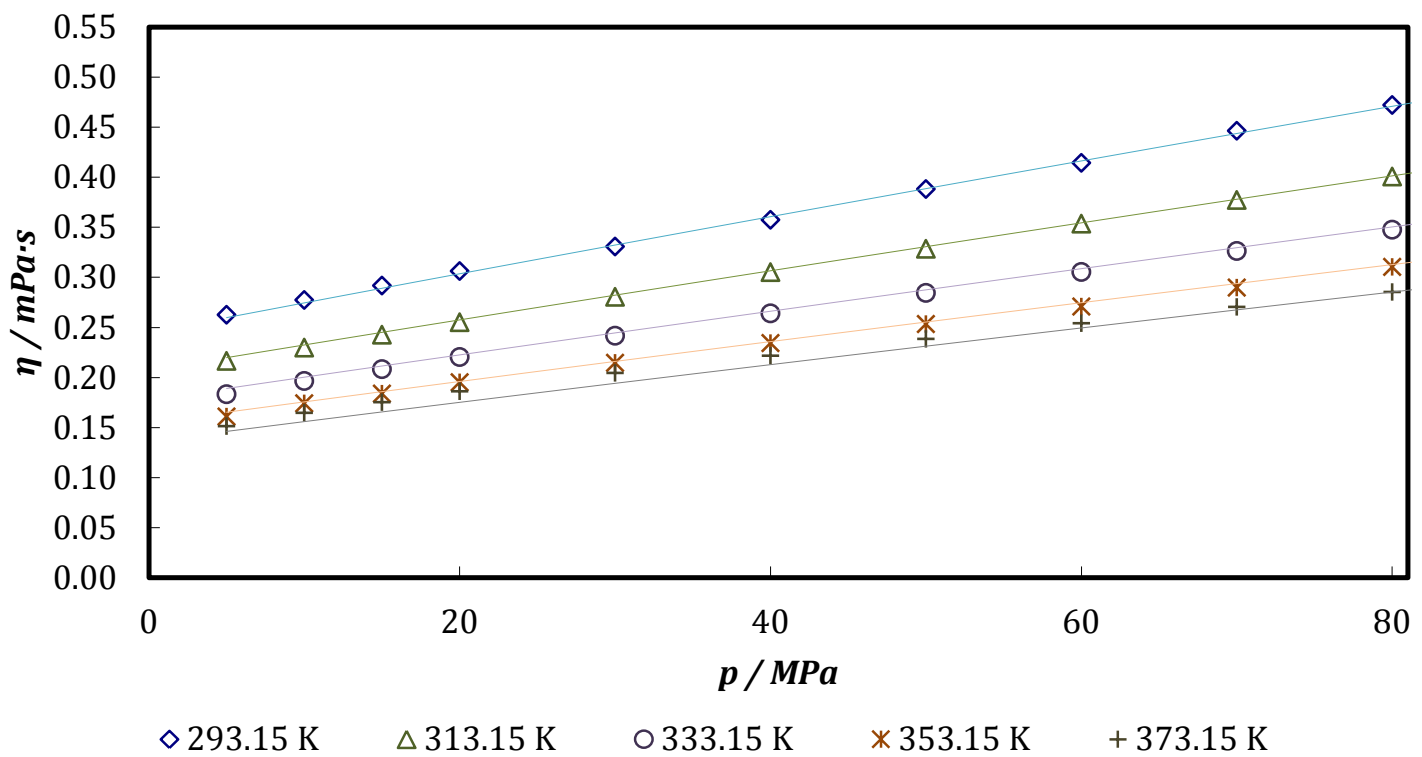

Figura 7.17. Valores experimentales de la viscosidad para Hexano $+\mathrm{CO}_{2}$ a $\mathrm{x}_{\mathrm{CO} 2}=0.1001$ a diferentes temperaturas en función de la presión. Líneas continuas valores ajustados con la ecuación VFT modificada y los puntos valores experimentales.

Tabla 7.30. Datos experimentales de viscosidad, $\eta(\mathrm{mPa} \cdot \mathrm{s})$, para la mezcla Hexano $+\mathrm{CO}_{2}$ a $\mathrm{x}_{\mathrm{CO} 2}=$ 0.2997 .

\begin{tabular}{cccccc}
\hline \multicolumn{5}{c}{$\eta(m P a \cdot s)$} \\
\hline \multicolumn{5}{c}{$T / \mathrm{K}$} \\
\hline 5 & 0.2302 & 0.1911 & 0.1576 & 0.1414 & \\
10 & 0.2430 & 0.2028 & 0.1699 & 0.1492 & 0.1363 \\
15 & 0.2571 & 0.2155 & 0.1808 & 0.1608 & 0.1464 \\
20 & 0.2697 & 0.2259 & 0.1936 & 0.1704 & 0.1571 \\
30 & 0.2956 & 0.2484 & 0.2119 & 0.1897 & 0.1754 \\
40 & 0.3200 & 0.2702 & 0.2310 & 0.2065 & 0.1906 \\
50 & 0.3449 & 0.2912 & 0.2495 & 0.2228 & 0.2060 \\
60 & 0.3682 & 0.3133 & 0.2686 & 0.2392 & 0.2199 \\
70 & 0.3924 & 0.3344 & 0.2871 & 0.2566 & 0.2345 \\
80 & 0.4159 & 0.3541 & 0.3045 & 0.2724 & 0.2482 \\
90 & 0.4432 & 0.3742 & 0.3240 & 0.2888 & 0.2620 \\
100 & 0.4774 & 0.4003 & 0.3408 & 0.3055 & 0.2754 \\
\hline
\end{tabular}


Resultados experimentales de las medidas de densidades y viscosidades en mezclas de $\mathrm{CO}_{2}+$ hidrocarburo

Tabla 7.31. Coeficientes y estadística de la ecuación VFT modificada para la mezcla Hexano $+\mathrm{CO}_{2}$ a $x_{C O 2}=0.2997, p_{r e f}=10 M P a$.

\begin{tabular}{cccccccc}
\hline \multicolumn{7}{c}{ Parámetros de la ecuación VFT modificada } \\
\hline $\mathrm{A}$ & $\mathrm{B}$ & $\mathrm{C}$ & $\mathrm{E}_{0}$ & $\mathrm{E}_{1}$ & $\mathrm{E}_{2}$ & $\mathrm{~F}$ & $\sigma$ \\
$\mathrm{mPa} \cdot \mathrm{s}$ & $\mathrm{K}$ & $\mathrm{K}$ & $\mathrm{MPa}$ & $\mathrm{MPa} \cdot \mathrm{K}^{-1}$ & $\mathrm{MPa} \cdot \mathrm{K}^{-2}$ & & $\mathrm{mPa} \cdot \mathrm{s}$ \\
\hline 0.011 & 1051.09 & -50.29 & -27.36 & 0.57 & -0.001 & 0.76 & 0.0034 \\
\hline
\end{tabular}

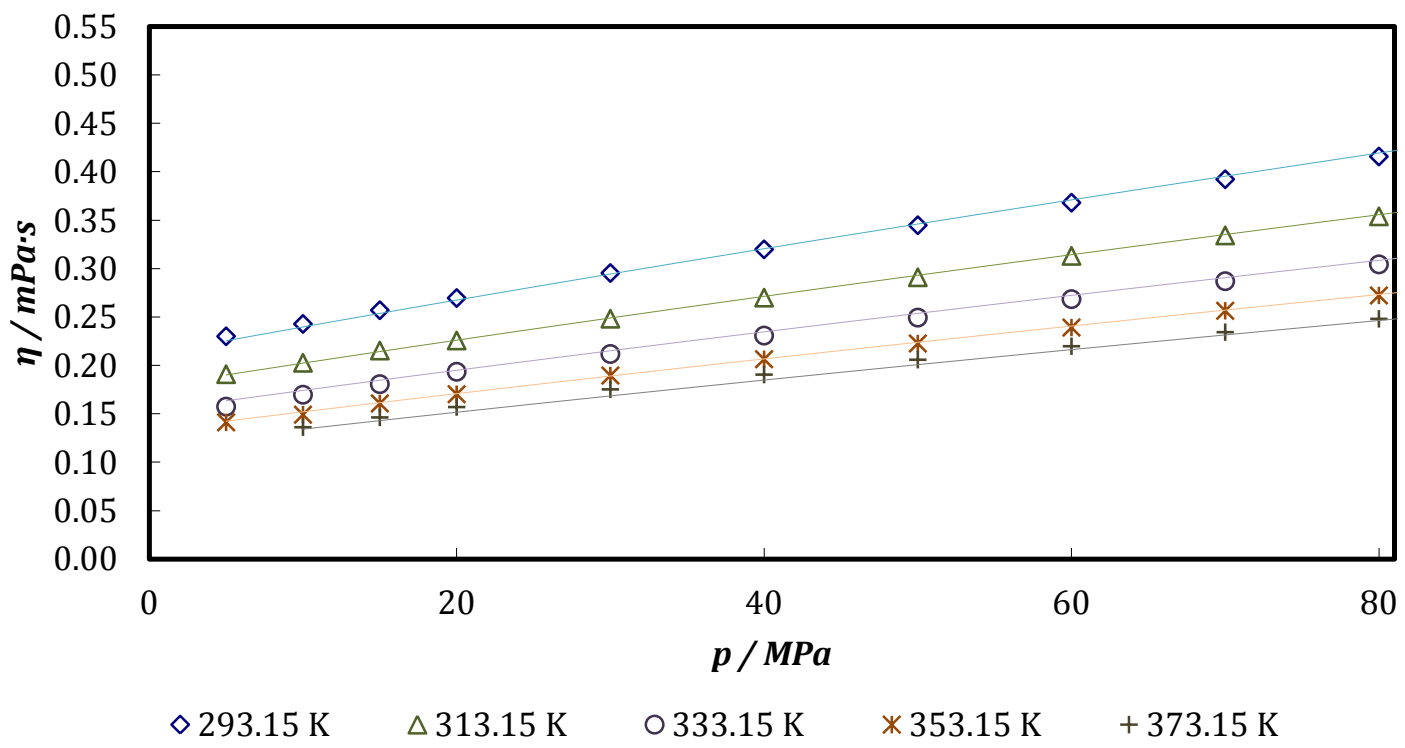

Figura 7.18. Valores experimentales de la viscosidad para Hexano $+\mathrm{CO}_{2}$ a $\mathrm{x}_{\mathrm{CO} 2}=0.2997$ a diferentes temperaturas en función de la presión. Líneas continuas valores ajustados con la ecuación VFT modificada y los puntos valores experimentales.

\subsection{Discusión de resultados de la viscosidad experimental.}

En este trabajo se determinaron las viscosidades del pentano y de dos mezclas binarias de hidrocarburos $+\mathrm{CO}_{2}$ realizadas en un rango de temperatura de $293.15 \mathrm{~K}$ a $373.15 \mathrm{~K}$ y en un rango de presiones de $5 \mathrm{MPa}$ hasta $100 \mathrm{MPa}$.

Los ajustes con la ecuación VFT modificada fueron buenos y del orden de la incertidumbre de medida de la viscosidad y composición. Las desviaciones estándar $(\sigma)$ varían entre $0.0020 \mathrm{mPa} \cdot \mathrm{s}$ y $0.0044 \mathrm{mPa} \cdot \mathrm{s}$.

Los valores experimentales obtenidos muestran una tendencia creciente de la viscosidad con la presión y decreciente al aumentar la temperatura. 
La viscosidad disminuye con el aumento de la fracción molar del $\mathrm{CO}_{2}$ para las mezclas en ambos hidrocarburos. Siendo el orden de los valores de viscosidad a una temperatura y presión constante el siguiente, Figura 7.19.

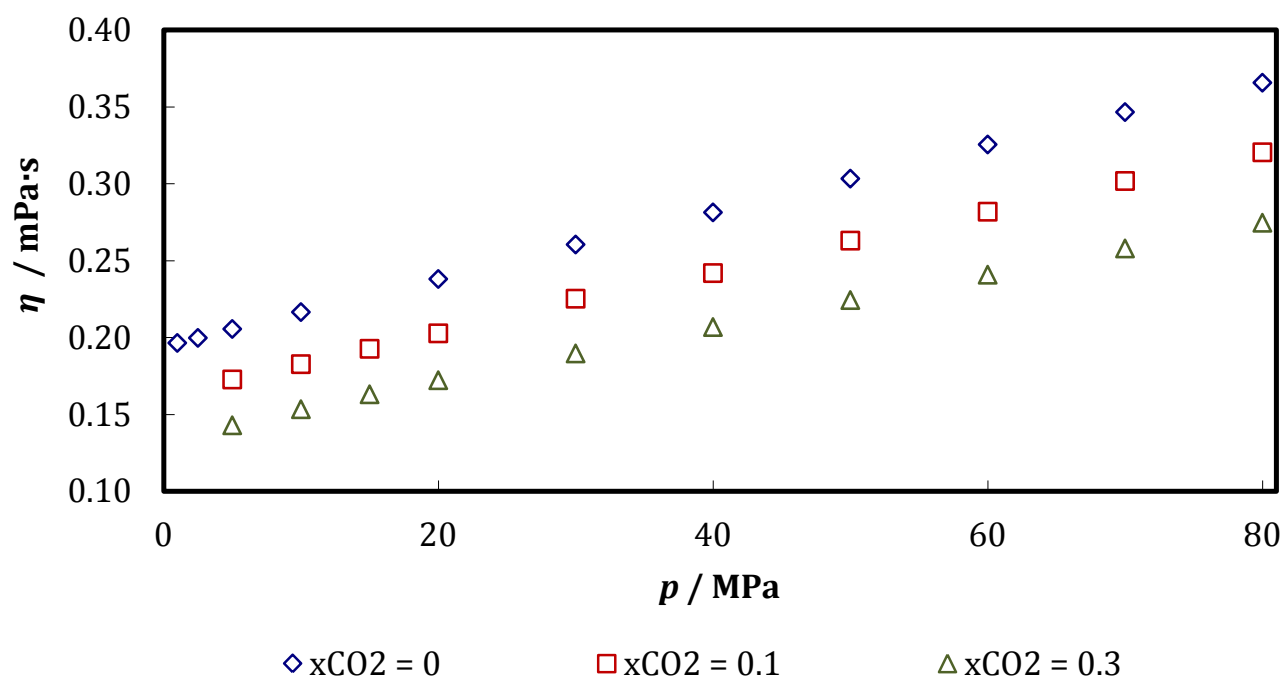

Figura 7.19. Valores experimentales de la viscosidad para la mezcla Pentano $+\mathrm{CO}_{2}$ a $313.15 \mathrm{~K}$ con diferentes concentraciones en $\mathrm{CO}_{2}$ en función de la presión.

Si comparamos diferentes concentraciones de $\mathrm{CO}_{2}$ a una misma temperatura, se observa que la disminución de la viscosidad con el aumento de la concentración en $\mathrm{CO}_{2}$ es ligeramente mayor a altas presiones, tanto en las mezclas de pentano como en las mezclas de hexano.

\subsection{Referencias.}

[1] M. J. P. Comuñas, A. Baylaucq, C. Boned, J. Fernández. "High-pressure measurements of the viscosity and density of two polyethers and two dialkyl carbonates at high pressures". Int. J. Thermophys. Vol. 22, (2001) 749-768.

[2] H. Vogel. "The law of the relation between the viscosity of liquids and the temperature". Phys. Z. Vol. 22, (1921) 645-646.

[3] G. S. Fulcher. "Analysis of recent measurements of the viscosity of glasses". J. Am. Ceram. Soc. Vol. 8, (1925) 339-355.

[4] G. Tammann, W. Hesse. "Die abhängigkeit der viscosität von der temperatur bie unterkühlten flüssigkeiten". Z. Anorg. Allg. Chem. Vol. 156, (1926) 245-257.

[5] C. A. Angell, W. Sichina. "Thermodynamics of the glass transition: Empirical aspects". Ann. N. Y. Acad. Sci. Vol. 279, (1976) 53-67. 
[6] C. A. Angell. "Thermodynamic aspects of the glass transition in liquids and plastic crystals". Pure Appl. Chem. Vol. 63, (1991) 1387-1392.

[7] G. Adam, J. H. Gibbs. "On the temperature dependence of cooperative relaxation properties in glass-forming liquids". J. Chem. Phys. Vol. 43, (1965) 139-146.

[8] X. Paredes, O. Fandiño, A. Pensado, M. J. P. Comuñas, J. Fernández. "Experimental density and viscosity measurements of di(2ethylhexyl) sebacate at high pressure". J. Chem. Thermodynamics (2012) 38-43.

[9] M. Sobrino, E. I. Concepción, A. Gómez-Hernández, M. C. Martín, J. J. Segovia. "Viscosity and density measurements of aqueous amines at high pressures: MDEA-water and MEAwater mixtures for $\mathrm{CO}_{2}$ capture". J. Chem. Thermodynamics. Vol. 98, (2016) 231-241.

[10] D. W. Marquardt. "An algorithm for least-squares estimation of nonlinear parameters". J. Soc. Indust. Appl. Math. Vol. 11 (2), (1963) 431-441.

[11] E. Kiran, H. Pöhler, Y. Xiong. "Volumetric properties of pentane + carbon dioxide at high pressures". J. Chem. Eng. Data. Vol. 41, (1996) 158-165.

[12] J. R. Zambrano. "Desarrollo de un viscosímetro de hilo vibrante para la caracterización termofísica a alta presión de nuevos biocombustibles". Universidad de Valladolid. Tesis Doctoral (2014).

[13] F. Audonnet, A. A. H. Pádua. "Simultaneous measurement of density and viscosity of npentane from 298 to $383 \mathrm{~K}$ and up to $100 \mathrm{MPa}$ using a vibrating-wire instrument". Fluid Phase Equilibria. Vol. 181, (2001) 147-161. 
Capítulo 8

\section{CONCLUSIONES}



Esta tesis se ha realizado en el laboratorio TERMOCAL (Laboratorio de Metrología y Calibración), del Instituto Universitario de Bioeconomía, BioEcoUva, de la Universidad de Valladolid.

En esta tesis se han desarrollado técnicas experimentales que han permitido la caracterización termodinámica de soluciones acuosas de aminas con $\mathrm{CO}_{2}$ a través de la densidad, viscosidad y solubilidad. Para validar las modificaciones efectuadas en los equipos se han estudiado mezclas binarias de: (a) hidrocarburos, alcoholes y cetonas; y (b) hidrocarburos con $\mathrm{CO}_{2}$.

Las conclusiones que se han alcanzado en este trabajo son:

1. Se ha modificado un equipo VLE para la medida de equilibrios isotermos a altas temperaturas y presiones, incorporando un nuevo sistema para la medida de la presión a alta temperatura. El equipo se ha validado a las siguientes temperaturas: (a) $313 \mathrm{~K}$ con la determinación del equilibrio del sistema binario 2-pentanol + ciclohexano; y (b) entre $313 \mathrm{~K}$ y $393 \mathrm{~K}$ con los sistemas binarios Ciclohexanona + 2-Heptanona y Ciclohexanona + Hexanal.

2. Los resultados experimentales han sido correlacionados con los modelos de comportamiento de soluciones de Margules, Wilson, NRTL y UNIQUAC. La desviación cuadrática media relativa encontrada con la ecuación de Margules fue mejor que en $0.12 \%$ para el sistema 2 -Pentanol + Ciclohexano, del $0.14 \%$ para la Ciclohexanona + 2-Heptanona y del $0.30 \%$ en el sistema Ciclohexanona + Hexanal. En el caso del modelo NRTL fue inferior al $0.5 \%$ para todos los sistemas.

3. Se han determinado las densidades de sistemas líquido-gas a alta presión y temperatura. Se han caracterizado los sistemas binarios formados por los hidrocarburos pentano y hexano con $\mathrm{CO}_{2}$. Los resultados se han correlacionado con la ecuación Tammann-Tait modificada, la desviación estándar relativa encontrada fue inferior al $0.3 \%$ en ambos sistemas a $x_{C O 2}=0.6$.

4. Se han medido las viscosidades de sistemas líquido-gas a alta presión y temperatura para los sistemas binarios formados por pentano $+\mathrm{CO}_{2}$ y hexano + $\mathrm{CO}_{2}$. Los resultados se han ajustado con la ecuación Vogel-Fulcher-Tammann modificada, la desviación estándar relativa encontrada fue inferior al $1.0 \%$ en ambos sistemas a la composición más alta $x_{\mathrm{CO}}=0.3$.

5. Se han determinado las densidades de soluciones acuosas de aminas $\mathrm{con}^{\mathrm{CO}_{2}}$ a alta presión y temperatura. Se han estudiado las disoluciones acuosas de tres aminas, en amplios rangos de temperatura desde $293 \mathrm{~K}$ hasta $373 \mathrm{~K}$ y de presión desde 0.1 MPa hasta $60 \mathrm{MPa}$. Los sistemas caracterizados han sido: (i) Monoetanolamina (MEA) con $\mathrm{CO}_{2}$; (ii) dimetilaminoetanol (DMAE) con $\mathrm{CO}_{2}$; y (iii) Trietanolamina (TEA) con $\mathrm{CO}_{2}$, con fracciones másicas de amina al 0.3 y con $\mathrm{CO}_{2}$ hasta $\alpha=1.5$. Los resultados se han correlacionado con la ecuación Tammann-Tait modificada, la desviación estándar relativa encontrada fue inferior al 0.05\%.

6. Se han caracterizado experimentalmente los equilibrios isotermos a altas temperaturas y presiones de soluciones acuosas de aminas con $\mathrm{CO}_{2}$. A partir de estos resultados se ha determinado la solubilidad del $\mathrm{CO}_{2}$ hasta presiones de 6 $\mathrm{MPa}$, y temperaturas de $313 \mathrm{~K}$ y $353 \mathrm{~K}$. La fracción másicas de amina es 0.3 y con índices de carga de $\mathrm{CO}_{2}$ hasta $\alpha=1.7$. 
7. Se han determinado las incertidumbres de medida para todas las propiedades termodinámicas estudiadas en esta tesis doctoral.

8. Por último y como resumen de este trabajo se ha contribuido con nuevos datos experimentales que mejorarán los procesos de captura de $\mathrm{CO}_{2}$ con disoluciones acuosas de aminas, permitiendo la disminución de emisiones de gases con efecto invernadero, y aportando soluciones para un futuro sostenible de nuestra sociedad. 
Universidad deValladolid

PROGRAMA DE DOCTORADO EN INGENIERÍA

TERMODINÁMICA DE FLUIDOS

PhD THESIS:

THERMODYNAMIC CHARACTERIZATION OF LIQUID + GAS MIXTURES FOR THE DEVELOPMENT OF $\mathrm{CO}_{2}$ CAPTURE TECHNOLOGIES

A Thesis submitted for the partial fulfillment of a Doctorate in Philosophy Jorge Rubio Gallego

Supervised by:

Dr. José Juan Segovia Puras

Dr. Alejandro Moreau Ortega

Valladolid, October 2019 

1.1 Introduction.

1.1 Objectives of the Doctoral Thesis.

2. Experimental determination of vapor-liquid equilibrium at different

temperatures.

2.1 Experimental determination of vapor-liquid equilibrium at different

temperatures

2.2 Pressure measurement system

2.3 Thermodynamic reduction of the liquid-vapor equilibrium

3. Experimental results of the vapor-liquid equilibrium

3.1 Experimental results of the vapor-liquid equilibrium

4. Determination of densities and solubilities of $\mathrm{CO}_{2}$ in aqueous amine solutions. 225

4.1 Description of the measurement method used to determine the solubility of $\mathrm{CO}_{2}$ in aqueous amines.

4.2 Experimental procedure for solubility measurement 228

4.3 Description of the measurement method used for the determination of

$\mathrm{CO}_{2}$ density in aqueous amines. 229

4.4 Experimental procedure for density measurement. 230

4.5 Densimeter calibration 
4.7 System validation

5. Experimental results of density and solubility of $\mathrm{CO}_{2}$ measurements in aqueous

amine solutions

5.1 Experimental results of $\mathrm{CO}_{2}$ density and solubility measurements in

aqueous amine solutions.

6. Determination of densities and viscosities of $\mathrm{CO}_{2}+$ hydrocarbon mixture

6.1 Viscosity measuring method

6.2 Experimental procedure for measuring viscosity

6.3 Calibration of the viscometer and validation of the technique 253

6.4 Measurement Uncertainty 254

6.5 Measuring method used to determine the density of $\mathrm{CO}_{2}+$ hydrocarbon.....

6.6 Measurement Uncertainty

7. Experimental results of density and viscosity measurements of $\mathrm{CO}_{2}+$

hydrocarbon mixtures.

7.1 Experimental results of density and viscosity measurements of $\mathrm{CO}_{2}+$

hydrocarbon mixtures. 259

8. Conclusions.

8.1 Conclusions 269

9. References. 
Chapter 1

INTRODUCTION 



\subsection{Introduction.}

As a consequence of the combustion of fossil fuels, concentrations of carbon dioxide in the atmosphere have been increasing and have recently exceeded $400 \mathrm{ppm}$. This upward trend is the main driving force behind a continuous process of climate change which, to date, has led to an increase in the average surface temperature of the planet of about $1{ }^{\circ} \mathrm{C}$ since pre-industrial times [1]. The objective of the Paris Agreement is "to keep the global average temperature increase below $2^{\circ} \mathrm{C}$ above pre-industrial levels and to seek to limit the temperature increase to $1.5^{\circ} \mathrm{C} "[2]$.

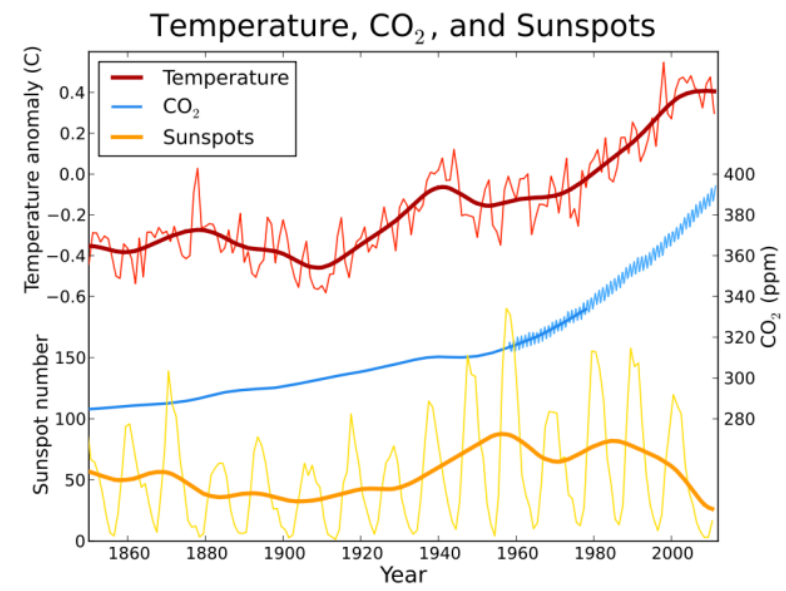

Figure 1.1. Variation of the global temperature and the $\mathrm{CO}_{2}$ concentration with the time [3].

Carbon dioxide sequestration is the separation of $\mathrm{CO}_{2}$ from other gaseous components, using physical or chemical separation techniques. The storage capability, the energy cost and other related expenses make impractical the transport and storage of gases with low $\mathrm{CO}_{2}$ concentrations. Therefore, it is necessary a sequestration technology able to produce an almost $\mathrm{CO}_{2}$ pure gaseous stream.

Capture is normally carried out at the main emission points of $\mathrm{CO}_{2}$, such as thermoelectric plants, where capture would be more profitable and would have the greatest environmental impact. Capturing $\mathrm{CO}_{2}$ directly from small and mobile sources such as transport, homes and businesses is more difficult and costly than from large and localized sources.

Various technologies can be applied for carbon capture according to the nature of the $\mathrm{CO}_{2}$ source. There are three main technologies for capturing $\mathrm{CO}_{2}$ whether they were generated from a primary fossil fuel (coal, natural gas or oil), biomass or a combination of these fuels.

Post-combustion systems. Post-combustion capture is a "final stage" technology that consists of separating $\mathrm{CO}_{2}$ from a combustion gas composed mainly of nitrogen, water, $\mathrm{CO}_{2}$ and other impurities.

Pre-combustion systems. Pre-combustion capture involves a reforming process with steam, partial oxidation or gasification of fossil fuels, producing a mixture known as synthetic gas or "syngas", consisting mainly of carbon monoxide, methane, hydrogen and $\mathrm{CO}_{2}$. 
Oxy-combustion systems. Oxy-combustion consists of burning fuel in a mixture of recycled $\mathrm{CO}_{2}$ and pure $\mathrm{O}_{2}$ instead of air, resulting in a combustion gas that mainly contains $\mathrm{CO}_{2}$ and water vapor.

One of the most widely applied technologies today for $\mathrm{CO}_{2}$ capture in the post-combustion process is through chemical absorption, being amine absorption one of the most developed technology in $\mathrm{CO}_{2}$ sequestration. The amine-based solvents used in $\mathrm{CO}_{2}$ sequestration are aqueous solutions of amines, between $10 \%$ and $30 \%$ by weight of amine.

Typical problems are solvent degradation, by-products formation and the corrosion issues caused by these $\mathrm{CO}_{2}$-rich solvents, along with the enormous regeneration energy needed for the solvent recovery.

In addition to the knowledge of the capture efficiency and selectivity of amines with $\mathrm{CO}_{2}$ in chemical absorption processes, it is necessary to have a greater knowledge of the thermophysical properties that intervene in $\mathrm{CO}_{2}$ capture processes due to the associated disadvantages of these processes.

\subsection{Objectives of the Doctoral Thesis.}

The specific objectives to be achieved in this Doctoral Thesis are the following:

1. Tuning Adaptation of a technique for measuring isothermal equilibrium at high temperatures and pressures.

a. Modify the equipment to measure vapor-liquid equilibria at high temperatures (up to $120^{\circ} \mathrm{C}$ ) and pressures (up to $6 \mathrm{MPa}$ ). This is necessary, because the original isothermal VLE technique was designed to measure between $25^{\circ} \mathrm{C}$ and $50{ }^{\circ} \mathrm{C}$ and low pressures (up to $125 \mathrm{kPa}$ ).

b. Measure the vapor-liquid equilibria of three systems: 2-Pentanol + Cyclohexane at $40{ }^{\circ} \mathrm{C}$, Cyclohexanone +2 -Heptanone and Cyclohexanone + Hexanal in a temperature range of $40^{\circ} \mathrm{C}$ up to $120^{\circ} \mathrm{C}$.

c. Estimate the uncertainties of the measurements carried out and adjust the experimental data to different models (Margules, Wilson, NRTL and UNIQUAC).

2. Tuning of a densimeter for the measurement of liquid-gas systems at high temperatures and pressures.

a. Fine-tune the Anton Paar DMA HPM vibrating tube densimeter available in the TERMOCAL laboratory of the University of Valladolid, for the measurement of mixtures of liquids with $\mathrm{CO}_{2}$.

b. Measure the systems: Pentane $+\mathrm{CO}_{2}$ and Hexane $+\mathrm{CO}_{2}$ in a temperature range from $0{ }^{\circ} \mathrm{C}$ up to $100{ }^{\circ} \mathrm{C}$, pressures from $5 \mathrm{MPa}$ up to $100 \mathrm{MPa}$ and $\mathrm{CO}_{2}$ concentrations, $x_{\mathrm{CO}_{2}}=[0.1,0.2,0.3,0.4$ and 0.6$]$. 
c. Determine density uncertainties and correlate density values with the modified Tammann-Tait equation.

3. Tuning of a viscometer for measuring liquid-gas systems at high temperatures and pressures.

a. Modify the vibrating wire viscometer developed in the TERMOCAL laboratory for the measurement of hydrocarbon $+\mathrm{CO}_{2}$ mixtures.

b. Measure the viscosities of the mixtures: Pentane $+\mathrm{CO}_{2}$ and Hexane $+\mathrm{CO}_{2}$ in a temperature range from $20{ }^{\circ} \mathrm{C}$ up to $100{ }^{\circ} \mathrm{C}$, pressures from $5 \mathrm{MPa}$ up to $80 \mathrm{MPa}$ and $\mathrm{CO}_{2}$ concentrations, $\mathrm{X}_{\mathrm{CO} 2}=[0.1$ and 0.3$]$.

c. Determine viscosity uncertainties and correlate dynamic viscosities with the modified Vogel-Fulcher-Tammann equation (VFT).

4. Determination of $p, V, T$ properties of aqueous amine solutions with $\mathrm{CO}_{2}$.

a. Measure densities of aqueous amine solutions with $\mathrm{CO}_{2}$ in wide temperature and pressure ranges (from $20{ }^{\circ} \mathrm{C}$ up to $100{ }^{\circ} \mathrm{C}$ and from $0.1 \mathrm{MPa}$ up to $60 \mathrm{MPa}$ ) using the Anton Paar DMA HPM vibrating tube densimeter. The amines studied are: Monoethanolamine (MEA), Dimethylaminoethanol (DMAE) and Triethanolamine (TEA). These densities are needed to calculate the solubility of $\mathrm{CO}_{2}$ in aqueous amine mixtures.

b. Determine density uncertainties and correlate density data with the modified Tammann-Tait equation.

5. Determination of $\mathrm{CO}_{2}$ solubility in aqueous amine solutions.

a. Measure $\mathrm{CO}_{2}$ solubilities in aqueous amine solutions with the reformed isothermal equilibrium cell at different temperatures and a maximum pressure of $6 \mathrm{MPa}$. In particular, perform measurements of aqueous solutions of $\mathrm{CO}_{2}$ with amines: MEA, DMAE and TEA.

b. Correlate the solubilities of $\mathrm{CO}_{2}$ in aqueous amine solutions with the $\mathrm{Xu}$ and Rochelle equation.

This doctoral thesis is included in the objectives proposed in the following projects and contracts:

(a) "Biogás renovable y procesos de captura del $\mathrm{CO}_{2}$ de combustión asociados como base a la sostenibilidad energética ambiental: Investigación Termodinámica Experimental (VA391A12-1)", funded by Junta de Castilla y León.

(b) "Revalorización de recursos renovables regionales biomásicos y eólicos para la producción de gases energéticos sostenibles (biogás e hidrógeno) y su incorporación en la red de gas natural (VA035U16)", funded by Junta de Castilla y León.

(c) "Gases energéticos: biogás y gas natural enriquecido con hidrógeno (ENE2013-47812R)", funded by the Ministry of Economy and Competitiveness of Spain. 
(d) "Caracterización termodinámica de mezclas de cetonas", financed by the company UBE Chemical.

(e) Pre-doctoral contracts from the European Social Fund (ESF) and Junta de Castilla y León. 


\section{Chapter 2}

\section{EXPERIMENTAL DETERMINATION OF VAPOR-LIQUID EQUILIBRIUM AT DIFFERENT TEMPERATURES}





\subsection{Experimental determination of vapor-liquid equilibrium at different temperatures.}

The technique used for the experimental determination of the vapor-liquid equilibrium is based on a static method of isothermal measurement of the total pressure. The experimental device was constructed according to the original design of Van Ness and his collaborators, Gibbs et al. [4]. The scheme of the equipment is shown in Figure 2.1.

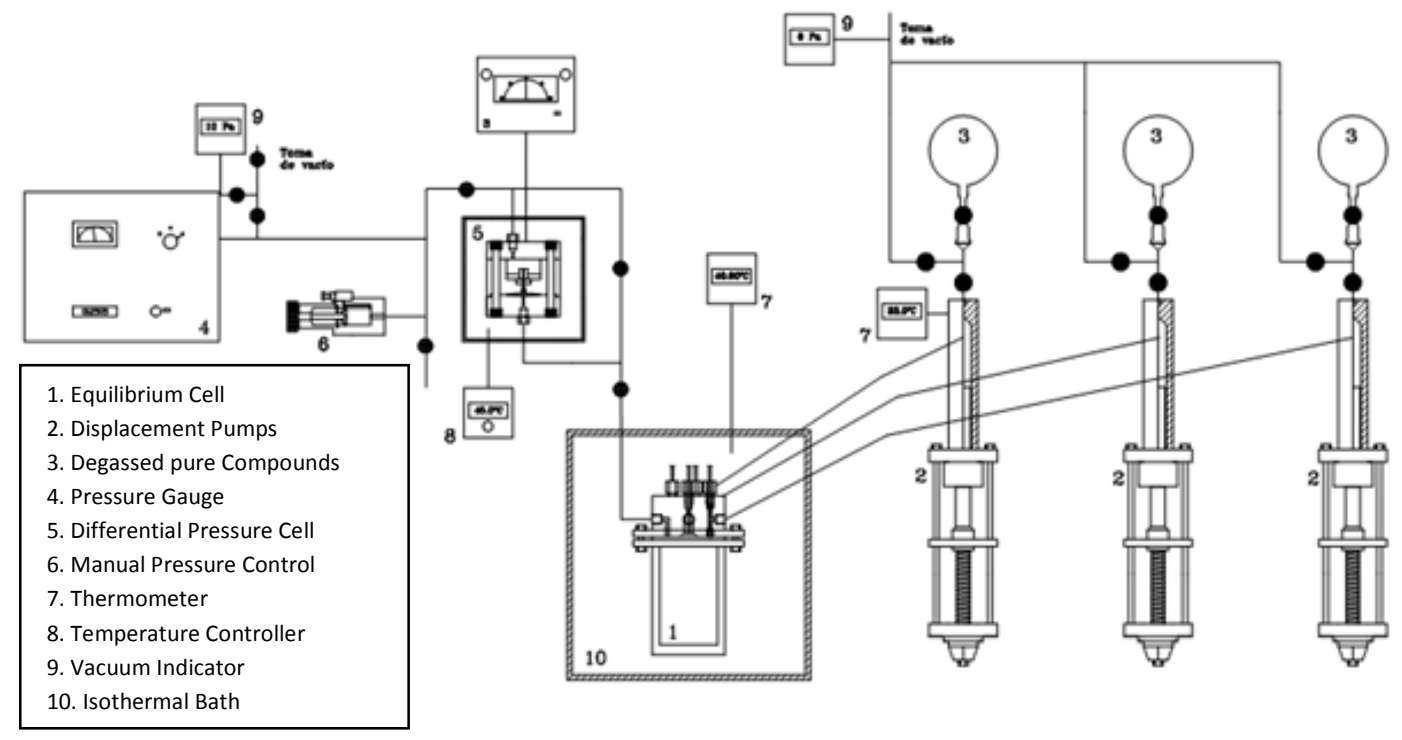

Figure 2.1. Diagram of the vapor-liquid equilibrium (VLE) measuring apparatus.

The equilibrium cell consists of a stainless-steel vessel with a capacity of approximately $180 \mathrm{~cm}^{3}$, inside which there is an externally operated magnetic stirrer to ensure rapid mixing of the pure components. The lid of the vessel is fixed and joined using a flange and an 0-ring. The equilibrium cell is immersed in a Hart Scientific, model 6020, thermostatic bath of great stability. The temperature of the bath is controlled and monitored using a controller with a platinum Pt100 thermoresistance, calibrated and traceable to international standards with an expanded uncertainty $(k=2)$ of $20 \mathrm{mK}$.

The entire system is under vacuum using a vacuum pump with a cryogenic trap. The compounds, previously degassed, are introduced into the positive displacement pumps. The pumps used are Ruska, model $2200-801$, with $100 \mathrm{~mL}$ capacity and graduated in intervals of $0.01 \mathrm{~mL}$ with an uncertainty of injected volume of $0.03 \mathrm{~mL}$. Right picture of Figure 2.2 shows the injection system.

The volumes to be injected into the cell are calculated considering the composition of the liquid phase to be obtained. Initially, the first component is injected and the vapor pressure is read in a pressure indicator. The injected volume of each component is measured by the difference between the initial and final volume in the load, after adjusting the pressure inside the cylinder and measured through a pressure transducer in each of them. Then, successive injected volumes of the second component will be added, which will modify the composition of the mixture and the corresponding vapor pressures until reaching an approximate final volume of the experiment of $120 \mathrm{~mL}$ with a $x_{2}=0.6$. 
The total amount of mass injected is accurately determined from the volume differences read between two stop-points of the piston, the temperature of the injector and the value of the density for that pure component.

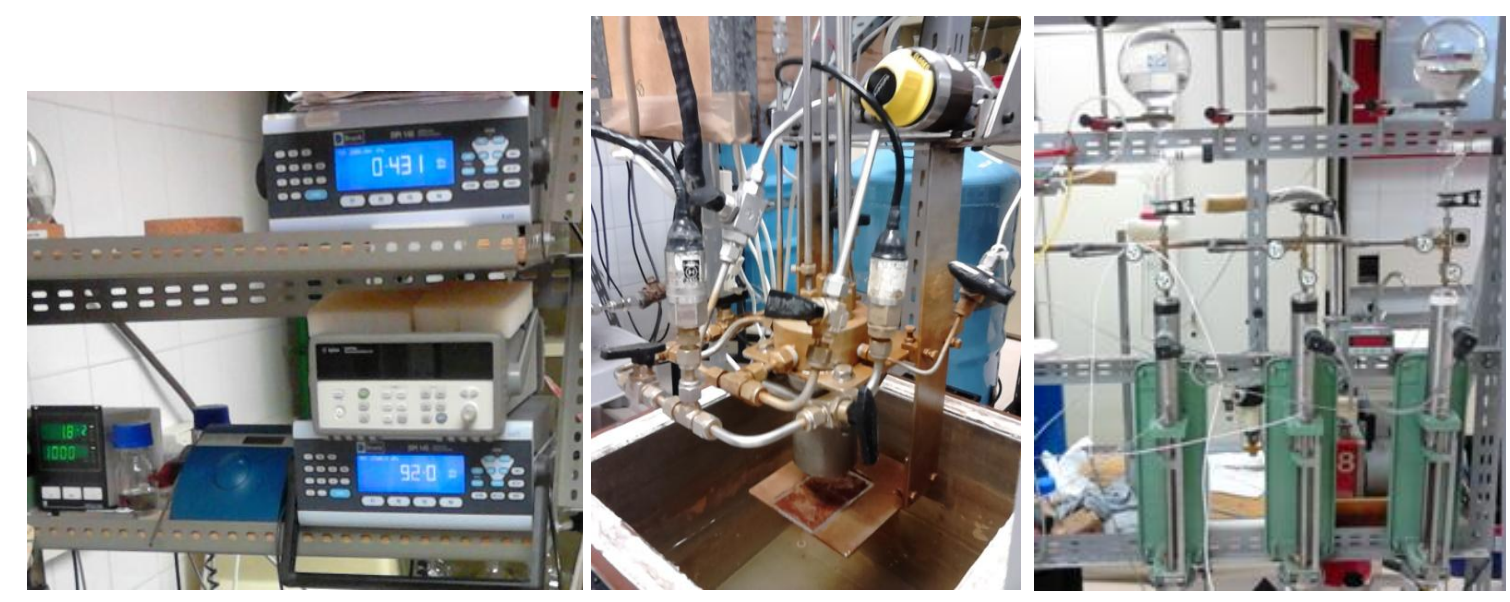

Figure 2.2. Photos of the vapor-liquid equilibrium apparatus:

The determination of VLE for a binary mixture is carried out by measuring two dilution lines or branches that overlap in the central part. The first branch begins with the first pure component, $x_{1}=1$, to which the second pure compound is added from $x_{2}=0.05$ to $x_{2}=0.6$ in 0.05 intervals. The second branch starts from the second pure compound $x_{2}=1$ and the first pure compound is added from $x_{1}=0.05$ to $x_{1}=0.6$, also in intervals of 0.05 . This completes the whole interval of compositions and the overlapping compositions are used to test the reliability of the measurements. This process will be repeated for each one of the temperatures, changing only the set of the thermostatic bath.

\subsection{Pressure measurement system}

One of the contributions of this thesis is the modification of the pressure measurement system in order to extend the pressure and temperature working ranges of the VLE technique.

\subsubsection{Bourdon type manometer}

This is the initial pressure system of the technique, it consists of a differential pressure manometer Texas Instruments, model PPG-149, with a Bourdon quartz capsule. The manometer reference is connected at a vacuum system therefore the manometer measures absolute pressure. The working temperature of this system is limited up to $50^{\circ} \mathrm{C}$. In this work, it was used for measuring VLE of the system 2-Pentanol + Cyclohexane at 40 ${ }^{\circ} \mathrm{C}$.

The device was calibrated using a pneumatic dead weight balance as primary standard traceable to international standards. The expanded uncertainty $(k=2)$ is better than $2 \cdot 10^{-4}$ $\cdot \mathrm{p} / \mathrm{Pa}+5 \mathrm{~Pa}$. 


\subsubsection{Druck pressure transducers}

For measuring VLE at higher temperatures, two pressure transductors (Druck model PDCR-910-1422) were coupled to the cell and immersed in the bath, one for the pressure range from 0.035 to 2 bar and the second for pressures between 2 to 20 bar, provided with and indicator Druck model DPI 145. They were calibrated in a temperature range from $40{ }^{\circ} \mathrm{C}$ to $120^{\circ} \mathrm{C}$ with a relative expanded uncertainty $(k=2)$ of $0.05 \%$.

\subsubsection{Resonant quartz Paroscientific transducer}

For the amine $+\mathrm{CO}_{2}$ solubility at high pressures it is required the installation of a third measuring instrument. A transducer Paroscientific, model $42 \mathrm{~K}-101$ connected to an indicator of the same Brand model 735. It was connected externally measuring the ambient temperature and being able to measure pressures up to $13.8 \mathrm{MPa}$.

\subsection{Thermodynamic reduction of the liquid-vapor equilibrium}

As a consequence of Duhem's theorem, the two-phase sampling is not necessary for the determination of the vapor-liquid equilibrium. The measurement technique used in this work is a static procedure from which data sets $\left(x_{i}, p\right)$ are obtained for a given temperature. Using a correlation equation, the parameters that lead to calculated pressure values as close as possible to the experimental pressure values are estimated. This method is known as the Barker method [5]. The equilibrium equation used is as follows:

$$
x_{i} \cdot \gamma_{i} \cdot p_{i}^{s a t}=y_{i} \cdot \Phi_{i} \cdot p
$$

Where,

$$
\Phi_{i}=\frac{\widehat{\phi}_{l}}{\phi_{i}^{s a t}} \exp \left[-\frac{\mathrm{V}_{\mathrm{i}}\left(p-p_{i}^{s a t}\right)}{\mathrm{R} T}\right]
$$

and using the virial equation truncated in the second term for taking into account the nonideality of the vapor phase it is obtained:

$$
\Phi_{i}=\exp \frac{B_{i i}\left(p-p_{i}^{s a t}\right)+p \frac{1}{2} \sum_{k} \sum_{l} y_{k} y_{l}\left(2 \delta_{k i}-\delta_{k l}\right)}{\mathrm{R} T}
$$

In which:

$$
\begin{aligned}
& \delta_{k i}=\delta_{i k}=2 B_{k i}-B_{k k}-B_{i i} \\
& \delta_{k l}=\delta_{l k}=2 B_{k l}-B_{k k}-B_{l l}
\end{aligned}
$$

The second viral coefficients, $B_{i i}, B_{i k}, \ldots$ can be obtained through a correlation by Tsonopoulos [6]. Activity coefficients $\left(\gamma_{i}\right)$ are calculated through correlation models for the excess Gibbs energy.

The VLE data were correlated using the six-parameters Margules equation: 


$$
\frac{G^{E}}{x_{1} x_{2} \mathrm{RT}}=\mathrm{A}_{21} x_{1}+\mathrm{A}_{12} x_{2}-\left(\lambda_{21} x_{1}+\lambda_{12} x_{2}\right) x_{1} x_{2}+\left(\eta_{21} x_{1}+\eta_{12} x_{2}\right) x_{1}^{2} x_{2}^{2}
$$

Where $A_{21}, A_{12}, \lambda_{21}, \lambda_{12}, \eta_{21}$ and $\eta_{12}$ are the adjustable parameters.

In addition to the above equation, the following models were used: Wilson [7], NRTL [8] and UNICUAC [9], whose $G^{E}$ expressions are equations $2.5,2.6$ and 2.7 respectively.

$$
\begin{gathered}
\frac{G^{E}}{\mathrm{R} T}=-\sum_{i} x_{i} \ln \left(\sum_{j} x_{j} \Lambda_{\mathrm{ij}}\right) \\
\frac{G^{E}}{\mathrm{R} T}=\sum_{i} x_{i} \frac{\sum_{j} \tau_{\mathrm{ji}} G_{j i} x_{j}}{\sum_{k} G_{k i} x_{k}} \\
\frac{G^{E}}{\mathrm{R} T}=\sum_{i} x_{i} \frac{\varphi_{i}}{x_{i}}+\frac{\mathrm{z}}{2} \sum_{i} \mathrm{q}_{\mathrm{i}} x_{i} \ln \frac{\vartheta_{i}}{\varphi_{i}}-\sum_{i} \mathrm{q}_{\mathrm{i}} x_{i} \ln \left(\sum_{j} \vartheta_{j} \tau_{\mathrm{ji}}\right)
\end{gathered}
$$

The calculations in all cases are iterative and the objective function (F.O.) is to minimize the sum of the differences between the calculated pressure $\left(p_{c a l}\right)$ and the experimentally determined pressure $\left(p_{\text {exp }}\right)$ squared.

$$
\text { F.O. }=\sum(\Delta p)^{2}=\sum\left(p_{\text {cal }}-p_{\text {exp }}\right)^{2}
$$

From this minimization, the parameters for all models are obtained and the excess Gibbs energy is calculated with the parameters of the best fit. 
Chapter 3

\section{EXPERIMENTAL RESULTS OF THE VAPOR-LIQUID EQUILIBRIUM}





\subsection{Experimental results of the vapor-liquid equilibrium.}

This chapter presents, in tables and figures, the experimental results of vapor-liquid equilibrium (VLE) obtained with a static and isothermal technique to measure vapor pressure at $313.15 \mathrm{~K}, 333.15 \mathrm{~K}, 353.15 \mathrm{~K}$ and $393.15 \mathrm{~K}$ described above. The pressure $p$, the composition of the liquid phase $x_{i}$, the composition of the vapour phase $y_{i}$, and the excess Gibbs energy $G^{E}$, obtained with different models, are shown in them.

For the tuning and validation of the measurements of the equipment, the binary system 2Pentanol (1) + Cyclohexane (2) was measured at 313.15 K.

The systems measured in this work are the following:

Binary B0: 2-Pentanol(1) + Cyclohexane(2) at 313.15 K.

Binary B1: Cyclohexanone(1) + 2-Heptanone(2) at 313.15 K, 333.15 K, 353.15 K, 393.15 K.

Binary B2: Cyclohexanone(1) + Hexanal(2) at 313.15 K, 333.15 K, 353.15 K, 393.15 K.

\subsubsection{Products used.}

The products to be used are of the highest possible purity for reliable results. In Table 3.1. (Spanish version, page 44), the products used, the origin and the purity given by the certificates of analysis are shown. No additional purification method has been used. The pure compounds were previously degassed using a vacuum rectification column with an ultrasonic agitation bath.

One of the ways to check the performance of the equipment and a correct degasification of the compounds, is to compare the experimental vapor pressures of the pure compounds at the different working temperatures with the literature values as shown in Table 3.2 (Spanish version, page 45).

\subsubsection{Experimental results of the vapor-liquid equilibrium.}

As an example of the experimental VLE data, the system Cyclohexanone (1) + 2-Heptanone (2) at $313.15 \mathrm{~K}$ is presented here; all the results are in section 3.3 (Spanish version, page 46). Table 3.1 presents the experimental data of total pressure $p$, as a function of the compositions of the liquid phase $x_{\mathrm{i}}$, and the vapour phase $y_{i}$, calculated using fourparameters Margules equation, as well as the thermodynamic function of the excess Gibbs energy, calculated using the NRTL model. Table 3.2 shows the data of the pure components, the vapor pressure $p_{\mathrm{i}}^{\text {sat, }}$, the molar volumes $V_{i}{ }^{L}$ (experimentally obtained), and the second virial coefficients of the pure components $B_{i i}$, and of the $B_{i j}$ mixture. Finally, Table 3.3 shows the results of the data reduction, being: the parameters adjusted using the Margules equation and different models, the root mean squared ( $r m s$ ) of the pressure residuals and the maximum deviation between the experimental pressure and the calculated pressure. 
The results shown graphically are: the experimental pressure as a function of the phase compositions $(p-x-y)$, the quality of the data reduction through the residuals of the pressure versus the molar fraction of the liquid phase $(\Delta p-x)$, the calculated composition of the vapor phase versus the molar fraction of the liquid phase $(x-y)$, and the excess Gibbs energy calculated by the parameters of NRTL model versus the composition of the liquid phase $\left(G^{E_{-}} X\right)$.

\subsubsection{Binary system B1: Cyclohexanone (1) + 2-Heptanone (2) at 313.15 K.}

Table 3.1. Experimental data $p-x-y$ calculated by Margules equation and excess Gibbs energy calculated by NRTL model for the binary system B1: Cyclohexanone (1) + 2-Heptanone (2) at $313.15 \mathrm{~K}$.

\begin{tabular}{cccccccc}
\hline $\mathbf{x}_{\mathbf{1}}$ & $\mathbf{\mathbf { y } _ { 1 } , \mathbf { c a l c }}$ & $\mathbf{p} / \mathbf{k P a}$ & $\begin{array}{c}\mathbf{G}^{\mathbf{E}} / \\
\mathbf{J} \mathbf{m o l} \mathbf{- 1}\end{array}$ & $\mathbf{x}_{\mathbf{1}}$ & $\mathbf{\mathbf { y } _ { 1 } , \mathbf { c a l c }}$ & $\mathbf{p} / \mathbf{k P a}$ & $\begin{array}{c}\mathbf{G}^{\mathbf{E}} / \\
\text { J-mol-1 }\end{array}$ \\
\hline 0.0000 & 0.0000 & 1.281 & 0.00 & 0.5007 & 0.5042 & 1.324 & 56.81 \\
0.0428 & 0.0484 & 1.289 & 11.70 & 0.5501 & 0.5523 & 1.328 & 54.96 \\
0.0965 & 0.1058 & 1.304 & 24.20 & 0.6002 & 0.6008 & 1.324 & 52.08 \\
0.1415 & 0.1521 & 1.303 & 32.94 & 0.6501 & 0.6490 & 1.320 & 48.27 \\
0.1966 & 0.2072 & 1.306 & 41.62 & 0.6967 & 0.6939 & 1.318 & 43.91 \\
0.2477 & 0.2576 & 1.310 & 47.86 & 0.7504 & 0.7457 & 1.320 & 38.00 \\
0.2981 & 0.3069 & 1.313 & 52.41 & 0.8009 & 0.7945 & 1.320 & 31.65 \\
0.3479 & 0.3555 & 1.316 & 55.46 & 0.8530 & 0.8455 & 1.316 & 24.34 \\
0.3977 & 0.4039 & 1.318 & 57.16 & 0.9045 & 0.8972 & 1.314 & 16.41 \\
0.4486 & 0.4536 & 1.325 & 57.61 & 0.9582 & 0.9535 & 1.306 & 7.44 \\
0.4502 & 0.4551 & 1.321 & 57.60 & 1.0000 & 1.0000 & 1.298 & 0.00 \\
\hline
\end{tabular}

Table 3.2. Experimental value of the vapor pressure of pure compounds $p_{i}^{\text {sat }}$, molar volumes $V_{i}^{L}$, and second viral coefficients of pure compounds $B_{i i}$ and mixture $B_{i j}$, for the binary system B1: Cyclohexanone (1) + 2-Heptanone (2) at $313.15 \mathrm{~K}$.

\begin{tabular}{|c|c|c|c|c|c|c|}
\hline $\begin{array}{l}p_{1}^{s a t} \\
\mathbf{k P a}\end{array}$ & $\begin{array}{l}p_{2}^{s a t} \\
\mathrm{kPa}\end{array}$ & $\begin{array}{c}V_{1}^{L} \\
\mathrm{~cm}^{3} \cdot \mathrm{mol}^{-1} \\
\end{array}$ & $\begin{array}{c}V_{2}^{L} \\
\mathrm{~cm}^{3} \cdot \mathrm{mol}^{-1} \\
\end{array}$ & $\begin{array}{c}B_{11} \\
\mathrm{~cm}^{3} \cdot \mathrm{mol}^{-1} \\
\end{array}$ & $\begin{array}{c}\quad B_{22} \\
\mathrm{~cm}^{3} \cdot \mathrm{mol}^{-1} \\
\end{array}$ & $\begin{array}{c}B_{12} \\
\mathrm{~cm}^{3} \cdot \mathrm{mol}^{-1} \\
\end{array}$ \\
\hline 1.298 & 1.281 & 105.61 & 142.97 & -2796 & -3571 & -2980 \\
\hline
\end{tabular}


Table 3.3. Adjustment parameters, root mean squared pressure residual (rms), maximum pressure residual (max $\Delta p$ ), and azeotrope determined for the binary system B1 adjustment parameters: Cyclohexanone (1) + 2-Heptanone (2) at $313.15 \mathrm{~K}$.

\begin{tabular}{lcccc}
\hline & Margules & Wilson & NRTL & UNIQUAC \\
\hline $\mathrm{A}_{12}$ & 0.1532 & 0.6281 & -0.0461 & 1.9409 \\
$\mathrm{~A}_{21}$ & 0.0398 & 1.3520 & 0.1629 & 0.4166 \\
$\lambda_{12}$ & 0.2567 & & & \\
$\lambda_{21}$ & -0.1776 & & & \\
$\alpha$ & & & 2.0778 & \\
$\mathrm{rms} \Delta \mathrm{p} / \mathrm{kPa}$ & 0.003 & 0.003 & 0.004 & 0.003 \\
$\mathrm{max}|\Delta \mathrm{p}| / \mathrm{kPa}$ & 0.009 & 0.009 & 0.009 & 0.009 \\
$\mathrm{x}_{1, \text { azeotrope }}$ & 0.601 & 0.560 & 0.560 & 0.574 \\
$\mathrm{p}_{\text {azeotrope }} / \mathrm{kPa}$ & 1.324 & 1.323 & 1.323 & 1.323 \\
\hline
\end{tabular}

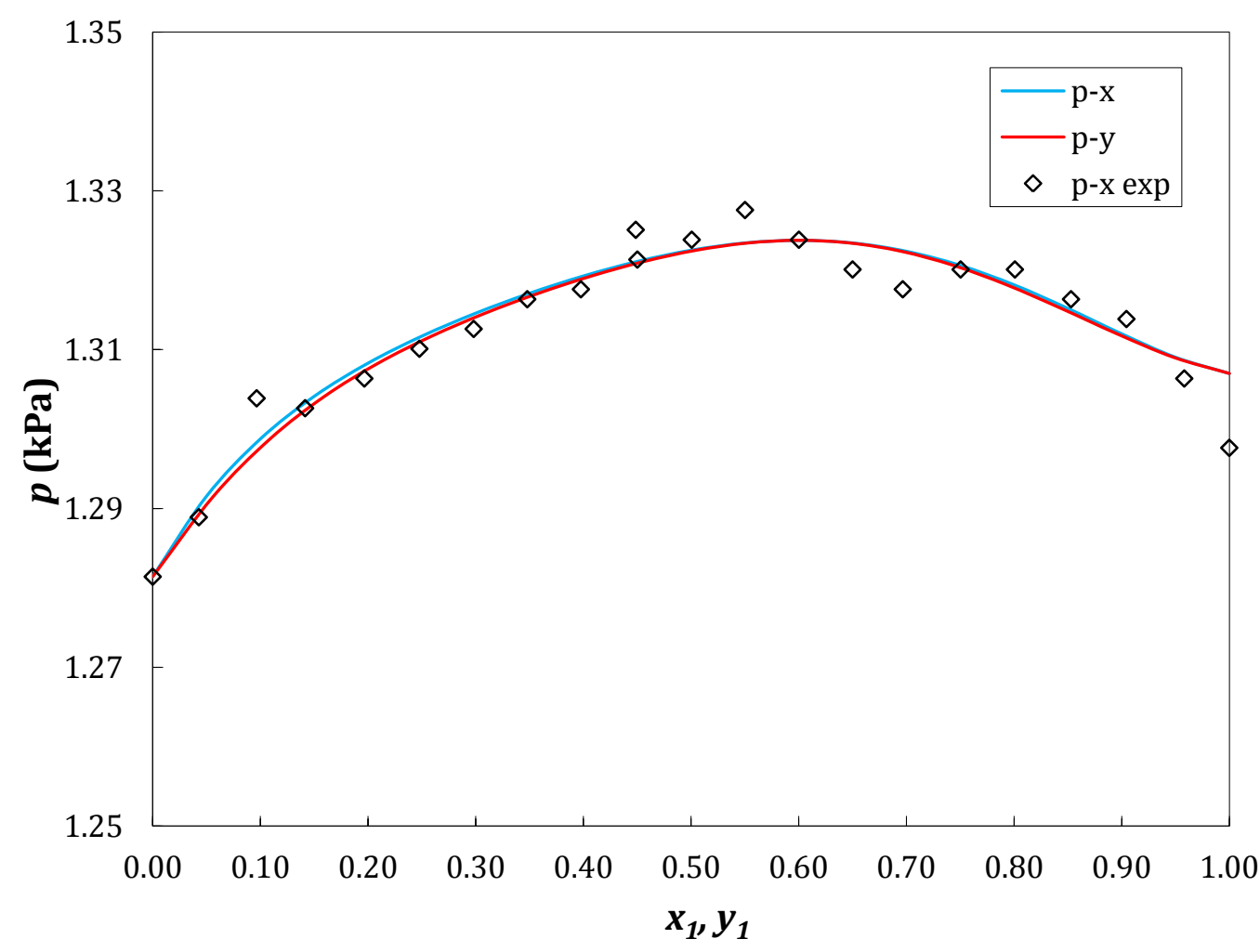

Figure 3.1. Vapor pressure $(p)$ as a function of the molar fractions of the liquid phase $x_{1}$ and vapor phase $y_{1}$, for the binary system B1: Cyclohexanone (1) + 2-Heptanone (2) at 313.15 K. ( 0 ) Experimental data. (-) Adjustment using the Margules equation. 


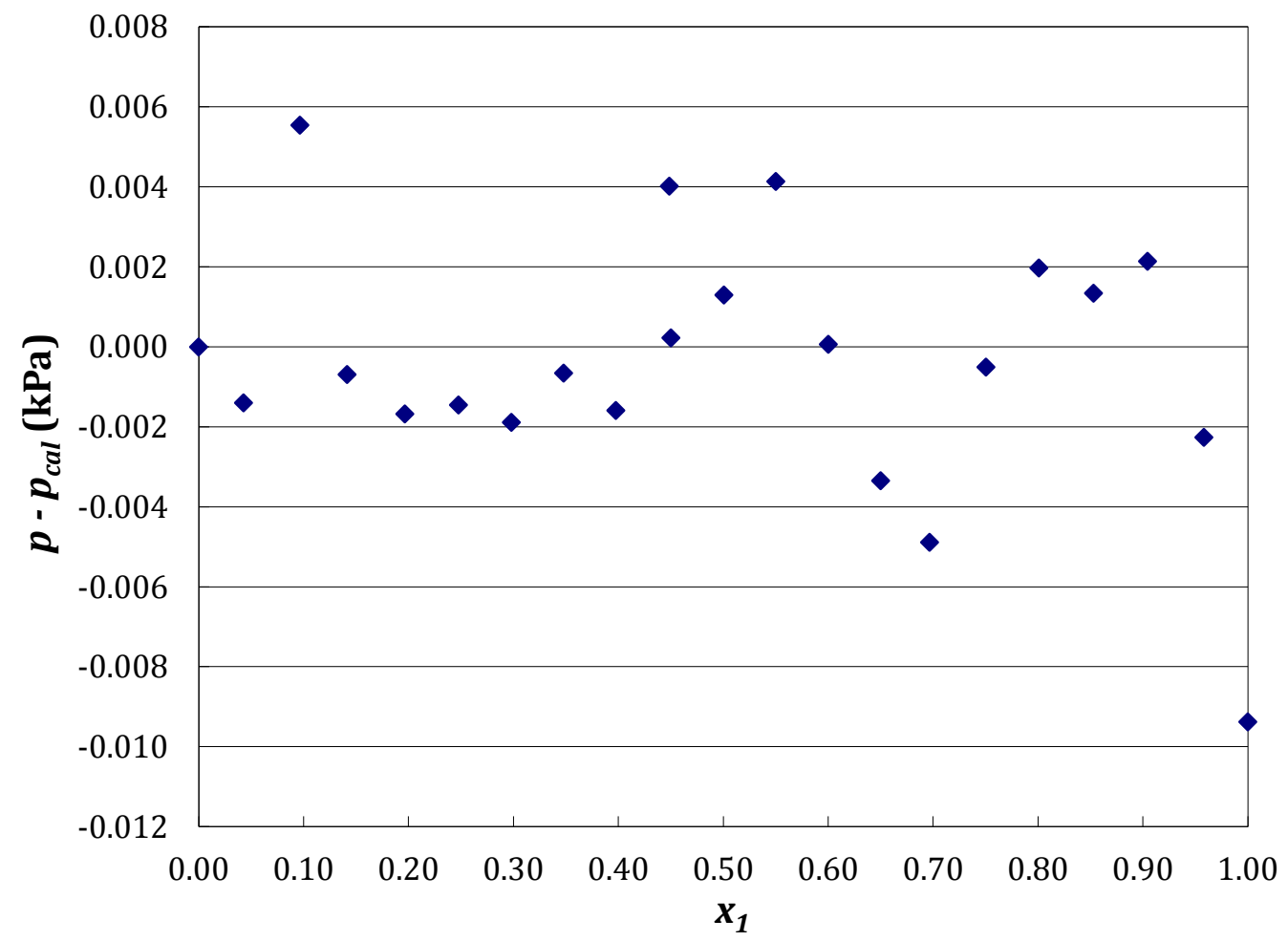

Figure 3.2. Comparison of differences between experimental pressure ( $p$ ) and the calculated pressure $\left(p_{\text {cal }}\right)$ using the Margules equation ( 1$)$, for the binary system B1: Cyclohexanone (1) + 2-Heptanone (2) at $313.15 \mathrm{~K}$.

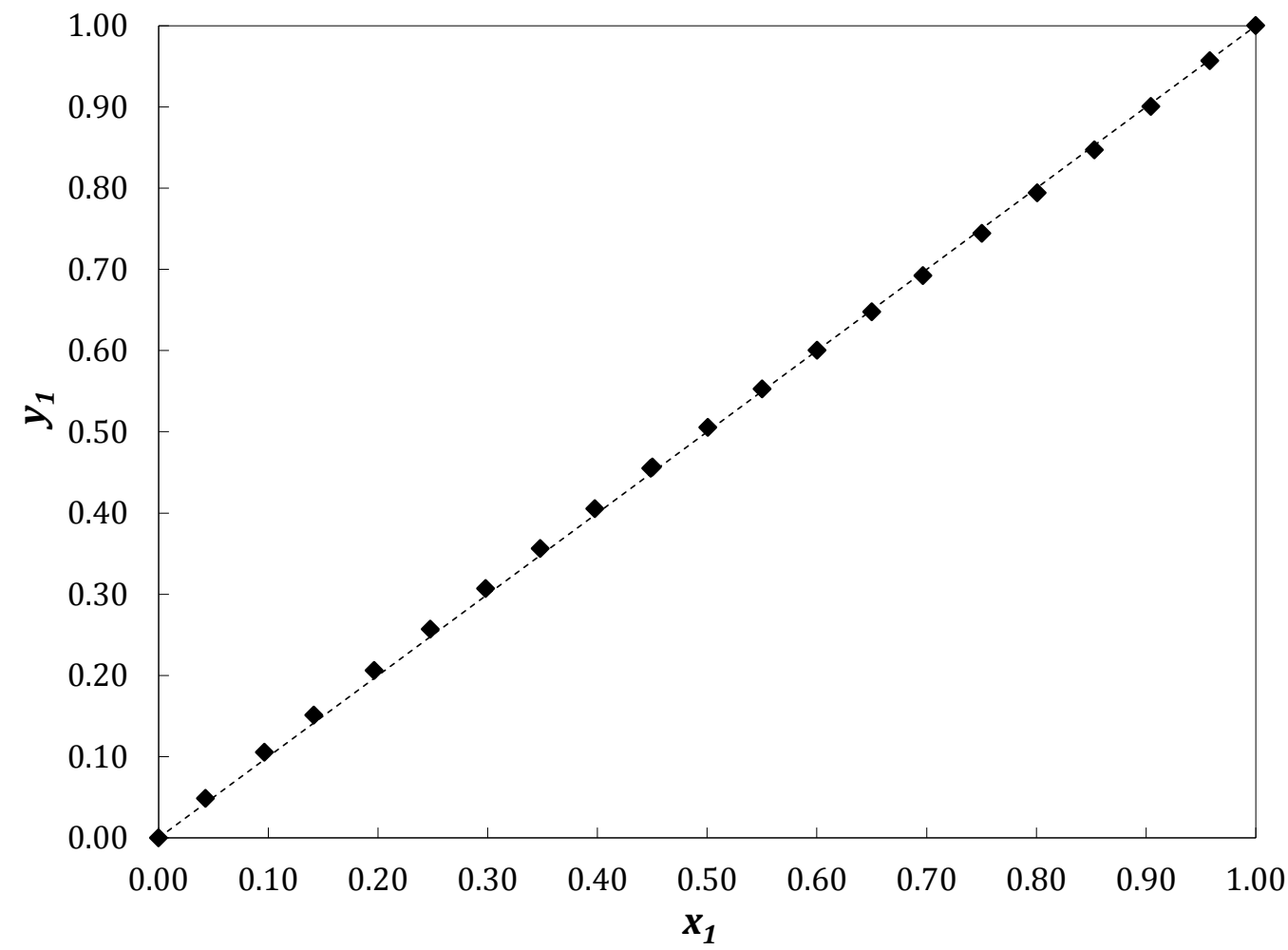

Figure 3.3. Mole fraction of the vapor phase $\left(y_{1}\right)$ as a function of the molar fraction of the liquid phase $\left(x_{1}\right)$ for the binary system B1: Cyclohexanone (1) + 2-Heptanone (2) at 313.15 K. Data calculated using the Margules equation ( 1 ). 


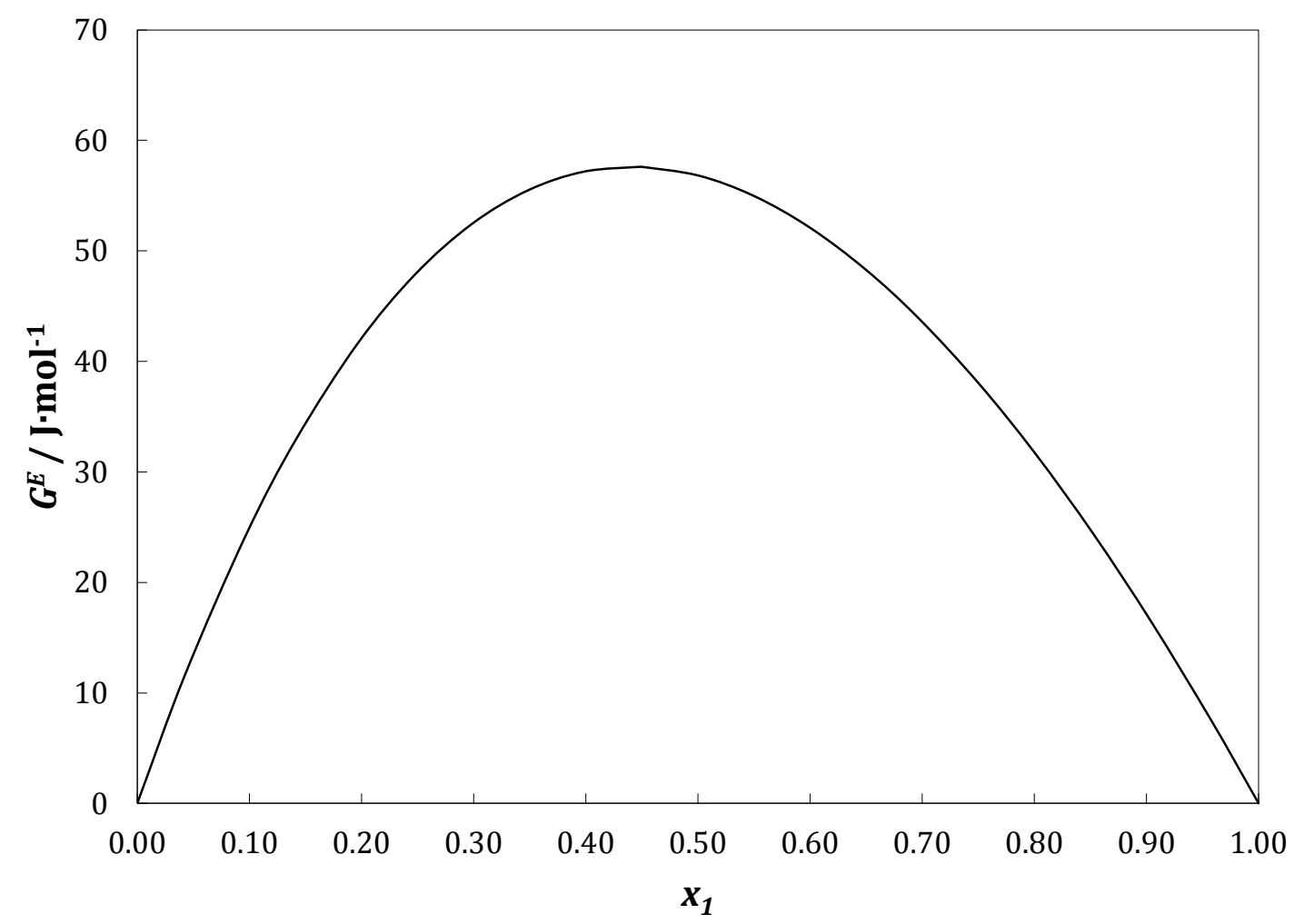

Figure 3.4. Excess Gibbs Energy $\left(G^{E}\right)$ calculated by NRTL model as a function of the molar fraction of the liquid phase $\left(x_{1}\right)$ for the binary system B1: Cyclohexanone (1) + 2-Heptanone (2) at $313.15 \mathrm{~K}$.

\subsubsection{Discussion.}

The binary system 2-Pentanol (1) + Cyclohexane (2) at 313.15 K was measured as an equipment tuning and validation. The data were published in Moreau et al. [10].

Table 3.4. summarizes the $r m s(\Delta P)$ values, equation 2.40 (Spanish version, page 38), from all the binary systems studied, where $\Delta p$ is the difference between the experimental and the calculated pressure, according to the lower expression:

$$
r m s(\Delta p)=\sqrt{\frac{\sum\left(p_{\text {exp }}-p_{\text {cal }}\right)^{2}}{n-m}}
$$

These values were always between $0.003 \mathrm{kPa}$ and $0.051 \mathrm{kPa}$ for the Margules equation in the binary systems formed by 2-Heptanone. For the binary systems formed by Hexanal, they were in the range from $0.010 \mathrm{kPa}$ to $0.133 \mathrm{kPa}$. For the rest of the models the rms $(\Delta p)$ values slightly increased.

The average relative deviations obtained by Margules equation were $0.14 \%, 0.09 \%, 0.12 \%$ and $0.07 \%$ for the system with 2-heptanone at $313.15 \mathrm{~K}, 333.15 \mathrm{~K}, 353.15 \mathrm{~K}$ and $393.15 \mathrm{~K}$ respectively. In the systems with hexanal, these values were $0.30 \%, 0.28 \%, 0.25 \%$ and $0.17 \%$ for $313.15 \mathrm{~K}, 333.15 \mathrm{~K}, 353.15 \mathrm{~K}$ and $393.15 \mathrm{~K}$. 
Table 3.4. Root mean squared pressure residual of the three binary systems studied, obtained for the Margules equation and Wilson, NRTL and UNIQUAC models.

rms $\Delta \mathbf{p} / \mathbf{k P a}$

\begin{tabular}{ccccc} 
Temperature & Margules & Wilson & NRTL & UNIQUAC \\
\hline \multicolumn{5}{c}{ 2-Pentanol (1) + Ciclohexane (2) } \\
$313.15 \mathrm{~K}$ & 0.031 & 0.137 & 0.092 & 0.264 \\
$313.15 \mathrm{~K}$ & 0.003 & 0.003 & 0.004 & 0.003 \\
$333.15 \mathrm{~K}$ & 0.008 & 0.009 & 0.009 & 0.009 \\
$353.15 \mathrm{~K}$ & 0.019 & 0.025 & 0.024 & 0.024 \\
$393.15 \mathrm{~K}$ & 0.051 & 0.075 & 0.061 & 0.089 \\
& \multicolumn{4}{c}{ Ciclohexanone (1) + 2-Heptanone (2) } \\
$313.15 \mathrm{~K}$ & 0.010 & 0.018 & 0.017 & 0.019 \\
$333.15 \mathrm{~K}$ & 0.025 & 0.035 & 0.036 & 0.035 \\
$353.15 \mathrm{~K}$ & 0.049 & 0.094 & 0.092 & 0.099 \\
$393.15 \mathrm{~K}$ & 0.133 & 0.197 & 0.181 & 0.165 \\
\hline
\end{tabular}

A maximum pressure azeotrope was found in the cyclohexanone +2 -heptanone system at three of the four temperatures studied whose data are summarized in Table 3.5.

Table 3.5. Azeotrope data for the system cyclohexanone (1) + 2-heptanone (2) calculated using different models.

\begin{tabular}{|c|c|c|c|c|c|c|c|}
\hline \multicolumn{2}{|c|}{ Margules } & \multicolumn{2}{|c|}{ Wilson } & \multicolumn{2}{|c|}{ NRTL } & \multicolumn{2}{|c|}{ UNIQUAC } \\
\hline $\begin{array}{c}\mathbf{x}_{1}, \\
\text { azeotrope }\end{array}$ & $\begin{array}{c}\text { pazeotrope }_{\text {a }} \\
\mathbf{k P a}\end{array}$ & $\begin{array}{c}\mathbf{x}_{1}, \\
\text { azeotrope }\end{array}$ & $\begin{array}{c}\mathbf{p}_{\text {azeotrope }} \\
\mathbf{k P a}\end{array}$ & $\begin{array}{c}\mathbf{x}_{1}, \\
\text { azeotrope }\end{array}$ & $\begin{array}{c}\mathbf{p}_{\text {azeotrope }} \\
\mathbf{k P a}\end{array}$ & $\begin{array}{c}\mathbf{x}_{1}, \\
\text { azeotrope }\end{array}$ & $\begin{array}{c}\mathbf{p}_{\text {azeotrope }} \\
\mathbf{k P a} \\
\end{array}$ \\
\hline \multicolumn{8}{|c|}{ Temperature at $313.15 \mathrm{~K}$} \\
\hline 0.601 & 1.324 & 0.560 & 1.323 & 0.560 & 1.323 & 0.574 & 1.323 \\
\hline \multicolumn{8}{|c|}{ Temperature at $333.15 \mathrm{~K}$} \\
\hline 0.311 & 3.754 & 0.276 & 3.753 & 0.283 & 3.753 & 0.266 & 3.753 \\
\hline \multicolumn{8}{|c|}{ Temperature at $353.15 \mathrm{~K}$} \\
\hline 0.292 & 9.356 & 0.224 & 9.352 & 0.238 & 9.349 & 0.238 & 9.346 \\
\hline
\end{tabular}

As can be seen, the azeotrope appears at lower cyclohexanone compositions when the temperature increases, and it disappears at $393.15 \mathrm{~K}$.

The systems studied do not present great deviations of the ideality in terms of excess Gibbs energy, being the less ideal system formed by the 2-heptanone, where the maximum values of $\mathrm{G}^{\mathrm{E}}$ are between $57.62 \mathrm{~J} / \mathrm{mol}$ and $90.30 \mathrm{~J} / \mathrm{mol}$, whereas the maximum values of $\mathrm{G}^{\mathrm{E}}$ are between $3.45 \mathrm{~J} / \mathrm{mol}$ and $86.24 \mathrm{~J} / \mathrm{mol}$ for the system with hexanal. 
Chapter 4

\section{DETERMINATION OF DENSITIES AND SOLUBILITIES OF $\mathrm{CO}_{2}$ IN AQUEOUS AMINE SOLUTIONS}





\subsection{Description of the measurement method used to determine the solubility of $\mathrm{CO}_{2}$ in aqueous amines.}

The measurement of $\mathrm{CO}_{2}$ solubilities in aqueous amine solutions was done using the equipment initially constructed for the measurement of vapor-liquid equilibrium [11] which was described in section 2.1 of this summary.

In order to carry out the $\mathrm{CO}_{2}$ solubility measurements, it was necessary to perform some changes to the initial technique, as shown in Figure 4.1. The main modification was made for the injection of $\mathrm{CO}_{2}$ into the cell, described in section 2.1 of this summary. These changes are described as follows:

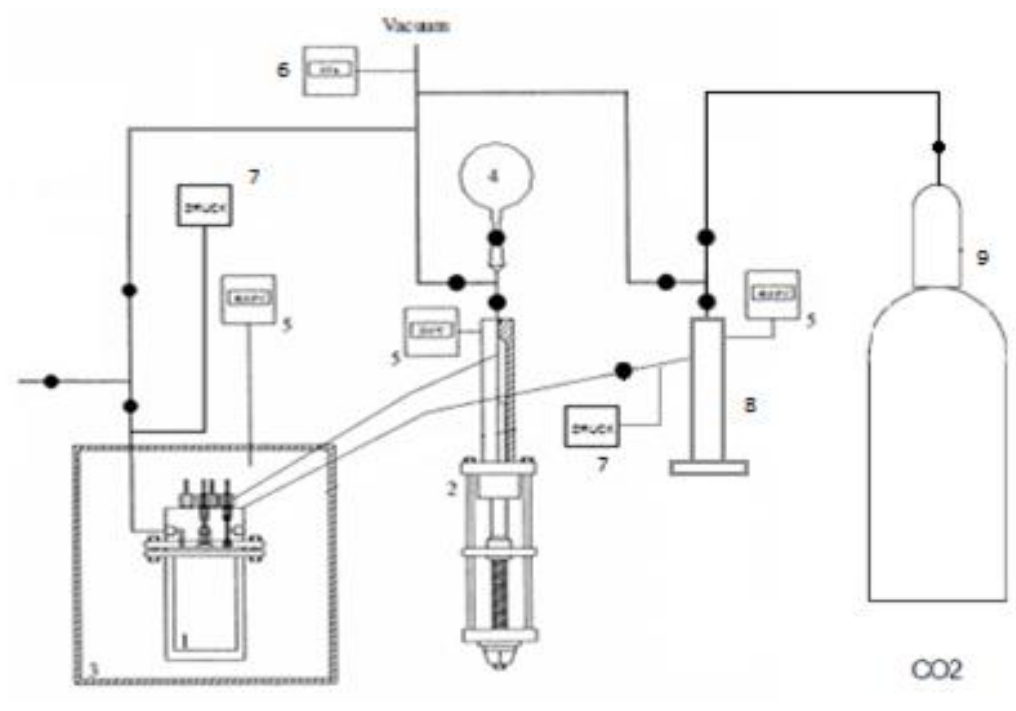

Figure 4.1. Scheme of the modified apparatus for measuring the vapor-liquid equilibrium of $\mathrm{CO}_{2}$ in aqueous amine solutions. (1) Measuring cell; (2) Injection pump; (3) Thermostatic bath; (4) Degassed water; (5) Temperature meter; (6) Vacuum gauge; (7) Pressure measurement; (8) ISCO injection pump; (9) Compressed gas cylinder $\left(\mathrm{CO}_{2}\right)$.

\section{$\mathrm{CO}_{2}$ injection system in the cell:}

The system consists of an ISCO positive displacement syringe pump. This is charged through a high purity $\mathrm{CO}_{2}$ bottle in liquid phase, at a constant temperature and pressure $(0$ ${ }^{\circ} \mathrm{C}$ and 55 bar). The pump is directly connected to our measuring cell through a ball valve. The pump is provided with a Druck 104 manometer for measuring the pressure of the injection branch.

\section{The pressure measuring system:}

Three sensors were used for measuring pressure at different ranges. For the range of measurements from 0 to 2 bar, a pressure sensor Druck, model PDCR-910-1422 was installed. Another identical sensor was installed for measurements from 2 to 20 bar. Finally, for measurements higher than 20 bar up to 138 bar a sensor Paroscientific, model 
42K-101, was installed. They were connected to their corresponding indicators (Druck DPI 145 and Paroscientific 735) as shown in Figure 4.2.

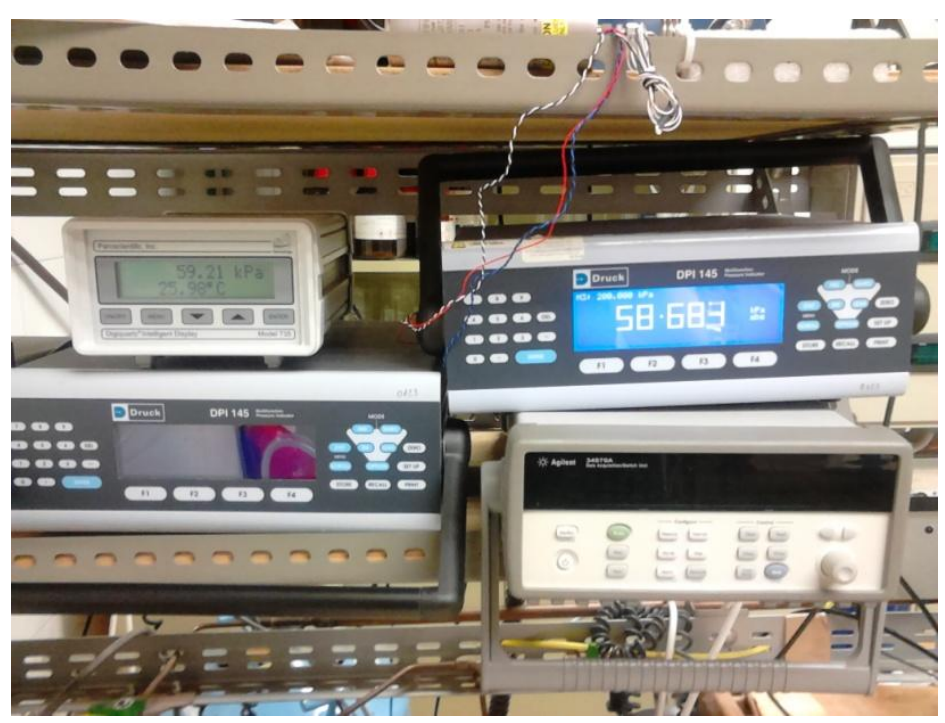

Figure 4.2. Pressure measuring system.

\subsection{Experimental procedure for solubility measurement.}

Before the measurements were undertaken, the volume of the equilibrium cell was calibrated considering the different sections of the different pressure gauges installed in the equipment. The calibration equations of the total cell volume $(\mathrm{mL})$ as a function of temperature $\left({ }^{\circ} \mathrm{C}\right)$ for each sensor are described as follows:

- Druck of 2 bar: The calibration volumen was $197.88 \mathrm{~mL}$ at $40^{\circ} \mathrm{C}$.

- Druck of 20 bar: $V_{\text {total }}=0.1047 \cdot T+194.3$

- Paroscientific up to 138 bar: $V_{\text {total }}=0.1047 \cdot T+190.9$

The pure compounds were previously degassed in the equilibrium cell through a series of cycles. First, solidifying the compound by cooling in a liquid nitrogen bath and then melting at room temperature. The amine introduced into the cell was obtained by weighing the required amount of amine up to have a final volume about $120 \mathrm{~mL}$ of the amine solution, at the concentration to be studied. In the case of water, it was degassed using a vacuum rectification column with an ultrasonic agitation bath.

Once the temperature and pressure of the cell are stable, the value of the amine vapor pressure is determined. Then, the cell is fulfilled with the degassed water using the injection cylinder until the concentration required. Once the pressure in the cell is stable, the vapor pressure of the mixture is obtained.

Finally, $\mathrm{CO}_{2}$ is loaded into the cell. The mass of $\mathrm{CO}_{2}$ injected is determined by the temperature of the pump, the pressure and the volume displaced at each load. Once equilibrium is reached, the total pressure of the cell is taken. 
At the end of a series of measurements, the equilibrium cell is disassembled for subsequent cleaning.

The concentration is expressed through the parameter $\alpha$ (load index) which is defined as the moles of $\mathrm{CO}_{2}\left(n_{\mathrm{CO} 2}\right)$ that are in the liquid phase divided by the moles of amine $\left(n_{\text {amine }}\right)$ in the liquid phase:

$$
\alpha=\frac{n_{\mathrm{CO}_{2}} \text { (liquid phase) }}{n_{\text {amine }} \text { (liquid phase) }}
$$

\subsection{Description of the measurement method used for the determination of $\mathrm{CO}_{2}$ density in aqueous amines.}

The experimental technique used to determine the density at high pressure of pure fluids and their mixtures is an automated vibrating tube densimeter. The system requires a set of elements that allow the filling with the samples, the emptying or cleaning. Also, other devices allow the control and measurement of pressure and temperature, and the record of the data obtained. The equipment diagram is shown in Figure 4.3.

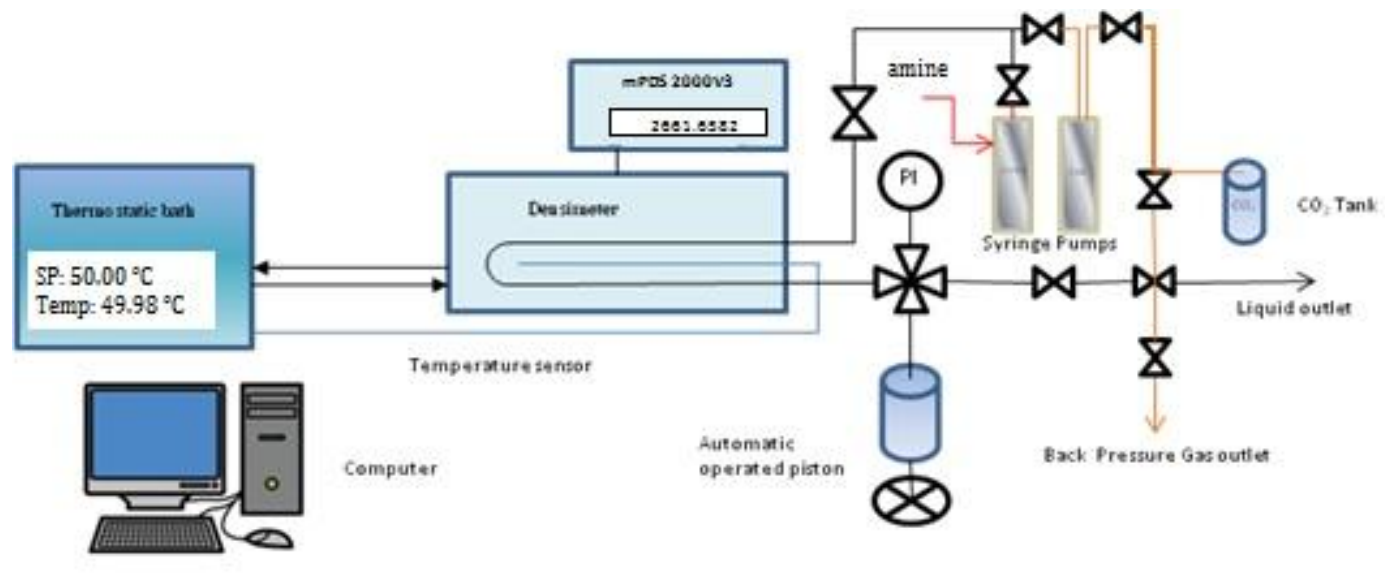

Figure 4.3. Diagram of the density measuring equipment.

The vibrating tube densimeter is Anton Paar HPM model, with an operation range of 0 - 3 $\mathrm{g} \cdot \mathrm{cm}^{-3}$ and a resolution of $10^{-5} \mathrm{~g} \cdot \mathrm{cm}^{-3}$. The range of temperature is from $263.15 \mathrm{~K}$ to 473.15 $\mathrm{K}$ and a maximum pressure of $140 \mathrm{MPa}$.

The evaluation unit mPDS 2000V3 measures the vibration period $\tau$ with an expanded uncertainty of $1 \cdot 10^{-6} \mu \mathrm{s}$, having periods around $2.6 \mu \mathrm{s}$, also measures the internal temperature of the cell and allows communication with a PC via RS232 connection. 


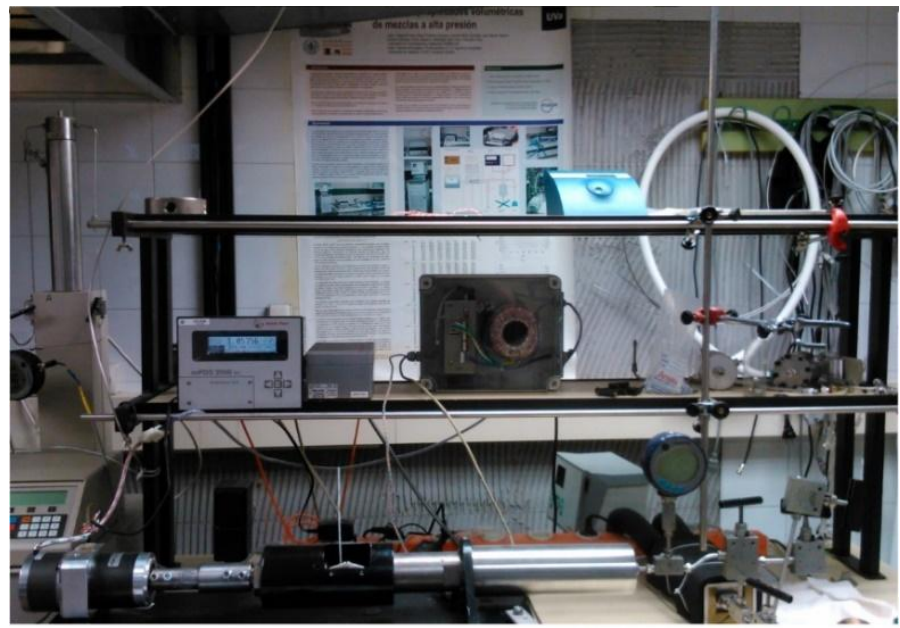

Figure 4.4. Picture of the densimeter and its components.

Except for filling, emptying and cleaning procedures, all temperature system, pressure control processes and data acquisition are fully automated, with a program developed in the Agilent VEE-Pro 7.0 programming environment.

The temperature control system consists of an external JULABO F25 HE bath, which keeps the temperature constant and uses a four-wire Pt100 control temperature probe. The probe is inserted inside the DMA HPM unit.

The pressure control system consists of a pressure measuring system, a pressure generator and a stepper motor. The pressure measurement system uses a Druck DPI 104 pressure gauge with a range $0.1-140 \mathrm{MPa}$, a resolution of $0.01 \mathrm{MPa}$, calibrated with a relative expanded uncertainty of $0.02 \%(k=2)$, connected to the PC with a USB. The pressure generator used in this work is a HiP piston (model 68-5.75-15), with a maximum volume of $35 \mathrm{~cm}^{3}$. The piston plunger is driven by a stepper motor ACP\&D 6530-R211.

The sample filling system is carried out using two positive displacement ISCO syringe pumps. Pure $\mathrm{CO}_{2}$ is introduced in one of them at liquid conditions and, in the other, the aqueous amine solution, prepared previously by weight in a RADWAG PS750/C/2 balance, which has an expanded uncertainty of $0.007 \mathrm{~g}(k=2)$. The $\mathrm{CO}_{2}$ pump is kept at a constant temperature and pressure of $0{ }^{\circ} \mathrm{C}$ and $55 \mathrm{bar}$, and for the second component the pump is at a constant temperature and pressure of $20^{\circ} \mathrm{C}$ and 55 bar. To ensure filling at constant pressure, a Mity-Mite backpressure valve (model S-91XW) is installed the outlet of the circuit. This enable a backpressure during filling of approximately 50 bar.

The evacuation of the sample is carried out through the valve connected to the backpressure valve. For cleaning, it is connected the pressure circuit to a vacuum line.

\subsection{Experimental procedure for density measurement.}

First, the pump that contains the $\mathrm{CO}_{2}$ is turned on at a constant flow, and then the second syringe pump which contains the second compound is switched on at also constant flow. 
Keeping the backpressure, the system is filled dynamically. The volumetric flow relationship allows to calculate the composition of the injected mixture.

The program developed in Agilent VEE language controls the entire measurement process. The pressures and temperatures at which the density is measured are introduced in the program. The program controls the pressure generator and the bath via control loops. The stability criterion in the pressure loop is established, when the standard deviation, $\sigma$, of the last ten period measurements is less than $1 \cdot 10^{-3} \mu$ s of the mean value, the data are recorded. The program jumps to the next pressure point for that isotherm, or starts a new isotherm, increasing the pressure or temperature as appropriate. At the end the sample is extracted, and the equipment is cleaned.

\subsection{Densimeter calibration.}

According to equation (4.2) the density $(\rho)$ can be expressed as a function of the parameters $(\mathrm{A}(T, p)$ and $\mathrm{B}(T, p))$ and the vibration period. For the calibration of the equipment it will be necessary to measure the vibration period of two reference fluids as a function of pressure and temperature, to obtain the constants $\mathrm{A}(T, p)$ and $\mathrm{B}(T, p)$.

$$
\begin{gathered}
\rho(T, p)=\mathrm{A}(T, p) \tau^{2}-\mathrm{B}(T, p) \\
\rho_{1}(T, p)=\mathrm{A}(T, p) \tau_{1}^{2}-\mathrm{B}(T, p) \quad \rho_{2}(T, p)=\mathrm{A}(T, p) \tau_{2}^{2}-\mathrm{B}(T, p)
\end{gathered}
$$

Where $\rho_{1}$ and $\rho_{2}$ are known for fluid 1 and 2 respectively at working temperatures and pressures, and their periods $\tau_{1}$ y $\tau_{2}$ are measured.

$$
\mathrm{A}(T, p)=\frac{\rho_{1}(T, p)-\rho_{2}(T, p)}{\tau_{1}^{2}(T, p)-\tau_{2}^{2}(T, p)} \quad \mathrm{B}(T, p)=\mathrm{A}(T, p) \tau_{2}^{2}(T, p)-\rho_{2}(T, p)
$$

Assuming the proposal of Lagourette et al. [12] vacuum and water were used for the calibration of the densimeter. The use of water is due toits availability and its high degree of purity. In addition, water density is known with very low uncertainty in the temperature and pressure ranges of our measurements, which is one of the requirements to minimize measurement error.

Considering equations (4.3) and (4.4), the relationships of the density with the period and the calibration parameters are expressed as:

$$
\begin{gathered}
\rho(T, p)=\rho_{w}(T, p)+\frac{\rho_{w}(T, 0.1 M P a)}{\rho_{w}^{2}(T, 0.1 M P a)-\rho_{v}(T)}\left(\tau^{2}(T, p)-\tau_{w}^{2}(T, p)\right) \\
\mathrm{A}(T)=\frac{\rho_{w}(T, 0.1 M P a)}{\tau_{w}^{2}(T, 0.1 M P a)-\tau_{v}^{2}(T)} \\
\mathrm{B}(T, p)=\frac{\rho_{w}(T, 0.1 M P a)}{\tau_{w}^{2}(T, 0.1 M P a)-\tau_{v}^{2}(T)} \tau_{w}^{2}(T, p)-\rho_{w}(T, p)
\end{gathered}
$$


Where $\rho_{w}(T, p)$ is the density of water at the same temperature $T$ and pressure $p$ of the fluid contained in the cell and $\rho_{w}(T, 0.1 \mathrm{MPa})$ is the density of water at the same temperature $T$ and atmospheric pressure; $\tau_{w}(T, p)$ y $\tau_{w}(T, 0.1 \mathrm{MPa})$ are the experimental periods of the fluid at the conditions referred above, $\tau_{v}(T)$ is the experimental period at the temperature $T$ of the vibrating tube with vacuum, $\tau^{2}(T, p)$ is the experimental period of the measured fluid. The densities of water as a calibration fluid were obtained with the Wagner and Pruss equation of state [13] due to its low uncertainty.

\subsection{Measurement uncertainty.}

The uncertainty calculation was carried out based on GUM 2008 [14]. The uncertainty of the $\mathrm{CO}_{2}$ composition of the aqueous solution of aqueous amine is associated to the precision balance with which the binary mixture amine $+\mathrm{H}_{2} \mathrm{O}$ was made, to the densities of the compounds of each pump and the flows of these pumps. The flow uncertainty for ISCO pumps is $0.5 \%$. Table 4.1 shows the uncertainty budget of the precision balance, and Table 4.2 shows the uncertainty budget of density of each compound.

Table 4.1. Calculation of the uncertainty budget of the precision balance for the mixing of the aqueous amine in the pump.

\begin{tabular}{|l|l|l|l|l|l|}
\hline & & Unit & Estimated & Divisor & $\mathrm{u}(x)$ \\
\hline & & & & & $\mathrm{g}$ \\
\hline \multirow{3}{*}{$\mathrm{u}(m)$} & Linearity & \multirow{3}{*}{$\mathrm{g}$} & 0.003 & 1 & \\
\cline { 2 - 2 } & Resolution & & 0.001 & 1 & 0.0035 \\
\cline { 2 - 5 } & Repeatability & & 0.0015 & 1 & \\
\hline \multicolumn{3}{|c|}{$\mathrm{g}(m)$} & $\mathrm{g}$ & $\mathrm{k}=1$ & 0.0035 \\
\hline $\mathrm{U}(m)$ & $\mathrm{g}=2$ & 0.007 \\
\hline
\end{tabular}

Table 4.2. Calculation of the uncertainty associated with the density of each compound in the pumps at the injection conditions of the mixture.

\begin{tabular}{|c|c|c|c|c|c|}
\hline & & Unit & Estimated & Divisor & $\mathrm{u}(\mathrm{x})$ \\
\hline & & & & & $\mathrm{kg} / \mathrm{m}^{3}$ \\
\hline \multirow{2}{*}{$\mathrm{u}(T) \mathrm{CO}_{2}$} & Calibration & \multirow{2}{*}{${ }^{\circ} \mathrm{C}$} & 0.020 & 2 & \multirow{2}{*}{0.064} \\
\hline & Resolution & & 0.010 & $2 \sqrt{3}$ & \\
\hline \multirow{2}{*}{$\mathrm{u}(p) \mathrm{CO}_{2}$} & Calibration & \multirow{2}{*}{$\mathrm{MPa}$} & 0.02 & 2 & \multirow{2}{*}{0.000047} \\
\hline & Resolution & & 0.01 & $2 \sqrt{3}$ & \\
\hline $\mathrm{u}(\rho) \mathrm{CO}_{2}$ & & $\mathrm{~kg} / \mathrm{m}^{3}$ & & $\mathrm{k}=1$ & 0.06 \\
\hline $\mathrm{U}(\rho) \mathrm{CO}_{2}$ & & $\mathrm{~kg} / \mathrm{m}^{3}$ & & $\mathrm{k}=2$ & 0.13 \\
\hline
\end{tabular}

\begin{tabular}{|c|c|c|c|c|c|}
\hline & & Unit & Estimated & Divisor & $\mathrm{u}(x)$ \\
\hline & & & & & $\mathrm{kg} / \mathrm{m}^{3}$ \\
\hline \multirow{2}{*}{$\mathrm{u}(T)$ Amine aq } & Calibration & \multirow{2}{*}{${ }^{\circ} \mathrm{C}$} & 0.020 & 2 & \multirow{2}{*}{0.007} \\
\hline & Resolution & & 0.010 & $2 \sqrt{3}$ & \\
\hline \multirow{2}{*}{$\mathrm{u}(p)$ Amine aq } & Calibration & \multirow{2}{*}{$\mathrm{MPa}$} & 0.02 & 2 & \multirow{2}{*}{0.000004} \\
\hline & Resolution & & 0.01 & $2 \sqrt{3}$ & \\
\hline $\mathrm{u}(\rho)$ Amine $\mathrm{aq}$ & & $\mathrm{kg} / \mathrm{m}^{3}$ & & $\mathrm{k}=1$ & 0.007 \\
\hline $\mathrm{U}(\rho)$ Amine aq & & $\mathrm{kg} / \mathrm{m}^{3}$ & & $\mathrm{k}=2$ & 0.01 \\
\hline
\end{tabular}


Table 4.3 shows the uncertainties associated at the composition of the mixture.

Table 4.3. Calculation of the uncertainty associated at the composition of the mixture.

\begin{tabular}{|c|c|c|l|l|l|}
\hline & & Unit & Estimated & Divisor & $\mathrm{u}(x)$ \\
\hline & & & & & $\mathrm{mol} / \mathrm{mol}$ \\
\hline $\mathrm{u}(\dot{n})$ Amine aq. & - & $\mathrm{mol} / \mathrm{min}$ & 0.0017 & 1 & 0.0004 \\
\hline $\mathrm{u}(\dot{m}) \mathrm{CO}_{2}$ & - & $\mathrm{g} / \mathrm{min}$ & 0.0022 & 1 & 0.0002 \\
\hline $\mathrm{u}(x) \mathrm{CO}_{2}$ & $\mathrm{~mol} / \mathrm{mol}$ & $\mathrm{k}=1$ & 0.0004 \\
\hline $\mathrm{U}(x) \mathrm{CO}_{2}$ & $\mathrm{~mol} / \mathrm{mol}$ & $\mathrm{k}=2$ & 0.0009 \\
\hline
\end{tabular}

Table 4.4 shows the uncertainties associated with the calibration parameters at our temperature range.

Table 4.4. Calculation of the uncertainty associated with the calibration parameters in the temperature range of (273.15-373.15) K and pressures 0.1-140 MPa.

\begin{tabular}{|c|c|c|c|c|c|}
\hline & & Unit & Estimated & Divisor & $\mathrm{u}(x)$ \\
\hline & & & & & $\mathrm{kg} / \mathrm{m}^{3}$ \\
\hline $\mathrm{u}\left(\rho_{w}\right)$ & $\begin{array}{c}\text { Reference } \\
\text { Material }\end{array}$ & $\mathrm{kg} / \mathrm{m}^{3}$ & 0.01 & $\sqrt{3}$ & 0.0058 \\
\hline \multirow{3}{*}{$\mathrm{u}(T)$} & Calibration & \multirow{3}{*}{${ }^{\circ} \mathrm{C}$} & 0.020 & 2 & \multirow{3}{*}{0.0046} \\
\hline & Resolution & & 0.010 & $2 \sqrt{3}$ & \\
\hline & Repeatability & & 0.0008 & 1 & \\
\hline \multirow{3}{*}{$\mathrm{u}(p)$} & Calibration & \multirow{3}{*}{$\mathrm{MPa}$} & 0.02 & 2 & \multirow{3}{*}{0.0041} \\
\hline & Resolution & & 0.01 & $2 \sqrt{3}$ & \\
\hline & Repeatability & & 0.002 & 1 & \\
\hline \multirow{2}{*}{$\mathrm{u}(\tau)$} & Repeatability & \multirow{2}{*}{$\mu \mathrm{s}$} & 0.0006 & 1 & \multirow{2}{*}{$8.5 \mathrm{E}-03$} \\
\hline & Resolution & & 1.00E-03 & $2 \sqrt{3}$ & \\
\hline $\mathrm{U}(\mathrm{A}(T))$ & \multicolumn{3}{|c|}{$\mathrm{kg} / \mathrm{m}^{3} \mu \mathrm{s}^{2}$} & $\mathrm{k}=2$ & 7.6E-08 \\
\hline $\mathrm{U}(\mathrm{B}(T, p))$ & \multicolumn{3}{|c|}{$\mathrm{kg} / \mathrm{m}^{3}$} & $\mathrm{k}=2$ & 0.43 \\
\hline
\end{tabular}

Table 4.5. shows the uncertainties associated with density measurement at different temperature ranges and the calculated expanded uncertainty with a coverage factor $k=2$ ( $95 \%$ confidence). 
Table 4.5. Uncertainty budget associated to the experimental density in the temperature range of (293.15-373.15) Kand pressures 0.1-60 MPa.

\begin{tabular}{|c|c|c|c|c|c|}
\hline & & Unit & Estimated & Divisor & $\mathrm{u}(x)$ \\
\hline & & & & & $\mathrm{kg} / \mathrm{m}^{3}$ \\
\hline \multirow{2}{*}{$\mathrm{u}(\tau)$} & Repeatability & \multirow{2}{*}{$\mu \mathrm{s}$} & 0.0006 & 1 & \multirow{2}{*}{$8.5 \mathrm{E}-03$} \\
\hline & Resolution & & $1.00 \mathrm{E}-03$ & $2 \sqrt{3}$ & \\
\hline \multirow{3}{*}{$\mathrm{u}(T)$} & Calibration & \multirow{3}{*}{${ }^{\circ} \mathrm{C}$} & 0.020 & 2 & \multirow{3}{*}{0.0046} \\
\hline & Resolution & & 0.010 & $2 \sqrt{3}$ & \\
\hline & Repeatability & & 0.0008 & 1 & \\
\hline \multirow{3}{*}{$\mathrm{u}(p)$} & Calibration & \multirow{3}{*}{$\mathrm{MPa}$} & 0.02 & 2 & \multirow{3}{*}{0.0041} \\
\hline & Resolution & & 0.01 & $2 \sqrt{3}$ & \\
\hline & Repeatability & & 0.002 & 1 & \\
\hline $\mathrm{u}(\mathrm{A}(T))$ & $\mathrm{kg} / \mathrm{m}^{3} \mu \mathrm{S}^{2}$ & & $7.6 \mathrm{E}-08$ & 2 & 0.26 \\
\hline $\mathrm{u}(\mathrm{B}(T, p))$ & $\mathrm{kg} / \mathrm{m}^{3}$ & & 0.43 & 2 & 0.21 \\
\hline $\mathrm{u}(\rho)$ & \multicolumn{3}{|c|}{$\mathrm{kg} / \mathrm{m}^{3}$} & $\mathrm{k}=1$ & 0.34 \\
\hline $\mathrm{U}(\rho)$ & \multicolumn{3}{|c|}{$\mathrm{kg} / \mathrm{m}^{3}$} & $\mathrm{k}=2$ & 0.7 \\
\hline $\operatorname{Ur}(\rho)$ & \multicolumn{3}{|c|}{$100 \cdot\left(\mathrm{kg} / \mathrm{m}^{3} / \mathrm{kg} / \mathrm{m}^{3}\right)$} & $\mathrm{k}=2$ & $0.07 \%$ \\
\hline
\end{tabular}

Table 4.5. shows that the greatest contribution to uncertainty comes from the calibration parameters $\mathrm{A}(T)$ and $\mathrm{B}(T, p)$. The expanded relative uncertainty of $0.07 \%$ was calculated for the lowest density within our measurement range.

\subsection{System validation.}

For the validation of the system, toluene densities were measured in a temperature range from $273.15 \mathrm{~K}$ to $373.15 \mathrm{~K}$ and a pressure range from $0.1 \mathrm{MPa}$ to $140 \mathrm{MPa}$. The maximum deviations from REFPROP were $0.07 \%$, which uses the equation described by Lemmon and Span [15]. These deviations are in agreement with the uncertainty. 
Chapter 5

\section{EXPERIMENTAL RESULTS OF DENSITY AND SOLUBILITY OF $\mathrm{CO}_{2}$ MEASUREMENTS IN AQUEOUS AMINE SOLUTIONS}





\subsection{Experimental results of $\mathrm{CO}_{2}$ density and solubility measurements in aqueous amine solutions.}

This chapter presents the experimental results of density measurements for ternary mixtures of aqueous amine solutions $+\mathrm{CO}_{2}$ in temperature ranges from $293.15 \mathrm{~K}$ to 373.15 $\mathrm{K}$ and pressure from $0.1 \mathrm{MPa}$ to $60 \mathrm{MPa}$. These were obtained by the vibrating tube densimeter technique described in the previous chapter. On the other hand, the experimental results of the $\mathrm{CO}_{2}$ solubilities in aqueous amine solutions are also reported. They were determined, using the vapor-liquid equilibrium equipment, at two different temperatures $313.15 \mathrm{~K}$ and $353.15 \mathrm{~K}$. The amines studied were Monoethanolamine (MEA), Dimethylethanolamine (DMAE) and Triethanolamine (TEA).

In this chapter, the adjustment to equations that describe the behaviour of the densities and solubility are presented together with the experimental data.

The experimental results are displayed in the form of tables and graphs. They show pressure $p$, temperature $T$ and density $\rho$. In the case of solubilities, the partial pressure of $\mathrm{CO}_{2}\left(p_{\mathrm{CO} 2}\right)$ is shown depending on the load index $\alpha$ (moles of $\mathrm{CO}_{2}$ per mole of amine) at different temperatures.

For knowing the solubility of $\mathrm{CO}_{2}$ in aqueous amine solutions, it is first necessary to measure the density of these ternary mixtures, to estimate the volume occupied by the mixture in the liquid phase within the measuring cell.

The mixtures carried out for the density measurement in this work were aqueous solutions at $10 \%$ and $30 \%$ by weight of MEA at different concentrations with $\mathrm{CO}_{2}$ for the validation of the technique. In addition, two different systems of aqueous solutions at $30 \%$ by weight with $\mathrm{CO}_{2}$, one with DMAE and other with TEA were measured. All measurements were performed at wide temperature ranges (from $293.15 \mathrm{~K}$ until $373.15 \mathrm{~K}$ ), pressure (from 0.1 $\mathrm{MPa}$ to $60 \mathrm{MPa}$ ) and at different compositions of $\mathrm{CO}_{2}$ depending on $\alpha$ from 0.1 up to 1.5.

The densities of three mixtures of aqueous amine solutions $+\mathrm{CO}_{2}$ at different concentrations were measured:

MEA $10 \% \mathrm{w}+\mathrm{CO}_{2}$ at $\alpha=0.8$ and 1.

MEA $30 \% \mathrm{w}+\mathrm{CO}_{2}$ at $\alpha=0.2,0.44,0.67$ and 1 .

DMAE $30 \% \mathrm{w}+\mathrm{CO}_{2}$ at $\alpha=0.3,0.7,1.1$ and 1.5 .

TEA $30 \% \mathrm{w}+\mathrm{CO}_{2}$ at $\alpha=0.3,0.6,0.9$ and 1.2 .

The solubilities carried out in this work were aqueous solutions at $10 \%$ and $30 \%$ by weight of MEA, aqueous solutions at $30 \%$ by weight of DMAE and aqueous solutions at $30 \%$ by weight of TEA. All systems except for MEA at $10 \%$ by weight were performed at two temperatures, $313.15 \mathrm{~K}$ and $353.15 \mathrm{~K}$, with pressures from the vapor pressure of the aqueous amine solution up to $6 \mathrm{MPa}$ :

MEA $10 \% \mathrm{w}+\mathrm{CO}_{2}$ at $313.15 \mathrm{~K}$. 
MEA 30\%w $+\mathrm{CO}_{2}$ at $313.15 \mathrm{~K}$ and $353.15 \mathrm{~K}$.

DMAE $30 \% \mathrm{w}+\mathrm{CO}_{2}$ at $313.15 \mathrm{~K}$ and $353.15 \mathrm{~K}$.

TEA $30 \% \mathrm{w}+\mathrm{CO}_{2}$ at $313.15 \mathrm{~K}$ and $353.15 \mathrm{~K}$.

\subsubsection{Products used.}

In Table 5.1. (spanish version, page 104), the products used, the provenance and the purity given by the certificates of analysis are shown and no additional purification method was used.

As explained in chapter 4, the pure amines were previously degassed as well as the water used in the solubility measurements. In addition, the aqueous amines solutions for the density measurements were previously degassed during at least 5 hours.

\subsubsection{Adjustment of density data.}

Density data at different temperatures and pressures have been adjusted to a modified Tammann-Tait equation of state [16] for each concentration as proposed in other works Comuñas et al. [17] or Sobrino et al. [18]:

$$
\rho(T, p)=\frac{\rho\left(T, p_{r e f}\right)}{1-\mathrm{C} \ln \left[\frac{\mathrm{B}(T)+p}{\mathrm{~B}(T)+p_{r e f}}\right]}
$$

where $\rho$ is the density, $T$ the temperature, $\mathrm{C}$ is a parameter which is considered temperature independent and $\rho\left(T, p_{\text {ref }}\right)$ is the dependence of the density with the temperature at the reference pressure, being in our case usually $5 \mathrm{MPa}$. This function is expressed in equation 5.2 .

$$
\rho\left(T, p_{\text {ref }}\right)=\sum_{\mathrm{i}=0} \mathrm{~A}_{\mathrm{i}} T^{\mathrm{i}}=\mathrm{A}_{0}+\mathrm{A}_{1} T+\mathrm{A}_{2} T^{2}
$$

Where $A_{i}$ values are determined from the experimental values of density at the reference pressure, and the function $\mathrm{B}(T)$ has a polynomial form according to equation 5.3:

$$
\mathrm{B}(T)=\sum_{\mathrm{i}=0} \mathrm{~B}_{\mathrm{i}} T^{\mathrm{i}}=\mathrm{B}_{0}+\mathrm{B}_{1} T+\mathrm{B}_{2} T^{2}
$$

The $\mathrm{B}_{i}$ values are determined from the experimental values. In this way, from equation 5.1, the density values for a wide range of temperature and pressure using the adjustable parameters $A_{i}, B_{i}$ and $C$ can be represented. 
In order to evaluate the adjustment, the standard deviation $\sigma$ and the maximum deviation $(M D \%)$ between experimental data and the calculated values by the model will be used, being equations 5.4 and 5.5 respectively.

$$
\begin{aligned}
& \sigma=\sqrt{\left[\frac{1}{N-m}\right] \sum_{i=0}^{N}\left(x_{\text {exp }}-x_{c a l}\right)^{2}} \\
& M D \%=\operatorname{Max} \cdot\left|\frac{\left(x_{c a l}-x_{\text {exp }}\right)}{x_{\text {exp }}} \cdot 100\right|
\end{aligned}
$$

where $N$ is the number of experimental data, $m$ is the number of adjustable parameters, $x_{\text {exp }}$ is the experimental value and $x_{c a l}$ is the value calculated using the modified Tammann-Tait equation 5.1 .

In addition, a simple equation was used to fit the density data as a function of the composition (load index, $\alpha$ ) and pressure at a given temperature for calculate the solubility, and the standard deviation $\sigma$ represented with equation 5.4, was also obtained.

$$
\rho_{\text {mixture }}=\mathrm{a}+\frac{\mathrm{b}}{\alpha}+\frac{\mathrm{c}}{\alpha^{2}}+\mathrm{d} \cdot p_{\text {total }}
$$

\subsubsection{Adjustment of the solubility data.}

The adjustment of the partial pressure of $\mathrm{CO}_{2}\left(p_{\mathrm{CO} 2}\right)$, against the load index $\alpha$ and the working temperature was performed using the $\mathrm{Xu}$ and Rochelle equation [19], with 6 parameters:

$$
\ln p_{\mathrm{CO}_{2}}(\mathrm{~Pa})=\mathrm{A}_{1}-\mathrm{A}_{2} /_{T}+\mathrm{A}_{3} \cdot \alpha+\mathrm{A}_{4} \cdot \alpha^{2}+\mathrm{A}_{5} \cdot \alpha / T+\mathrm{A}_{6} \cdot \alpha^{2} / T
$$

where $p_{\mathrm{CO} 2}$ is the partial pressure of $\mathrm{CO}_{2}, T$ the temperature and $\alpha$ is the load index in moles of $\mathrm{CO}_{2}$ per mole of amine and $\mathrm{A}_{\mathrm{i}}$ are theadjustable parameters.

\subsubsection{Experimental results of density measurements.}

As an example of the experimental data obtained for the densities of the mixtures studied, the system MEA $30 \% \mathrm{w}+\mathrm{CO}_{2}$ is presented at $\alpha=0.67$, the other results are in Chapter 5 , Section 5.5 (Spanish version, page 105). The experimental data are shown both as tables and graphs. As well as the results of the adjustment of the experimental data to the Tammann-Tait model for each one of the studied compositions, depending on temperature and pressure. In addition, the standard deviation $\sigma$ (equation 5.4) and the maximum deviation ( $M D \%$ ) (equation 5.5) are shown. Finally, the adjustable parameters of equation 5.6 where adjustment of the experimental data of the density are represented as a function of $\alpha$ and the total pressure and the standard deviation are also presented. 


\subsubsection{System MEA $30 \% \mathrm{w}+\mathrm{CO}_{2}$.}

Table 5.1. Experimental density data, $\rho\left(\mathrm{kg} \cdot \mathrm{m}^{-3}\right)$, for the mixture $\mathrm{MEA} 30.000 \% \mathrm{w}+\mathrm{CO}_{2}$ at $\alpha=0.6661$.

\begin{tabular}{cccccc}
\hline \multicolumn{5}{c}{$\rho\left(\mathrm{kg} \cdot \mathrm{m}^{-3}\right)$} \\
\hline$p / \mathrm{MPa}$ & 293.15 & 313.15 & 333.15 & 353.15 & 373.15 \\
\hline 0.1 & 1132.3 & 1123.5 & & & \\
0.5 & 1132.3 & 1123.5 & 1113.2 & 1100.8 & \\
1 & 1132.5 & 1123.7 & 1113.4 & 1101.0 & 1086.9 \\
5 & 1133.8 & 1125.0 & 1114.8 & 1102.5 & 1088.5 \\
10 & 1135.4 & 1126.7 & 1116.5 & 1104.3 & 1090.5 \\
15 & 1137.1 & 1128.3 & 1118.2 & 1106.1 & 1092.4 \\
20 & 1138.7 & 1129.9 & 1119.9 & 1107.8 & 1094.3 \\
30 & 1141.8 & 1133.1 & 1123.2 & 1111.3 & 1097.9 \\
40 & 1145.0 & 1136.2 & 1126.4 & 1114.7 & 1101.6 \\
50 & 1147.9 & 1139.3 & 1129.6 & 1118.0 & 1105.1 \\
60 & 1151.0 & 1142.3 & 1132.8 & 1121.3 & 1108.6 \\
\hline
\end{tabular}

Table 5.2. Coefficients and statistics from the modified Tammann-Tait equation (eq. 5.1) for the mixture MEA 30.000\%w $+\mathrm{CO}_{2}$ at $\alpha=0.6661, p_{\text {ref }}=1 \mathrm{MPa}$.

Parameters of the modified Tammann-Tait equation

\begin{tabular}{ccccccccc}
\hline $\begin{array}{c}\mathrm{A}_{0} \\
\mathrm{~kg} \cdot \mathrm{m}^{-3}\end{array}$ & $\begin{array}{c}\mathrm{A}_{1} \\
\mathrm{~kg} \cdot \mathrm{m}^{-3} \cdot \mathrm{K}^{-1}\end{array}$ & $\begin{array}{c}\mathrm{A}_{2} \\
\mathrm{~kg} \cdot \mathrm{m}^{-3} \cdot \mathrm{K}^{-2}\end{array}$ & $\begin{array}{c}\mathrm{B}_{0} \\
\mathrm{MPa}\end{array}$ & $\begin{array}{c}\mathrm{B}_{1} \\
\mathrm{MPa} \cdot \mathrm{K}^{-1}\end{array}$ & $\begin{array}{c}\mathrm{B}_{2} \\
\mathrm{MPa} \cdot \mathrm{K}^{-2}\end{array}$ & $\mathrm{C}$ & $\begin{array}{c}\sigma \\
\mathrm{kg} \cdot \mathrm{m}^{-3}\end{array}$ & $\begin{array}{c}M D \\
(\%)\end{array}$ \\
\hline 1064.8 & 0.860 & $-2.14 \mathrm{E}-03$ & 596.8 & 0.1450 & $-1.93 \mathrm{E}-03$ & 0.1345 & 0.12 & 0.02 \\
\hline
\end{tabular}




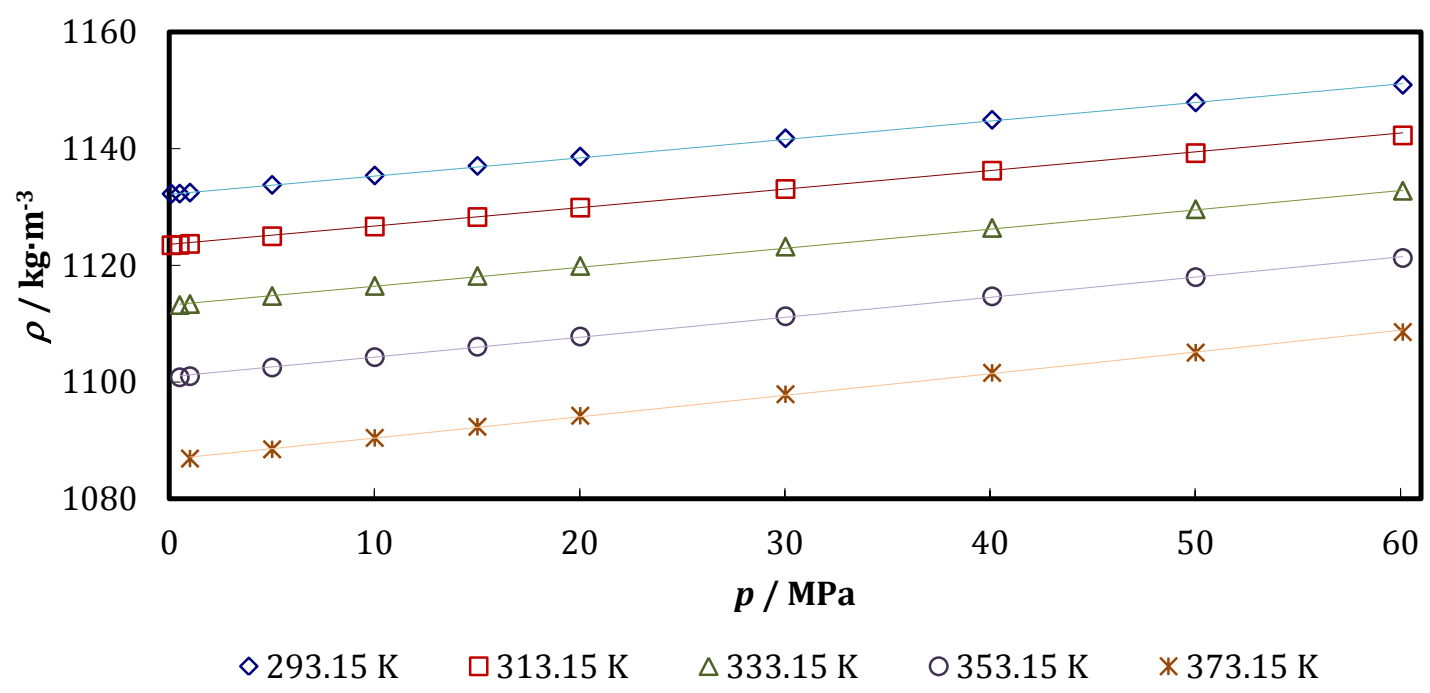

Figure 5.1. Experimental density values (symbols) for the mixture $\mathrm{MEA} 30.000 \% \mathrm{w}+\mathrm{CO}_{2}$ at $\alpha=0.6661$ and different temperatures depending on the pressure. The continuous lines represent the modified Tammann-Tait equation.

Table 5.3. Parameters and standard deviation of the adjusted equation of the density with $\alpha$ and $p_{\text {total }}$ (eq. 5.6) for the mixture $\mathrm{MEA} 30.000 \% \mathrm{w}+\mathrm{CO}_{2}$.

\begin{tabular}{cccccc}
\hline \multicolumn{5}{c}{ Equation 5.6 parameters } \\
\hline $\mathrm{T}$ & $\mathrm{a}$ & $\mathrm{b}$ & $\mathrm{c}$ & $\mathrm{d}$ & $\sigma$ \\
$\mathrm{K}$ & $\mathrm{kg} \cdot \mathrm{m}^{-3}$ & $\mathrm{~kg} \cdot \mathrm{m}^{-3}$ & $\mathrm{~kg} \cdot \mathrm{m}^{-3}$ & $\mathrm{~kg} \cdot \mathrm{m}^{-3} \cdot \mathrm{bar}^{-1}$ & $\mathrm{~kg} \cdot \mathrm{m}^{-3}$ \\
\hline 313.15 & 1148.38 & -15.71 & -0.71 & 0.0345 & 0.76 \\
353.15 & 1118.8 & -7.6 & -2.06 & 0.0377 & 1.95 \\
\hline
\end{tabular}

The density results obtained for mixtures of MEA $30 \% \mathrm{w}+\mathrm{CO}_{2}$ have been compared with those found in the literature [20], [21] and [22]. Our experimental density data were interpolated to the concentrations and pressures using eq. 5.6 that appear for each author in order to compare them with literature values.

Figure 5.2 shows the relative deviations obtained between the experimental data and those of the literature at $313.15 \mathrm{~K}$. The average absolute deviation obtained (AAD\%) for the mixture at $313.15 \mathrm{~K}$ was $1.08 \%$ with [20], $1.57 \%$ with [21] and $1.24 \%$ with [22]. 


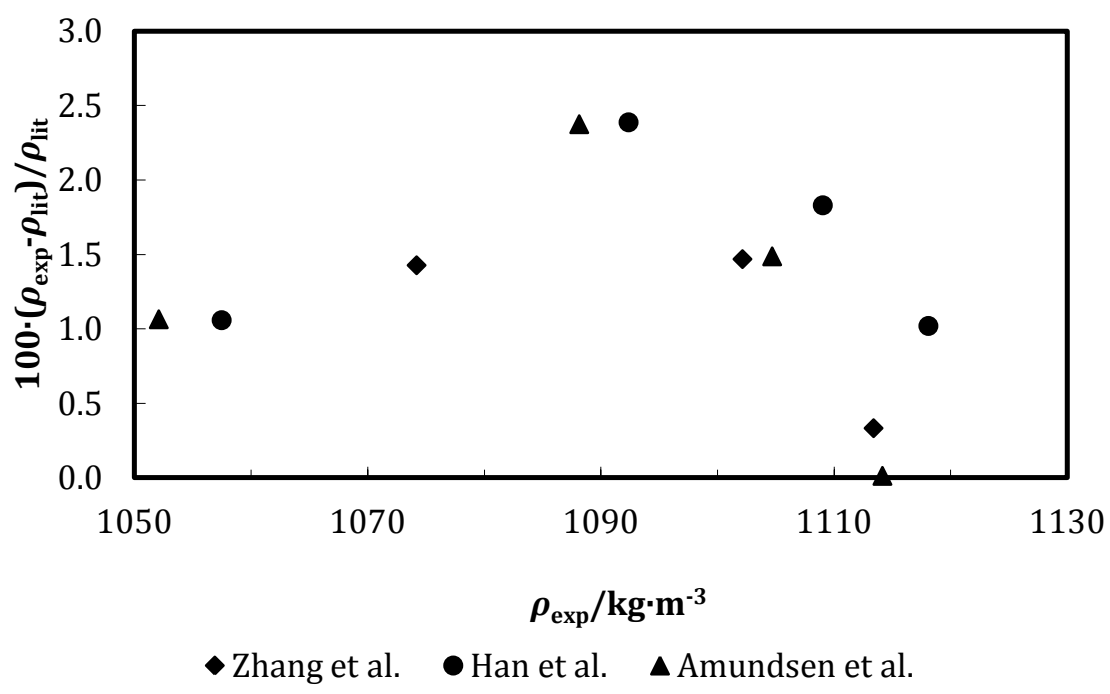

Figure 5.2. Relative percentage deviation of experimental densitiy $\rho_{\text {exp }}$ from the literature data of Zhang et al. [20], Han et al. [21] and Amundsen et al. [22] for the system $\mathrm{MEA} 30 \% \mathrm{w}+\mathrm{CO}_{2}$ at 313.15 K.

Figure 5.3 shows the relative deviations obtained between the experimental data and those of the literature at $333.15 \mathrm{~K}$. The average absolute deviation obtained (AAD\%) for the mixture at $313.15 \mathrm{~K}$ was $1.95 \%$ with [20], $1.99 \%$ with [21] and $1.64 \%$ with [22].

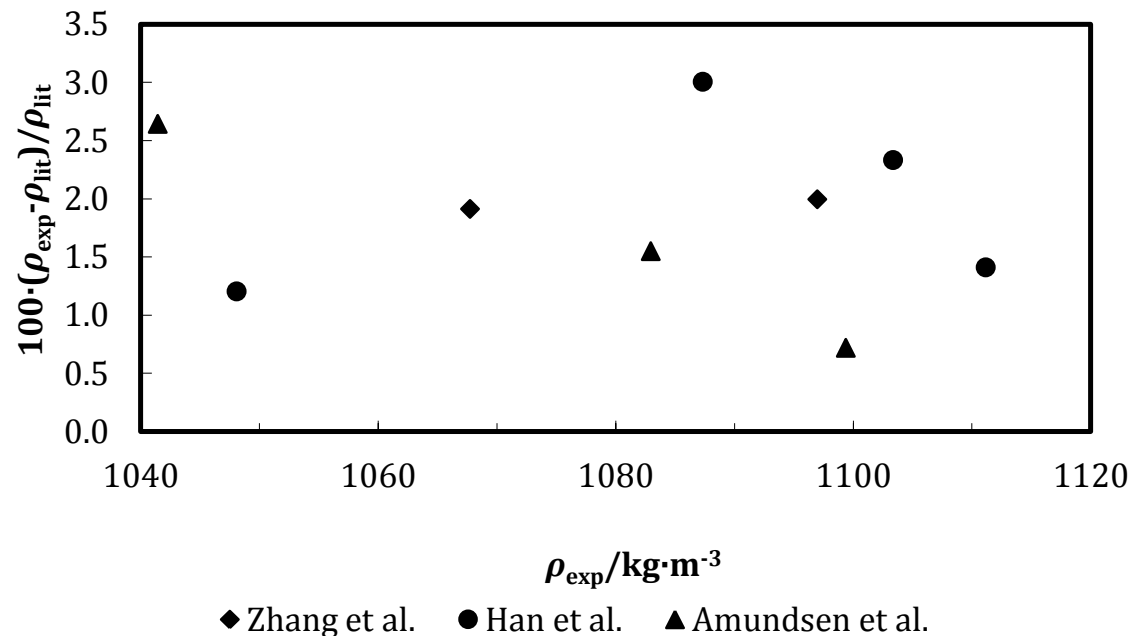

Figure 5.3. Relative percentage deviation of the experimental $\rho$ to $333.15 \mathrm{~K}$ with respect to the data of the bibliography of Zhang et al. [20], Han et al. [21] and Amundsen et al. [22].

Figure 5.4 shows the relative deviations obtained between the experimental data and those in the literature at $353.15 \mathrm{~K}$. The average absolute deviation obtained $(A A D \%)$ for the mixture at $313.15 \mathrm{~K}$ was $1.96 \%$ with [20], 2.05\% with [21] and $2.02 \%$ with [22]. 


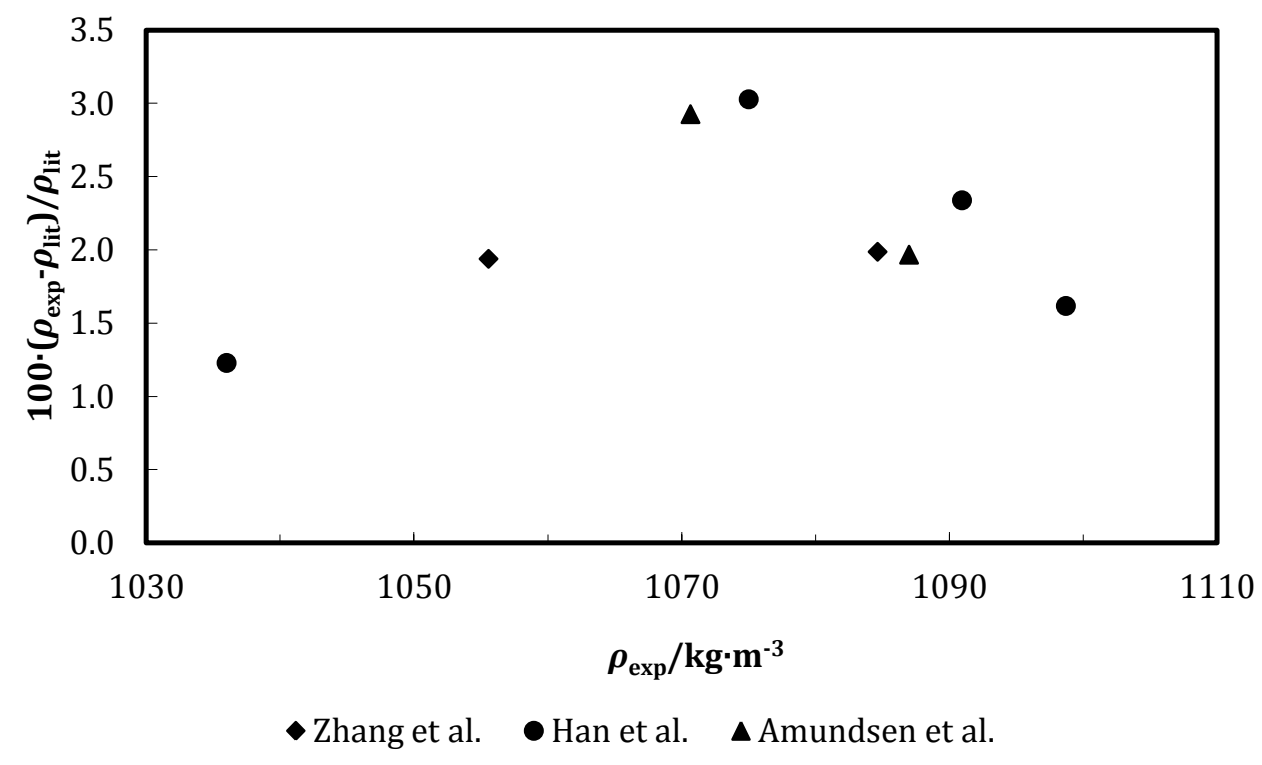

Figure 5.4. Relative percentage deviation of experimental $\rho$ to $353.15 \mathrm{~K}$ from the bibliographic data of Zhang et al. [20], Han et al. [21] and Amundsen et al. [22].

\subsubsection{Discussion of density results.}

In this work, the densities of three ternary mixtures of aqueous amines solutions with $\mathrm{CO}_{2}$ were performed in a temperature range from $293.15 \mathrm{~K}$ to $373.15 \mathrm{~K}$ for mixtures with MEA and e from $313.15 \mathrm{~K}$ to $353.15 \mathrm{~K}$ for mixtures with DMAE and TEA. All in a pressure range from $0.1 \mathrm{MPa}$ up to $60 \mathrm{MPa}$.

The fitting results using the modified Tammann-Tait equation were good considering the complexity of the mixtures. This is due to the reaction of $\mathrm{CO}_{2}$ with the amine, which needs a very long reaction time until reaching the equilibrium. The maximum deviation $(M D \%)$ is less than $0.12 \%$ in all cases and the standard deviations $(\sigma)$ vary between $0.03 \mathrm{~kg} \cdot \mathrm{m}^{-3}$ and $0.70 \mathrm{~kg} \cdot \mathrm{m}^{-3}$ for all the mixtures studied.

The best adjustments of the modified Tammann-Tait equation present a standard deviation of $0.03 \mathrm{~kg} \cdot \mathrm{m}^{-3}$ and a maximum deviation $(M D \%)$ of $0.004 \%$ for the system composed of MEA $10 \% \mathrm{w}+\mathrm{CO}_{2}$ at $\alpha=0.8$.

The experimental values obtained show an increasing tendency of density with pressure and decreasing with increasing temperature. As regards the concentration of $\mathrm{CO}_{2}$, the density always increases as it increases for all the different mixtures with amines.

The density also increases as a function of the amine at the same pressure, temperature, and $\mathrm{CO}_{2}$ concentration; Comparing different amines at constant conditions, the highest density corresponds to MEA:

$$
\rho_{D M A E 30 \%_{W}}<\rho_{\text {TEA 30\%W }}<\rho_{M E A 30 \% W}
$$


Concerning comparison with literature data, the deviations are consistent with the literature taking into account that these measures present the added difficulty of ensuring that the reaction reached the chemical equilibrium.

It is observed that the density obtained is greater than the values in the bibliography for all temperatures. For this reason, a stability study was carried out in order to know the end of the reaction. The results show that the equilibrium needs a minimum of 24 hours but, in others works [23], the waiting time was less than 6 hours. In the Figure 5.5 can be observed that if the reaction time of the other authors were 42 hours, the differences between our data and literature would have been reduced by half. This figure shows the density variation with time for a mixture of MEA to $30 \% \mathrm{w}$ and $\alpha=0.2$ moles of $\mathrm{CO}_{2}$ per mole of MEA, taking the times in periods of 0.5 hours. As seen, the reaction has not already reached the equilibrium after $35.5 \mathrm{~h}$ andthe density is still increasing. In our case, the end-of-reaction criterion established that the slope between measurements measured in intervals of 5 hours was less than $10 \%$ of the measurement standard uncertainty of the equipment, resulting in an uncertainty value less than $0.36 \mathrm{~kg} / \mathrm{m}^{3}$ for waiting times between 24 and 48 hours, depending on the amine type, $\mathrm{CO}_{2}$ concentration and temperature.

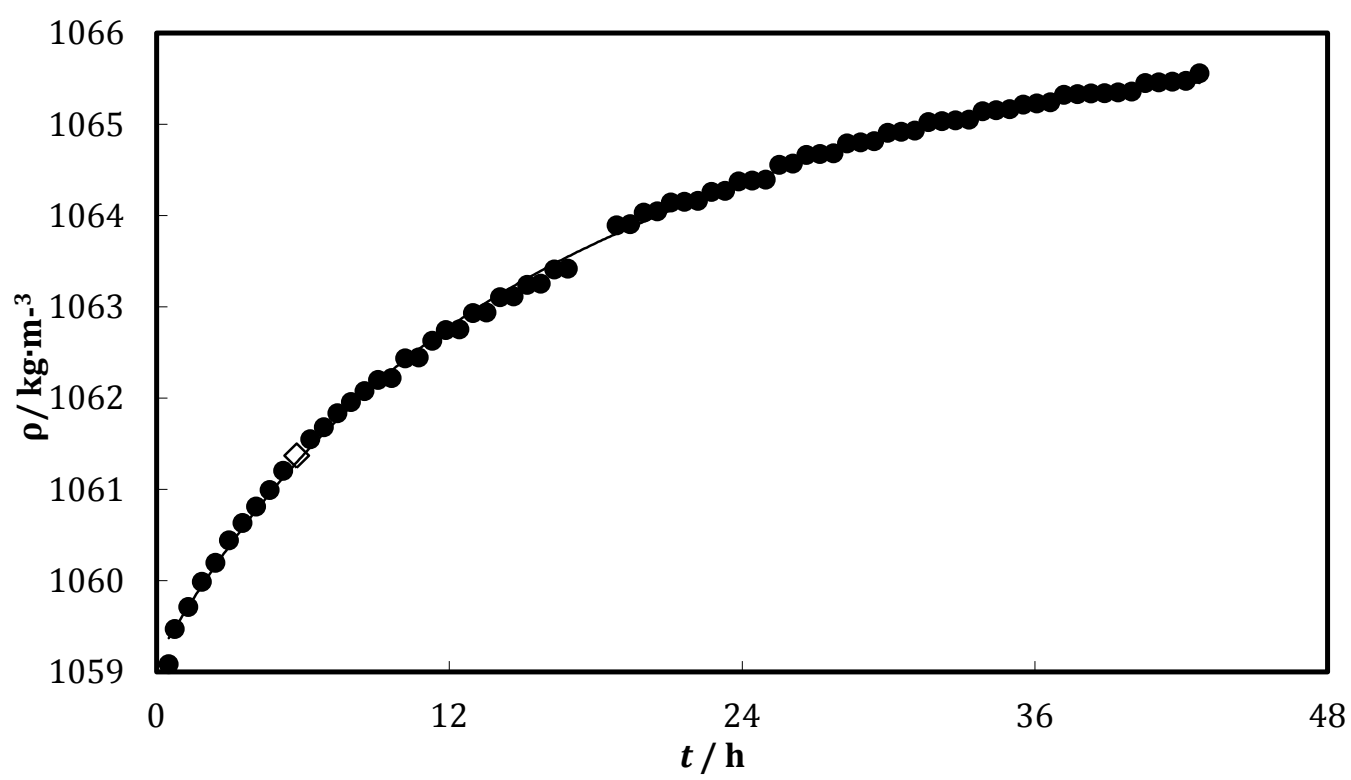

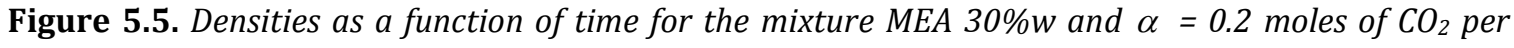
mole of MEA at $313.15 \mathrm{~K}$ and 50 bar. $(\diamond)$ Represents the density measured for a time close to 6 hours.

\subsubsection{Experimental results of solubility measurements.}

As an example of the experimental data obtained for the solubility of the mixtures studied, the system MEA $30 \% \mathrm{w}+\mathrm{CO}_{2}$ is presented at $313.15 \mathrm{~K}$ and $353.15 \mathrm{~K}$, the other results are in Chapter 5, Section 5.7 (Spanish version, page 130). The experimental data are shown both as tables and graphs. As well as the results of the adjustment of the experimental data to the $\mathrm{Xu}$ and Rochelle equation (eq. 5.7) in section 5.1.3. In addition, the standard deviation of the adjustment $\sigma$ (equation 5.4) between experimental data and the model used are shown. 


\subsubsection{System MEA $30 \% \mathrm{w}+\mathrm{CO}_{2}$ at $313.15 \mathrm{~K}$ and $353.15 \mathrm{~K}$.}

Table 5.4. Experimental solubility of $\mathrm{CO}_{2}$ in aqueous $\mathrm{MEA} 30.015 \% \mathrm{w}$ at $313.15 \mathrm{~K}$ and $\mathrm{MEA} 29.999 \% \mathrm{w}$ at $353.15 \mathrm{~K}$.

\begin{tabular}{cccc}
\hline \multicolumn{3}{c}{$T / \mathrm{K}$} \\
\hline$p_{\mathrm{CO}_{2}} / \mathrm{kPa}$ & $\alpha_{\mathrm{CO}_{2}}$ & $p_{\mathrm{CO}_{2}} / \mathrm{kPa}$ & $\alpha_{\mathrm{CO}_{2}}$ \\
\hline 0.232 & 0.462 & 22.031 & 0.462 \\
4.891 & 0.529 & 76.793 & 0.516 \\
29.938 & 0.583 & 236.9 & 0.567 \\
82.310 & 0.630 & 362.5 & 0.590 \\
91.632 & 0.636 & 547.1 & 0.615 \\
146.1 & 0.664 & 1061.4 & 0.662 \\
185.0 & 0.679 & 1736.2 & 0.704 \\
284.4 & 0.710 & 4207.2 & 0.804 \\
452.0 & 0.748 & & \\
734.9 & 0.792 & & \\
1232.4 & 0.846 & & \\
\hline
\end{tabular}

Table 5.5. Adjustable parameters and standard deviation of Xu and Rochelle equation for the solubility of $\mathrm{CO}_{2}$ in aqueous $\mathrm{MEA} 30 \%$ w at $313.15 \mathrm{~K}$ and $353.15 \mathrm{~K}$.

\begin{tabular}{ccccccc}
\hline \multicolumn{7}{c}{ Parameters of the Xu and Rochelle equation } \\
\hline $\begin{array}{c}\mathrm{A}_{1} \\
\mathrm{~kg} \cdot \mathrm{m}^{-3}\end{array}$ & $\begin{array}{c}\mathrm{A}_{2} \\
\mathrm{~kg} \cdot \mathrm{m}^{-3} \cdot \mathrm{K}^{-1}\end{array}$ & $\begin{array}{c}\mathrm{A}_{3} \\
\mathrm{~kg} \cdot \mathrm{m}^{-3} \cdot \mathrm{K}^{-2}\end{array}$ & $\begin{array}{c}\mathrm{A}_{4} \\
\mathrm{MPa}\end{array}$ & $\begin{array}{c}\mathrm{A}_{5} \\
\mathrm{MPa} \cdot \mathrm{K}^{-1}\end{array}$ & $\begin{array}{c}\mathrm{A}_{6} \\
\mathrm{MPa} \cdot \mathrm{K}^{-2}\end{array}$ & $\sigma$ \\
\hline 22.13 & 15563.50 & 49.07 & -67.19 & 9119.34 & 7247.48 & 0.27 \\
\hline
\end{tabular}




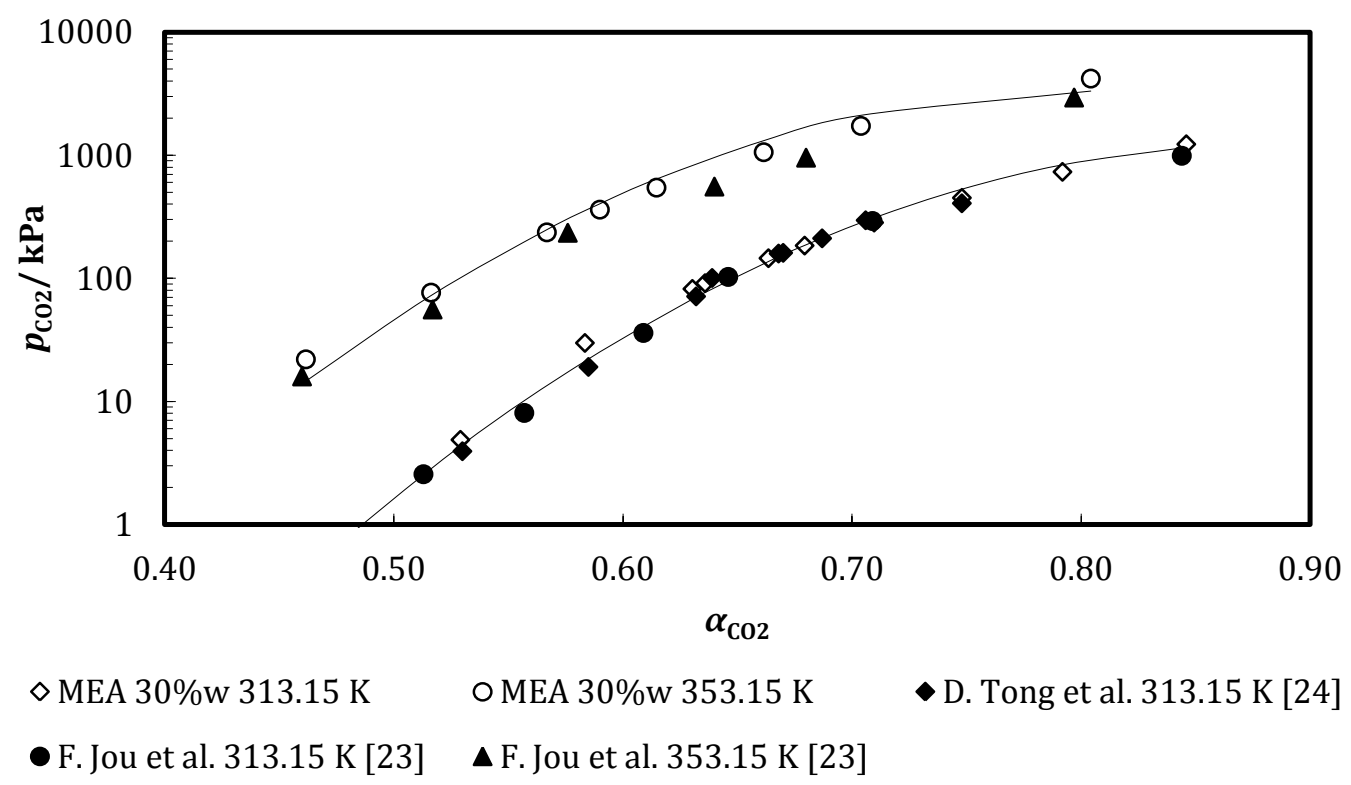

Figure 5.6. Comparison of solubilities of $\mathrm{CO}_{2}$ in aqueous $\mathrm{MEA} 30 \% \mathrm{w}$ at two temperatures. The continuous lines show the values calculated with the coefficients of $X u$ and Rochelle equation and the symbols show the experimental values of this work and literature [23, 24].

Figure 5.7 shows the relative deviations obtained between the literature data and those calculated using equation 5.7 with the parameters of Table 5.5. The average absolute deviations obtained $(A A D \%)$ were $17.2 \%$ and $11.2 \%$ for the mixture measured at $313.15 \mathrm{~K}$ by Jou et al. [23] and by Tong et al. [24], respectively. The average absolute deviation $(A A D \%)$ was $25.9 \%$ for the mixture at $353.15 \mathrm{~K}$ measured by [23]. It can be observed that the differences between authors have the same order of magnitude than the ones obtained in this work.

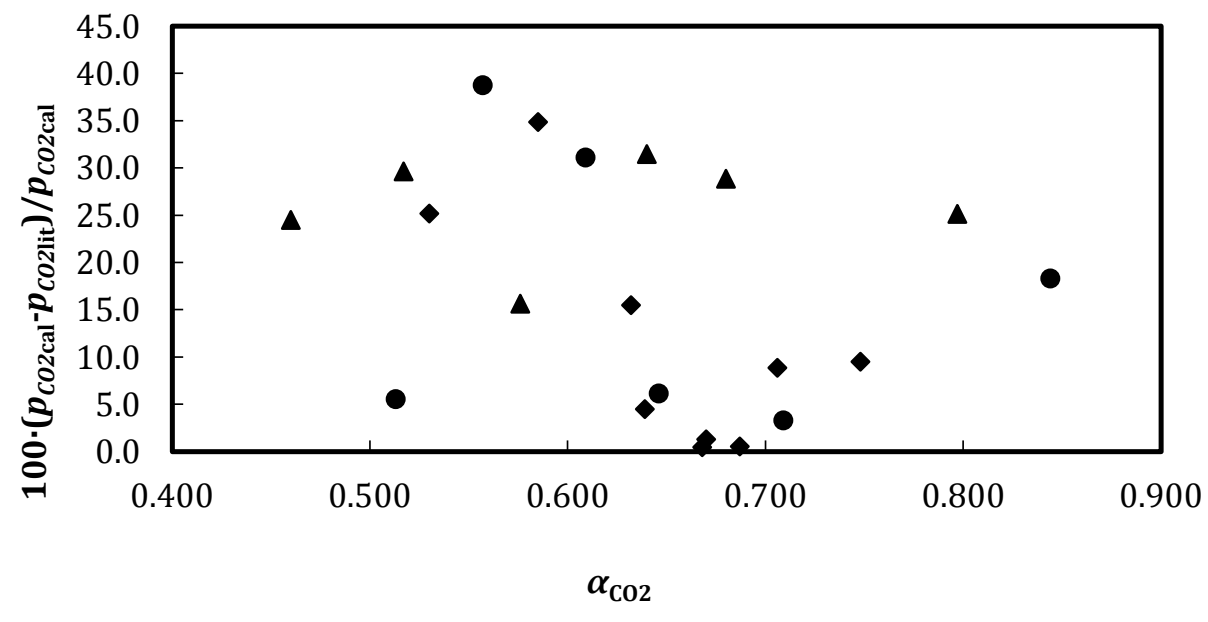

$\bullet$ D. Tong et al. $313.15 \mathrm{~K} \quad \bullet$ F. Jou et al. $313.15 \mathrm{~K} \quad \boldsymbol{\Delta}$ F. Jou et al. $353.15 \mathrm{~K}$

Figure 5.7. Percentage relative deviation of the literature experimental solubility of $\mathrm{CO}_{2}$ in aqueous MEA $30 \% \mathrm{w}$ of Jou et al. at $313.15 \mathrm{~K}$ and $353.15 \mathrm{~K}$ [23], and Tong et al. $313.15 \mathrm{~K}$ [24] in comparison with the values calculated with Eq. 5.7 and parameters of Table 5.5. 


\subsubsection{Discussion of solubility results.}

In this work, the solubilities of three ternary mixtures of aqueous amine solutions with $\mathrm{CO}_{2}$ (MEA 10\%w, MEA 30\%w, DMAE 30\%w and TEA 30\%w) were determined at $313.15 \mathrm{~K}$ and $353.15 \mathrm{~K}$. The pressure range varies from vapor pressures of the amine-water mixture up to $6 \mathrm{MPa}$, and load index up to 1.7 moles of $\mathrm{CO}_{2}$ per mole of amine.

Adjustments with $\mathrm{Xu}$ and Rochelle equation were good, adjusting worse at elevated pressures, above $5 \mathrm{MPa}$, and pressures close to the vapor pressure of the mixture amine + $\mathrm{H}_{2} \mathrm{O}$. The standard deviation $(\sigma)$ is lower to 0.27 for all the mixtures studied. The best adjustment of the Xu and Rochelle equation obtained a standard deviation $(\sigma)$ of 0.07 for the system TEA $30 \% \mathrm{w}+\mathrm{CO}_{2}$.

The experimental values show a tendency of increasing solubility with pressure and decreasing with increasing temperature. Being the most marked increase in solubility at low pressures for all mixtures at any temperature.

The behavior of the three amines at $30 \% \mathrm{w}$ and $313.15 \mathrm{~K}$ is compared in the figure 5.8.

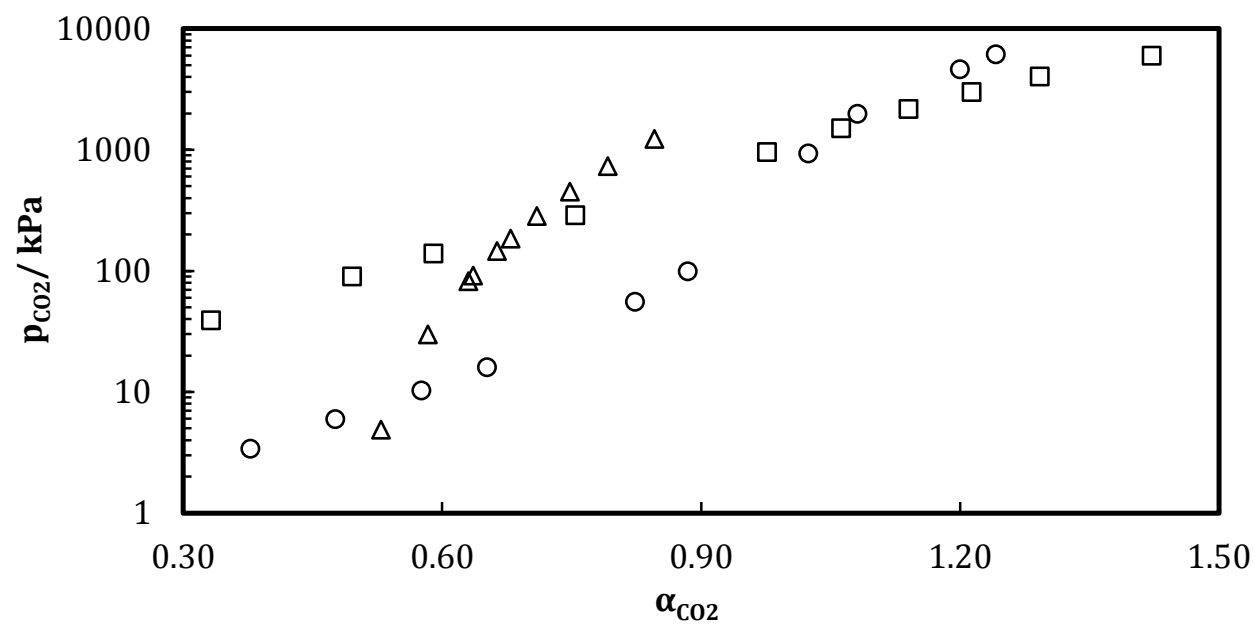

Figure 5.8. Experimental solubility values at $313.15 \mathrm{~K}$ for the aqueous mixtures: $M E A 30 \% \mathrm{w}(\triangle), D M A E$ $30 \% w(\bigcirc)$ and TEA 30\%w (口).

In the Figure 5.8 can be observed that below $100 \mathrm{kPa}$, the solubiliy of $\mathrm{CO}_{2}$ in TEA is lower than in MEA and DMAE. However, above $150 \mathrm{kPa}$, the MEA is the amine with lowest solubility and TEA is the most efficient above $2000 \mathrm{kPa}$, being DMAE the best between 150 $\mathrm{kPa}$ and $2000 \mathrm{kPa}$. 

Chapter 6

\section{DETERMINATION OF DENSITIES AND VISCOSITIES OF $\mathrm{CO}_{2}+$ HYDROCARBON MIXTURES}





\subsection{Viscosity measuring method}

The experimental technique used to determine the viscosity of $\mathrm{CO}_{2}+$ hydrocarbon mixtures is a vibrating wire viscometer. The equipment requires a set of elements which are shown in Figure 6.1.
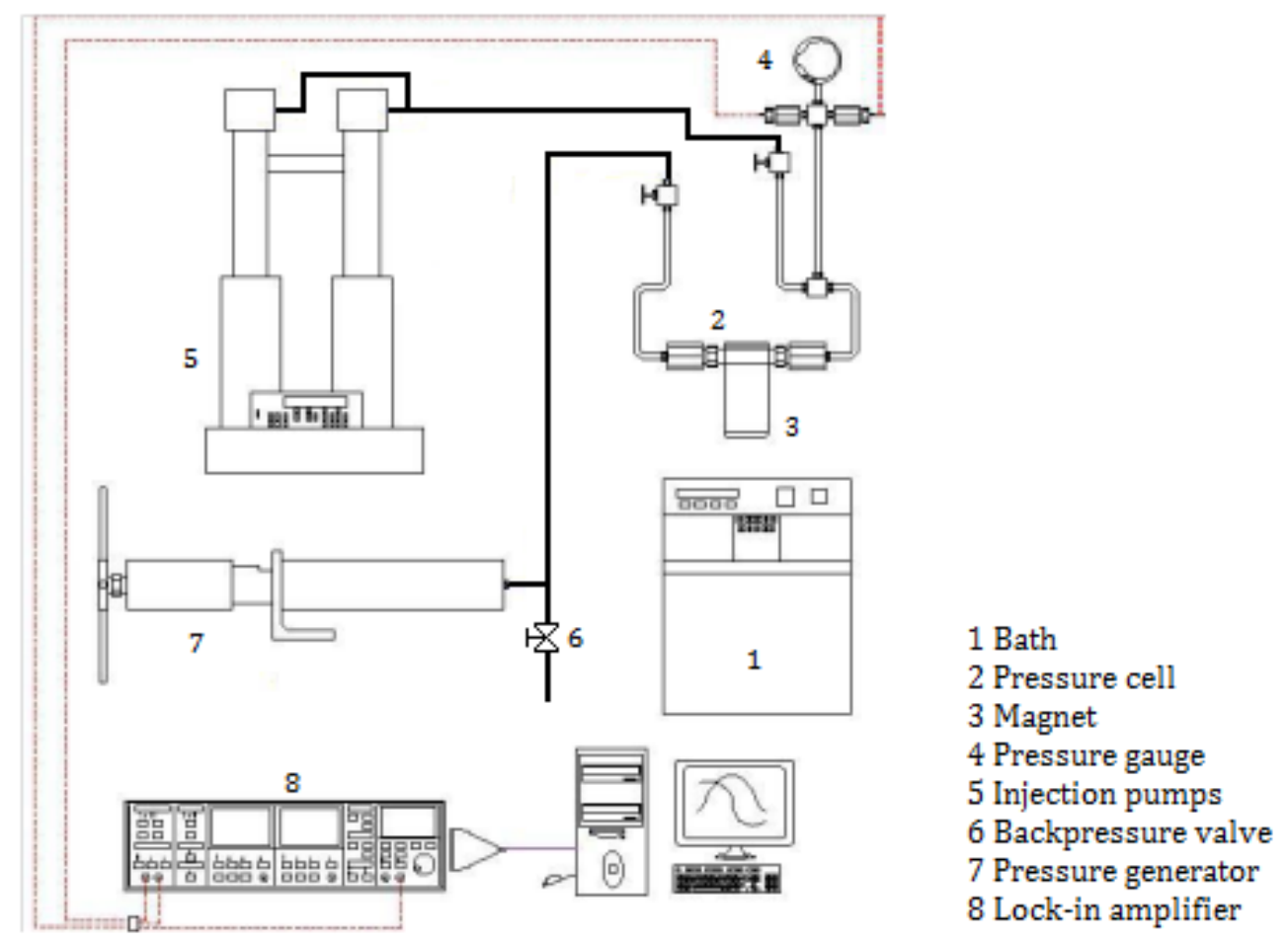

Figure 6.1. Schematic diagram of the vibrating wire viscometer technique for fluid mixtures.

The techniqueuses as a sensor a tungsten wire of length $L=50 \mathrm{~mm}$ and nominal radius $R=$ $75 \mu \mathrm{m}$. The sensor is housed inside a pressure cell placed in such a way that it is centered and oriented in a constant magnetic field. The magnetic field is generated using of a twopole C-shaped magnet, Eclipse Magnetics brand, Figure 6.2. a. A constant alternating current passes through the wire, generating a transversal oscillation, and the induced voltage produced in the wire is measured using a lock-in amplifier (Stanford Research Systems model SR380 DPS) Figure 6.2. c). The steady state frequency response of the oscillating wire is measured near the resonance of the fundamental transversal mode.

The power supply provides a maximum output of $5 \mathrm{~V}_{\mathrm{AC}}$. In this study a measurement sensitivity of $500 \mu \mathrm{V}$, an amplitude of $0.8 \mathrm{~Hz}$ with a time constant of $300 \mathrm{~ms}$ was generally used. These parameters were chosen according to the mixture to be measured.

The interface with the computer is done by means of an Agilent model 82357A USB-GPIB adapter, Figure 6.2. d), allowing the acquisition of the measured data and the assignment of initial reference values. And the graphic interface for control and data processing is done with Agilent VEE-Pro V7.0 software, Figure 6.2. b). 
a)

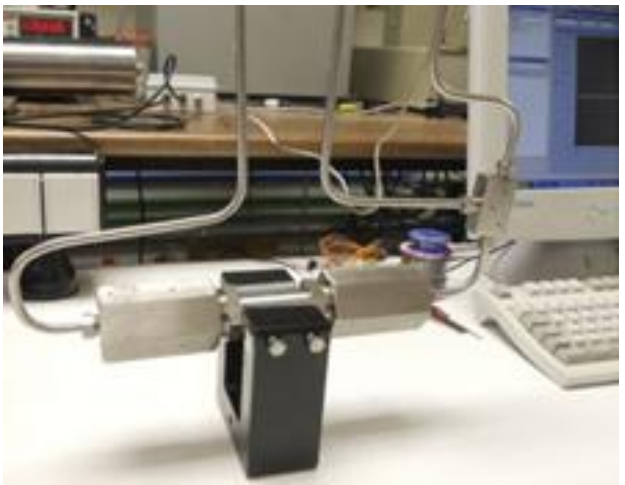

b)

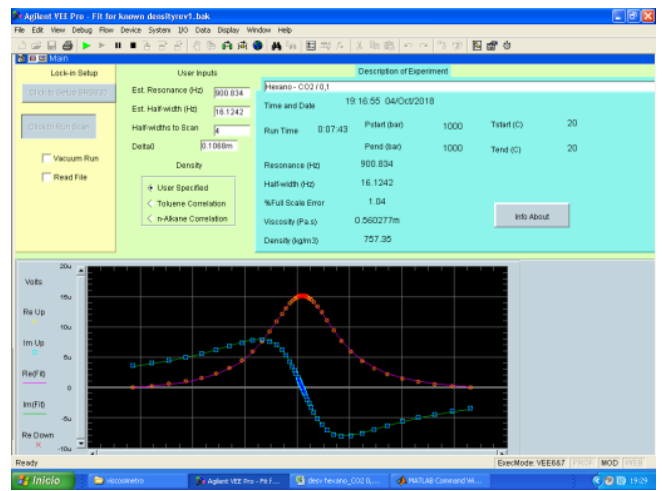

c)
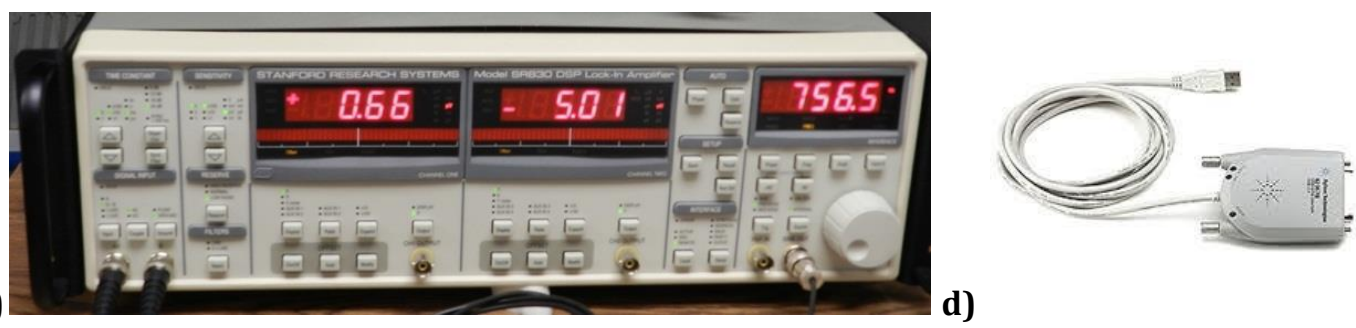

d)

Figure 6.2. Pictures of the vibrating wire viscometer: a) centered and oriented of the pressure cell, $b$ ) graphic interface for control and data processing, c) lock-in amplifier, d) USB-GPIB interface.

Signal detection system. The wire supplied with alternating current and under the action of a magnetic field generates an oscillation. In this way, a Lorentz force acts in alternating direction over the wire causing its forced oscillation. This movement generates an induced voltage according to Faraday's Law that can be detected through the lock-in amplifier Figure 6.2. c), capable of measuring small alternating current signals. This amplifier produces a continuous signal by amplifying the input and mixing it with a reference signal.

The temperature control system. The pressure cell together with the magnet are immersed in a Hart Scientific bath model 5004 iso-therm. For the improvement of stability and the possibility of working at temperatures below $40{ }^{\circ} \mathrm{C}$, this bath is coupled with a cooling system through a second external bath JULABO model F25 HE. The temperature is measured with an ASL F-100 and two Pt100 probes inside the bath.

The pressure control system. The vibrating wire sensor is housed in the inside of a pressure cell which connected to a pressure generator system. A Druck DPI 104 is used to measure pressure in the range from 1.0 bar to 1400.0 bar, with a resolution of 0.1 bar. It was calibrated with a relative expanded uncertainty $(k=2)$ of $0.02 \%$. The pressure generator used in this work is a 68-5.75-10 $\mathrm{HiP}$ piston, with a maximum volume of $35 \mathrm{~cm}^{3}$.

The sample filling system. The filling is carried out with two positive displacement ISCO syringe pumps. The pump containing $\mathrm{CO}_{2}$ is at constant temperature and pressure of $0{ }^{\circ} \mathrm{C}$ and 55 bar, and the pump with the second component (pure hydrocarbon previously degassed) is at a temperature and pressure of $10{ }^{\circ} \mathrm{C}$ and 55 bar. To ensure filling at a constant high pressure, a Mity-Mite backpressure valve model S-91XW is installed at the outlet of the circuit. A back pressure of 50 bar is Applied. 
The evacuation and cleaning system of the equipment. The evacuation of the sample fluid is carried out through the valve connected to the backpressure valve. For cleaning, it is connected the pressure circuit to a vacuum line.

\subsection{Experimental procedure for measuring viscosity.}

The hydrocarbons were previously degassed in a Branson ultrasonic bath, model DTH3210 , with a frequency of $47 \mathrm{kHz}$ and a power of $335 \mathrm{~W}$ during at least 5 hours.

First, $\mathrm{CO}_{2}$ is loaded into the densimeter by applying constant flow in the pump. Once the circuit is filled with $\mathrm{CO}_{2}$, the second syringe pump is put into operation sending the hydrocarbon. A large enough volume is expected to pass through the equipment. In this way, the equipment is loaded dynamically ensuring the homogenization of the compounds.

Different programs, developed in Agilent VEE-Pro V7.0, are used to measure dynamic viscosity. The "Quick Scan modified" program is used to quickly explore the approximate resonance frequency $\left(f_{\mathrm{r}}\right)$. The program "Fit for known density $(T, p)$ " serves to calculate the dynamic viscosity and the program "Find Radius" finds the "true" radius of the wire for the calibration of the technique. Before measurements, the resonance frequency $\left(f_{r}\right)$, the estimated average bandwidth $\left(f_{\mathrm{b}}\right)$, the average bandwidth number to scan, the pressure, the temperature at which you want to measure the viscosity and density of the fluid being measured are entered into the program. When the standard deviation $\sigma$, of five viscosity measurements, is less than $1 \%$, the mean value of them is taken.

\subsection{Calibration of the viscometer and validation of the technique.}

The vibrating wire viscometer is an absolute device, that, in theory, does not require calibration with reference fluids. However, in practice, the wire radius $R$ and the internal damping (or natural logarithmic decrease in vacuum) $\Delta_{0}$ cannot be established with sufficient precision by independent methods, hence these two parameters are fixed by calibration. This initial calibration and the obtaining of the true wire radius was previously done in Johnny Zambrano's thesis [25].

In this work, a new calibration of the damping constant was carried out $\left(\Delta_{o}\right)$, making high vacuum in the equipment. Through the Vacuum Run option of the "Fit for Know Density" program, the new $\Delta_{0}$ was obtained and used in our study. In our case, the value increased from $44.8 \cdot 10^{-6}$ to $106.8 \cdot 10^{-6}$.

To check the technique, the viscosity of toluene was measured at a pressure of 10 bar and a temperature of $25^{\circ} \mathrm{C}$, comparing the results with REFPROP and it was verified that the deviation of the experimental data did not exceed $1.5 \%$. The maximum deviations from REFPROP were $1.17 \%$ and the mean deviation obtained (AAD\%) was $0.75 \%$. 


\subsection{Measurement Uncertainty.}

The uncertainty calculation is based on GUM 2008 [14]. The uncertainty in the $\mathrm{CO}_{2}$ composition of the mixture with the hydrocarbon, is associated with the densities of the compounds of each pump and the flows of these pumps, being uncertainty of the flow rate of the pumps $0.5 \%$.

The uncertainties of the densities of the compounds associated with the temperature and pressure conditions of each pump are presented in Table 6.1, and Table 6.2. shows the uncertainties associated at the composition in the mixture.

Table 6.1. Calculation of the uncertainty associated with the density of each compound in the pumps at the injection conditions of the mixture.

\begin{tabular}{|c|c|c|c|c|c|}
\hline & & Unit & Estimated & Divisor & $\mathrm{u}(x)$ \\
\hline & & & & & $\mathrm{kg} / \mathrm{m}^{3}$ \\
\hline \multirow{2}{*}{$\mathrm{u}(T) \mathrm{CO}_{2}$} & Calibration & \multirow{2}{*}{${ }^{\circ} \mathrm{C}$} & 0.02 & 2 & \multirow{2}{*}{0.064} \\
\hline & Resolution & & 0.01 & $2 \sqrt{3}$ & \\
\hline \multirow{2}{*}{$\mathrm{u}(p) \mathrm{CO}_{2}$} & Calibration & \multirow{2}{*}{$\mathrm{MPa}$} & 0.02 & 2 & \multirow{2}{*}{0.00005} \\
\hline & Resolution & & \begin{tabular}{|l|}
0.01 \\
\end{tabular} & $2 \sqrt{3}$ & \\
\hline $\mathrm{u}(\rho) \mathrm{CO}_{2}$ & & $\mathrm{~kg} / \mathrm{m}^{3}$ & & $\mathrm{k}=1$ & 0.06 \\
\hline $\mathrm{U}(\rho) \mathrm{CO}_{2}$ & & $\mathrm{~kg} / \mathrm{m}^{3}$ & & $\mathrm{k}=2$ & 0.13 \\
\hline
\end{tabular}

\begin{tabular}{|l|l|l|l|l|l|}
\hline & & Unit & Estimated & Divisor & \multicolumn{1}{c|}{$\mathrm{u}(x)$} \\
\hline & & & & & $\mathrm{kg} / \mathrm{m}^{3}$ \\
\hline \multirow{2}{*}{$\mathrm{u}(T)$ Hydrocarbon } & Calibration & \multirow{2}{*}{${ }^{\circ} \mathrm{C}$} & 0.02 & 2 & \multirow{2}{*}{0.009} \\
\cline { 2 - 2 } & Resolution & & 0.01 & $2 \sqrt{3}$ & \\
\hline \multirow{2}{*}{$\mathrm{u}(p)$ Hydrocarbon } & Calibration & \multirow{2}{*}{$\mathrm{MPa}$} & 0.02 & 2 & \multirow{2}{*}{$\mathrm{k}$} \\
\cline { 2 - 2 } & Resolution & & 0.01 & $2 \sqrt{3}$ & \\
\hline $\mathrm{y}(\rho)$ Hydrocarbon & $\mathrm{kg} / \mathrm{m}^{3}$ & $\mathrm{k}=1$ & 0.009 \\
\hline $\mathrm{U}(\rho)$ Hydrocarbon & $\mathrm{kg} / \mathrm{m}^{3}$ & $\mathrm{k}=2$ & 0.02 \\
\hline
\end{tabular}

Table 6.2. Calculation of the uncertainty associated with the composition of the mixture.

\begin{tabular}{|c|c|c|c|c|c|}
\hline & & Unit & Estimated & Divisor & $\mathrm{u}(x)$ \\
\hline & & & & & $\mathrm{mol} / \mathrm{mol}$ \\
\hline $\mathrm{u}(\dot{m})$ Hydrocarbon & - & $\mathrm{g} / \mathrm{min}$ & 0.005 & 1 & 0.0004 \\
\hline $\mathrm{u}(\dot{m}) \mathrm{CO}_{2}$ & - & $\mathrm{g} / \mathrm{min}$ & 0.014 & 1 & 0.0004 \\
\hline $\mathrm{u}(x) \mathrm{CO}_{2}$ & $\mathrm{~mol} / \mathrm{mol}$ & $\mathrm{k}=1$ & 0.0006 \\
\hline $\mathrm{U}(x) \mathrm{CO}_{2}$ & $\mathrm{~mol} / \mathrm{mol}$ & $\mathrm{k}=2$ & 0.0013 \\
\hline
\end{tabular}

Table 6.3. shows the uncertainty budget of the viscosity measurement at different temperature ranges. The expanded uncertainty is calculated with a coverage factor $k=2$ ( $95 \%$ confidence). It shows that the greatest contribution to uncertainty comes from the density of the calibration fluid and the frequency of the bandwidth. 
Table 6.3. Uncertainty budget of the experimental viscosity in a temperature range of (293.15353.15) Kand pressures 0.1-100 MPa.

\begin{tabular}{|c|c|c|c|c|c|}
\hline & & Unit & Estimated & Divisor & $\mathrm{u}(x)$ \\
\hline & & & & & $\mathrm{mPa} \cdot \mathrm{s}$ \\
\hline \multirow{3}{*}{$\mathrm{u}\left(f_{r}\right)$} & Calibration & \multirow{3}{*}{$\mathrm{Hz}$} & 0.010 & 2 & \multirow{3}{*}{0.000006} \\
\hline & Repeatability & & 0.0010 & $2 \sqrt{3}$ & \\
\hline & Resolution & & 0.04 & 1 & \\
\hline \multirow{3}{*}{$\mathrm{u}(T)$} & Calibration & \multirow{3}{*}{${ }^{\circ} \mathrm{C}$} & 0.020 & 2 & \multirow{3}{*}{0.000048} \\
\hline & Resolution & & 0.010 & $2 \sqrt{3}$ & \\
\hline & Repeatability & & 0.03 & 1 & \\
\hline \multirow{3}{*}{$\mathrm{u}(p)$} & Calibration & \multirow{3}{*}{$\mathrm{MPa}$} & 0.02 & 2 & \multirow{3}{*}{0.000025} \\
\hline & Resolution & & 0.01 & $2 \sqrt{3}$ & \\
\hline & Repeatability & & 0.00 & 1 & \\
\hline \multirow{2}{*}{$\mathrm{u}(\rho)$} & Solid & $\mathrm{kg} / \mathrm{m}^{3}$ & 19.0 & 2 & 0.0003 \\
\hline & Fluid & $\mathrm{kg} / \mathrm{m}^{3}$ & 4.7 & 2 & 0.0011 \\
\hline $\begin{array}{l}\text { Calibration } \\
\text { of radius }\end{array}$ & & $\mathrm{mm}$ & 0.08 & 2 & 0.0004 \\
\hline \multirow{3}{*}{$\mathrm{u}\left(f_{b}\right)$} & Calibration & \multirow{3}{*}{$\mathrm{Hz}$} & 0.01 & 2 & \multirow{3}{*}{0.001} \\
\hline & Repeatability & & 0.018 & 1 & \\
\hline & Resolution & & 0.001 & $2 \sqrt{3}$ & \\
\hline \multicolumn{2}{|l|}{$\mathrm{u}(\eta)$} & \multicolumn{2}{|l|}{$\mathrm{mPa} \cdot \mathrm{s}$} & $\mathrm{k}=1$ & 0.0016 \\
\hline \multicolumn{2}{|l|}{$\mathrm{U}(\eta)$} & \multicolumn{2}{|c|}{$\mathrm{mPa} \cdot \mathrm{s}$} & $\mathrm{k}=2$ & 0.0032 \\
\hline \multicolumn{2}{|l|}{$\mathrm{U}_{\mathrm{r}}(\eta)$} & \multicolumn{2}{|c|}{$100 \cdot(\mathrm{mPa} \cdot \mathrm{s} / \mathrm{mPa} \cdot \mathrm{s})$} & $\mathrm{k}=2$ & $1.5 \%$ \\
\hline
\end{tabular}

\subsection{Measuring method used to determine the density of $\mathrm{CO}_{2}+$ hydrocarbon.}

The measurement of densities of $\mathrm{CO}_{2}+$ hydrocarbon mixtures was done with the same vibrating tube densimeter described in section 4.1 of this summary.

\subsection{Measurement Uncertainty.}

The uncertainty calculation was based on GUM 2008 [14]. The uncertainty of the $\mathrm{CO}_{2}$ composition of the mixture with the hydrocarbon, is associated with the densities of the compounds of each pump and the flows of these pumps being the uncertainty of the pump flow rate of $0.5 \%$.

The uncertainties of the densities of the compounds associated with the temperature and pressure of each pump was presented in Table 6.1. Table 6.4. shows the uncertainties associated with the composition in the mixture.

Table 6.4. Calculation of the uncertainty associated with the composition of the mixture.

\begin{tabular}{|c|c|c|l|l|l|}
\hline & & Unit & Estimated & Divisor & $\mathrm{u}(x)$ \\
\hline & & & & & $\mathrm{mol} / \mathrm{mol}$ \\
\hline $\mathrm{u}(\dot{m})$ Hydrocarbon & - & $\mathrm{g} / \mathrm{min}$ & 0.005 & 1 & 0.0012 \\
\hline $\mathrm{u}(\dot{m}) \mathrm{CO}_{2}$ & - & $\mathrm{g} / \mathrm{min}$ & 0.014 & 1 & 0.0012 \\
\hline $\mathrm{u}(x) \mathrm{CO}_{2}$ & $\mathrm{~mol} / \mathrm{mol}$ & $\mathrm{k}=1$ & 0.0017 \\
\hline $\mathrm{U}(x) \mathrm{CO}_{2}$ & $\mathrm{~mol} / \mathrm{mol}$ & $\mathrm{k}=2$ & 0.0034 \\
\hline
\end{tabular}


Tables 6.5. and 6.6. show the uncertainty budgets of density measurement where the expanded uncertainty is calculated with a coverage factor $k=2$ (95\% confidence).

Table 6.5. Calculation of the uncertainty associated with the calibration parameters in a temperature range of (273.15-373.15) Kand pressures 0.1-140 MPa.

\begin{tabular}{|c|c|c|c|c|c|}
\hline & & Unit & Estimated & Divisor & $\mathrm{u}(x)$ \\
\hline & & & & & $\mathrm{kg} / \mathrm{m}^{3}$ \\
\hline $\mathrm{u}\left(\rho_{w}\right)$ & $\begin{array}{l}\text { Material } \\
\text { reference }\end{array}$ & $\mathrm{kg} / \mathrm{m}^{3}$ & 0.01 & $\sqrt{3}$ & 0.0058 \\
\hline \multirow{3}{*}{$\mathrm{u}(T)$} & Calibration & \multirow{3}{*}{${ }^{\circ} \mathrm{C}$} & 0.020 & 2 & \multirow{3}{*}{0.0046} \\
\hline & Resolution & & 0.010 & $2 \sqrt{3}$ & \\
\hline & Repeatability & & 0.0008 & 1 & \\
\hline \multirow{3}{*}{$\mathrm{u}(p)$} & Calibration & \multirow{3}{*}{$\mathrm{MPa}$} & 0.02 & 2 & \multirow{3}{*}{0.0041} \\
\hline & Resolution & & 0.01 & $2 \sqrt{3}$ & \\
\hline & Repeatability & & 0.002 & 1 & \\
\hline \multirow{2}{*}{$\mathrm{u}(\tau)$} & Repeatability & \multirow{2}{*}{$\mu \mathrm{s}$} & 0.0006 & 1 & \multirow{2}{*}{$8.5 \mathrm{E}-03$} \\
\hline & Resolution & & $1.00 \mathrm{E}-03$ & $2 \sqrt{3}$ & \\
\hline $\mathrm{U}(\mathrm{A}(T))$ & & $\mathrm{kg} / \mathrm{m}^{3} \mu \mathrm{s}^{2}$ & & $\mathrm{k}=2$ & $7.6 \mathrm{E}-08$ \\
\hline $\mathrm{U}(\mathrm{B}(T, p))$ & & $\mathrm{kg} / \mathrm{m}^{3}$ & & $\mathrm{k}=2$ & 0.43 \\
\hline
\end{tabular}

Table 6.6. Calculation of the uncertainty associated to the experimental density in a temperature range of (273.15-373.15) Kand pressures 5-100 MPa.

\begin{tabular}{|c|c|c|c|c|c|}
\hline & & Unit & Estimated & Divisor & $\mathrm{u}(x)$ \\
\hline & & & & & $\mathrm{kg} / \mathrm{m}^{3}$ \\
\hline \multirow{2}{*}{$\mathrm{u}(\tau)$} & Repeatability & \multirow{2}{*}{$\mu \mathrm{s}$} & 0.0006 & 1 & \multirow{2}{*}{$8.5 \mathrm{E}-03$} \\
\hline & Resolution & & $1.00 \mathrm{E}-03$ & $2 \sqrt{3}$ & \\
\hline \multirow{3}{*}{$\mathrm{u}(T)$} & Calibration & \multirow{3}{*}{${ }^{\circ} \mathrm{C}$} & 0.020 & 2 & \multirow{3}{*}{0.0046} \\
\hline & Resolution & & 0.010 & $2 \sqrt{3}$ & \\
\hline & Repeatability & & 0.0008 & 1 & \\
\hline \multirow{3}{*}{$\mathrm{u}(p)$} & Calibration & \multirow{3}{*}{$\mathrm{MPa}$} & 0.02 & 2 & \multirow{3}{*}{0.0041} \\
\hline & Resolution & & 0.01 & $2 \sqrt{3}$ & \\
\hline & Repeatability & & 0.002 & 1 & \\
\hline $\mathrm{u}(\mathrm{A}(T))$ & $\mathrm{kg} / \mathrm{m}^{3} \mu \mathrm{S}^{2}$ & & $7.6 \mathrm{E}-08$ & 2 & 0.26 \\
\hline $\mathrm{u}(\mathrm{B}(T, p))$ & $\mathrm{kg} / \mathrm{m}^{3}$ & & 0.43 & 2 & 0.21 \\
\hline $\mathrm{u}(\rho)$ & \multicolumn{3}{|c|}{$\mathrm{kg} / \mathrm{m}^{3}$} & $\mathrm{k}=1$ & 0.34 \\
\hline $\mathrm{U}(\rho)$ & \multicolumn{3}{|c|}{$\mathrm{kg} / \mathrm{m}^{3}$} & $\mathrm{k}=2$ & 0.7 \\
\hline $\operatorname{Ur}(\rho)$ & \multicolumn{3}{|c|}{$100 \cdot\left(\mathrm{kg} \cdot \mathrm{m}^{-3} / \mathrm{kg} \cdot \mathrm{m}^{-3}\right)$} & $\mathrm{k}=2$ & $0.1 \%$ \\
\hline
\end{tabular}

Table 6.6. shows that the greatest contribution to uncertainty comes from the calibration parameters $\mathrm{A}(T)$ and $\mathrm{B}(T, p)$. 
Chapter 7

\section{EXPERIMENTAL RESULTS OF DENSITY AND VISCOSITY MEASUREMENTS OF $\mathrm{CO}_{2}+$ HYDROCARBON MIXTURES}





\subsection{Experimental results of density and viscosity measurements of $\mathrm{CO}_{2}+$ hydrocarbon mixtures.}

In this chapter the experimental results of the measurement of density and viscosity of binary mixtures of hydrocarbon $+\mathrm{CO}_{2}$ are presented. They were obtained by means of the vibrating tube densimeter and the vibrating wire viscometer respectively, described in the previous chapter, in the temperature range from $273.15 \mathrm{~K}$ to $373.15 \mathrm{~K}$ and pressures from $5 \mathrm{MPa}$ to $100 \mathrm{MPa}$.

Densities were measured for the following mixtures:

Mixture: Pentane $+\mathrm{CO}_{2}$ at $x_{\mathrm{CO} 2}=0.1,0.2,0.3,0.4$ and 0.6.

Mixture: Hexane $+\mathrm{CO}_{2}$ at $x_{\mathrm{CO} 2}=0.1,0.2,0.3,0.4$ and 0.6.

And viscosities were measured for:

Mixture: Pentane $+\mathrm{CO}_{2}$ at $x_{\mathrm{CO} 2}=0.1$ and 0.3 .

Mixture: Hexane $+\mathrm{CO}_{2}$ at $x_{\mathrm{CO} 2}=0.1$ and 0.3 .

\subsubsection{Products used.}

Table 7.1. (Spanish version, page 169) shows the products used, their origin and their purity given by the certificates of analysis. No additional purification method was used. The hydrocarbons were previously degassed by means of a Branson ultrasonic bath, model DTH-3210, with a frequency of $47 \mathrm{kHz}$ and a power of $335 \mathrm{~W}$.

\subsubsection{Adjustment of density data}

Density data at different temperatures and pressures were adjusted through modified Tammann-Tait equation for each concentration. This type of adjustment was also used in chapter 5 . The equation used was the proposed by Comuñas et al. [20]:

$$
\rho(T, p)=\frac{\rho\left(T, p_{r e f}(T)\right)}{1-\mathrm{C} \ln \left[\frac{\mathrm{B}(T)+p}{\mathrm{~B}(T)+p_{r e f}(T)}\right]}
$$

To evaluate the adjustment the standard deviation $\sigma$ and the maximum deviation $M D \%$ between experimental data and the model used were calculated, using equations 5.4 and 5.5 respectively. 


\subsubsection{Adjustment of viscosity data}

One of the most commonly used equations for correlating dynamic viscosity data at atmospheric pressure is the VFT model (Vogel [26], Fulcher [27] and Tammann [28]) equation 7.2. The equation is obtained making some assumptions [29, 30] from AdamGibbs' theory of configurational entropy [31].

$$
\eta(T)=A \cdot \exp \left(\frac{B}{T-C}\right)=A \cdot \exp \left(\frac{D \cdot T_{0}}{T-T_{0}}\right)
$$

Where $\mathrm{D}$ is the fragility index (or Angell parameter) and $\mathrm{T}_{0}$ the temperature of Vogel. This equation has been used for wide temperature ranges and isobaric conditions. In order to establish a relationship of the viscosity with the pressure, some authors have extended this equation. One of the proposed modifications is published by Comuñas et al. [17] and Paredes et al. [32]:

$$
\eta(T, p)=A \cdot \exp \left(\frac{B}{T-C}\right) \cdot\left(\frac{p+E(T)}{p_{r e f}+E(T)}\right)^{F}
$$

Where:

$$
E(T)=E_{0}+E_{1} T+E_{2} T^{2}
$$

where $\mathrm{A}, \mathrm{B}$ and $\mathrm{C}$ are determined by a preliminary adjustment of the viscosity as a function of the temperature at the reference pressure in this work at $5 \mathrm{MPa}$ and the coefficients $\mathrm{F}$, $\mathrm{E}_{0}, \mathrm{E}_{1}$ and $\mathrm{E}_{2}$ are adjusted to the viscosity values at pressures different from the reference. In the equation 7.3, the parameters $\mathrm{C}=\mathrm{T}_{0}, \mathrm{~B}=\mathrm{D}-\mathrm{T}_{0}$ are independent of the pressure. This model was also used in previous works such as Sobrino et al. [18].

The coefficients $A, B, C, F, E_{0}, E_{1}$ and $E_{2}$ of equation 7.3 were determined by adjusting the experimental data using an algorithm for estimation of nonlinear parameter minimal squares [33]. To evaluate the fit, the standard deviation $\sigma$ in equation 5.4 was calculated.

\subsubsection{Results of density measurements.}

As an example of the experimental densities obtained for the mixtures studied, the system Pentane $+\mathrm{CO}_{2}$ is presented at $x_{\mathrm{CO} 2}=0.1$, the other results are in section 7.5 (Spanish version, page 169). The experimental data are shown both as tables and graphs, as well as the results of the adjustment to the Tammann-Tait model. This adjustment was made for each composition studied, depending on temperature and pressure. In addition, is shown the standard deviation of the adjustment $\sigma$ (equation 5.4), the maximum deviation (MD\%) (e.g. 5.5) are shown in Table 7.2. 


\subsubsection{Mixture: Pentane $+\mathrm{CO}_{2}$.}

Table 7.1. Experimental density data, $\rho\left(\mathrm{kg} \cdot \mathrm{m}^{-3}\right)$, for the mixture Pentane $+\mathrm{CO}_{2}$ at $x_{\mathrm{CO}}=0.1004$.

\begin{tabular}{ccccccc}
\hline \multicolumn{7}{c}{$\rho\left(\mathrm{kg} / \mathrm{m}^{3}\right)$} \\
\hline \multicolumn{7}{c}{$T / \mathrm{K}$} \\
\hline$p / \mathrm{MPa}$ & 273.15 & 293.15 & 313.15 & 333.15 & 353.15 & 373.15 \\
\hline 5 & 661.1 & 642.9 & 622.7 & 600.6 & 576.8 & 551.1 \\
10 & 666.3 & 649.1 & 630.5 & 609.9 & 588.2 & 565.7 \\
15 & 671.3 & 655.0 & 637.2 & 618.0 & 597.9 & 577.4 \\
20 & 675.8 & 660.3 & 643.4 & 625.2 & 606.4 & 587.4 \\
30 & 684.2 & 669.8 & 654.3 & 637.7 & 620.7 & 603.8 \\
40 & 691.8 & 678.4 & 663.9 & 648.4 & 632.7 & 617.4 \\
50 & 698.7 & 686.0 & 672.3 & 657.8 & 643.1 & 628.8 \\
60 & 705.1 & 693.0 & 680.0 & 666.3 & 652.4 & 638.8 \\
70 & 711.0 & 699.5 & 687.1 & 673.9 & 660.6 & 647.8 \\
80 & 716.6 & 705.6 & 693.6 & 681.1 & 668.2 & 656.1 \\
90 & 721.9 & 711.2 & 699.8 & 687.6 & 675.3 & 663.7 \\
100 & 726.8 & 716.5 & 705.4 & 693.7 & 681.8 & 670.6 \\
\hline
\end{tabular}

Table 7.2. Coefficients of the modified Tammann-Tait equation, standard deviation $\sigma$ and maximum deviation $\mathrm{MD} \%$ for the mixture Pentane $+\mathrm{CO}_{2}$ at $x_{\mathrm{CO} 2}=0.1004, p_{\text {ref }}=5 \mathrm{MPa}$.

Parameters of the modified Tammann-Tait equation

\begin{tabular}{ccccccccc}
\hline $\begin{array}{c}\mathrm{A}_{0} \\
\mathrm{~kg} \cdot \mathrm{m}^{-3}\end{array}$ & $\begin{array}{c}\mathrm{A}_{1} \\
\mathrm{~kg} \cdot \mathrm{m}^{-3} \cdot \mathrm{K}^{-1}\end{array}$ & $\begin{array}{c}\mathrm{A}_{2} \\
\mathrm{~kg} \cdot \mathrm{m}^{-3} \cdot \mathrm{K}^{-2}\end{array}$ & $\begin{array}{c}\mathrm{B}_{0} \\
\mathrm{MPa}\end{array}$ & $\begin{array}{c}\mathrm{B}_{1} \\
\mathrm{MPa} \cdot \mathrm{K}^{-1}\end{array}$ & $\begin{array}{c}\mathrm{B}_{2} \\
\mathrm{MPa} \cdot \mathrm{K}^{-2}\end{array}$ & $\mathrm{C}$ & $\begin{array}{c}\Sigma \\
\mathrm{kg} \cdot \mathrm{m}^{-3}\end{array}$ & $\begin{array}{c}M D \\
(\%)\end{array}$ \\
\hline 723.06 & 0.413 & $-2.34 \mathrm{E}-03$ & 302.84 & -1.32 & $1.42 \mathrm{E}-03$ & 0.090 & 0.15 & 0.05 \\
\hline
\end{tabular}




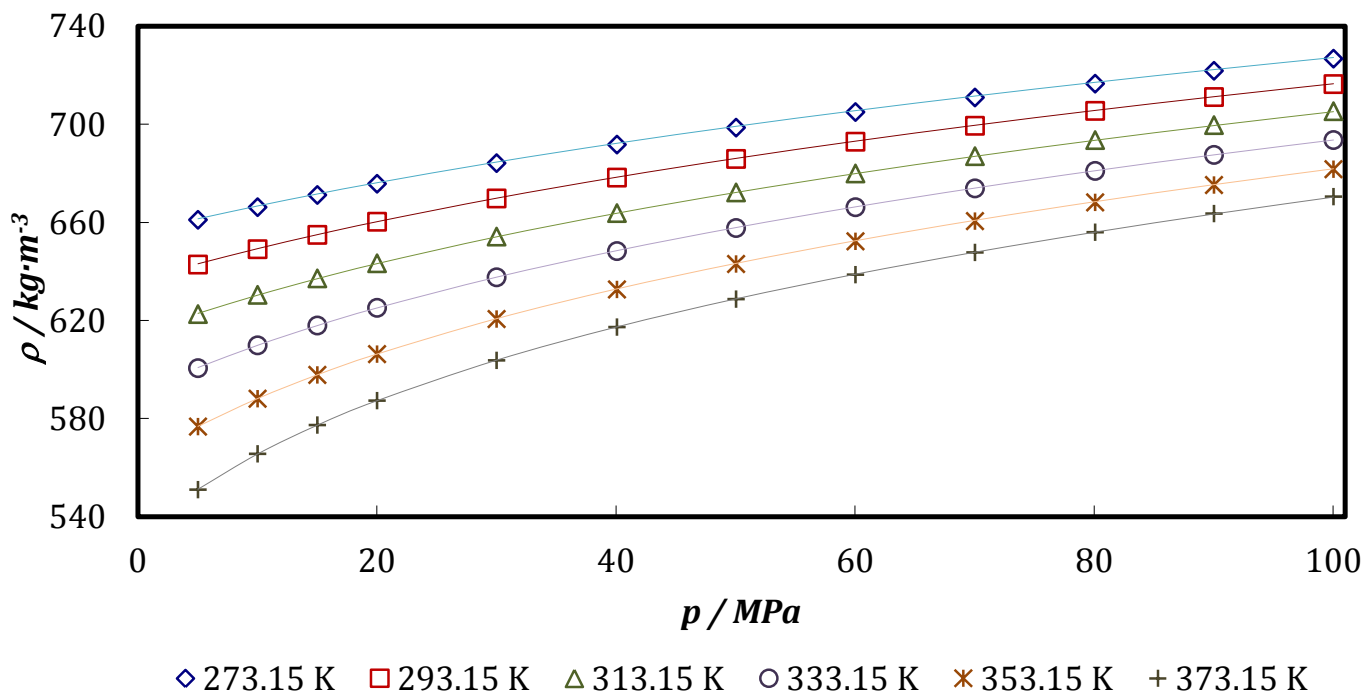

Figure 7.1. Experimental density values for mixture Pentane $+\mathrm{CO}_{2}$ at $\mathrm{x}_{\mathrm{CO} 2}=0.1004$ at different temperatures depending on pressure. The continuous lines represent adjusted with the modified Tammann-Tait equation and the symbols the experimental values.

The density results obtained for mixtures of Pentane $+\mathrm{CO}_{2}$ have been compared with those found in the literature [34], where the system was measured at $x_{C O 2}=0.3$ and $\mathrm{T}=$ $373.15 \mathrm{~K}$

Figure 7.2 shows the relative deviations obtained between the experimental data and those of the literature being the average deviation obtained $(A A D \%)$ of $1.2 \%$. As a result of these deviations, it can be concluded that our values are consistent with the literature.

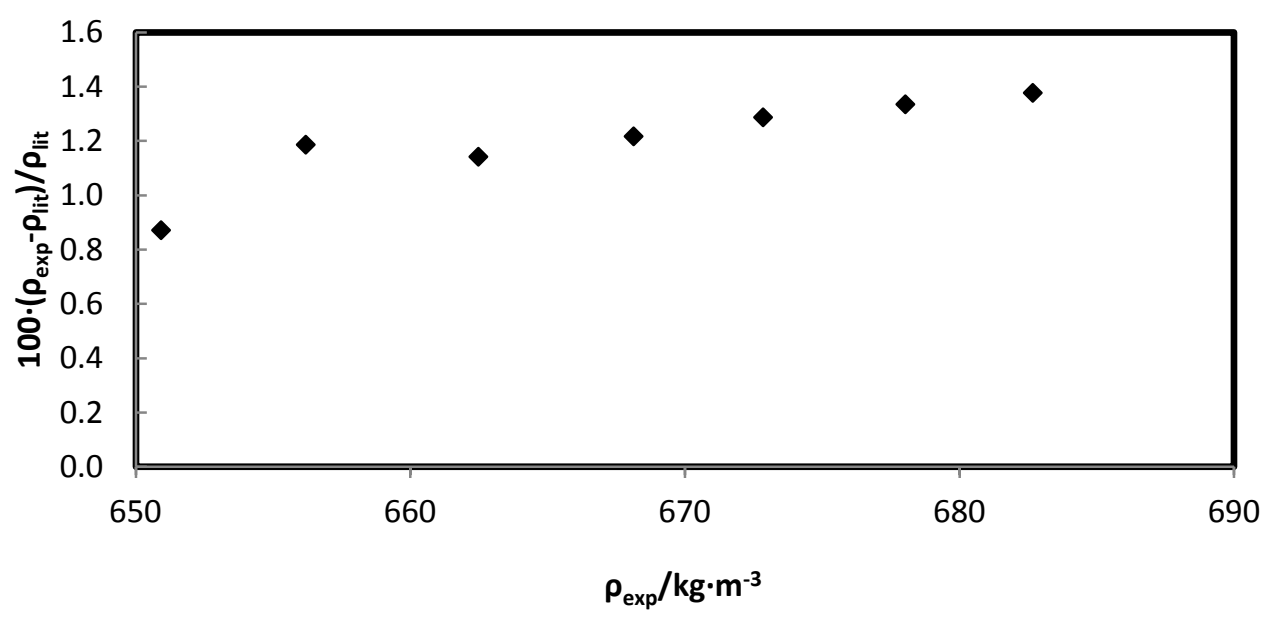

$\bullet \mathrm{xCO} 2=0.3$ Kiran et al.

Figure 7.2. Relative percentage deviation of experimental $\rho$ from Kiran et al. [34] literature data. 


\subsubsection{Discussion of experimental density results.}

In this work, the densities of two binary mixtures of hydrocarbons with $\mathrm{CO}_{2}$ have been determined, carried out in a temperature range from $273.15 \mathrm{~K}$ to $373.15 \mathrm{~K}$ and in a pressure range from $5 \mathrm{MPa}$ up to $100 \mathrm{MPa}$.

The adjustments with the modified Tammann-Tait equation were good considering the complexity of the mixtures. The maximum deviation $(M D \%)$ in all cases was less than $0.41 \%$ and the standard deviations $(\sigma)$ varied between $0.15 \mathrm{~kg} \cdot \mathrm{m}^{-3}$ and $1.37 \mathrm{~kg} \cdot \mathrm{m}^{-3}$, for all the mixtures studied with the exception of the mixtures to $x_{C O 2}=0.6$.

In section 7.5 of chapter 7 (Spanish version, page 169) Table 7.11 and Table 7.21 shows that for the system Pentane $(0.4)+\mathrm{CO}_{2}(0.6)$ the standard deviation is $2.30 \mathrm{~kg} \cdot \mathrm{m}^{-3}$ with a maximum percentage deviation of $0.97 \%$ and an average percentage deviation of $0.23 \%$. For the Hexane $(0.4)+\mathrm{CO}_{2}(0.6)$ a standard deviation of $2.43 \mathrm{~kg} \cdot \mathrm{m}^{-3}$ with a maximum percentage deviation of $1.16 \%$ and an average percentage deviation of $0.26 \%$ were obtained.

The experimental values show a trend of increasing density with pressure and decreasing density with increasing temperature. The density increases with the increase of the molar fraction of $\mathrm{CO}_{2}$ for both hydrocarbons $+\mathrm{CO}_{2}$ as shown in Figure 7.3 for the mixture pentane $+\mathrm{CO}_{2}$ at $313.15 \mathrm{~K}$.

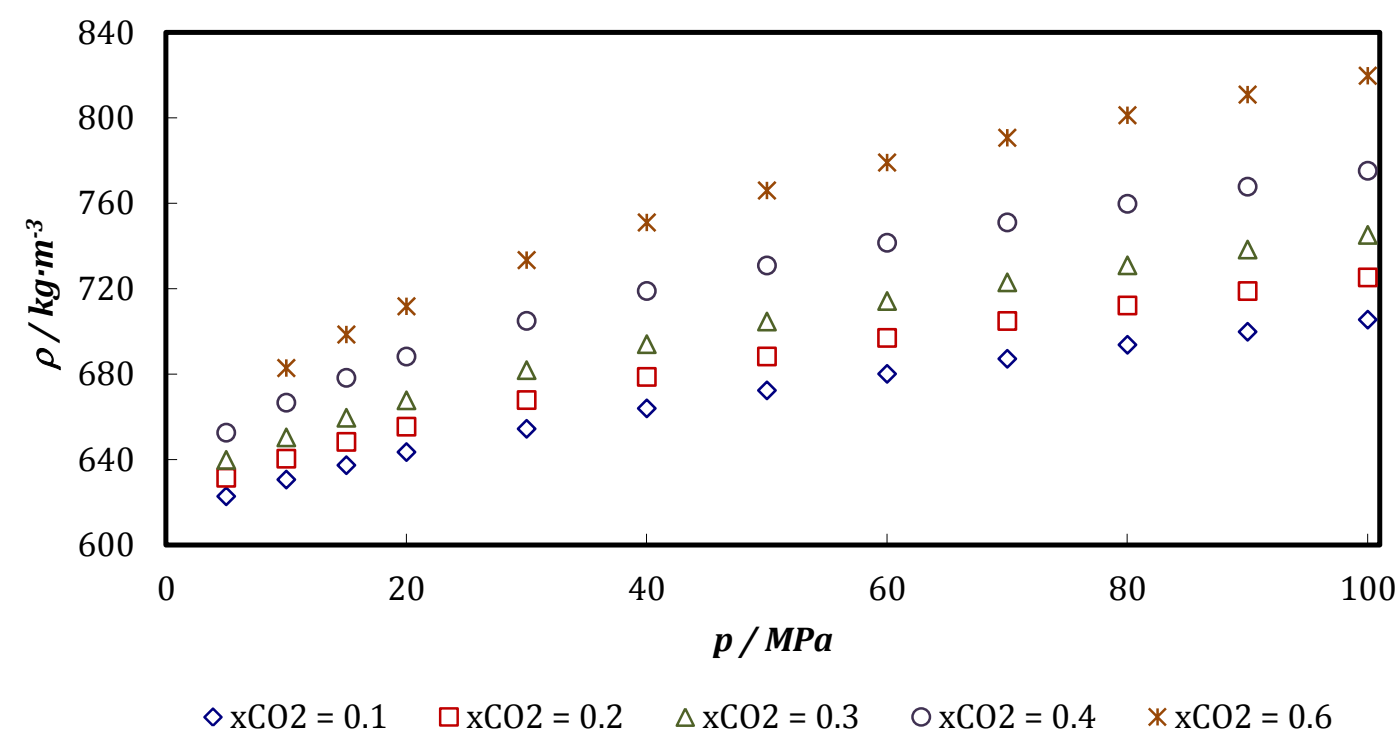

Figure 7.3. Experimental density values for the mixture Pentane $+\mathrm{CO}_{2}$ at $313.15 \mathrm{~K}$ and different $\mathrm{CO}_{2}$ concentrations depending on the pressure. 


\subsubsection{Experimental results of viscosity measurements.}

As an example of the experimental viscosity data obtained from of the mixtures studied, the system Pentane (0.9) $+\mathrm{CO}_{2}(0.1)$ is presented being the other results in section 7.7 (Spanish version, page 186). The experimental data are shown both as tables and graphs. As well as the results of the adjustment of the experimental data to the VFT model described in section 7.1.3. This adjustment was made for each composition of the mixtures studied, depending on temperature and pressure. In addition, the standard deviation of the adjustment $\sigma$ (equation 5.4) and maximum deviation MD (eq. 5.5).

\subsubsection{Mixture: Pentane $+\mathrm{CO}_{2}$.}

Table 7.3. Experimental viscosity data, $\eta(\mathrm{mPa} \cdot \mathrm{s})$, for the mixture Pentane $+\mathrm{CO}_{2}$ at $x_{\mathrm{CO} 2}=0.0950$.

\begin{tabular}{cccccc}
\hline \multicolumn{5}{c}{$\eta(m P a \cdot s)$} \\
\hline$p / \mathrm{MPa}$ & 293.15 & 313.15 & 333.15 & 353.15 & 373.15 \\
\hline 5 & 0.2049 & 0.1727 & 0.1436 & 0.1240 & 0.1111 \\
10 & 0.2169 & 0.1827 & 0.1539 & 0.1341 & 0.1197 \\
15 & 0.2282 & 0.1927 & 0.1637 & 0.1434 & 0.1296 \\
20 & 0.2400 & 0.2027 & 0.1740 & 0.1519 & 0.1370 \\
30 & 0.2610 & 0.2253 & 0.1931 & 0.1695 & 0.1539 \\
40 & 0.2835 & 0.2419 & 0.2110 & 0.1868 & 0.1691 \\
50 & 0.3040 & 0.2630 & 0.2292 & 0.2028 & 0.1840 \\
60 & 0.3249 & 0.2818 & 0.2479 & 0.2193 & 0.1982 \\
70 & 0.3469 & 0.3018 & 0.2652 & 0.2341 & 0.2120 \\
80 & 0.3686 & 0.3204 & 0.2818 & 0.2518 & 0.2273 \\
\hline
\end{tabular}

Table 7.4. Coefficients of the modified VFT equation, standard deviation $\sigma$ and maximum deviation $M D \%$ for the mixture Pentane $+\mathrm{CO}_{2}$ at $\mathrm{X}_{\mathrm{CO} 2}=0.0950, p_{\text {ref }}=5 \mathrm{MPa}$.

Parameters of the modified VFT equation

\begin{tabular}{ccccccccc}
\hline $\mathrm{A}$ & $\mathrm{B}$ & $\mathrm{C}$ & $\mathrm{E}_{0}$ & $\mathrm{E}_{1}$ & $\mathrm{E}_{2}$ & $\mathrm{~F}$ & $\begin{array}{c}\Sigma \\
\mathrm{mPa} \cdot \mathrm{s}\end{array}$ & $\begin{array}{c}\mathrm{MD} \\
\mathrm{mPa} \cdot \mathrm{s}\end{array}$ \\
$\mathrm{K}$ & $\mathrm{K}$ & $\mathrm{MPa}$ & $\mathrm{MPa} \cdot \mathrm{K}^{-1}$ & $\mathrm{MPa} \cdot \mathrm{K}^{-2}$ & & 0.92 & 1.91 \\
\hline 0.009 & 1051.69 & -38.17 & 6.27 & 0.56 & -0.001 & 0.84 & 0.92 \\
\hline
\end{tabular}




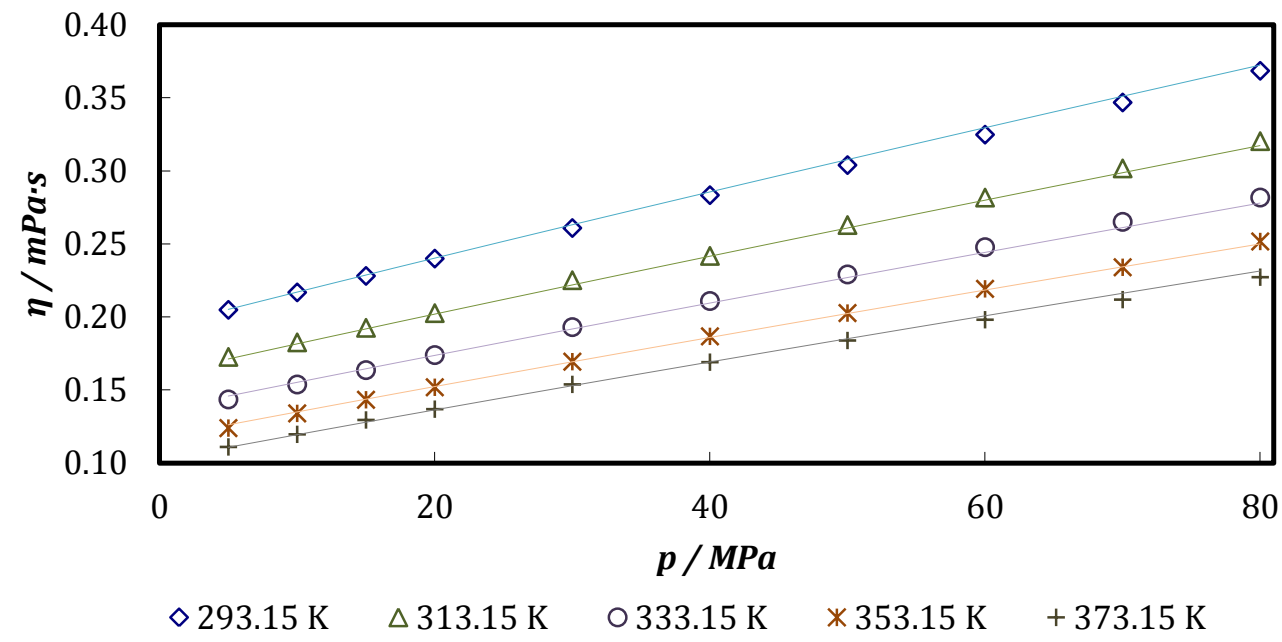

Figure 7.4. Experimental viscosity values for mixture Pentane $+\mathrm{CO}_{2}$ at $\mathrm{x}_{\mathrm{CO} 2}=0.0950$ at different temperatures as a function of the pressure. The continuous lines are adjusted values with the modified VFT equation, and the dots are experimental values.

For the calculation of pentane viscosities, the density data of the pure pentane reported in J. Zambrano's thesis [25] were used. For the mixtures were used the densities measured in this thesis.

The viscosity results for pentane were compared with those found in the literature [35], where the viscosity measurements at two temperatures $303.15 \mathrm{~K}$ and $353.15 \mathrm{~K}$ were found.

Figure 7.5 shows the relative deviations obtained between the experimental data and those of the literature. The average deviations obtained $(A A D \%)$ for the pentane were $1.2 \%$ and $2.6 \%$ at $303.15 \mathrm{~K}$ and $353.15 \mathrm{~K}$ respectively.

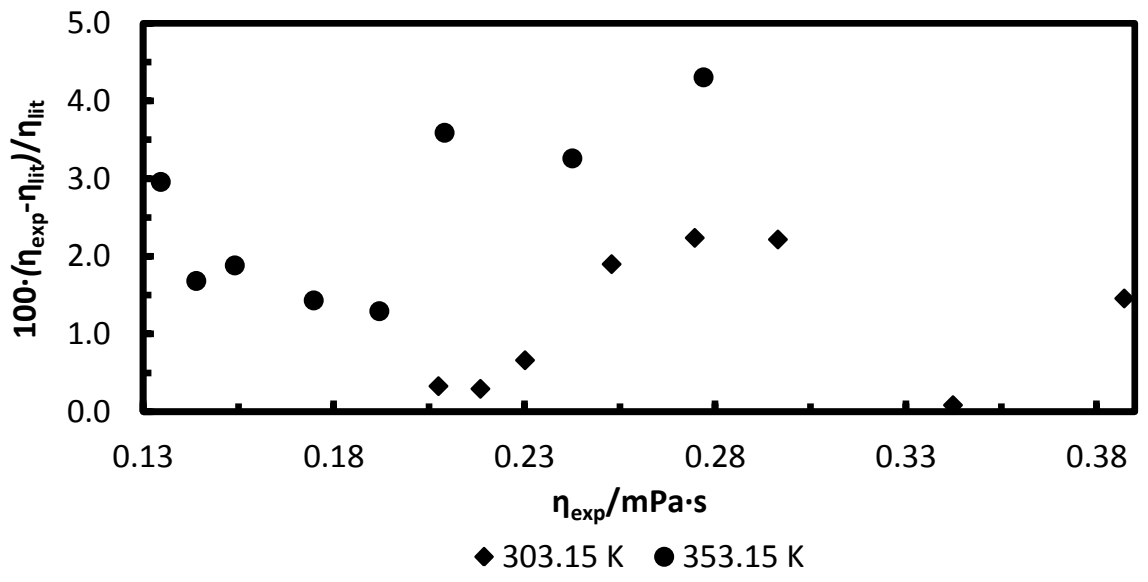

Figure 7.5. Percentage relative deviation of the experimental viscosities $\eta_{\text {exp }}$ with respect to the data of Audonnet and Pádua, $\eta_{\text {lit, }}$ [35]. 


\subsubsection{Discussion of experimental viscosity results.}

In this work, the viscosities of pentane and two binary hydrocarbon $+\mathrm{CO}_{2}$ mixtures have been determined, carried out in a temperature range of $293.15 \mathrm{~K}$ to $373.15 \mathrm{~K}$ and in a pressure range from $5 \mathrm{MPa}$ up to $100 \mathrm{MPa}$.

The adjustments with the modified VFT equation were good and within the uncertainty of viscosity measurement. The standard deviations $(\sigma)$ varied between $0.0020 \mathrm{mPa} \cdot \mathrm{s}$ and $0.0044 \mathrm{mPa} \cdot \mathrm{s}$.

The experimental values obtained show a trend of increasing viscosity with pressure and decreasing viscosity with increasing temperature. The viscosity decreases with increasing $\mathrm{CO}_{2}$ molar fraction for both hydrocarbons. In Figure 7.6 the effect of $\mathrm{CO}_{2}$ concentration on pentane viscosities is depicted at $313.15 \mathrm{~K}$.

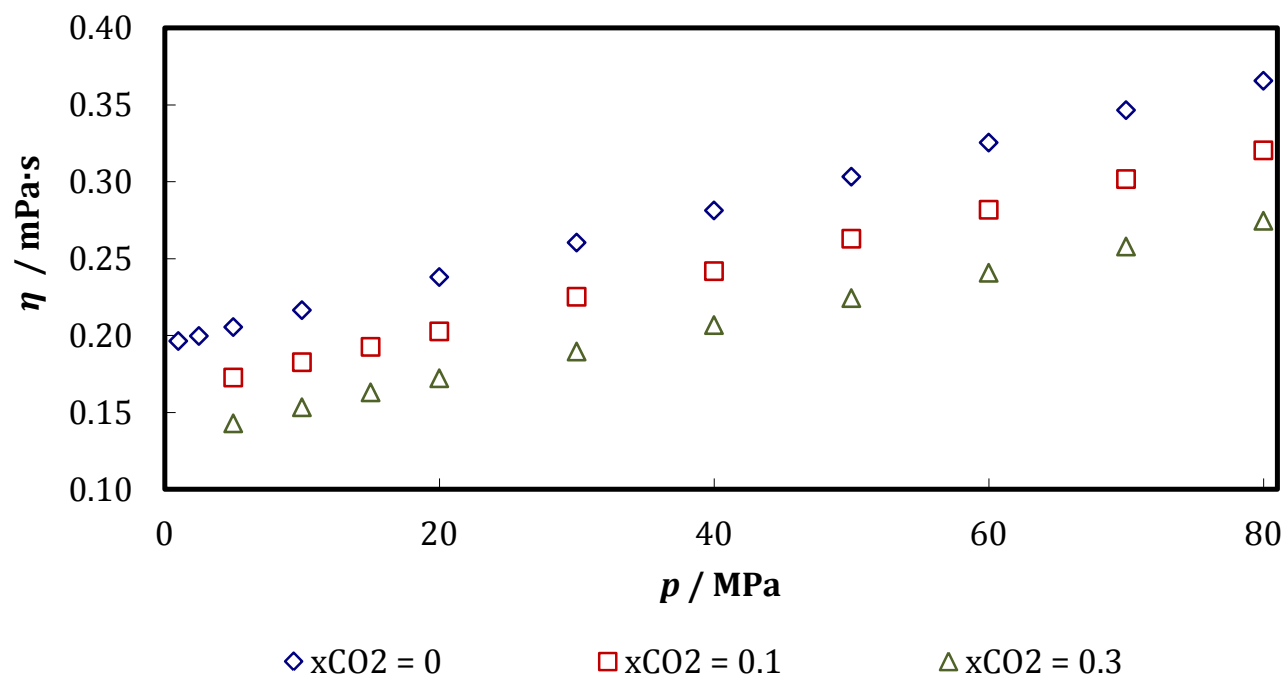

Figure 7.6. Experimental viscosity values for the mixture Pentane $+\mathrm{CO}_{2}$ at $313.15 \mathrm{~K}$ with different $\mathrm{CO}_{2}$ concentrations depending on the pressure.

Comparing different concentrations of $\mathrm{CO}_{2}$ at the same temperature, the decrease in viscosity with the increase in $\mathrm{CO}_{2}$ concentration is slightly higher at high pressures, in both pentane and hexane mixtures. 
Chapter 8

CONCLUSIONS 



\subsection{Conclusions.}

This thesis has been carried out in the laboratory TERMOCAL (Laboratory of Metrology and Calibration), of the Research Institute on Bioeconomy, BioEcoUva, of the University of Valladolid.

In this thesis, different experimental techniques have been developed to allow the thermodynamic characterization of mixtures of aqueous solutions of amines with $\mathrm{CO}_{2}$ through density, viscosity and solubility. In order to validate the modifications made to the equipment, the following mixtures have been studied: (a) hydrocarbons, alcohols and ketones, (b) hydrocarbons $+\mathrm{CO}_{2}$.

The goals achieved in the work have been the following:

1. VLE equipment has been modified for the measurement of isothermal equilibria at high temperatures and pressures, using a new system for the measurement of the pressure at high temperature. The equipment was validated with the determination of VLE of the binary system 2-Pentanol + Cyclohexane at $40{ }^{\circ} \mathrm{C}$ and the binary systems Cyclohexanone +2 -Heptanone and Cyclohexanone + Hexanal both temperatures from $40^{\circ} \mathrm{C}$ up to $120^{\circ} \mathrm{C}$.

2. The experimental results have been correlated with the models of Margules, Wilson, NRTL and UNIQUAC. The relative mean square deviation found with the Margules equation was better than $0.12 \%$ for the 2-Pentanol + Cyclohexane system, $0.14 \%$ for Cyclohexanone +2 -Heptanone and $0.30 \%$ for the Cyclohexanone + Hexanal system. In the case of the NRTL model it was less than $0.5 \%$ for all systems.

3. The densities of liquid-gas systems at high pressure and temperature have been determined. The binary systems formed by pentane and hexane hydrocarbons with $\mathrm{CO}_{2}$ have been characterized. The results were correlated with the modified Tammann-Tait equation, being the relative standard deviation less than $0.3 \%$ in both systems at $x_{\mathrm{CO} 2}=0.6$.

4. The viscosities of liquid-gas systems have been measured at high pressure and temperature for binary systems formed by pentane $+\mathrm{CO}_{2}$ and hexane $+\mathrm{CO}_{2}$. The results were adjusted with the modified Vogel-Fulcher-Tammann equation, being the relative standard deviation less than $1.0 \%$ in both systems at the highest composition $x_{\mathrm{CO} 2}=0.3$.

5. The densities of aqueous amine solutions with $\mathrm{CO}_{2}$ have been determined at high pressures and temperatures in wide ranges of temperature from $293 \mathrm{~K}$ up to $373 \mathrm{~K}$ and pressure from $0.1 \mathrm{MPa}$ up to $60 \mathrm{MPa}$. The characterized systems have been: (i) Monoethanolamine (MEA) with $\mathrm{CO}_{2}$; (ii) Dimethylaminoethanol (DMAE) with $\mathrm{CO}_{2}$; and (iii) Triethanolamine (TEA) with $\mathrm{CO}_{2}$, with amine mass fractions of 0.3 and loading $\mathrm{CO}_{2}$ up to $\alpha=1.5$. The results have been correlated with the modified Tammann-Tait equation, the relative standard deviation found was less than $0.05 \%$.

6. Isothermal equilibria at high temperatures and pressures of aqueous solutions of amines with $\mathrm{CO}_{2}$ have been characterized experimentally. From these results, the solubility of $\mathrm{CO}_{2}$ up to pressures of $6 \mathrm{MPa}$, and temperatures of $313 \mathrm{~K}$ and $353 \mathrm{~K}$ 
has been determined, for aqueous amines $30 \% \mathrm{w}$ and $\mathrm{CO}_{2}$ loading up and with load indexes of $\mathrm{CO}_{2}$ up to $\alpha=1.4$.

7. The measurement uncertainties have been calculated for all the thermodynamic properties studied in this doctoral thesis.

8. Finally, as a summary of this work, we have contributed with new experimental data that will improve the processes of $\mathrm{CO}_{2}$ capture with aqueous amine solutions, allowing the reduction of greenhouse gas emissions, and providing solutions for a sustainable future of our society. 
Chapter 9

\section{REFERENCES}





\subsection{References.}

[1] Y. Sanchez-Vicente, W. J. Tay, S. Z. Al Ghafri, J. P. M. Trusler. "Thermodynamics of carbon dioxide-hydrocarbon systems". Applied Energy. Vol. 220, (2018) 629-642.

[2] United Nations. Framework convention on climate change (FCCC). "Adoption of the Paris Agreement". FCCC/CP/2015/L.9/Rev.1.

[3] L. McInnes. " $\mathrm{CO}_{2}$, temperature, and sunspot activity since 1850". Available in: $<$ https://commons.wikimedia.org/wiki/File:Temp-sunspot-co2.svg> [accessed 19/08/2019].

[4] R. E. Gibbs and H. C. Van Ness. "Vapor-liquid equilibria from total-pressure measurements. A new apparatus". Industrial and Engineering Chemistry Fundamentals. Vol. 11, (1972) 410- 413.

[5] J. A. Barker. "Determination of activity coefficients from total pressure measurements". Australian Journal of Chemistry. Vol. 6, (1953) 207-210.

[6] C. Tsonopoulos. "An empirical correlation of second virial coefficients". AIChE Journal. Vol. 20, (1974) 263-272.

[7] G. M. Wilson. "Vapor-liquid equilibrium XI. A new expression for the excess free energy of mixing". J. Amer. Chem. Soc. Vol. 86, (1964) 127-130.

[8] H. Renon, J. M. Prausnitz. "Local compositions in thermodynamic excess functions for liquid mixtures". AIChE Journal. Vol. 14, (1968) 135-144.

[9] D. S. Abrams, J. M. Prausnitz. "Statistical thermodynamics of liquid mixtures: A new expression for the excess Gibbs energy of partly or completely miscible systems". AIChE Journal. Vol. 21, (1975) 116-128.

[10] A. Moreau, J. J. Segovia, J. Rubio, M. C. Martín. "Thermodynamics properties, VLE and $H^{E}$, of the systems 2-pentanol and cyclohexane or methylbenzene for contributing to the knowledge of new biofuels". Fluid Phase Equilibria. Vol. 409, (2016) 92-97.

[11] A. Moreau. "Caracterización termodinámica de combustibles ambientalmente sostenibles: determinación experimental del equilibrio líquido-vapor y entalpías de exceso de mezclas de interés para la formulación de biogasolinas". Universidad de Valladolid. Tesis doctoral (2014).

[12] B. Lagourette, C. Boned, H. Saint-Guirons, P. Xans, H. Zhou. "Densimeter calibration method versus temperature and pressure". Meas. Sci. Technol. Vol. 3, (1992) 699-703.

[13] W. Wagner, A. Pruss. "The IAPWS formulation 1995 for the thermodynamic properties of ordinary water substance for general and scientific use". J. Phys. Chem. Vol. 31, (2002) 387-535.

[14] JCGM, "Evaluation of measurement data-Guide to the expression of uncertainty in measurement". BIPM (2008). 
[15] E. W. Lemmon and R. Span. "Short fundamental equations of state for 20 industrial fluids". J. Chem. Eng. Data. Vol. 51, (2006) 785-850.

[16] G. Tammann. "Tammann compressibility equation for liquids". Z. Phys. Chem. Vol. 17, (1895) 620.

[17] M. J. P. Comuñas, A. Baylaucq, C. Boned, J. Fernández. "High-pressure measurements of the viscosity and density of two polyethers and two dialkyl carbonates at high pressures". Int. J. Thermophys. Vol. 22, (2001) 749-768.

[18] M. Sobrino, E. I. Concepción, A. Gómez-Hernández, M. C. Martín, J. J. Segovia. "Viscosity and density measurements of aqueous amines at high pressures: MDEA-water and MEAwater mixtures for $\mathrm{CO}_{2}$ capture". J. Chem. Thermodynamics. Vol. 98, (2016) 231-241.

[19] Q. Xu, G. Rochelle. "Total pressure and $\mathrm{CO}_{2}$ solubility at high temperature in aqueous amines". Energy Procedia. Vol. 4, (2011) 117-124.

[20] J. Zhang, P. S. Fennell, J. P. Trusler. "Density and viscosity of partially carbonated aqueous tertiary alkanolamine solutions at temperatures between (298.15 and 353.15) K". J. Chem. Eng. Data. Vol. 60, (2015) 2392-2399.

[21] J. Han, J. Jin, D. A. Eimer, M. C. Melaaen. " Density of water (1) + monoethanolamine (2) $+\mathrm{CO}_{2}$ (3) from (298.15 to 413.15) K and surface tension of water (1) + monoethanolamine (2) from (303.15 to 333.15) K". J. Chem. Eng. Data. Vol. 57, (2012) 1095-1103.

[22] T. G. Amundsen, L. E. Øi, D. A. Eimer. "Density and viscosity of monoethanolamine + water + carbon dioxide from (25 to 80) ${ }^{\circ} \mathrm{C}^{\prime \prime}$. J. Chem. Eng. Data. Vol. 54, (2009) 3096-3100.

[23] F. Y. Jou, A. E. Mather, F. D. Otto. "The solubility of $\mathrm{CO}_{2}$ in a 30 mass percent monoethanolamine solution". Can. J. Chem. Eng. Vol. 73, (1995) 140-147.

[24] D. Tong, J. P. M. Trusler, G. C. Maitland, J. Gibbins, P. S. Fennell. "Solubility of carbon dioxide in aqueous solution of monoethanolamine or 2-amino-2-methyl-1-propanol: Experimental measurements and modelling". International Journal of Greenhouse Gas Control. Vol. 6, (2012) 37-47.

[25] J.R. Zambrano, "Desarrollo de un viscosímetro de hilo vibrante para la caracterización termofísica a alta presión de nuevos biocombustibles". Universidad de Valladolid. Tesis Doctoral (2014).

[26] H. Vogel. "The law of the relation between the viscosity of liquids and the temperature". Phys. Z. Vol. 22, (1921) 645-646.

[27] G. S. Fulcher. "Analysis of recent measurements of the viscosity of glasses". J. Am. Ceram. Soc. Vol. 8, (1925) 339-355.

[28] G. Tammann, W. Hesse. "Die abhängigkeit der viscosität von der temperatur bie unterkühlten flüssigkeiten". Z. Anorg. Allg. Chem. Vol. 156, (1926) 245-257.

[29] C. A. Angell, W. Sichina. "Thermodynamics of the glass transition: Empirical aspects". Ann. N. Y. Acad. Sci. Vol. 279, (1976) 53-67. 
[30] C. A. Angell. "Thermodynamic aspects of the glass transition in liquids and plastic crystals". Pure Appl. Chem. Vol. 63, (1991) 1387-1392.

[31] G. Adam, J. H. Gibbs. "On the temperature dependence of cooperative relaxation properties in glass-forming liquids". J. Chem. Phys. Vol. 43, (1965) 139-146.

[32] X. Paredes, O. Fandiño, A. Pensado, M. J. P. Comuñas, J. Fernández. "Experimental density and viscosity measurements of di(2ethylhexyl)sebacate at high pressure". J. Chem. Thermodynamics (2012) 38-43.

[33] D. W. Marquardt. "An algorithm for least-squares estimation of nonlinear parameters". J. Soc. Indust. Appl. Math. Vol. 11 (2), (1963) 431-441.

[34] E. Kiran, H. Pöhler, Y. Xiong. "Volumetric properties of pentane + carbon dioxide at high pressures". J. Chem. Eng. Data. Vol. 41, (1996) 158-165.

[35] F. Audonnet, A. A. H. Pádua. "Simultaneous measurement of density and viscosity of npentane from 298 to $383 \mathrm{~K}$ and up to $100 \mathrm{MPa}$ using a vibrating-wire instrument". Fluid Phase Equilibria. Vol. 181, (2001) 147-161. 